\title{
Geochemical Characterization of Tea Leaves (Camellia sinensis) and Soils for Provenance Studies based on Compositional Data Analysis
}

\author{
Dissertation \\ zur Erlangung des mathematisch-naturwissenschaftlichen Doktorgrades \\ "Doctor rerum naturalium" \\ der Georg-August-Universität Göttingen \\ im Promotionsprogramm Geowissenschaften / Geographie \\ der Georg-August University School of Science (GAUSS)
}

vorgelegt von

Solveig Pospiech

aus Heidelberg

Göttingen 2018 
Betreuungsausschuss:

Prof. Hans Ruppert

Abteilung Sedimentologie und Umweltgeologie

Geowissenschaftliches Zentrum

Georg-August-Universität Göttingen

Dr. Raimon Tolosana-Delgado

Abteilung Modellierung und Bewertung

Helmholtz-Institut Freiberg für Ressourcentechnologie

Helmholtz-Zentrum Dresden-Rossendorf

Mitglieder der Prüfungskommission

Referent: Prof. Hans Ruppert

Korreferent: Dr. Raimon Tolosana-Delgado

weitere Mitglieder der Prüfungskommission:

Prof. Hilmar von Eynatten

Prof. Dirk Hölscher

Dr. Andreas Kronz

Prof. Katharina Pawlowski

Tag der mündlichen Prüfung: 17.09.2018 


\section{Contents}

\begin{tabular}{ll}
\hline 1. Introduction & 21
\end{tabular}

2. Tea 25

2.1. Tea Plant . . . . . . . . . . . . . . . . . . . . . . . . . . . 25

2.1.1. Camellia sinensis . . . . . . . . . . . . . . . . . 25

2.1.2. Aspects of Tea Physiology . . . . . . . . . . . . . . . 27

2.1.3. Tea lonome . . . . . . . . . . . . . . . . . . . . . 31

2.2. Tea Soils . . . . . . . . . . . . . . . . . . . . . . . . . . . . . 35

2.3. Tea Production . . . . . . . . . . . . . . . . . . . . . . 36

2.3.1. Fertilizers and Fungicides . . . . . . . . . . . . . . 36

2.3.2. Plucking . . . . . . . . . . . . . . . . . . . . 37

2.3.3. Tea Manufacturing . . . . . . . . . . . . . . . . . . 40

2.4. Summary . . . . . . . . . . . . . . . . . . . 42

3. Characterization of Sampling Areas 45

3.1. India . . . . . . . . . . . . . . . . . . . . . . 45

3.1.1. Darjeeling . . . . . . . . . . . . . . . . . . . . 45

3.1.2. Assam . . . . . . . . . . . . . . . . . . . 6 60

3.1.3. Nilgiris . . . . . . . . . . . . . . . . . . . . . . . . . 64

3.2. Brazil $\ldots \ldots \ldots \ldots 6 \ldots$

3.2.1. São Paulo . . . . . . . . . . . . . . . . . . . . . . . 666

3.2.2. Paraná . . . . . . . . . . . . . . . . . . . . 68 68

4. Description of Samples and Methods 71

4.1. Samples . . . . . . . . . . . . . . . . . . . . . . . . . . . . 71

4.1.1. Types and Groups of Samples . . . . . . . . . . . . . . . . . 71

4.1.2. Origin of Samples . . . . . . . . . . . . . . . . . . 73

4.1.3. Sampling . . . . . . . . . . . . . . . . . . . . . . 74

4.2. Sample Preparation and Analysis . . . . . . . . . . . . . . . . . . 76

4.2.1. Sample Preparation and Digestion . . . . . . . . . . . 76 


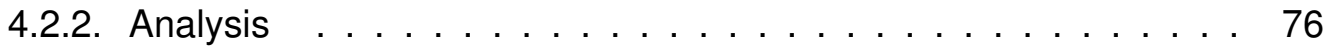

4.2.3. Limit of Detection, Precision and Accuary . . . . . . . . . 77

4.3. Data Handling . . . . . . . . . . . . . . . . . . . 80

4.3.1. Selection of Element Data . . . . . . . . . . . . . . 80

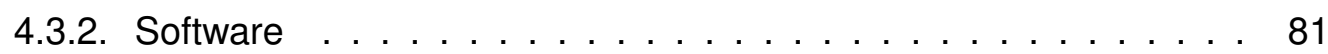

5. Mathematical Tools 83

5.1. Compositional Data and Log-ratio Transformations . . . . . . . . 83

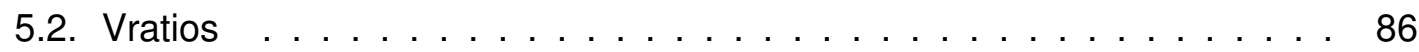

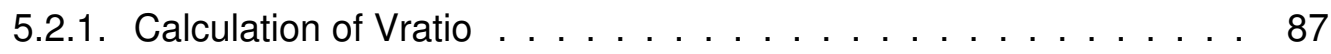

5.2.2. Comparison of Vratio Representations . . . . . . . . . . . 88

5.2.3. Summary . . . . . . . . . . . . . . . . . 91

5.3. Statistical Tools . . . . . . . . . . . . . . . . . . . . . . . . . 94

5.4. Visualization Tools . . . . . . . . . . . . . . . . . . . . . . . . 95

6. Correction for Adhering Particles 99

6.1. Method . . . . . . . . . . . . . . . . . . 99

6.1.1. Materials and Methods . . . . . . . . . . . . . . . . . 101

6.1.2. Theory of the Correction. . . . . . . . . . . . . . . . . 103

6.1.3. Calculation of the Correction . . . . . . . . . . . . . . 104

6.1.4. Results and Discussion of the Method . . . . . . . . . . . . 105

6.2. Results for Tea Plant Data . . . . . . . . . . . . . . . . . . 111

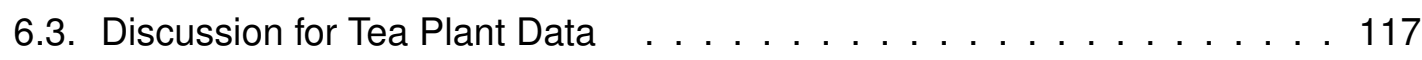

6.4. Summary . . . . . . . . . . . . . . . . . . . 119

7. Tonome and Elemental Distribution of Tea Leaf 121

7.1. Ionome of Shoot Tip . . . . . . . . . . . . . . . . . . 122

7.2. Ionome of Mature Leaf . . . . . . . . . . . . . . . . . . . . . 129

7.3. Tonome of Processed Tea . . . . . . . . . . . . . . . . . 133

7.4. Aspects of Shoot Tip/Mature Leaf Ratio in Tea Leaf Samples . . . . . . 140

7.4.1. Element Distribution between Shoot Tip and Mature Leaf . . . . . 140

7.4.2. Estimating the Average Age of Tea Leaf Samples . . . . . . . 148

7.5. Comparison between Shoot Tip and Processed Tea . . . . . . . . 151

7.5.1. Effects of Black Tea Manufacturing . . . . . . . . . . . 152

7.5.2. Comparability of Shoot Tips and Processed Teas within a Garden 156

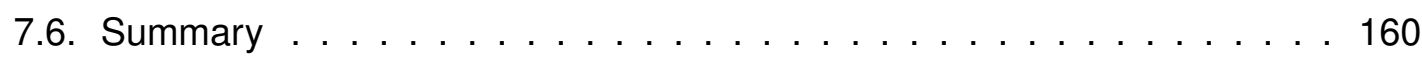


8. Influence of Soil on the Tea lonome 163

8.1. Soil . . . . . . . . . . . . . . . . . . . . . . . . . . 163

8.1.1. Geochemical Composition of Subsoils . . . . . . . . . . . . . . . . 164

8.1.2. Comparison between Topsoils and Subsoils . . . . . . . . . . . 166

8.2. Relation between Soil Geochemistry and Shoot Tip lonome . . . . . . . . 171

8.3. Summary . . . . . . . . . . . . . . . . . . . . . . . . . . . 179

$\begin{array}{lr}\text { 9. Provenance Analysis } & 181\end{array}$

9.1. Countries . . . . . . . . . . . . . . . . . . . . . . . . 183

9.2. India . . . . . . . . . . . . . . . . . . . . . . . . . . . . . . . 189

9.3. Darjeeling . . . . . . . . . . . . . . . . . . . . . . . 197

9.4. Summary . . . . . . . . . . . . . . . . . . . 206

\begin{tabular}{lr}
\hline 10. Conclusions and Outlook & 209
\end{tabular}

10.1. Conclusions . . . . . . . . . . . . . . . . . . . . . . . 209

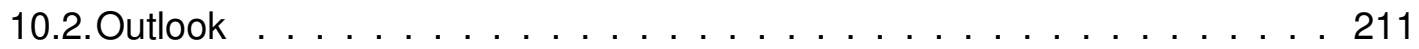

\begin{tabular}{ll}
\hline Bibliography & 211
\end{tabular}

\begin{tabular}{ll}
\hline Appendix & 231
\end{tabular}

A. Tea and Sampling Areas 233

\begin{tabular}{|l}
\hline B. Sample Preparation and Analysis \\
\hline
\end{tabular}

B.1. Digestion Protocol . . . . . . . . . . . . . . . . . . . . . . . . . . . . 239

B.2. Protocol for Tea Brewing . . . . . . . . . . . . . . . . . . . . . 239

B.3. Protocol for Plant Digestion . . . . . . . . . . . . . . . . . . . . 239

B.4. Protocol for Soil Digestion . . . . . . . . . . . . . . . . . . . . . . . . . 240

B.5. Calibration Solutions . . . . . . . . . . . . . . . . . . 241

B.6. Reference Samples . . . . . . . . . . . . . . . . . . . . . 250

\begin{tabular}{ll}
\hline C. Mathematical Tools & 265
\end{tabular}

D. Geochemical Characterization of Tea and Soil 267

\begin{tabular}{ll}
\hline E. Provenance Analysis & 271
\end{tabular}

\begin{tabular}{ll}
\hline Curriculum Vitae & 275
\end{tabular} 



\section{List of Figures}

2.1. Leaves of C. sinensis var. sinensis . . . . . . . . . . . . . . . . . . 26

2.2. Sketch of a shoot of C. sinensis . . . . . . . . . . . . . . . . . . . . . 29

2.3. Schematic figure of the basic elements of xylem and phloem . . . . . . 30

2.4. Compilation of published element concentrations of C. sinensis . . . . . . 34

2.5. Tea bushes - manual plucking . . . . . . . . . . . . . . . 38

2.6. Tea bushes - mechanical plucking . . . . . . . . . . . . . 38

2.7. Tea bushes manually plucked . . . . . . . . . . . . . . . . . . 39

2.8. Tea bushes mechanically plucked . . . . . . . . . . . . . . . . . . 39

3.1. Map of tea estates in Darjeeling . . . . . . . . . . . . . . . . . . 47

3.2. Geological map of the eastern Himalaya . . . . . . . . . . . . . . . . . . 48

3.3. Geological map of the Darjeeling-Sikkim Himalaya . . . . . . . . . . . . 50

3.4. Daling Group, outcrop . . . . . . . . . . . . . . . . . . . . . . . . 51

3.5. Daling Group, outcrop close up . . . . . . . . . . . . . . . . . . . . . 51

3.6. MCTZ, Grt-Mu-zone, outcrop . . . . . . . . . . . . . . . . . . . 52

3.7. MCTZ, Grt-Mu-zone, schist . . . . . . . . . . . . . . . . . . . . . . . 52

3.8. MCTZ, St-zone, outcrop . . . . . . . . . . . . . . . . . . 53

3.9. MCTZ, St-zone, hand specimen . . . . . . . . . . . . . . 53

3.10. MCTZ, Ky-zone, outcrop . . . . . . . . . . . . . . . . . . . . 54

3.11.MCTZ, Ky-zone, hand specimen . . . . . . . . . . . . . . . . . 54

3.12. MCTZ Kangchenjunga Gneiss, outcrop . . . . . . . . . . . . . . . . . 55

3.13.MCTZ Kangchenjunga Gneiss, outcrop . . . . . . . . . . . . . . . . 55

3.14. Geological map of Darjeeling . . . . . . . . . . . . . . . . 57

3.15.Soil map of Darjeeling . . . . . . . . . . . . . . . . . . . . . . 59

3.16. Map of tea estates in Assam . . . . . . . . . . . . . . . . 62

3.17.Soil profile, Assam . . . . . . . . . . . . . . . . . . . 63

3.18. Tea plantation in Assam showing grain size variability of soils . . . . . . 63

3.19. Geological section of Ribeira Depression . . . . . . . . . . . . . 67 
5.1. Biplots of the PCA of vratios . . . . . . . . . . . . . . 93

6.1. Correction for AP, histogram of field crop samples . . . . . . . . . . . 107

6.2. Correction for AP, distribution of corrected/original data of field crop samples 109

6.3. Coviariance biplots of original and corrected data of field crop samples. . 110

6.4. Correction for AP, histogram of tea leaf samples . . . . . . . . . . . . 111

6.5. Correction for AP, distribution of corrected/original data of tea leaf samples 114

6.6. Coviariance biplots of original and corrected data of tea leaf samples. . . 115

6.7. Dendrograms (HCA) of elements of original and corrected leaf data . . . 116

6.8. Correction for AP, heatmap of $p$-values . . . . . . . . . . . . . . 120

7.1. Element concentrations of shoot tip ionome . . . . . . . . . . . . . . . 125

7.2. Comparison of shoot tip ionomes of three samples per location . . . . . 127

7.3. Element concentrations of mature leaf ionome . . . . . . . . . . . 131

7.4. Element concentrations of processed tea ionome . . . . . . . . . . . . . 136

7.5. Comparison of processed tea ionomes of samples from seven gardens . 138

7.6. Comparing shoot tip and mature leaf, heatmap of $p$-values . . . . . . . 141

7.7. Comparing shoot tip and mature leaf, clr-vratios . . . . . . . . . . . . . . 147

7.8. Estimating the leaf age by [Cu K| Al Ca Mn] balance. . . . . . . . . . . . 148

7.9. Sorting machine . . . . . . . . . . . . . . . . . . . . 150

7.10. Sorting machine, output of sieve No. 18 . . . . . . . . . . . . 150

7.11.Sorting machine, output of sieve No. 14 . . . . . . . . . . . . . . 151

7.12. Sorting machine, output of sieve No. 10 . . . . . . . . . . . . . . 151

7.13. Comparing shoot tip and processed tea, heatmap of p-values . . . . . . 153

7.14. Ir-ANOVA of shoot tip and black processed tea . . . . . . . . . . . . . . 154

7.15. Comparing shoot tip with corresponding processed teas, biplot of clr-vratios 157

7.16. Comparing shoot tip with corresponding processed teas, heatmap p-values 158

7.17.Comparing shoot tip with corresponding processed teas, biplot of alr-vratios 159

8.1. Element concentrations of subsoils . . . . . . . . . . . . . . . . . . 168

8.2. Comparing topsoil and subsoil . . . . . . . . . . . . . . . . . . . 169

8.3. Comparing shoot tip and subsoil, simple vratio . . . . . . . . . . . . . 172

8.4. IIr-ANOVA of shoot tip and subsoil . . . . . . . . . . . . . . . . 173

8.5. IIr-ANOVA of shoot tip and subsoil of Darjeeling . . . . . . . . . . . . . . . 174

8.6. IIr-ANOVA of shoot tip and subsoil of all regions except Darjeeling . . . . 175

8.7. Comparing shoot tip and corresponding topsoil, heatmap of p-values . . . 176 
8.8. Comparing shoot tip and corresponding subsoil, heatmap of p-values . . 177

9.1. LDA discriminating tea ionome by countries . . . . . . . . . . . . . . . 184

9.2. Clr-biplot of tea ionomes for standard subcomposition with $\mathrm{Ni}, \mathrm{Mg}, \mathrm{Fe}$ and Lil 186

9.3. LDA discriminating tea ionome by countries, with $\mathrm{K}, \mathrm{P}$ and $\mathrm{Li}$. . . . . . 188

9.4. LDA discriminating tea ionome by regions of India . . . . . . . . . . . 189

9.5. LDA discriminating tea ionome by regions of India, including $\mathrm{K}$ and $\mathrm{Mg}$. . 192

9.6. Clr-biplot of soils from Darjeeling and Assam . . . . . . . . . . . . . . 195

9.7. LDA discriminating tea ionome between Darjeeling and Assam with Al, Mgand T . . . . . . . . . . . . . . . . . 196

9.8. Clr-biplot of soils from Darjeeling . . . . . . . . . . . . . . . . . . . 199

9.9. LDA discriminating tea ionome between metamorphic zones in Darjeeling with $\mathrm{K}$ and $\mathrm{Ca} \ldots \ldots \ldots \ldots$. . . . . . . . . . . . . . . . . . . . . . . . . .

9.10.LD1 of $\mathrm{Ca}, \mathrm{K}, \mathrm{Mn}, \mathrm{Sr}$ and $\mathrm{Zn}$ versus elevation . . . . . . . . . . . . . . . 202

9.11. Geological map of Darjeeling with tea sampling locations . . . . . . . . . 205

A.1. MCTZ, Grt-Mu-zone, saprolitic schist . . . . . . . . . . . . . . . . . . . 233

A.2. MCTZ, Grt-Mu-zone, hand specimen . . . . . . . . . . . . . . . . . 233

A.3. MCTZ, St-zone, gneiss with crenulated matrix . . . . . . . . . . . . . . . 234

A.4. MCTZ, St-zone, outcrop . . . . . . . . . . . . . . . . . . . . . 234

A.5. MCTZ, Ky-zone, gneiss with folded texture . . . . . . . . . . . . . . 234

A.6. MCTZ, Ky-zone, . . . . . . . . . . . . . . . . . . . . . . . . . 234

A.7. MCTZ Kangchenjunga Gneiss, weathered texture . . . . . . . . . . . 237

A.8. MCTZ Kangchenjunga Gneiss, fist-sized garnet . . . . . . . . . . . . 237

A.9. Location map of soil samples in Assam, Karak et al. (2015a) . . . . . . 237

A.10.Overview map of sampling region in Brazil . . . . . . . . . . . . . . . . 238

C.1. Biplots of the PCA of 'ar' and 'alr' vratios . . . . . . . . . . . . . . 266

D.1. IIr-ANOVA of shoot tip and black processed tea, including Sn and Al . . . 267

D.2. Vratios of subsoil/upper crust . . . . . . . . . . . . . . . . . . 270

E.1. Histogram of weighted discrimination of origin by regions of India with Mg,

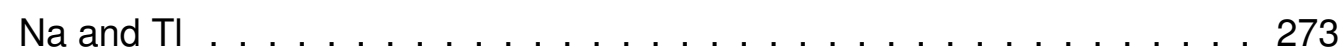

E.2. LDA discriminating tea ionome between metamorphic zones in Darjeeling

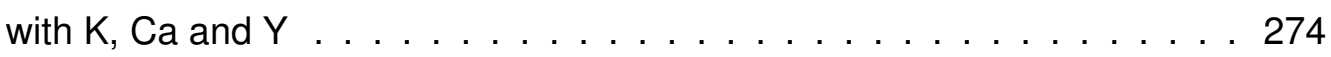





\section{List of Tables}

4.1. Overview of samples in respect of type, group and subgroups . . . . . . . 72

4.2. Overview of samples per type, country and region . . . . . . . . . . . . 74

4.3. Averaged accuracy and precision of the plant and rock standards . . . . . 79

5.1. Median of vratios of selected elements . . . . . . . . . . . . . . . . . 92

5.2. Variance of vratios of selected elements. . . . . . . . . . . . . . . 92

6.1. List of plants used for correction for adhering particles . . . . . . . . . . 101

6.2. Number of tea samples plotted in Fig. 6.6 . . . . . . . . . . . . . . . . 112

7.1. Element concentrations of shoot tip ionome . . . . . . . . . . . . . . 126

7.2. Standard deviation of clr-values of shoot tip per garden . . . . . . . . . 128

7.3. Element concentrations of mature leaf ionome . . . . . . . . . . . . . 132

7.4. Element concentrations of processed tea ionome . . . . . . . . . . . . 137

7.5. Standard deviation of clr-values of processed tea per garden . . . . . . 139

7.6. Average of $p$-values of comparison between mature leaf and shoot tip . . 144

7.7. Element concentration of $\mathrm{Cr}, \mathrm{Pb}, \mathrm{Sb}$ and $\mathrm{Zr}$ for processed tea and shoot tip. 152

8.1. Sum of nutrient concentrations in subsoils . . . . . . . . . . . . . . 165

8.2. Soil concentrations . . . . . . . . . . . . . . . . . . . . . . . . . . 170

9.1. Clr-scalings of LDA and misclassifications of tea ionome discrimination by

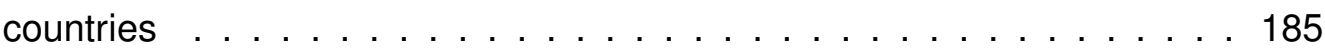

9.2. Clr-scalings of LDA and misclassifications of tea ionome discrimination by

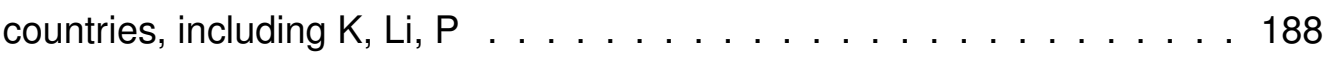

9.3. Clr-scalings of LDA and misclassifications of tea ionome discrimination by Indian regions . . . . . . . . . . . . . . . . . . . . . 190

9.4. Clr-scalings of LDA and misclassifications of tea ionome discrimination by Indian regions, including $\mathrm{K}$ and $\mathrm{Mg}$. . . . . . . . . . . . . . . . . . 191

9.5. Clr-scalings of LDA and misclassifications of tea ionome discrimination for Assam and Darjeeling, including Al, Tl and Mg . . . . . . . . . . . 196 
9.6. Number of tea samples by geological source rocks in Darjeeling . . . . 198

9.7. Clr-scalings of LDA and misclassifications of tea ionome discrimination for metamorphic zones in Darjeeling, including $\mathrm{K}$ and $\mathrm{Y}$. . . . . . . . . . . 201

A.1. List of tea estates in Darjeeling . . . . . . . . . . . . . . . . . . 235

A.2. List of leave sizes of orthodox black tea . . . . . . . . . . . . . . . . . 236

B.1. Compostion of the Internal Standard . . . . . . . . . . . . . . . . . . 241

B.2. Concentration of calibration solution of ICP-MS . . . . . . . . . . . . . . . 242

B.3. Concentration of old calibration solution of ICP-OES . . . . . . . . . . . 243

B.4. Concentration of new calibration solution of ICP-OES . . . . . . . . . . 244

B.5. Concentration of new maximal calibration solution of ICP-OES . . . . . . 245

B.6. Selected element datum . . . . . . . . . . . . . . . . . . . . 246

B.7. Selected element datum, continued from table $\mid$ B.6 . . . . . . . . . . . 247

B.8. Selected element datum, continued from table|B.6 . . . . . . . . . . . 248

B.9. Selected element datum, continued from table B.6 . . . . . . . . . . . 249

B.10.Certified values of the international reference materials $\ldots . . . .251$

B.11.Results of references samples . . . . . . . . . . . . . . . . . . 252

B.12.Reference samples . . . . . . . . . . . . . . . . . . . . . 253

B.13.Reference samples, continued from table|B.11 . . . . . . . . . . . . . 254

B.14.Reference samples, continued from table|B.11 . . . . . . . . . . . . 255

B.15.Accuracy of references samples . . . . . . . . . . . . . . . . 256

B.16.Accuracy, continued from table $\mathrm{B} .15$. . . . . . . . . . . . . 257

B.17.Accuracy, continued from table B.15 . . . . . . . . . . . . . 258

B.18.Accuracy, continued from table|B.15 . . . . . . . . . . . . . . . 259

B.19.Precision of references samples . . . . . . . . . . . . . . . . 260

B.20.Precision, continued from table|B.19 . . . . . . . . . . . . . 261

B.21.Precision, continued from table B.19 . . . . . . . . . . . . . . . 262

B.22.Precision, continued from table $\mid$ B.19 . . . . . . . . . . . . . . 263

D.1. Overview of element lists . . . . . . . . . . . . . . . . . . . 268

D.2. Overview of element lists for nutrients . . . . . . . . . . . . . . . . . . . . 269

E.1. List of elements for suitability for provenance analysis. . . . . . . . . . . . 272

E.2. Clr-scalings of weighted LDA and misclassifications of tea ionome discrimination for Assam and Darjeeling, including Al, $\mathrm{Tl}$ and $\mathrm{Mg}$. . . . . . . 273 
List of Tables

E.3. Clr-scalings of LDA and misclassifications of tea ionome discrimination for metamorphic zones in Darjeeling, including $\mathrm{K}, \mathrm{Ca}$ and $\mathrm{Y}$. . . . . . . . . 274 



\begin{abstract}
Provenance studies on tea (Camellia sinensis) are an important tool to reconstruct the origin of tea products. This thesis explores the potential of using solely the ionome (main and trace element concentrations) of tea shoot tips for provenance studies. The emphasize of the thesis is to find element subcompositions which are robust in respect to the various parameters of tea cultivation and production, such as the area, their soils, soil fertilizer and applications of foliar sprays, tea cultivars, plucking/harvesting techniques, manufacturing or leaf grade of the processed tea. For specific discrimination tasks these robust subcompositions can be combined with element subcompositions which are sensitive to one or several tea cultivation and production parameters. The data set consists of ca. 300 leaf and processed tea samples and ca. 130 soil samples. The sampling areas are located in Darjeeling, Assam and Nilgiris in India, in Paraná and São Paulo in Brazil and in Uji and Shizuoka in Japan. All samples, plants and soils, had been treated with four acid digestion methods with $\mathrm{HNO}_{3}, \mathrm{HCl}, \mathrm{HClO}_{4}$ and $\mathrm{HF}$ to achieve a total dissolution. The sample solutions had been analyzed by Inductively Coupled Plasma Mass Spectrometry (ICP-MS) and Inductively Coupled Plasma Optical Emission Spectroscopy (ICP-OES). The element concentrations of the leaf and processed tea samples had been corrected for adhering (soil) particles. Hence, all statistical analysis are based on the corrected concentrations values of $\mathrm{Al}, \mathrm{Ba}, \mathrm{Ca}, \mathrm{Cd}, \mathrm{Co}, \mathrm{Cr}, \mathrm{Cs}, \mathrm{Cu}, \mathrm{Fe}, \mathrm{K}, \mathrm{La}, \mathrm{Li}$, $\mathrm{Mg}, \mathrm{Mn}, \mathrm{Mo}, \mathrm{Na}, \mathrm{Ni}, \mathrm{P}, \mathrm{Pb}, \mathrm{Rb}, \mathrm{S}, \mathrm{Sb}, \mathrm{Sc}, \mathrm{Sr}, \mathrm{Tl}, \mathrm{Y}$ and $\mathrm{Zn}$. The element concentrations are converted into log-ratios by additive, centered or isometric log-ratio transformations prior to statistical analysis to avoid spurious correlations and to enhance the signal-noise ratio for e.g. the trace elements concentrations. The comparison of the geochemical composition of topsoils, subsoils, mature leaves, shoot tips and processed tea samples is used to establish for each element a qualitative index of robustness with respect to cultivation and production parameters. The elements with a high robustness are considered as very suitable for a provenance analysis without further knowledge about the tea samples. The thesis exemplary shows that with already small element subcompositions a good discrimination by geographical origin is possible if the elements are chosen in terms of their suitability for provenance studies of tea including their sensitivity in respect to specific parameters. The geological source rocks of the tea plantations is one of the major factors for discrimination of tea origin.
\end{abstract}





\section{Acknowledments}

Not even one of these chapters would have been possible without the help, encouragement, knowledge and support of literally hundreds of people. I experienced amazing, generous, idealistic and surprising humans since I started 'working in tea'. I would like to mention all, but limited space forces me to present only few by name, and sadly, I have to omit names of persons who also contributed to this work. Still, I feel that these following pages are one of most important parts of this thesis.

I thank my Doktorvater Hans Ruppert for being my Doktorvater, in fact for being a Doktorvater in its most positive and valuable sense. In this atmosphere where intense scientific discussions go together with mutual respect and personal warmth the creativity for developing new ideas is at home and made it so much easier for me to find the courage to embark on and go on with this project off the main roads of research. I am also very thankful for his active support during the field campaigns in India and Brazil.

I thank Raimon Tolosana-Delgado, my co-mentor, not only for introducing me to the concept of compositional data analysis, $\mathrm{R}$ and statistics, or reading, correcting and commenting hundreds of manuscript pages. I am especially thankful that he became my mentor despite all - at that point already emerged - obstacles, because I could not have wished for any better, more reliable, inspiring and humorous mentor.

I thank the Stiftung der deutschen Wirtschaft for granting me the PhD-scholarship. Although the financial support was crucial for the project the educational and academic support became important pieces of experiences which helped me on the non-scientific parts of conducting a doctoral thesis.

Without the backup of my whole family nothing would have been possible at all. My husband Stephan, my parents, my sisters and my late grandparents have their input in between every line, lovingly, emotionally, supportingly, encouragingly, helpfully, scientifically, inspiring. There are no words to express the incredible blessing to have this family.

This thesis would certainly have been a different one without my friends and colleagues in our department. I am very thankful to Wiebke Fahlbusch for taking up the endeavor to work together on our data handling, which means coding, coding, coding and enduring my crazy moments when I felt I should really restructure the whole code. All this work of building up a data bank of plant and soil analysis has only been fruitful thanks to the good and heartily support, both scientifically and by providing data sets, from Benedikt Sauer and Tino Pasold. I thank Klaus Simon for the ICP-MS analysis and 
Volker Karius, Judith Dunkle-Nagy and Irina Ottenbacher for assisting with preparing solutions and analysis at the ICP-OES. Ines Ringel was indispensable - for finding the right trail in the jungle of forms but even more for the feeling to be in the right spot in this institute. For the latter, the credit goes also to the whole department with its appreciative interaction between all.

The sampling takes up only few lines in this document but it was - in my eyes - the key to this thesis. I am very thankful to all persons who made my sampling campaigns not simply possible, but an eye-opening, instructive experience.

In India I was strongly supported by the Tea Board of India, namely Dr. Biswajit Bera, who opened the doors wide for this international cooperation. Among many other things I owe this trustful and personal relation a very valuable and complete data set which was sampled 2013 in Darjeeling in cooperation with the Darjeeling Tea Research and Development Centre by Rajesh Kumar Chauhan and the field assistant Mazhar Ibrahimi. Without Dr. Edward Sayed Kabir the whole project would not have started in the first place, because without knowing me he helped me getting started with knowledge, contacts and infrastructure. Ashok Lohia intensely supported me since our first meeting in 2011 and made a great contribution to sampling campaign in Darjeeling through welcoming me and letting me stay in all gardens of the Chamong Group. For my first samples I thank the Goodrike Company for permitting me to visit all gardens of this group. I thank all managers, section managers, soil workers, pluckers and drivers of the visited tea gardens. They took their time, provided cars, jeeps, material, joined to help with their knowledge, prepared awesome food, welcomed me in their houses and work every day really hard to produce this amazing Darjeeling tea. I thank United Planter's Association of Southern India for supporting me during the sampling campaign in Nilgiris by scientific discussion, providing contacts and laboratory infrastructure.

In Brazil the laboratory infrastructure was provided by Miguel Basei, Departamento de Mineralogia e Geotectônica, and Elke Cardoso, Department of Soil Science, from the Universidade de São Paulo (USP). I received a warm welcoming and lot of help from the family Amaya of the Amaya Tea in Registro, who would take their time to turn over tea bushes for studying the root system. I thank the company Yamamotoyama do Brasil to let me sample both of their tea estates and visit their factories.

In Japan the sampling in Shizuoka would have been so much harder without Masato Kikutani, who took me around in all Shizouka area, organized, translated, helped and got the best food in Japan without knowing me before. I also thank Obubu Tea Garden in Uji for their time, interns and cars to help sampling. 
Of course, the field work could not be done in the limited times of the stays abroad with a single person. I warmly thank everyone who assisted me in the fields. Special thanks go to Beatrix Heller, who provided a house in São Paulo and did a great job with translating and plucking in the Brazilian tea gardens. In India, she strongly supported me to manage this extensive sampling campaign in 2015. I am also thankful to Mr. Heller for arranging the visit to and sampling opportunities in Arunachal Pradesh, India.

Several sample sets had been provided by tea companies. I would like to thank Fa. Lebensbaum, Fa. TeeGeschwendner and Fa. Teekampagne for sending samples directly into my office. I had interesting, personal and sometimes very open talks and discussions about the tea trade, which helped me to connect my scientific work to the practical aspects of tea production and trade. I would like to thank Katrin Gassert and Thomas Räuchle from Fa. Teekampagne, Wolfgang Wilhelm from Fa. Kloth und Köhnken, Rajiv Gupta from Fa. Chamong and Swaraj Kumar (Rajah) Banerjee Fa. Makaibari. Ajay Kichlu and Sujoy Sengupta from Fa. Chamong did not only answer all my questions, but helped me in all, logistics, contacts, friendship.

At several points, I got important impulses from scientific discussions with friends and colleagues. I thank Julian Dehne for the basic understanding what is possible with computers and what is not, Katharina Pawlowski for intensive discussions and major and decades long input of plant sciences, Jens Walter for discussions about provenance studies and prove reading of the geological parts of this thesis, Annette Süssenberger for the now decade long inspiration and the paper we finally write together, Klaus Wemmer for his back up in all means, Susanne Ruppert for her art of opening the hearts (and doors), Ingo Hache for walks and talks, Melanie Brandmeier for sharing her extensive GIS-knowledge and all our tours, Karak Tanmoy and Simon Blotevogel.

Of course I can not mention all my friends, but some had made very important contribution to this thesis. I deeply thank Hrishikesh and his parents for giving me a second family in Kolkata and the incredible selfless help. I warmly thank Vandana Lohia for giving me 'my' home in Kolkata, which was also my 'base' for field work, and letting me so heartily into her family. I thank Gourab Sen and Ananya who made me live among friends in Kolkata. And last but not least, I thank Jan and Özlem for putting in the kindest way a deadline to this thesis. 



\section{Introduction}

The fascination for provenance analysis on tea comes from the experience that a simple question like "Can we determine the origin of the tea we just bought from the supermarket shelf?" leads to a broad, challenging and interdisciplinary field of research. After compiling this thesis, dedicated only to this question, the answer will still not be an unconditional "Yes, we can!", but rather "We got long strides closer.".

Why is it so challenging to determine the geographical origin of tea? First of all, tea is a plant and its interaction with the environment is influenced by soil, climate, slope of the field, neighboring plants, pest attacks, age, cultivar, treatment, etc. Secondly, tea is produced nearly all over Asia, in large parts of Africa and small parts of South America, and also in minor quantities in Australia, North America and Europe. Thirdly, there are many different ways how to manufacture tea, and each type of manufacturing will give its own imprint on the product tea. And last but not least, tea is traded worldwide. It is sorted, blended, sold and resold, until it is finally packed, labeled and put onto the shelf of the supermarket. Considering this complicated, multi-variate setting, it might not even be possible to determine the one tea field in the world where the tea was harvested.

Nevertheless, there is a strong motivation to work on provenance analysis for tea. Tea products cover a wide range of qualities and also a wide range of pricing. Tea is produced under very diverse working and environmental conditions, and, unfortunately, not everywhere the conditions are acceptable. Especially when it comes to human health and human labor no compromises should be made, nor by producers, nor by traders, nor by consumers. Ideally, the price of tea matches approximately the quality, the label on the tea box matches the box' contents and the certificates for environmental and labor conditions conform with the actual local situation. Needless to say, the tea box from the supermarket shelf is not always in accordance with this ideal; and it is not always easy or even possible to point with the finger on who caused the deviation from the ideal. The underlying structures of the tea production and trade which results into the nicely designed tea boxes of small (too small?) prices is too complex to easily pin down one guilty person. Developing a tool to determine the geographical origin of tea will not be 


\section{Introduction}

the solution to guarantee always the ideal of tea production and trading. But if questions are asked about the tea product which can be related to its geographical origin, a provenance tool could help to make the relations between producers, traders and consumers a bit fairer.

It is mandatory to use a multi-variate approach to provenance analysis. No single parameter will ever answer provenance questions in respect to the huge variety of tea products. In this thesis, the analyzed variables cover a broad spectrum of the inorganic elements of the tea leaves. Other parameters, like isotope ratios or organic components, are deliberately excluded: The methods of multi-element analysis are well established, comparably simple and low in cost, and therefore a good deployability for practical purposes is to be expected. Certainly, combining techniques of multi-element analysis with other techniques will increase robustness of the provenance determination, but this would also increase the costs of the provenance tool.

One major obstacle for determining the geographical origin is the blending of tea. Tea is nearly always blended to achieve bigger lots with homogeneous characteristics. These lots can be very small, with sizes of few kilogram, containing only the harvest of one section of one garden, up to sizes of dozens of tons, containing sometimes even leaf material from different continents. The issue of blending is not addressed in this thesis, but discussed in the last chapter 'Conclusions and Outlook'.

Most researchers working on provenance of food items use a reference data set to establish discriminating functions between the groups of their specific data set. In the case of a world-spanning provenance analysis, which should cover all types of tea, this approach would require an enormous reference data set. This project follows an approach which will ultimately help to reduce the necessary sample size for meaningful provenance studies on tea: The elemental composition of tea is caused by parameters of the tea cultivation and the tea production.

The first aim of this thesis is to determine element subcompositions which are influenced by one of the parameters. This opens up two possibilities: a) Previously to the provenance analysis the samples can be grouped by one or more of these parameters by means of their elemental composition. If the sample groups are more homogeneous in terms of e.g. type of manufacturing, type of plucking or type of cultivation, less element variables are needed for robust discrimination functions. b) To establish element subcompositions which are not influenced by critical parameters. These 'robust' element subcompositions are 'first choice' or 'standard' elements to calculate discrimination functions for provenance analysis. 
The second aim of this thesis is to demonstrate the possibilities of filtering sample groups and establishing specific element subcomposition for provenance analysis. The prospect of a following project would be to combine various statistical methods with the results of generating element subcompositions and sample subgroups into a holistic model (see also introduction of Chapter 9).

The dissertation is structured by consecutive chapters. Each chapter has a short introduction and a summary of the main findings. The first five chapters give an overview of the tea plant and tea production, the sampled areas, the analytical methods, the data refinement methods and the mathematical tools. Chapter 2 about tea is mainly compiled of published data and occasionally completed by field observations and personal communications from tea garden managers or producers. Chapter 3 describes the sampling areas to provide the basis to interpret the elemental composition of tea in terms of its geographical origin. For this purpose all available geological maps and soil maps had been taken into account for the description of the areas. The section 'Darjeeling' incorporates an own geological mapping, because the published geology of this region was not detailed enough to explain the discriminating parameters for tea samples of Darjeeling. The description of Japan is not included in this thesis, because the data set of Japanese samples consists only of leaf material without the corresponding soils and therefore the Japanese data are not interpreted with respect to their geographical, pedological and geological background. Chapter 4 describes the analytical methods, the data set and the data refinement. All data, which are not represented in the appendix, are provided on the enclosed CD. The sample set from Darjeeling includes 24 sampling spots which had been samples from joint collaboration with the Tea Board. In order to achieve highest possible accuracy and precision for the data, two in-house R-packages had been developed, co-authored by Wiebke Fahlbusch. Both are not further presented within this thesis, but the packages are provided on the enclosed CD. The mathematical tools explained in Chapter 5 are all based on compositional data analysis. The different representations of vector ratios (Section 5.2) are developed within the compass of this thesis to handle element ratios between two samples on a basis of compositional data analysis. In this thesis it is used for element ratios of plant/soil and of young leaf/mature leaf.

The main findings of this thesis are presented in the last five chapters. Chapter 6 evaluates the impact of adhering particles on the elemental composition of tea leaves. All plant data are corrected for adhering particles, and elements with high correction factors are excluded from further analysis. The method of the correction is published in Pos- 
piech et al. (2017) and is included with small adaptions in Section 6.1. The R-package to calculate the correction for adhering particles is published on CRAN (Pospiech and Fahlbusch 2017). Chapter 7 describes the elemental composition of the plant samples and the differences of elemental distribution between the various groups of plant samples. Element subcompositions are determined which are sensitive to the age of the tea leaf, which indicate a contamination through manufacturing, and which have small spread of concentration within one tea garden. Chapter 8 describes the elemental composition of subsoils and the difference to topsoils. This is used to determine element subcompositions which are sensitive to measures of soil management. The second part of the chapter describes which element log-ratios are comparable for soil and leaf samples. In Chapter 9 all findings of the previous three chapters are applied to demonstrate how the various element subcompositions can be deployed for provenance studies. The last chapter gives a summary of the results of this thesis and addresses future tasks.

Throughout the document the publications are cited with the surname of the first author and year of the publication. Initials are only given to disambiguate authors with identical surnames. For Chinese authors the last name in the respective publication is used as author name, in correspondence to the surnames of English author names. All units used in this thesis follow the International System of Units (SI-units). For names of tea gardens the font family 'typewriter' (monospace) is used to disambiguate between geographical names and garden names. For example, 'Tukdah' is a village in Darjeeling District, Tukdah is the tea garden Tukdah Tea Estate, Chamong Tea in Darjeeling District. Generally, all plant data presented after Chapter 6 are corrected for adhering soil particles. The appendix is split into two parts: Selected data tables and all additional figures are provided within this document. The analytical results and further data such as the element selection or limit of detections are provided on the enclosed CD. The 'readme' file contains information about the file types and access. 


\section{Tea}

The word 'tea' has ambiguous meanings because it refers as well as to the plant Camellia sinensis (L.) O. Kuntze ( $C$. sinensis), to the product and to the beverage. In this thesis the term $C$. sinensis - or simply 'tea' - is used for the tea plant in general. If specification for the species or varieties of the plant is needed, it is referred to as $C$. sinensis var. sinensis, $C$. sinensis var. assamica or $C$. sinensis 'yabukita' respectively. The term 'processed tea' is used for the agricultural product of the leaves, leaf buds and internodes of the plant $C$. sinensis, prepared and cured by various methods (Gupta et al. 2010).

The chapter describes aspects of $C$. sinensis and processed tea samples (processed teas) which are important with regard to the following chapters: This encompasses the basics about the tea plant and its physiology as well as the main element sources and processes which contribute to the elemental composition of $C$. sinensis and processed teas. Organic compounds of $C$. sinensis are left aside because they are irrelevant for this thesis.

\subsection{Tea Plant}

\subsubsection{Camellia sinensis}

The tea plant Camellia sinensis (L.) O. Kuntze belongs to the genus Camellia in the family Theaceae and the species sinensis. The plant is indigenous to South-East Asia. In China its use for tea brewing is reported for more than 3500 years (Heiss and Heiss 2011). Nowadays the plant is cultivated in many regions of Asia, Africa and partly America (I. A. Ross 2007; Barua 2008). The tea plant grows in a broad range of climatic conditions (Bhagat et al. 2010) but in general Camellia sinensis var. sinensis ( $C$. sinensis var. sinensis) grows well under tropical to subtropical climate conditions with high annual rainfall of minimum $1150 \mathrm{~mm}$ (Carr 1972) and high humidity (Squire and Callander 2012). The numerous varieties of the plant have slightly different preferences towards their climatic optimum. 


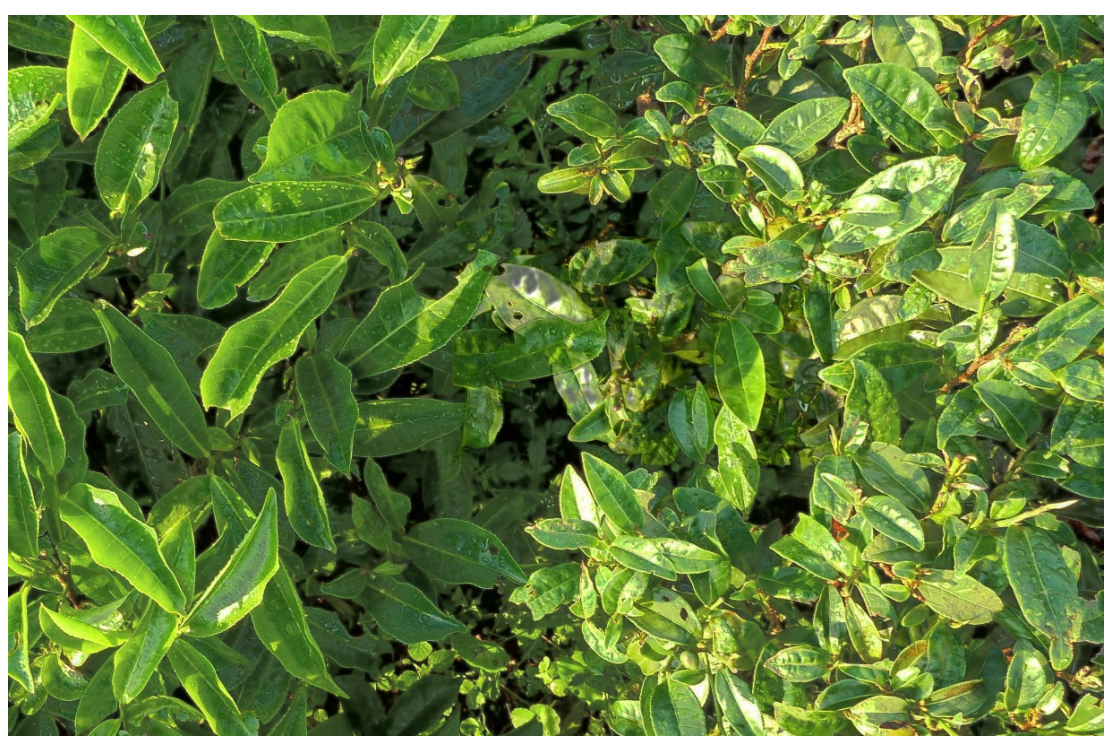

Figure 2.1.: Photo of $C$. sinensis var. assamica, left side, and $C$. sinensis var. sinensis, right side in Darjeeling. C. sinensis var. assamica develops $8-14 \mathrm{~cm}$ long leaves which are thin and glossy green, while $C$. sinensis var. sinensis-plants have smaller leaves $(1.6-4 \mathrm{~cm}$ long), which are hard, thick and leathery with a matte surface.

The plant is an evergreen small tree which grows in natural environment as high as up to $5 \mathrm{~m}$ in height. Occasionally heights up to $9 \mathrm{~m}$ are also reported (FOC 14.12.2011). Generally, leaves of $C$. sinensis are light green when emerging from the bud and become dark green as fully grown leaves. Young branches and older roots show a typical purplish red bark while older branches change into a yellowish gray color (Wachira et al. 2013, and references therein). The tea plant is a very heterogeneous outbreeder and most vegetative characteristics of tea show a continuous variation which makes it difficult to differentiate taxa (Banerjee 1992). According to botanical classification there are three main distinct varieties used for tea cultivation, which in some publications are also referred to as own species (Wachira et al. 2013, and references therein). These are $C$. sinensis var. sinensis, Camellia sinensis var. assamica (C. sinensis var. assamica) and C. assamica ssp. Lasiocalyx (Cambod race). The numerous types of clones, hybrids and occasionally seed plants are called 'Assam', 'Chinese' or 'Cambod' depending on their morphological proximity to one species (Banerjee 1992). Even for well studied clonal varieties it is sometimes difficult to assign the tea plant exactly to one of the main botanical classified species.

The species $C$. sinensis var. sinensis is characterized by small, serrate leaves (1.6$4 \mathrm{~cm}$ long), which are hard, thick and leathery with a matte surface (Figure 2.1). The leaf 
is directed upwards and is placed at an angle of less than $50^{\circ}$ from the stem (Wachira et al.2013). C. sinensis var. sinensis prefers the climate characteristic for higher elevations with $500 \mathrm{~m}$ NN up to $2000 \mathrm{~m} \mathrm{NN}$ in subtropical regions and is thus mostly cultivated in hilly regions (Bhagat et al. 2010).

The species $C$. sinensis var. assamica is taller and develops long, broad, less serrated leaves (8-14 cm long) which are thin and glossy green. The leaf grows more horizontally with an angle less than $70^{\circ}$ from the stem (Wachira et al. 2013). The species prefers higher air temperatures (Tanton 1982) and is therefore mostly cultivated in lowlands and plains such as Assam, NE-India, or Kenya. In this thesis all samples originate from plants which are morphological between $C$. sinensis var. sinensis and $C$. sinensis var. assamica.

Normally in agriculture the plants of $C$. sinensis are trimmed to approximately waisthigh shrubs according to the cultivation techniques. Some farmers in Vietnam are using fully grown trees for tea production (personal communication). Tea plants grown from seeds (seed plants) are generally very robust and can be used for production for about 100 years. Single bushes are reported to be up to 150 years old (personal communication, Ging, Darjeeling). Clonal tea bushes in intensely used tea fields are normally replaced after 40 to 60 years, or as soon as the yield per hectare decreases.

\subsubsection{Aspects of Tea Physiology}

This section will give a summary of the most important aspects of the tea plant related to this thesis. It is not intended as a comprehensive description of the physiology of the tea plant, especially the often debated topic of detoxification of Al is deliberately excluded.

\section{Roots}

The root system and its rhizosphere determines mainly the depth and location of the element source. Microorganisms, e.g. arbuscular myccorhizal fungi (AMFs), may help the plant to extend its reach considerably beyond the root system (George et al. 1995; Hodge et al. 2001; S. Singh et al. 2008). Typical fungal symbionts of the AMF are Glomus, Gigaspora and Acaulospora (Barua 2008, p.98).

The uptake of elements is confined to the youngest parts of the roots, close to the root apex in the creamy-white, fine feeder-roots. Two third of the feeder roots are located in the upper 30 to $40 \mathrm{~cm}$ depth of the soil. The quantity of fibrous roots decreases with depth (Sinha 2010, pp. 100), but feeder roots are also present in 90-100 cm depth (field 
observation in Darjeeling, India, and Araucária, Brazil). Depth and distribution of feeder and mature roots depends largely on local conditions, such as soil properties, rainfall pattern and application of manure or fertilizers (field observations and Marschner 2002; Sinha 2010). If tea plantations are treated with fertilizer applied directly under the bushes the plant develops a shallow and dense fibrous root system down to a depth to $25 \mathrm{~cm}$ and a second, less dense fibrous root system located below $40 \mathrm{~cm}$ (field observation Brazil, personal communication Masumi Moriuchi, Moriuchi Tea Farm, Shizuoka (Moriuchi)). In Darjeeling District at tea plantations with very steep slopes, manure is applied downhill of the bushes as close to the stem as possible. Through the high erosion rates the manure has less time assimilate with the soil at that specific location. Hence, the fibrous root system of these bushes is more evenly distributed and it is difficult to determine at which depth the quantity of feeder roots notably decreases. As the roots mature, they become woody, shed the root cortex and their epidermis is replaced by a periderm. The periderm has a typically reddish, sometimes red-purple color.

The seminal root or taproot, which exists only for tea plants grown from seeds, is developed as a meter-long, thick, reddish root extending deep into the C-horizon of soils (field observation in Darjeeling). The importance of the seminal root diminishes with plant age, e.g. in Japan it is reported to reach down to only $40-60 \mathrm{~cm}$ (Aono et al. 1981). Seed-grown plants develop from the base of the stem with increasing stand age few thick roots which resemble taproots (field observation Darjeeling, India, Sinha 2010 , pp. 100). The branches of these roots often penetrate to greater depths than the seminal root (Sinha 2010, pp. 100). Root depth of these roots may extend down to $4-5 \mathrm{~m}$, as reported from Central Africa (Fordham 1972) and from Tanzania (Carr and Carr 1971, pp. 21-47). In flat areas their depth is limited by water-saturated horizons in the soil where the taproot continues growing horizontally above this horizon (field observation in Registro, Brazil, and Assam, India). Root systems located at areas with a water-saturated horizon at rather low depths show a much greater horizontal spread (Sinha 2010, pp. 100). Clonal plants, which don't have a taproot because they are not grown from seeds, develop seven to eight thick adventitious roots growing at an angle from the vertical, which leads to a shallower root system than for seed plants (personal communication of tea planters in Darjeeling, Yamashita et al. 1997).

The root exudation of organic acids, such as oxalic, citric and malic acid, from the tea plant roots is mentioned by many authors based on results obtained by the analysis of root exudates from other plants (Wang et al. 2010; Karak et al. 2015b; Li et al. 2016, and references therein). Morita et al. (2001, 2011) showed that small amounts of citrate 
and malate are always secreted while oxalate is secreted to chelate $\mathrm{Al}^{3+}$-ions in the soil solution. Wan et al. (2012) showed in culture experiments that also $C$. sinensis releases protons at its roots during the uptake of cations as a function of the $\mathrm{pH}$ of the nutrition solution. It is also reported that the plant's root elongation and growth of tea plant under acidic conditions with high $\mathrm{Al}^{3+}$ contents in the soil solution was stimulated rather than suppressed (Konishi et al. 1985; Morita et al. 2001; Tolra et al. 2011). For example Y. M. Chen et al. (2011) showed in hydroponic experiments for $C$. sinensis that Al stimulates the uptake of $\mathrm{Ca}^{2+}, \mathrm{Mg}^{2+}, \mathrm{K}^{+}$and $\mathrm{Mg}^{2+}$ and diminishes the uptake of $\mathrm{Fe}^{2+}, \mathrm{Cu}^{2+}$ and $\mathrm{Zn}^{2+}$.

\section{Leaves}

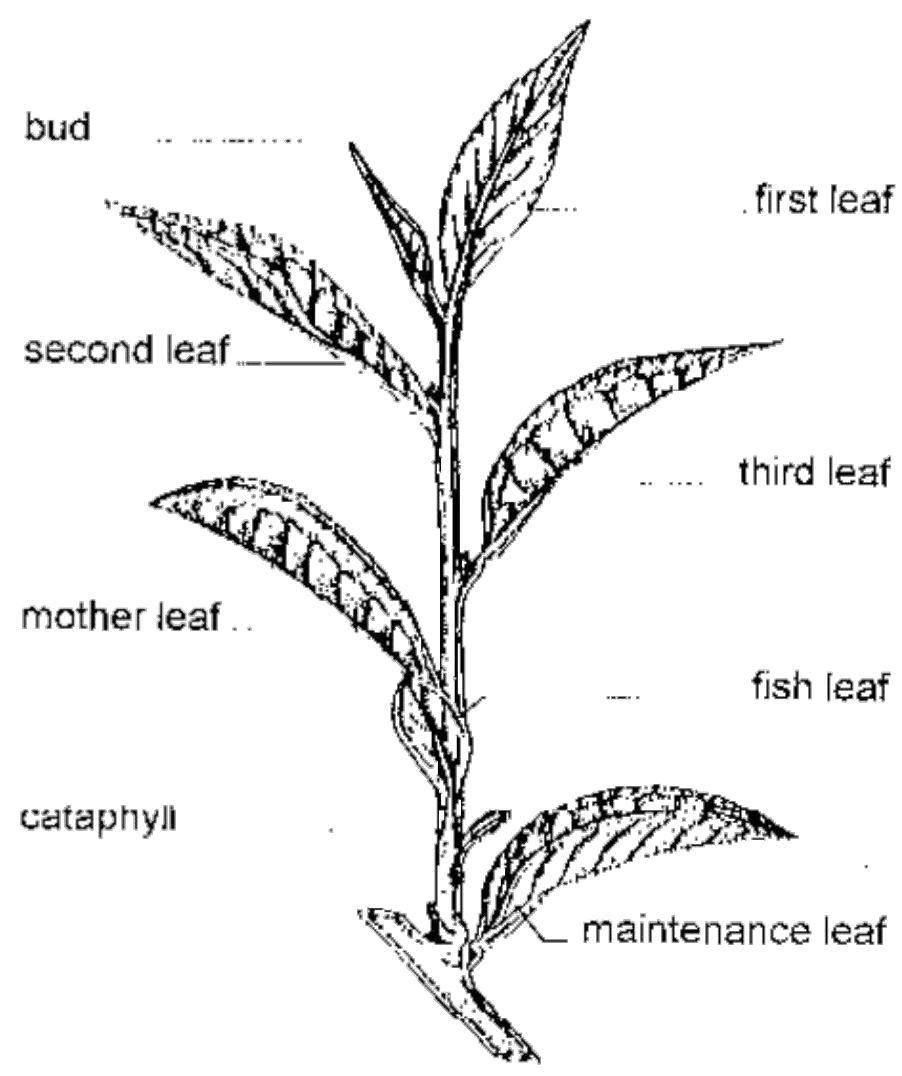

Figure 2.2.: Sketch of a shoot of $C$. sinensis (source Kumar et al. 1998).

The majority of tea is produced from the apical shoots of the tea plant. Under the point of view of the plant's metabolism this encloses two types of plant organs, the sink and 


\section{Tea}

source leaves, respectively. Source leaves are leaves which are 'autotrophic' because they are actively performing photosynthesis and produce sugars which are moved to the sink tissues through the phloem part of the vascular system. The source leaves obtain nutrients and water through the xylem part of the vascular system, in which a watery solution containing ions and some organic components moves from the roots to the source leaves, drawn by the evapotransporation from the leaves (Figure 2.3). Sink leaves, on the other hand, are 'heterotrophic' because they depend for their development on the sugars produced and/or stored in other tissues of the plant.

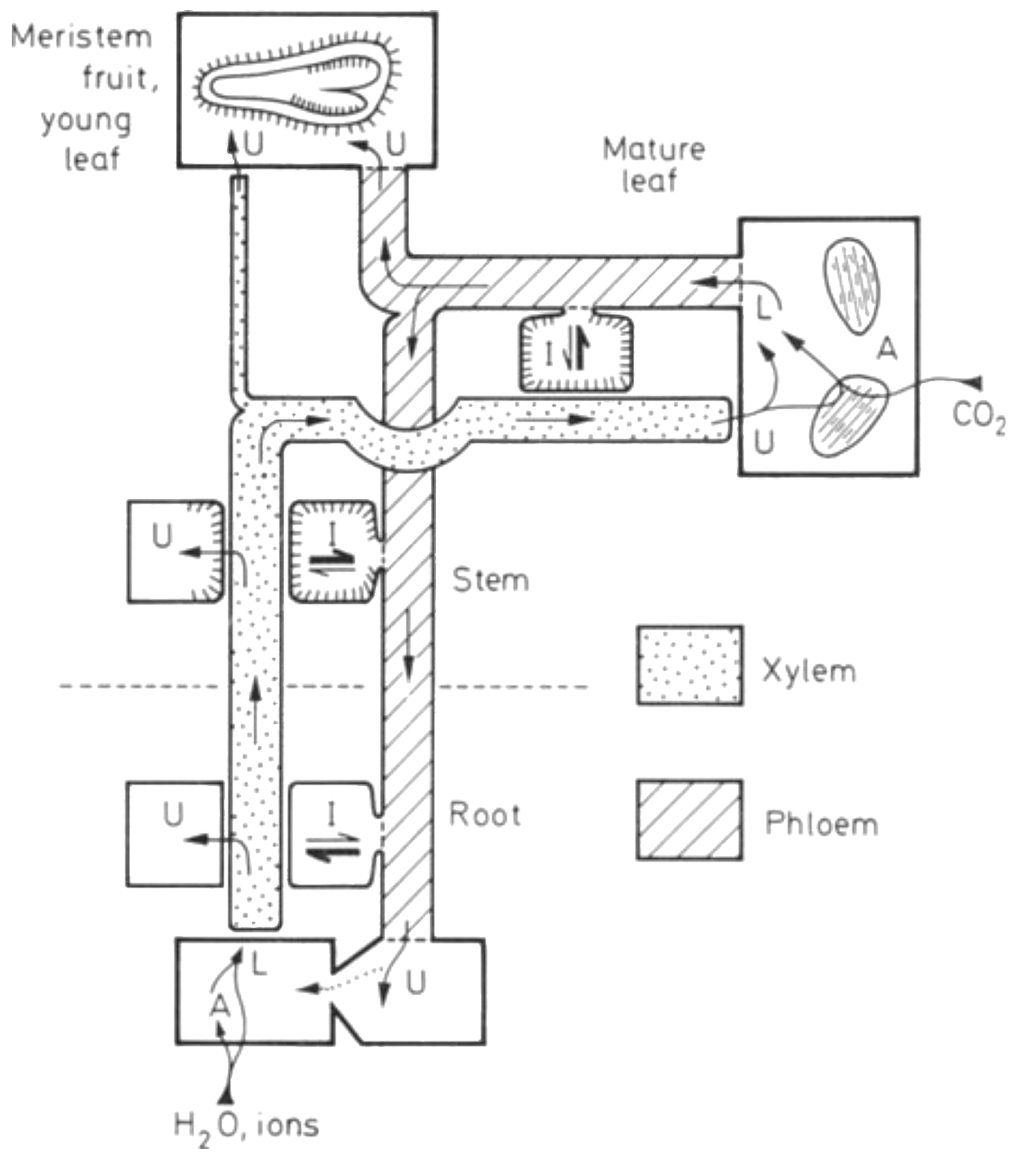

Figure 2.3.: Schematic figure of the basic elements of the transport systems xylem and phloem (reprinted with permission from Springer Customer Service Centre: Pate (1975)). Principal interchange points between xylem and phloem labeled $I$, regions of loading and unloading of transport channels labeled $L$ and $U$ respectively, areas specializing in assimilation of raw materials labeled $A$. Directions of flux of solutes are indicated by arrows.

The conversion from sink to source tissue is a developmental transition which results 
into a characteristically different chemical composition between autotrophic and heterotrophic leaves (Walworth and Sumner 1987; Turgeon 1989; H. P. Carr et al. 2003 [1, During the development of the leaf from the apical bud to a fully grown leaf the conversion gradually takes place in many cases when the leaves are expanded by 30-60\% (Turgeon 1989, and references therein). Hence, at the apical shoot of tea plants the bud is purely heterotrophic (sink leaf), the first and second leaf after the bud are mainly heterotrophic with beginning photosynthesis (mainly sink), and the third leaf after the bud is in the middle of the transient stage neither exporting nor importing sugars (Marimuthu et al. 1994; Kumar et al. 1998). The fourth leaf is already nearly fully converted into a source leaf (Marimuthu et al. 1994; Kumar et al. 1998). The timing of the conversion depends on climatic conditions including altitude, on cultivars, on the season, on the time since the last pruning and on the plucking standard (Section 2.3.2), because these parameter determine the rate of the tea shoot development (Smith et al. 1990; Durairaj et al. 2015).

A tea leaf has a life span of approximately two years (Durairaj et al. 2015). The active participation in photosynthesis lasts for six to nine months, depending on cultivar and plantation region (Sinha 2010, pp.102, Durairaj et al. 2015, Kabir and Das 2015, pp. 8-17). The active mature leaf samples (mature leaves) serve in commercially exploited tea bushes as maintenance foliage for the next generation of shoot tip samples (shoot tips).

\subsubsection{Tea lonome}

The ionome of a plant is defined as all the mineral nutrients and trace elements found in a plant (Salt 2004; Salt et al. 2008, and references therein) through element uptake or respiration. This includes all metals, metalloids and nonmetals such as N, P, S, Se Cl and I (Salt 2004). The term ionome was developed to link the plant nutrient signatures with other signatures and profiles, e.g. of genetics, environmental factors, geographic region or farming methods (Parent et al. 2013; Baxter 2015; Huang and Salt 2016, and references therein). In this thesis the term ionome for the elemental composition is used to emphasize the significance of the multi-element approach: thinking beyond individual elements allows the more sensitive detection for elemental variations for discriminating between groups of tea samples (Buccianti et al. 2006; Baxter 2015).

\footnotetext{
${ }^{1}$ In table I in H. P. Carr et al. $\sqrt{2003}$ the values for $\mathrm{K}$ are displayed in reverse order, showing the higher concentration for the old leaves while in the text of the article it is described that young leaves show higher concentrations of $\mathrm{K}$ than old leaves. The latter is correct, because it agrees with findings of other authors.
} 
The ionome of $C$. sinensis is influenced by the type of cultivar (Y. Chen et al. 2009; Fung et al. 2009; Y. Chen et al. 2010), geographical origin (Marcos et al. 1998; FernándezCáceres et al. 2001; Moreda-Piñeiro et al. 2003; Q. Chen et al. 2009), agricultural methods and soil conditions (regulation of soil $\mathrm{pH}$, application of fertilizers, etc.), stand age of the tea plantation (Fung 2003; Fung et al. 2009) and climate, e.g. rainfall patterns. The relative variation of element concentration ranges is small for the nutrients and rather high for trace elements. Despite these differences, there are typical element ranges for tea (Figure 2.4, Szymczycha-Madeja et al. 2012, and references therein).

As a response to the highly acidic soil solution and its toxic $\mathrm{Al}^{3+}$ concentration, $C$. sinensis uses a sequestration mechanism by forming Al complexes with organic ligands such as catechins and phenolic acids (Samac and Tesfaye 2003; Morita et al. 2008, 2011, Poschenrieder et al. 2008, and references therein) which leads to the accumulation of Al in the vacuoles of the leaves' epidermal cells (H. P. Carr et al. 2003; Tolra et al. 2011; Matsumoto et al. 1976); Accordingly, C. sinensis is considered as an Alaccumulating plant. Some authors have suggested that $A l$ even has a nutritive role in tea physiology (H. P. Carr et al. 2003).

The ionome differs between tissues (Section 2.1.2) and therefore every plant organ has its own characteristic elemental composition (H. P. Carr et al. 2003; Shu et al. 2003; Fung et al. 2009). The description of the ionome is here confined to shoot tips and mature leaves as these plant parts are the relevant ones for this thesis.

\section{Shoot tip}

Processed tea is for most tea types produced from shoot tips of $C$. sinensis (Section 2.3.3 and therefore the elemental composition of processed tea is for most elements similar to shoot tips. However, for the elements $\mathrm{Al}, \mathrm{Cr}, \mathrm{Cu}, \mathrm{Pb}$ and $\mathrm{Zn}$ elevated levels had been reported in processed tea, depending on the production method (Wong et al. 1998; Fung 2003; Ashraf and Mian 2008; Jin et al. 2008; Seenivasan et al. 2008; Salahinejad and Aflaki 2010). Possible sources of contamination are described in Section 2.3.3. Hence, for these elements the ionome of processed teas is potentially altered during production and is not necessarily comparable to the elemental composition of shoot tip.

The main inorganic elements present in shoot tips and processed teas are $\mathrm{Al}, \mathrm{Ca}$, $\mathrm{K}, \mathrm{Mg}, \mathrm{P}$ and $\mathrm{S}$ (Karak et al. 2015b, and references therein); trace elements with high abundance are $\mathrm{Ba}, \mathrm{Cu}, \mathrm{Fe}, \mathrm{Mn}, \mathrm{Na}, \mathrm{Rb}, \mathrm{Sr}$ and $\mathrm{Zn}$, while elements like $\mathrm{B}, \mathrm{Cd}$, Co, Cr, $\mathrm{Cs}, \mathrm{Hg}$, Li, Ni Pb, rare earth elements (LAs + Y) (REE), Sc, Se, Sn, Ti, V, Y and Zr are usually present at very low concentrations (Figure 2.4, Szymczycha-Madeja et al. 2012 , 
and references therein, McKenzie et al. 2010). The elements Ag, As, Be, Bi, Cd, Ce, Co, Cs, Ga, Hg, In, Li, Mo, Pb, REE, Sb, Se, Sn, Te, TI, U, V, Y and Zr are sometimes present at concentrations below the detection limit of analytical methods (Szymczycha-Madeja et al. 2012, and references therein). For these elements exist very few published data.

Shoot tips, consisting mainly of sink tissue, are compared to source tissue relatively enriched in phloem-mobile nutrients, such as K, P and Zn (Schachtman et al. 1998). On the other hand, they are compared to source tissue relatively depleted in elements which are phloem-immobile, like $\mathrm{Ca}$ and $\mathrm{Al}$ (Figure 2.4.

\section{Mature leaves}

As the leaf matures, the ionome changes from the typical sink leaf to the source leaf composition (Wong et al. 1998; H. P. Carr et al. 2003; Shu et al. 2003; Fung et al. 2009). Mature leaf samples have higher levels of $\mathrm{Ca}, \mathrm{Mg}$ and $\mathrm{Mn}$ than shoot tip samples (H. P. Carr et al. 2003; Szymczycha-Madeja et al. 2012, and references therein). Mature leaves progressively accumulate Al (H. P. Carr et al. 2003; Fung et al. 2009; Tolra et al. 2011; Hajiboland et al. 2013); hence the highest Al-concentrations are found in the oldest leaves. Apart from Al there are very few published data for mature leaf samples, e.g. in Figure 2.4 all mature leaf data points originate from H. P. Carr et al. (2003). 


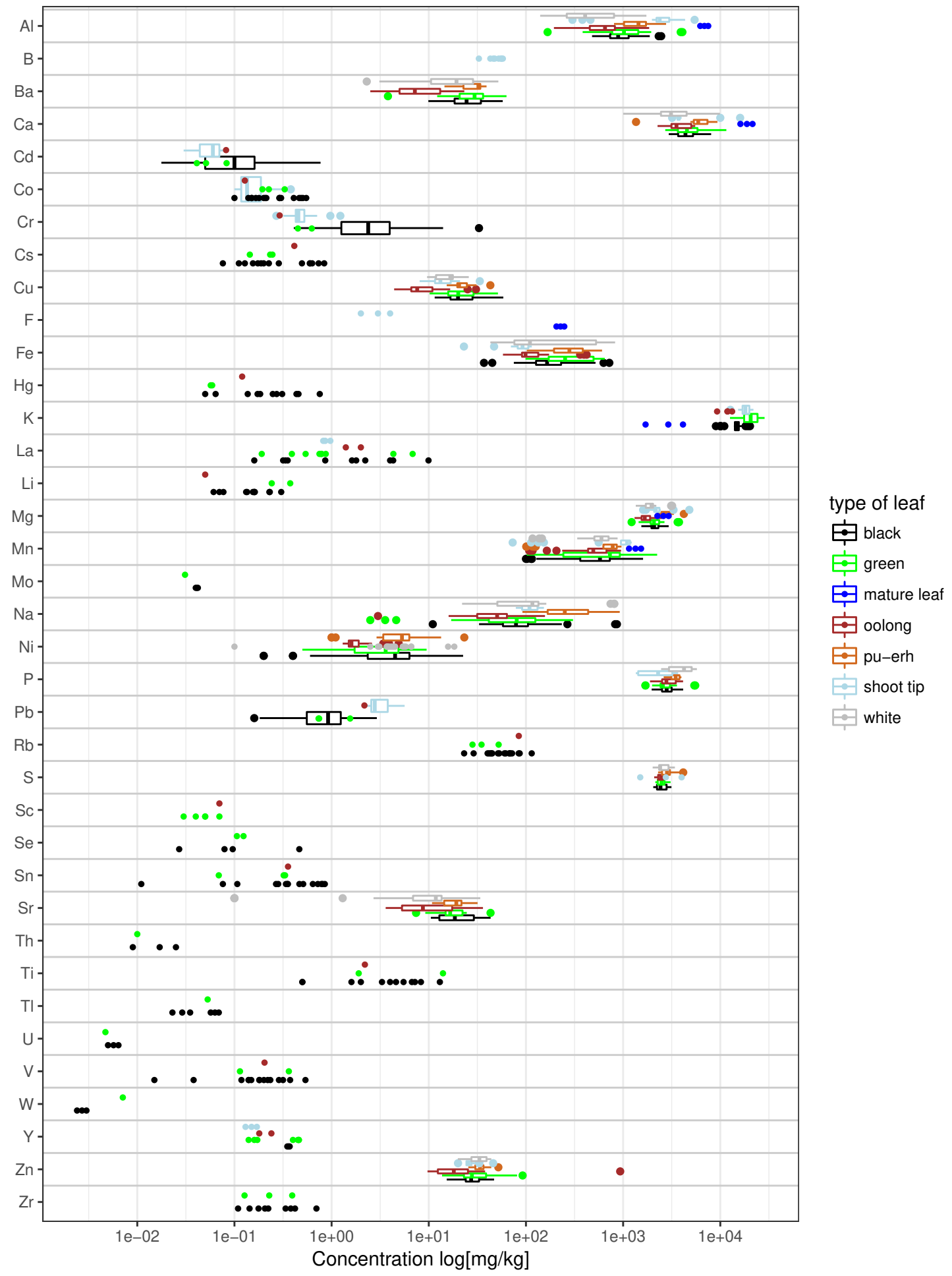

Figure 2.4.: Compilation of published element concentrations of $C$. sinensis. Boxplots are used if there are more than 20 data points per group. For some elements there are data of three mature leaf samples. Number of samples per publication: Ødegård and Lund 1997, $N=3$, Cao et al. 1998 $\mathrm{N}=4$, Marcos et al. 1998, $\mathrm{N}=15$, Herrador and González 2001, $\mathrm{N}=48$, Matsuura et al. 2001, N=4, H. P. Carr et al. 2003, N=6, Gallaher et al. 2006, N=3, Jin et al. 2008 $N=20$, Y. Chen et al. 2009, $N=8$, Salahinejad and Aflaki 2010, $N=11$, McKenzie et al. 34 2010 $N=135$, Hosseinzadegan et al. 2016, $N=10$ 


\subsection{Tea Soils}

The commercial cultivation of tea requires moderately acidic soils with $\mathrm{pH}$ optimum between 4 - 4.5 and 5 - 5.5 (Harler 1971; Eden 1976; Ma et al. 2000; Ruan et al. 2007; Paix Mupenzi et al. 2011; Karak et al. 2015b; Pandey 1997; Barua 2008) which have been developed under mesic conditions such as high rainfall and humidity (Fung et al. 2009). Typical soils for tea cultivation are for example in South India laterites or latosols (Ranganatha and Natesan 1985), in China red-yellow podzolic or red-yellow lateritic soils (Fung et al. 2009) or in Kenya soils of volcanic origin (M. K. V. Carr et al. 1987; Othieno et al. 1992).

The acidity of soils in tea gardens is reported to increase with time (Jayman and Sivasubramaniam 1975; Ruan et al. 2004; Zhang and Fang 2007; Li et al. 2016, and references therein), and with the stand age of the plantation (Xue et al. 2006; Han et al. 2007). One reason for the tea soil acidification is obviously excessive application of $\mathrm{N}$-fertilizers containing $\mathrm{NH}_{4}{ }^{+}$, especially in tea gardens located in China (Ruan et al. 2000; Guo et al. 2010; Wang et al. 2010; Cheng et al. 2015; Karak et al. 2015b). Root exudates as a source for acidification are assumed by many authors based on results for other plants (Wang et al. 2010; Karak et al. 2015b; Li et al. 2016, and references therein). Indeed, results of one cultivation experiment had been interpreted that relatively higher $\mathrm{pH}$ conditions in tea garden soils, e.g. if the $\mathrm{pH}$ increases from 4.0 to 4.5 , result into a higher rate of proton release from the tea plant roots (Wan et al. 2012). Other reasons, such as increased organic content through organic manure and litterfall (Fung 2003) or disturbing the soil structure through plowing, may also have a minor impact on acidification (Li et al. 2016).

The very low $\mathrm{pH}$ of the tea garden soils has a major impact on bio-availability as well on nutrients as on non-nutrients, which can be toxic at high concentrations, e.g. heavy metals, F or I (Jayman and Sivasubramaniam 1975; Fung et al. 1999; Ma et al. 2000; Ruan et al. 2004, 2006; Han et al. 2007; Zhang and Fang 2007; Y. Chen et al. 2010; Karak et al. 2011; Seenivasan et al. 2016). Because most tea plantations have high stand ages, are situated in regions of leached soils or the geologic source rocks of the region support low $\mathrm{pH}$ in the soils, the bio-availability of most elements is comparably high, except for leached elements, for example $\mathrm{Ca}$ and $\mathrm{Sr}$. If $\mathrm{pH}$ decreases notably below 4 the high concentrations of $\mathrm{Al}^{3+}$ and $\mathrm{H}^{+}$ions can cause toxicity to the plants and the plant significantly reduces its proton release by the roots (Wan et al. 2012).

Acidification is generally corrected by applying carbonate rocks, such as limestone or 
dolomite, the so-called 'liming'. The frequency and amount of applied carbonate rocks vastly differs between regions and companies. In a garden where carbonate rocks are applied, the frequency ranges between $10 \mathrm{y}$ to $25 \mathrm{y}$ (personal communication of 26 tea garden managers). If recently carbonate containing rock powder had been applied the bio-availability decreases for trace elements and heavy metals, e.g. Al, Fe, REE, Pb, Cd and $\mathrm{F}$.

\subsection{Tea Production}

In this thesis 'tea production' comprises the treatment of tea bushes like pruning or re-planting, plucking seasons and techniques and tea manufacturing. All steps of the production have an impact on the $C$. sinensis's ionome. It remains difficult to assign certain element pattern to a specific production step because available data sets are too small-sized or not systematically collected for quantification. This section will give a theoretical overview of which element concentrations of shoot tip and processed tea alter during the steps of tea production with regard to the various production systems included in the present data set.

\subsubsection{Fertilizers and Fungicides}

Intensive research had been carried out about the tea plant's requirements for nutrients and suitable fertilizers and manure. This section is limited to general effects on the ionome of $C$. sinensis by using fertilizer or manure and possible sources of contamination in analysis of tea soils and leaves.

Commercial production of tea requires intensive fertilizer or compost application in tea fields, usually by several splits per year, especially if the harvest takes place throughout the year (Durairaj et al. 2015; Kabir and Das 2015). Fertilizers can be applied either as foliar spray or as supplement for the soil. Frequency, timing in the year, dose and type of fertilizer varies for each region and between organic and conventional managed tea gardens. Regions with dormancy periods have fertilizer application cycles adjusted to the harvesting periods. However, the frequency and amount fertilizer application is sometimes also used to regulate dormancy periods; For example in some regions the producer can determine the end of the dormancy period by applying fertilizer e.g. four weeks before first plucking round of the harvest period (personal communication Milton Amaya, Amaya Chás, Registro (Amaya), and Masumi Moriuchi, Moriuchi). 
The main difference in fertilizing and pest control is obviously found between conventional and organic tea gardens. Because conventional gardens mostly rely on artificial fertilizers with high $\mathrm{N}, \mathrm{P}$ and $\mathrm{K}$ contents, the tea plants are often very well supplied with the major nutrients. On the other hand organic gardens mainly use compost and manure and deficiencies or replacement of nutrients by trace elements with similar chemical characteristics occur more easily.

The regulations about compost composition and production vary considerably between countries and regions. Tea garden compost nearly always contains tea pruning, tea waste, cuttings from weeding and, in the case of India, cow dung. Garden in Darjeeling additionally harvest cuttings of citronella grass or Guatemala grass (Tripsacum andersonii J. R. Gray or Tripsacum laxum Nash) or other Tripsacum species for composting. Some tea gardens enrich the compost with oil cakes as $\mathrm{N}$-source, like castor (Lima et al. 2011, and references therein), coconut, neem, etc., chicken manure, paddy straw, lime or other organic components containing additional N, P, K and S and microorganisms (personal communication, Kabir and Das (2015)). Accordingly, tracking the input and availability of nutrients and other trace elements in tea soils is for organic tea gardens often impossible.

Foliar sprays are potentially a major source of contamination while sampling tea leaves. Typical foliar fertilizer sprays are $\mathrm{ZnSO}_{4}, \mathrm{MgSO}_{4}, \mathrm{MnSO}_{4}$ and $\mathrm{H}_{3} \mathrm{BO}_{3}$ (Seenivasan et al. 2008; Durairaj et al. 2015). In organic gardens foliar sprays the Bordeaux mixture, a mixture containing $\mathrm{CuSO}_{4}$ and $\mathrm{Ca}(\mathrm{OH})_{2}$, or Copper oxychloride, $\mathrm{Cu}_{2}(\mathrm{OH})_{3} \mathrm{Cl}$, is often used as fungicide. Extensive use of the Bordeaux mixture can have a significant impact on Cu concentrations in processed teas (Han et al. 2005).

For most visited tea gardens there are no data collected about the exact timing and frequency of fertilizer application. Whenever possible a general overview of the fertilizers and manure used in the specific tea garden had been recorded. For this thesis, samples had only be taken in sections without very recent treatment (Section 4.1.3) to avoid contamination of the leaves or soil samples by remnants of fertilizers or foliar sprays.

\subsubsection{Plucking}

The plucking technique determines the ratio between apical buds, mature leaves and leaves in transition in the harvested leaves. As discussed in the sections 2.1.2 and 2.1.3 the ionome differs for nutrients and some trace elements considerably between sink and source tissue and therefore the plucking techniques influence the elemental composition of processed teas. 


\section{Tea}

The tea plants are usually to cut to bushes ('training') with an flat, often horizontal surface, the so called 'plucking table'. Below the plucking table there is a dense layer of mature foliage. From the uppermost centimeters of the plucking table the tea shoots grow from the leaf axils and are harvested in several rounds per season. With each harvest round the plucking table is cut slightly higher to leave a new layer of mature leaves for higher photosynthesis activity (Figure 2.5). Every 2 to 5 years the whole canopy is pruned to remove all younger branches and set up a new plucking table (Squire and Callander 2012).

For each type of processed tea (Section 2.3.3) and for maintenance of the tea bushes there are certain requirements in terms of plucking standard: The most common plucking standard includes the apical bud plus the first two unfolded leaves, called 'two and a bud'. Other plucking standards include plucking only buds - for the highest tea qualities down to 'hard plucking', where the whole shoot is plucked including mature leaves from the maintenance foliage. The plucking standard has therefore a major impact on the quality of processed tea.

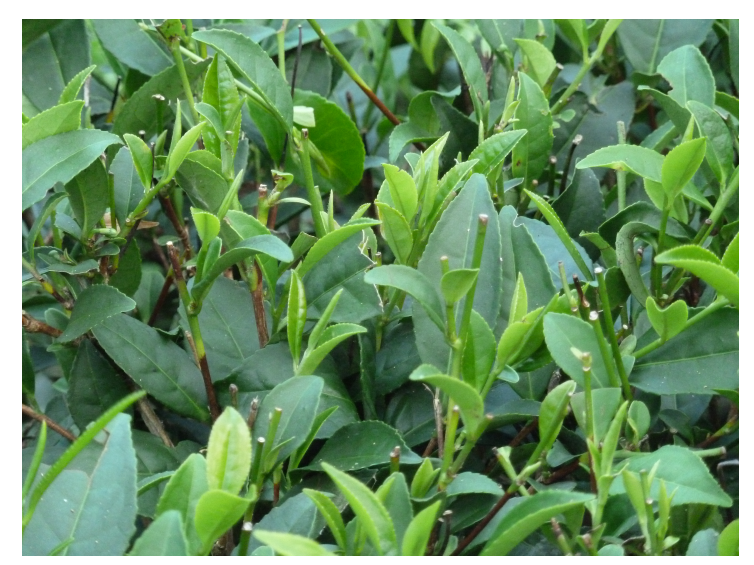

Figure 2.5.: C. sinensis var. assamica, hand plucked, late in harvest season, Ging, Darjeeling. The actual plucking level can be recognized by the uppermost level of rupture location of shoot remnants. Between the original maintenance leaves (dark green) and the actual plucking table a layer of this season's leaves had been left as maintenance foliage (mature leaves of light green color). From the level of actual maintenance foliage shoot tips in different development stages emerge.

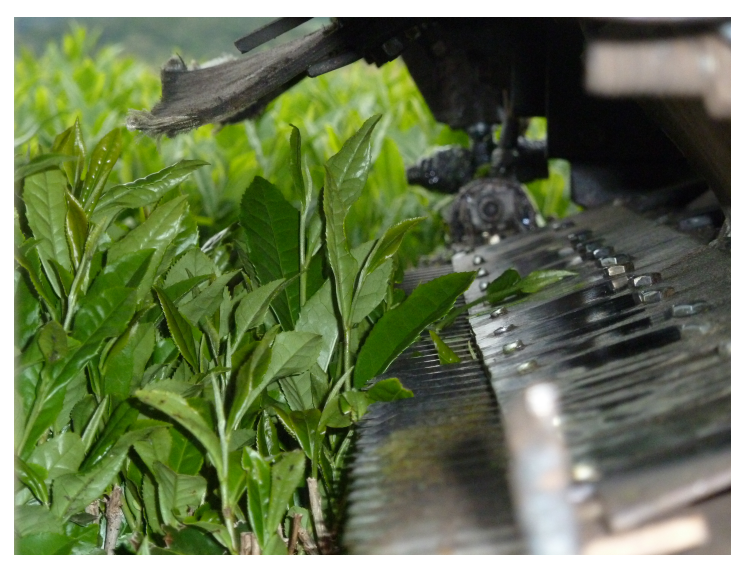

Figure 2.6.: C. sinensis 'yabukita', mechanical plucking, begin of harvest season, S. Miguel de Arcañjo, Paraná. The plucking table is set by the level of the cutting machine. The shoot tips are mostly in phase and are therefore all approximately at the same stage of development. The plucking standard is set to ' 2 and bud', but cutting of further developed leaves can not be avoided. 
C. sinensis can be plucked either manual, 'hand-plucking', or mechanical, 'machineplucking', respectively semi-mechanical, e.g. 'shear-plucking'. Hand-plucked tea contains, if pluckers are well trained, only the leaves which should be plucked according to the respective plucking standard. For example, good quality black tea (orthodox black tea) is in Darjeeling produced by 'two and a bud' plucking standard and hence the processed tea contains only buds, the first two leaves and internodes of the shoots (Figures 2.5 and 2.7). For mechanical plucking the growth of shoots developing from axillary buds has to be brought in phase so that the shoots, which are cut at the actual plucking level, match the plucking standard (Figure 2.6). If the shoots are not perfectly in phase, with the occurrence of aperiodic shoots or if the plucking machine is adjusted slightly above or below the actual plucking level, more matured leaves or too young leaves alter the sink-source leaf ratio of the resulting processed tea. Additionally maintenance foliage leaves extend during their development above the actual plucking level and get partly cut during the next round of harvest (Figure 2.8).

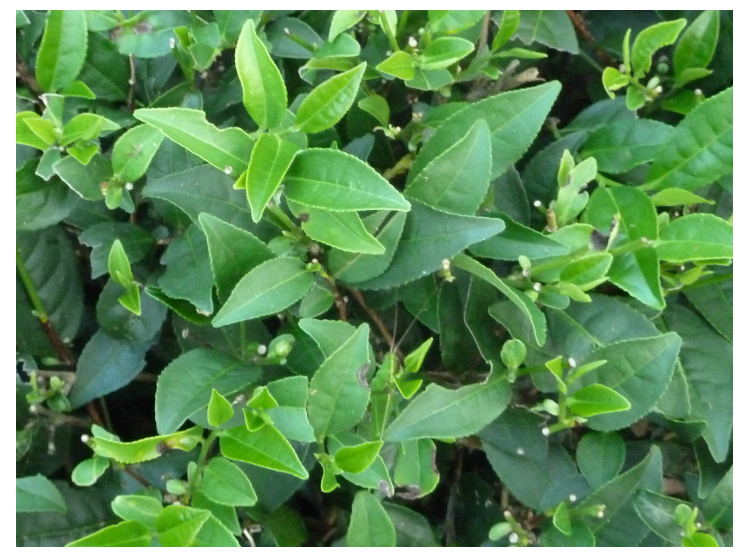

Figure 2.7.: C. sinensis var. sinensis, a ter hand plucking, late in harvest season, Ging, Darjeeling. The mature leaves of the maintenance foliage are all complete and had been carefully avoided during plucking.

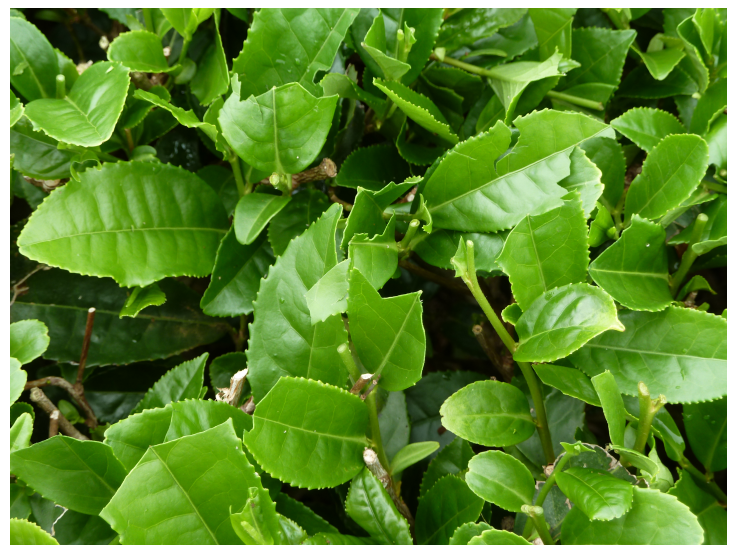

Figure 2.8.: $\quad$ C. sinensis 'yabukita', after mechanical plucking, S. Miguel de Arcañjo, Paraná. The mature leaves of the maintenance foliage are partly cut and had been accidentally harvested. On the other hand some shoot tips had not been completely harvested.

In the various tea plantation regions different types of machines are used for mechanical plucking. Highest precision in terms of matching a specific plucking standard achieve the fully mechanical plucking methods combined with hormone induced in phase shoot growth as applied in some Japanese companies (personal communication, $\mathrm{N}$. Hashimoto). 
Plucking Seasons At Darjeeling the raw material is harvested during four plucking periods in a year: first flush (1st flush) is harvested in early spring after the bushes' winter dormancy. During late spring and early summer the second flush (2nd flush) is plucked producing a slightly stronger tea. Tea picked in the time between 1st flush and 2nd flush is labeled in between. During the raining season the rains tea and in October the autumnal flush (atm flush) (if the weather allows it) is harvested. High quality teas are manually plucked ensuring that only the freshly grown bud with the first two leaves are collected. The quality of the product tea is also defined by the different grades of tea (Table A.2).

\subsubsection{Tea Manufacturing}

Each type of processed tea requires its, sometimes specific, plucking standard and plucking season. For example, a 1st flush FTGFOP Darjeeling is plucked manual between March and end of April with the plucking standard strictly '2 and bud', while a stalky OF CTC Assam can be the result of manual or semi-mechanical coarse plucking. In China green tea and oolong of normal leaf grade is produced from ' 3 and bud' up to ' 4 and bud' (Wong et al. 1998), whereas high quality leaf grades are made from ' 1 and a bud' to '2 and bud' (Han et al. 2006).

The tea processing is a multistage procedure to transform the fresh leaves of $C$. sinensis into processed tea ready for brewing. There is a multitude of different ways to process the leaves of $C$. sinensis to processed tea depending on which type, quality and taste of processed tea should be achieved as outcome. Moreover, every company or even every tea factory have their own variations in manufacturing. This chapter focuses only on the general effect of machines used for sencha tea, green tea, black orthodox tea and CTC tea on possible sources of contamination.

Withering For sencha, green tea, orthodox and CTC the plucked leaves are filled into troughs and air is ventilated in order to remove excess moisture from the leaves. If the air is not filtered prior to be pushed through the withering leaves, air borne dust may pollute the plant material (Figure 6.4). Jabeen et al. (2015) describes an increase in $\mathrm{Al}, \mathrm{Ni}$ and $\mathrm{Pb}$ concentration with withering time and explains this increase with pollution from the surrounding area.

Disruption/Rolling The disruption of the wilted leaves destroys the leaves' internal structures at a desired degree to quicken and enhance the oxidation process. For or- 
thodox black tea leaves are extensively rolled on steel, gun metal or brass rolling tables (Sinha 2010). In Darjeeling some rolling tables are made of brass with unusual high copper content (personal communication factory managers). The abrasion of the brass rolling tables can reduce the thickness of the rolling tables in the center by millimeter to centimeters per season (personal communication Mr. Pancholi, manager of Marybong Tea Estate, Chamong Tea (Marybong)). For CTC (crush, tear, curl) the leaves are passed, occasionally after a short period of rolling on a table roller, into two stainless steel rollers with a dense mesh of sharp 'teeth'. This procedure cuts and tears the leaves into small parts which ensures a full oxidation in the next step. The rollers of a CTC machinery are especially prone to metal abrasion (Seenivasan et al. 2008). For green tea the disruption of cells is achieved by steaming or pan frying of the leaves. This procedure has no notable source of contamination.

Oxidation/Fermentation For the oxidation process the ruptured leaves are exposed to warm, humid air, either on oxidation benches (black tea) or in several intervals between steaming (green tea). Generally the phase of oxidation/fermentation is of comparable short duration and involves no to very minor invasive machinery. Accordingly no source of contamination is expected by the oxidation step.

Fixation/Rolling/Shaping For some tea varieties the oxidation process is stopped by moderately heating of the leaves, the so called fixation. Between fixation and the drying of the tea the damp tea leaves can then be further processed by rolling, shaping, frying, pressing on bamboo, etc. to achieve a certain shape or special taste. Both procedure steps, fixation and shaping, provide the possibility only for minor metal abrasion but for delicate and/or manual shaping there might be various sources of contamination, depending on local production settings.

Drying Drying removes all remaining moisture of the tea leaves and finishes the tea. Black tea is baked at high temperature by transporting the leaves on a conveyor through a dryer. For green tea each green tea type is dried with a different technique, including dryers similar for black tea production to manual pan frying. In India most orthodox black tea factories use dryers which are heated by wood, coal or sometimes liquefied petroleum gas, which is a possible source of contamination by micro-ash particles or other organic components of the combustion. 
Sorting The last step of black tea production is the sorting by particle size, respectively leaf grades (Table A.2), and in CTC additionally by particles density: After drying the tea is loaded into a chained machinery of separators, shakers and sieves of different meshes to separate the various grades of leaves from fiber components and dust grades (Figure 7.9). The sorting line is an additional possible but minor source of metal abrasion. The sorting process may also concentrate non-tea particles in certain leaf grades: For example, fannings or dust grades consists of leaf material separated from all bigger leaf parts by continuously decreasing sieve meshes. The fine particles of machine abrasion are probably found at the leaf grade of similar particle size and which did not got separated by density in a last step, like extensive fanning.

\subsection{Summary}

The tea plant grows in a broad range of climatic conditions but in general $C$. sinensis var. sinensis grows well under tropical to subtropical climate conditions with high annual rainfall and high humidity. The plant is an evergreen small tree which in agriculture fields is trimmed to approximately waist-high shrubs. It is a very heterogeneous outbreeder and most vegetative characteristics of tea show a continuous variation which makes it difficult to differentiate taxa. The numerous types of clones, hybrids and occasionally seed plants are called 'Assam', 'Chinese' or 'Cambod' depending on their morphological proximity to one species.

The root system and its rhizosphere determines mainly the depth and distribution of the element source and depends largely on local conditions, such as soil properties, rainfall pattern and application of manure or fertilizers. The seminal root or taproot is developed as a meter-long, thick, reddish root extending deep into the C-horizon of soils. In flat areas their depth is limited by water-bearing horizons in the soil where the taproot continues growing horizontally above this horizon. Two third of the feeder roots are located in the upper 30 to $40 \mathrm{~cm}$ depth of the soil, but feeder roots are also present in $90-100 \mathrm{~cm}$ depth. The commercial cultivation of tea requires acidic soils with $\mathrm{pH}$ optimum between $4-4.5$ and $5-5.5$.

The majority of tea is produced from the apical shoots of the tea plant. Under the point of view of the plant's metabolism, this encloses two types of plant organs, the sink and source leaves, respectively, because the plucking technique determines the ratio of sink to source leaves in the harvested leaves. The apical shoot of tea plants the bud is purely heterotrophic (sink leaf), the first and second leaf after the bud are mainly 
heterotrophic with beginning photosynthesis (mainly sink), and the third leaf after the bud is in the middle of the transient stage neither exporting nor importing sugars. The fourth leaf is already nearly fully converted into a source leaf and its active participation in photosynthesis lasts for six to nine months.

The ionome of $C$. sinensis is influenced by the type of cultivar, geographical origin, agricultural methods and soil conditions (regulation of soil $\mathrm{pH}$, application of fertilizers, etc.), stand age of the tea plantation and climate, e.g. rainfall patterns. The relative variation of element concentration ranges is small for the nutrients and rather high for trace elements. The ionome differs between tissues (Section 2.1.2) and therefore every plant organ has its own characteristic elemental composition. Shoot tips, consisting mainly of sink tissue, are relatively enriched compared to source tissue in nutrients which are phloem-mobile, like K, P and $\mathrm{Zn}$. They are relatively depleted compared to source tissue in elements which are phloem-immobile, like $\mathrm{Ca}$ and Al. Mature leaf samples have higher levels of $\mathrm{Ca}, \mathrm{Mg}$ and $\mathrm{Mn}$ than shoot tip samples. Mature leaves progressively accumulate $\mathrm{Al}$ and the highest $\mathrm{Al}$-concentrations are found in the oldest leaves: As a response to the highly acidic soil solution and its toxic $\mathrm{Al}^{3+}$ concentration, $C$. sinensis uses a sequestration mechanism of forming $\mathrm{Al}$ complexes which leads to the accumulation of $\mathrm{Al}$ in the leaves. Accordingly $\mathrm{C}$. sinensis is considered as an Al-accumulating plant and some authors have suggested that $\mathrm{Al}$ even has a nutritive role in tea physiology.

Commercial production of tea requires intensive fertilizer or compost application in tea fields, usually by several splits per year, especially if the harvest takes place throughout the year. Fertilizers can be applied either as foliar spray or as supplement for the soil. Compost composition and production vary considerably between countries and regions and accordingly input and availability of nutrients and other trace elements in tea soils is for organic tea gardens difficult to estimate. Foliar sprays are potentially a major source of contamination while sampling tea leaves. Acidification of soils is generally corrected by applying carbonate rocks, such as limestone or dolomite, the so-called 'liming'.

The plucking technique determines the ratio between apical buds, mature leaves and leaves in transition in the harvested leaf material. The ionome differs for nutrients and some trace elements considerably between sink and source tissue and therefore the plucking techniques influence the elemental composition of processed teas. The most common plucking standard for high quality tea products includes the apical bud plus the first two unfolded leaves, called 'two and a bud'.

The tea processing is a multistage procedure to transform the fresh leaves of $C$. sinensis into processed tea ready for brewing. Tea manufacturing includes all or several 
2. Tea

production steps of withering, disruption/rolling, oxidation/fermentation, green fixation/ second rolling/ shaping, drying and sorting. The machinery is known to be one source of metal contamination in processed tea through abrasion or chipping. Especially during the step of disruption/rolling some manufacturing methods require very impacting machineries, like the CTC rollers. 


\section{Characterization of Sampling Areas}

Out of the numerous tea growing areas in the world five different areas have been sampled for this study: Darjeeling, Assam and Nilgiris in India, SE-Brazil, and central Japan. These five working areas represent contrasting tea plantation areas which have few parameters of tea cultivation and production in common. This combination of contrasts and common parameter is the key to discriminate between local and general aspects of element uptake by the plants.

The main area is the Darjeeling District of the federal state of West Bengal, NEIndia. It is used as a case study for demonstrating the relation between tea ionome and geographical origin. The basis of this case study is a detailed geological map which had been compiled for this thesis from field work and maps and descriptions from other publications.

\subsection{India}

\subsubsection{Darjeeling}

The term 'Darjeeling' has ambiguous meanings because it is used as a name for a district, a city, a tea and a tea producing area. In this thesis the district Darjeeling will be always named 'Darjeeling District', the city Darjeeling 'Darjeeling City', the tea Darjeeling 'Darjeeling Tea'. The term 'Darjeeling' is reserved for the tea producing area defined by the areas of the tea gardens which are listed by the Tea Board of India (Tea Board) as Darjeeling Tea Producers. The list of these gardens is in the appendix Table A.1.

\section{Geography}

The Darjeeling District is the northernmost district of the federal state of West Bengal in the north-eastern part of India. The district covers the foothills of the Himalaya orogen, also called the Lesser Himalaya, enclosed by Nepal in the west, Bhutan in the east and Sikkim in the north. Capital of Darjeeling District is Siliguri, which is situated in the 


\section{Characterization of Sampling Areas}

southern part of the district in the lowland at $120 \mathrm{~m}$ a.s.I. The most important hill station is Darjeeling City which is located $45 \mathrm{~km}$ north of Siliguri at $2100 \mathrm{~m}$ a.s.l. Other important cities are Kurseong (1500 m a.s.I.), halfway between Siliguri and Darjeeling City, Mirik (1600 $\mathrm{m}$ a.s.I.) west of Kurseong and Kalimpong (1150 M a.s.I.), $20 \mathrm{~km}$ east of Darjeeling City. Between Darjeeling City and Kalimpong the river Teesta cuts a deep, S-N striking valley (ca. $200 \mathrm{~m}$ a.s.I.) which divides Darjeeling District into a western and an eastern part.

The climate is influenced by the monsoon causing an alternate wet and dry season (Bhagat et al. 2010). The annual rainfall in Darjeeling varies between $2500-5000 \mathrm{~mm}$ (Bhagat et al. 2010). The average temperatures are strongly influenced by the altitude.

The southern part of Darjeeling District is characterized by alluvial fans resulting into plains only $120 \mathrm{~m}$ a.s.I. These lowland with recent sedimentation controlled by the synorogenetic processes consists in very young soils which are relatively coarse grained and rich in pebbles. The main part of the district lies within the Himalayan orogeny and is characterized by steep slopes $\left(20^{\circ}-40^{\circ}\right)$ and narrow deep valleys. The heavy rainfalls during monsoon season and huge deforested areas cause serious landslide hazards all over Darjeeling District (Froehlich and Starkel 1993). Due to the frequent landslides and heavy erosion soils are often reworked and seldom show typical soil layering.

Tea is cultivated in the plains of Darjeeling District as well as in the mountainous parts up to $2000 \mathrm{~m}$ a.s.l., but only the gardens located in the hilly areas, roughly above $500 \mathrm{~m}$ a.s.I., are listed by the Tea Board as Darjeeling Tea Producers (Figure 3.1, Table A.1). Tea plantations are covering $30-40 \%$, locally up to $80 \%$, while forest cover occupies $38 \%$ of the total area (Soja and Starkel 2007). Most of the tea gardens are located in the western part of Darjeeling. As most tea estates are situated in the hilly areas their topography is often dominated by steep slopes with only small areas of relative flatness. 


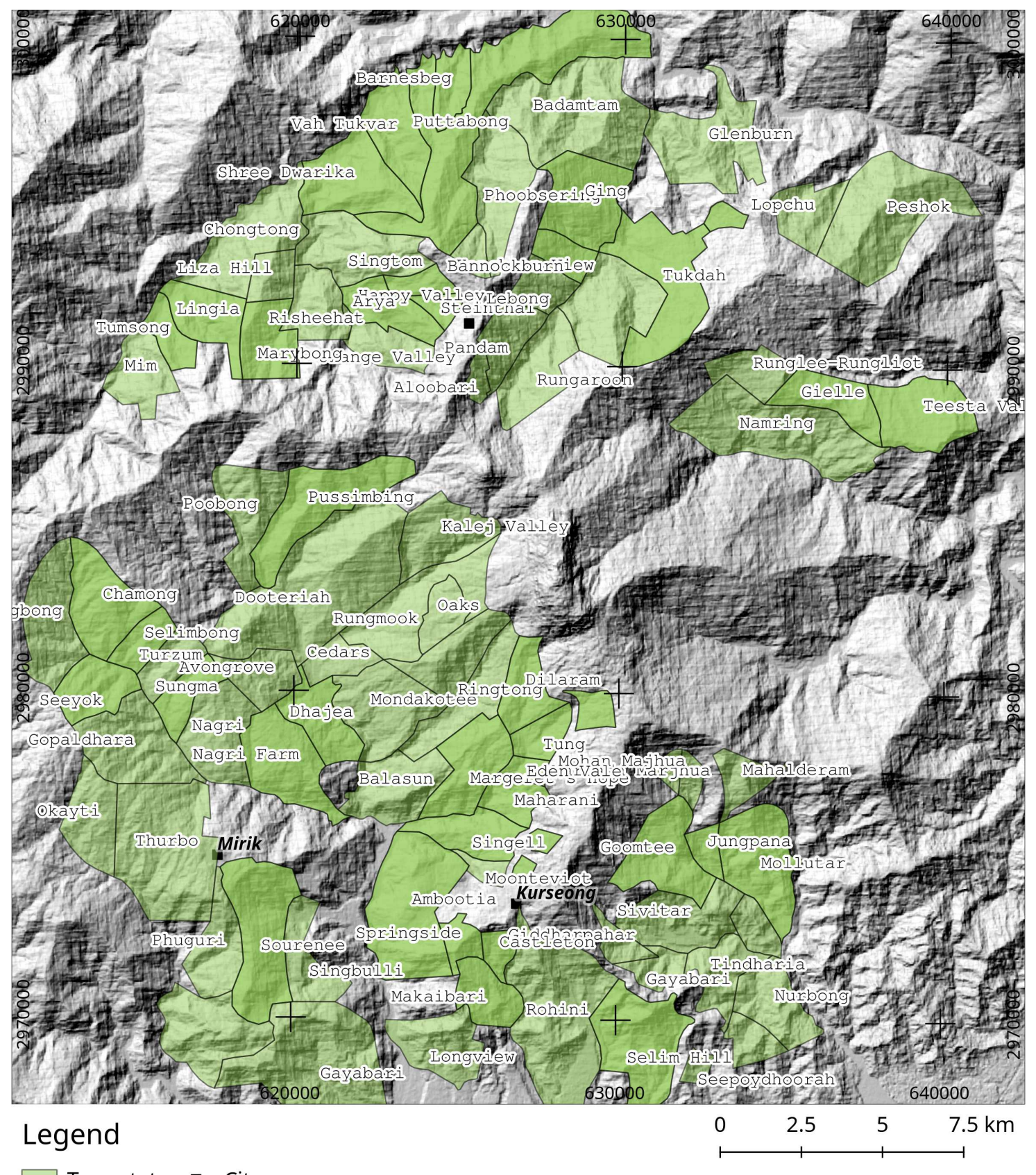

Figure 3.1.: Map of tea estates in Darjeeling District. DEM downloaded from http://bhuvannoeda.nrsc.gov.in, CartoDEM Ver.3, Toposheet No. G45K, tea estates redrawn after Indian Tea Association 1952, projection WGS 84 UTM Zone 45R Pseudo Mercator 


\section{Geology}

Darjeeling District is located at the southern-eastern part of the Himalayan orogen (Figure 3.2.

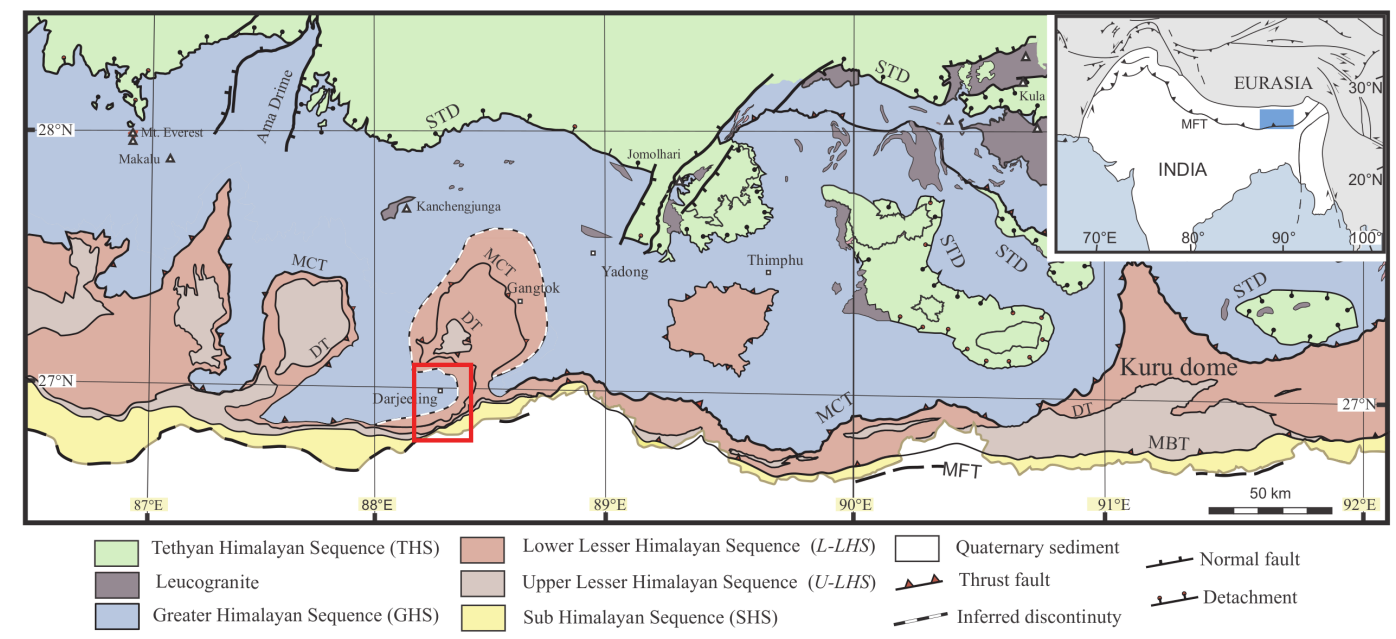

Figure 3.2.: Geological map of eastern Himalaya (modified from Acharyya et al. 2017). The red rectangular shows the area of Darjeeling District. The transition between LHS and GHS is drawn as a dashed line because the exact location of the MCT for this region is under debate.

The onset of the formation of the Himalayan orogen started approximately $48 \mathrm{Ma}$ ago with the collision of the Indian and the Eurasian Plate. During the orogeny the geological units now building the Himalayas got thrusted and imbricated mainly parallel to the continental margin in northern direction. This resulted into an approximately $2400 \mathrm{~km}$ long arc of the Himalayas range with all four domains being present the whole lateral extension.

Himalayas is generally divided into four geologic domains (Heim and Gansser 1939; Gansser 1964; Le Fort 1975; Yin 2006). These are from south to north: (1) the subHimalaya Sequence (sub-HS) of Cenozoic age, (2) the LHS, Palaeoproterozoic metasedimentary rocks which are non-fossiliferous low-grade metamorphic, (3) the GHS, Neoproterozoic and early Paleozoic gneisses and aplitic granites, and (4) the Tethyan Himalaya Sequence (THS), a marine, fossiliferous sequence.

Structurally, there are four major thrusts systems between the geologic domains. These are from south to north: The (1) Main Frontal Thrust (MFT) as the thrust placing the Neogene Siwalik of the sub-HS above the Quaternary deposits of the Alluvium (e.g. Gansser 1964; Yeats and Lillie 1991; K. A. Singh et al. 2001; Yin 2006); the (2) 
Main Boundary Thrust (MBT), defined by Heim and Gansser (1939) as the fault placing the LHS above the sub-HS; the (3) MCT, very roughly defined as a fault and thrust zone between the LHS and GHS (Heim and Gansser 1939; Gansser 1964; Le Fort 1975; Searle et al. 2008); and the (4) South Tibet Detachment (STD) juxtaposing the THS over the GHS (Burg and Chen 1984; Burchfiel et al. 1992; Ding et al. 2001; Yin 2006).

The definition and exact location of the MCT is subject of an ongoing debate (Ghosh 1956; Acharyya and Ray 1977; Lal et al. 1981; Ray 2000; Catlos et al. 2004; Dasgupta et al. 2004; Harris et al. 2004; Searle and Szulc 2005; Searle et al. 2008; Anczkiewicz et al. 2014; Mottram et al. 2014a). In the eastern Himalaya between the Daling group of the LHS in the footwall and high grade gneisses of the GHS in the hanging wall there is a zone of an inverted Barrovian sequence (Lal et al. 1981; Acharyya 1989; Dasgupta et al. 2004; Harris et al. 2004; Mottram et al. 2014a; Chakraborty et al. 2016), from some authors also referred to as 'zone of inverted metamorphism': The grade of metamorphism increases from the topographically lower areas with increasing altitude. This zone of inverted metamorphism has in the Darjeeling-Sikkim area a thickness of about $2000 \mathrm{~m}$ (Goscombe et al. 2006 and field observations). Based on field observation and the definition of Dasgupta et al. (2004), Goscombe et al. (2006) and Gupta et al. (2010) here, this zone will be addressed as the Main Central Thrust Zone (MCTZ) (Figure 3.3). This zone is either interpreted as being a sequence or partly a sequence in the hanging wall of the LHS, or that parts of the sequence are within the GHS implying that the MCT is located also within this zone (e.g. Goscombe et al. 2006).

Due to high weathering rates and the steep topography with frequently occurring landslides and heavy erosion rocks on the surface are often displaced. Huge blocks gradually sliding down the hills can be easily mistaken for outcrops and lead to misinterpretation of orientation of bedding and/or foliation as well as location of formations. Poor road conditions and dense forest areas or tea plantations makes it diffult to find reliable outcrops.

The geological map of Darjeeling compiled for this thesis is mainly based on field observations of 2011, 2012 and 2015 with the help of a compilation of petrology described by Mottram et al. (2014b) and Bhattacharyya and Mitra (2014) and maps from Mohan et al. (1989), citing Roy (1976) and Lal et al. (1981), Mukul (2000), citing Schwan (1980), and Goscombe et al. (2006). For field observation outcrops of recent road works, small streams and fresh landslides had been chosen if the total setting of the location indicated that the rocks are still originally placed. The map is shown in Figure 3.14. 


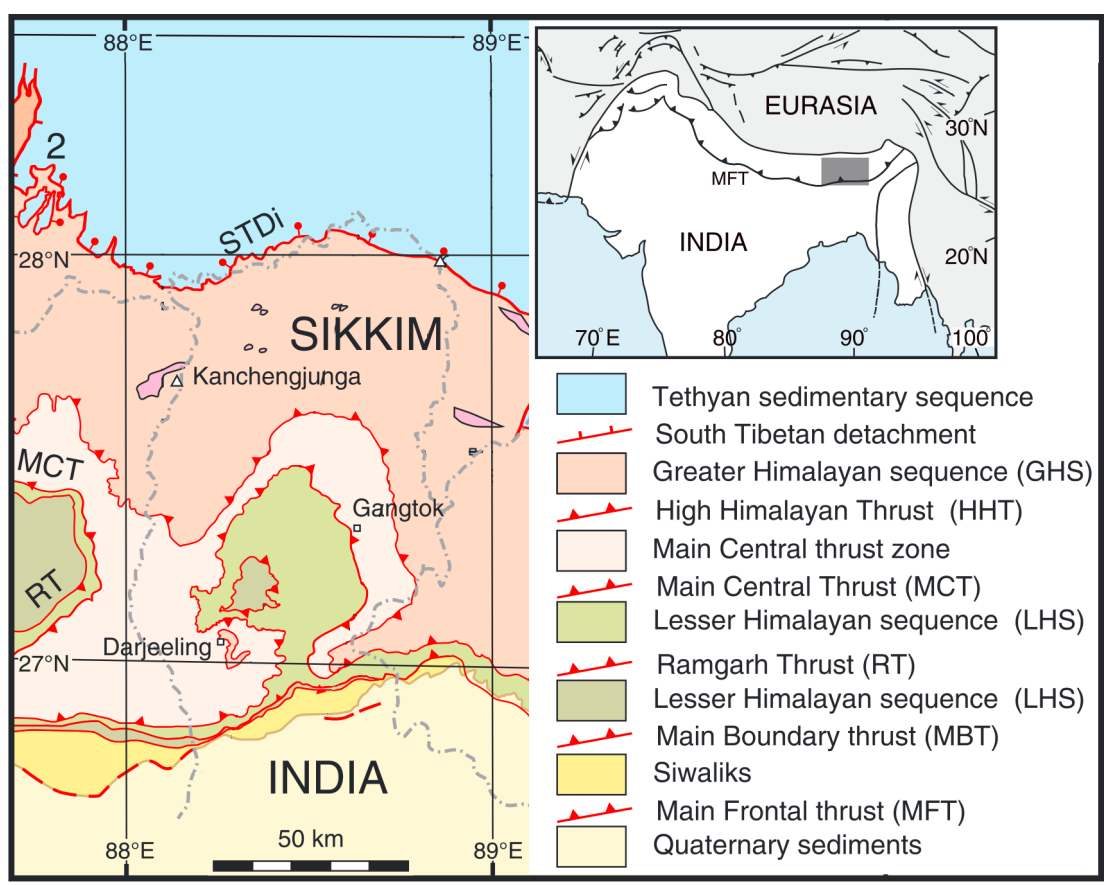

Figure 3.3.: Geological map of Darjeeling-Sikkim Himalaya (modified from Grujic et al. 2011 which is a map compiled of Schelling (1992), Goscombe and Hand (2000), Goscombe et al. (2006), Bhattacharyya and Mitra (2009), Kali et al. (2010), and Kellett et al. (2010)). The transition between the LHS and the GHS is marked as MCTZ interpretating the inverted Barrovian sequence as an $\sim 2000 \mathrm{~m}$ thick shear and thrust zone.

In Darjeeling District there are three to four geological units:

1. Quaternary: The valley of the river Teesta and the plains of the Darjeeling District are covered by alluvium and debris originating from the Himalaya hinterland as a result of the ongoing Himalayan orogeny.

2. Daling Group (LHS): In the topographically lower areas the Daling unit of the LHS is cropping out

3. Main Central Thrust Zone: The part of Darjeeling District which is situated in the the Himalayan orogenic belt is dominated by the sheared and thrusted package of amphibolite facies metapelite schists and gneisses of the MCTZ.

4. Kangchenjunga Gneiss (GHS) ?: Whether or not in the topographically highest peaks the Kangchenjunga Gneiss (GHS) (Kangchenjunga Gneiss) as a unit of the GHS is cropping out remains under debate. 
Quaternary The Quaternary sediments of Darjeeling Districts are built by the proximal parts of the Teesta Mega Fan, lobe $1 \mathrm{~A}$ and 3 (Chakraborty and Ghosh 2010), and the fans of the rivers (from east to west) Mahananda, Balason and Mechi. These alluvial sediments formed during the last 2.0 Ma in response to the thrusting of the Siwalik of the sub-HS along the MFT above the foreland sediments MFT (Powers et al. 1998; Wesnousky et al. 1999). Close to the mountain front there are coarse gravely piedmont sediments, along the rivers there are channel fills of gravel, sand and clay and related to the megafans there are pebbly, coarse to fine sand and mud sediments (Chakraborty and Ghosh 2010). The setting in Darjeeling District is generally dominated by sheetbraided sandy stream deposits (Tunbridge 1981; Chakraborty and Ghosh 2010, and field observations).

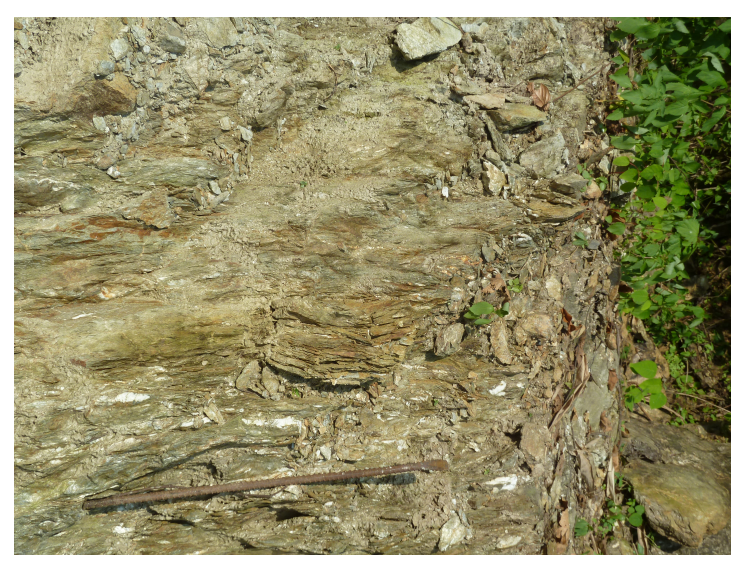

Figure 3.4.: Outcrop of Daling Group (LHS). See meter-sized stick on the right rim for scale.

N $27^{\circ} 06^{\prime} 16$, E $88^{\circ} 14 ' 30$, WGS 84

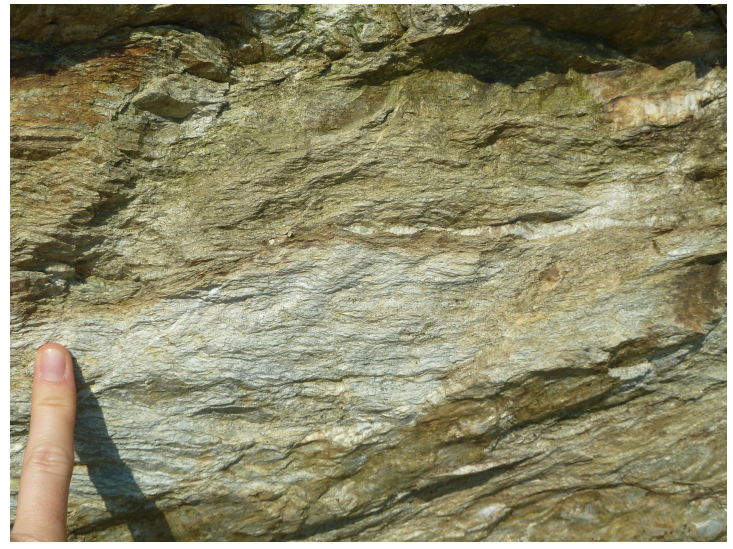

Figure 3.5.: Detail of outcrop from Figure 3.4. The greenschist grade phyllites have often a crenutaled cleavage.

N $27^{\circ} 06^{\prime} 16$, E $88^{\circ} 14^{\prime} 30$, WGS 84

Daling Group (LHS) The Daling Group (LHS) (Daling Group) is mainly composed of low grade metamorphic rocks such as greenish phyllite, massive quartzite, and (not exposed in Darjeeling District) greywacke and epidiorite (Acharyya et al. 2017 and references therein). In Darjeeling District it crops out as greenish colored, fine-grained mica-schist, interbedded with up to $15 \mathrm{~cm}$ thick quartzite layers. In some locations the rocks seem to be of relatively higher metamorphic grade which was determined by the occurrence of small garnets in the schist layers.

The Daling Group had been mapped in the north of Darjeeling District in the adjacent valley of the river Rammam north of Shree Dwarika Tea Estate, Chamong Tea (Shree Dwarika), in the west in the lower sections of Teesta Valley Exports Limited (Teesta 
Valley) and in the south in the lower section of Makaibari Tea Estates (Makaibari).

Main Central Thrust Zone The MCTZ is the major unit in Darjeeling District and nearly all tea estates are located on its gneissic rocks. The metamorphic grade of this zone of inverted metamorphism gradually changes from greenschist facies in the structural lowest levels up to amphibolite facies with abundant kyanite in the structural highest levels (Goswami 2005; Goscombe et al. 2006; Dasgupta et al. 2009; Mottram et al. 2014ab; Gaidies et al. 2015 and field observations). For this study this sequence had been subdivided into three metamorphic zones by isogrades of indicator minerals. These are from the structural lowest levels of the MCTZ to the higher levels a) the garnet-muscovite zone (Grt-Mu-zone), b) the staurolith zone (St-zone) and c) the kyanite zone (Ky-zone).

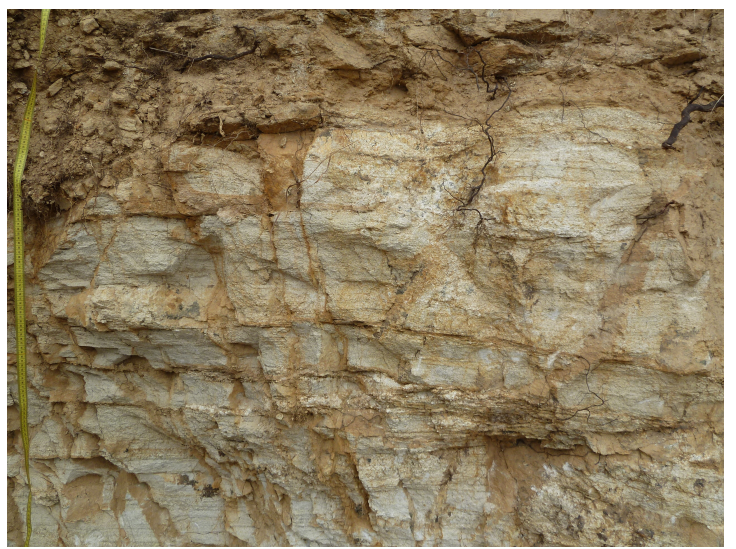

Figure 3.6.: Outcrop of metasandstone of garnet-muscovite zone with preserved sedimentary layering. See tapeline on left margin for scale.

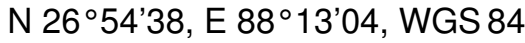

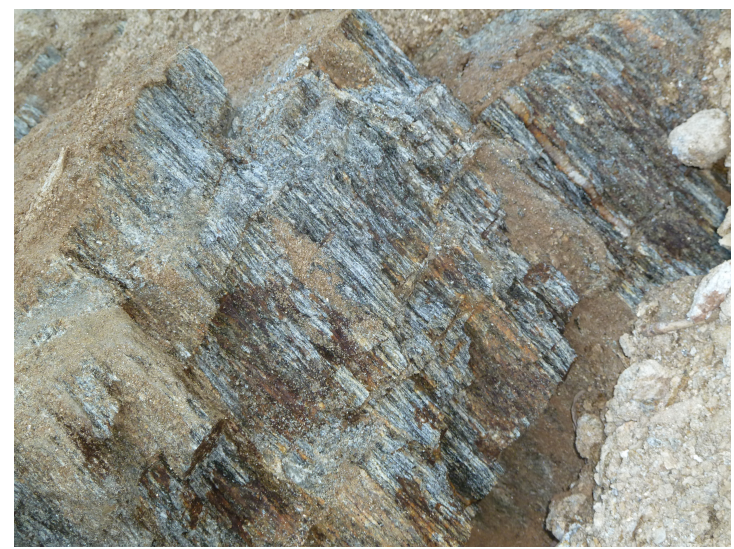

Figure 3.7.: Outcrop of schist of garnetmuscovite zone.

N $27^{\circ} 06^{\prime} 10$, E $88^{\circ} 14^{\prime} 43$, WGS 84

The structurally lowest Grt-Mu-zone consists of low- to middle-grade fine to medium grained garnet-staurolite schist intercalated with metasiltstones and occasionally metasandstones. The original sedimentary layering is well preserved in metasiltstones and metasandstones (Figure 3.6), while metapelitic layers show a well developed schistosity defined by aligned mica and chlorite minerals (Figure 3.7). The schistosity is planar towards the footwall of the Grt-Mu-zone and changes to more crenulated cleavage in structurally higher parts. In layers of undulated micaceous cleavage occur deformed quartz lenses and fine to middle grained garnets (Mottram et al. 2014b). The thickness of the layers reaches from several millimeter up to meter scale.

The mineral content varies strongly depending on the original sedimentary rocks. The 
main minerals are: quartz \pm biotite \pm muscovite \pm garnet \pm staurolite \pm chlorite \pm feldspar and accessories. In a example sample of Mottram et al. (2014b) located on the ridge between Gielle Tea Estate (Gielle) and Namring Tea Estate (Namring) (Location N $27^{\circ} 00^{\prime} 21$, E $88^{\circ} 10^{\prime} 22$, WGS 84) the mineral composition is $50 \% \mathrm{Mu}, 30 \% \mathrm{Qu}$, $10 \% \mathrm{Bi}, 3 \% \mathrm{Grt}, 2.5 \% \mathrm{Chl}$ and $1.7 \% \mathrm{St}$. In an example sample of Bhattacharyya and Mitra (2014) located in Sivitar Tea Estate, Ambootia Tea Group (Sivitar) (Location N $26^{\circ} 52^{\prime} 45$, E $88^{\circ} 20^{\prime} 05$, WGS 84) the mineral composition is $47 \% \mathrm{Qz}, 20 \% \mathrm{Fsp}, 20 \% \mathrm{Bi}$, $8 \% \mathrm{Grt}$ and $5 \% \mathrm{Mu}$.

The garnet minerals have a grain size of $1 \mathrm{~mm}$ up to maximum $3 \mathrm{~mm}$. The staurolite occurs as fine, max. $0.8 \mathrm{~cm}$ long needles oriented along the foliation (Figure A.2).

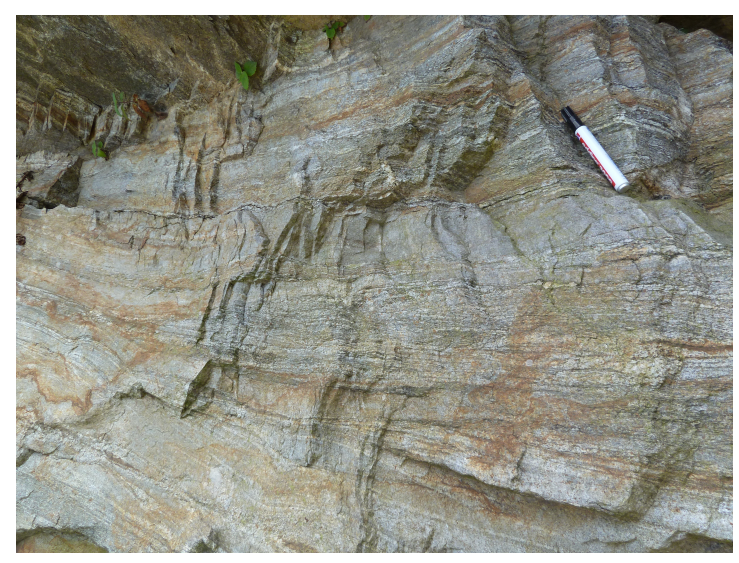

Figure 3.8.: Outcrop of staurolith zone along the street between Tumsong and Lingia. N $27^{\circ} 01^{\prime} 59$, E $88^{\circ} 11^{\prime} 10$, WGS 84

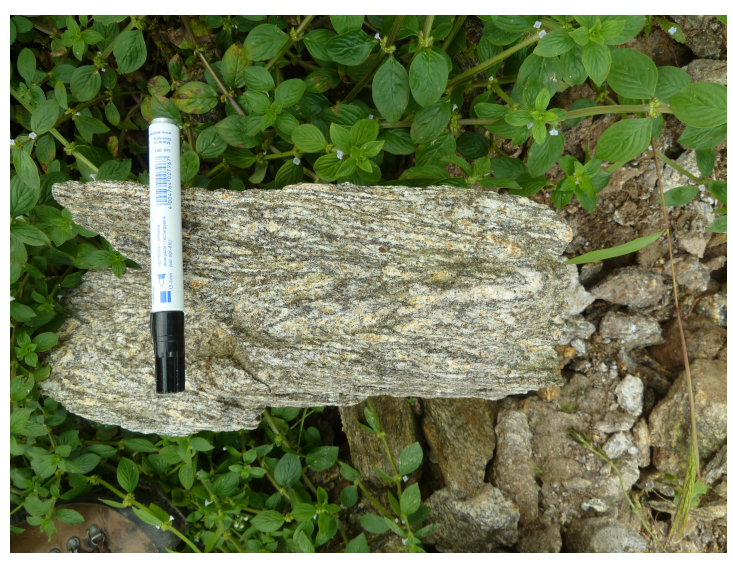

Figure 3.9.: Hand specimen from outcrop in Tumsong. The macrostructure of the rock shows small scale folding. The gneiss includes small, ubiquitous garnet grains, visible by brownish weathered remnants. $\mathrm{N} 27^{\circ} 02^{\prime} 16, \mathrm{E} 88^{\circ} 10^{\prime} 30$, WGS 84

The St-zone begins with the occurrence of middle grained staurolite minerals, i.e. when the $\{001\}$ plane of staurolite grains is visible with the eye. The zone consists of medium to coarse grained garnet-staurolite(-kyanite) gneisses with very distinct foliation (Figure 3.8). The gneisses consist of crenulated mica-rich foliation bands consisting mainly of biotite. These micaceous foliated bands with a well defined foliation are intercalated with quartzitic layers (Figure A.4). There are frequently elongated deformed porphyroclasts of quartzo-feldspathic material. In some localities the main foliation is superimposed by a second schistosity resulting into a crenulated cleavage in foliated bands with high mica content (Figure A.3) or, in layers of higher content in quartzite and feldspar, folding of the original layering (Figure 3.9. 


\section{Characterization of Sampling Areas}

The main minerals are: quartz \pm garnet \pm feldspar \pm staurolite \pm biotite \pm muscovite \pm kyanite and accessories. In an example sample of Mottram et al. (2014b) located on the south side of Tukdah Tea Estate, Chamong Tea (Tukdah) (Location N 2701'58, E $88^{\circ} 20$ '30, WGS 84) the mineral composition $40 \% \mathrm{Qz}, 20 \% \mathrm{Plg}, 19 \% \mathrm{Mu}, 10 \% \mathrm{Bi}, 5 \%$ Grt, $5 \%$ St and 1\% Ky. Quartz and feldspar grains show a shape preferred orientation within the foliation. Garnets are ubiquitous and isomorph developed with grain sizes up to $1 \mathrm{~cm}$. Generally the abundance of muscovite is notably decreased compared to the Grt-Mu-zone and the gneisses of the St-zone have a darker aspect. The staurolite and the higher content in biotite is probably a result of the breakdown of light micas and chloritoids (Deer et al. 1992). In more iron-rich layers of the schist this would be

Chloritoid $+\mathrm{Qz} \rightleftharpoons \mathrm{Gr}+\mathrm{St}+\mathrm{H}_{2} \mathrm{O}$

and in more magnesium-rich layers of the schist this would be

$$
\text { Chloritoid }+\mathrm{Chl}+\mathrm{Mu} \rightleftharpoons \mathrm{St}+\mathrm{Bi}+\mathrm{Qz}+\mathrm{H}_{2} \mathrm{O}
$$

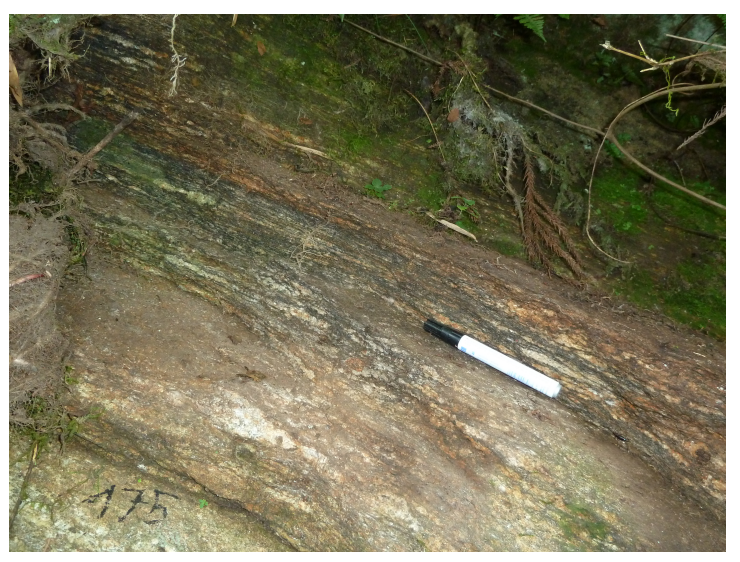

Figure 3.10.: Outcrop of kyanite zone along a street. Block is probably slightly displaced which results into a rotated foliation compared to original setting. N 265 $58^{\prime} 35$, E $88^{\circ} 09^{\prime} 02$, WGS 84

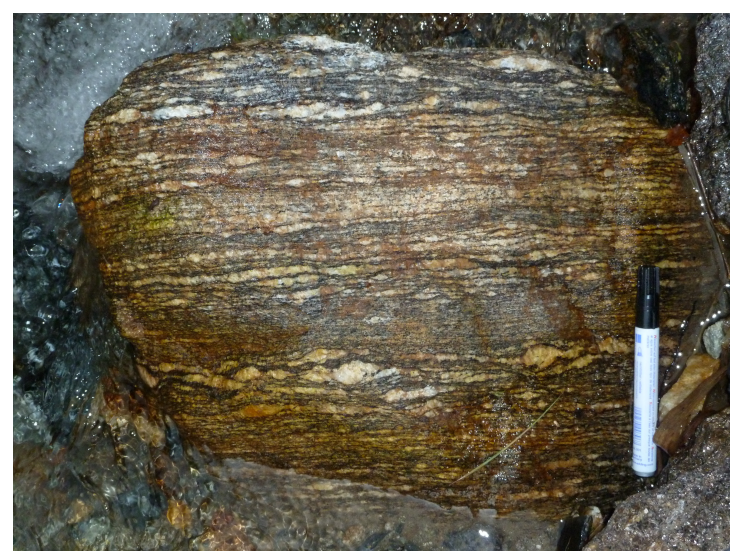

Figure 3.11.: Boulder of kyanite zone in a stream $3.5 \mathrm{~km}$ distance to uppermost point of the ridge. The reddish colors result from iron-oxides and weathered garnets. In the upper part of the boulder kyanites can be seen by bright blueish colors).

$\mathrm{N} 26^{\circ} 58^{\prime} 56, \mathrm{E} 88^{\circ} 08^{\prime} 34$, WGS 84

The Ky-zone starts with the occurrence of kyanite minerals with grain sizes up to $1 \mathrm{~cm}$. The zone consists of fine to coarse grained kyanite-biotite-staurolite gneisses which are partly folded (Figure A.5). In most outcrops the gneisses show a strong foliation defined by the general orientation of the quartz ribbons of $\mathrm{cm}$-scale to $\mathrm{dm}$-scale in a dark biotite(staurolite) matrix (Figure 3.10 and Figure 3.11. Dark, biotite-rich layers are alternating with gray layers of fine-grained quartzo-feldspathic material, porphyroblasts of kyanite 
and rare porphyroclasts of feldspars. In leucosomes or more felsic layers the kyanite, quartz and feldspar content is significantly higher. The main minerals are: biotite \pm kyanite \pm garnet \pm quartz \pm feldspar \pm staurolite \pm sillimanite and accessories. In an example sample of Mottram et al. (2014b) located on the ridge $5 \mathrm{~km}$ east of the

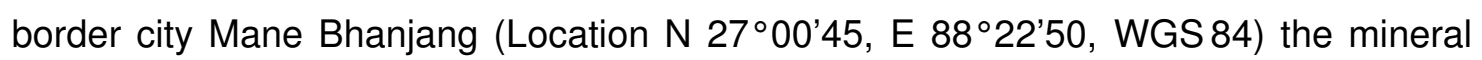
proportions are $40 \% \mathrm{Qz}, 17 \% \mathrm{Plg}, 17 \% \mathrm{~K}-\mathrm{Fsp}, 17 \% \mathrm{Bi}, 3.5 \% \mathrm{Grt}, 3 \% \mathrm{Ky}$ and $0.5 \%$ Sil. Like in the St-zone garnet grains are ubiquitous with varying grain size distribution, but may reach up to $5 \mathrm{~cm}$ grain size in $\mathrm{m}$-scale quartz lenses. Muscovite is (nearly) not present in the gneisses, probably to further breakdown due to higher metamorphic peak conditions.

Kangchenjunga Gneiss In the topographically highest areas of Darjeeling District, in Darjeeling City and around Tiger Hill, outcrops show amphibolite grade gneisses. They are very poorly exposed and often highly weathered. The gneisses are characterized by elongated ( $\mathrm{cm}$ - m scale), undulating and deformed felsic lenses of varying composition embedded in grayish to dark-grayish, melanocratic matrix. The coarse grained gneiss appears (locally) as Augengneiss. There are meter-sized quartzite lenses with garnets up to $6 \mathrm{~cm}$ (Figure 3.13).

Some researchers describe this amphibolite grade gneisses as a klippe of the Kangchenjunga Gneiss of the GHS. This could presumably only be confirmed by dating the gneisses which has not been accomplished yet.

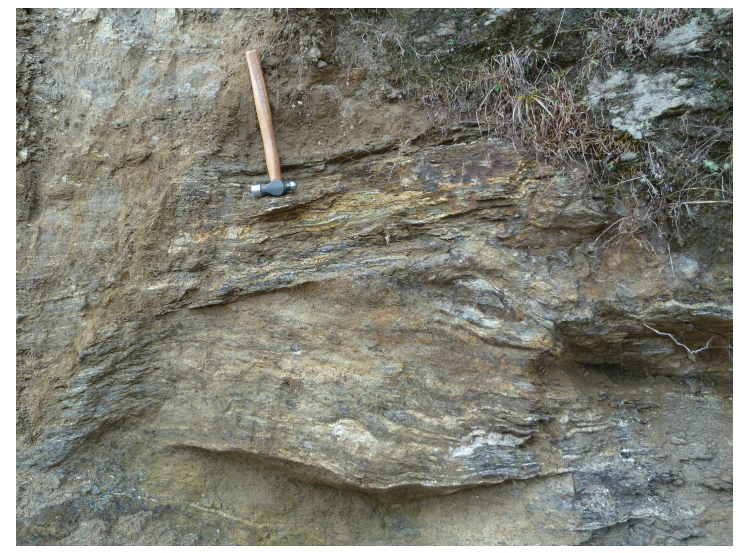

Figure 3.12.: Outcrop of amphibolite gneiss in Darjeeling city.

N $27^{\circ} 00^{\prime} 53$, E $88^{\circ} 15^{\prime} 07$, WGS 84

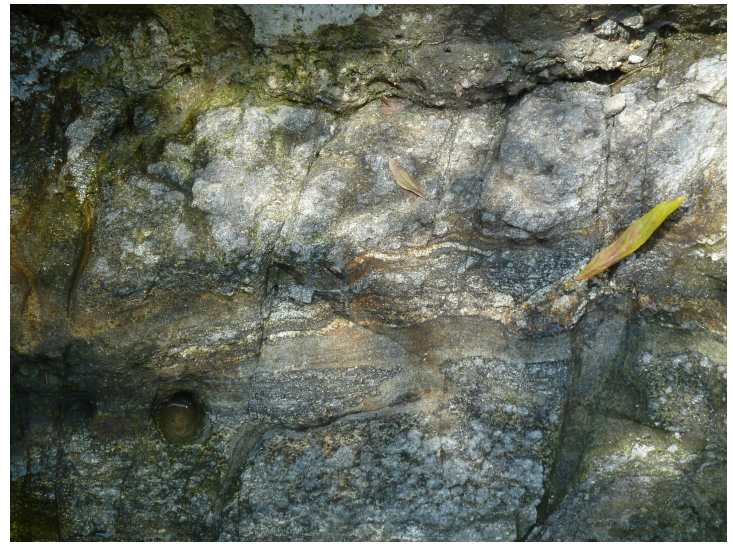

Figure 3.13.: Outcrop of amphibolite gneiss in Darjeeling city. On the left lower corner of the foto the hole is caused by weathered garnet (for unweathered garnet see Figure A.8. See leaf for scale. N $27^{\circ} 01^{\prime} 34$, E $88^{\circ} 15^{\prime} 45$, WGS 84 
Structure The foliation of the Barrovian sequence in Darjeeling is slightly dipping to NE (Gupta et al. 2010 and field observation). Bhattacharyya and Mitra (2014) describes the leading edge of the thrust between MCTZ and Daling Group as a $\sim 2 \mathrm{~m}$ thick NNEdipping greenschist facies phyllonite zone with the foliation dipping $35^{\circ}$ towards $037^{\circ}$ (Location N 2652'45, E 88²0'05, WGS 84). This is about parallel to the observed foliation for the whole region. The contact between MCTZ and Daling Group is in most locations associated with a topographic break.

In the northwestern part of Darjeeling District the valley stretching between the village Mane Bhanjang and the village Jorethang is defined by a major strike-slip fault zone: In Tumsong the gneisses facing the valley exhibit a nearly vertical dipping foliation striking parallel to he valley NE-SW. 


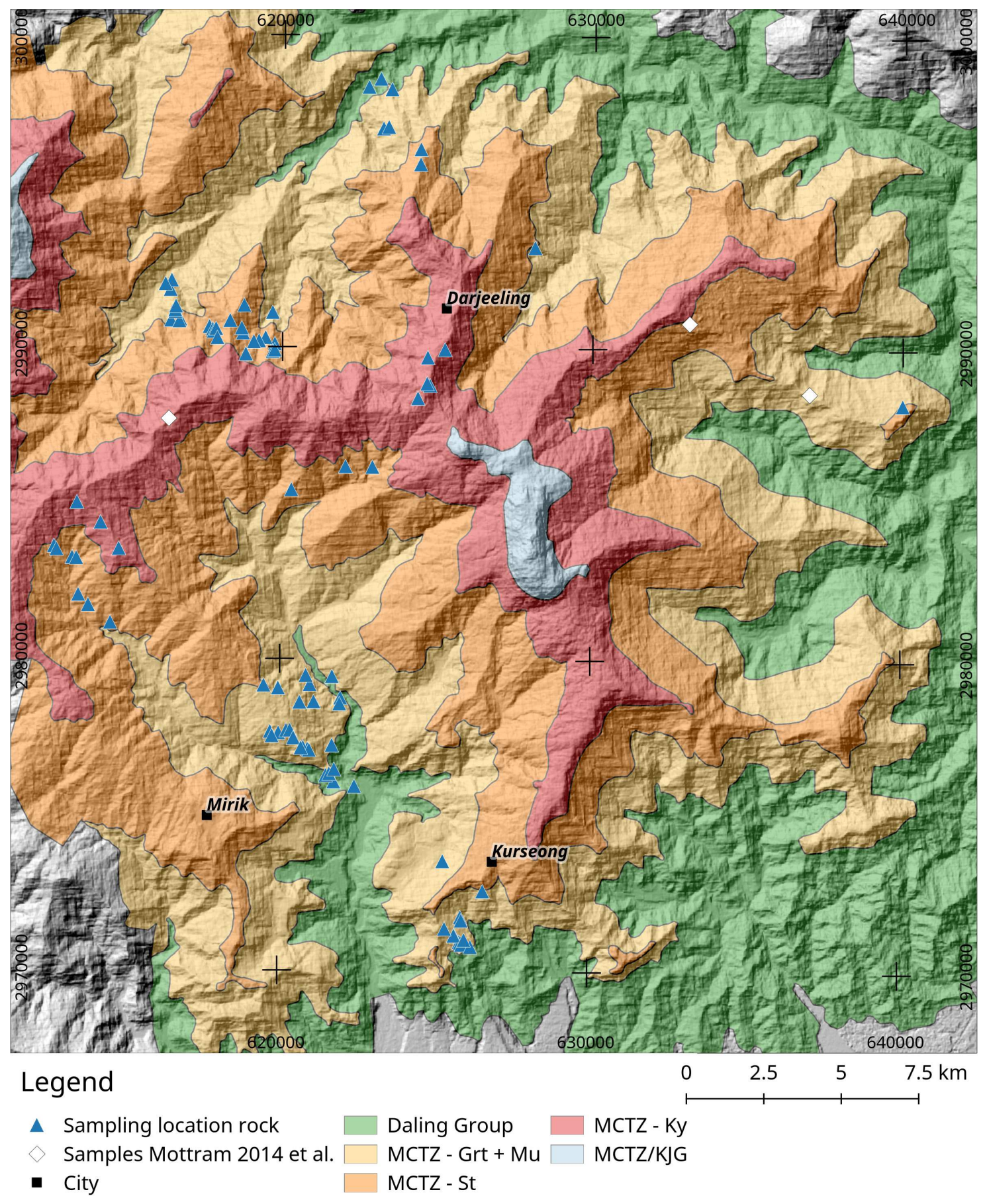

Figure 3.14.: Map of geological units in Darjeeling District. DEM downloaded from http://bhuvannoeda.nrsc.gov.in, CartoDEM Ver.3, Toposheet No. G45K, projection WGS 84 UTM Zone 45R Pseudo Mercator 


\section{Characterization of Sampling Areas}

\section{Soils}

The soils of Darjeeling hill areas are mainly sandy-silty regoliths showing notable variations, depending on elevation, degree of slope and geological source material (Soja and Starkel 2007; Governement of West Bengal 2012 and field observations). They do usually not exceed 1-2 $\mathrm{m}$ thickness and are very permeable with high infiltration rates (Froehlich and Starkel 1993). Generally the soils have very low $\mathrm{pH}$ values and are predominantly reddish till brownish in color: Reddish color can mainly be found in soils with greenschist facies gneisses or sandstone as source rock. Typical soils developed of the greenschist facies gneisses are found for example north of Darjeeling in Shree Dwarika, or north of Mirik, in Nagri Farm Tea Estate, Chamong (Nagri Farm) and Dhajea Tea Estate, Chamong Tea (Dhajea), or in the south of the district, in Castleton Tea Estates, Goodrike (Castleton) and Makaibari: They are deep red to orange red and have locally a very high content in light micas. Brownish colors developed at amphibolite facies gneisses, schist or shale or/and with high organic content in soils, like in forests (Governement of West Bengal 2012; Kundu et al. 2012 and field observations). Soil developed of amphibolite facies gneisses are found for example in western part of Darjeeling District, in topsoil sample (topsoil), Marybong or Chamong Tea Estate, Chamong Tea (Chamong), or in the southern part of the district, in Margeret's Hope Tea Estate, Goodrike (Margeret's Hope), Singell Estate (Singell) or Castleton. Also the grain size of soil components is regulated by the source rock: Units with high content of quartzite and sandstones produce very coarse grained, skeletal soils. Units of mica schists or garnet schists like the Daling series, soils become sandy with high content in coarse particles. On the the low-grade metasediments like the Siwaliks soils become silty and clay rich (Figure 3.15. 


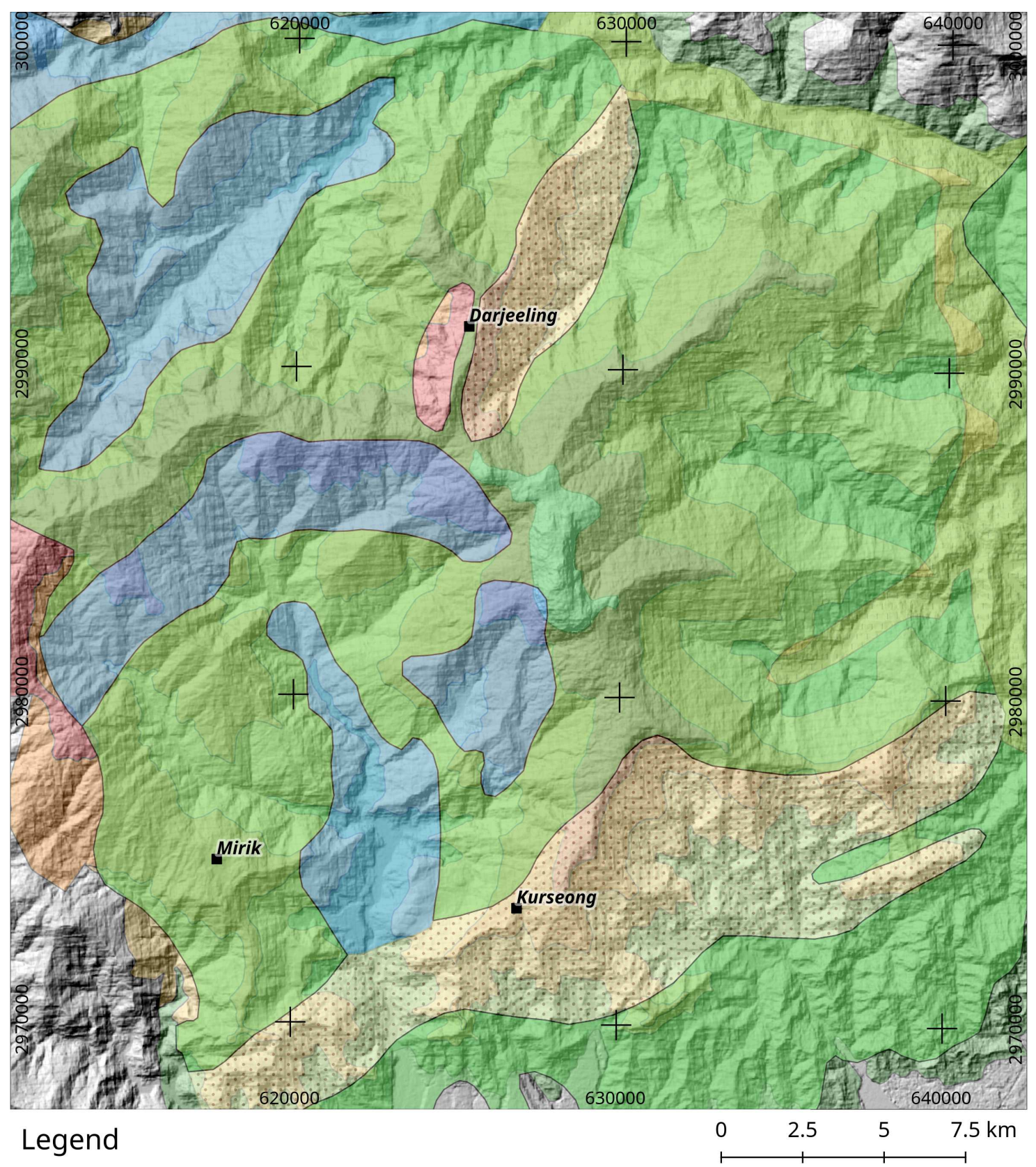

- City Loamy skeletal, lithic Udorthents Coarse loamy, Udorthents
Fine loamy, Dystrochrepts

Loamy skeletal, Haplubrepts

Fine loamy, Fluventic Dystrochrepts

Figure 3.15.: Map of soils in Darjeeling District. Map of tea estates in Darjeeling District. DEM downloaded from http://bhuvan-noeda.nrsc.gov.in, CartoDEM Ver.3, Toposheet No. G45K. Soil map redrawn after Seghal (1991) (Selvaradjou et al. 2005). Projection WGS 84 UTM Zone 45R Pseudo Mercator 


\subsubsection{Assam}

The term 'Assam' has ambiguous meanings because it is used as a name for the federal state in India, a type of tea plant, a tea and a tea producing area. In this thesis the federal state of India will be named 'Assam State', the tea plant ' $C$. sinensis var. assamica', the tea Assam 'Assam Tea'. The term 'Assam' is reserved for the tea producing area which covers the broad plains of the Brahmaputra river in Assam State.

\section{Geography}

Assam is a federal state of Republic of India situated in the north-eastern part of the country with an area of approximately $79000 \mathrm{~km}^{2}$. The state covers the valley of Brahmaputra river in the north and the Barak river in the south. The Brahmaputra valley is defined by the eastern Himalaya in the north, the Mishmi Hills in the north-east, the Nagar-Patkai range in the south-east and the Shillong plateau with the Mikir Hills in the south. The capital of Assam State is the city Dispur which lies within the city region of Guwahati in central Assam State. Along the river Brahmaputra there are the cities Tezpur, $130 \mathrm{~km}$ east of Guwahati, Jorhat, $160 \mathrm{~km}$ east of Tezpur, Dibrugarh, $120 \mathrm{~km}$ north-east of Jorhat, and Tinsukia, $50 \mathrm{~km}$ east of Dibrugarh. At the border between Assam State and the federal State Arunachal Pradesh lies the city Namsai, which is located $65 \mathrm{~km}$ north-east-north of Tinsukia. Tea is produced mainly in the Cachar region in the southern part of Assam State and along the valley of the Brahmaputra river in the northern part. Because all samples originate from the region around Tinsukia and Namsai the following description will focus on this tea producing area in Upper Assam (Figure 3.16).

In the Brahmaputra valley the annual precipitation varies from 2100 to $2900 \mathrm{~mm}$ (Bhagat et al. 2010). During the summer months the precipitation pattern is characterized by the south-west monsoon while the winter is the dry season. The heavy precipitation during monsoon causes surplus water and the regularly occurrence of floods. During the dry season occasionally the tea producing areas suffer water deficit due to very low precipitation (Bhagat et al. 2010).

\section{Geology}

The Upper Assam Basin is structurally a basin between two convergent margins (Raju and Mathur 1995). It is enclosed in the north by the eastern Himalayas through the MFT, in the east by the Mishmi Hills through a thrust and in the south by Patkai Hills through 
the Naga thrust (Lahiri and Sinha 2012 and references therein). The interpretation of seismic data acquired by the oil companies reveals that the basin is disturbed by several NE-SW trending faults. The upper layers of the basin fillings are alluvial sediments from the mountain-fed river system of the Brahmaputra river (Sinha and Friend 1994). The sources of the alluvial material is mainly the tectonically active Himalaya (Singh 2006) and consequently the parent rocks of the alluvium include igneous, metamorphic and sedimentary rocks. The mineralogy of the alluvium is dominated by resistant minerals, like quartz and the comparably higher amount of zircons, while easier weathering minerals like mica, feldspar and chlorites are notably decreased, especially in older parts of the alluvium due to the high rate of surface weathering (Chakravarty et al. 1979)

\section{Soils}

Soils in Assam are mainly alluvium-derived soils which are moderately developed with occasionally minor content of clay in weak argillic horizons (Baruah et al. 1983; Chakraborn et al. 1984; Chakravarty et al. 1985; Pal et al. 2001). They are generally acidic and rich in organic carbon with the concentration of the nutrients being fairly high in the upper soil layers (J. Singh et al. 1995). The dominant clay mineral is illite with chlorite and/or kaolinite as a minor phases (Chakraborn et al. 1984).

In Upper Assam Karak et al. (2015a) carried out a study about soils in tea estates with a total of 991 samples from 15 tea estates (Figure A.9): The authors classified the soils as Oxisols (Histic Haplaquox) according to the USDA classification. The $\mathrm{pH}$ values, measured with a soil:water ratio of $1: 2.5$, range in median between 4 and 5 , with a minimum at 3.6 and a maximum at 6.8 . Soils with a pH higher than 5.6 are probably less acidic due to water-logging or application of lime. Litterfall and regular pruning of tea bushes and shade trees in combination with weak decomposition leads to relatively high contents of Total Organic C (TOC) in most analyzed tea estates (Karak et al. 2015a).

In the visited tea estates (Maud/Sewpur Tea Estate, Chamong Tea (Maud), Sankar Tea Estate, Division Gohain, Chamong Tea (Gohain), Platzhalter (Platzhalter)) the grain size distribution of the soils is sandy to silty and well drained. Additionally meter-deep drainage trenches in the tea gardens help to prevent water-logging. On a km-scale the soils are very homogeneous. But there is a strong variability in grain size within $\mathrm{m}$-scale due to old channels and benches of former tributaries of Brahmaputra river (Figure 3.18) while the regularly occurring floods erode and re-sediment material in the topsoil layer. 


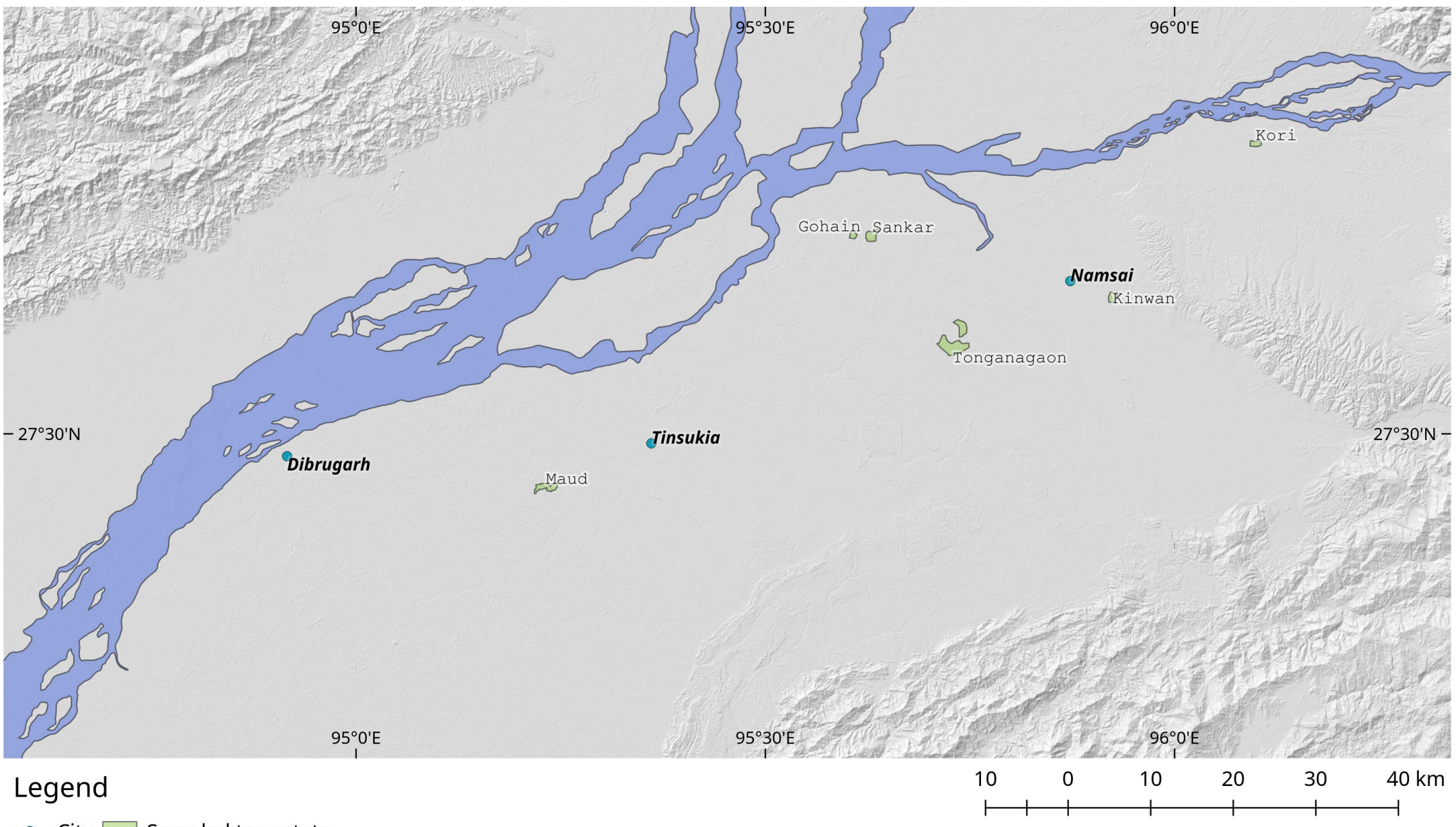

- City $\square$ Sampled tea estate

Figure 3.16.: Map of tea estates in Assam State and Arunachal Pradesh. DEM downloaded from http://bhuvan-noeda.nrsc.gov.in, CartoDEM Ver.3, Toposheets No. G46E and G46F. Tea estate locations and river redrawn after satellite image 2018 (Google satellite), projection WGS 84 UTM Zone 46R Pseudo Mercator 


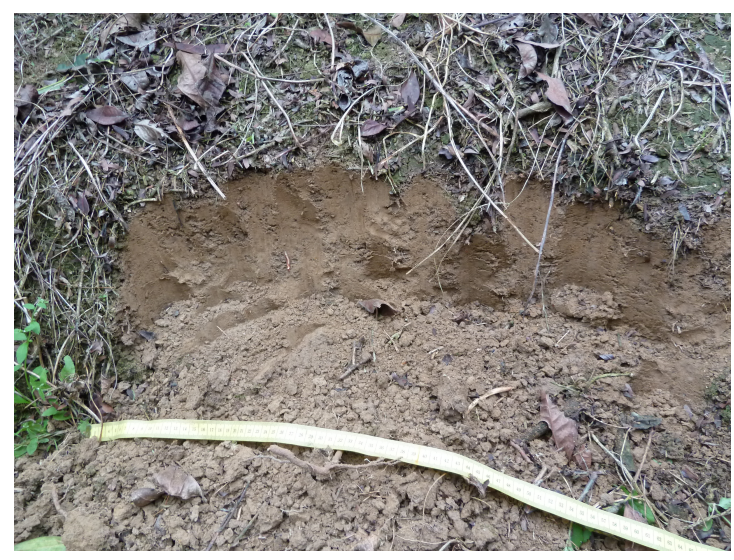

Figure 3.17.: Soil profile in Gohain.

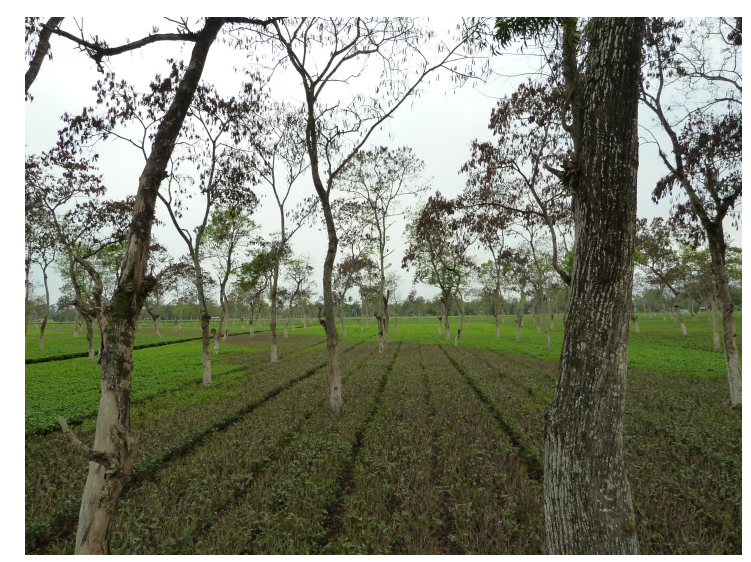

Figure 3.18.: Tea plantation in Gohain. Tea bushes, which are still in winter dormancy are without young shoots and appear as dark green, while flushing tea bushes are light green colored. The time offset of the 1st flush is caused by grain size variability of soils which leads to different water availability for tea bushes. 


\subsubsection{Nilgiris}

The term 'Nilgiris' has ambiguous meanings because it is used as a name the Nilgiris District in the federal state Tamil Nadu in South India and a tea producing area. In this thesis the term 'Nilgiris' is reserved for the tea producing area which covers the southern area of the Nilgiri Hills.

\section{Geography}

The Nilgiris district is the westernmost district of the federal state Tamil Nadu in the South India. The neighboring federal states are Karnataka to the North and Kerala to the West. The main cities are Udagamandalam (Ooty) in the central part, Coonoor in the southern part, Kotagiri lying $10 \mathrm{~km}$ South-West of Coonoor and Gudalur $25 \mathrm{~km}$ North-East of Ooty. The district is situated within the Nilgiri Massif which is located at the juncture of the Eastern and Western Ghats mountain ranges (Samuel et al. 2014). North of the the Nilgiri Massif lies the Mysore plateau, and towards the North-West its bounded to the continuation of the Western Ghats by high scarps (Rajakumar et al. 2007; Valdiya and Sanwal 2017). In the South-West and the South-East the Nilgiri mountains rise abruptly from the surrounding plains from approximately $300 \mathrm{~m}$ a.s.l. to plateaus of hilly ranges and broad valleys between $1000 \mathrm{~m}$ and $2000 \mathrm{~m}$ a.s.I. (Valdiya and Sanwal 2017). The prominent peaks rise up to $2500 \mathrm{~m}$ to $2600 \mathrm{~m}$ (Seshagiri et al. 1982).

The climate of the district is dominated by the South West Monsoon and the North East Monsoon. The total annual rainfall ranges between $5000-2500 \mathrm{~mm}$ in the western regions, 2000-1500 $\mathrm{mm}$ in the eastern and southern regions and in average 1200$900 \mathrm{~mm}$ (Caner et al. 2007). Due to the elevation the temperatures are moderate with a mean minimum of $5^{\circ} \mathrm{C}$ in winter and $24^{\circ} \mathrm{C}$ in spring and summer (Caner et al. 2007).

\section{Geology}

The Nilgiris Massif is an Archaean granitoid-gneissic crustal domain of the extensive South Indian granulite terrain (M. M. Raith et al. 1999, and references therein). It is bounded in the north by the Moyar shear zone and in the south by the Bhavani shear zone (M. M. Raith et al. 1999; Samuel et al. 2014, and references therein). The Nilgiris Massif is predominantly constituted of hypersthene-bearing, medium-coarse grained enderbitic to charnockitic rocks (M. Raith et al. 1988; C. Srikantappa et al. 1988). The charnockites are of intermediate, tonalitic to granodioritic, composition (Rajesh and Santosh 2004, and references therein). The enderbites are described as garnetiferous and 
non-garnetiferous layered gneisses (M. Raith et al. 1988; Rajesh and Santosh 2004, and references therein). The garnetiferous intermediate charnockite exhibit calc-alkine characteristics and are geochemically close to the tholeiitic series (Rajesh and Santosh 2004). The Nilgiris Massif is dissected by frequently occurring, ENE-WSW trending pyroxene-bearing doleritic dykes. There are rare metasedimentary intercalations of amphibolite facies, including quartzites and gneisses, and granulitic xenoliths of mafic composition (C. Srikantappa et al. 1986; M. M. Raith et al. 1999; Rajesh and Santosh 2004).

The sampled tea gardens are all located within the area which is predominantly composed of charnockites. At the eastern slopes of the Nilgiri Massif two outcrops are describes by Samuel et al. (2014): Close to Kil Kotagiri Tea Estates (Kil Kotagiri), about $1 \mathrm{~km}$ north of the garden, the outcrop consists of mafic granulite of clinopyroxene + garnet + plagioclase + amphibole. About $5.5 \mathrm{~km}$ of north of Havukal Tea Estate (Havukal) and $6 \mathrm{~km}$ north of Kil Kotagiri a charnockitic outcrop is described to contain quartz + plagioclase + kalifeldspar + orthopyroxene + garnet \pm biotite. In the central part close to the garden Highfield Tea Estate (Highfield) charnockite of the same composition is cropping out. About $7 \mathrm{~km}$ north of Coonoor the composition of the charnokites are described to be slightly different with quartz + plagioclase + kalifeldspar + garnet + biotite \pm orthopyroxene. They are intercalated with the mafic granulites.

\section{Soils}

Road cuts and boreholes reveal that the weathering profile of soils in the Nilgiris ranges from less than a meter to $20 \mathrm{~m}$, with a maximum of $32 \mathrm{~m}$ (Seshagiri et al. 1982; Jaiswal et al. 2011) The sub-tropical climate with its intense weathering altered the gneissic source rocks into lateritic clays, clayey loams and loams (Natarajan et al. 1997). The weathered zone between the clayey soils and the source rock is approximately one meter thick (Parthasarathy and Vaidyanathn 1974). Some areas reveal also patches of red sandy soils (Parthasarathy and Vaidyanathn 1974).

The tea plantations are mainly based on sandy loam to sandy clay loam which are very deep and well drained (Natarajan et al. 1997). The topsoils reach down to 0.3-1 m and the subsoils have a thickness between 3-4 m (Natarajan et al. 1997, and personal communication). They are characterized by very low $\mathrm{pH}$ around 4 (personal communication) and have generally a poor nutrient status. In the eastern parts the tea soils are classified as Inceptisols according to the USDA classification, and in the western parts as Ultisols (Natarajan et al. 1997). 


\subsection{Brazil}

\subsubsection{São Paulo}

\section{Geography}

São Paulo is a federal state in the east central part of Brazil with its south-eastern border being the coast line. North and north-west of São Paulo lie the federal states Minas Gerais and Rio de Janeiro, north-east the federal state Mato Grosso do Sul and south-east neighboring lies the federal state Paraná. The state capital and the largest municipality is São Paulo City, located in the south eastern part close to the coast. Tea is cultivated in two communities ca. $180 \mathrm{~km}$ south of São Paulo City, i) south of the city Registro and ii) close the village São Miguel de Arcanjo.

Registro is situated in the Vale do Ribeira, $30 \mathrm{~km}$ west of the coast. The average elevation is 25 - $30 \mathrm{~m}$ a.s.I. and the morphology is characterized by the alluvial plains with dense ramification of the river Ribeira. The tea fields are planted on slightly elevated terraces of clay-sandy sediments, locally enriched with gravels (J. L. S. Ross n.d.). Being close to the coast with very low topographical gradient the area is regularly affected by floods. The climate, subtropical and with generally high humidity, is influenced by Registro's location between coast and the coastal range to its north-west.

São Miguel de Arcanjo is situated $150 \mathrm{~km}$ west of coast and inside the coastal range (Cinturão Orogênico do Atlântico) at 700 - $900 \mathrm{~m}$ a.s.l. The neighboring cities are Registro, $70 \mathrm{~km}$ south, Capão Bonito, $40 \mathrm{~km}$ south-west, Pilar do Sul, $30 \mathrm{~km}$ north-east and Itapetininga, $30 \mathrm{~km}$ north. The climate is subtropical with average temperatures between $22^{\circ} \mathrm{C}$ and $14^{\circ} \mathrm{C}$. Although the tea fields are situated in a mountainous setting slopes are moderate. In some fields meter-sized boulders are very frequent.

\section{Geology}

The city Registro is located in the Ribeira Tectonic Depression area (J. L. S. Ross n.d.). North of Registro this depression is limited by an ENE-WSW elongated block of gneissic, biotite rich monzogranite, the Gnaisse Itapeúna of the Atuba unit (Perrotta et al. 2005). South and southeast of this granitic block the Ribeira Tectonic Depression is filled with fluvial Cenozoic sediments (Embrapa Solos 2013; Souza Lima et al. 2016). The sampled tea fields are located on a terrace of sandy to loamy and fanglomeratic sediments of the Pariquera-Açu Formation (Melo 1990; Perrotta et al. 2005, Figure A.10). These fanglomeratic sediments have a low ZTR (zircon-turmaline-rutile) index (Hubert 
1962), indicating that these are rather immature sediments which formed in alluvial fans (Moraes et al. 1991).

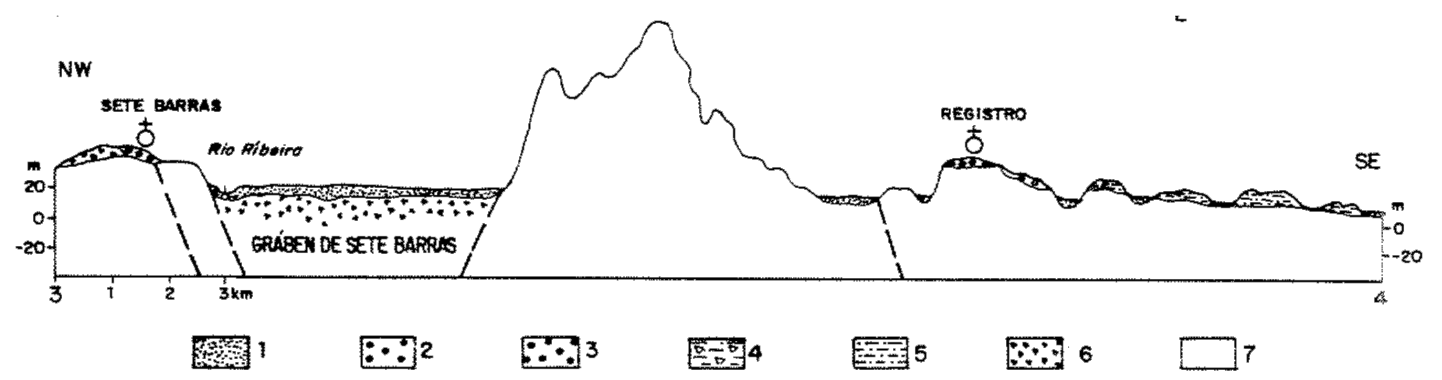

Figure 3.19.: Geological section of Ribeira Depression, source: Melo 1990, Figure 7). 1 - Quarternary sediments $2 \& 3$ - rubbles of terraces 4 - Pariquera-Açu Formation, fanglomeratic sediments 5 - Pariquera-Açu Formation, fluvial sediments 6 - Siete Barras Formation 7 Precambrian basement

In the village São Miguel de Arcanjo the base rocks of the two tea fields are constituted of two very distinct geologies. The main field, the division 'Matriz', is located very close to the northern limit of the Apiaí terrane of the western part of the Ribeira Fold Belt (Campanha and Sadowski 1999). It is located inside a small occurrence of the São Miguel Arcanjo Granite (Perrotta et al. 2005), a post-tectonic granite of the Neoproterozoic orogenies (Janasi et al. 2001). The outcrop consists of a slightly foliated porphyritic to porphyroid, hornblende-biotite, K-feldspar-rich monzogranite (Leite et al. 2007). The second tea field, the division 'Filia' approximately $10 \mathrm{~km}$ WNW of 'Matriz', is located in the Phanerozoic Paraná Sedimentary Basin (Perrotta et al. 2005).

\section{Soils}

The soils around Registro are mainly organosols in and along the recent and ancient river channels, and cambisols in the rest. The sediment terrace, on which the sampled tea fields are located, is covered by latosols (Oliveira et al. 1999). The soils are redyellow to deep red colored, intensely weathered, silty to sandy with minor contents of clay consisting mainly of quartz grains and kaolinite. The latosols in this area are described to have a very low capacity to retain cations and need frequent fertilizer application to be usable for agriculture (Oliveira et al. 1999; Oliveira 2014).

At São Miguel de Arcanjo the soils of the tea gardens are also intensely weathered, dystrophic, brownish-red latosols (Oliveira et al. 1999). They are silty to clayey with a varying sand component and minor amount of small pebbles. Source rocks are of 


\section{Characterization of Sampling Areas}

igneous (granitic) and metamorphic origin and they are former forest soils (Oliveira et al. 1999). The top soils (ca. $20 \mathrm{~cm}$ ) are rich in organic matter and are regularly treated with compost of saw mill waste and chicken feces (3:2).

\subsubsection{Paraná}

\section{Geography}

Paraná is a federal state south eastern part of Brazil, its southern border being the coast line of the Atlantic Ocean. North of Paraná lies the federal state São Paulo, northwest Mato Grosso do Sul and south Santa Catarina. West of Paraná lie neighboring the states Argentine Republic and the Republic of Paraguay. The state capital and the largest municipality is Curitiba, located in the eastern part close to the coast. Tea is cultivated close to the community Araucária, ca. $30 \mathrm{~km}$ south-west of the city Curitiba.

The tea fields of Paraná are located $13 \mathrm{~km}$ south-west of the community Araucária. The altitude is around $930 \mathrm{~m}$ a.s.l, at the southern rim of the Curitiba Basin. The plateau is gently undulating due to a dense drainage system of sediment filled stream beds.

\section{Geology}

The area is part of the Curitiba Tectonic Domain. In the community Auracária crop out the units of the Atuba Complex and Cenozoic deposits (Sato et al. 2003). The Atuba Complex is characterized as a tonalite-trondhjemite-granodiorite (TTG) suite (Faleiros et al. 2011). It consists of mainly mylonitic, migmatic orthogneisses which alternate with tonalitic-trondhjemitic to granodioritic leucosomes and plagioclase-rich, mesocratic neosomes (Sato et al. 2003; Faleiros et al. 2011). There are frequently occurring amphibolitic lenses. The mylonitic, migmatic gneisses are composed of hornblende, microcline, plagioclase, quartz and biotite. The mesocratic neosomes contain additionally hypersthene and/or diopside and garnet (Sato et al. 2003) with titanite as very frequent accessory mineral (Sato et al. 2003; Faleiros et al. 2011).

In the area of the sampling locations the gneisses are joined by the schists of the Atuba Complex (Figure A.10): They are often rich in biotite, partly migmatic, and intercalated with quartzites and arkoses. The cenozoic deposits are mostly alluvial sediments of sandy to coarse-sandy grain size. 


\section{Soils}

The tea garden in Araucária is mostly situated on latosols, and only on the alluvial sediments are argisols (Embrapa and Emater 2006).

The latosols are deeply weathered (up to $2 \mathrm{~m}$ ), yellowish to reddish soils, with generally low fertility and low $\mathrm{pH}$. They are porous and well drained and poor in rocky components (Santos et al. 2008; Embrapa Solos 2013). The argisols are characterized by a accumulation of clayey components in the $B$ horizon while the $A$ horizon may contain high percentages of sandy components. This leads in combination with low organic content to a reduced capacity to retain nutrients. 



\section{Description of Samples and Methods}

The section 'Samples' gives an overview of sampling and various sample groups, which will be used as grouping parameter for statistical discrimination techniques. The section 'Methods and Analysis' describes all steps of data acquisition from the original sample material to the raw data output of the instruments. The section 'Data Handling' gives details about how the raw data are processed and selected for the final geochemical data set which is used for the provenance analysis.

\subsection{Samples}

Samples have been collected between 2011 and 2016. In total there are 438 analyzed samples of which 96 samples are also used in Pospiech (2012).

\subsubsection{Types and Groups of Samples}

The sample set consists of two types of samples, plant tissue and soil. For most soil samples there are leaf samples of one or two bushes growing exactly above the soil sampling location, but not for each leaf sample exists a corresponding soil sample. In total there are 81 sampling locations with corresponding leaf and soil samples.

\section{Plant Samples}

The sample type 'plant' splits into two groups: Processed tea sample (processed tea) refers the ready made product 'tea', which are processed and dried leaves, and plucked leaf sample (plucked leaf) refers to the leaf samples plucked from the tea bushes. The latter group is divided into two subgroups: The shoot tip sample (shoot tip) refer to the young shoots of tea bushes and mature leaf sample (mature leaf) refer to leaves which are fully matured.

The plucked leaf sample 'shoot tip' consists of the first two to three leaves of a shoot, leaf buds and internodes of the shoot (Figure 2.2). These are the parts of $C$. sinensis, 
Table 4.1.: Overview of number of analyzed samples in respect of type, group and subgroups.

\begin{tabular}{lllr}
\hline Type & Group & Subgroup & Number of samples \\
\hline \multirow{4}{*}{ Plant } & plucked leaf & shoot tip & 160 \\
& & mature leaf & 27 \\
\cline { 2 - 3 } & & green & 187 \\
\cline { 2 - 4 } & processed tea & black & 26 \\
& & gunpowder & 85 \\
& & oolong & 1 \\
\cline { 2 - 3 } & & & 2 \\
\cline { 2 - 3 } & & & 114 \\
\hline \multirow{3}{*}{ Soil } & topsoil & 301 \\
\cline { 2 - 4 } & & subsoil & 41 \\
& & & 96 \\
\hline
\end{tabular}

which are harvested for tea production according to plucking standards 'two and a bud' and 'three and a bud' (Section 2.3.2). Depending on the climate conditions and the cultivar of $C$. sinensis the second and third leaf of the shoots could already have undergone the transition from sink to source leaf (Section 2.1.2). This implies that the sample subgroup shoot tip is a mixed sample of sink and source leaves with unknown mixing ratio under the point of view of plant physiology. It can be assumed that the percentage of sink leaves is higher than that of source leaves, especially in shoot tip samples plucked 'two and a bud'.

The sample subgroup 'mature leaf' consist of leaves which are at least two months old and at maximum four years. The latter are plucked leaves which consist only of source leaves. Mature leaves are generally not plucked for tea production, but during machine plucking (Figure 2.6), semi-mechanical/ shear plucking and rough hand plucking parts of mature leaves come into the mass of harvested shoot tips.

For the sample group of processed tea it has to be distinguished between single garden processed tea and blend. The single garden processed teas have been either collected directly from the tea garden's factories, covering most of the visited gardens, or had been provided by head offices of the tea producers or official tasting samples for tea traders. Blends can be either I) samples originating from trusted sources like head offices from tea producers or II) unconfirmed sources like packaged teas from supermarkets. 


\section{Soil Samples}

The sample type 'soil' splits into two subgroups: Topsoil refers to the upper layers of soil while subsoil sample (subsoil) refers to the soil sampling volume underneath the topsoil. The transition between topsoil and subsoil is approximately where the influence of the soil management ceases, indicated e.g. by changing of the soil color.

Due to very different soil characteristics of the four sample regions the transition zone between topsoil and subsoil varies: In Darjeeling, having generally shallow soil coverage due to very steep slopes, topsoil reaches down to approximately $15 \mathrm{~cm}$ and subsoil is sampled from $15 \mathrm{~cm}$ to approximately $30 \mathrm{~cm}$ depth. In the alluvial sediments of Assam the depth of transition between topsoil and subsoil varies strongly between the locations, but generally topsoil reaches down to approximately $25 \mathrm{~cm}$ and subsoil is sampled from $25 \mathrm{~cm}$ up to $45 \mathrm{~cm}$ depth. In the strongly weathered soils of Brazil the transition between topsoil and subsoil is approximately at $15-20 \mathrm{~cm}$ depth.

\subsubsection{Origin of Samples}

Plucked leaf samples originate from seven regions: Darjeeling, Nilgiris and Assam in India, São Paulo and Paraná in Brazil and Uji and Shizuoka in Japan. Soil samples originate only from four regions: Darjeeling and Assam in India and São Paulo and Paraná in Brazil. Processed tea samples originate from six of the seven regions of plucked leaf samples, only of Paraná in Brazil there are no processed tea samples, because production had not yet started during sampling. Additionally there are processed tea samples of several regions of Nepal $(N=12)$, China $(N=1)$, Sri Lanka $(N=1)$, Iran $(N=1)$ and Uganda $(\mathrm{N}=1)$.

The samples are not equally distributed in terms of their origin (Table 4.2). The majority of samples originates from Darjeeling, while from the other six regions the number of samples is comparatively small. Results of regions with less than six samples are considered as tentative studies.

Darjeeling had been sampled four times: in May 2011, April 2012, April and May 2014 and October 2015. Assam had been samples twice, in late March 2012 and November 2015. All other regions had been sampled only once: São Paulo and Paraná during September 2013, Nilgiris during late October 2015 and Shizuoka and Uji during June 2016. 
Table 4.2.: Overview of number of analyzed samples in respect of type, country and region.

\begin{tabular}{|c|c|c|c|}
\hline Type & Country & Region & Number of samples \\
\hline \multirow{16}{*}{ Plant } & \multirow{4}{*}{ India } & Assam & 26 \\
\hline & & Darjeeling & 167 \\
\hline & & Nilgiris & 25 \\
\hline & & & 218 \\
\hline & \multirow{3}{*}{ Brazil } & Paraná & 5 \\
\hline & & São Paulo & 37 \\
\hline & & & 42 \\
\hline & \multirow{4}{*}{ Japan } & & 1 \\
\hline & & Shizuoka & 10 \\
\hline & & Uji & 13 \\
\hline & & & 24 \\
\hline & \multirow{3}{*}{ Nepal } & & 7 \\
\hline & & Dhankuta & 5 \\
\hline & & & 12 \\
\hline & \multirow[t]{2}{*}{ Others } & & 5 \\
\hline & & & 301 \\
\hline \multirow{7}{*}{ Soil } & & Assam & 13 \\
\hline & India & Darjeeling & 90 \\
\hline & & & 103 \\
\hline & \multirow{4}{*}{ Brazil } & Paraná & 12 \\
\hline & & São Paulo & 22 \\
\hline & & & 34 \\
\hline & & & 137 \\
\hline
\end{tabular}

\subsubsection{Sampling}

Sampling took place during different seasons of the year, spanning all three high quality shoot seasons 1st flush, 2nd flush and atm flush (Section 2.3.2). The majority of the samples had been collected during the flushing seasons of the respective tea producing area.

All plucked leaf and soil samples originate from tea gardens. Tea gardens are organized in sections implying that the area of one section is treated exactly the same and that the field and factory management is very similar throughout the garden. Only few processed tea samples from Nilgiris might include leaf material from fields of so called 
'small growers' (Tea Board India 2017), which have their own and undocumented field management.

Sampling locations were chosen due to following considerations:

- at least $30 \mathrm{~m}$ distance to streets and field paths

- at least $100 \mathrm{~m}$ distance to busy streets

- minimum distance to other sampling location should be more than $50 \mathrm{~m}$

- location should be "inside" a section to avoid effects of the field margin, like more exposure of the bushes, other competitive plants, etc.

- avoiding direct proximity of small streams, villages, compost sites, etc.

- even bush density, avoiding patchy sections

- representativity of the section of the garden

- sections without recent fertilizer application (Section 2.3.1

For the sampling of the soils first step was to spade a hole of $30-45 \mathrm{~cm}$ depth, and describe layering, gradation, grain size, skeleton and quantity of roots of the fresh soil exposure. In Brazil a tool equivalent to the Pürckhauer driller was used for sampling. Then topsoil sample was drawn from homogenized soil material of the upper $15-20 \mathrm{~cm}$ and subsoil sample was drawn from the homogenized soil material from $15-20 \mathrm{~cm}$ down to $30-45 \mathrm{~cm}$ depth. The soil samples were stored in fresh and labeled bags of stable plastic and as soon as possible dried at $105^{\circ} \mathrm{C}$. Each soil sample contains more than $100 \mathrm{~g}$ dry mass.

Plucked leaves had been sampled with clean and dry hands imitating hand plucking for tea production. Seed plant bushes had been sampled separately, while for clonal bushes shoot tips and mature leaves are plucked from several adjacent bushes. Shoot tips and mature leaves were kept in separate clean, unused and labeled paper bags. Plucked leaf samples had been air dried during field campaign and as soon as possible oven dried at $105^{\circ} \mathrm{C}$. Each plucked leaf sample contained at least $12 \mathrm{~g}$ dry mass.

Processed tea samples had been partly derived from head offices or official retailers of the respective companies, but mostly directly from the tea garden's factories. Processed teas from factories are either from before sorting ('drier mouth'), after sorting, or after reblending. Reblended samples are in most cases blends of the day's production and only in few cases blends of several days' production. Reblended samples are very comparable to processed tea samples from the head offices. 


\subsection{Sample Preparation and Analysis}

\subsubsection{Sample Preparation and Digestion}

The aim of the sample preparation is to obtain a residual free, clear sample solution. The reproducibility of the element measurements is better for completely digested samples because the concentrations are independent of the interaction time between sample and the acids as compared to partial digestion procedures (Ruppert 1991; Dean 2014). According to Hoenig (1995), Matsuura et al. (2001), and Welna et al. (2013, and references therein) the following protocol is highly suitable for the tea plant samples. 32 samples are dissolved together as series of which at least one sample is a blind sample (blind) and one to three samples are international reference materials. The blinds are included to ensure that the handling was clean and without remarkable contamination during the digestion process.

The plant samples were dried unwashed at $105^{\circ} \mathrm{C}$ and grounded to $<60 \mu \mathrm{m}$ grain size in an agate ball mill in order to avoid contamination through metals. For the digestion process $400-500 \mathrm{mg}$ of the homogenized powder of plant material were used.

The soil samples were dried and split into three parts. For pH measurement one part were sieved to $<2 \mathrm{~mm}$ in grain size. The $\mathrm{pH}$ was measured in $25 \mathrm{~mL} 0.01 \mathrm{~mol} / \mathrm{L} \mathrm{CaCl}_{2}$ solution according to DIN 19684 Part 1 with $10 \mathrm{~g}$ of the soil. For geochemical analysis one part were dried at $105^{\circ} \mathrm{C}$ and then grounded in an agate ball mill to $<60 \mu \mathrm{m}$ grain size. Out of the homogenized powder $100-120 \mathrm{mg}$ of soil were used for the digestion process. The aliquots of plant and soil samples were fully digested with a mixture of ultrapure concentrated $\mathrm{HNO}_{3}, \mathrm{HClO}_{4}$ and $\mathrm{HF}$ in closed ultra clean PTFE vessels (PicoTrace Göttingen). Detailed description is enclosed in the appendix (B.1).

\subsubsection{Analysis}

The clear solutions (soils and plants) were analyzed in batches of 90-150 samples, including the blinds of the digestion series and the international reference materials samples. Analysis were done by ICP-OES and ICP-MS of about 50 elements. For several elements there are more than one analysis, because the measurement routines on ICP-MS and ICP-OES include several masses (ICP-MS) and spectral lines (ICP-OES). Because the routines do not always include exactly the same masses or spectral lines samples may have different number of analysis for each element. For example, for Barium there are for some samples in total eight analysis, the masses Ba 135 and $\mathrm{Ba} 137$ 
of the ICP-MS and the spectral lines Ba233.527 (axial and radial), Ba 455.403 (axial and radial) and $\mathrm{Ba} 493.408$ (axial and radial) of the ICP-OES. Out of these one to eight element data for each sample one data point per element is selected as analytical result (Section 4.3.1). The selection is listed in Table B.6 in the appendix.

The calibration solutions for the ICP-MS and ICP-OES are given in the appendix (Table B.5). For the ICP-MS analysis (machine Elan DRC II, Perkin Elmer) the same calibration solution was used for all samples (Table B.2). For the ICP-OES analysis for the sample types soil and plant two different calibration solutions had been used in order to adjust the characteristics of the calibration solution to the type of sample solution. Both types of ICP-OES calibration solutions had been slightly changed according to adjustments to general elemental composition of plant samples and varying detection ranges of the machines Optima 3300 DV (Perkin Elmer) (Table B.3, until May 2015) and the machine 5100 VDV (Agilent) (Table B.4, from June 2015 onwards).

For ICP-MS analysis all samples had been spiked with an Internal Standard (IS) (Table B.1. . Accordingly the elements $\mathrm{Be}, \mathrm{Ge}, \mathrm{Rh}, \mathrm{In}$ and $\mathrm{Re}$ are not included in the results.

\subsubsection{Limit of Detection, Precision and Accuary}

The limit of detection (LOD) is calculated on basis of the blinds of each batch of samples. Each batch of samples includes between 3 to 12 blinds. Because blinds are samples the resulting LOD takes into account the handling of the samples and not only the LOD of the machines.

$$
L O D=3 * \operatorname{stdev}\left(\text { blind }_{1}, \text { blind }_{2}, \ldots, \text { blind }_{n}\right)
$$

Only results above the LOD had been accepted.

Precision and accuracy are ensured by using the international plant reference materials GBW 10052, BCR-129, NCS DC 73349, NJV 94-5 as well as the in-house standard TW-45. Each analysis of a reference sample includes the process of digestion and analysis via ICP-MS and ICP-OES. The referenced values of the 27 analyzed reference samples are given in the appendix (Table B.10).

For each reference sample precision and accuracy had been calculated to assess the quality of analytics for each series and each sample batches analyzed by ICP-MS and ICP-OES. Accuracy is the relative proximity of the measured value from the reference 


\section{Description of Samples and Methods}

value in percent and is calculated by

$$
\text { Accuracy }[\%]=\frac{\mid \text { Conc }_{\text {measured }}-\text { Conc }_{\text {reference }} \mid}{\text { Conc }_{\text {reference }}} * 100
$$

Precision is calculated the relative deviation from the median of all measured values of the respective reference sample $\left(\right.$ Conc $\left._{\text {median }}\right)$ :

$$
\text { Precision }[\%]=\frac{\mid \text { Conc }_{\text {measured }}-\text { Conc }_{\text {median }} \mid}{\text { Conc } c_{\text {median }}} * 100
$$

The precision is calculated by the relative deviation, instead of using the range or the standard deviation, because the total data set of analyzed reference material samples contains between 3 to max. 45 analyses per each type of reference material (Gorard 2005). The calculation of the precision is based on a compiled data set of 104 analysis from several similar projects. Hence the precision is based on 5 analysis for BCR-129, 5 for NJV 94-5, 49 for NCS DC 73349, 24 for GBW 10052 and 21 for TW-45.

The LOD, accuracy and precision of each analysis batch are the basis to select for each element the best analysis for the respective sample type out of the rawdata of ICP-MS (different masses) and the rawdata of ICP-OES (different spectral lines). The accuarcy and precision of the selected element analysis of each reference sampled are given in the appendix (Table B.15 and B.19). Average accuracy and precision for the elements was better than $5 \%$ for all main elements and better than $10 \%$ for most trace elements (Table 4.3). For plants the elements $\mathrm{Cr}$, Co, Mo, Sn and ultra-trace elements like e.g. the heavy REE show an average precision between 10\%-20\%. 
Table 4.3.: Averaged accuracy and precision of the international reference plant and rock standards used for this thesis. All values are given in \%. Averages (mean) are calculated for plants by BCR-129 ( $\mathrm{N}=2)$, GBW $10052(\mathrm{~N}=11)$, NCS DC $73349(\mathrm{~N}=3)$, NJV 94-5 $(\mathrm{N}=3)$ and for soils by TW-45 $(\mathrm{N}=5), \mathrm{TW}-59(\mathrm{~N}=2)$ and JLK-1 $(\mathrm{N}=1)$.

\begin{tabular}{|c|c|c|c|c|}
\hline & Plant Acc. & Plant Prec. & Soil Acc. & Soil Prec. \\
\hline $\mathrm{Al}$ & 5.8 & 3.7 & 1.1 & 0.7 \\
\hline As & 20.6 & 15.4 & 13.4 & 19.8 \\
\hline $\mathrm{Ba}$ & 4.0 & 2.4 & 2.6 & 2.2 \\
\hline $\mathrm{Ca}$ & 11.7 & 2.3 & 3.1 & 1.6 \\
\hline $\mathrm{Cd}$ & 21.6 & 6.6 & 26.4 & 24.9 \\
\hline $\mathrm{Ce}$ & 10.4 & 12.3 & 6.9 & 5.6 \\
\hline Co & 14.1 & 6.7 & 6.3 & 2.9 \\
\hline $\mathrm{Cr}$ & 36.1 & 29.2 & 35.3 & 28.2 \\
\hline Cs & 9.1 & 10.7 & 7.8 & 20.7 \\
\hline $\mathrm{Cu}$ & 8.1 & 5.2 & 6.3 & 2.3 \\
\hline Dy & 11.5 & 10.0 & 8.3 & 21.7 \\
\hline Er & 7.9 & 9.3 & 10.1 & 21.8 \\
\hline $\mathrm{Eu}$ & 26.4 & 27.6 & 8.9 & 20.5 \\
\hline $\mathrm{Fe}$ & 13.6 & 10.4 & 1.8 & 2.6 \\
\hline $\mathrm{Gd}$ & 13.1 & 12.2 & 6.1 & 23.3 \\
\hline $\mathrm{Hf}$ & 44.0 & 23.6 & 18.9 & 22.9 \\
\hline $\mathrm{Ho}$ & 9.9 & 11.3 & 8.6 & 21.6 \\
\hline K & 12.5 & 11.4 & 3.5 & 2.5 \\
\hline La & 10.4 & 11.6 & 14.8 & 8.9 \\
\hline $\mathrm{Li}$ & 8.5 & 9.8 & 7.3 & 8.1 \\
\hline Lu & 11.8 & 12.2 & 7.3 & 16.8 \\
\hline $\mathrm{Mg}$ & 9.8 & 2.3 & 3.2 & 2.2 \\
\hline $\mathrm{Mn}$ & 13.7 & 8.1 & 2.8 & 1.8 \\
\hline Mo & 26.6 & 21.1 & 21.4 & 24.7 \\
\hline $\mathrm{Na}$ & 8.9 & 7.4 & 2.7 & 1.7 \\
\hline $\mathrm{Nb}$ & 98.0 & 19.4 & 11.0 & 2.6 \\
\hline $\mathrm{Nd}$ & 8.0 & 9.3 & 8.7 & 21.3 \\
\hline $\mathrm{Ni}$ & 10.1 & 7.4 & 3.1 & 1.8 \\
\hline$P$ & 12.5 & 9.6 & 3.4 & 1.0 \\
\hline $\mathrm{Pb}$ & 13.4 & 9.4 & 5.7 & 16.2 \\
\hline $\mathrm{Pr}$ & 11.0 & 11.0 & 9.4 & 21.1 \\
\hline $\mathrm{Rb}$ & 12.9 & 12.6 & 11.8 & 21.6 \\
\hline$S$ & 12.1 & 9.0 & 11.1 & 2.5 \\
\hline $\mathrm{Sb}$ & 14.8 & 13.2 & 15.2 & 22.9 \\
\hline Sc & 18.4 & 98.8 & 6.3 & 3.7 \\
\hline Sm & 9.9 & 10.8 & 9.7 & 20.8 \\
\hline Sn & 45.2 & 35.7 & 16.6 & 18.9 \\
\hline $\mathrm{Sr}$ & 7.1 & 4.2 & 2.7 & 3.0 \\
\hline $\mathrm{Ta}$ & & 42.2 & 45.2 & 23.3 \\
\hline $\mathrm{Tb}$ & 10.2 & 10.8 & 9.5 & 20.5 \\
\hline Th & 21.0 & 19.8 & 32.3 & 22.5 \\
\hline $\mathrm{Ti}$ & 35.4 & 10.6 & 1.8 & 1.5 \\
\hline $\mathrm{TI}$ & 17.5 & 8.3 & 16.0 & 25.9 \\
\hline $\mathrm{Tm}$ & 10.8 & 10.0 & 13.3 & 22.7 \\
\hline$U$ & 28.9 & 19.9 & 29.5 & 9.4 \\
\hline V & 158.9 & 29.8 & 2.5 & 2.2 \\
\hline$Y$ & 29.5 & 11.2 & 11.6 & 21.0 \\
\hline $\mathrm{Yb}$ & 20.4 & 10.8 & 24.9 & 20.4 \\
\hline $\mathrm{Zn}$ & 4.9 & 3.3 & 3.0 & 3.1 \\
\hline $\mathrm{Zr}$ & & 27.6 & 20.8 & 8.1 \\
\hline
\end{tabular}




\subsection{Data Handling}

The aim of the data processing is to make the final concentration data independent of the sample groups, sample lots, type of machines and analytical protocols: A sample of processed tea, sampled in 2013 and analyzed in 2014, should be comparable to a shoot tip, sampled in 2015 and analyzed in 2016.

Data processing includes all steps of data treatment from the raw data export from the machine until the final concentrations used in the statistical analysis. These steps are:

1. Calculation of element concentrations for the sample solutions from the raw data of ICP-MS and ICP-OES. For ICP-MS the IS (Table B.1) is used for calculating the concentration.

2. Drift-correction

3. Subtraction of the values of the blinds from all other samples in order to remove background values.

4. Corrected concentration of the sample solution $(\mu \mathrm{g} / \mathrm{L})$ are converted into dry weight (d.w.) concentration $(\mathrm{mg} / \mathrm{kg})$.

5. Selection of one datum for each element and each sample based on accuracy and precision of adjacent reference samples and range between LOD and sample concentration (Section 4.3.1).

6. Check for outliers and irregularities

7. In case of plant samples: Correction for adhering particles (Section 6)

\subsubsection{Selection of Element Data}

As mentioned in Section 4.2.2 for each sample and for each element there are several data points which have to be reduced to one datum as result. The selection of data points depends on a) the matrix of sample solution, e.g. plant or soils, b) the concentration range, $c$ ) the comparability between calibration solution and sample matrix, d) the LOD of the respective spectral lines of ICP-OES or masses of ICP-MS and e) the measurement itself, e.g. if there had been enhanced oxidation rate in the ICP-MS for certain elements or minor plasma instabilities at the ICP-OES for certain elements on that specific day of analysis. Hence, there is no general selection for each type sample matrix but the selection is calculated for each digestion series and sample type based and accuracy 
and precision of the measurement. The accuracy and precision is determined for each reference sample for all elemental data points (Section 4.2.3) and weighted by 1:3, e.g. if the ICP-OES spectral line $\mathrm{Ba} 455.403$ for the reference sample $X Y$ had an accuracy of $5 \%$ and a precision of $3 \%$ the ratio is $3.5 \%$. The spectral line or the mass with the smallest ratio is selected as the best data point for this element in this reference sample, e.g. if $\mathrm{Ba} 455.403$ (axial) of ICP-OES has a ratio of $3.5 \%$ and $\mathrm{Ba} 135$ of ICP-MS has a ratio of $4.1 \%$ the $\mathrm{Ba} 455.403$ is chosen.

For each element and each sample type there is a preferred data point. For example, out of the eight Ba data points $\mathrm{Ba} 455.403$ (axial) is the preferred line for choosing the Ba concentration. The preferred data point is determined for each sample type by the best accuracy:precision ratio over all analyzed reference samples for the respective sample type. In order to use for one sample type as often as possible the same line or mass for one element there is a flexibility of $5 \%$ in the ratio in favor to the preferred data point: If the best accuracy:precision ratio is not the preferred data point and less than $5 \%$ smaller than the preferred data point then the preferred data point is selected, e.g. if $\mathrm{Ba} 455.403$ has a ratio of $6 \%$ and $\mathrm{Ba} 135$ of $4 \%$ then still $\mathrm{Ba} 455.403$ is selected if it is the preferred data point for the respective sample type.

The selection of data points is calculated per digestion series because series of samples (Section 4.2.1) are combined to different sample batches for ICP-MS and ICP-OES analyses (Section 4.2.2). The combination of series is often different for ICP-MS and for ICP-OES analyses. If there is more than one international reference sample included in the series the accuracy:precision ratios are averaged for the data points. The selection of data points calculated for the reference samples is applied on all non-reference samples of the same series. The selection was calculated by the in-house R-packages Pospiech and Fahlbusch (2018) and Pospiech (2017) and is listed in the electronic Appendix.

The prerequisite of this selection technique is a very good comparability of reference material and sample material within one series. Consequently for this thesis all series of plant material include at least one sample of GBW 10052 and all series of soil material include at least one sample of TW-45.

\subsubsection{Software 1}

Maps had been produced by QGIS (QGIS Development Team 2018). All data processing and plotting had been carried out within the free statistical software R Core Team (2017). The R packages used for data handling are 'ICPData' (Pospiech and Fahlbusch

\footnotetext{
'The package 'ratios' is published on CRAN (Pospiech and Fahlbusch 2017)
} 
2018), 'ICPSubfunctions' (Pospiech 2017), 'data.table' (Dowle and Srinivasan 2017), 'dplyr' (Wickham et al. 2017), 'tidyr' (Wickham and Henry 2018), 'gnumeric' (Antal 2017), for representation 'ggplot2' (Wickham 2009) and 'xtable' (Dahl 2016) and for calculations are 'compositions' (Boogaart and Tolosana-Delgado 2008), 'MASS' (Venables and Ripley 2002), 'ratios' (Pospiech and Fahlbusch 2017). The calculations done by the package 'ratios' are described in the sections 5.2 and 6.1 . 


\section{Mathematical Tools}

The results of this thesis are derived mainly of concentration data. Concentrations are values which represent parts of the whole, e.g. parts per million (ppm) or weight percent (wt\%), and contain only relative information. Following the notation introduced by Aitchison (1986) the elemental composition of samples is represented by a $D$-dimensional, positive vector, the concentration of each element being one of the $D$ vector components. Vectors are written in bold font, $\mathbf{x}$, which are denoted by $\mathbf{x}=\left[x_{1}, x_{2}, \ldots, x_{D}\right]=$ $\left[x_{i}\right]_{i=1, \ldots, D}$. Such vectors are subject to several constraints and therefore do not belong to the space o D-tuples of real numbers, $R^{D}$, but to the $D$-part simplex $\left(S^{D}\right)$ : the set of vectors of positive components and constant sum. Hence, before using statistical tools and algorithms developed for numbers of $\mathbb{R}$ the vectors have to be moved from the $S^{D}$ into the $\mathbb{R}$ by logarithmic ratio (log-ratio) transformations. In this thesis various log-ratio transformations are used and all results of concentration values will be presented as log-ratios. Another reason to use log-ratios is to enhance the signal-noise ratio for trace elements: Ratios help avoiding artefacts through analytical errors, e.g. machine error or weighing inaccuracy 1 . The logarithm allows to compare very different concentration levels, e.g. wt\% with ppb. As a rule calculations are based on the natural logarithm (In), only for certain figures the decimal logarithm (log) was used for ease of understanding.

\subsection{Compositional Data and Log-ratio Transformations}

Properties and characteristics of numbers in the $S^{D}$, called compositions, were first time fully described by Aitchison (1982, 1986). Elemental concentrations are one type of compositional data with the characteristic that they are so called "closed" compositional data: Each element concentration is a component of a D-part composition, all parts of the composition are constrained to a constant sum and in this thesis all components are considered as strictly positive. For example, if concentrations are given in wt\%, they

\footnotetext{
data

${ }^{1}$ tea powder is highly hydrophile and gaining up to $5 \mathrm{wt} \%$ within $30 \mathrm{~min}$ of exposure to air, unpublished
} 


\section{Mathematical Tools}

have values between 0 and 100 and the sum of all components will be 100 . Accordingly, this type of compositional data provide only relative information.

If compositional data are closed the components of a vector are algebraically linked with each other, e.g. if one part increases from $10 \%$ to $20 \%$ at least one other part has to diminish in order to keep the constant sum. Hence, if concentration data are used without taking the linkage of parts into account spurious correlations and biases in the results may occur (Filzmoser and Hron 2008). Furthermore, it is necessary that results are consistent between an analysis with all components of a composition and an analysis of a subcomposition (selection of components). This 'subcompositional coherence' is one important requirement which has to be fulfilled for all analysis. For example the relation between the elements $\mathrm{Na}, \mathrm{Ca}$ and $\mathrm{K}$ should be identical for all possible subcompositions, e.g. if the analysis was carried out with all elements of a sample, or the subcomposition $\mathrm{Na}, \mathrm{Ca}, \mathrm{K}, \mathrm{P}, \mathrm{S}$ or the subcomposition $\mathrm{Na}, \mathrm{Ca}, \mathrm{K}$. For detailed description of compositional data please refer to e.g. Aitchison (1986, 2003), Buccianti et al. (2006), Pawlowsky-Glahn and Egozcue (2006), Pawlowsky-Glahn and Buccianti (2011), and Pawlowsky-Glahn et al. (2015).

There are several possible log-ratio transformations. Here only a small overview of the basic equations is given for the transformations which are used in this thesis. For a detailed description of properties, advantages and disadvantages of the various log-ratio transformations please refer to Egozcue and Pawlowsky-Glahn (2011).

The simplest log-ratio is between two parts, $x_{i}$ and $x_{j}$, of a composition:

$$
\operatorname{pwlr}(\mathbf{x})=\left[\ln \left(\frac{x_{i}}{x_{j}}\right)\right]_{i, j=1,2, \ldots, D}
$$

The calculation of pairwise logarithmic ratios (pairwise log-ratios) of a data set can be understood as producing for each sample a matrix which contains log-ratios of each element against each element. If a data set consists of $n$ samples with $y$ elements the result of a pairwise log-ratio transformation are $n$ matrices of $y \times y$ entries.

If this simple log-ratio is applied for a fixed denominator $x_{D}$ it is called additive log-ratio (alr) (Aitchison 1986):

$$
\operatorname{alr}(\mathbf{x})=\left[\ln \left(\frac{x_{i}}{x_{D}}\right)\right]_{i=1, \ldots, D-1}
$$

The alr of $\mathrm{x}$ has one component less than $\mathrm{x}$ and each part is algebraically independent. Using a centered log-ratio (clr) transformation (Aitchison 1986) all parts of the compo- 
sition are set into relation to the geometric mean $\left(g_{m}\right)$ of the composition:

$$
\operatorname{clr}(\mathbf{x})=\left[\ln \left(\frac{x_{i}}{g_{m}(\mathbf{x})}\right)\right]_{i=1, \ldots, D} \quad, \quad g_{m}(\mathbf{x})=\left(\prod_{i=1}^{D} x_{i}\right)^{\frac{1}{D}}
$$

While the clr transformation produces a log-ratio croe for each component and is easy to interpret it has the disadvantage of a singular covariance matrix, which precludes the use of some of the standard statistical tools.

The isometric log-ratio (ilr) transformation, introduced by Egozcue et al. (2003), is related to the clr by a set of $D-1$ linear combinations of the clr coefficients

$$
\operatorname{ilr}_{\mathrm{j}}(\mathbf{x})=\sum_{i=1}^{D} \beta_{i j} \operatorname{clr}_{\mathrm{i}}(\mathbf{x})
$$

in such a way that the vectors of coefficients $\boldsymbol{\beta}_{j}=\left[\beta_{1}, \beta_{2}, \ldots, \beta_{D}\right]$ are orthogonal by pairs, and they are all normalized to length $1=\beta_{1}^{2}+\beta_{2}^{2}+\cdots+\beta_{D}^{2}$. The advantage of an ilr transformation above the clr transformation is that the covariance matrix can be inverted, which is required by most of the statistical tools used in this dissertation. The disadvantage of ilr values is that they are in general difficult to interpret.

One way of producing an interpretable ilr transformation is to use one special case of ilr, the so called balanced log-ratios (Egozcue and Pawlowsky-Glahn 2005; Parent et al. 2013). A balanced log-ratio is the log-ratio contrast between two non-overlapping subcompositions of one composition. It is expressed as the normalized quotient between the geometric mean of one subcomposition of elements against the geometric mean of a second subcomposition of elements. With the first subcomposition $\mathbf{r}$ containing $D_{r}$ elements and the second subcomposition s containing $D_{s}$ elements, the balanced logratio is calculated by:

$$
\left[\mathrm{r}_{1}, \ldots, \mathrm{r}_{\mathrm{D}_{\mathrm{r}}} \mid \mathrm{s}_{1}, \ldots, \mathrm{s}_{\mathrm{D}_{\mathrm{s}}}\right]=\sqrt{\frac{D_{r} \cdot D_{s}}{D_{r}+D_{s}}} \cdot \ln \left(\frac{g_{m}(\mathbf{r})}{g_{m}(\mathbf{s})}\right)
$$

Another way to make ilr transformed data interpretable is calculate the ilr by a known vector space base, e.g. as used for the ilr-ANOVA in Section 5.3 . 


\subsection{Vratios 2}

For certain questions not only the concentration data are used but also the ratios of element concentrations between two samples, like $K_{\text {sample } 1} / K_{\text {sample2 }}$ for the element Potassium. To avoid ambiguities with ratios of parts within one sample composition, for example between two elements of one sample $K_{\text {sample } 1} / P_{\text {sample } 1}$, we will call these ratios between two samples 'vratio' (vector-ratio). These vratios are calculated for example to the describe uptake of elements through plants by the vratio plant/soil, translocation of elements within a plant by the vratio leaf/root, the relation between mineral composition versus host rock by the vratios mineral/rock, etc.

This simple approach of dividing one composition by the other describes the proportion of one element concentration between two samples and is for some research questions a good and correct approach. However, this approach might lead to biased conclusions or blurred results if the focus is laid upon the relation between elements of vratios: The simple vratios, being ratios of intrinsically linked proportions, are affected by the constraints of compositional data (e.g. Duce et al. 1975; Reimann and Caritat 2000). Especially if statistical tools like principal component analysis (PCA), linear discriminant analysis (LDA), analysis of variance to create an ilr-base (ilr-ANOVA), clustering, biplots, etc. are used to explore relations between multiple variables of vratios, the results can be affected by the untreated skewness of the distribution of vratios, the chosen subcomposition of variables, or by one or more dominating variables.

In order to reduce the biases, support visibility of trends or correlations or to allow quantification of trends vratios can be transformed into less skewed and/or unconstrained data. Equivalent to transformations developed for vectors of compositional data the same method of transformations can be used for vratios. One method often used is to normalize each part of a composition to a reference part for calculating the vratio: vratio $_{i}=\mathbf{x}\left[x_{i}\right] / \mathbf{y}\left[x_{i}\right] \times \mathbf{y}\left[x_{r e f}\right] / \mathbf{x}\left[x_{r e f}\right]$ (e.g. Walker 1972; Zoller et al. 1974). The choice of the reference part has to be done with great care and the constraints of each ratio of $x_{i} / x_{\text {ref }}$ for the setting of the study must be taken into account (Duce et al. 1975; Reimann and Caritat 2000).

In this dissertation we will introduce logarithmic vratios, centered vratios and centered logartihmic vratios, equivalent to the additive log-ratio (alr) and the centered log-ratio (clr), introduced by Aitchison (1986) for compositional data vectors. These transformation of data can be seen like applying different 'filters' which could help to see further

\footnotetext{
${ }^{2}$ Parts of this section has been presented at Goldschmidt Conference 2017 (Pospiech and TolosanaDelgado 2017)
} 
aspects and trends in a mutli-variate setting of vratios. The aim, advantages and disadvantage will be described and discussed on the example of ionome of shoot tips compared to the geochemical composition of corresponding soils. These five filters are: the normalization to one reference element, 'ar'-filter, the normalization to the geometric mean, 'cr'-filter, and logarithmic transformation of the simple ratios and these normalizations, 'log', 'alr' and 'clr'- filters.

There are several terms used for the process of elements moving from the soil into plant tissue, like 'element uptake', 'transfer-factors', 'soil-plant partition coefficient', 'bioconcentration factor', 'concentration ratio', 'enrichment factor' or 'biological accumulation coefficient' (Hope 1995; Roca and Vallejo 1995; Dinelli and Lombini 1996; Rodríguez et al. 2002; Tomé et al. 2003; Chojnacka et al. 2005; Reimann et al. 2007; Goni et al. 2014). The definitions may refer to the total content in the soil, to the bio-available element solution, to the total plant, to some specific part of the plant, like stems, fruits, etc, or even to an estimation coefficient in order to predict the uptake of an element by a certain species (Chojnacka et al. 2005). Because this thesis focuses on the element concentration in the plant in respect to the corresponding soil, the neutral, descriptive term plant-soil-ratio is used. For 'soil' the total concentration of either top- or subsoil is used, and for 'plant' the total concentration of either shoot tip or mature leaf is used.

\subsubsection{Calculation of Vratio}

The simple vratio between two compositions, $\mathrm{x}$ and $\mathbf{y}$, is defined as:

$$
\text { vratio }=\left[\frac{x_{i}}{y_{i}}\right]_{i=1, \ldots, D}=\frac{\mathbf{x}}{\mathbf{y}}
$$

If each part of a composition is related to a reference part and vratio are then calculated by these 'normalized' parts, as used by e.g. Walker (1972) and Zoller et al. (1974), in the following it will referred to as representation 'ar' - by analogy to the additive logratio without the logarithmic transformation:

$$
\text { vratio_ar }=\left[\frac{\frac{x_{i}}{x_{r e f}}}{\frac{y_{i}}{y_{r e f}}}\right]_{i=1, \ldots, D}=\frac{\mathbf{x}}{\mathbf{y}} \times \frac{y_{r e f}}{x_{r e f}}
$$

Following the analogy of the definition of 'alr' for compositional data by Aitchison 
(1986), the 'alr' representation will be:

$$
\text { vratio_alr }=\left[\ln \left(\frac{\frac{x_{i}}{x_{r e f}}}{\frac{y_{i}}{y_{r e f}}}\right)\right]_{i=1, \ldots, D}=\ln \left(\frac{\mathbf{x}}{\mathbf{y}} \times \frac{y_{r e f}}{x_{r e f}}\right)
$$

Normalizing the parts to the geometric mean $\left(g(\mathbf{x})=\sqrt[D]{x_{1} \cdot x_{2} \cdots x_{D}}\right)$ and then calculating ratios of these 'centered' compositions will be referred to as representation 'cr' (centered ratio):

$$
\text { vratio_cr }=\left[\frac{\frac{x_{i}}{g(\mathbf{x})}}{\frac{y_{i}}{g(\mathbf{y})}}\right]_{i=1, \ldots, D}=\frac{\mathbf{x}}{\mathbf{y}} \times \frac{g(\mathbf{y})}{g(\mathbf{x})}
$$

Applying the logarithm on the representation 'cr' leads to the representation 'clr' (centered log-ratio), analogical to the definition of 'clr' for compositional data by Aitchison (1986):

$$
\text { vratio_clr }=\left[\ln \left(\frac{\frac{x_{i}}{g(\mathbf{x})}}{\frac{y_{i}}{g(\mathbf{y})}}\right)\right]_{i=1, \ldots, D}=\ln \left(\frac{\mathbf{x}}{\mathbf{y}} \times \frac{g(\mathbf{y})}{g(\mathbf{x})}\right)
$$

\subsubsection{Comparison of Vratio Representations}

To visualize the differences between the six representations of vratio calculation, 13 elements had been selected to generate PCA-biplots (Section 5.4) in order to demonstrate the spread of vratios and the relations among them. Figure 5.1 shows on the left side biplots of the non-logarithmic representations, and on the right side the biplots of the logarithmic representations. For the representations 'ar' and 'alr' the element $\mathrm{K}$ had been chosen as reference element for illustration, because $\mathrm{K}$ is one of the elements which has very different uptake between regions. In the appendix there are further examples with $\mathrm{P}$ and $\mathrm{Ca}$ as reference element for the representations 'ar' and 'alr' (Figure C.1).

The plots in Figure 5.1 reveal that for this data set all non-log-representations have in general roughly a similar pattern and all log-representations have roughly a similar pattern. Vratios calculated via non-log-representations have very different values and variances, as well between elements as between representations. On the other hand, log-representations have more homogeneous distributed values (Tables 5.1 and 5.2).

Non-log-representations Among the non-log-representations the representation 'simple' gives the factor between the concentration in the first data set compared to the 
concentration in the second data set. Table 5.1 displays the medians vratios for eight different representations. In the example data set the element $S$ has highest vratios with median $=19$, while $\mathrm{La}$ and Fe have lowest vratios, both with median $=0.0016$. The element $\mathrm{K}$ with vratios of median $=1.2$ is in average similarly concentrated in shoot tips as in the corresponding soils. All 'ar' representations are similar between each other, because each 'ar' representation has the same denominator and between the various 'ar' representations only the denominator differs. For the representation 'cr' the homogeneity of this normalization factor is determined by the scatter of the geometric means of both data sets.

The advantages of the non-log normalizations take effect if statistical tools are used which incorporate e.g. the variance of the vratio distributions. For nearly all non-logrepresentations the variance of the vratios is highest for the elements $\mathrm{S}$ and $\mathrm{K}$, only in the representation 'ar Ca' the vratios of $P$ show also notably high variance (Table 5.2). For all other elements the distribution of vratios have minor variances. The coloring by region in the left plots of Figure 5.1 reveal that the regions Arcanjo and Registro have very high variances of plant-soil-ratios for $\mathrm{K}$ compared to the plant-soil-ratios for $\mathrm{K}$ in Darjeeling and Assam. On the other hand, the high variance of vratios of $S$ is provoked by large differences in plant-soil-ratios within the regions Darjeeling and Assam.

The general pattern of the non-log-representation biplots is similar, except for the representation 'ar K': The component 1 is mainly controlled by the link between $S$ and the origin of the biplot, while the spread of samples along the component 2 is mainly caused by the variance of the vratios of $\mathrm{K}$; this also explains why the biplot of 'ar $\mathrm{K}$ ' is very different in its pattern compared to all other non-log-representations. Differences between the representations can be seen in the sample grouping, e.g. compare 'simple' vs. 'cr' vs. 'ar Ca' (Figure C.1). Through applying these various mathematical filters the differences of the representations visually highlight different distributions of the respective elements. For example, in the plot of representation 'simple' it becomes clear that the $\mathrm{K}$ - uptake in shoot tips in Brazilian tea gardens differs notably compared to the $\mathrm{K}$ - uptake in Indian tea gardens and the $\mathrm{K}$ - uptake differs between the Brazilian regions, too. Additionally, within the Brazilian sample group there is a slight offset between the regions parallel to the component 1 axis (Figure 5.1, left side, method 'simple' and method 'cr'). This suggests that differences in $\mathrm{K}$ - uptake could be the main parameter which discriminates the various regions in Brazil, but not the only parameter. Of the displayed non-log-representations it is indeterminable which else vratios distinguishes between the regions. The biplots suggest that a further investigation on $\mathrm{Sr}$ or $\mathrm{Rb}$ uptake could 
lead into more satisfying results. Another example of how a different normalization may help to find trends and discrimination parameter can be seen in the biplot of representation 'ar Ca' (upper left plot in Figure C.1). In this plot most samples from Assam form a distinguished group, and, by the position of the variable labels, presumably the $\mathrm{S} / \mathrm{Ca}$ and $\mathrm{P} / \mathrm{Ca}$ ratios in soils and shoot tips are important proxies. The interpretation of this plot could be: The content of $P$ in leaves compared to $P$ in soil is, compared to the ratio of $\mathrm{Ca}$ in leaves to $\mathrm{Ca}$ in soil, higher for Assam than in the other regions.

Log-representations The log-representations have the advantage that through the logarithm the spread of data is more comparable between values of different orders of magnitude. Hence, the approach of using a log-representation helps to see differences in vratios of generally very small values compared to vratios of other variables in the data set. Table 5.1 displays the difference in orders of magnitude: The medians of vratios of non-log-representations span up to five orders of magnitude, while the values of log-representations have a much smaller range. Accordingly, the variances of logrepresentations also span less order or magnitudes (Table 5.2).

The general pattern of log-presentation biplots is similar for the methods 'log', 'alr K' and 'clr' (Figure 5.1, right side), but are slightly different for the methods 'alr Ca' and 'alr $\mathrm{P}^{\prime}$ (Figure C.1). As discussed for the non-log-representations, the variance of vratios involving the element $\mathrm{K}$ discriminates between Brazilian and Indian regions. Only for the method 'alr P' (Figure C.1) samples from the region Registro cannot be clearly separated from Indian sample groups. In contrast to the non-log-representations, the biplots of logrepresentations allow a clear discrimination of sample groups of the regions within India and Brazil: For nearly all filters samples from Darjeeling, Assam, Araucária, Arcanjo and Registro plot in distinguished groups. Only samples of Araucária and Arcanjo are indistinguishable for certain log-representations, like 'clr' and 'alr Ca'. Similar to the nonlog-representations, each log-representations reveals a slightly different aspect of the sample set.

As a visual tool the biplots of the log-representation reveal following results: i) all regions are different from each other concerning element uptake pattern, because for several filters the samples group by regions. This could not be seen as clearly from the non-log-representation biplots. ii) $\mathrm{K}$ - uptake is one the main differences between Indian and Brazilian samples. This could be seen more clearly for the non-log-representations. iii) Various other details, e.g. tea in Assam has the lowest $\mathrm{Ca}$ and Al - uptake, tea in Registro shows the highest $\mathrm{Sr}$ - uptake, the uptake of $\mathrm{Ni}$ and $\mathrm{Fe}$ does not discriminate 
between the regions, etc.

In summary, the log-representations show less clearly the main players of differences in vratios of a data set, but rather reveal the spread of vratios of small values in a data set. The choice of representation depends upon the question: For example, if the focus of the visual approach should be lain upon differences of the uptake of $\mathrm{Ca}$ by tea in various regions, the representation 'alr Ca' (Figure C.1, upper right plot) displays that tea in Assam, Araucária and Arcanjo has less uptake of $\mathrm{Ca}$ than tea in Registro and Darjeeling.

\subsubsection{Summary}

Vratios (vector-ratios) are element-wise ratios between two samples. There are six different options of representing vratios, the simple ratio, a ratio normalized to one element, the ratio normalized to the geometric mean and the three corresponding logarithmic representations. Each representation focuses on different aspect of the data set of vratios. The choice of a representation depends vastly upon the question asked for the specific data sets. Generally, non-log-representations emphasize on the vratios of great values and high variance. On the other log-representations are less sensitive to the total spread of data and allow to see spread of vratios of different orders of magnitude. Especially the representation of clr-vratios proved to be a very handy tool for a first exploratory approach. For the visual exploration of the vratio data sets biplots based on PCA transformation are a powerful tool to identify possible trends for further statistical analysis. 


\begin{tabular}{lrrrrrrrrrrrrr}
\hline Method & $\mathrm{Al}$ & $\mathrm{Ca}$ & $\mathrm{Co}$ & $\mathrm{Fe}$ & $\mathrm{K}$ & $\mathrm{La}$ & $\mathrm{Mg}$ & $\mathrm{Mn}$ & $\mathrm{Ni}$ & $\mathrm{P}$ & $\mathrm{Rb}$ & $\mathrm{S}$ & $\mathrm{Sr}$ \\
\hline simple & 0.0076 & 2.9 & 0.0096 & 0.0016 & 1.2 & 0.0016 & 0.37 & 0.59 & 0.21 & 6.2 & 0.36 & 19 & 0.42 \\
ar K & 0.0056 & 1.9 & 0.0062 & 0.0011 & - & 0.00094 & 0.26 & 0.42 & 0.15 & 4.4 & 0.24 & 13 & 0.24 \\
ar Ca & 0.003 & - & 0.0032 & 0.00061 & 0.54 & 0.00054 & 0.17 & 0.23 & 0.093 & 2 & 0.13 & 5.8 & 0.15 \\
ar P & 0.0012 & 0.5 & 0.0016 & 0.00025 & 0.23 & 0.00029 & 0.063 & 0.1 & 0.035 & - & 0.069 & 2.6 & 0.082 \\
cr & 0.038 & 15 & 0.046 & 0.0079 & 7 & 0.0081 & 2 & 3.3 & 1 & 32 & 2 & 89 & 2.1 \\
log & -4.9 & 1.1 & -4.6 & -6.4 & 0.18 & -6.5 & -1 & -0.53 & -1.6 & 1.8 & -1 & 2.9 & -0.88 \\
alr K & -5.2 & 0.62 & -5.1 & -6.8 & - & -7 & -1.3 & -0.87 & -1.9 & 1.5 & -1.4 & 2.6 & -1.4 \\
alr Ca & -5.8 & - & -5.7 & -7.4 & -0.62 & -7.5 & -1.8 & -1.5 & -2.4 & 0.7 & -2 & 1.8 & -1.9 \\
alr P & -6.7 & -0.7 & -6.4 & -8.3 & -1.5 & -8.1 & -2.8 & -2.3 & -3.4 & - & -2.7 & 0.96 & -2.5 \\
clr & -3.3 & 2.7 & -3.1 & -4.8 & 1.9 & -4.8 & 0.72 & 1.2 & 0.015 & 3.5 & 0.7 & 4.5 & 0.74 \\
\hline
\end{tabular}

Table 5.2.: Variance of vratios of six different representations. Table displays only selected elements. Each variance is calculated by 106 vratios.

\begin{tabular}{|c|c|c|c|c|c|c|c|c|c|c|c|c|c|}
\hline Method & $\mathrm{Al}$ & $\mathrm{Ca}$ & Co & $\mathrm{Fe}$ & $\mathrm{K}$ & $\mathrm{La}$ & $\mathrm{Mg}$ & $\mathrm{Mn}$ & $\mathrm{Ni}$ & $P$ & $\mathrm{Rb}$ & $S$ & $\mathrm{Sr}$ \\
\hline simple & $5.2 e-05$ & 21 & $7.6 e-05$ & $.7 e-06$ & 110 & $1.6 e-05$ & 1 & 1 & 0.016 & 11 & 0.9 & 540 & 0.54 \\
\hline ar & 2 & $4 . \varepsilon$ & $5.7 e$ & & & 1 & & & & & 32 & 0 & 73 \\
\hline ar & $1.6 \mathrm{e}-0$ & - & 0.000 & & 20 & 1 & & & & & 1 & 570 & 0.016 \\
\hline $\operatorname{ar} \mathrm{F}$ & $1.9 e-06$ & 0.28 & $4.5 \mathrm{e}-\mathrm{c}$ & & 3.2 & & 3 & 5 & & - & 56 & 18 & 0.008 \\
\hline $\mathrm{cr}$ & 00044 & 130 & 0.0018 & $1.4 \mathrm{e}-05$ & 390 & 0.00015 & 4.1 & 7.5 & 0.34 & 240 & 6.4 & 5000 & 2.2 \\
\hline $\log$ & 0.27 & .94 & 0.48 & 0.4 & 1.5 & 1.1 & 0.84 & 0.54 & 033 & 0.28 & 086 & 0.83 & 0.75 \\
\hline alr K & 1.7 & 1.6 & 1.6 & 1.1 & & 1.2 & 0.23 & 1 & 1.4 & 1.4 & 0.45 & 3 & 1.1 \\
\hline alr $\mathrm{Ca}$ & 0.67 & & 1.7 & 0.99 & 1.6 & 1.9 & 1.1 & 1.3 & 0.85 & 0.93 & 1.7 & 1.7 & 0.37 \\
\hline alr $\mathrm{P}$ & 0.54 & 0.93 & 0.72 & 0.48 & 1.4 & 1.5 & 0.82 & 0.82 & 0.53 & - & 1 & 0.66 & 0.99 \\
\hline $\mathrm{clr}$ & 0.32 & 0.65 & 0.53 & 0.23 & 0.71 & 0.79 & 0.31 & 0.35 & 0.28 & 0.3 & 0.47 & 1.1 & 0.42 \\
\hline
\end{tabular}



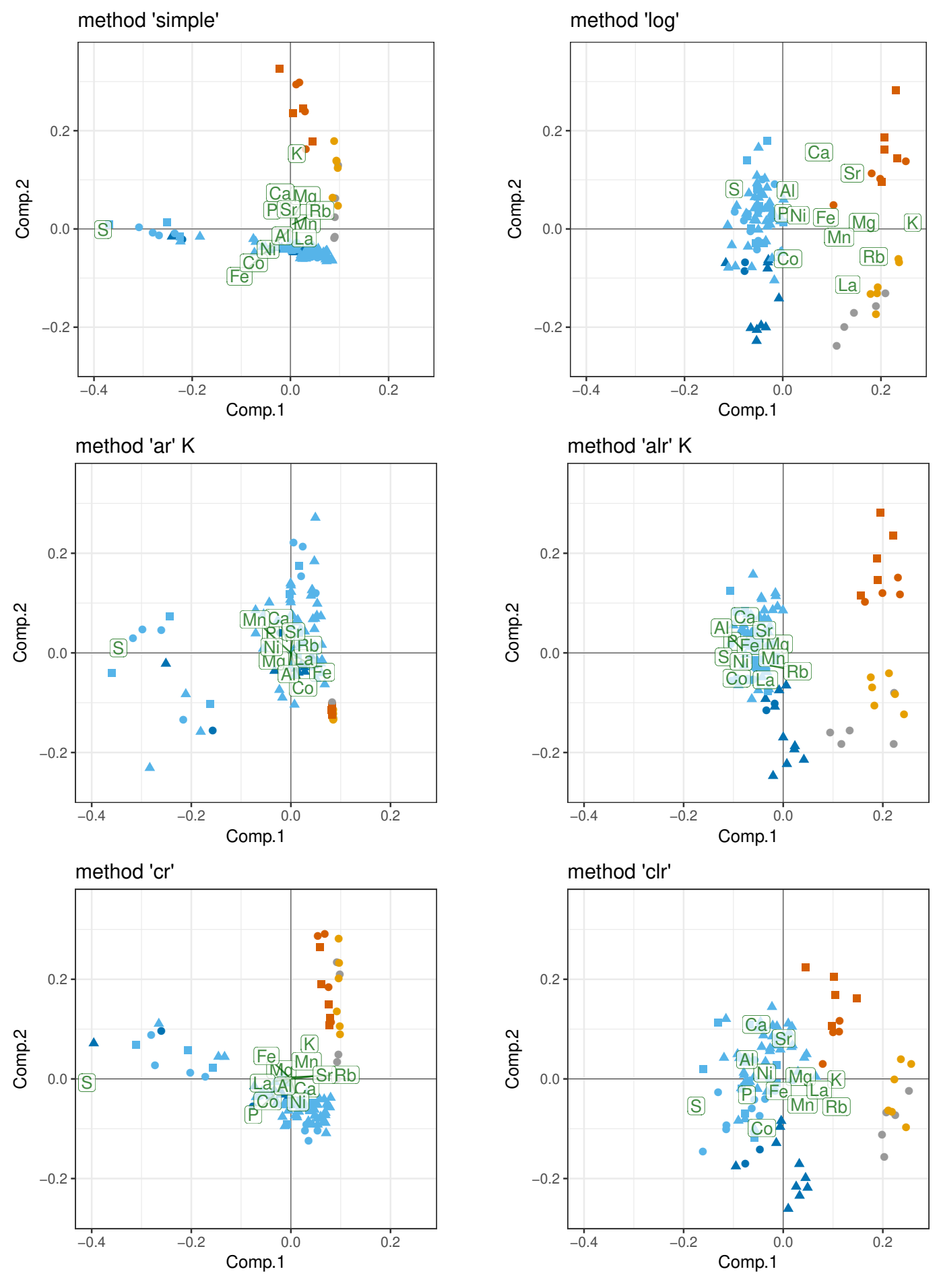

Region

Cultivation

- Darjeeling - Assam - Curitíba - Arcanjo, SP • Registro, SP

- conventional $\Delta$ organic - under conversion

Figure 5.1.: Biplots of the PCA of vratios between shoot tips of $C$. sinensis and corresponding soil analysis for six different representations. Left side of the plot are all non-log-representations, on the right side of the plot are the corresponding log-representations. Labels of elements do not overlap for visibility. 


\subsection{Statistical Tools}

This section gives an overview of the statistical tools used for the multivariate data in this thesis. Detailed explanations of the principles of HCA, PCA, LDA and ANOVA can be found for example in Fahrmeir and Hamerle (1984).

\section{Hierarchical cluster analysis}

For the agglomerative hierarchical cluster analysis (HCA) a measure of dissimilarity between sets of observations is used to calculate which groups or individual observations are most similar and can be merged into one group. The dissimilarity between observation can be expressed for example as the distance between observations, which can be calculated by various methods. Starting from each of the $D$ observations being an individual group the groups are incrementally merged into bigger groups. The results of the clustering are displayed as dendrograms. In this thesis all HCA are calculated with the Rfunction hclust of package 'stats' with the method ward.D2, which implements Ward's (1963) clustering criterion (Murtagh and Legendre 2014). At every agglomeration step, the ward.D2 clusters the two groups which produces a minimal loss of homogeneity through the merging. The algorithm calculates the loss of homogeneity by subtracting the sum of total variances within each group before merging from the variance of the group after merging (Boogaart and Tolosana-Delgado 2013).

\section{Principal component analysis}

A principal component analysis (PCA) is used to find associations between variables through reducing the dimension of the data set: The data are projected into a new coordinate system by an orthogonal linear transformation. The first coordinate (first principal component) of this coordinate system points along the greatest variance of the observations, the second coordinate (second principal component) along the second greatest variance and so on. In this thesis the PCA is calculated from the R-function princomp of package 'stats' which derives the new coordinate system by using the eigenvalue decomposition. For princomp the columns in the matrix 'loadings' contain the eigenvectors while the 'scores' are the coordinates of the individuals (observations) projected onto the principal components.

If a PCA is used for concentration data the PCA is always applied on clr-transformed compositions. This transformation is the best choice for PCA due to its inherent prop- 
erties and because results are intuitively interpretable based on the scores for each original variable.

\section{Linear discriminant analysis}

The linear discriminant analysis (LDA) is a technique based on singular value decomposition to find a coordinate system which maximizes the variance between categories and minimizes the variance within categories. It is used to distinguish groups of samples by a categorical variable, e.g. the region of sample origin. The LDA is calculated from the function lda of the package 'MASS'.

\section{Analysis of variance to create an ilr-base}

Generally, ANOVA denotes a regression-like technique where the covariables are of categorical type. For complying the necessities of compositional data the linear regression model is applied on log-ratio transformed element data, e.g. ilr (Eq. 5.1, with categorical covariables. In this thesis the built-in ANOVA function of $R$ is used to create an ilr-base with a specific structure which captures the grouping of element variables in relation to the categorical variable: By means of the regression model the total variance of the data set is decomposed in the variance due to the differences between group means and the variance within each group. This very specific application of ANOVA technique is used as an exploratory tool to assess whether groups of samples have similar or different behavior within a certain subcomposition of elements.

The ilr-ANOVA is calculated from the R-function $\operatorname{lm}$ of package 'stats' with $\operatorname{lm}(\mathrm{x} \sim \mathrm{g})$, $\mathrm{x}$ being the ilr transformed concentration data and $\mathrm{g}$ begin the categorical variable. The resulting ilr-coefficients are converted into clr-coefficients, and, based on these, a new ilr-base is calculated. The structure of this new ilr-base is visualized by dendrograms from the function CoDaDendrogram of the package 'compositions'. For more detailed explanation please refer to Tolosana-Delgado and et. al. (2019).

\subsection{Visualization Tools}

\section{Biplot}

Biplots (Gabriel 1971, 1972) are one possibility to visualize a multivariate data set in one plot. In a classical biplot the plot is based on a PCA (Section 5.3) of the variables. 


\section{Mathematical Tools}

The biplot is a scatter plot of two coordinates of the PCA, generally the first coordinate versus the second coordinate. The loadings of princomp are plotted as arrows or labels of the variables and the scores of princomp are plotted as dots or labels representing the observations which are projected onto the coordinate system of the two PCAcomponents.

The classical PCA-biplots are row-metric-preserving, so-called "form biplots" (Greenacre 2010): The length of the arrows approximates the variance of each element variable. The angle between two arrows approximates the correlation between two elemental scores.

In biplots based on log-ratio transformed data the rays are seldom interpretable and one should focus instead on the links between apexes which represent the log-ratio between two elements. The length of a link will be proportional to the variance of the log-ratio of the two variables involved. Two orthogonal links will indicate uncorrelated data respectively parallel links will indicate good correlation between the log-ratios of the several pairs of elements affected (Aitchison and Greenacre 2002; Greenacre 2010).

\section{Dendrogram}

Dendrograms are hierarchical, tree-like graphs to illustrate clustering and/or grouping of samples or variables. In this thesis two different types of dendrograms are used:

HCA dendrogram Dendrograms illustrating the result of an HCA, which is the result of the R-function plot.hclust of the package 'stats'.

CoDa dendrogram Dendrograms illustrating the hierarchy of groups underlying the ilr structure determined by the ilr-ANOVA (Section 5.3). These graphs are produced from the R-function CoDaDendrogram of the package 'compositions': Each horizontal axis represents one coordinate of the vector field of an ilr-transformed data set (Section 5.1 and the contact point the coordinate mean. The length of the vertical bars are representing the variance of the specific elements or element subcompositions.

\section{Heatmap}

A heatmap is a visualization tool to simultaneously show the similarity or associations between the rows and between the columns of a matrix. In this thesis heatmaps are used to answer two questions: 
1. For which pairwise log-ratios is the distribution of data set $A$ similar to the pairwise log-ratio distribution of data set $B$ ?

2. Which groups of elements have similar log-ratio distributions between data set $A$ and data set $\mathrm{B}$ ?

To answer the first question the distributions of the pairwise log-ratios of data set $A$ and data set $B$ are compared via tests for equal means of two distributions. These are, depending on the sample sets $A$ and $B$, either the Student's $t$-test, Wilcoxon signed rank test or Mann-Whitney test. The heatmap is a map of tiles with each tile colored by the probability value ( $p$-value) of the respective test: $p$-values below 5\% (hypothesis is rejected) are colored reddish, and $p$-values above $5 \%$ ()the means of the log-ratio distributions are considered as equal) are colored blueish.

To answer the second question the elements are grouped by their pairwise log-ratio p-values. The grouping is calculated from distfun $(X) \quad \%>\%$ hclustfun $\%>\%-$ as . dendrogram of package 'stats', whereof $X$ is the matrix of $p$-values. 



\section{Correction for Adhering Particles}

Plants grown in open field, such as tea, are prone to also contain adhering particles, such as airborne dust or spluttered soil particles. During digestion and analysis of the samples adhering particles (AP) alter the elemental composition of the plant material and introduce a bias on the tea's ionome. The amount of adhering particles on tea might vastly differ due to local climate conditions, e.g. frequent raining versus longer period of dryness or wind, distance to road or a track across fields and local manufacturing conditions, e.g. whether a filter is placed in front the factory's air intake tube. But processed tea cannot be washed or cleaned prior to analysis because it is already steamed, dried, milled or otherwise processed. To make the sample group of processed tea and plucked leaves better comparable between seasons, regions and manufacturing conditions a correction for adhering particles (correction for AP) should be calculated.

The method of the correction for AP was developed on a data set of 1040 plant samples grown in open field and pot trials in Germany. Accordingly, the Section 6.1 contains data of field plants from several projects conducted by Benedikt Sauer, Wiebke Fahlbusch, Tino Pasold and Hans Ruppert. The application of the method on tea data is presented in the Sections 6.2 and 6.3.

\subsection{Method 1}

Exact and reproducible analysis of element concentrations in plant tissue is the basis for many research fields such as environmental, health, phytomining, agricultural or provenance studies. Unfortunately plant samples collected in the field will always contain particles on their tissue surfaces such as airborne dust or soil particles (Chamberlain 1983; Harrison and Chirgawi 1989; Faucon et al. 2007). If not removed these particles may induce a bias to the element concentrations measured in plant samples. The influence of adhering particles on element concentration in plants is negligible for elements

\footnotetext{
${ }^{1}$ This section was published in Solveig Pospiech et al. (2017). Alteration of trace element concentrations in plants by adhering particles - Methods of correction. Chemosphere, 182, 501-508
} 


\section{Correction for Adhering Particles}

which have a much higher concentration in the plant tissues compared to the adhering material. This is the case for most main or minor nutrient elements such as $\mathrm{P}, \mathrm{K}, \mathrm{Ca}$, Mg, S, Mn, B, Mo, Zn or Cu (Arkley et al. 1960; Mitchell 1960; Bargagli et al. 1995). But elements with typically very low concentrations in plant tissue such as $\mathrm{Al}, \mathrm{Co}, \mathrm{Fe}, \mathrm{Li}$, $\mathrm{Ni}, \mathrm{Ti}, \mathrm{Sc}, \mathrm{Zr}$, or REEs, may show significantly altered concentrations measured in the plants due to adhering particles. Mitchell (1960) proposed that elements with concentration ratios of soil to plant above 100 might show biased concentrations in the measured samples.

Reducing the impact of adhering particles on trace element concentration in plants is crucial in order to be able to compare elemental composition of plants, e.g. between sampling periods or slightly different sampling methods (MacNaeidhe 1995), or for biomonitoring studies. It is also important in studies for plant nutrition to calculate the real uptake of an element by a plant, e.g. for phytoremediation/phytomining (Baker et al. 1994; Fässler et al. 2010; Wiche et al. 2016), or in studies on the trace elements Co, $\mathrm{Ni}, \mathrm{Mn}$ and Mo which are required by the microorganisms in biogas plants (Sahm 1981; Demirel and Scherer 2011).

Most authors recommend washing or carefully rinsing of the plant material to clean the samples prior to analysis. Washing methods were tested by several authors, e.g. ultrapure water, washing detergents, complexing agents like EDTA, slightly acidic solutions or combinations of these (Husted et al. 2004; Faucon et al. 2007; Cook et al. 2009). The disadvantages of the washing method are (1) particles may remain in leaf axils or on rough foliar (or epidermal) surfaces of the plants (Cook et al.2009) and (2) elements may get leached from the plant tissue. Washing is sometimes not possible if the plant material was already processed prior to sampling, e.g. by fermentation, drying, cutting or mixing.

Although several authors were aware of the problem of irremovable adhering particles and their bias to trace element concentrations in plant tissue (Mitchell 1960; Tölgyesi and Nagy 1990; Markert 1995; Reimann et al. 2001; Anke and Seifert 2004; Faucon et al. 2007; Verbruggen et al. 2009; Censi et al. 2017), or showed by experimental studies the influence of adhering particles on plant tissue (Cary 1992; Gerzabek et al. 1992), only Bargagli et al. 1995 and Sauer and Ruppert 2013 provided a mathematical approach using aluminum or titanium as a reference element to estimate the amount of adhering material and calculate "true" element concentrations in plant tissue.

Based on the model that the analyzed plant material is a mixture of plant tissue and a very minor amount of adhering particles we developed more general methods to calcu- 
late a correction term for adhering material. We will present three methods of calculating a correction term and show the effect of these correction terms on the trace element concentrations of plant matter.

\subsubsection{Materials and Methods}

\section{Study Sample Set}

The sample set consist of plants and corresponding soil samples from different research projects between 2011 and 2015. In all projects a broad element range was measured focusing on trace elements. The plants in these studies comprised forage and energy crops (for biogas production), perennial and catch crops (Table 6.1). In this study the element data of a total of 1040 plant samples of 19 species and at least 10 samples for each species is presented.

Table 6.1.: List of plants in the data set used for correction for adhering particles.

\begin{tabular}{llcc}
\hline Plant Species & Scientific name & Nr. of samples & Nr. of locations \\
\hline Amaranth & Amaranthus spp. & 255 & 12 \\
Barley & Hordeum vulgare L. & 11 & 2 \\
Beet (leaves) & Beta vulgaris L. & 18 & 7 \\
Buckwheat & Fagopyrum esculentum & 47 & 16 \\
Catnip & Nepeta cataria L. & 14 & 11 \\
Cup Plant & Silphium perfoliatum L. & 13 & 8 \\
Faba Bean & Vicia faba L. & 59 & 11 \\
Maize & Zea mays L. & 151 & 23 \\
Mustard & Sinapsis alba L. & 15 & 12 \\
Oat & Avena sativa L. & 24 & 2 \\
Pea & Pisum sativum L. & 14 & 3 \\
Quinoa & Chenopodium quinoa & 50 & 16 \\
Rye & Secale cereale L. & 102 & 20 \\
Ryegrass & Lolium perenne L. & 28 & 4 \\
Sorghum & Sorghum bicolor L. & 13 & 3 \\
Sunflower & Helianthus annuus L. & 52 & 14 \\
Triticale & Triticosecale Wittm. & 92 & 19 \\
Vetch & Vicia villosa L. & 55 & 6 \\
Wheat & Triticum aestivum L. & 27 & 15 \\
\hline & & & \\
\hline
\end{tabular}




\section{Correction for Adhering Particles}

The plant and soil samples were retrieved from open arable field trials and from (open) pot trials filled with differently soils. Of the 1040 plant samples 789 plants were from open field (20 locations in southern Lower Saxony and Bavaria, Germany), and 251 from open pot trials (a total of 13 different locations in Lower Saxony). The pots were located close to the sedimentology and environmental geology department of the Georg-August University in Göttingen. None of the plant samples were rinsed. The soil types in the field ranged between silt, silty loam and sandy loam with a pH of 5.8 to 7.0 (measured in $0.01 \mathrm{~mol} / \mathrm{L} \mathrm{CaCl}_{2}$ ).

\section{Sampling, Sample Preparation and Analysis}

The plant samples were cut at ca. $10 \mathrm{~cm}$ above soil surface at the stage of lactic ripeness or end of flowering. The roots are not included in the analysis. About $500 \mathrm{~g}$ of soil samples were taken in the upper part of the soil (up to $30 \mathrm{~cm}$ depth) and are air dried and sieved to $<2 \mathrm{~mm}$ in grain size. All samples, soils and plants, were dried at $105^{\circ} \mathrm{C}$. A minimum of $100 \mathrm{~g}$ of the soils and ca. $500 \mathrm{~g}$ of plants were ground in an agate ball mill to avoid metal contamination.

Aliquots of $150 \mathrm{mg}$ of soil respectively $700 \mathrm{mg}$ of plant powder were fully digested with a mixture of ultra pure concentrated $\mathrm{HNO}_{3}, \mathrm{HClO}_{4}$ and $\mathrm{HF}$ in closed ultra clean PTFE vessels (PicoTrace, Bovenden, Acid sample digestion system DAS 30). In the soil samples a small amount of $\mathrm{HCl}$ is added. Soil samples were diluted to $100 \mathrm{ml}$ the plant samples to $50 \mathrm{ml}$ for measurement. In the resulting clear sample solutions 47 elements could be quantified by ICP-OES and ICP-MS.

To ensure clean handling and quality of analysis for every 30 samples one to two blank solutions and one or two international reference standards were digested and analyzed together with the samples. For plant samples the following international reference materials were used: GBW 10052, BCR-129, WEPAL-IPE-126, WEPAL-IPE-168. For soils the geochemical standard TW-45 was used. Results were only accepted if concentrations were above the limit of detection (3-fold standard deviation of the blank concentrations for each analysis batch) and if the corresponding reference samples showed concentrations within the certified values. Average precisions for the elements were better than $5 \%$ for all main elements and most of the trace elements; for plants the ultratrace elements $\mathrm{Cr}$, Co, Mo, Sn, and the heavy REE showed average precision between $10 \%-20 \%$. All concentration data are given in $\mathrm{mg} / \mathrm{kg}$ d.w.. 


\subsubsection{Theory of the Correction}

All correction terms are based on the following simplified model: The plant samples collected from open field trials $(\mathbf{P})$ consist of the pure plant material, the ionome $(\mathbf{I})$, and a minor content of deposited solid material from different sources, the adhering particles (A). With a digestion method ensuring complete and total dissolution of the sample the analytically determined element concentration, $\mathbf{P}$, contains both components, $\mathbf{I}$ and $\mathbf{A}$. Hence the concentrations of the ionome I could be calculated by subtracting adhering particles $\mathbf{A}$ from the analyzed plant sample $\mathbf{P}$ (Eq. 6.1).

The most important sources of adhering particles are airborne dust, soil particles from wind or rain splash. In this study the adhering particles are assumed to be mainly soil particles (S). Especially the lower parts of plants will be strongly affected by splashed soil particles, but this holds also for the higher parts of the plants even if less affected.

We are aware that a fractionation of soil particles may occur during transport through the air or that collected atmospheric dust could locally be a better proxy for adhering material, but to simplify our calculations we take into account only data of the soils corresponding to the plants. Air dust collection and analysis would be extremely laborious and time-consuming.

The content of adhering particles can be approximated by elements with good analytical precision and accuracy and with negligible uptake by plants, hence concentrations approach zero, but high concentrations in the adhering particles. We call these elements "indicator elements", e.g. $\mathbf{P}_{\text {ind }}, \mathbf{S}_{\text {ind }}$ or $\mathbf{A}_{\text {ind }}$. In order to estimate the content of adhering soil $k$ (Eq. 6.1) to the plant samples we propose three different methods:

Method 1: Using one pre-defined element indicator element for calculating the content of adhering particles in plant samples From all elements fulfilling all requirements of one indicator element chosen to calculate $k$ in Eq. 6.4 (Ti, Al, Zr, Sc...). For example using Ti as indicator the content of $\mathbf{A}$ is calculated through $k=\mathbf{P}_{T i} / \mathbf{S}_{T i}$ and this $k$ is then used in Eq. 6.2. Resulting negative concentrations should be set to zero.

Method 2: Subtracting the smallest possible content of adhering particles (smallest $\boldsymbol{k}$ ) For each sample the element with the smallest $k$ of all ratios $k=\mathbf{P}_{i} / \mathbf{S}_{i}$ of each sample is taken as indicator element, hence every sample is corrected based on a different $i$. With this method only the smallest possible content of $\mathbf{A}$ is subtracted from 


\section{Correction for Adhering Particles}

$\mathbf{P}$ (Eq. 6.2). Typical indicator elements are Ti, Al, Th, Cs, Zr etc., if they can be measured with high sensitivity and reproducibility. With this method there are no negative concentrations.

Method 3: Using the median of several elements with a very small $k$ for calculating the content of adhering particles In order to reduce the uncertainty of the content of adhering particles based on only one element as in method 1 and 2 an average of several $k$ of indicator elements can be calculated. With $\Delta k$ being the absolute error of $k$ we suggest to take the median of the $k$ of all elements which values $k-\Delta k$ are smaller than $k_{\text {smallest }}+\Delta k_{\text {smallest }}$. The value of the median $\bar{k}$ is then used as $k$ in Eq. 6.2. Negative concentrations should be set to zero, because statistically the $k$ of all elements, which error overlaps the error of the element with smallest $k$, are indistinguishable. For the same reason, all elements contributing to the computation of the median $\bar{k}$ should be set to zero, because these small values should not be interpreted.

\subsubsection{Calculation of the Correction}

With $k$ as the variable for the content of $\mathbf{A}$ in $\mathbf{P}, 0<k<1$, the composition of $\mathbf{P}$ can be expressed as the sum of $\mathbf{I}$ and $\mathbf{A}$ :

$$
\mathbf{P}=\mathbf{I} \cdot(1-k)+\mathbf{A} \cdot k
$$

Resolving equation 6.1 for I results in equation 6.2 .

$$
\mathbf{I}=\frac{\mathbf{P}-k \cdot \mathbf{A}}{1-k}
$$

of which the composition of $\mathbf{A}$ and the content of the adhering particles, expressed as $k$, are unknown and assumptions have to be made.

Assuming that the concentration of one of the indicator elements in $\mathbf{I}$ is zero, $\mathbf{I}_{\text {ind }}=0$, equation 6.2 can be used for solving $k$, the content of $\mathbf{A}$ :

$$
\begin{gathered}
\mathbf{I}_{i n d}=\frac{\mathbf{P}_{i n d}-k \cdot \mathbf{A}_{i n d}}{1-k}=0 \\
k=\frac{\mathbf{P}_{i n d}}{\mathbf{A}_{i n d}}
\end{gathered}
$$

Note that for the calculation of $k$ only elements of the group of indicator elements are 
allowed. Otherwise the correction term will lead to unrealistic element concentrations in I.

The absolute error of $k, \Delta k$, is calculated through the general formula for error propagation for random and uncorrelated errors of $\mathbf{P}$, with $\Delta \mathbf{P}$ as the absolute error range for analyzed plant values, and $\mathbf{A}, \Delta \mathbf{A}$ as the absolute error range of the adhering particles:

$$
\begin{aligned}
\Delta k & =\Delta \mathbf{P} \cdot \frac{\partial k}{\partial \mathbf{P}}+\Delta \mathbf{A} \cdot \frac{\partial k}{\partial \mathbf{A}} \\
& \approx \Delta \mathbf{P} \cdot \frac{1}{\mathbf{A}}-\Delta \mathbf{A} \cdot \frac{\mathbf{P}}{\mathbf{A}^{2}}
\end{aligned}
$$

To estimate the absolute error $\Delta \mathbf{P}$ we use the relative standard deviation of more than 30 measurements of the international reference sample GBW10052 as relative error $\delta \mathbf{P}$ : $\Delta \mathbf{P}=\delta \mathbf{P} \cdot \mathbf{P}$. To simplify the error calculation $\Delta \mathbf{I}$ for equation 6.6 we suggest to use in the error-equation as $\Delta \bar{k}$ the standard deviation of $\bar{k}$.

For the results we used Method 3 explained in theory part. We assume that the major source of adhering particles are particles of the soil on which the plants grew $(\mathbf{A}=\mathbf{S})$. Hence based on equation 6.2 the correction equation is

$$
\mathbf{I}=\frac{\mathbf{P}-\bar{k} \cdot \mathbf{S}}{1-\bar{k}}
$$

If an element has a low concentration in the soils the term $\bar{k} \cdot \mathbf{S}$ becomes close to zero. With $\bar{k} \cdot \mathbf{S} \approx 0$ and the term $\mathbf{P} /(1-\bar{k})$ being always larger than $\mathbf{P}$ this may result for main elements in plants into higher concentration in these elements after correction.

\subsubsection{Results and Discussion of the Method}

\section{Discussion of the Methods}

Calculating the content of adhering particles via Method 1 The advantage of the method with a pre-defined element is a rather easy calculation because for all samples the same indicator element is used. The disadvantage is that an overcorrection may occur if the chosen indicator element has a notable higher $\mathbf{P} / \mathbf{S}$-ratio than the smallest ratio. That might be the case if due to fractionation of the $\mathbf{A}$ the chemical composition of the adhering particles is not exactly the same as the chemical composition of $\mathbf{A}$ used in the correction term or if the indicator element is (unexpectedly) taken up by a plant. This is the case for Al in buckwheat Fagopyrum esculentum or in tea Camellia sinensis (Flaten 2002; H. P. Carr et al. 2003; Zheng et al. 1998). Even Ti, considered as highly immobile in soils, might be taken up by plants in very small quantities and by some 


\section{Correction for Adhering Particles}

authors is considered as beneficial to plants (Dumon and Ernst 1988; Cigler et al. 2010; Haghighi et al. 2012).

Calculating the content of adhering particles via Method 2 Subtracting only the smallest possible content of adhering particles minimizes the risk of overcorrection. Disadvantageous is that the correction depends on one element only (lowest ratio of $\mathbf{P} / \mathbf{S}$ ) and not on analytical reasoning. If the chosen element is an (ultra-)trace element with concentrations close to the detection limit with poor reproducibility and high analytical uncertainty, this results into a high uncertainty of the smallest $k$ and that directly determines the uncertainty of the applied correction.

The content of subtracted A can therefore vary strongly on the set of trace elements considered for the group of indicator elements. For example plants contain very low concentrations in $\mathrm{Hf}$ and therefore it is difficult to determine $\mathrm{Hf}$ with high accuracy and precision. If $\mathrm{Hf}$ represents the smallest $k$ and $\mathrm{Ti}$ second smallest $k$ but considerably higher, the content of $\mathbf{A}$ would be calculated by $\mathrm{Hf}$. If $\mathrm{Hf}$ is excluded from the group of indicator elements due to analytical reasons, the content of $\mathbf{A}$ would be calculated by $\mathrm{Ti}$ and would result into a stronger correction. So the calculated content of $\mathbf{A}$ would depend upon if $\mathrm{Hf}$ whether included to the group of indicator elements or not. Additionally if the indicator element has large errors, these will propagate trough the correction to all elements.

Calculating the content of adhering particles via Method 3 This method is particularly interesting if several elements of the group of indicator elements had been analyzed with very small analytical uncertainties. The value of $\bar{k}$ is based on several elements with overlapping error $\Delta k_{i}$. That avoids on the one hand the problem of method 1 that the element might be taken up by the plant and on the other hand avoids the problem of method 2 that it is quite random which element is the element used for correction and that the analytical error might be quite high. The disadvantage is that the implementation of method 3 on a routine basis is not as simple as for example in method 1 , especially if error calculation is included.

\section{Evaluation of the Calculated Content of Adhering Particles}

The content of adhering particles in $\mathbf{P}$ had been calculated via method 3 for 1040 whole crop samples from fields in Germany. As shown in Figure 6.1 proportions of adhering 
particles vary among samples on the range between $0 \mathrm{wt} \%$ (no particles) and $6.1 \mathrm{wt} \%$, with median values of about $0.09 \mathrm{wt} \%$ in the pot trials and $0.16 \mathrm{wt} \%$ in open field trials.

Although the content of adhering material seems to be rather small in comparison with the dry weight mass of the plant samples, it may have a notable impact on many elements. For each sample and for each element the ratio of corrected values to measured values $(\mathbf{I} / \mathbf{P})$ are calculated, hence for each element there are over 1000 ratios. The distribution of ratios for each element after applying method 3 is displayed in Figure 6.2 . Figure 6.2 shows that - as expected - the concentrations of most nutrients, S, P, Ca, K, $\mathrm{Mo}, \mathrm{Mg}, \mathrm{Zn}, \mathrm{Cu}$, and some trace elements with chemical characteristics analogous to a nutrient, such as $\mathrm{Cd}, \mathrm{Sr}$ (analogous to $\mathrm{Ca}$ ) and $\mathrm{Ba}, \mathrm{Rb}$ (analogous to $\mathrm{K}$ ), are only slightly affected through adhering soil particles with ratios close to 1 (Figure 6.2).

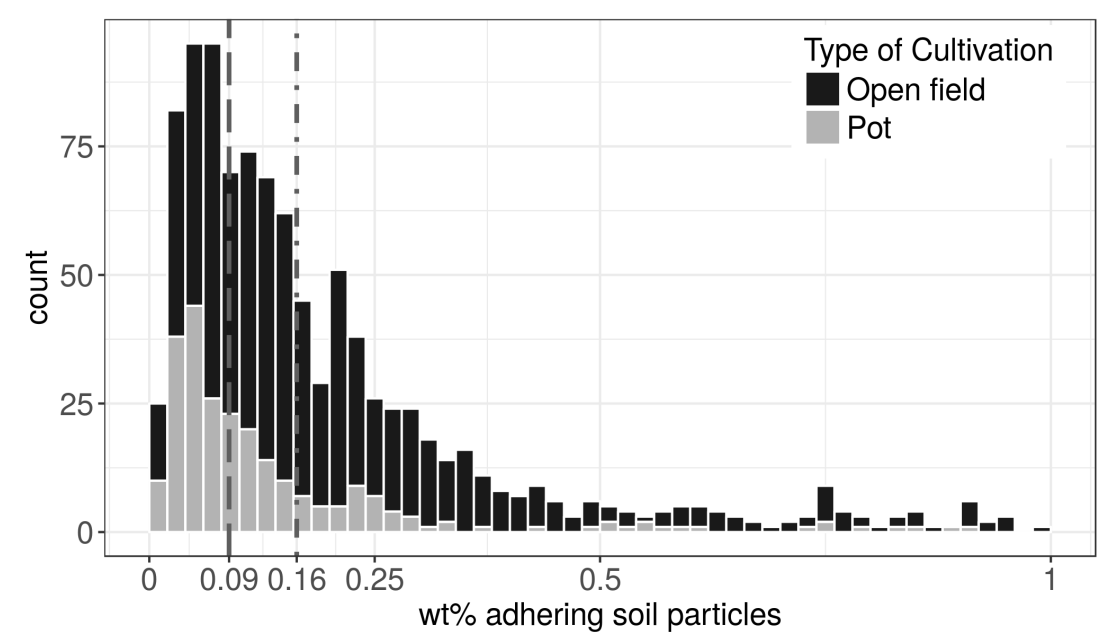

Figure 6.1.: Distribution (bin width $=0.02$ ) of wt $\%$ of adhering soil particles in 1040 crop samples grown in open field $(n=789)$ and open pot trials $(n=251)$. The content of adhering particles calculated via method 3. Right skewed distribution shows that all samples contain at least minor amounts of adhering particles. The median of adhering particles in open field is bigger (dotdash line) than in open pot trials (dashed line) maybe because of severe weather events. The plot is cut at $1 \mathrm{wt} \%$, the maximum value of $6.1 \mathrm{wt} \%$ not shown.

The strongest impact of adhering particles is on the trace elements $\mathrm{Y}, \mathrm{Li}$, La (representative for all REEs), U, Nb, Ta, Al, Ti, Sc, Th, $\mathrm{Zr}$ and Hf: The concentrations after the correction are clearly less than $50 \%$ of the measured value. These elements belong to the typical "indicator elements". For $\mathrm{Mn}, \mathrm{Ni}, \mathrm{Ba}, \mathrm{Cr}, \mathrm{Sb}, \mathrm{TI}, \mathrm{Co}, \mathrm{Na}, \mathrm{Cs}, \mathrm{Pb}$ and $\mathrm{Fe}$ the distribution of the ratios shows that the impact of adhering soil particles varies among sample: for some the effect is barely measurable, for others the impact is notable. For example, Fe shows a ratio is significantly smaller than 1 for nearly all samples and half 


\section{Correction for Adhering Particles}

of the samples contain less than $64 \%$ of the measured concentration after correction. In case of essential trace elements for the microorganisms in biogas plants ( $\mathrm{Co}, \mathrm{Ni}, \mathrm{Mn}$, Mo) (Sahm 1981; Demirel and Scherer 2011) we see a diverse picture: The correction slightly affects the concentration of Mo, because Mo is a micro-nutrient for plants and is fairly mobile in the soil solution (Kaiser et al. 2005). It is taken up easily by the plant so that we observe a negligible correction of the measured values (Median $=1.0$ ) only. The median values of $\mathrm{Mn}$ and $\mathrm{Ni}$ are reduced by $3 \%(\mathrm{Mn})$ and $5 \%(\mathrm{Ni})$. Co shows for most samples a significant reduction of concentration after applying the correction. On average the Co concentrations are reduced by $16 \%$ (Figure6.2).

\section{Effect of Correction}

There is no direct evidence to assess the correctness of the method. But by looking at the grouping of elements via principal component analysis (PCA) on log-ratios it can be seen that some elements show a different behavior before and after the correction.

In Figure 6.3 the upper plot represents the correlation and variance of log-ratios of the uncorrected plants (data set $\mathbf{P}$ ) and the lower plot of the corrected plants (data set $\mathbf{I}$ ). For the original sample data (P, Figure 6.3, upper) the elements $\mathrm{Nb}, \mathrm{Zr}, \mathrm{U}, \mathrm{Ti}$, $\mathrm{Al}, \mathrm{La}, \mathrm{Li}, \mathrm{Pb}$ and $\mathrm{Sb}$ are lying close together which indicates a correlation between these typical indicator elements. This group of elements forms long links to all nutrient elements, except for $\mathrm{Fe}$ and $\mathrm{Na}$, indicating no correlation between the group of indicator elements and the nutrients. The ratio $\mathbf{P} / \mathbf{S}$ of the nutrient Fe lies between the ratios $\mathbf{P} / \mathbf{S}$ of indicator elements and nutrients and also in Figure 6.3 in the data set $\mathbf{P}$ the element Fe plots between the two groups. That means that the concentration of $\mathrm{Fe}$ is notably influenced by adhering soil particles. A similar situation applies to $\mathrm{Na}$.

For the corrected samples (I, Figure 6.3, lower) the grouping of elements is notably reduced and the links between elements are generally shorter. Indicator elements plot in one corner of the plot. Especially $\mathrm{Zr}$ and $\mathrm{Nb}$ show a high variation with respect to all other elements. This suggests that the log-ratios between most indicator elements are still quasi-constant but do not form a distinct group. The nutrients $\mathrm{Fe}$ and $\mathrm{Na}$ have moved towards the nutrient group indicating that their log-ratios are decoupled from the group of indicator elements. Additionally, the shorter links between elements indicate that logratios between all elements are now more constant between all samples: Corrected concentrations should better quantify the mechanisms of element uptake into the plants than concentrations including the bias through contamination by adhering particles. 


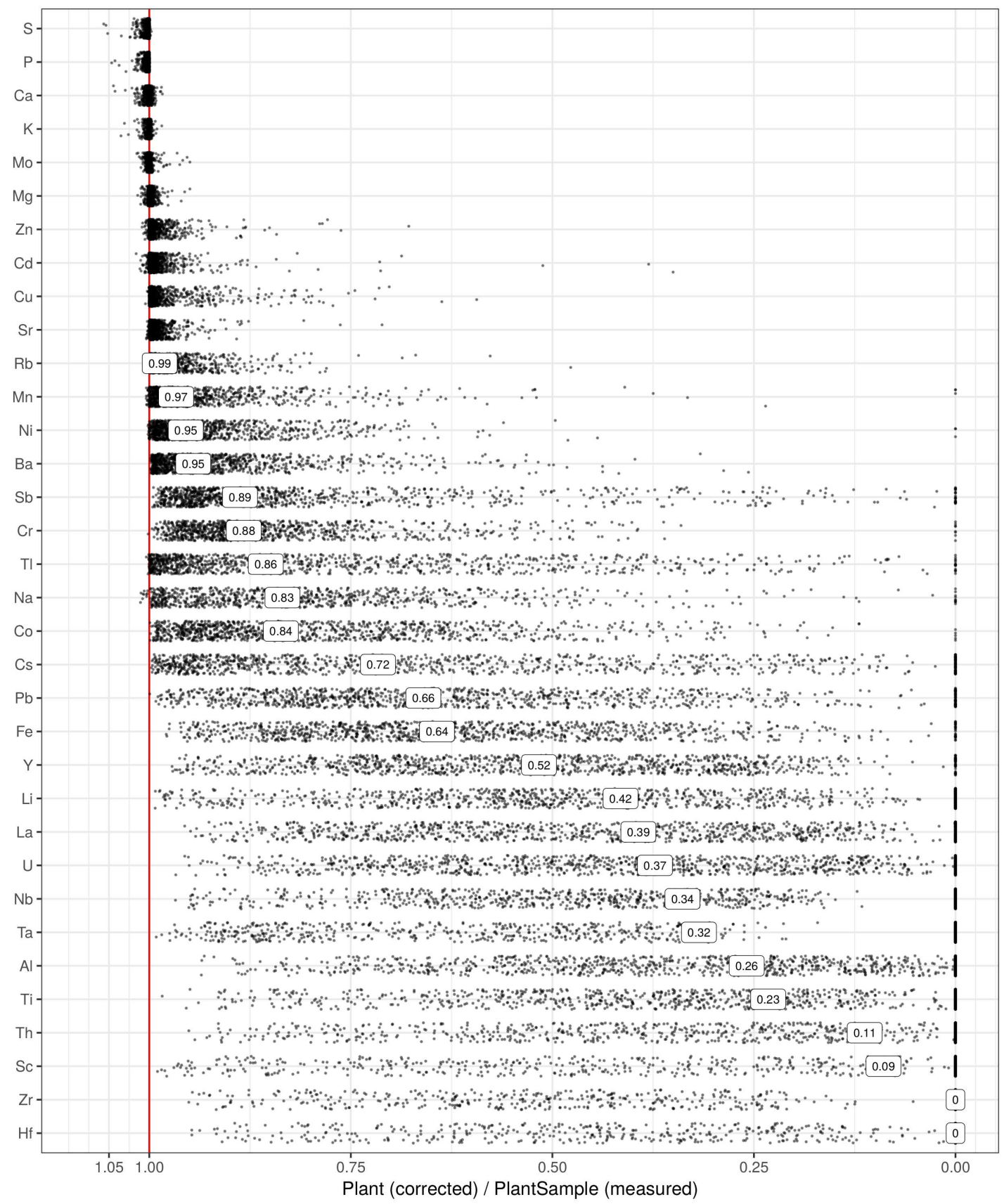

Figure 6.2.: Distribution of ratios (1040 samples) of $\mathbf{I} / \mathbf{P}$ (corrected/original) for main and trace elements. Corrected values of I calculated via Method 3. Each dot in element rows represents one ratio so that in each row there are $\sim 1040$ dots. The dots jitter vertically to reduce overplotting. The median is plotted as label at the position of the median, and rows are sorted by median. For $\mathrm{S}$ to $\mathrm{Sr}$ the median label is omitted because the median is close to 1. Main elements may show slightly higher concentrations after applying the correction (see explanation for Eq. 6.6. Concentrations of elements which contributed to $\bar{k}$ (see explanation method 3) are set to zero to avoid overinterpretation of these small residual values. 

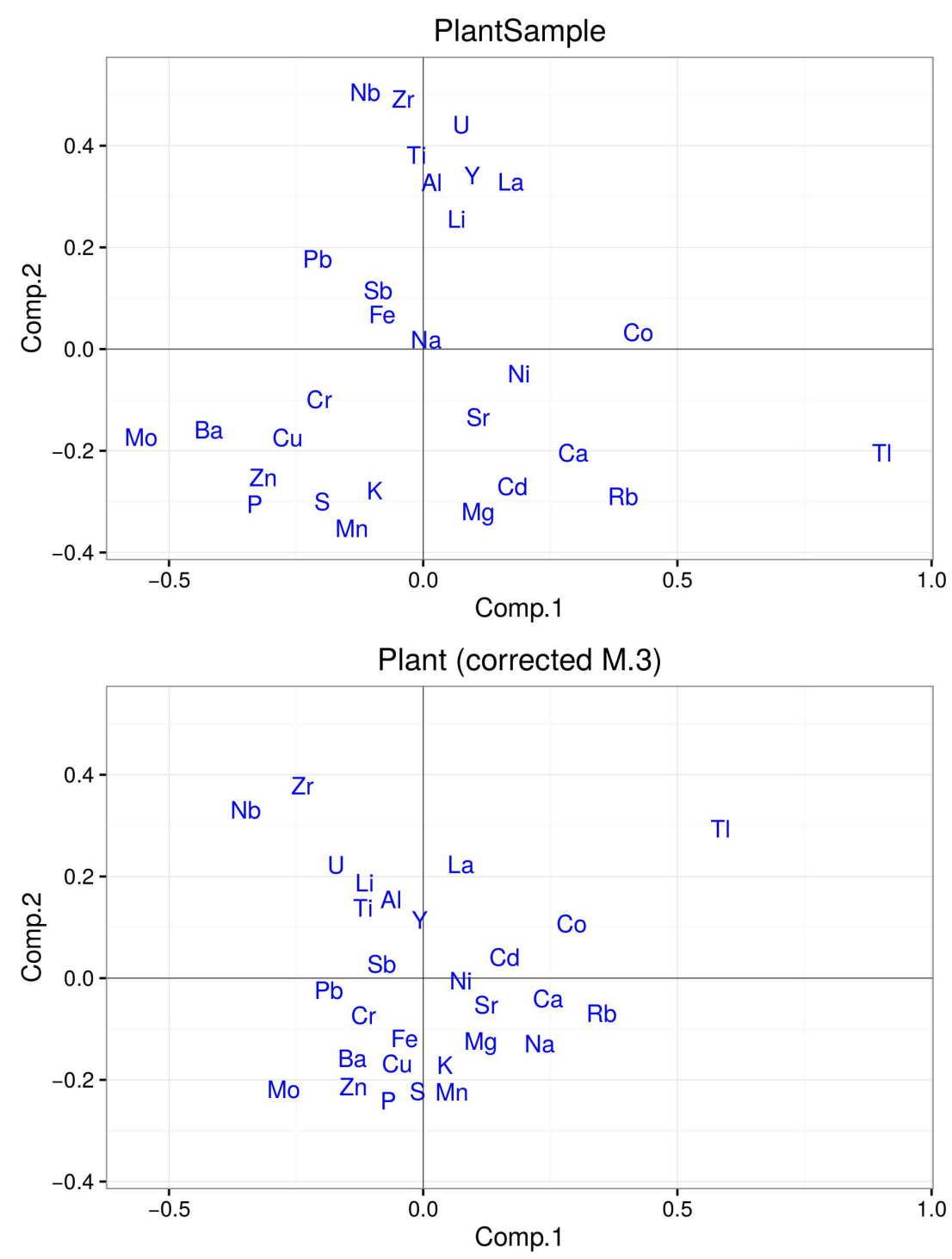

Figure 6.3.: Coviariance biplots of $\mathbf{P}$ (upper, original) and $\mathbf{I}$ (lower, corrected) of a principal component analysis of log-ratio transformed (clr) data (712 samples with 29 elements). The proportion of the explained variance is 0.52 for the upper plot and 0.49 for the lower plot. The position of the element labels can be interpreted as follows (Greenacre 2010; Boogaart and Tolosana-Delgado 2013): the link between two element labels represents the log-ratio between these two elements. A short link indicates that the elements have a quasi-constant log-ratio. An angle of $90^{\circ}$ at the origin means that the log-ratios of the two elements are uncorrelated. Several samples and elements had to be excluded from this examination because of special circumstances (i.e. heavy metal contaminated locations.) The general pattern of the links between elements remains unchanged by this adaption. 

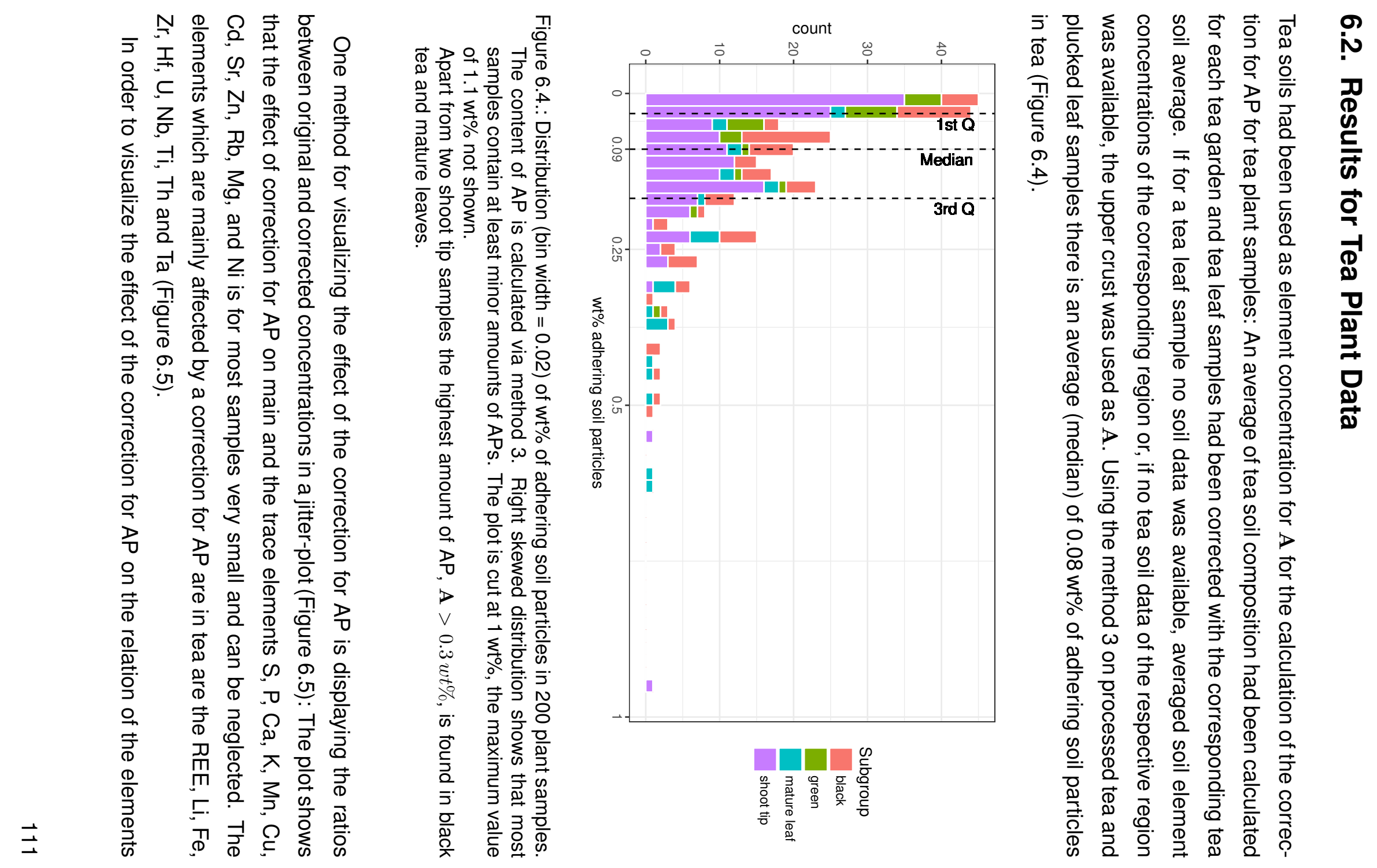

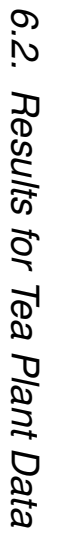




\section{Correction for Adhering Particles}

in the tea ionome the log-ratios are plotted by biplots (Figure 6.6. In Figure 6.6 the left plot represents the correlation and variance of log-ratios of the uncorrected tea leaf samples and the right plot of the corrected tea leaf samples. The calculation of the biplots is based on samples of several origins (Table 6.2). The distribution of the sample

Table 6.2.: Number of tea samples in respect of country and region plotted in Fig. 6.6.

\begin{tabular}{llr}
\hline Country & Region & N \\
\hline Brazil & São Paulo & 37 \\
Brazil & Paraná & 5 \\
India & Darjeeling & 155 \\
India & Assam & 26 \\
India & Nilgiris & 25 \\
Iran & & 2 \\
Japan & Uji & 13 \\
Japan & Shizuoka & 10 \\
Japan & & 1 \\
Nepal & Dhankuta & 4 \\
Nepal & & 7 \\
Sri Lanka & & 1 \\
Uganda & & 1 \\
\hline
\end{tabular}

dots in the right biplot in Figure 6.6 shows that the grouping of samples has no visible effect by the origin of samples or whether the samples had been corrected via soil data or upper crust. Samples are in both biplots mainly grouped by the subgroup of the samples, especially the subgroup of the mature leaf samples is clearly distinct from the other samples. The correction for AP does not affect the general grouping of samples.

On the other hand some element ratios are visibly affected by the correction for AP: For the original sample data (Figure 6.6, left) the nutrient and micro-nutrient elements $\mathrm{K}$, $\mathrm{P}, \mathrm{S}, \mathrm{Mg}, \mathrm{Zn}, \mathrm{Cu}, \mathrm{Co}, \mathrm{Ni}$ and trace element $\mathrm{Rb}$ are lying close together which indicates a correlation between these elements. Trace elements $\mathrm{Ti}, \mathrm{Nb}, \mathrm{U}, \mathrm{Cr}, \mathrm{Zr}, \mathrm{Pb}$ and $\mathrm{Sb}$, all elements affected by correction for AP, are loosely plotting in one group which is in the biplot located opposite to the nutrient group. Between these two groups plot the element Fe plots in line with $\mathrm{Zr}$ and $\mathrm{Ti}$, which hints a correlation between the pairwise log-ratios of $\mathrm{Ti}, \mathrm{Zr}$ and Fe. A third group is composed of the elements $\mathrm{Ca}, \mathrm{Sr}, \mathrm{Ba}, \mathrm{TI}, \mathrm{Mn}$ and $\mathrm{Cd}$. The group of Sc, La, Y, Al and $\mathrm{Li}$ is placed in the field of vastly spreading mature leaf samples.

For the corrected samples the right plot in Figure 6.6 exhibits a slightly different relation between the elements. The shorter links between some elements indicate that the 
log-ratios between these elements are more constant for the corrected tea data. For example in the corrected data (right plot) Fe plots closer to the group of nutrients, $\mathrm{Ba}$, $\mathrm{Sr}$ and $\mathrm{Ca}$ are now in-line hinting a correlation between the elements, and Sc - a typical indicator for adhering soil, plots in a close group with Al, La and Y, which are typical for mature leaves. In general the distance between the group of nutrients, which stay unaffected by the correction, and the group of elements, which are intensely affected by the correction, is smaller after the correction for adhering particles.

For displaying the difference in grouping of elements before and after the correction for AP the method HCA is used (Figure 6.7). In both dendrograms there are three main groups of elements. After applying the correction for AP the three groups are:

1. Th, Ta, Hf, Zr, U, Nb, Ti, $\mathrm{Pb}$ and $\mathrm{Sb}$

2. Al, La, Y, Sc, and $\mathrm{Ca}, \mathrm{Sr}, \mathrm{Ba}$

3. TI, nutrients, micro-nutrients, and elements with nutrient-similar characteristics, such as $\mathrm{Rb}$, Cs and Cd

The first group are elements which are heavily affected by soil AP (Figure 6.2 and 6.5), the second group are elements which are typically influenced by the developmental stage of the leaf (Figure 6.6) and the last group are the mainly nutrients. Through the correction for AP the elements $\mathrm{Fe}, \mathrm{Na}, \mathrm{Cd}$ and $\mathrm{Mn}$ move into the third group, Cr moves out of the first group and $\mathrm{Sb}$ and $\mathrm{Pb}$ into the first group. Hence, after the correction for AP the element groups are better explainable by plant physiology, such as nutrients vs. non-nutrients, leaves development stages, etc. 


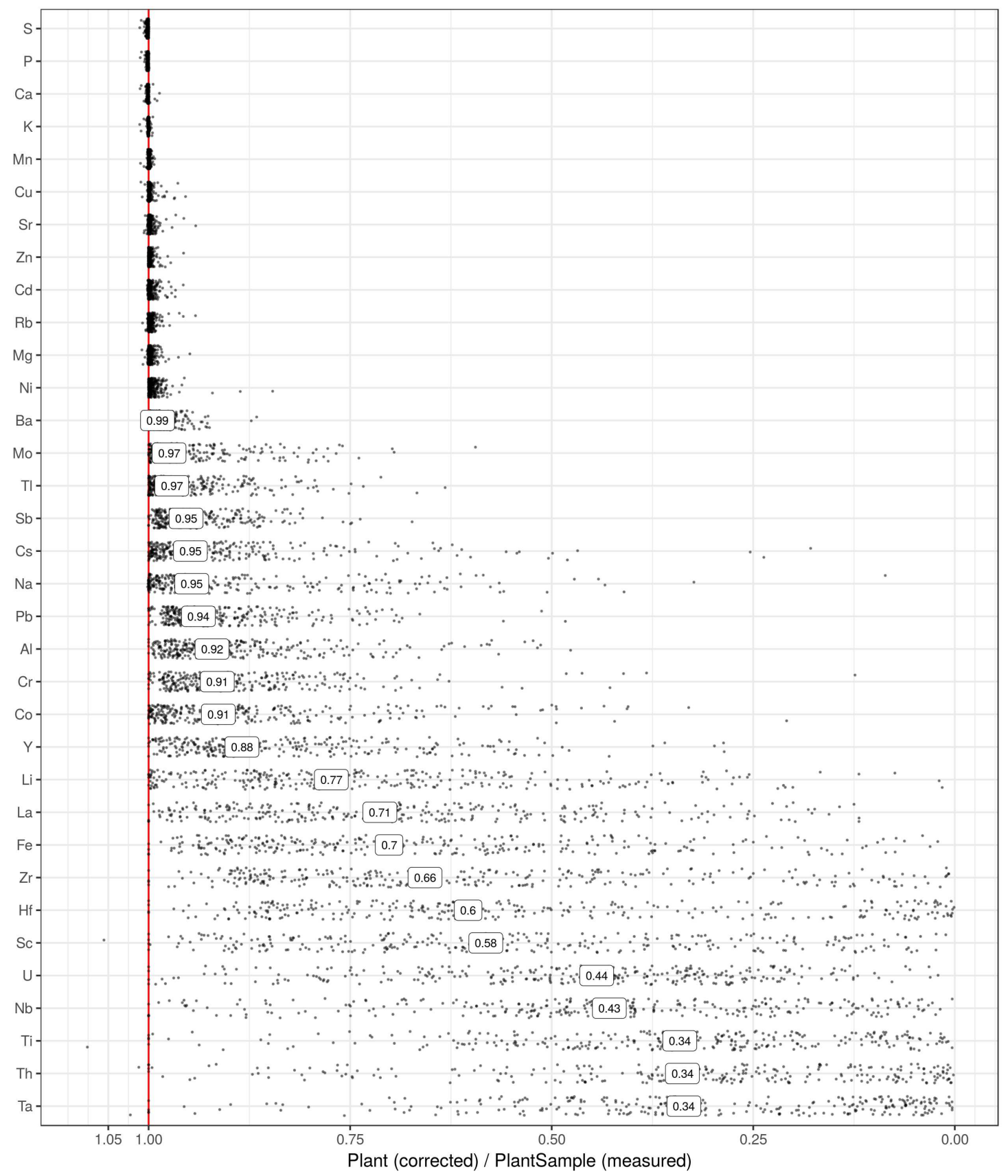

Figure 6.5.: Distribution of ratios (200 tea leaf samples) of $\mathbf{I} / \mathbf{P}$ (calculated ionome/original) for main and trace elements. Corrected values of $\mathbf{I}$ is calculated via Method 3 . Each dot in element rows represents one ratio so that in each row there are maximum 200 dots. The dots jitter vertically to reduce overplotting. The median is plotted as label at the position of the median, and rows are sorted by median. For $\mathrm{S}$ to $\mathrm{Ni}$ the median label is omitted because the median is close to 1 . Main elements may show slightly higher concentrations after applying the correction (see explanantion in section 'Method'). 

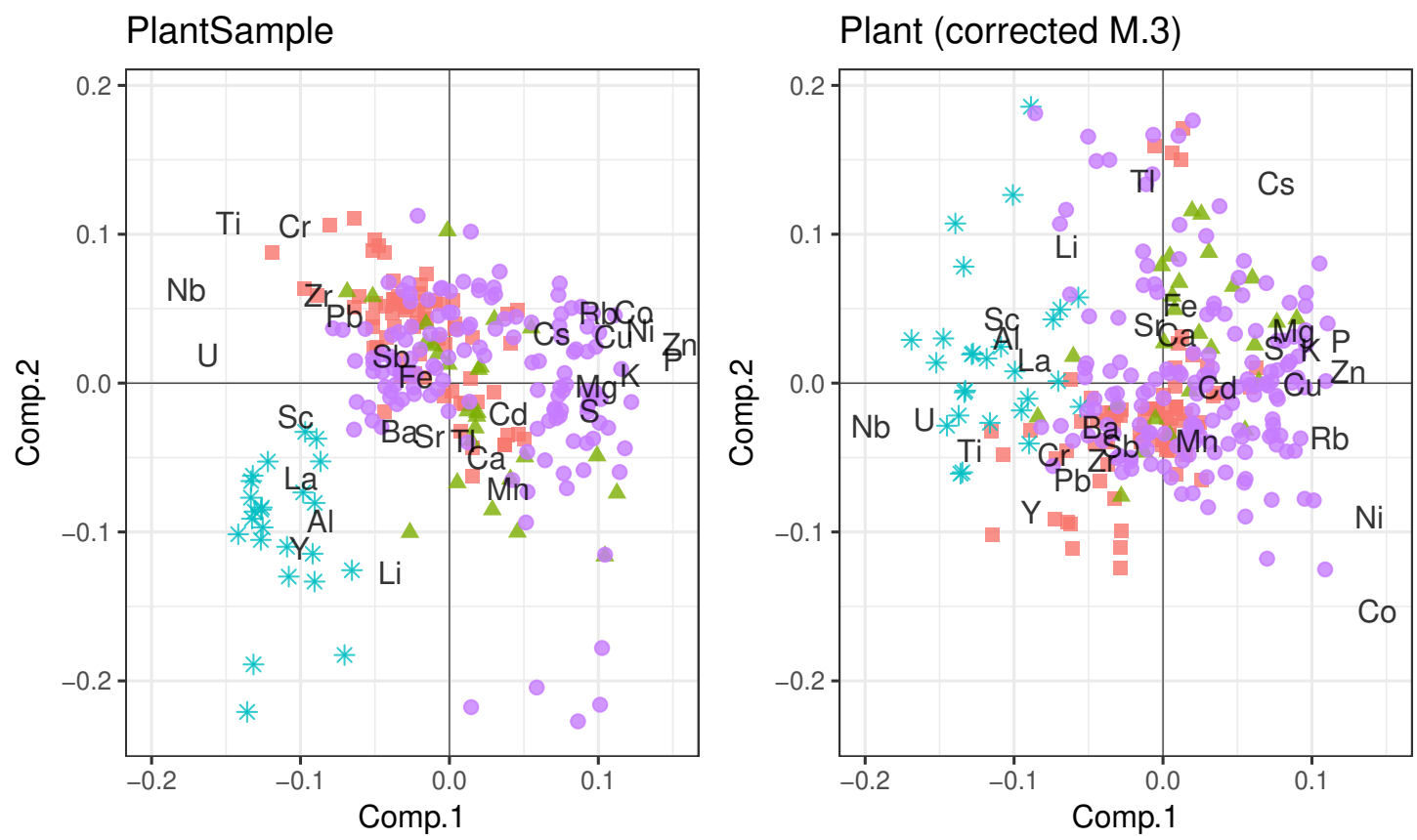

Subgroup " black $\Delta$ green $*$ mature leaf $\bullet$ shoot tip

Figure 6.6.: Coviariance biplots of $\mathbf{P}$ (left, original data) and $\mathbf{I}$ (right, corrected data) of principal component analysis of log-ratios transformed (clr) data (200 tea leaf samples with 28 elements). The proportion of the explained variance is 0.38 for the left plot and 0.52 for the right plot. The position of the element labels can be interpreted as follows (Greenacre 2010; Boogaart and Tolosana-Delgado 2013): the link between two element labels represents the log-ratio between these two elements. A short link indicates that the elements have a quasiconstant log-ratio. An angle of $90^{\circ}$ at the origin means that the log-ratios of the two elements are uncorrelated. 


\section{Correction for Adhering Particles}

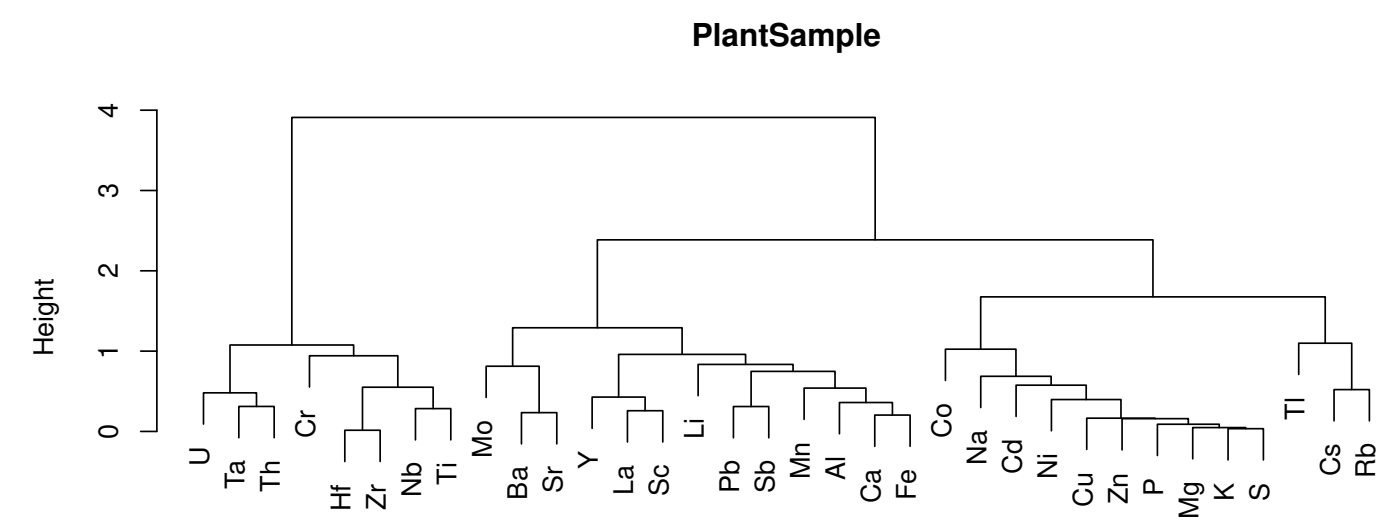

Plant (corrected M.3)

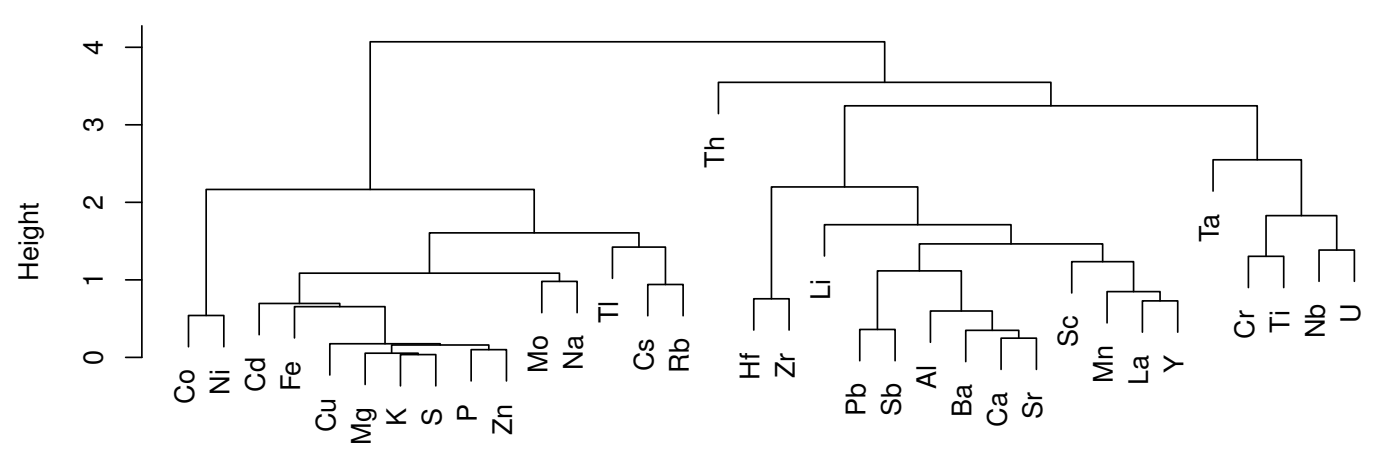

Figure 6.7.: Dendrograms of elements of $\mathbf{P}$ (upper plot, original data) and $\mathbf{I}$ (lower plot, corrected data) of hierarchical cluster analysis (Section 5.3 and 5.4) of closed compositional data using R-function acomp of package 'compositions'. 


\subsection{Discussion for Tea Plant Data}

Tea samples consist mainly in shoot tips which are harvested at waist hight level at the tea bushes. These harvested plant parts are only few days to maximum 3 weeks old and, accordingly, had been exposed only comparably short time to airborne dust accumulation. In China a study of 1225 C. sinensis and processed tea samples showed that $\mathrm{Pb}$ contamination of tea increased with increasing proximity to roads, although leaded gasoline was prohibited $1.5 \mathrm{y}$ before sampling (Han et al. 2006). A washing of samples had nearly no effect on $\mathrm{Pb}$ concentration for very young leaves and buds, but up $23 \%$ removal for mature leaves and the authors of the study concluded that soil dust is one of major source of APs.

Only mature leaf samples have notably longer duration of exposure, and accordingly can have high amount of APs (Figure 6.4). Processed tea samples may have been contaminated by AP by ventilation during the steaming or the withering process (Jabeen et al. 2015): During the withering process (Section 2.3.3) air, often unfiltered, is pushed for several hours through a thick layer of withering leaves, so that air borne dust remains in the tea leaves on the withering tables. In most factories the ventilation air is sucked in well above ground and therefore it has to be assumed that, apart from machine abrasion, airborne dust makes up the main contribution of AP for processed teas.

The correction's assumption, that the adhering particles are mainly soil particles, is especially for tea leaf samples a rather rough approximation. But in absence of data of airborne dust for each region an average of closely located soil data is considered still as the best approximation for correcting the plant data. This assumptions is reinforced by the results of the correction for AP: The element Fe, which should as nutrient only be influenced by uptake of the plant, is after the correction closer to the group of nutrients while not before (Figure 6.7 and 6.5). The element Al, an indicator for the leaves' age (Section 2.1.3), is after correction grouped within the group of $\mathrm{Ca}, \mathrm{Ba}$ and $\mathrm{Sr}$, which are typically higher concentrated in mature leaves than in shoot tips, while before correction it is grouped with the REEs, represented by La and Y, and Sc. In summary comparing the grouping of elements before and after the correction for AP and the overall smaller variance of the log-ratios of elements strongly indicates that the correction successfully reduced the bias induced by airborne particles on certain elements.

Due to the very rough approximation for the elemental composition of adhering particles the corrected concentrations of the mainly affected elements have to interpreted with caution. There are three ways of handling the plant data: 


\section{Correction for Adhering Particles}

a) using original plant data

b) using corrected values and include uncertainty into the results

c) exclude elements which are a strongly affected by the correction

Option a) has the disadvantage that local events, such as a period of drought or heavy rain, before sampling might have a major effect on the elemental composition of the specific sample which may cause difficulties to interpret the results. On the other hand the chemical signal of the adhering particles, although it is prone to a certain fluctuation, might also contain information.

Option b) has the disadvantage that the errors and uncertainties have to be calculated or estimated and are very high for certain elements. In a second step the errors have to be incorporated into all statistical tools used for the analysis. As a consequence the estimation of error is prone to biases, the incorporation of errors is sometimes cumbersome and the overall result will be that elements with high uncertainty have low impact on the results. The advantage of option $b$ ) is that for elements which are only moderately affected by the correction for AP the approach of incorporating the corrected values in combination with their uncertainty is more "honest" approach for interpreting the results.

Option c) has the disadvantage that elements which might contain information for some - clean - samples, are excluded from the results.

In this thesis options b) and c) are used:

Option c) is used for all elements with very high correction factors. For determining the group of elements which should be skipped due to potentially high bias by adhering particles the results of Figure 6.5 and Figure 6.8 are used.

Figure 6.8 shows that the elements $\mathrm{Ti}, \mathrm{Th}, \mathrm{U}, \mathrm{Nb}, \mathrm{Zr}, \mathrm{Hf}$ and $\mathrm{Sc}$ are a group of elements which are similarly affected by the correction for AP. All other elements are differently affected by the correction. Combining the results of Figure 6.5 and Figure 6.8 elements with a generally high correction factor and which are similarly affected by the correction for AP are going to be considered as mainly determined by airborne soil dust. This could also explain findings described in previous publications: For example Matsuura et al. (2001) noted that the elements $\mathrm{Fe}, \mathrm{Zr}$ and Th have high variance between samples and explained this with inhomogeneity of black tea leaf samples. This could also well be the effect of contamination by APs. In summary the elements $\mathrm{Ta}, \mathrm{Ti}, \mathrm{Th}, \mathrm{U}, \mathrm{Nb}, \mathrm{Zr}, \mathrm{Hf}$ and Sc will be excluded in the following, as well for the ionome description as for further statistical analysis. 
Option b) is used for all remaining elements. For elements with a very small correction factor, such as the elements of group 3 of the dendrograms (Figure 6.7), original and corrected data are very similar. If a subset of elements is used which consists only in these elements the additional error induced by correction for AP can be neglected while using the statistical tools. If a subset of elements is used containing one element which has a high probability to be notably affected by the correction for AP statistical tools should incorporate these errors, e.g. as weights.

\subsection{Summary}

Air borne dust on tea leaves can have a major effect on concentrations of some trace elements and the nutrients $\mathrm{Fe}, \mathrm{Na}$ and $\mathrm{Mn}$. To be able to compare tea data from different regions of climatic conditions, plucking periods, etc. a correction for AP should be calculated. Averages of tea soils of the region or upper crust may serve as an adequate approximation for the elemental composition of the APs in absence of aerial dust data. There are three methods to handle element concentrations after applying the correction for AP:

1. The element concentrations of $\mathrm{Ta}, \mathrm{Ti}, \mathrm{Th}, \mathrm{U}, \mathrm{Nb}, \mathrm{Zr}, \mathrm{Hf}$ and $\mathrm{Sc}$ will be excluded because they have a high probability to be intensely affected by APs.

2. If a subset of elements is used which contains one of the elements Mo, $\mathrm{Tl}, \mathrm{Sb}$, $\mathrm{Cs}, \mathrm{Pb}, \mathrm{Na}, \mathrm{Co}, \mathrm{Al}, \mathrm{Cr}, \mathrm{Y}, \mathrm{Li}$, La or Fe ther errors induced by the correction for AP should be incorporated into statistical analysis and interpretations

3. If a subset of elements is used which consists only of elements of $\mathrm{S}, \mathrm{P}, \mathrm{Ca}, \mathrm{K}, \mathrm{Mn}$, $\mathrm{Cu}, \mathrm{Cd}, \mathrm{Zn}, \mathrm{Sr}, \mathrm{Rb}, \mathrm{Mg}, \mathrm{Ni}$ and $\mathrm{Ba}$ the correction for AP can be neglected. 


\section{Correction for Adhering Particles}

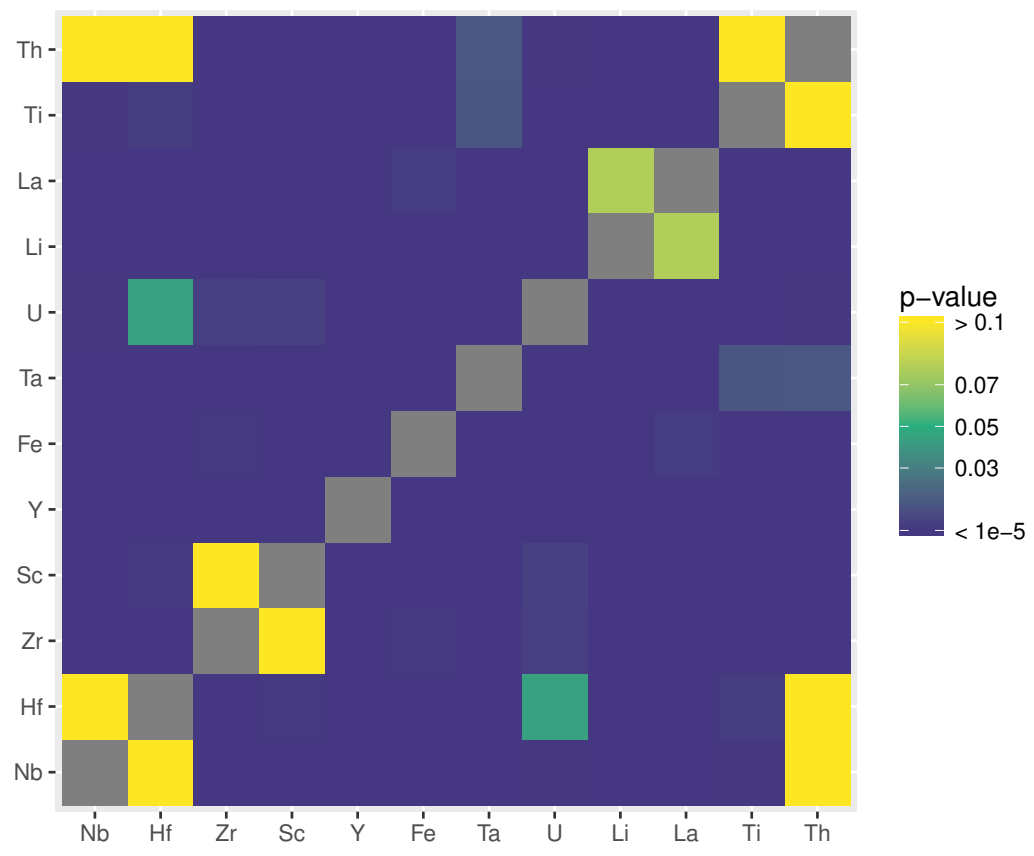

Figure 6.8.: Heatmap of $p$-values calculated by paired t-test between distribution of pairwise logratio of original tea data versus corrected tea data. P-values of log-ratios of element pairs are colored blueish if they are below 0.05 (alternative hypothesis: true difference in means is not equal to 0 ) and are colored greenish to yellowish if values are above 0.05 (the means of two populations are equal).

The distribution of log-ratios of $\mathrm{Zr} \sim \mathrm{Sc}, \mathrm{Ti} \sim \mathrm{Th}, \mathrm{Nb} \sim \mathrm{Th}, \mathrm{Nb} \sim \mathrm{Hf}$ and $\mathrm{U} \sim \mathrm{Hf}$ have the same mean in original and corrected data. That indicates that the correction factor for these elements is always very similar, e.g. if $\mathrm{Zr}$ has a high correction factor in one sample, Sc will have a very similar correction factor, and vice versa. The log-ratios of $\mathrm{Li} \sim \mathrm{La}$ and $\mathrm{Hf} \sim \mathrm{Th}$ have $\mathrm{p}$ values slightly above 0.05 indicating a high probability that the means of the two log-ratio distributions are the same before and after the correction. Additionally the log-ratios Ta Ti, $\mathrm{Ta} \sim \mathrm{Th}, \mathrm{Sc} \sim \mathrm{U}, \mathrm{Fe} \sim \mathrm{La}, \mathrm{Fe} \sim \mathrm{Zr}$ and $\mathrm{Hf} \sim \mathrm{Sc}$ have p-values only slightly below 0.05 indicating that the probability for equal means is low but still notable.

For $\mathrm{Y}$ all displayed $\mathrm{p}$-values of the t-tests of pairwise log-ratio between original and corrected data have values close to zero, hence the distribution of log-ratios containing one of these elements have different means before and after the correction for AP. 


\section{Ionome and Elemental Distribution of Tea Leaf}

The elemental composition of plucked leaf samples differs by type of tea plant tissue and is additionally influenced by environmental parameters (Chapter 2). The processed tea samples are a melange of tissue types (Section 4.1.1) and are often blended with processed leaves which had been grown in heterogeneous environmental conditions. Hence, the ionome of processed tea samples of a tea garden can be subject to considerable variation despite originating from the same provenance.

The aim of this chapter is to unravel which element subcompositions are suitable for which sample group to serve as possible proxy for the provenance of the tea leaves. This is achieved through the following steps: A) The ionome of plucked leaf samples and processed tea samples are described, compared with published data and discussed. Attention is also paid to within-garden variability. B) The ionomes of the sample groups are compared, namely shoot tips vs. mature leaves and shoot tips vs. processed teas. The results of the first comparison help to assign samples with higher probability into one of the two sample groups or a subsets of a group. The results of the latter comparison help to determine subcompositions of which all element log-ratios are independent of the sample groups or subsets of a sample group.

Tea leaf samples will be described by sets of elements which are divided into macronutrients, micro-nutrients, beneficials and non-nutrients (Table D.2). The subcomposition of analyzed macro-nutrients consists of $\mathrm{Ca}, \mathrm{K}, \mathrm{Mg}, \mathrm{P}$ and $\mathrm{S}$, the set of analyzed micronutrients in $\mathrm{Cu}, \mathrm{Fe}, \mathrm{Mn}, \mathrm{Mo}, \mathrm{Ni}, \mathrm{Zn}$, and the subcomposition of analyzed beneficial elements in Al, Co, Na and REE (e.g. H. P. Carr et al. 2003; Barker and Pilbeam 2015). All other analyzed elements are considered as non-nutrients. These are $\mathrm{Ba}, \mathrm{Cd}, \mathrm{Cr}$, $\mathrm{Cs}, \mathrm{Li}, \mathrm{Pb}, \mathrm{Rb}, \mathrm{Sb}, \mathrm{Sc}, \mathrm{Sr}$ and TI. Elements which are strongly influenced by adhering particles (Section 6.4), are excluded from this list. 


\subsection{Ionome of Shoot Tip}

The highest element concentrations are found for the macro-nutrients $\mathrm{K}, \mathrm{P}, \mathrm{Ca}, \mathrm{S}$ and $\mathrm{Mg}$ (Table 7.1, Figure 7.1). Out of the micro-nutrients the elements $\mathrm{Mn}, \mathrm{Fe}, \mathrm{Zn}, \mathrm{Cu}$ and $\mathrm{Ni}$ are found in medium concentrations, while Mo is found in minor concentrations. The beneficial elements show medium concentrations for $\mathrm{Al}$ and $\mathrm{Na}$ and minor concentrations for $\mathrm{Co}$ and REE. Out of the non-nutrients the elements $\mathrm{Rb}, \mathrm{Sr}$ and $\mathrm{Ba}$ are found in medium concentrations and all remaining elements, $\mathrm{Cr}, \mathrm{Cs}, \mathrm{Pb}, \mathrm{Li}, \mathrm{TI}, \mathrm{Cd}, \mathrm{Sc}$ and $\mathrm{Sb}$ are found in minor concentrations.

Comparing with published data the concentrations of the macro-nutrients are consistent with literature data within the standard deviations (Table 7.1). For the micro-nutrients the concentrations of $\mathrm{Mn}, \mathrm{Fe}, \mathrm{Zn}$ and $\mathrm{Cu}$ coincide within one order of magnitude. Among the beneficial elements $\mathrm{Al}$ and $\mathrm{Na}$ show a magnitude less average concentration than published in previous works. The beneficial elements Co and the REE, on the other hand, are found in comparable concentrations within the standard deviation. Out of the non-nutrients the elements $\mathrm{Cr}$ and $\mathrm{Cd}$ have the same order of magnitude as published by Y. Chen et al. (2009). For the other micro-nutrients Mo and $\mathrm{Ni}$ and the non-nutrients there are no published data for shoot tips yet.

In summary, measured concentrations in shoot tip samples agree generally well with previous published tea leaf data. Al has presumably higher concentrations because the samples of Y. Chen et al. (2009) are considerably older, approximately one month of age, and therefore the Al-accumulating process in leaves had been active longer (Sections 2.1.3 and 7.4.1). The samples of H. P. Carr et al. (2003) have about the same age as shoot tips of this thesis and show comparable concentrations with $381 \pm 80 \mathrm{mg} / \mathrm{kg}$. The differences in $\mathrm{Na}$ concentrations can not be explained.

The spread of concentrations is calculated by standard deviation of clr values (clr calculated by all elements, Table 7.1. The smallest spread is found for the nutrients and Al. Only the micro-nutrients $\mathrm{Ni}$ and Mo show comparably wide scatter of concentrations. For $\mathrm{Ni}$ the spread is produced by highly different $\mathrm{Ni}$ concentrations between samples originating from Paraná and São Paulo (Figure 7.1). Mo concentrations are highly variable in all listed tea growing areas. All beneficial elements have high spread of data - except for Al which shows a similar spread of concentrations as the nutrients $S$ and $P$. Al has probably a similar small spread in concentration compared to other nutrients because $\mathrm{Al}$ is reaching mature leaves by xylem transport and is further exported to the shoot tips by phloem transport (Tolra et al. 2011). Only a certain proportion of the Al is stored in the 
vacuole or cell walls of epidermal cells, similar to e.g. potentially toxic elements. Out of the non-nutrients $\mathrm{Sb}, \mathrm{Cd}, \mathrm{Rb}, \mathrm{Sr}$ and $\mathrm{Ba}$ show moderate spread of concentrations while all other non-nutrients have high spread, with Cs having the highest (Table 7.1).

Ionome variability with respect to provenance analysis For the main research region Darjeeling a case study was conducted to determine element subcompositions which have a smaller intra-garden variability than the variability between the tea growing areas in Darjeeling. Eight sampling locations at different tea gardens had been sampled three times at spots which form a triangle of approximately $50 \mathrm{~m}$ side length. At seven of the sampling location the tea bushes are plants grown from seeds of more than $110 \mathrm{y}$ of age. Only in Makaibari the plant material originates from $30 \mathrm{y}$ old tea plant clones of type 'Dushara'. To compare the ionomes of all 24 shoot tip samples the concentrations had been converted into clr-values by using all elements, including REE but excluding elements influenced by the correction for adhering particles (Section 6).

Figure 7.2 displays the clr-values for all 24 shoot tip samples. For each element the samples originating from the same sampling locations are plotted in same color in one row, so that the dots dodge vertically by garden. If the three dots of one garden are plotting closely, the intra-location variability of the respective element concentration is small, and vice versa. Table 7.2 gives the standard deviation of clr-values for each element for each sampling location as a measure for the spread of clr-values within one sampling location. The average spread (median of the stdev per element) gradually increases in this order: $\mathrm{Ca}, \mathrm{Al}, \mathrm{Y}, \mathrm{Ba}, \mathrm{Li}, \mathrm{K}, \mathrm{Sb}, \mathrm{Cu}, \mathrm{La}, \mathrm{S}, \mathrm{Rb}, \mathrm{Ni}, \mathrm{P}, \mathrm{Mg}, \mathrm{Mo}, \mathrm{Mn}, \mathrm{Pb}$, $\mathrm{Sr}, \mathrm{Zn}, \mathrm{Tl}, \mathrm{Na}, \mathrm{Cr}, \mathrm{Fe}, \mathrm{Co}, \mathrm{Sc}, \mathrm{Cd}, \mathrm{Cs}$. Hence, $\mathrm{Ca}, \mathrm{Y}$ and $\mathrm{Al}$ show in general only minor spread of element concentration at one location and the intra-location variability is often smaller than the between-location variability. On the other hand, for elements like Fe, $\mathrm{Cs}, \mathrm{Sc}, \mathrm{Cd}$ or Co the intra-location variability might exceed the spread of data between the locations.

For discriminating between regions the element subcompositions are important which have small intra-location variability but potentially large spread between regions. For example Ba fulfills these conditions: $\mathrm{Ba}$ has a average small spread of concentrations within one location (Table 7.2), but the spread for tea samples from various regions is notably bigger (clr-stdev in Table 7.1). Figure 7.2 confirms that $\mathrm{Ba}$ has distinguishable average clr-values for certain tea gardens in Darjeeling: Tumsong and Seeyok have the same average Ba-clr-values, Mullootar, Sungma and Tumsong, and Makaibari and Sourenee. For Darjeeling, the element $Y$ has the largest spread of clr-values in ratio 
to average clr-standard deviation of samples from one location (ratio $=3.5$ ). This ratio gradually decreases from $\mathrm{Y}$ in this order: $\mathrm{Tl}, \mathrm{Cs}, \mathrm{Pb}, \mathrm{Na}, \mathrm{Ca}, \mathrm{Ni}, \mathrm{K}, \mathrm{Mn}, \mathrm{Co}, \mathrm{Sb}, \mathrm{La}, \mathrm{Rb}$, $\mathrm{Cr}$, Li, Ba, Mo, P, Fe, Zn, Cu, Mg, S, Sc, Sr, Al and Cd. According to this parameter, in Darjeeling element subcompositions containing the non-nutrients $\mathrm{Tl}, \mathrm{Cs}, \mathrm{Pb}, \mathrm{Sb}$, the beneficial elements REE, $\mathrm{Na}, \mathrm{Co}$, and the nutrients $\mathrm{Ca}, \mathrm{Ni}, \mathrm{K}$ and $\mathrm{Mn}$ may well serve as proxies for provenance analysis. On the contrary, the elements $\mathrm{Cd}, \mathrm{Al}, \mathrm{Sr}, \mathrm{Sc}, \mathrm{S}$, $\mathrm{Mg}, \mathrm{Cu}, \mathrm{Zn}, \mathrm{Fe}$ and $\mathrm{P}$ have probably less impact for discriminating between regions in Darjeeling. 


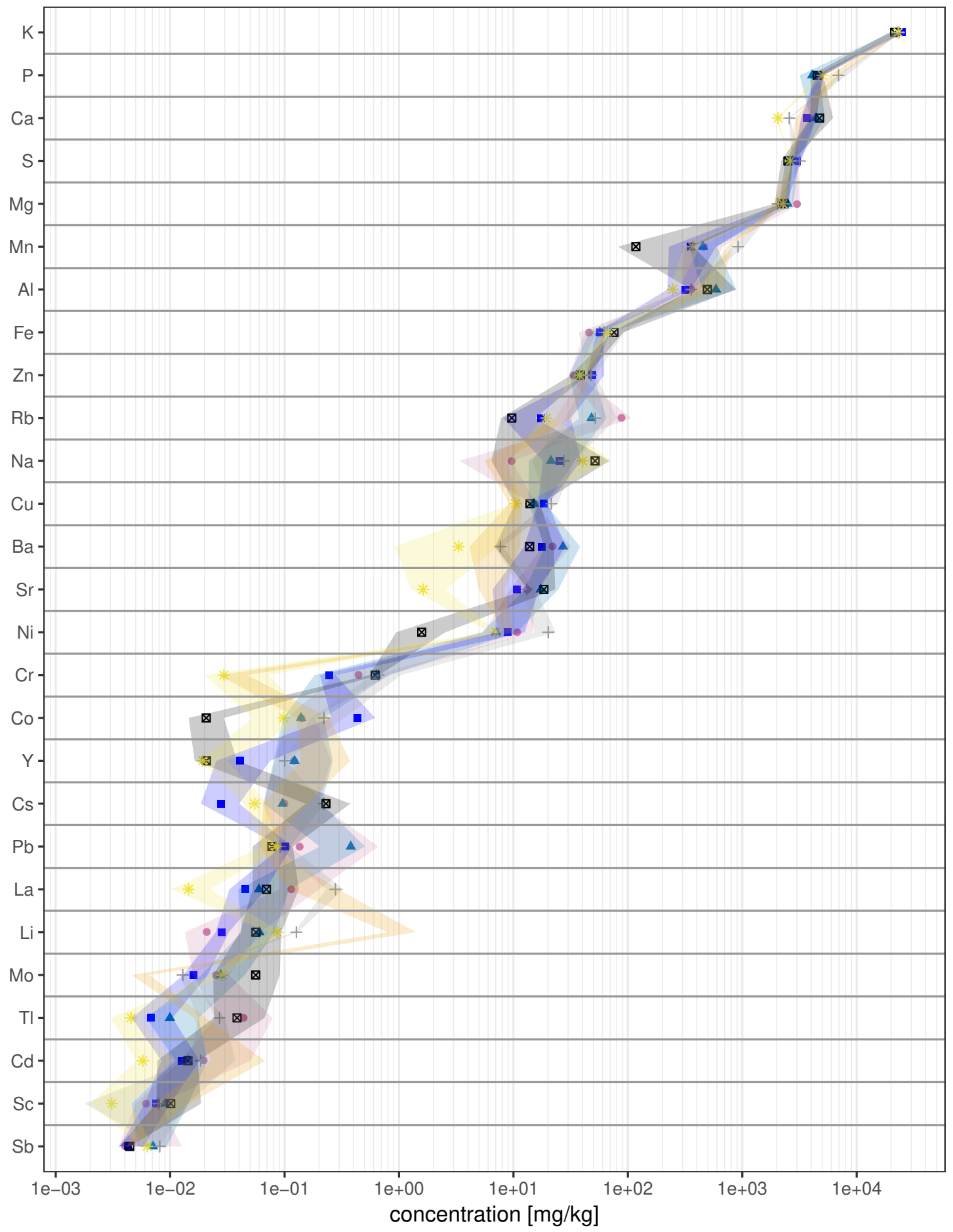

Region - Assam \ Darjeeling - Nilgiris + Paraná $₫$ São Paulo * Shizuoka Uji

Figure 7.1.: Ionomes of shoot tip samples ( $N=160)$, grouped by tea growing regions. Elements are ordered by median of all samples. Median for each sample group is marked by a colored symbol, the shaded areas mark the second and third quartile. Median values are listed in Table 7.1. 
Table 7.1.: Shoot tip concentrations [mg/kg] and clr-values, compilation of data of this thesis $(N=160)$ and data from Cao et al. $(1998)(\mathrm{N}=3)$, H. P. Carr et al. (2003) ( $\mathrm{N}=3$ ), Y. Chen et al. (2009) ( $\mathrm{N}=24)$ and Jin et al. (2008) $(\mathrm{N}=20)$. The compilation of published data is displayed in the columns left of the marker 'Publ.'. The 'mad' denotes the median absolute deviation of the concentration values and the 'stdev' the standard deviation. The geometric mean for calculating the clr-values is based on all elements listed in this table. Nutrients are marked with an asterisk, beneficial elements with a dagger.

\begin{tabular}{|c|c|c|c|c|c|c|c|c|c|c|c|c|}
\hline & median & mad & mean & stdev & clr-median & clr-stdev & Publ.: & median & mad & mean & stdev & $N$ \\
\hline $\mathrm{Al}^{\dagger}$ & $4.9 \mathrm{e}+02$ & $2.5 e+02$ & $6.2 e+02$ & $4.1 e+02$ & 6.80 & 0.47 & & $2.4 \mathrm{e}+03$ & $5.1 e+02$ & $2.5 e+03$ & $1.1 e+03$ & 27 \\
\hline $\mathrm{Ba}$ & $2.3 e+01$ & $1.3 e+01$ & $2.4 \mathrm{e}+01$ & $1.3 e+01$ & 3.60 & 0.64 & & & & & & \\
\hline $\mathrm{Ca}^{*}$ & $4.2 e+03$ & $1.1 e+03$ & $4.3 e+03$ & $1.1 e+03$ & 8.90 & 0.40 & & $3.7 e+03$ & $1.6 e+02$ & $4.3 e+03$ & $2.5 e+03$ & 30 \\
\hline $\mathrm{Cd}$ & $1.4 \mathrm{e}-02$ & $8.6 e-03$ & $2.0 e-02$ & $1.8 \mathrm{e}-02$ & -3.60 & 0.63 & & $6.0 e-02$ & $1.5 e-02$ & $5.6 e-02$ & $1.8 \mathrm{e}-02$ & 24 \\
\hline $\mathrm{Co}^{\dagger}$ & $1.4 \mathrm{e}-01$ & $1.2 \mathrm{e}-01$ & $2.9 e-01$ & 7.7e-01 & -1.40 & 1.10 & & $1.4 \mathrm{e}-01$ & 3.7e-02 & $1.7 e-01$ & 8.3e-02 & 24 \\
\hline $\mathrm{Cr}$ & $5.5 e-01$ & $4.5 e-01$ & $5.2 e-01$ & $3.5 e-01$ & -0.15 & 0.96 & & $4.6 e-01$ & $6.7 e-02$ & $5.2 e-01$ & $2.1 \mathrm{e}-01$ & 24 \\
\hline Cs & $9.6 e-02$ & $8.9 e-02$ & $1.6 \mathrm{e}-01$ & $1.6 e-01$ & -1.80 & 0.99 & & & & & & \\
\hline $\mathrm{Cu}^{*}$ & $1.6 e+01$ & $4.1 e+00$ & $1.8 e+01$ & $1.9 e+01$ & 3.40 & 0.54 & & $1.3 e+01$ & $3.8 e+00$ & $1.4 e+01$ & $4.4 e+00$ & 47 \\
\hline $\mathrm{Fe}^{*}$ & $5.9 e+01$ & $1.7 e+01$ & $6.4 e+01$ & $3.1 e+01$ & 4.60 & 0.56 & & $9.2 e+01$ & $1.7 e+01$ & $9.1 e+01$ & $2.1 e+01$ & 27 \\
\hline $\mathrm{K}^{*}$ & $2.3 e+04$ & $3.0 \mathrm{e}+03$ & $2.3 e+04$ & $3.3 e+03$ & 11.00 & 0.42 & & $1.8 e+04$ & $2.6 e+03$ & $1.8 e+04$ & $2.2 e+03$ & 27 \\
\hline $\mathrm{La}^{\dagger}$ & 6.3e-02 & $5.0 e-02$ & $1.1 \mathrm{e}-01$ & $1.7 e-01$ & -2.20 & 0.64 & & $8.6 e-01$ & $5.9 e-02$ & $8.8 e-01$ & $7.8 \mathrm{e}-02$ & 3 \\
\hline $\mathrm{Li}$ & $5.6 e-02$ & $3.9 e-02$ & $1.0 \mathrm{e}-01$ & $2.1 e-01$ & -2.30 & 0.92 & & & & & & \\
\hline $\mathrm{Mg}^{*}$ & $2.4 \mathrm{e}+03$ & $4.7 e+02$ & $2.4 \mathrm{e}+03$ & $4.6 e+02$ & 8.40 & 0.44 & & $2.2 e+03$ & $2.1 e+02$ & $2.3 e+03$ & $5.6 e+02$ & 30 \\
\hline $\mathrm{Mn}^{*}$ & $4.4 \mathrm{e}+02$ & $2.4 \mathrm{e}+02$ & $4.8 e+02$ & $2.9 e+02$ & 6.60 & 0.65 & & $1.1 e+03$ & $1.6 e+02$ & $9.5 e+02$ & $3.6 e+02$ & 30 \\
\hline Mo* & $2.5 e-02$ & $1.7 e-02$ & $3.8 \mathrm{e}-02$ & $3.7 e-02$ & -3.20 & 0.94 & & & & & & \\
\hline $\mathrm{Na}^{\dagger}$ & $2.0 e+01$ & $1.5 e+01$ & $2.9 e+01$ & $2.8 \mathrm{e}$ & 3.50 & 1.10 & & $1.1 e+02$ & $3.0 e+01$ & $1.1 e+02$ & $2.2 e+01$ & 24 \\
\hline $\mathrm{Ni}^{*}$ & $7.3 e+00$ & $4.4 e+00$ & $9.0 e+00$ & $9.4 e+00$ & 2.60 & 0.84 & & & & & & \\
\hline $\mathrm{P}^{*}$ & $4.5 e+03$ & $1.1 e+03$ & $4.5 e+03$ & $1.2 \mathrm{e}+03$ & 9.00 & 0.53 & & $2.3 e+03$ & $1.3 e+03$ & $2.4 e+03$ & $9.0 e+02$ & 24 \\
\hline $\mathrm{Pb}$ & $1.7 e-01$ & $1.5 e-01$ & $2.9 e-01$ & $2.8 e-01$ & -0.98 & 0.78 & & $2.8 e+00$ & $5.2 e-01$ & $3.3 e+00$ & $1.1 e+00$ & 24 \\
\hline $\mathrm{Rb}$ & $4.2 \mathrm{e}+01$ & $2.6 e+01$ & $4.9 e+01$ & $3.7 e+01$ & 4.20 & 0.75 & & & & & & \\
\hline $\mathrm{S}^{*}$ & $2.8 e+03$ & $5.1 e+02$ & $2.8 e+03$ & $4.6 e+02$ & 8.50 & 0.44 & & $2.8 e+03$ & $1.9 e+03$ & $2.8 e+03$ & $1.2 e+03$ & 3 \\
\hline $\mathrm{Sb}$ & $5.7 e-03$ & $2.8 e-03$ & 7.0e-03 & 4.7e-03 & -4.50 & 0.53 & & & & & & \\
\hline Sc & 8.2e-03 & $6.3 e-03$ & 1.1e-02 & $9.1 \mathrm{e}-03$ & -4.10 & 0.82 & & & & & & \\
\hline Sr & $1.5 e+01$ & $8.8 e+00$ & $1.6 e+01$ & $8.7 e+00$ & 3.20 & 0.62 & & & & & & \\
\hline TI & $1.2 \mathrm{e}-02$ & $1.1 \mathrm{e}-02$ & $2.2 \mathrm{e}-02$ & $2.6 e-02$ & -3.90 & 0.97 & & & & & & \\
\hline $\mathrm{Y}^{\dagger}$ & $9.8 e-02$ & $8.5 e-02$ & $1.5 e-01$ & $1.8 e-01$ & -1.80 & 0.66 & & & $3.0 \mathrm{e}-02$ & $1.5 e-01$ & $2.0 \mathrm{e}-02$ & 3 \\
\hline Zn* & $3.9 e+01$ & $1.2 \mathrm{e}+01$ & $4.5 e+01$ & $2.9 e+01$ & 4.30 & 0.62 & & $2.6 e+01$ & $1.9 e+00$ & $2.7 e+01$ & $4.8 e+00$ & 27 \\
\hline
\end{tabular}




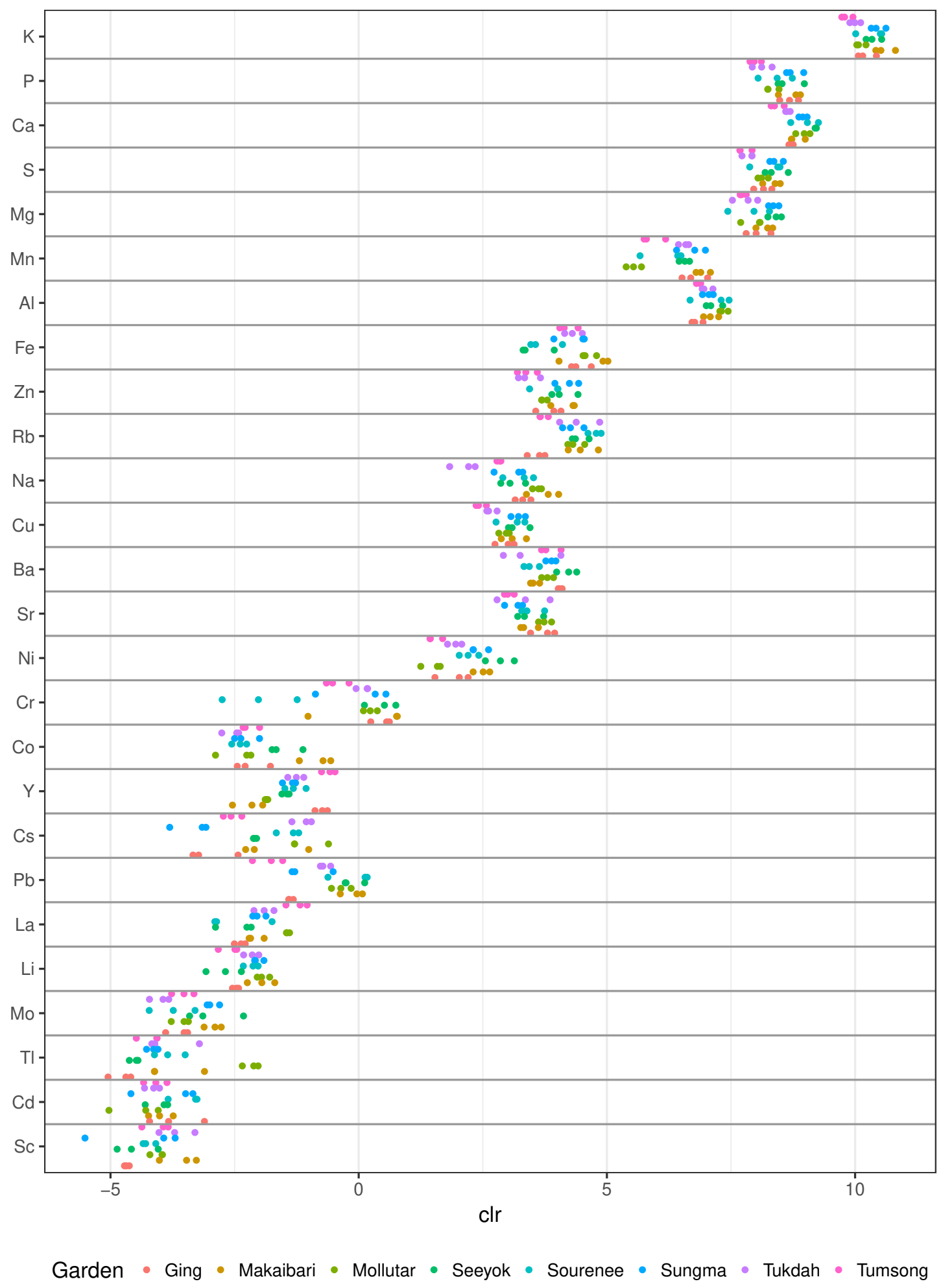

Figure 7.2.: Clr-values of shoot tip ionomes of eight sampling location in tea gardens of Darjeeling. Each sampling location consists of three closely located sampling spots, represented by the colored dots $(\mathrm{N}=24)$. The dots are vertically dodged by garden: For each element the upper row of dots represents Tumsong and the lowest row of dots represents Ging. Clr-values are calculated by all listed elements. Elements are ordered as in Figure 7.1. 


\begin{tabular}{lrrrrrrrrrrrrrr}
\hline Garden & $\mathrm{N}$ & $\mathrm{Mo}$ & $\mathrm{Na}$ & $\mathrm{Ni}$ & $\mathrm{P}$ & $\mathrm{Pb}$ & $\mathrm{Rb}$ & $\mathrm{S}$ & $\mathrm{Sb}$ & $\mathrm{Sc}$ & $\mathrm{Sr}$ & $\mathrm{Tl}$ & $\mathrm{Y}$ & $\mathrm{Zn}$ \\
\hline Ging & 3 & 0.24 & 0.16 & 0.35 & 0.19 & 0.05 & 0.18 & 0.18 & 0.26 & 0.05 & 0.25 & 0.24 & 0.13 & 0.26 \\
Makaibari & 3 & 0.17 & 0.33 & 0.17 & 0.23 & 0.23 & 0.31 & 0.18 & 0.11 & 0.39 & 0.19 & 0.58 & 0.31 & 0.27 \\
Mollutar & 3 & 0.18 & 0.09 & 0.21 & 0.13 & 0.20 & 0.17 & 0.11 & 0.18 & 0.14 & 0.13 & 0.16 & 0.03 & 0.06 \\
Seeyok & 3 & 0.57 & 0.25 & 0.29 & 0.29 & 0.22 & 0.18 & 0.24 & 0.30 & 0.42 & 0.27 & 0.09 & 0.07 & 0.27 \\
Sourenee & 3 & 0.46 & 0.32 & 0.20 & 0.35 & 0.44 & 0.13 & 0.34 & 0.37 & 0.13 & 0.24 & 0.31 & 0.22 & 0.33 \\
Sungma & 3 & 0.13 & 0.31 & 0.18 & 0.18 & 0.46 & 0.22 & 0.14 & 0.11 & 0.99 & 0.19 & 0.12 & 0.14 & 0.24 \\
Tukdah & 3 & 0.20 & 0.27 & 0.14 & 0.20 & 0.11 & 0.41 & 0.12 & 0.11 & 0.36 & 0.53 & 0.54 & 0.16 & 0.23 \\
Tumsong & 3 & 0.23 & 0.04 & 0.14 & 0.12 & 0.31 & 0.10 & 0.14 & 0.08 & 0.28 & 0.10 & 0.24 & 0.14 & 0.20 \\
\hline
\end{tabular}




\subsection{Ionome of Mature Leaf}

The ionome of the source (mature) leaves differs from the ionome of shoot tips, although the total concentration of analyzed elements is about the same. Compared to sink leaves (shoot tips), they are depleted in elements which are transported via the phloem into the sink tissue. The phloem-immobile elements are higher concentrated in the source leaves compared to the sink leaves. The element Al is significantly enriched in mature leaves, as has been frequently reported in previous publications (Sections 2.1.3 and 7.4.1).

The highest concentrations in mature leaves are found for macro-nutrients and $\mathrm{Al}$ (Table 7.3). Out of the micro-nutrients $\mathrm{Mn}$ is highly concentrated, Fe, $\mathrm{Zn}, \mathrm{Cu}$ and $\mathrm{Ni}$ are medium concentrated and $\mathrm{Mo}$ is found at minor concentrations. The beneficial elements are all found at minor concentrations, except for $\mathrm{Na}$, which is medium concentrated and $\mathrm{Al}$, which is found at concentrations similar to the macro-nutrients. The non-nutrients $\mathrm{Ba}, \mathrm{Sr}$ and $\mathrm{Rb}$ are found at medium concentrations, while all other non-nutrients occur only at minor concentrations. The spread of concentrations is in general higher than calculated for the shoot tip samples. This is probably a result of the very few analysis of mature leaves and therefore will not be further discussed here.

Compared to the data published by H. P. Carr et al. (2003) Al, Ca, Mg and Mn have the same order of magnitude in concentration, while $\mathrm{K}$ is found in one order of magnitude less concentration (Table 7.3). Han et al. (2006) describes an increasing accumulation of $\mathrm{Pb}$ with progressing leaf development up to $3.5 \mathrm{mg} / \mathrm{kg}$ in one year old mature leaves 1 . For all remaining elements no data have been published yet.

Regional differences of the ionomes with respect to provenance analysis Figure 7.3 shows that mature leaves sampled in Darjeeling are found in lower concentrations of the macro-nutrients $K$ and $P$ than the other three regions. This pattern can not be seen in for the shoot tips in Figure 7.1. Presumably the lower concentrations in $\mathrm{K}$ and $\mathrm{P}$ in mature leaves of Darjeeling reflects a lower availability of these two macro-nutrients compared to the other three regions: All mature leaf samples of Darjeeling originate from tea gardens which had farmed without artificial fertilizers for several years, and only relied on self-made compost and dung. The gardens of Brazil and Japan had regularly applied fertilizers which comply with USDA organic standards (USDA organic 2018).

The elements $\mathrm{Al}, \mathrm{Rb}, \mathrm{Sr}, \mathrm{Ba}, \mathrm{Ni}$ and $\mathrm{Cr}$ are found to have higher concentrations in mature leaves from Darjeeling than from the other regions. The high $\mathrm{Rb}$ contents could be a reaction of the plant to the low $K$ availability if $R b$ is replacing $K$ due its similar 
physicochemical characteristics like valence and ion diameter (Tyler 1997; Vinichuk et al. 2010, and references therein). A similar reaction could explain the higher contents of $\mathrm{Sr}$ and $\mathrm{Ba}$ in Darjeeling mature leaves: If the soils provide a poor availability of the main nutrient $\mathrm{Ca}$ the plants might activate ion transporters which are less sensitive to macro-nutrients with respect to their chemically similar elements.

It remains unclear whether the high content in $\mathrm{Al}$ is an effect of less fertilizer application. On the one side, poor availability of macro-nutrients might stimulate the plant to release more root exudates of acidic composition. This locally reduces the $\mathrm{pH}$ in the rhizosphere and would increase the availability of required nutrients for the plant uptake and, along with it, increase concentration of Al-ions in the soil solution (Section 2.1.2). On the other side, the Al concentration is strongly influenced by the age of the mature leaf (Section 2.1.3 and small differences in the plucking standard during sampling can have a notable impact on the average Al concentration for these small numbers of samples.

The differences in concentration of nutrients and their respective replacement elements between regions is clearly visible for mature leaf samples but not for shoot tip samples. This suggests that mature leaves may 'buffer' the element flux from soils into the shoot tips: The mature leaves, doing actively respiration, receive via the xylem transport the ions from the root-system and export the nutrients and proteins required by the shoots into the phloem tissue. This process obviously seems to favor a stable ratio of the nutrients $\mathrm{K}, \mathrm{P}, \mathrm{S}$ and $\mathrm{Mg}$ to $\mathrm{C}, \mathrm{H}, \mathrm{N}$ and $\mathrm{O}$ for sink tissue. For provenance analytics a possible 'buffering' by mature leaves implies that a high percentage sink tissue in the analyzed plant material reduces the geochemical signal derived by element uptake from the underlying soils. 


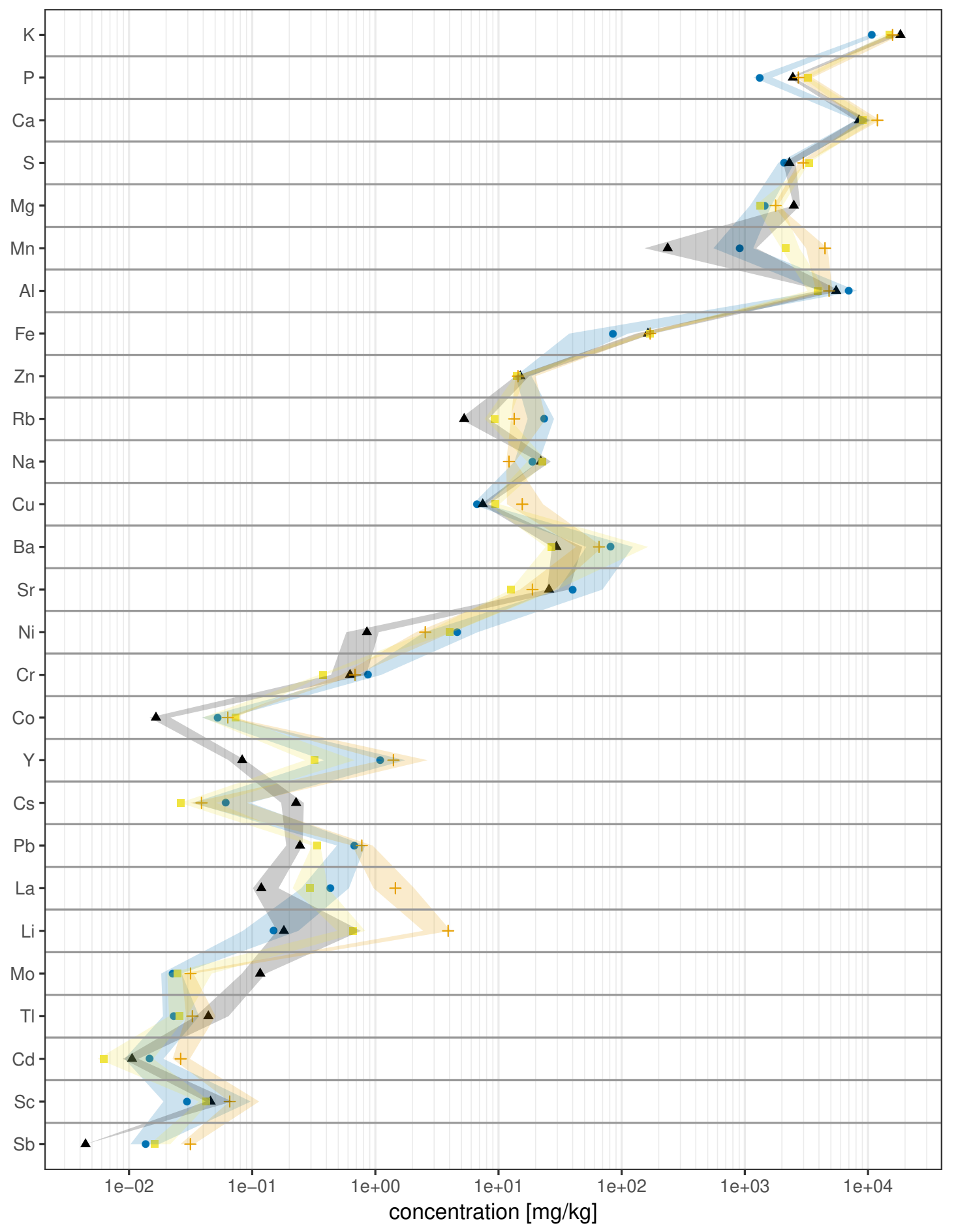

Region - Darjeeling $\Delta$ São Paulo $\backsim$ Shizuoka + Uji

Figure 7.3.: lonomes of mature leaf $(\mathrm{N}=27)$, grouped by tea growing regions. Elements are ordered by median of shoot tip. Median for each sample group is marked by a colored dot, the shaded areas mark the second and third quartile. Median values are listed in Table 7.3 . 
Table 7.3.: Mature leaf concentrations [mg/kg] and clr-values, compilation of data of this thesis $(\mathrm{N}=27$ ) and data from H. P. Carr et al. (2003). The compilation of published data is displayed in the columns left of the marker 'Publ.'. The 'mad' denotes the median absolute deviation of the concentration values and the 'stdev' the standard deviation. The geometric mean for calculating the clr-values is based on all elements listed in this table. Nutrients are marked with an asterisk, beneficial elements with a dagger.

\begin{tabular}{|c|c|c|c|c|c|c|c|c|c|c|c|c|}
\hline & median & mad & mean & stdev & clr-median & clr-stdev & Publ.: & median & mad & mean & stdev & $\mathrm{N}$ \\
\hline $\mathrm{Al}^{\dagger}$ & $5.5 e+03$ & $3.1 e+03$ & $5.9 e+03$ & $2.5 e+03$ & 8.40 & 0.45 & & $6.9 e+03$ & $9.6 e+02$ & $6.9 e+03$ & $6.4 e+02$ & 3 \\
\hline $\mathrm{Ba}$ & $6.6 e+01$ & $4.8 e+01$ & $8.2 e+01$ & $6.0 e+01$ & 4.10 & 0.59 & & & & & & \\
\hline $\mathrm{Ca}^{*}$ & $9.1 e+03$ & $2.7 e+03$ & $9.6 e+03$ & $3.0 e+03$ & 8.90 & 0.44 & & $1.9 e+04$ & $4.0 e+03$ & $1.9 e+04$ & $2.7 e+03$ & 3 \\
\hline $\mathrm{Cd}$ & $1.4 \mathrm{e}-02$ & $9.5 e-03$ & $1.6 e-02$ & $8.5 e-03$ & -4.60 & 0.48 & & & & & & \\
\hline $\mathrm{Co}^{\dagger}$ & $5.6 e-02$ & $2.6 \mathrm{e}-02$ & $5.0 \mathrm{e}-02$ & $2.3 e-02$ & -3.40 & 0.56 & & & & & & \\
\hline $\mathrm{Cr}$ & $7.8 e-01$ & $4.3 e-01$ & $7.4 \mathrm{e}-01$ & $3.3 e-01$ & -0.61 & 0.63 & & & & & & \\
\hline Cs & $6.2 \mathrm{e}-02$ & $5.4 \mathrm{e}-02$ & $7.9 e-02$ & $6.7 e-02$ & -3.00 & 1.10 & & & & & & \\
\hline $\mathrm{Cu}^{*}$ & $7.1 e+00$ & $1.6 e+00$ & $8.5 e+00$ & $4.9 e+00$ & 1.60 & 0.58 & & & & & & \\
\hline $\mathrm{Fe}^{*}$ & $1.0 \mathrm{e}+02$ & $5.5 e+01$ & $1.0 \mathrm{e}+02$ & $5.6 e+01$ & 4.10 & 0.91 & & & & & & \\
\hline $\mathrm{K}^{*}$ & $1.2 e+04$ & $3.1 e+03$ & $1.3 e+04$ & $3.2 e+03$ & 9.00 & 0.61 & & $2.9 e+03$ & $1.8 e+03$ & $2.9 e+03$ & $1.2 e+03$ & 3 \\
\hline $\mathrm{La}^{\dagger}$ & 4.3e-01 & $3.3 e-01$ & $5.5 e-01$ & $5.7 e-01$ & -1.30 & 0.52 & & & & & & \\
\hline $\mathrm{Li}$ & $1.8 \mathrm{e}-01$ & $1.9 e-01$ & $5.9 e-01$ & $1.1 e+00$ & -2.10 & 1.20 & & & & & & \\
\hline Mg* & $1.6 e+03$ & $5.7 e+02$ & $1.7 e+03$ & $6.3 e+02$ & 7.10 & 0.63 & & $2.6 e+03$ & $5.1 e+02$ & $2.6 e+03$ & $3.4 e+02$ & 3 \\
\hline$M n^{*}$ & $1.1 \mathrm{e}+03$ & $9.6 e+02$ & $1.4 \mathrm{e}+03$ & $1.2 e+03$ & 6.60 & 0.78 & & $1.3 e+03$ & $2.8 e+02$ & $1.3 e+03$ & $1.9 e+02$ & 3 \\
\hline Mo* & $2.4 \mathrm{e}-02$ & $9.1 \mathrm{e}-03$ & $3.5 e-02$ & $3.0 \mathrm{e}-02$ & -4.10 & 0.84 & & & & & & \\
\hline $\mathrm{Na}^{\dagger}$ & $1.9 e+01$ & $6.8 e+00$ & $1.9 e+01$ & $7.8 e+00$ & 2.50 & 0.75 & & & & & & \\
\hline $\mathrm{Ni}^{*}$ & $4.0 \mathrm{e}+00$ & $3.3 e+00$ & $3.9 e+00$ & $2.4 e+00$ & 0.88 & 0.65 & & & & & & \\
\hline $\mathrm{P}^{*}$ & $1.7 e+03$ & $7.9 e+02$ & $1.9 e+03$ & $8.7 e+02$ & 7.10 & 0.72 & & & & & & \\
\hline $\mathrm{Pb}$ & $5.9 e-01$ & $3.7 e-01$ & $5.9 e-01$ & $2.8 \mathrm{e}-01$ & -0.87 & 0.41 & & & & & & \\
\hline $\mathrm{Rb}$ & $2.0 e+01$ & $1.1 e+01$ & $2.1 e+01$ & $1.2 e+01$ & 2.60 & 0.68 & & & & & & \\
\hline $\mathrm{S}^{*}$ & $2.4 e+03$ & $7.4 e+02$ & $2.4 e+03$ & $5.4 e+02$ & 7.30 & 0.60 & & & & & & \\
\hline $\mathrm{Sb}$ & $1.4 \mathrm{e}-02$ & $6.3 e-03$ & $1.5 e-02$ & $7.8 \mathrm{e}-03$ & -4.60 & 0.39 & & & & & & \\
\hline $\mathrm{Sc}$ & $3.8 e-02$ & $3.3 e-02$ & $6.3 e-02$ & $5.7 e-02$ & -3.40 & 0.86 & & & & & & \\
\hline $\mathrm{Sr}$ & $3.5 e+01$ & $1.6 e+01$ & $4.1 \mathrm{e}+01$ & $2.2 e+01$ & 3.40 & 0.65 & & & & & & \\
\hline $\mathrm{TI}$ & $2.6 e-02$ & $1.1 \mathrm{e}-02$ & $3.7 e-02$ & $2.7 e-02$ & -3.70 & 0.87 & & & & & & \\
\hline $\mathrm{Y}^{\dagger}$ & $8.8 e-01$ & $9.4 \mathrm{e}-01$ & $1.1 e+00$ & $1.0 e+00$ & -0.59 & 0.75 & & & & & & \\
\hline $\mathrm{Zn}^{*}$ & $1.5 e+01$ & $2.3 e+00$ & $1.7 e+01$ & $6.5 e+00$ & 2.60 & 0.54 & & & & & & \\
\hline
\end{tabular}




\subsection{Ionome of Processed Tea}

This section describes the ionomes of black tea, green tea, oolong and pu-erh tea, which are produced from shoot tips. Most types of processed teas are produced from shoot tips, harvested with the plucking standard ' 2 and bud' or ' 3 and bud'. White tea is in this section excluded because white tea in sensu strictu consists only of tea buds without the second and third leaves and accordingly is not directly comparable to the sampled shoot tip sample. The elemental composition of processed teas may differ from the ionome of plucked leaf samples due to different sink/source leaf ratio and contamination through the processing. The elemental composition of processed tea samples is similar to the averaged ionome of shoot tip samples for subcompositions of elements, which are unaffected by leaf development stage (Section 7.4.1 and Table D.1).

Table 7.4 displays the average concentrations for the analyzed elements in processed tea samples: For all macro-nutrients and most micro-nutrients the values of shoot tips and processed teas are alike. The micro-nutrient $\mathrm{Mo}$ is in average higher concentrated in shoot tips and the micro-nutrient $\mathrm{Fe}$ is in average higher concentrated in processed teas. Out of the beneficial and non-nutrient elements, the Cr concentration is on average slightly greater in shoot tips while REE, Pb, Sb, Sc, Cs and $\mathrm{Tl}$ concentrations are considerably greater on average in processed teas.

The concentrations of the macro-nutrients, micro-nutrients, beneficial elements and non-nutrients are generally very well consistent with published data (Table 7.1, Szymczycha-Madeja et al. (2012) and references therein). Only the elements $\mathrm{Fe}, \mathrm{Pb}, \mathrm{Cr}$, $\mathrm{La}, \mathrm{Cd}$ and slightly $\mathrm{Li}$ and $\mathrm{Na}$ show notably higher concentrations in the compilation of literature data. Fe and La are elements typically influenced by contamination of adhering particles (Figure 6.5). The plant data of this thesis are corrected for adhering particles (Chapter 6) and therefore are expected to show lower concentrations than uncorrected data. $\mathrm{Pb}$ and $\mathrm{Cr}$ are elements typically influenced by contamination through machine abrasion (Section 7.5.1). Processed tea data of this thesis are mainly derived of whole leaf grades, like TGFOP (Table A.2) and originating mainly of Darjeeling. The sample material at the publications is often described as "tea bags", which are commonly filled with tea leaf grades of smaller particles sizes, like blends of fannings and dust grades. Hence, it can be assumed that these processed tea samples had a higher percentage of the fine particles of machine abrasion which are inherent to finer grained leaf material (Section 2.3.3). Alternatively, the processed tea samples blends analyzed by the other authors contained higher percentage of teas similar to Assam: Figure 7.4 shows that 
Assam has notably higher concentrations e.g. in $\mathrm{Pb}, \mathrm{Cr}$ and $\mathrm{Cu}$ than the Japanese teas of this thesis, which had been produced very delicately and semi-manual. The in overall greater concentrations of $\mathrm{Cd}, \mathrm{Na}$ and $\mathrm{Li}$ can not be explained by one of the parameters discussed in this thesis.

The spread of data is calculated by the standard deviation of the clr-values (Table 7.4). For most nutrient elements the ionomes of processed teas have spread similar to data of the shoot tip samples. Only Fe, Mn, Mo and Ni have differing spread between shoot tips and processed teas. Among the beneficial elements and non-nutrients the elements $\mathrm{Cd}, \mathrm{Cr}$, Sc, TI, Rb, Al, Li show typically a spread of data comparable to shoot tips, while $\mathrm{Pb}, \mathrm{Sb}, \mathrm{Cs}, \mathrm{REE}, \mathrm{Co}, \mathrm{Sr}$ and $\mathrm{Na}$ show less comparable spread. Generally, shoot tip samples exhibit a greater spread of concentration than processed tea samples, only $\mathrm{Cr}$, $\mathrm{Fe}, \mathrm{Pb}$ and $\mathrm{Sb}$ show a notably greater spread in processed tea samples. The $\mathrm{Cr}, \mathrm{Pb}$ and $\mathrm{Sb}$ concentrations in processed tea samples display a wide spread which are well consistent with the results of ilr-ANOVA in Section 7.5.1. It is most probable an effect of varying contamination by metal abrasion, either during the manufacturing itself or by airborne dust. The great spread of Fe concentration in processed tea samples could be caused by combined effects of metal abrasion, airborne dust, accumulated either in the field or during the withering process, and lower sink-source leaf ratio of the raw leaf material: all three aspects may contribute to the Fe concentration (Sections 2.3.3, 6.2 and 7.5.1.

lonome variability with respect to provenance analysis The spread of processed tea data within one garden gives a hint of the variability of processed tea ionomes between years, plucking seasons and plucking sections in the garden. Exemplary, for seven tea gardens with more than three analysis per garden the spread of clr-values is visualized in Figure 7.5 and calculated in Table 7.5. For most gardens the processed tea samples had been derived of one year, only Tumsong was sampled in 2010, 2011, and 2015 and Jun Chiyabari Tea Garden Pvt. Ltd. (Jun Chiyabari) in 2014 and 2015. For all gardens the raw leaf material for the processed tea samples had been derived of different plucking sections, but only for Yamamotoyama do Brasil, São Miguel de Arcañjo (S. Miguel de Arcañjo) nearly all sections of one division are included into the standard deviation, covering approximately 90 ha of tea plantation. For most elements the spread of clr-values ranges in the same order of magnitude than the spread of clr-values for shoot tips within one sample location (Table 7.2). For the nutrients $\mathrm{Co}, \mathrm{Cu}, \mathrm{Fe}, \mathrm{K}, \mathrm{Mo}$, $\mathrm{Na}, \mathrm{P}, \mathrm{S}$ and $\mathrm{Zn}$ and the non-nutrients $\mathrm{Cr}, \mathrm{Pb}$ and $\mathrm{Sb}$ the standard deviation is higher 
compared to shoot tips of one sample location. $\mathrm{Cr}, \mathrm{Pb}$ and $\mathrm{Sb}$ could be an effect of high fluctuation of contamination of metal abrasion from machines. $\mathrm{K}, \mathrm{P}, \mathrm{S}, \mathrm{Co}, \mathrm{Cu}$ and Zn show high standard deviation for the two tea gardens Kyoto Obubu Tea Farm, Uji (Obubu) and S. Miguel de Arcañjo, the only two gardens in this list which regularly apply fertilizers. For Mo and $\mathrm{Na}$ there is no good explanation for high spread of clr-values for some garden, e.g. Tumsong. 


\section{Ionome and Elemental Distribution of Tea Leaf}

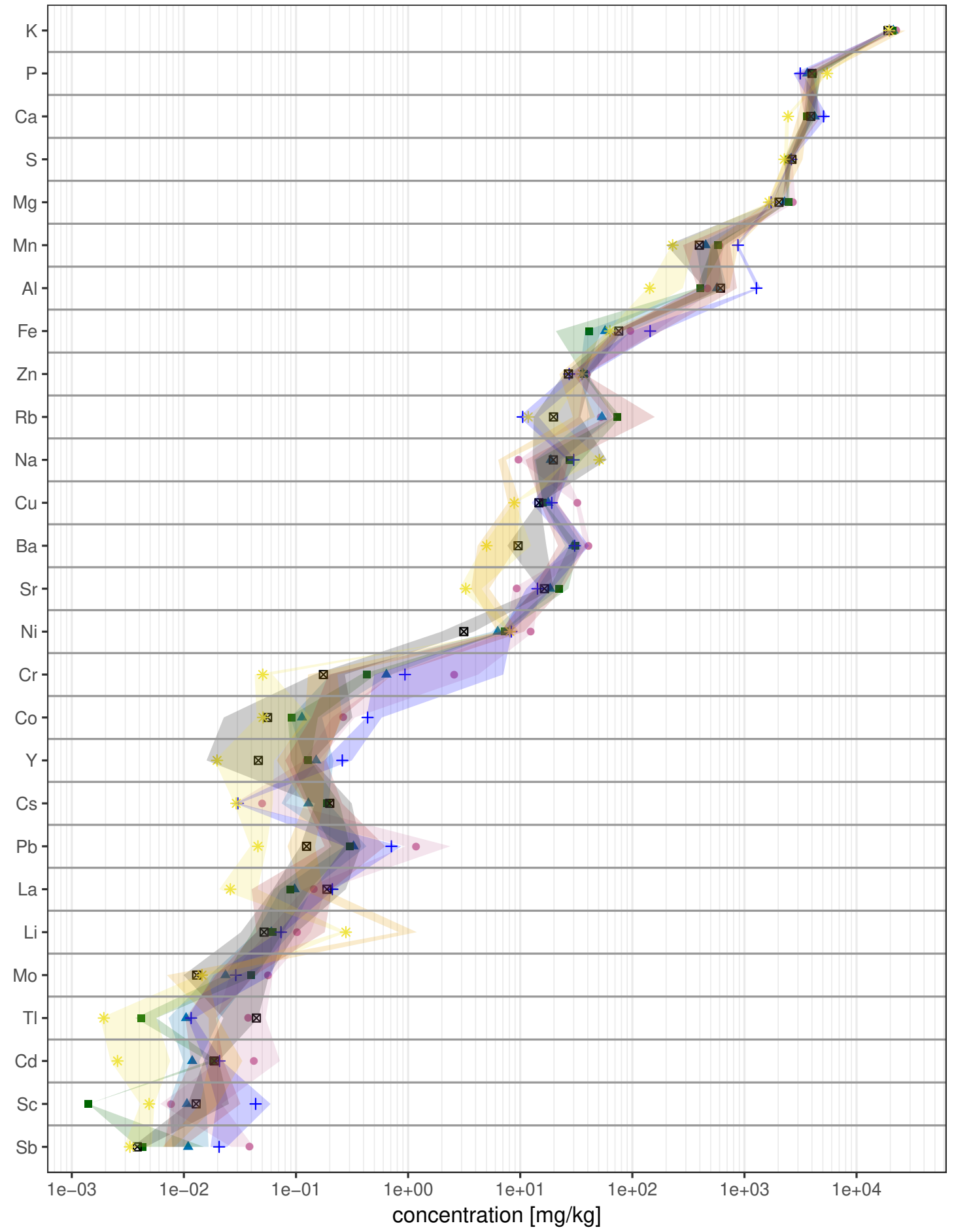

Region - Assam \ Darjeeling - Dhankuta + Nilgiris 凶 São Paulo * Shizuoka Uji unspecified

Figure 7.4.: lonomes of processed tea samples $(N=101)$, grouped by tea growing regions. Elements are ordered by median of all samples. Median for each sample group is marked by a colored dot, the shaded areas mark the second and third quartile. Median values are listed in Table 7.4 . 
Table 7.4.: Processed tea concentrations, compilation of data of this thesis $(N=101)$ and data from Ødegård and Lund (1997) $(N=3)$, Marcos et al. (1998) $(\mathrm{N}=15)$, Matsuura et al. (2001) $(\mathrm{N}=4)$, Gallaher et al. (2006) $(\mathrm{N}=3)$, McKenzie et al. (2010) $(\mathrm{N}=135)$, Salahinejad and Aflaki (2010) $(N=11)$ and Seenivasan et al. (2008) $(N=21)$. The compilation of published data is displayed in the columns left of the marker 'Publ. '. The 'mad' denotes the median absolute deviation of the concentration values and the 'stdev' the standard deviation. The geometric mean for calculating the clr-values is based on all elements listed in this table. Nutrients are marked with an asterisk, beneficial elements with a dagger.

\begin{tabular}{|c|c|c|c|c|c|c|c|c|c|c|c|c|}
\hline & median & mad & mean & stdev & clr-median & clr-stdev & Publ.: & median & mad & mean & stdev & $\mathrm{N}$ \\
\hline $\mathrm{Al}^{\dagger}$ & $5.4 e+02$ & $2.2 e+02$ & $6.0 e+02$ & $2.9 e+02$ & 6.60 & 0.35 & & $9.2 e+02$ & $3.6 e+02$ & $1.1 \mathrm{e}+03$ & $5.5 e+02$ & 188 \\
\hline $\mathrm{Ba}$ & $2.8 e+01$ & $1.3 e+01$ & $2.8 e+01$ & $1.5 e+01$ & 3.70 & 0.61 & & $2.4 e+01$ & $1.3 e+01$ & $2.5 e+01$ & $1.2 e+01$ & 177 \\
\hline $\mathrm{Ca}^{*}$ & $4.0 e+03$ & $5.9 e+02$ & $4.1 e+03$ & $9.0 e+02$ & 8.70 & 0.38 & & $4.6 e+03$ & $1.4 \mathrm{e}+03$ & $4.9 e+03$ & $1.7 e+03$ & 191 \\
\hline $\mathrm{Cd}$ & $1.4 \mathrm{e}-02$ & 7.1e-03 & $1.9 e-02$ & $1.5 e-02$ & -3.90 & 0.63 & & $9.0 e-02$ & $5.9 e-02$ & $1.7 e-01$ & $2.0 \mathrm{e}-01$ & 45 \\
\hline $\mathrm{Co}^{\dagger}$ & $1.4 \mathrm{e}-01$ & $1.0 \mathrm{e}-01$ & $2.0 e-01$ & $1.9 e-01$ & -1.60 & 0.69 & & $2.1 \mathrm{e}-01$ & $1.1 \mathrm{e}-01$ & $2.7 e-01$ & $1.4 \mathrm{e}-01$ & 21 \\
\hline $\mathrm{Cr}$ & $5.1 e-01$ & $4.6 e-01$ & $1.8 e+00$ & $5.4 e+00$ & -0.41 & 1.10 & & $2.3 e+00$ & $2.0 e+00$ & $3.6 e+00$ & $5.2 e+00$ & 47 \\
\hline Cs & $1.3 e-01$ & $9.9 e-02$ & $1.5 e-01$ & $1.1 \mathrm{e}-01$ & -1.80 & 0.94 & & $2.3 e-01$ & $1.6 e-01$ & $3.4 \mathrm{e}-01$ & $2.4 \mathrm{e}-01$ & 19 \\
\hline $\mathrm{Cu}^{*}$ & $1.6 e+01$ & $3.4 e+00$ & $1.9 e+01$ & $7.2 e+00$ & 3.20 & 0.43 & & $2.0 e+01$ & $8.4 e+00$ & $2.1 e+01$ & $9.6 e+00$ & 212 \\
\hline $\mathrm{Fe}^{*}$ & $7.2 e+01$ & $3.7 e+01$ & $9.0 \mathrm{e}+01$ & $7.5 e+01$ & 4.70 & 0.75 & & $1.8 e+02$ & $1.1 e+02$ & $2.4 e+02$ & $1.5 e+02$ & 143 \\
\hline $\mathrm{K}^{*}$ & $2.1 e+04$ & $2.1 e+03$ & $2.1 e+04$ & $2.4 e+03$ & 10.00 & 0.44 & & $1.6 e+04$ & $4.3 e+03$ & $1.7 e+04$ & $4.9 e+03$ & 55 \\
\hline $\mathrm{La}^{\dagger}$ & $1.1 \mathrm{e}-01$ & $7.5 e-02$ & $1.5 e-01$ & $1.5 e-01$ & -1.80 & 0.59 & & $8.6 e-01$ & $9.1 e-01$ & $2.0 \mathrm{e}+00$ & $2.5 e+00$ & 22 \\
\hline $\mathrm{Li}$ & $6.5 e-02$ & $4.2 e-02$ & $1.3 e-01$ & $2.0 e-01$ & -2.40 & 0.99 & & $1.6 e-01$ & $1.1 \mathrm{e}-01$ & $1.7 e-01$ & $9.3 e-02$ & 15 \\
\hline $\mathrm{Mg}^{*}$ & $2.2 e+03$ & $2.9 e+02$ & $2.2 e+03$ & $3.2 e+02$ & 8.10 & 0.40 & & $2.1 e+03$ & $3.6 e+02$ & $2.2 e+03$ & $4.6 e+02$ & 191 \\
\hline $\mathrm{Mn}^{*}$ & $4.9 e+02$ & $2.0 e+02$ & $5.3 e+02$ & $2.5 e+02$ & 6.50 & 0.38 & & $6.4 e+02$ & $3.1 e+02$ & $6.0 e+02$ & $3.7 e+02$ & 191 \\
\hline Mo* & $2.4 \mathrm{e}-02$ & $1.7 \mathrm{e}-02$ & $4.4 \mathrm{e}-02$ & $6.8 \mathrm{e}-02$ & -3.30 & 0.76 & & $4.0 \mathrm{e}-02$ & $1.5 e-03$ & $3.8 e-02$ & $5.1 e-03$ & 4 \\
\hline $\mathrm{Na}^{\dagger}$ & $1.8 e+01$ & $1.1 e+01$ & $2.2 e+01$ & $1.7 e+01$ & 3.30 & 0.89 & & $8.8 e+01$ & $6.8 e+01$ & $1.6 e+02$ & $2.0 \mathrm{e}+02$ & 114 \\
\hline $\mathrm{Ni}^{*}$ & $6.8 e+00$ & $2.3 e+00$ & $7.2 e+00$ & $3.2 e+00$ & 2.20 & 0.46 & & $3.8 e+00$ & $3.1 e+00$ & $4.4 e+00$ & $3.5 e+00$ & 139 \\
\hline $\mathrm{P}^{*}$ & $3.9 e+03$ & $6.8 e+02$ & $4.0 e+03$ & $7.2 e+02$ & 8.60 & 0.54 & & $2.9 e+03$ & $6.2 e+02$ & $3.0 e+03$ & $7.0 e+02$ & 114 \\
\hline $\mathrm{Pb}$ & $3.0 \mathrm{e}-01$ & $1.9 e-01$ & $5.0 e-01$ & $5.5 e-01$ & -0.79 & 0.82 & & $9.2 e-01$ & $5.3 e-01$ & $1.0 \mathrm{e}+00$ & $6.1 \mathrm{e}-01$ & 51 \\
\hline $\mathrm{Rb}$ & $4.8 e+01$ & $2.7 e+01$ & $5.5 e+01$ & $3.5 e+01$ & 4.10 & 0.71 & & $5.3 e+01$ & $2.2 e+01$ & $5.7 e+01$ & $2.2 e+01$ & 22 \\
\hline $\mathrm{S}^{*}$ & $2.6 e+03$ & $1.7 e+02$ & $2.6 e+03$ & $2.4 \mathrm{e}+02$ & 8.20 & 0.43 & & $2.5 e+03$ & $3.3 e+02$ & $2.6 e+03$ & $3.8 e+02$ & 107 \\
\hline $\mathrm{Sb}$ & $9.0 e-03$ & $6.9 e-03$ & $1.3 e-02$ & $1.2 \mathrm{e}-02$ & -4.20 & 0.72 & & & & & & \\
\hline Sc & $1.1 e-02$ & $8.0 e-03$ & $1.7 e-02$ & $1.5 e-02$ & -4.10 & 0.71 & & $5.0 e-02$ & $2.2 e-02$ & $5.2 e-02$ & $1.6 e-02$ & 6 \\
\hline $\mathrm{Sr}$ & $1.6 e+01$ & $6.8 e+00$ & $1.8 e+01$ & $1.2 e+01$ & 3.20 & 0.61 & & $1.7 e+01$ & $7.9 e+00$ & $1.9 e+01$ & $8.3 e+00$ & 129 \\
\hline $\mathrm{TI}$ & $1.5 e-02$ & $1.1 \mathrm{e}-02$ & $2.2 \mathrm{e}-02$ & $2.1 \mathrm{e}-02$ & -3.90 & 0.85 & & $5.3 e-02$ & $2.4 \mathrm{e}-02$ & $4.7 e-02$ & $1.8 e-02$ & 7 \\
\hline$Y^{\dagger}$ & $1.3 e-01$ & $7.5 \mathrm{e}-02$ & $1.6 e-01$ & $1.3 e-01$ & -1.70 & 0.54 & & 3.6e-01 & $1.6 e-01$ & $3.1 \mathrm{e}-01$ & $1.3 e-01$ & 12 \\
\hline $\mathrm{Zn}^{*}$ & $3.6 e+01$ & $6.3 e+00$ & $3.6 e+01$ & $7.4 e+00$ & 3.90 & 0.50 & & $2.7 e+01$ & $8.1 e+00$ & $3.4 e+01$ & $6.6 e+01$ & 191 \\
\hline
\end{tabular}




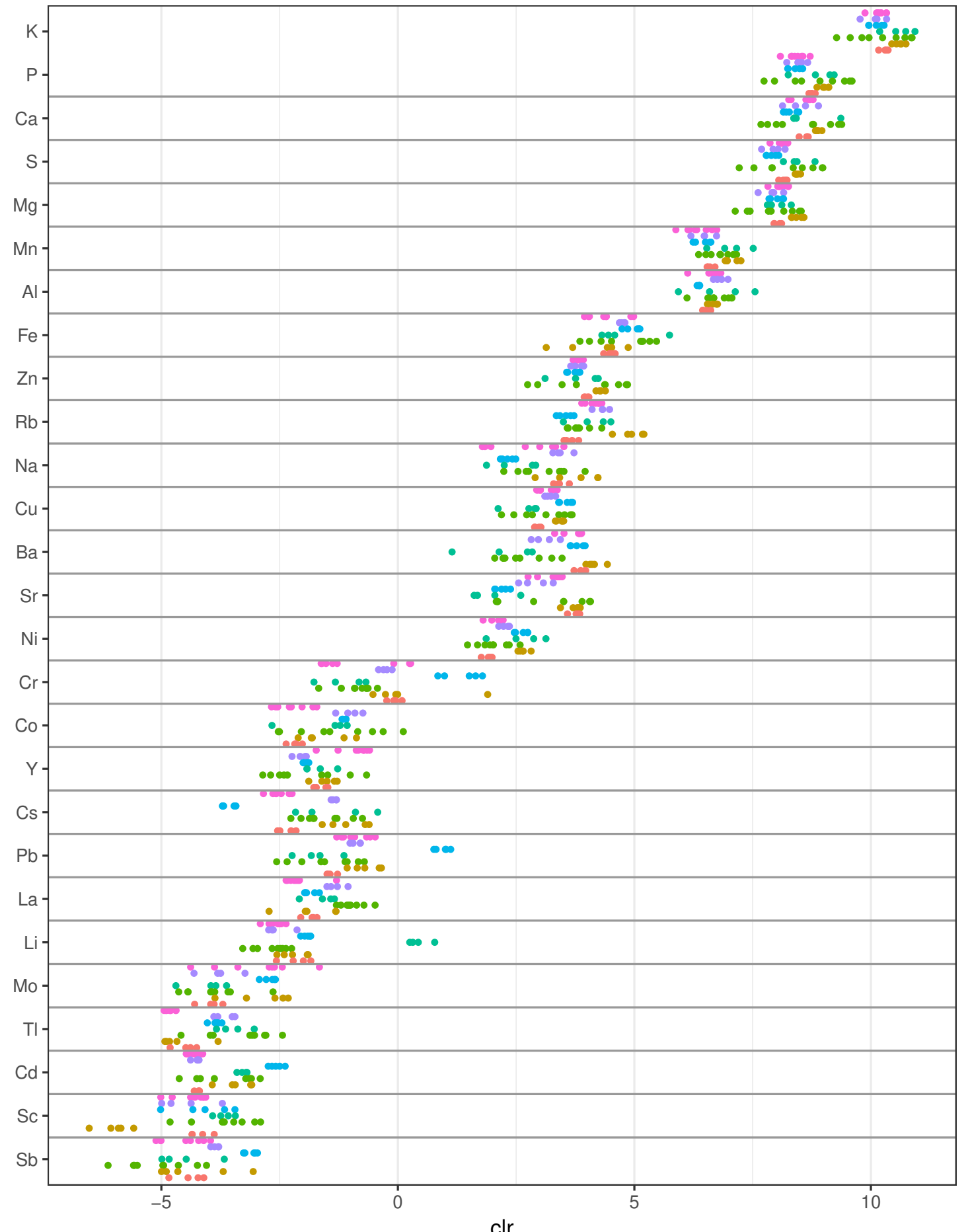

Garden - Ging - Jun Chiyabari - Midori-Arcanjo - Obubu • Sankar • Teesta Valley • Tumsons

Figure 7.5.: Clr-values of processed tea ionomes of samples from seven gardens of different tea growing regions. The dots are vertically dodged by garden: For each element the upper row of dots represents Tumsong and the lowest row of dots represents Ging. Clr-values are calculated by all listed elements. Elements are ordered as in Figure 7.1 . 
Table 7.5.: Standard deviation of clr-values of processed tea analysis per garden. Clr-values had been calculated by all elements unaffected by correction for adhering particles (Table D.1). ' $N$ ' is number of analysis for calculating the standard deviation.

\begin{tabular}{|c|c|c|c|c|c|c|c|c|c|c|c|c|c|c|c|}
\hline Garden & $N$ & $\mathrm{Al}$ & $\mathrm{Ba}$ & $\mathrm{Ca}$ & $\mathrm{Cd}$ & Co & $\mathrm{Cr}$ & Cs & $\mathrm{Cu}$ & $\mathrm{Fe}$ & $\mathrm{K}$ & La & $\mathrm{Li}$ & $\mathrm{Mg}$ & $\mathrm{Mn}$ \\
\hline Tumsong & 8 & 0.23 & 0.21 & 0.19 & 0.12 & 0.36 & 0.86 & 0.21 & 0.17 & 0.39 & 0.14 & 0.34 & 0.17 & 0.14 & 0.29 \\
\hline Sankar & 5 & 0.02 & 0.15 & 0.14 & 0.14 & 0.04 & 0.42 & 0.14 & 0.14 & 0.17 & 0.15 & 0.14 & 0.09 & 0.14 & 0.17 \\
\hline Ging & 4 & 0.08 & 0.11 & 0.09 & 0.05 & 0.14 & 0.13 & 0.19 & 0.07 & 0.10 & 0.09 & 0.15 & 0.32 & 0.08 & 0.07 \\
\hline Jun Chiyabari & 5 & 0.10 & 0.17 & 0.05 & 0.34 & 0.52 & 0.97 & 0.43 & 0.08 & 0.70 & 0.11 & 0.58 & 0.29 & 0.12 & 0.15 \\
\hline Teesta Valley & 4 & 0.13 & 0.27 & 0.32 & 0.08 & 0.24 & 0.12 & 0.06 & 0.10 & 0.05 & 0.23 & 0.19 & 0.28 & 0.22 & 0.22 \\
\hline Midori-Arcanjo & 9 & 0.31 & 0.51 & 0.66 & 0.63 & 0.96 & 0.37 & 0.51 & 0.54 & 0.60 & 0.58 & 0.25 & 0.35 & 0.51 & 0.26 \\
\hline Obubu & 4 & 0.70 & 0.78 & 0.49 & 0.10 & 0.73 & 0.50 & 0.80 & 0.38 & 0.66 & 0.32 & 0.33 & 0.23 & 0.23 & 0.41 \\
\hline
\end{tabular}

\begin{tabular}{|c|c|c|c|c|c|c|c|c|c|c|c|c|c|c|}
\hline Garden & $\mathrm{N}$ & Mo & $\mathrm{Na}$ & $\mathrm{Ni}$ & $P$ & $\mathrm{~Pb}$ & $\mathrm{Rb}$ & $S$ & $\mathrm{Sb}$ & Sc & $\mathrm{Sr}$ & $\mathrm{TI}$ & $Y$ & $\mathrm{Zn}$ \\
\hline Tumsong & 8 & 0.87 & 0.71 & 0.14 & 0.19 & 0.30 & 0.15 & 0.11 & 0.45 & 0.35 & 0.25 & 0.09 & 0.38 & 0.07 \\
\hline Sankar & 5 & 0.14 & 0.14 & 0.14 & 0.14 & 0.15 & 0.15 & 0.12 & 0.13 & 0.62 & 0.14 & 0.11 & 0.05 & 0.13 \\
\hline Ging & 4 & 0.25 & 0.14 & 0.10 & 0.05 & 0.10 & 0.14 & 0.08 & 0.32 & 0.22 & 0.11 & 0.24 & 0.15 & 0.04 \\
\hline Jun Chiyabari & 5 & 0.65 & 0.57 & 0.11 & 0.10 & 0.31 & 0.27 & 0.04 & 0.85 & 0.35 & 0.17 & 0.47 & 0.24 & 0.07 \\
\hline Teesta Valley & 4 & 0.44 & 0.19 & 0.10 & 0.19 & 0.11 & 0.15 & 0.21 & 0.08 & 0.57 & 0.33 & 0.22 & 0.14 & 0.12 \\
\hline Midori-Arcanjo & 9 & 0.61 & 0.55 & 0.35 & 0.69 & 0.66 & 0.24 & 0.64 & 0.69 & 0.61 & 0.86 & 0.69 & 0.78 & 0.80 \\
\hline Obubu & 4 & 0.46 & 0.50 & 0.55 & 0.44 & 0.45 & 0.44 & 0.28 & 0.59 & 0.21 & 0.45 & 0.35 & 0.31 & 0.52 \\
\hline
\end{tabular}




\subsection{Aspects of Shoot Tip/Mature Leaf Ratio in Tea Leaf Samples}

The leaf age determines the transition state of the leaves from sink to source leaf (Section 2.1.2). Because the ionomes of sink leaves and source leaves differ considerably for certain elements (Section 7.1 and 7.2) the sink/source leaf ratio, respectively shoot tip/mature leaf ratio influences specific element ratios. For processed tea samples mainly the plucking technique determines the sink/source leaf ratio (Section 2.3.2). To ensure that provenance analytics is independent of the plucking technique or the harvesting method, the analyzed sample set should either contain only samples of similar average leaf age or only subcomposition of elements should be considered where element ratios are unaffected by the leaf development stage. The aim of this section is I) to determine element ratios, which are independent of the leaf development stage and II) to estimate the average leaf age of a tea sample.

\subsubsection{Element Distribution between Shoot Tip and Mature Leaf}

The distribution of elements between sink and source tissue, i.e. between shoot tips and mature leaves, is described by pairwise log-ratios (Figure 7.6) and clr-vratios (Figure 7.7). The results of comparing the pairwise log-ratio distribution shows which elements are similarly distributed between sink and source leaves. To visualize how the elements are distributed without spurious effects clr-vratios are calculated and displayed in Figure 7.7.

Conclusions from pairwise logarithmic ratios 27 pairs of samples, each pair consisting of one mature leaf sample and one shoot tip sample of the same tea bush, are used for comparing the difference of elemental composition between sink and source leaf tissue. For each sample a matrix of pairwise log-ratios is calculated (Equation 5.1). Then for each element pair the log-ratio distribution of mature leaves is compared with the log-ratio distribution of shoot tips by a paired Wilcoxon signed rank test. The probability-values ( $p$-values) of the result are displayed in Figure 7.6: Greenish to yellowish colored $p$-values indicate that the mean of the log-ratio distribution of mature leaf samples is equal to the mean of the log-ratio distribution of shoot tip samples, while blueish colored $p$-values indicate that the means are different. For example, one could interpret that $\mathrm{Fe}$ and $\mathrm{Cr}$ have similar log-ratios in mature leaves and in shoot tips, but log-ratios of Fe to Ba differ with different leaf development stage. 


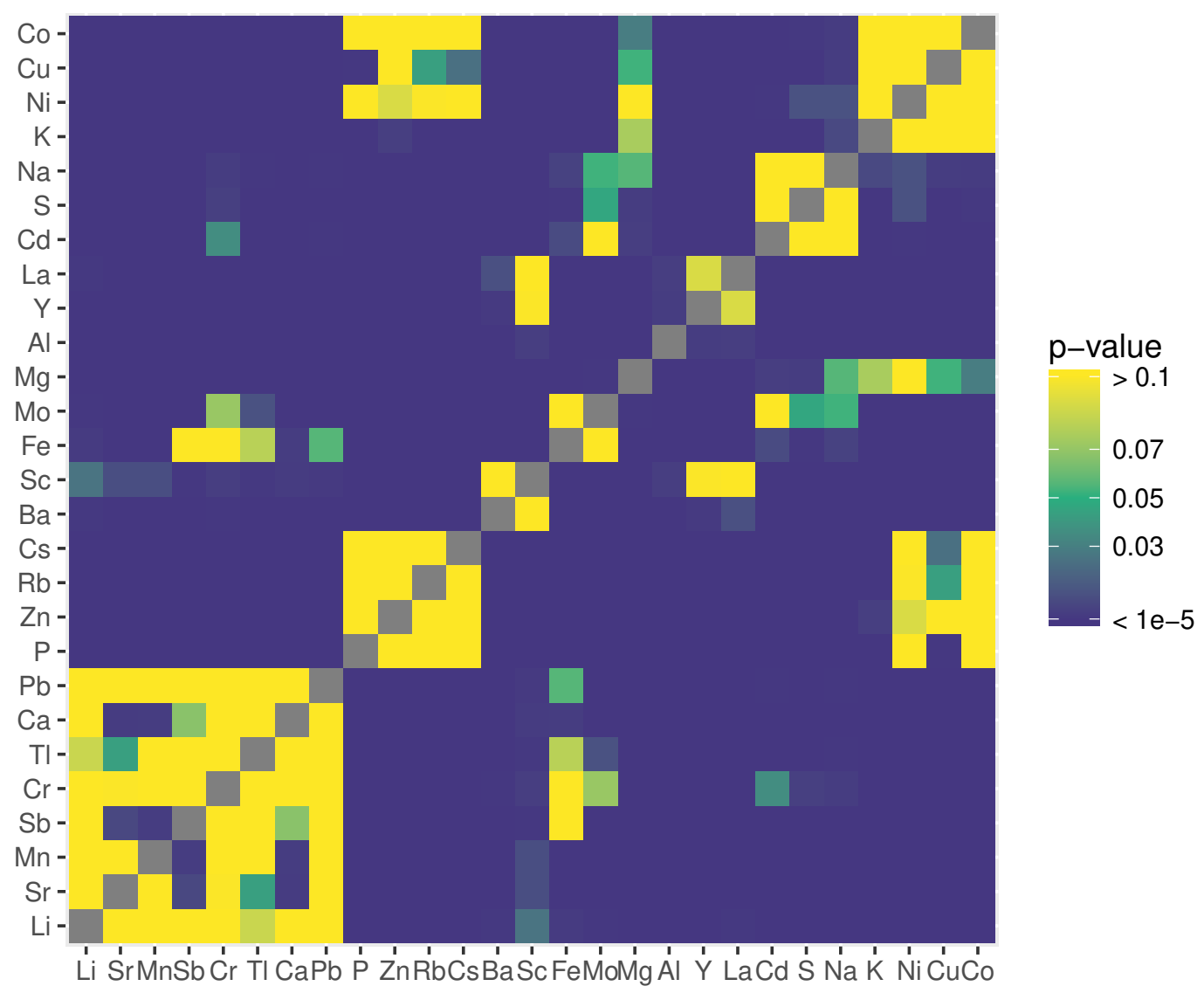

Figure 7.6.: Heatmap of $p$-values calculated by paired Wilcoxon signed rank test with continuity correction between distribution of pairwise log-ratio of shoot tip samples versus mature leaf samples $(\mathrm{N}=27)$. P-values of log-ratios of element pairs are colored blueish if they are below 0.05 (alternative hypothesis: true location shift is not equal to 0 ) and are colored greenish to yellow if values are above 0.05 (the means of two populations are equal).

Figure 7.6 exhibits following relations between mature leaves and shoot tips:

- All log-ratios with Al are different between the two sample groups

- The REE (represented by La and Y) have slightly similar log-ratio distributions between the two sample groups, but are different to all other elements, except for Sc

- The element subcomposition $\mathrm{K}, \mathrm{Rb}, \mathrm{Cs}, \mathrm{Cu}, \mathrm{Ni}, \mathrm{Co}, \mathrm{Zn}$ and $\mathrm{P}$ have similar logratios between the two groups, including $\mathrm{Mg} \sim \mathrm{K}, \mathrm{Mg} \sim \mathrm{Cu}$ and $\mathrm{Mg} \sim \mathrm{Ni}$, excluding the log-ratios $\mathrm{K} \sim \mathrm{Cs}, \mathrm{K} \sim \mathrm{Rb}, \mathrm{K} \sim \mathrm{Zn}, \mathrm{K} \sim \mathrm{P}, \mathrm{P} \sim \mathrm{Cu}$ and $\mathrm{Cs} \sim \mathrm{Cu}$.

- All log-ratios with Ba are different between the two sample groups except for Sc 
- All log-ratios with Fe are different between the two sample groups except for $\mathrm{Pb}$, $\mathrm{TI}, \mathrm{Mo}, \mathrm{Cr}$ and Sb.

- The element subcomposition $\mathrm{Na}, \mathrm{S}$ and $\mathrm{Cd}$ have similar log-ratios between the two groups and additionally there are similar log-ratios for $\mathrm{Na} \sim \mathrm{Mo}, \mathrm{Na} \sim \mathrm{Mg}, \mathrm{S} \sim \mathrm{Mo}$, $\mathrm{Cd} \sim \mathrm{Mo}$ and $\mathrm{Cd} \sim \mathrm{Cr}$.

- The element subcomposition Li, TI, Sb, Pb, Sr, Mn, $\mathrm{Ca}$ and $\mathrm{Cr}$ have similar logratios between the two groups, except for the log-ratios $\mathrm{Mn} \sim \mathrm{Ca}, \mathrm{Mn} \sim \mathrm{Sb}, \mathrm{Sr} \sim \mathrm{Ca}$ and $\mathrm{Sr} \mathrm{Sb}$. Additionally there is similar log-ratios between Mo $\mathrm{Cr}$.

The concentration of Al changes with age of the leaves, independent of all other element concentrations. This observation can be very well explained by the Al-accumulating physiological processes of $C$. sinensis (Section 2.1.3). The concentrations of REE also change significantly with respect to all other element concentrations with longer life span of the leaves, but differently than Al. There are two possible explanations for this observation: I) Uptake of Al and La could be correlated in Al-accumulating plants (unpublished data of buckwheat, Sauer and Fahlbusch). II) The correction for adhering particles (Section 6.2 did not fully remove the REE-signal of the AP, and the mature leaves, which are longer time exposed to airborne dust, show therefore higher REE concentrations. This second option would also explain the similar log-ratio distributions of La Sc ( $p$ value $=0.082$ ), because $S c$ is also a typical element which is in plants an indicator for APs (Section 6.1.4. Figure 6.2). Probably both options contribute to the REE concentration in mature leaves and therefore the log-ratio distributions are different from shoot tip samples and the log-ratio distribution of $\mathrm{Al} \sim \mathrm{La}$ is also different between the two sample groups.

The elements $\mathrm{K}, \mathrm{Zn}$ and $\mathrm{P}$ are typically enriched in sink tissue (Section 2.1.3 because they are phloem-mobile. They play a pivotal role in leaf development. The elements $\mathrm{Rb}$ and $\mathrm{Cs}$ have roughly similar characteristics as $\mathrm{K}$ and accordingly are probably also phloem-mobile. But the $\mathrm{p}$-values of the log-ratios of $\mathrm{K} \sim \mathrm{Cs}$ and $\mathrm{K} \sim \mathrm{Rb}$ (Figure 7.6) are close to zero, suggesting that the enrichment of $\mathrm{K}$ in shoot tips with respect to the mature leaves differs from the enrichment factor of $\mathrm{Cs}$ and $\mathrm{Rb}$ (see also Figure 7.7). The elements $\mathrm{Cu}, \mathrm{Ni}$ and $\mathrm{Co}$ are micro-nutrients, which are in the same group as the phloem-mobile elements $\mathrm{K}, \mathrm{Zn}$ and $\mathrm{P}$, indicating that these elements are also enriched in the shoot tips with respect to the mature leaves. In conclusion, the element subcomposition of $\mathrm{K}, \mathrm{Mg}, \mathrm{Cu}, \mathrm{Ni}, \mathrm{Co}, \mathrm{Cs}, \mathrm{Rb}, \mathrm{Zn}$ and $\mathrm{P}$ can be considered as set of phloem-mobile elements, which are enriched in shoot tips with respect to mature leaves. 
The elements $\mathrm{Mo}, \mathrm{Li}, \mathrm{Tl}, \mathrm{Sb}, \mathrm{Pb}, \mathrm{Sr}, \mathrm{Mn}, \mathrm{Ca}, \mathrm{Fe}$ and $\mathrm{Cr}$ can be grouped into one subcomposition. This subcomposition does not overlap with elements of the 'phloemmobile' subcomposition. Because $\mathrm{Ca}$ is known to be phloem-immobile, this subcomposition can be considered as phloem-immobile and accordingly is relatively enriched in mature leaves with respect to shoot tips.

The elements $\mathrm{Ba}, \mathrm{Na}, \mathrm{S}, \mathrm{Cd}$ and Fe have log-ratio distributions different from those of most other elements. There had no research been conducted yet why these elements in $C$. sinensis have similar log-ratio distributions between mature leaves and shoot tips among each other (except for $\mathrm{Ba}$ ), but have different distributions with respect to all other elements. $\mathrm{Ba}$, for example, seems to be sensitive to specific soil parameters (unpublished data, Pospiech et al. (2016)).

The two subcompositions of 'phloem-mobile' and 'phloem-immobile' elements have in common that the log-ratio distributions within these element subcompositions are similar between the two sample groups. The grouping of the element subcompositions is also displayed in Figure 6.6 (right plot): The elements of the set $\mathrm{K}, \mathrm{Mg}, \mathrm{Cu}, \mathrm{Ni}, \mathrm{Co}, \mathrm{Cs}, \mathrm{Rb}$, $\mathrm{Zn}$ and $\mathrm{P}$ are plotting closely suggesting quasi-constant log-ratios between elements. The elements $\mathrm{Ca}, \mathrm{Sr}, \mathrm{Ba}, \mathrm{Cd}, \mathrm{Tl}, \mathrm{Sb}, \mathrm{Pb}, \mathrm{Mn}, \mathrm{Fe}$ and $\mathrm{Cr}$ are plotting away from the first element subcomposition as a loose group, with slightly longer links between each other. $\mathrm{Al}, \mathrm{REE}, \mathrm{Sc}$ and Li are plotting as a third set of element, clearly influenced by the mature leaf samples.

Table 7.6 gives the averaged $p$-values between the log-ratio distributions of mature leaves and shoot tips. The average $p$-values indicate how probable it is that a log-ratio with this element is comparable between mature leaves and shoot tips:

- Log-ratio distributions with Al and REE (represented by La and Y) have generally very low $p$-values between the two groups

- Log-ratio distributions with $\mathrm{Mg}, \mathrm{S}, \mathrm{Ba}, \mathrm{Cd}, \mathrm{Sc}, \mathrm{Fe}$ and $\mathrm{Na}$ have in average p-values below the threshold of significance of 0.05

- Log-ratio distributions with all other elements have in average $p$-values above the threshold of significance of 0.05

The results from Figure 7.6 and Table 7.6 lead to the following conclusions:

- Log-ratios with $\mathrm{Al}, \mathrm{Ba}, \mathrm{Sc}$ and the REE are very sensitive to the developmental stage of the leaf 
Table 7.6.: Average of $p$-values: P-values are calculated by paired Wilcoxon signed rank test between distribution of pairwise log-ratio of shoot tip samples versus mature leaf samples (Figure 7.6. Then $p$-values of all log-ratios of one element had been averaged. Table is sorted by mean of $p$-values.

\begin{tabular}{lrrr}
\hline Element & Mean & Median & Var \\
\hline $\mathrm{Al}$ & 0.000 & $3.0 \mathrm{e}-08$ & $7.9 \mathrm{e}-07$ \\
$\mathrm{Y}$ & 0.007 & $3.0 \mathrm{e}-08$ & $6.3 \mathrm{e}-04$ \\
$\mathrm{La}$ & 0.011 & $6.0 \mathrm{e}-08$ & $1.7 \mathrm{e}-03$ \\
$\mathrm{Mg}$ & 0.015 & $1.6 \mathrm{e}-06$ & $1.6 \mathrm{e}-03$ \\
$\mathrm{Mo}$ & 0.022 & $2.3 \mathrm{e}-05$ & $3.8 \mathrm{e}-03$ \\
$\mathrm{Ba}$ & 0.027 & $3.0 \mathrm{e}-08$ & $2.0 \mathrm{e}-02$ \\
$\mathrm{Fe}$ & 0.032 & $1.3 \mathrm{e}-05$ & $7.8 \mathrm{e}-03$ \\
$\mathrm{Sc}$ & 0.040 & $2.3 \mathrm{e}-05$ & $2.1 \mathrm{e}-02$ \\
$\mathrm{Cd}$ & 0.054 & $4.1 \mathrm{e}-06$ & $2.4 \mathrm{e}-02$ \\
$\mathrm{~S}$ & 0.055 & $1.5 \mathrm{e}-07$ & $3.8 \mathrm{e}-02$ \\
$\mathrm{Na}$ & 0.058 & $9.5 \mathrm{e}-05$ & $3.8 \mathrm{e}-02$ \\
$\mathrm{Sr}$ & 0.062 & $2.1 \mathrm{e}-07$ & $4.1 \mathrm{e}-02$ \\
$\mathrm{Ca}$ & 0.066 & $6.0 \mathrm{e}-08$ & $2.4 \mathrm{e}-02$ \\
$\mathrm{~K}$ & 0.072 & $1.5 \mathrm{e}-07$ & $4.7 \mathrm{e}-02$ \\
$\mathrm{Cu}$ & 0.073 & $3.0 \mathrm{e}-07$ & $3.5 \mathrm{e}-02$ \\
$\mathrm{Sb}$ & 0.075 & $9.8 \mathrm{e}-07$ & $3.9 \mathrm{e}-02$ \\
$\mathrm{Mn}$ & 0.079 & $5.7 \mathrm{e}-07$ & $5.4 \mathrm{e}-02$ \\
$\mathrm{P}$ & 0.081 & $8.9 \mathrm{e}-08$ & $4.3 \mathrm{e}-02$ \\
$\mathrm{Ni}$ & 0.089 & $3.0 \mathrm{e}-07$ & $4.6 \mathrm{e}-02$ \\
$\mathrm{Rb}$ & 0.092 & $3.0 \mathrm{e}-08$ & $4.9 \mathrm{e}-02$ \\
$\mathrm{Li}$ & 0.093 & $2.3 \mathrm{e}-05$ & $3.8 \mathrm{e}-02$ \\
$\mathrm{Co}$ & 0.100 & $7.5 \mathrm{e}-07$ & $4.0 \mathrm{e}-02$ \\
$\mathrm{~Pb}$ & 0.100 & $5.2 \mathrm{e}-05$ & $4.8 \mathrm{e}-02$ \\
$\mathrm{Zn}$ & 0.100 & $8.9 \mathrm{e}-08$ & $5.6 \mathrm{e}-02$ \\
$\mathrm{Cs}$ & 0.110 & $6.0 \mathrm{e}-08$ & $6.8 \mathrm{e}-02$ \\
$\mathrm{Tl}$ & 0.110 & $7.5 \mathrm{e}-06$ & $5.4 \mathrm{e}-02$ \\
$\mathrm{Cr}$ & 0.130 & $5.2 \mathrm{e}-04$ & $5.7 \mathrm{e}-02$ \\
\hline
\end{tabular}

- Log-ratios of elements of the subcomposition $\mathrm{Co}, \mathrm{Cs}, \mathrm{Cu}, \mathrm{K}, \mathrm{Ni}, \mathrm{P}, \mathrm{Rb}$ and $\mathrm{Zn}$ vs. elements of the subcomposition $\mathrm{Ca}, \mathrm{Li}, \mathrm{Mn}, \mathrm{Mo}, \mathrm{Pb}, \mathrm{Sb}, \mathrm{Sr}$ and $\mathrm{TI}$ are sensitive to the developmental stage of the leaf

- Log-ratios with $\mathrm{Cd}, \mathrm{Cr}, \mathrm{Fe}, \mathrm{Na}, \mathrm{Mg}$ and $\mathrm{S}$ are slightly sensitive to the developmental stage of the leaf

Hence, $\mathrm{Al}, \mathrm{Ba}, \mathrm{Sc}$ and the REE should be excluded from element subcompositions for sample groups mixing shoot tip, processed tea and/or mature leaf samples. 
Conclusions from clr-vratios Figure 7.7 displays the clr-vratios of the 27 pairs of samples, each pair consisting of one mature leaf sample and one shoot tip sample of the same tea bush. All elements identified in the previous paragraph as primarily phloemmobile, $\mathrm{K}, \mathrm{Mg}, \mathrm{Cu}, \mathrm{Ni}, \mathrm{Co}, \mathrm{Cs}, \mathrm{Rb}, \mathrm{Zn}$ and $\mathrm{P}$, are enriched in shoot tips compared to mature leaves. Additionally $\mathrm{S}, \mathrm{Na}$ and $\mathrm{Cd}$ are for most sampled bushes enriched in shoot tips. Most elements identified in the previous paragraph as phloem-immobile, Mo, $\mathrm{Li}, \mathrm{TI}, \mathrm{Sb}, \mathrm{Pb}, \mathrm{Sr}, \mathrm{Mn}, \mathrm{Ca}, \mathrm{Fe}$ and $\mathrm{Ba}$, are enriched in mature leaves compared to shoot tips. For Mo, Fe, Sb, $\mathrm{Pb}$ and $\mathrm{Tl}$ it varies whether the elements are higher concentrated in mature leaves or in shoot tips. Al, Sc and REE are always clearly higher concentrated in mature leaves.

The standard deviation of the clr-vratios is lowest for $\mathrm{Ca}(0.26)$, increasing via $\mathrm{Mn}, \mathrm{Cu}$, K, S, Al, Cs, Rb, Sb, P, Sr, Mg, Cd, Fe, Y, Mo, Zn, TI, Ba, Li, La, Na, Pb, Co, Ni, Sc, up to $\mathrm{Cr}(1.0)$. A small spread of vratios indicates that for this element the distribution between shoot tips and mature leaves is similar for all sink-source-leaf sample pairs. A large spread of data indicates the contrary. The variability of clr-vratios could either be caused by the plucking technique, plant cultivar or environmental settings. The plucking technique determines the ratio of sink to source leaves (Section 2.3.2) while the pruning technique determines the approximate age of the remaining leaves. Sampling was done carefully according to the plucking standard 'two and bud' (Section 2.3.2) and for mature leaf samples approximately one year old foliage leaves had been chosen. The tea plant samples of Japan originate from different subspecies and cultivars than the tea plant samples from São Paulo or Darjeeling Additionally, the samples had been harvested at different seasons, which also affects the exact age and developmental stage of the leaf material. That implies that the between regions all three parameters, average development stage of leaves at '2 and bud' plucking standard, plant type and environmental parameters, change at the same time.

There are five sample pairs of identical cultivars, two sample pairs of Camellia sinensis var. sinensis 'yabukita' (C. sinensis 'yabukita') and three sample pair of Camellia sinensis var. sinensis 'AV2' (C. sinensis 'AV2'). The two sample pairs of $C$. sinensis 'yabukita' originate from Uji and in Shizuoka in Japan. The three sample pairs of $C$. sinensis 'AV2' originate all from Makaibari in Darjeeling, India. These five sample pairs are a very small sample group for statistical purposes and results will be statistically uncertain. But the distribution of vratios of this sample group compared to all other sample pairs suggests, that the type of cultivar is not the main source for differences in clr-vratios. For example, for the clr-vratios of $\mathrm{Ca}$ the two $C$. sinensis 'yabukita' sample pair clearly plot 
within their respective regional group, while the three sample pairs of $C$. sinensis 'AV2' are spread through the whole group of Darjeeling samples. The third, more probable possibility for differences in the element distribution between source and sink tissue between regions are environmental parameters such as nutrient state of the soils, climate, timing of last pruning, etc. This data set is too small to make assumptions or estimations which of these numerous parameters contribute notably to the differences in the distribution of elements between sink and source leaves.

Figure 7.7 shows that apart from the nutrients $\mathrm{P}, \mathrm{Zn}, \mathrm{Co}, \mathrm{Cu}, \mathrm{K}, \mathrm{Ni}, \mathrm{Mg}$ and $\mathrm{S}$ the nonnutrients $\mathrm{Rb}$ and $\mathrm{Cs}$ are enriched in shoot tips compared to mature leaves. The log-ratio analysis (Section 7.4.1 and Figure 7.6) describes that the enrichment factors of Rb, Cs and $\mathrm{K}$ are different and $\mathrm{Rb}$ and $\mathrm{Cs}$ are even higher enriched than the physiochemically similar nutrient $\mathrm{K}$. Ca, $\mathrm{Sr}$ and $\mathrm{Ba}$ are all enriched in mature leaves compared to shoot tips, with $\mathrm{Ba}$ showing the highest enrichment and $\mathrm{Ca}$ the lowest. $\mathrm{Rb}$ and $\mathrm{Cs}$ are both potentially toxic to plants (Gough et al. 1979; Kabata-Pendias 2010). On the other hand as monovalent cations they can serve as substitutes for $\mathrm{K}$, especially on acidic soil, but apparently without replacing $\mathrm{K}$ in its metabolic role (Tyler 1997; Kabata-Pendias 2010). There is no explanation yet why during the continuous translocation of ions via the phloem $\mathrm{Rb}$ and $\mathrm{Cs}$ are preferred over $\mathrm{K}$, while $\mathrm{Ca}$ is preferred over $\mathrm{Sr}$ and $\mathrm{Ba}$.

In summary, the results of the clr-vratios lead to the following conclusions:

- The distribution of elements is approximately constant for all sample pairs for mainly nutrients, e.g. $\mathrm{Ca}, \mathrm{Mn}, \mathrm{Cu}, \mathrm{K}, \mathrm{S}, \mathrm{P}$, but also for $\mathrm{Al}, \mathrm{Rb}, \mathrm{Cs}, \mathrm{Sb}$ and $\mathrm{Sr}$

- The distribution of elements differs between sample pairs for trace elements, e.g. $\mathrm{Cr}, \mathrm{Sc}, \mathrm{Ni}, \mathrm{Co}, \mathrm{Pb}, \mathrm{Na}, \mathrm{REE}$, Li, etc.

- The type of cultivar does not seem to have an influence on the element distribution patterns.

- $\mathrm{Rb}$ and $\mathrm{Cs}$ are stronger enriched in sink leaves compared to source leaves than $\mathrm{K}$.

The varying distribution patterns of trace elements could be an effect of the 'buffering' of elements in mature leaves (Section 7.2). In that case, the inhomogeneous distribution of trace elements might veil the geographical fingerprint, especially in the sink leaf tissue. Elements with a constant distribution pattern may serve as proxy for the average age of the leaf tissue (Section 7.4.2). 


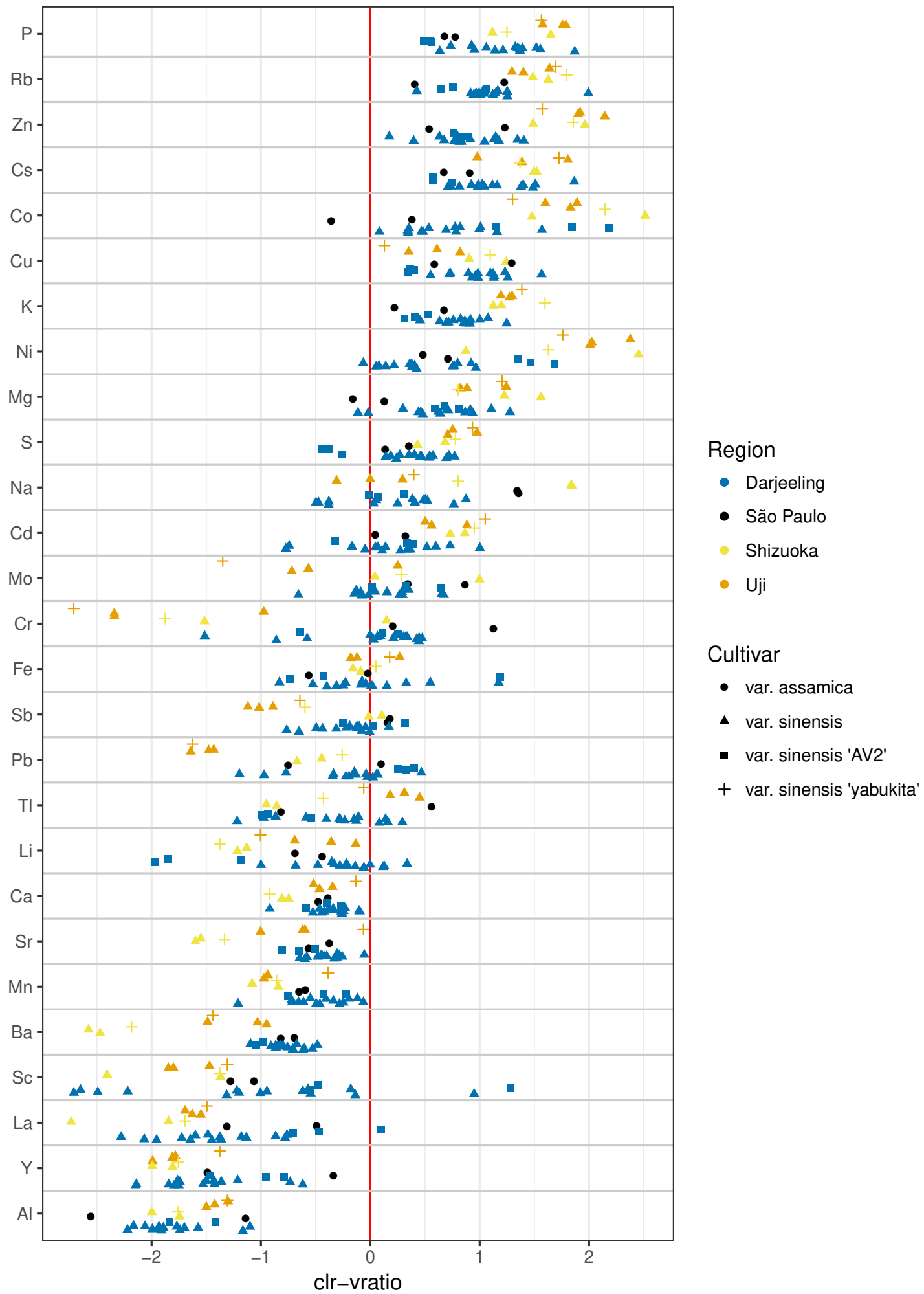

Figure 7.7.: Clr-vratios of shoot tip/mature leaf of corresponding pairs of samples $(\mathrm{N}=27$ ), grouped by tea growing regions. Elements are ordered by median of clr-vratio. Elements with negative clr-vratios are higher concentrated in mature leaves, elements with positive clr-vratios are enriched in shoot tips. 


\subsubsection{Estimating the Average Age of Tea Leaf Samples}

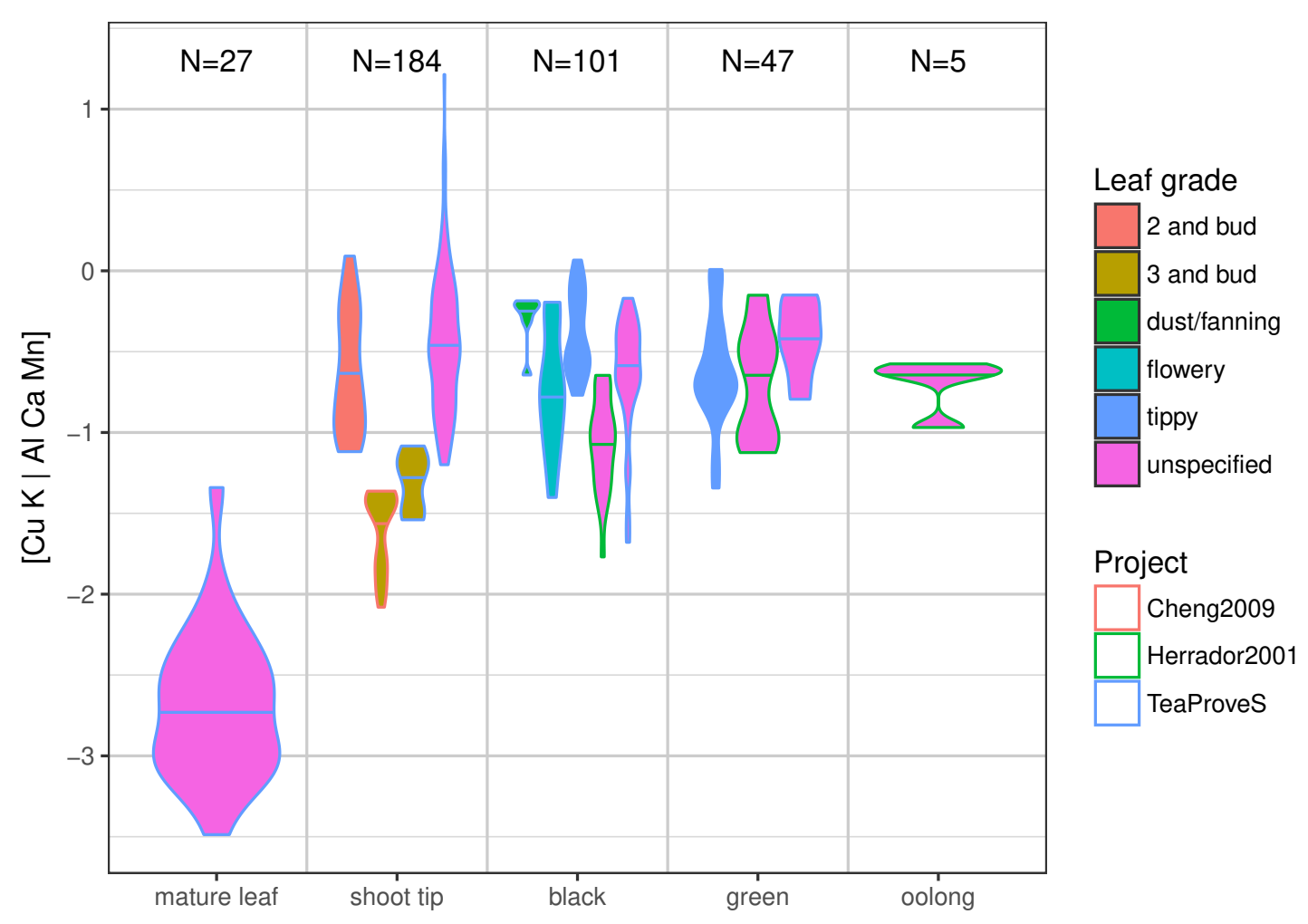

Figure 7.8.: The value of the balance [ $\mathrm{CuK} \mid \mathrm{Al} \mathrm{Ca} \mathrm{Mn]} \mathrm{vs.} \mathrm{the} \mathrm{subgroup} \mathrm{of} \mathrm{samples,} \mathrm{further}$ divided by plucking standard and leaf grade. For the shoot tip samples the plucking standard is mostly unspecified, but sampling was similar to the plucking standard '2 and bud'. For black tea all orthodox whole leaf and broken leaf grades containing "fine tippy" and "tippy" are subsumed under 'tippy' (Table A.2). All whole leaf and broken leaf grades containing "golden flowery" and "flowery" are subsumed under 'flowery'. All fannings and dust grades are subsumed under 'dust/fanning'. CTC and green tea leaf grades, which correspond to 'tippy' or 'flowery' leaf grades of the orthodox black tea, had been also subsumed under the respective groups. The plot is based on data collected in this thesis and previously published data (Section 2.1.3, Figure 2.4.

The distribution of elements between source and sink tissue can be used to get a rough estimation of the sink/source leaf ratio of the respective sample. The elements $\mathrm{Ca}, \mathrm{Mn}, \mathrm{Cu}, \mathrm{K}$ and $\mathrm{Al}$ have the smallest standard deviation of the clr-vratios describing the translocation from source to sink leaves (Section 7.4.1). This suggests that the distribution of these elements between sink and source tissue is less influenced by environmental parameters than other elements. Al and $\mathrm{Ca}$ are enriched in source leaves, while $\mathrm{Cu}, \mathrm{K}$ and $\mathrm{Mn}$ are enriched in sink leaves (Figure 7.7). A balanced ratio of these two element sets (Section 5.1, Equation 5.4, noted as [Cu K | Al Ca Mn], calculates the 
log-ratio contrast between $\mathrm{K}$ and $\mathrm{Cu}$ to $\mathrm{Al}, \mathrm{Ca}$ and $\mathrm{Mn}$ without spurious effects. The biggest contrast between the two element subcompositions exists for mature leaves and the lowest for shoot tips (Figure 7.8): Mature leaves have an average balanced ratio of -2.7 and shoot tips an averaged balanced ratio of -0.5 . Within the group of shoot tips the samples collected with '2 and bud' plucking standard have a smaller log-ratio contrast than samples collected with the ' 3 and bud' plucking standard; the latter includes one more and further developed leaf. The shoot tip samples marked as 'unspecified' in Figure 7.8 are mostly plucked at '2 and bud' plucking standard, but occasionally with higher percentage of buds. This explains the on average higher balanced ratio value compared to the samples which had been plucked strictly '2 and bud'.

Black, green, oolong and pu-erh tea are all produced from different leaf grades. For the data of pu-erh (McKenzie et al. 2010) and oolong (Herrador and González 2001; McKenzie et al. 2010, and this study) no further specification of leaf grades was available. The authors of Wong et al. (1998) compared oolong, which was produced from tea flushes harvested at ' 3 and bud' to ' 4 and bud' plucking standard, with other types of processed tea produced from '2 and bud' plucking standard and proposed to use Al as an age indicator. Black and green teas exhibit different means of [Cu K $\mid \mathrm{Al} \mathrm{Ca} \mathrm{Mn]} \mathrm{val-}$ ues by leaf grades. For black and green teas the leaf grades of 'whole leaf' and 'broken' had been unified into 'tippy' if leaf grade description indicated a high percentage of buds and unified into 'flowery', if description indicated a small percentage of buds. The fine grained leaf grades 'fanning' and 'dust' had been subsumed under 'dust/fanning' (see caption Figure 7.8. It can be seen that processed tea with leaf grades corresponding to "fine tippy" or "tippy" (Table A.2) have a notable higher values for the [Cu K| Al Ca Mn] balance than processed teas of leaf grades corresponding to "golden flowery" or "flowery". The highest leaf qualities for orthodox black teas from Darjeeling, e.g. SFTGFOP or FTGFOP-1, have the highest balanced ratio with a median value $(\mathrm{N}=12)$ of -0.25 . This clearly reflects a very highest percentage of immature leaves and buds and accordingly a high sink/source leaf ratio.

It has to be noted that the six samples of leaf grade 'dust/fannings' have also a very high [Cu K | Al Ca Mn] balance. The trend of a small log-ratio contrast between $\mathrm{K}$ and $\mathrm{Cu}$ against $\mathrm{Al}, \mathrm{Ca}$ and $\mathrm{Mn}$ for the fine leaf grades could be confirmed by four processed tea samples of Tumsong sampled during sorting. All four samples originate from the same withering table batch and had been sampled within 10 min at one sorting machine (Figure 7.9) to ensure that the raw processed tea is comparable in its elemental composition. The finest grained sample contained a very high percentage of trichomes, visible 


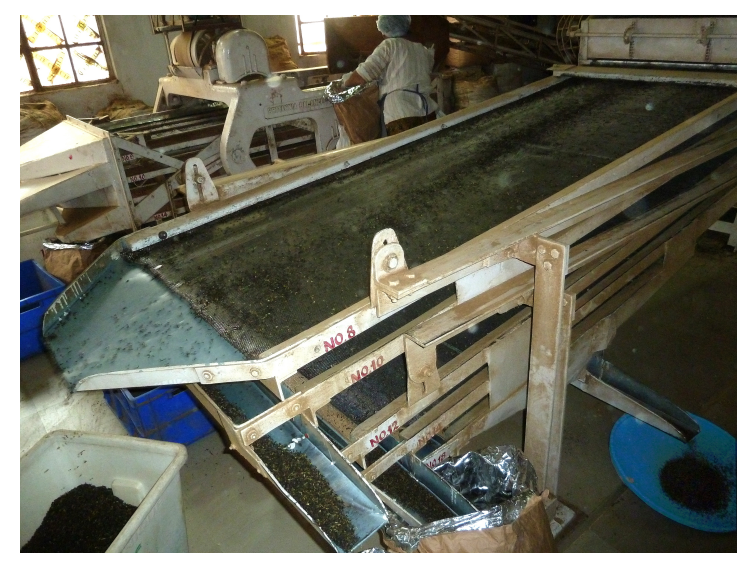

Figure 7.9.: Sorting machine with 10 sieves for black tea (orthodox) production in Darjeeling. The sieve with the coarsest mesh is on the top (No. 8), the sieve with the finest mesh is on the bottom (No. 18). For Sieve No. 8 the balance is

[Cu K | Al Ca Mn] = -0.82

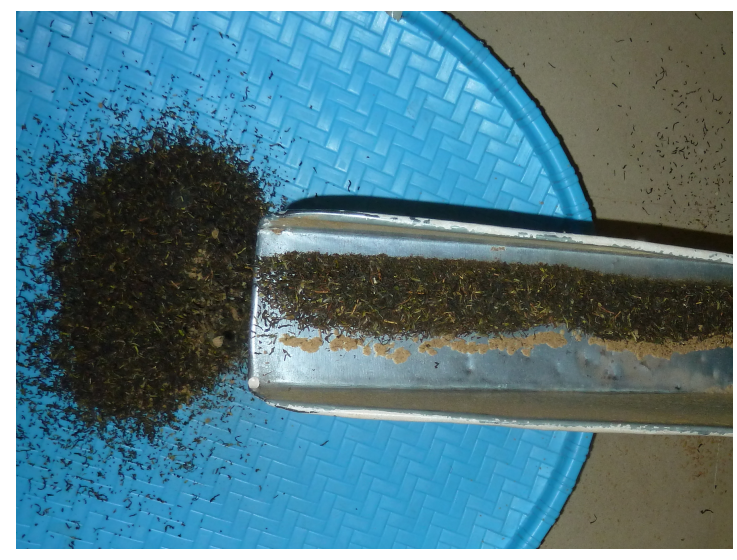

Figure 7.10.: Detail of Figure 7.9. Output of sieve No. 18. The tea hairs are enriched in this leaf grade and accumulated as the beige band on the metal outlet splint.

[Cu K | Al Ca Mn] = -0.52

in Figure 7.10 as yellowish band, and has a [Cu K | Al Ca Mn] value of -0.52 . The other three samples, from fine to coarse, are derived from sieve Nr. 14 (Figure 7.11), Nr. 10 (Figure 7.12) and Nr. 8 (Figure 7.9). Their [Cu K| Al Ca Mn] values are $-0.68,-0.74$ and -0.82 , respectively. The last value corresponds very well to the log-ratio contrast between $\mathrm{Cu}$ and $\mathrm{K}$ to $\mathrm{Al}, \mathrm{Ca}$ and $\mathrm{Mn}$ of the 'flowery' leaf grades of black teas (Figure 7.8.

There are two possible sources of high $\mathrm{Cu}$ and $\mathrm{K}$ in relation to $\mathrm{Al}, \mathrm{Ca}$ and $\mathrm{Mn}$ in the fine grained leaf grades: I) The abundant trichomes of the leaves, which for tea of high quality plucking standards can make up a significant portion in dust and fanning grades. II) Young leaves and buds have a more delicate tissue which breaks easier under the mechanical stress of production and therefore 'young' tissue is enriched in the finer grained leaf grades. This preliminary study of [Cu K $\mid \mathrm{Al} \mathrm{Ca} \mathrm{Mn]} \mathrm{balance} \mathrm{in} \mathrm{dependence}$ of leaf grade do not explain why there is a shift in the [Cu K | Al Ca Mn] values by leaf grade. Ajay Kichlu (Chamong Tea Exports Pvt. Ltd.) confirmed that broken and fanning leaf grades contain higher percentages of small leaves and shoot tips than the whole leaf grades, which can also be tasted by experienced tea sommeliers. In summary, not only the plucking standard but also the leaf grade influences the average development stage of leaf particles in a processed tea sample.

This approximation of average developmental stage of the leaf material of the samples can be used to filter sample sets for a specific range of [Cu K | Al Ca Mn] values: The 


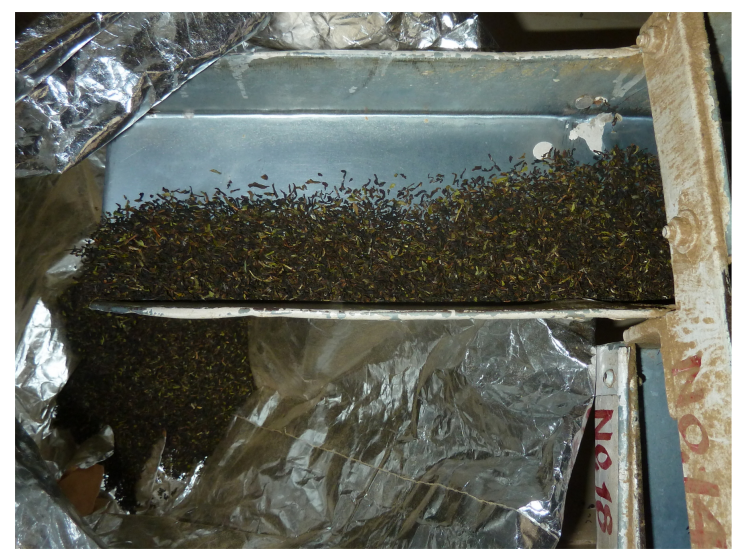

Figure 7.11.: Detail of Figure 7.9. Output of sieve No. 14.

$[\mathrm{Cu} \mathrm{K} \mid \mathrm{Al} \mathrm{Ca} \mathrm{Mn]}=-0.68$

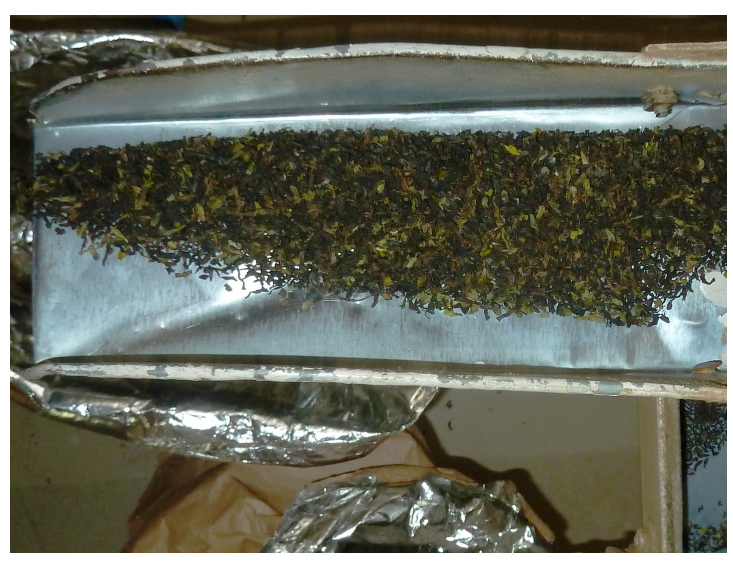

Figure 7.12.: Detail of Figure 7.9. Output of sieve No. 10.

[Cu K $\mid \mathrm{Al} \mathrm{Ca} \mathrm{Mn]} \mathrm{=}-0.74$

higher the minimum [ $\mathrm{Cu} \mathrm{K} \mid \mathrm{Al} \mathrm{Ca} \mathrm{Mn]} \mathrm{values} \mathrm{are} \mathrm{the} \mathrm{higher} \mathrm{is} \mathrm{the} \mathrm{probability} \mathrm{that} \mathrm{the}$ sample set contains only sink tissue samples, e.g. if the filter is set to [Cu K| $\mathrm{Al} \mathrm{Ca} \mathrm{Mn]}$ $>-1$. On the other hand if the filter is set to [Cu K $\mid \mathrm{Al} \mathrm{Ca} \mathrm{Mn]}<-2$ the probability is very high, that the sample set consists only of mature leaves.

This filtering can be used for three objectives: I) For statistical analysis where one sample group should consist strictly of sink tissue, II) to decide about the element subcomposition used for analysis, e.g. should elements being sensitive to the developmental stage of the leaves be included or not and III) estimating for processed tea samples the plucking standard as proxy for provenance analysis. For the latter the influence of the leaf grade on the [Cu K | Al Ca Mn] values has to be taken into account.

\subsection{Comparison between Shoot Tip and Processed Tea}

In contrast to plucked leaf samples, the sampling location of which can be documented by meter-scale, processed teas are often an average of garden section, and often the exact plucking section of the respective production batch is unknown. Additionally, batches of tea are often blended with other batches, and the resulting lots could even consist of tea material from the whole garden area. Moreover, abrasion of the machinery during the tea processing is an other possible source of contamination (Section 2.3.3) affecting certain element ratios. Every type of processed tea requires its own set of machines and accordingly green tea will have different sources of abrasion than black orthodox tea or black CTC. For provenance analysis of the ready-made product 'tea' the shift in elemen- 
tal composition between plucked leaves and processed teas has to be characterized. This section discusses in detail differences between the sample group of plucked leaves and processed teas.

\subsubsection{Effects of Black Tea Manufacturing}

This section focuses on metal abrasion sources for black orthodox and black CTC tea by comprising all samples of the subgroups 'black' and 'shoot tip': 74 processed tea samples and 160 shoot tip samples are used as unpaired data set for the analysis. Three methods are used to determine the elements which have a high probability to be altered by machinery abrasion: I) A covariance biplot of clr-transformed data, II) the Mann-Whitney test on distribution of pairwise log-ratios, similar to the method used in Section 7.4.1, and III) an ilr-ANOVA.

I) In Figure 6.6 (right plot) the sample dots of black teas concentrate in the upper left quadrant while the sample dots of green tea are mainly found in the lower right quadrant. On the one hand, the position of the element labels $\mathrm{Pb}, \mathrm{Zr}$, $\mathrm{Cr}$ and $\mathrm{Sb}$ indicate that the log-ratio of one of these elements to one of the other elements is probably in average higher for samples projected into the upper left quadrant. This observation excludes log-ratios with $\mathrm{Nb}, \mathrm{Ti}$ and $\mathrm{U}$, On the other hand, samples projected into the opposite quadrant, the lower right quadrant, have probably the lowest log-ratios with $\mathrm{Pb}, \mathrm{Zr}, \mathrm{Cr}$ and $\mathrm{Sb}$. This suggests that the concentrations of the elements $\mathrm{Pb}, \mathrm{Cr}$ and $\mathrm{Sb}$ vary between black processed tea samples and green processed tea samples or shoot tip samples. The averaged concentrations of these elements confirm that black tea samples show notably higher concentrations compared to the majority of shoot tip samples and green tea samples (Table 7.7).

Table 7.7.: Averaged (median) element concentration [mg/kg] of $\mathrm{Cr}, \mathrm{Pb}, \mathrm{Sb}$ and $\mathrm{Zr}$ by type of processed tea and shoot tip. Table is ordered by median concentration of $\mathrm{Pb}$. ' $\mathrm{N}$ ' denotes the number of analysis. In this data set exist for oolong and gunpowder only one sample each, but values coincides with published data (McKenzie et al. 2010; Szymczycha-Madeja et al. 2012.

\begin{tabular}{lrrrrr}
\hline Subgroup & $\mathrm{N}$ & $\mathrm{Pb}$ & $\mathrm{Cr}$ & $\mathrm{Sb}$ & $\mathrm{Zr}$ \\
\hline green & 26 & 0.133 & 0.198 & 0.005 & 0.052 \\
shoot tip & 160 & 0.170 & 0.546 & 0.006 & 0.039 \\
oolong & 1 & 0.207 & 0.377 & 0.004 & 0.010 \\
black & 74 & 0.375 & 0.661 & 0.011 & 0.081 \\
gunpowder & 1 & 1.602 & 0.516 & 0.051 & 0.408 \\
\hline
\end{tabular}


II) The Mann-Whitney test is used to calculated the p-values if the means of the pairwise log-ratio distribution between the two sample groups are equal (Figure 7.13). The figure clearly shows that $\mathrm{Pb}$ and $\mathrm{Sb}$ have similar log-ratio distributions in both sample groups, but differing log-ratio distributions with all other elements. The p-values of all other log-ratio distributions do not permit a clear grouping of elements, but the two main element subcompositions, Mo, Na, Mg, K, S, Ni, Co, Cd, Zn and Ba, REE, Sc, Li, TI, $\mathrm{Sr}, \mathrm{Ca}, \mathrm{Rb}, \mathrm{Al}, \mathrm{Cs}, \mathrm{Cr}, \mathrm{Mn}, \mathrm{Fe}, \mathrm{Cu}$, are roughly corresponding to the phloem-mobile/ phloem-immobile element subcompositions (Section 7.4.1).

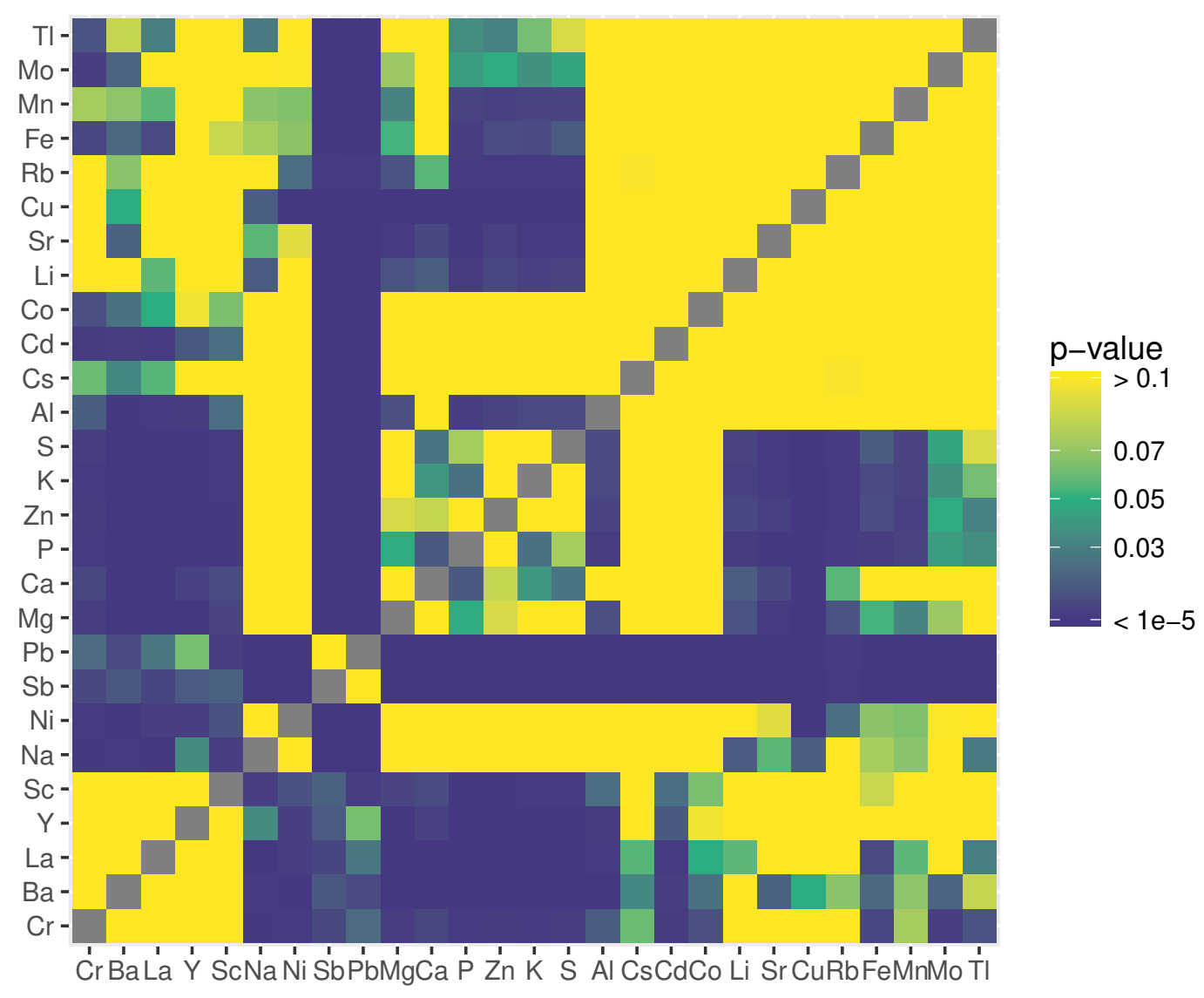

Figure 7.13.: Heatmap of $p$-values calculated by (unpaired) Mann-Whitney test between distribution of pairwise log-ratio of shoot tip samples $(N=160)$ versus black processed tea samples $(\mathrm{N}=74)$. P-values of log-ratios of element pairs are colored blueish if they are below 0.05 (alternative hypothesis: true difference in means is not equal to 0 ) and are colored greenish to yellow if values are above 0.05 (the means of two populations are equal).

III) The ilr-ANOVA of two sample groups shows that $\mathrm{Pb}, \mathrm{Sb}$ and $\mathrm{Cr}$ build one element subcomposition. This subcomposition has the highest variance between the two sample groups black tea and shoot tips (Figure 7.14). Most nutrients are part of the 
element subcomposition of Mo, Co, P, Zn, Ca, Cs, Fe, Mg, K, and Ni. The third element subcomposition consists of $\mathrm{Sc}, \mathrm{Sr}, \mathrm{Mn}, \mathrm{Cu}, \mathrm{Rb}$, $\mathrm{Li}$ and $\mathrm{Tl}$, whereof Sc builds an own subcomposition. Summarizing the results of the three methods the concentrations of

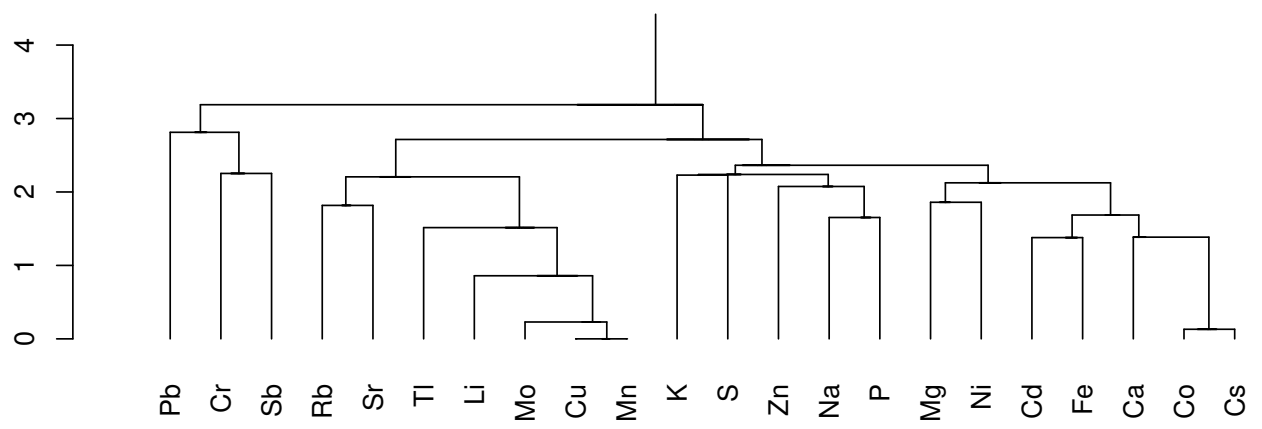

Figure 7.14.: Graph of ilr-ANOVA (CoDa dendrogram) of shoot tip samples ( $\mathrm{N}=159)$ and black processed teas $(\mathrm{N}=74)$ as categorical variable to explore which element subcompositions have a similar behavior with respect to the two sample groups. For calculating the ilr-ANOVA the element subcomposition was used which excludes elements with high probability to be altered by leaf development stage (Table D.1 unaffected element subcomposition of the parameter 'leaf development stage').

$\mathrm{Cr}, \mathrm{Pb}$ and $\mathrm{Sb}$ are clearly altered in black processed tea compared to shoot tips. The concentrations of Sc have a less probability to be significantly altered but the methods of covariance biplots and ilr-ANOVA suggest to include this element to set of elements which could be potential biased by black tea manufacturing.

The source of $\mathrm{Pb}$ and $\mathrm{Sb}$ is probably lead containing solders. A very frequently used solder alloy consists of $\mathrm{Pb}$ and $\mathrm{Sn}$ with varying minor contents in $\mathrm{Fe}, \mathrm{Sb}, \mathrm{Cu}$ and $\mathrm{Ni}$ (Council of the European Union 2011b). Analysis of Sn does generally not meet the quality criteria to be used for provenance analysis (Section 4.3.1, Table 4.3). But if Sn is included for the ilr-ANOVA, the grouping of $\mathrm{Sn}$ with $\mathrm{Pb}$ and $\mathrm{Sb}$ (Figure D.1, upper graph) supports the assumption that abrasion of solder is the major source of $\mathrm{Pb}$ and $\mathrm{Sb}$ in processed teas. There are two possible sources of solder abrasion: a) The machinery in the tea factories: Lead containing solders are now restricted by several legislations, e.g. in the EU (Council of the European Union 2011b) and Japan, but for most tea producing areas lead containing solders are still commonly utilized for building or maintaining tea factories. b) Airborne dust: Darjeeling is very close to the cities of Siliguri and Bagdogra 
(Section 3.1.1), both home to thriving industry with a high rate of combustion residues in the air. Other tea growing areas, like Nilgiris, Registro or Shizuoka, are also exposed to airborne dust of industry and road traffic. During the hours of withering the wilted leaves of the tea could act as an air filter (Section 2.3.3) retaining also the particles of solders from industries located in the proximity of the tea growing areas.

One source of $\mathrm{Cr}$ is likely to be the metal abrasion of the machines, especially CTC production is prone to (too) high $\mathrm{Cr}$ abrasion during cutting and tearing the leaves due to affected teeth of the steel rollers (Section 2.3.3). A study of $\sim 800$ Chinese processed tea samples (Han et al. 2005) reveals that $\mathrm{Cr}$ in black tea samples $(\mathrm{N}=148)$ are found in significantly higher concentrations, median $=1.73 \mathrm{mg} / \mathrm{kg}$, than green tea $(\mathrm{N}=537)$, median $=0.48 \mathrm{mg} / \mathrm{kg}$, or oolong tea $(\mathrm{N}=81)$, median $=0.27 \mathrm{mg} / \mathrm{kg}$. Because black tea production requires more invasive machinery than oolong or green tea, this study confirms that black tea manufacturing processes are one possible source of $\mathrm{Cr}$ in black teas. In the data set used for this thesis the effect of machinery on $\mathrm{Cr}$ concentration can not be seen as clearly because the majority of black tea samples are Darjeeling orthodox black teas. The manufacturing procedure used for orthodox black teas, similar to the one used for oolong, does not involve machinery as rough or invasive as used for CTC black tea production. In Figure 7.15 (left plot) the projection of sample dots onto the variable rays indicates, that the clr-vratio of $\mathrm{Cr}$ is induced mainly by the samples from Matigara Tea Estate (Matigara) and Ord Terai Tea Garden (Ord Terai). In 2011, both gardens used the oldest CTC machinery of all visited factories; it can be presumed that this caused higher abrasion rates than the other CTC factories in the Maud and Amaya.

Sources of Sc could be a) metal abrasion of Al-Sc-alloys, b) airborne dust during withering - the same effect as described for $\mathrm{Pb}$ and $\mathrm{Sb}-$, or $\mathrm{c}$ ) the accumulation of $\mathrm{Sc}$ in mature leaf sample during their life span combined with the fact that the ratio of sink to source leaves is lower in processed teas than in the shoot tip samples. The latter option is supported by the observation that the Sc concentration is slightly sensitive to the leaf development stage (Figure 6.6 and Section 7.4.1). On the other hand, the effect of the leaf age is estimated to be too small to account for an effect of differences in the sink-source leaf ratio between processed teas and shoot tips on the Sc concentration. Additionally the ilr-ANOVA including Al - a strong indicator for leaf age - shows no grouping with Sc, but with Mn (Figure D.1, lower graph). Therefore, it is likely that the Sc concentration in processed teas is due to a combination of all three effects: metal abrasion, airborne dust and ratio of sink leaves to source leaves. 


\subsubsection{Comparability of Shoot Tips and Processed Teas within a Garden}

To assess if there is a compositional shift between shoot tips and processed teas within a tea garden, 53 sample pairs of processed tea and the corresponding shoot tips have been calculated: For processed tea samples with known origin of sections or divisions of the tea garden, the average ionome of all shoot tip samples of the respective section was calculated. For processed tea samples of unknown section or division but known plucking period, the average ionome of all shoot tip samples of the respective plucking period of this garden was calculated. If for processed tea samples only the garden was known as origin, the average ionome of all shoot tip samples of the respective garden was calculated. For each sample pair of processed tea and corresponding average, ionome of shoot tips vratios and pairwise log-ratios were calculated. For calculation of the clr-vratios all elements were included (Table D.1).

Figure 7.15 displays in the left plot the results of PCA of clr-vratios with all elements (Table D.1), the REEs represented by La and Y. The biplot is dominated by the spread of clr-vratio values for $\mathrm{Cr}$. The projection of two sample pairs, one of Ord Terai and one of Matigara, close to the label of $\mathrm{Cr}$, suggests that for these two sample pairs the ratio for $\mathrm{Cr}$ of processed tea versus shoot tip is relatively higher than for most other samples and elements. Both tea estates produce mainly CTC black tea. This coincides very well with the results of the previous section that $\mathrm{Cr}$ is a typical contaminant through metal abrasion in CTC black tea production (Section 7.5.1). A third sample pair of green tea (matcha) vs. shoot tips from the tea garden Obubu, which also plots relatively close to $\mathrm{Cr}$, was slightly contaminated by $\mathrm{Cr}$ by an unknown source: For the sencha samples of Obubu $\mathrm{Cr}$ is found in a median concentration of $0.10 \mathrm{mg} / \mathrm{kg}$, the bancha sample shows a $\mathrm{Cr}$ concentration of $0.13 \mathrm{mg} / \mathrm{kg}$, and the matcha sample shows a $\mathrm{Cr}$ concentration of $0.52 \mathrm{mg} / \mathrm{kg}$. Sencha and bancha production in Japan generally excludes machinery which is prone to high metal abrasion. For the matcha sample the production step of pulverization may include machines which are the source of $\mathrm{Cr}$. Element concentrations of $\mathrm{Sc}, \mathrm{Pb}$ and $\mathrm{Sb}$, which are typically altered through tea production (Section 7.5.1), do not seem to be significantly altered in processed teas compared to shoot tips for the samples of this data subset.

To visualize differences in element ratios of processed tea samples compared to the corresponding shoot tips other than by contamination through the typical machine abrasion, the right plot of Figure 7.15 displays the results of PCA of clr-vratios of all elements without the elements $\mathrm{Sc}, \mathrm{Sb}, \mathrm{Pb}, \mathrm{Cr}$ and the two nutrients $\mathrm{S}$ and $\mathrm{P}$. These two nutrients had been omitted for better visibility of the other labels in the plot, because since they 

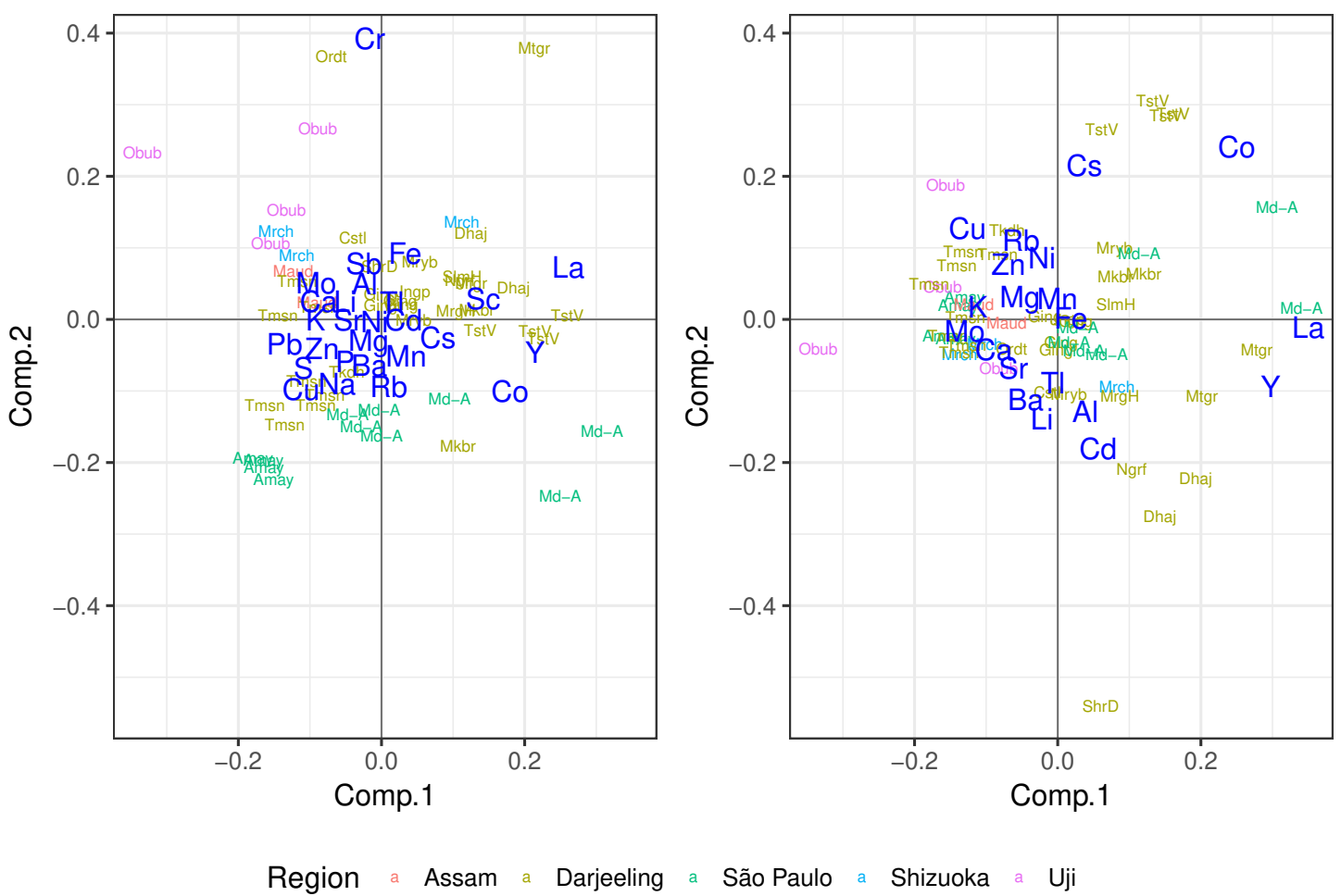

Figure 7.15.: Biplots of clr-vratios of processed teas to corresponding ionome averages of shoot tip $(\mathrm{N}=53)$. For calculation of the clr-vratios all elements are included (Table D.1). The left biplot displays all elements, REEs represented by La and $Y$. The right biplot excludes elements affected mainly by black tea manufacturing (Table D.1) and $S$ and $P$ for better visibility. Abbreviations for tea gardens: Amay: Amaya, Md-A: S. Miguel de Arcañjo, Maud: Maud, Obub: Obubu, Mrch: Moriuchi, Mkbr: Makaibari, Tmsn: Tumsong, TstV: Teesta Valley, Cstl: Castleton, Dhaj: Dhajea, Jngp: Jungpana, Mryb: Marybong, Ging: Ging, MrgH: Margeret 's Hope, Mtgr: Matigara, SImH: Selim Hill, Ordt: Ord Terai, Ngrf: Nagri Farm, ShrD: Shree Dwarika, Tkdh: Tukdah.

are macro-nutrients, their concentrations are not influenced by a production step after plucking. The biplot indicates that ratio of $\mathrm{Co}$ and the REE between processed tea and shoot tip have a larger variance than the other element vratios. This Co variance seems to be induced mainly by the samples of Teesta Valley. The source of a possible alteration of Co concentration between shoot tips and processed teas in Teesta Valley remains unknown. The REE variance seems to be influenced by several samples of Matigara and S. Miguel de Arcañjo. The source of the variance in REE could be incomplete removal of the signature of adhering particles (Section 6.2).

To show which element ratios systematically differ between the processed tea samples and shoot tip samples the distribution of pairwise log-ratios between sample pairs of the 


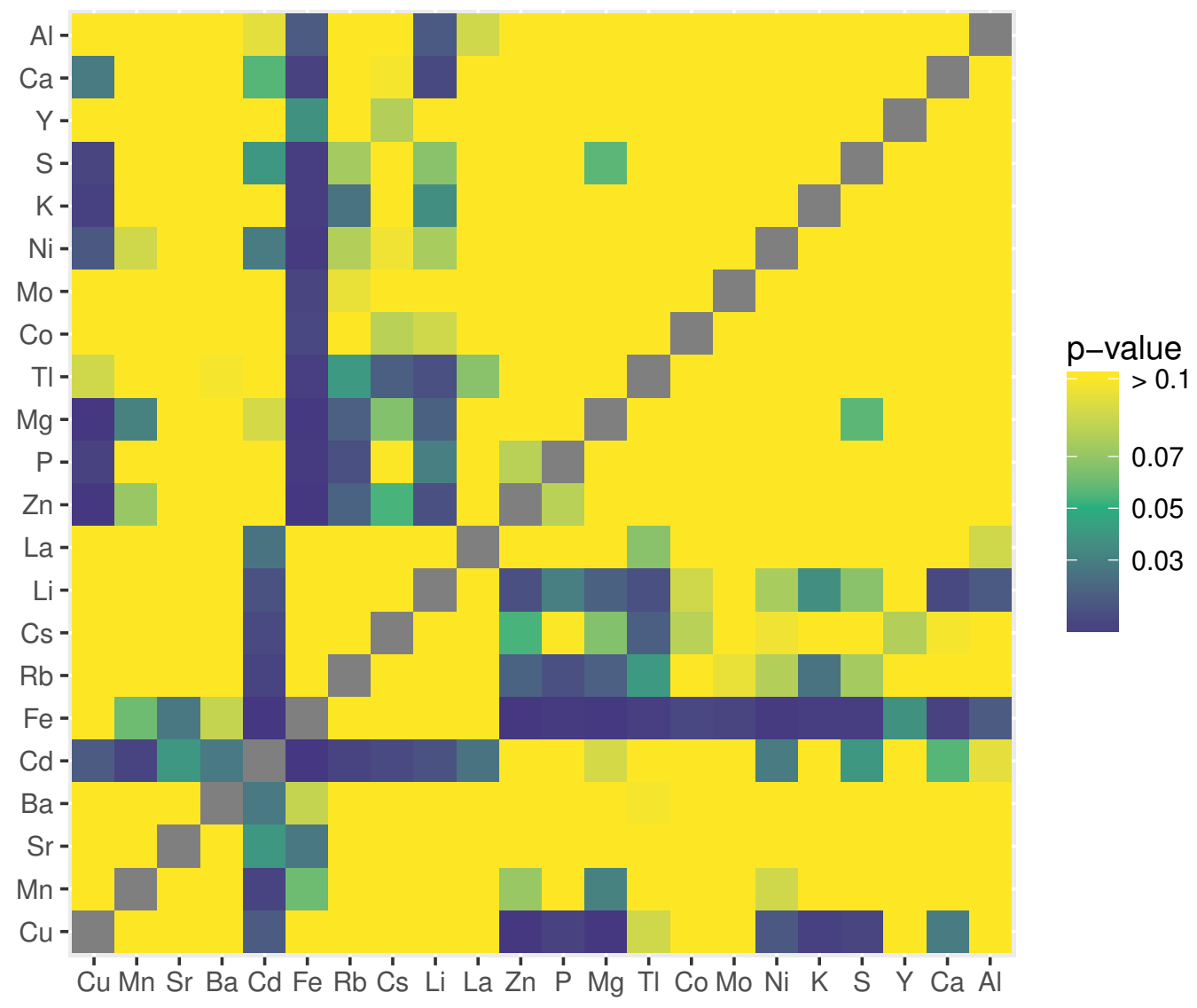

Figure 7.16.: Heatmap of $p$-values calculated by paired Wilcoxon signed rank test with continuity correction between distribution of pairwise log-ratio of shoot tip samples versus processed tea samples (sample pairs: $N=53$ ). P-values of log-ratios of element pairs are colored if they are below 0.05 (alternative hypothesis: true location shift is not equal to 0 ) and are colored greenish to yellow if values are above 0.05 (the means of two populations are equal).

two sample groups are compared by a paired Wilcoxon signed rank test (Figure 7.16. The $p$-values of this test reveal that only few element log-ratios have a location shift not equal to 0 , respectively differ between processed teas and shoot tips. These are several log-ratios with $\mathrm{Fe}$, Cu and La, followed by $\mathrm{Cd}, \mathrm{Cs}$, Li and Rb (sorted by average p-value). Of these elements, $\mathrm{Fe}$ and $\mathrm{Cu}$ display the biggest offset in their log-ratios between the two sample groups.

The vratio representation 'alr' with the reference element Fe (Section 5.2) puts the focus on differences of element ratios with Fe between shoot tip samples and the corresponding processed tea samples. The 'alr Fe' is visualized as biplot in the left plot of Figure 7.17: the plot reveals that especially processed tea samples of Selim Hill, 

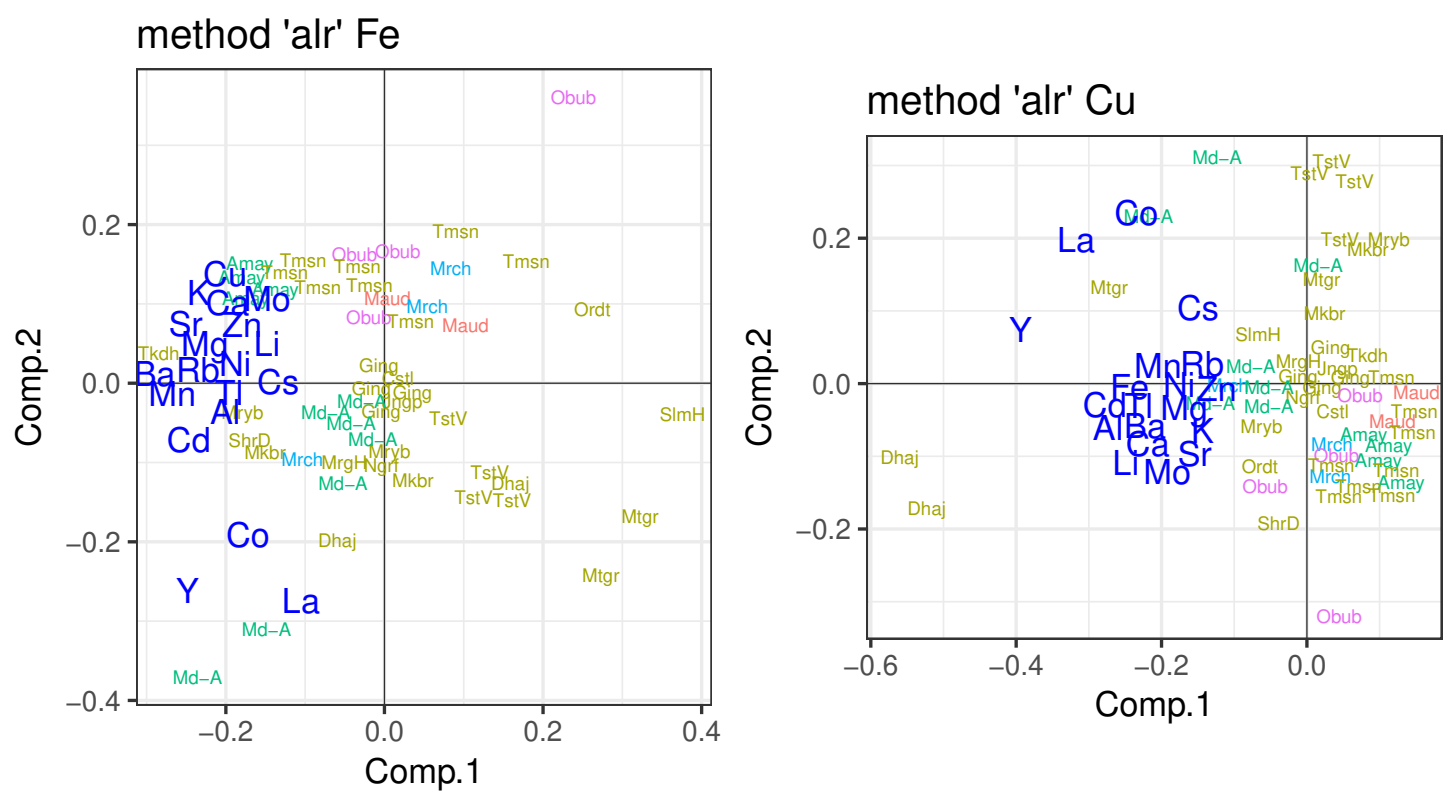

Region a Assam a Darjeeling a São Paulo a Shizuoka a Uji

Figure 7.17.: Biplots of alr-vratios of processed teas to corresponding ionome averages of shoot tip $(\mathrm{N}=53)$. The displayed alr-vratios excludes elements affected by tea manufacturing $\mathrm{Sb}$, $\mathrm{Pb}, \mathrm{Cr}, \mathrm{Sc}$ (Table D.1) and S and P. In the left biplot the reference element for the alr-vratios is $\mathrm{Fe}$, for the right plot the reference element is $\mathrm{Cu}$. Abbreviations for tea gardens are listed in Figure 7.15

Matigara, Teesta Valley and Ord Terai show higher Fe concentrations relative to their corresponding shoot tip samples compared to most other sample pairs. A higher Fe concentration in processed tea samples could be on the one hand, similarly to the REEs, caused by too weak correction for AP or, on the other hand, be caused by contamination through metal abrasion of machinery. Based on the findings of the previous biplots in Figure 7.15, the Fe signal is probably mainly caused by incomplete mathematical removal of APs.

The left biplot in Figure 7.17 displays the PCA calculated on basis of the vratio representation 'alr' with the reference element $\mathrm{Cu}$. The projection of the sample dots onto the PC1/PC2 plane suggests that processed teas of the tea gardens Tumsong, Maud, Tukdah, Marybong and some of Amaya $\mathrm{Cu}$ is found in slightly higher concentrations than shoot tips of the respective gardens. In contrast, processed tea samples from Dhajea seem to be considerably less concentrated in $\mathrm{Cu}$ in ratio to shoot tips compared to all other gardens. A possible source of $\mathrm{Cu}$ could be rolling tables during the step of disruption/rolling (Section 2.3.3). The factories of Tumsong, Tukdah and Marybong both 
have mainly old $(>40 \mathrm{y}$ ) gun metal rolling tables and only few steel rollers. For Maud and Amaya the types of rolling table is unknown, but the tea makers of Amaya eventually add traces of $\mathrm{Cu}$ to their processed tea products. The tea factory of Dhajea has rolling tables made of modern brass metal alloys, installed in 2001. The results indicate that teas rolled on rolling tables made of old brass metal alloys may be slightly contaminated by $\mathrm{Cu}$, while teas rolled on rolling tables of modern brass alloys, or recently installed rolling tables, respectively, or rolled on rolling tables made of steel alloys, have no or only a minor contamination of $\mathrm{Cu}$.

These results do only partly confirm previous works, where for $\mathrm{Al}, \mathrm{Cr}, \mathrm{Cu}, \mathrm{Pb}$ and Zn elevated levels had been reported for processed tea (Wong et al. 1998; Fung 2003; Ashraf and Mian 2008; Jin et al. 2008; Seenivasan et al. 2008; Salahinejad and Aflaki 2010, Section 2.1.3. In the data set of this thesis no indication for elevated levels of Al and $\mathrm{Zn}$ in processed teas could be statistically ascertained and $\mathrm{Cu}$ concentrations are elevated only in very specific samples. Elevated levels of Al could be an effect of the sink/source leaf ratio in the two groups of samples: If the processed tea samples had on average higher contents of mature leaves than the shoot tip samples with which they were compared, i.e. if the processed teas had a smaller sink/source leaf ratio, then Al concentrations could be slightly higher than in shoot tip samples (Section 7.4.1). Elevated levels of $Z n$ could only be explained by a strong contamination of unclear source, because $\mathrm{Zn}$, being a micro-nutrient, is already rather high concentrated in the leaves (Section 7.1, Table 7.1). The results of a statistical approach for all shoot tip samples and black processed tea samples do not confirm on a general basis machine abrasion as possible source of contamination for the elements $\mathrm{Al}, \mathrm{Cu}$ and $\mathrm{Zn}$. Hence, these elements are not included into the element subcomposition that was possibly altered during tea manufacturing (Table D.1.

\subsection{Summary}

The measured concentrations in shoot tip, mature leaf and processed tea samples generally agree well with previously published data. For all macro-nutrients and most micronutrients the values of shoot tips and processed teas are similar. The smallest spread of data is found for the nutrients and Al.

Mature leaves of Darjeeling show lower concentrations for the macro-nutrients $\mathrm{K}$ and $P$ than the other three regions. This probably reflects a lower availability of these two macro-nutrients compared to the other three regions due to the organic soil management 
in Darjeeling. Moreover, for mature leaf samples there are clearly visible differences in concentration of nutrients and their respective replacement elements between regions. These differences are not visible for shoot tip samples, suggesting that mature leaves 'buffer' the element flux from soils into the shoot tips. This process seems to favor a constant ratio of the nutrients $\mathrm{K}, \mathrm{P}, \mathrm{S}$ and $\mathrm{Mg}$ to $\mathrm{C}, \mathrm{H}, \mathrm{N}$ and $\mathrm{O}$ for sink tissue. For provenance analytics the possible 'buffering' by mature leaves implies that a high percentage of sink tissue in the analyzed plant material reduces the geochemical signal derived by element uptake from the soils.

The comparison between intra-location variability versus inter-location variability of element concentrations shows that in Darjeeling the element subcompositions containing $\mathrm{TI}, \mathrm{Cs}, \mathrm{Pb}, \mathrm{Sb}, \mathrm{REE}, \mathrm{Na}, \mathrm{Co}, \mathrm{Ca}, \mathrm{Ni}, \mathrm{K}$ and $\mathrm{Mn}$ can serve as proxies for provenance analysis. On the contrary, the elements $\mathrm{Cd}, \mathrm{Al}, \mathrm{Sr}, \mathrm{Sc}, \mathrm{S}, \mathrm{Mg}, \mathrm{Cu}, \mathrm{Zn}, \mathrm{Fe}$ and $\mathrm{P}$ have probably less impact for discriminating between regions in Darjeeling.

The distribution of elements between source and sink leaves is as follows:

- The distribution of elements is approximately constant for the nutrients, e.g. Ca, $\mathrm{Mn}, \mathrm{Cu}, \mathrm{K}, \mathrm{S}, \mathrm{P}$, and $\mathrm{Al}, \mathrm{Rb}, \mathrm{Cs}, \mathrm{Sb}$ and Sr.

- The distribution of elements varies for trace elements, e.g. Cr, Sc, Ni, Co, $\mathrm{Pb}, \mathrm{Na}$, REE, Li, etc.

- The type of cultivar does not seem to have an influence on the distribution patterns.

- $\mathrm{Rb}$ and $\mathrm{Cs}$ are stronger enriched in sink leaves compared to source leaves than $\mathrm{K}$.

The variations of distribution patterns of trace elements could be an effect of the 'buffering' of elements in mature leaves (Section 7.2): This inhomogeneous distribution of trace elements might veil the geographical fingerprint, especially in the sink leaf tissue. Elements with a less variable distribution pattern can serve as proxy for the average age of the leaf tissue.

- Log-ratios with $\mathrm{Al}, \mathrm{Ba}, \mathrm{Sc}$ and the REE are very sensitive to the developmental stage of the leaf.

- Log-ratios of elements of the subcomposition Co, Cs, Cu, K, Ni, P, Rb and Zn vs. elements of the subcomposition $\mathrm{Ca}, \mathrm{Li}, \mathrm{Mn}, \mathrm{Mo}, \mathrm{Pb}, \mathrm{Sb}, \mathrm{Sr}$ and $\mathrm{Tl}$ are sensitive to the developmental stage of the leaf.

- Log-ratios with $\mathrm{Cd}, \mathrm{Cr}, \mathrm{Fe}, \mathrm{Na}, \mathrm{Mg}$ and S are slightly sensitive to the developmental stage of the leaf. 
Hence, if shoot tip samples are grouped together with processed tea samples and/or mature leaf samples, the used element subcomposition should always exclude the elements $\mathrm{Al}, \mathrm{Ba}, \mathrm{Sc}$ and the REE.

A balanced ratio of elements which are very sensitive to the developmental stage of the leaf and have a less variable sink/source leaf distribution can help to estimate the average leaf age of a tea sample. In this thesis the balanced log-ratio contrast between $\mathrm{K}$ and $\mathrm{Cu}$ to $\mathrm{Al}, \mathrm{Ca}$ and $\mathrm{Mn}$ is used, noted as [Cu K|Al Ca Mn]. Mature leaves have an average balanced ratio of -2.7 and shoot tips an averaged balanced ratio of -0.5 . This trend is also visible for orthodox black teas from Darjeeling, e.g. the highest leaf qualities have the highest balanced ratio with a median value $(N=12)$ of -0.25 . This clearly reflects a high percentage of immature leaves and buds, i.e. a high sink/source leaf ratio, in processed teas of high quality.

The fine grained leaf grades of processed tea samples have also notably high $\mathrm{Cu}$ and $\mathrm{K}$ in relation to $\mathrm{Al}, \mathrm{Ca}$ and $\mathrm{Mn}$. There are two possible reasons: I) The abundant trichomes of the leaves, which can make up a significant portion in dust and fanning grades of high quality teas. II) Young leaves and buds have a more delicate tissue which breaks easier under the mechanical stress of production and therefore 'young' tissue is enriched in the finer grained leaf grades. In summary, not only the plucking standard but also the leaf grade influences the [Cu K $\mid \mathrm{Al} \mathrm{Ca} \mathrm{Mn]} \mathrm{ratio.}$

The values of this balanced ratio can be used to filter samples in order to make sample groups more homogeneous. This is important I) for statistical analysis for known sink/source leaf ratio, II) to compose the element subcomposition used for analysis, e.g. if elements which are sensitive to the leaf development stage should be included or not and III) estimating the plucking standard as proxy for provenance analysis for processed tea samples. For the latter the influence of the leaf grade on the [ $\mathrm{Cu} \mathrm{K} \mid \mathrm{Al} \mathrm{Ca} \mathrm{Mn]}$ values has to be taken into account.

Processed tea samples, especially black teas, are prone to contamination of Fe and heavy metals. The source of $\mathrm{Pb}$ and $\mathrm{Sb}$ is probably lead containing solders. One source of $\mathrm{Cr}$ is likely to be the metal abrasion of the machines. The source of $\mathrm{Sc}$ is probably a combination of metal abrasion of Al-Sc-alloys, airborne dust, accumulation in the field or accumulation during the withering process. The same possible sources apply for Fe. Additionally, different plucking standards could cause the large spread of Fe concentration in processed tea samples by varying sink/source leaf ratios of the leaf material. 


\section{Influence of Soil on the Tea Ionome}

Element uptake by plants is since decades a major research topic. The nutrient uptake by $C$. sinensis had been intensely studied, too, in order to optimize tea cultivation for commercial purposes. But for provenance analysis it is less important why or how elements are transferred into the plant, but rather which element ratios remain constant during the uptake. Aggravatingly, the geochemical composition of tea soils and the bioavailability of elements is subject to seasonal and yearly changes due to measures of soil management. Accordingly, there are a) element ratios influenced by the soil management and b) element ratios which are independent of soil management. The aim of this chapter is to distinguish element subcompositions which are suitable for provenance analysis because they reflect the geochemical composition of the soils and are stable in respect to measures of soil management. The second conditions includes that certain soil management is often subject to the soil type of specific tea growing regions: Eventually, subcompositions of elements influenced by soil management can also serve as a proxy for tea growing regions.

\subsection{Soil}

Tea soils, and especially the rhizosphere of tea plants, is a very complex and still poorly understood environment. To use the soil data for refining provenance analytics a radical simplification is applied on the soils, deliberately excluding soil parameters like type of soil, $\mathrm{pH}$, etc. The tea soils are characterized solely as an element reservoir for the tea plants and the description therefore focuses only on the geochemical composition. Because the $\mathrm{pH}$ of the tea soils is between 3.9-4.5 (Section 2.2, Pospiech 2012) it can be assumed that a high percentage of the total element concentration in soils is potentially available to the tea plant.

As described in Section 4.1.1, topsoil samples are a melange of soil of the upper $15-25 \mathrm{~cm}$ and subsoil samples are a melange from below topsoil down to $30-45 \mathrm{~cm}$. Several element concentrations can be subject of strong fluctuation due to longterm, 
recent or changing agricultural measures, such as fertilizer applications, remnants of fungicides, application of carbonate/dolomite, plowing in tea litter, etc. (Section 2.2). The geochemistry of topsoils is supposedly stronger affected by these measures than the geochemistry of subsoils. Hence, subsoils have supposedly less variability between years and represent better the geochemical composition of the total soil volume underneath the plant. The contrast between topsoil and subsoil gives an indication of which elements are affected by agricultural measures. These elements are less sound for provenance analysis on tea. In the following sections the subsoil composition is taken as reference.

\subsubsection{Geochemical Composition of Subsoils}

Figure 8.1 and Table 8.2 give the geochemical composition of all subsoils. The elements $\mathrm{Al}, \mathrm{Fe}, \mathrm{P}$ and $\mathrm{Sc}$ are found in approximately the same concentration in all subsoil samples, independent of the region. The elements $\mathrm{Zn}, \mathrm{Cr}, \mathrm{Pb}$ and $\mathrm{Cd}$ show minor variations between the regions. All remaining elements are differently concentrated between the regions, whereof the biggest contrast exists between India and Brazil. The biggest contrast between regions in terms of concentration have $\mathrm{S}, \mathrm{Cs}, \mathrm{Ca}, \mathrm{Na}, \mathrm{Sr}, \mathrm{Rb}$, and $\mathrm{K}$. Except for $\mathrm{S}$, for these elements Brazilian soils are found in lower concentrations than the Indian soils.

Table 8.1 displays the sum of the concentration of nutrient elements. The element Fe is excluded from this sum because it is an abundant element in tropical soils but $C$. sinensis requires it in minor quantities. The Brazilian soils show lower concentration of nutrients than the soils from Darjeeling and Assam in India. Lowest concentration of nutrients is found in Registro, Brazil, with only $0.2 \mathrm{wt} \%$, followed by the soils in São Miguel de Arcanjo with $0.4 \mathrm{wt} \%$ and $0.6 \mathrm{wt} \%$ and Araucária with in average $0.7 \mathrm{wt} \%$. In Darjeeling and Assam the average concentration of nutrients is approximately three to four times as high. The highest average of nutrient concentration is found in the sampled gardens close to the city Dibrugarh in Assam with $4.3 \mathrm{wt} \%$. The total organic carbon had not been determined, but is in tea soils approximately between $5 \%-15 \%$ (Wang et al. 2010; Karak et al. 2011, 2015b; Li et al. 2016).

Compared to the upper continental crust (Rudnick and Gao 2003) (Figure D.2) the soils are only in S significantly enriched. They are all depleted in the macro-nutrients $\mathrm{Ca}, \mathrm{Mg}$ and $\mathrm{K}$ as well as in the elements $\mathrm{Ba}, \mathrm{Cd}, \mathrm{Na}, \mathrm{Sr}$ and $\mathrm{TI}$. Except for Darjeeling the soils are also depleted in the macro-nutrient $\mathrm{P}$. Other elements which are depleted in the soils of Brazil, but not in all soils of India are Cs, Cr, Mn, P, Co and Sc. 
Table 8.1.: Sum of nutrient concentrations $\mathrm{Ca}, \mathrm{Cu}, \mathrm{K}, \mathrm{Mg}, \mathrm{Mn}, \mathrm{Mo}, \mathrm{Ni}, \mathrm{P}, \mathrm{S}$ and $\mathrm{Zn}$ (without Fe) in subsoils by sampling location. Sums are given in \%wt $(10 \mathrm{~g} / \mathrm{kg})$.

\begin{tabular}{llllr}
\hline Country & Region & Location & Division & $\sum$ nutrients \\
\hline \multirow{3}{*}{ Brazil } & Paraná & Araucária & & 0.67 \\
\cline { 2 - 4 } & \multirow{3}{*}{ São Paulo } & Registro & \\
& & São Miguel Arcanjo & Filia & 0.20 \\
& & São Miguel Arcanjo & Matriz & 0.56 \\
& \multirow{2}{*}{ Assam } & Dibrugarh & 0.36 \\
\cline { 3 - 4 } & & Tinsukia & 4.31 \\
& & Darjeeling City & 3.02 \\
\hline \multirow{4}{*}{ India } & Darjeeling East & 2.95 \\
& & Darjeeling North & 3.11 \\
& & Darjeeling South & 3.41 \\
& \multirow{2}{*}{ Darjeeling } & 3.03 \\
& & Darjeeling West & 3.05 \\
& & Kurseong & 3.59 \\
& & Kurseong East & 3.44 \\
& & Mane Bhanjang & 2.37 \\
& & Mirik & 2.96 \\
& Siliguri & 2.92 \\
& & Teesta Valley & 3.43 \\
& & Tung & 2.96 \\
\hline
\end{tabular}

The soils sampled in the region São Paulo are all deeply weathered latosols (Section 3.2.1). Therefore they show small concentrations of nutrients and show the a strong depletion compared to upper crust. The tea fields in Registro are lowest in nutrients because the fields are located on sandy to silty alluvial deposits (Section 3.2.1). This type of source rock material is generally poor in nutrients, especially if the sand and silt fraction consists mainly of quartzitic grains. The other tea fields in Brazil are slightly less depleted than Registro, probably because the source rocks consisted of minerals with higher contents of nutrients: In São Miguel de Arcanjo the tea fields of the garden's division 'Matriz' are situated on source rock of K-feldspar-rich monzogranite. The tea fields of the division 'Filia' are situated in source rock of Permo-Carboniferous sediments of the Paraná-Basin. The soils of Araucária are derived from migmatic gneisses with biotite-amphibole mesosomes and tonalitic-granodioritic leucosomes, mafic rocks, like amphibolites, and biotite rich, magnesian schists (Section 3.2.2, Sato et al. (2003)). Accordingly, these soils show for several elements greater concentrations in comparison to the other Brazilian soils: For example the large ion lithophile (LIL) elements like K, Rb 
and $\mathrm{Ba}$, typical for TTG suites (Condie 2005), Ni and Co due to the amphibolite lenses and $\mathrm{Mg}$ due to the magnesian rich schists. Nevertheless, the nutrient status in the Brazilian is very low and tea soils require intense application of fertilizer and/or compost for commercial tea cultivation (Section 3.2.1.

All subsoils of Darjeeling have approximately similar total content in nutrients. Only for $\mathrm{Ca}$ and $\mathrm{Mo}$ there is a notably higher spread in the concentrations (Figure 8.1). Although the weathering rate of the source rocks is high due to the subtropical climate conditions, especially in the lower elevations of Darjeeling, the steep slopes with high erosion rates lead to shallow soils with relatively fresh material. This is reflected by less depletion of nutrients compared to the upper crust than the Brazilian soils and accordingly, a much higher total content in nutrients. All soils in Darjeeling are regoliths from the gneisses of the MCTZ and greenschists from the LHS (Section 3.1.1).

The subsoils of the alluvial plain in India, the sampling locations in Assam and Siliguri in Darjeeling, display for most elements a similar geochemical signature than Darjeeling. Only the $\mathrm{Ca}$ and $\mathrm{Sr}$ concentration are notably greater while the $\mathrm{Al}$ and $\mathrm{Cs}$ concentrations are notably smaller (Figure 8.1. This shows that the soils in Assam, being fluviatile sediments originating from the East-Himalaya, do not significantly weather during their transport from the mountainous parts into the plains of the Brahmaputra river. The higher concentration of $\mathrm{Ca}$ could either be an affect of slightly difference in the geological setting of the origin of the sediments of an affect of applying carbonate/dolomite in the sampled gardens.

\subsubsection{Comparison between Topsoils and Subsoils}

Topsoils and corresponding subsoils have for most elements approximately the same geochemical composition. To identify elements which are influenced by soil management the upper soil volume will be compared with the less influenced, lower soil volume. Figure 8.2 displays the difference between the element concentrations in topsoils and subsoils, subdivided by regions in India and locations in Brazil.

In Assam and Darjeeling there are only very few elements notably different concentrated in top- and subsoils: $\mathrm{Ca}, \mathrm{P}$ and $\mathrm{S}$ are systematically higher concentrated in Indian topsoils than in the subsoils. The heavy metals $\mathrm{Cd}, \mathrm{Cr}$ and $\mathrm{Pb}$ show a wide spread of distribution between topsoil and subsoils. The greater concentration of $\mathrm{Ca}$ in topsoils is probably caused by the (irragular) application of carbonate containing rock powder for mitigating the soil $\mathrm{pH}$. The difference of $\mathrm{P}$ and $\mathrm{S}$ is an effect of the regular application of manure. The elevated $S$ concentration in topsoils could be a result of using compost, 
which was enriched with animal feces, e.g. cow dung and chicken feces, or sulphur containing products (personal communication from tea garden managers). Remarkably, other nutrients like $\mathrm{K}, \mathrm{Mg}, \mathrm{Mn}$ or $\mathrm{Zn}$ similarly concentrated in the whole soil volume. The greater $\mathrm{Cu}$ concentration in the topsoils of same gardens is most probably remnants of copper containing fungicides, which are regularly used in organic farming. The reason for the spread of distribution of heavy metals remains unclear.

In Araucária, Paraná, and São Paulo there is for more elements a significant difference between top- and subsoils: The nutrients $\mathrm{Ca}$ and $\mathrm{P}$ are strongly enriched in the topsoils. Additionally, the elements $\mathrm{S}, \mathrm{Na}, \mathrm{Ba}, \mathrm{Zn}, \mathrm{La}, \mathrm{Sr}, \mathrm{Cu}$ and in Paraná also Y and Cd are found at higher concentrations in topsoils. Partly elevated are $\mathrm{K}, \mathrm{Mg}, \mathrm{Mn}, \mathrm{Cr}$ and $\mathrm{Cd}$. The greater concentrations of $\mathrm{K}, \mathrm{P}, \mathrm{Mg}, \mathrm{Mn}$ and $\mathrm{Zn}$ in topsoils results from the regular application of fertilizers according to USDA standards. $\mathrm{Ca}, \mathrm{Sr}$ and $\mathrm{Ba}$ are probably enriched due to th irregular application of carbonate containing rock powder, similar to Darjeeling and Assam. No reasons could be determined for the differences in distribution between topsoil and subsoil for the named heavy metals and $\mathrm{Y}$.

The differences between topsoils and corresponding subsoils give a qualitative approach about which elements are affected by soil management. These are in strongly weathered soils, e.g. the Brazilian soils, especially $\mathrm{P}, \mathrm{K}$ and $\mathrm{Ca}$, and with less affected $\mathrm{S}$, $\mathrm{Mg}, \mathrm{Na}, \mathrm{Mn}, \mathrm{Ba}, \mathrm{Sr}$ and $\mathrm{Cu}$. For less intensely weathered soils, respectively organically treated soils, e.g. the soils of Darjeeling and few in Assam, this affects mainly $P$ and $S$, and less $\mathrm{K}, \mathrm{Ca}$ and $\mathrm{Cu}$. The varying distribution of heavy metals in the soil volume have to be taken in consideration for provenance analysis.

The impact of fertilizer and manure application can not be quantified in this study, because the soils had been sampled at onlyone point of time. Hence, the enrichment factors between topsoil and subsoil in Figure 8.2 must not be used for quantification. 


\section{Influence of Soil on the Tea lonome}

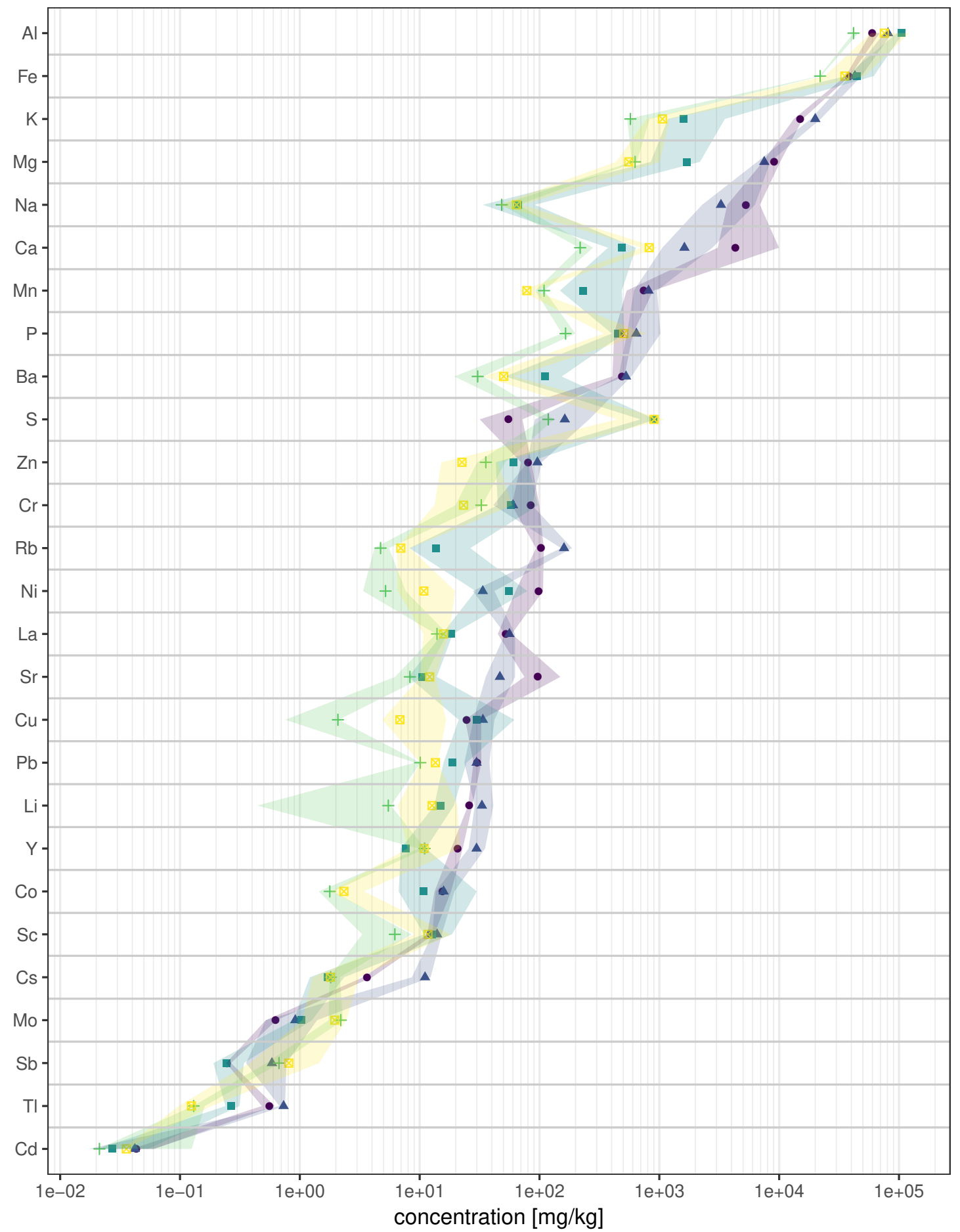

Region - Assam $\Delta$ Darjeeling $\|$ Pr Loc: Araucaria + SP Loc: Registro \SP Loc: São Miguel Arcanjo

Figure 8.1.: Element concentrations of subsoils, grouped by tea growing regions and locations in Brazil. Elements are ordered by median of concentrations of all subsoil samples. Median for each sample group is marked by a colored symbol, the shaded areas mark the second and third quartile. Median values are listed in Table 8.2. 


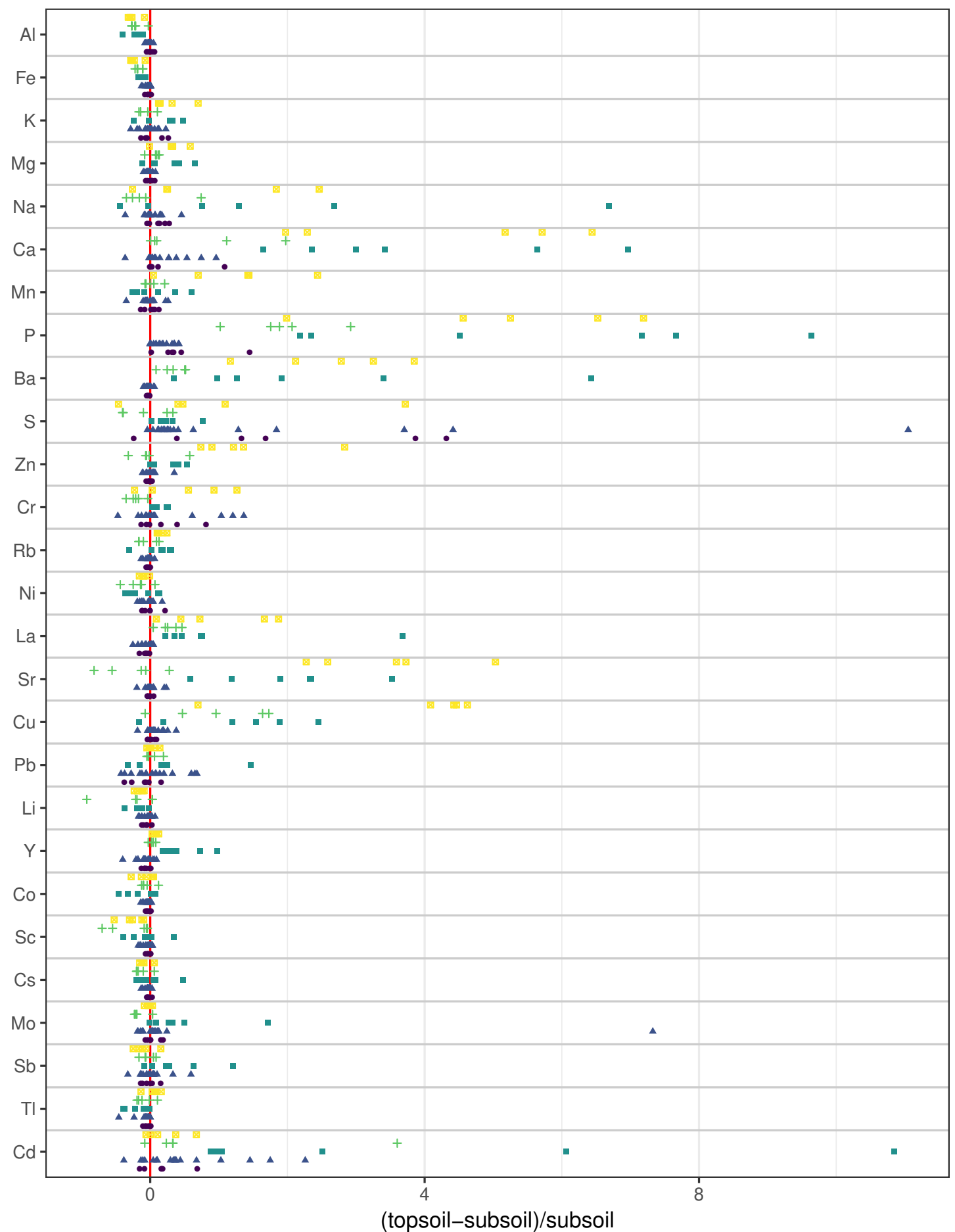

Region - Assam \ Darjeeling - Pr Loc: Araucaria + SP Loc: Registro \SP Loc: São Miguel Arcanjo

Figure 8.2.: Comparison of element concentrations in topsoil versus subsoil, grouped by tea growing regions. Each point represents a pair of topsoil and corresponding subsoil sample. If values are above zero the element is higher concentrated in the topsoil, and if the the values are below zero the element is higher concentrated in the subsoil. Elements are ordered by median element concentration of the subsoils. 
Table 8.2.: Topsoil and subsoil concentrations, compilation of data of this thesis and data from H. P. Carr et al. (2003). Elements are ordered by median of subsoil concentrations of this thesis. RMD denotes the median absolute deviation normalized by the median and RSD the relative standard deviation. The compilation of published data is displayed in the columns left of the marker 'Publ.'. Nutrients are marked with an asterix, beneficial elements with a dagger.

\begin{tabular}{|c|c|c|c|c|c|c|c|c|c|c|c|c|c|c|}
\hline & Top: & Median & MAD & RMD \% & Mean & SD & RSD \% & Sub: & Median & MAD & RMD \% & Mean & SD & RSD \% \\
\hline $\mathrm{Al}^{\dagger}$ & & $7.4 e+04$ & $1.5 e+04$ & 21 & $6.9 e+04$ & $2.1 e+04$ & 31 & & $8.0 e+04$ & $1.1 e+04$ & 14 & $8.0 e+04$ & $1.8 e+04$ & 22 \\
\hline $\mathrm{Fe}^{*}$ & & $3.8 e+04$ & $8.1 e+03$ & 21 & $3.7 e+04$ & $1.6 e+04$ & 42 & & $4.2 e+04$ & $8.5 e+03$ & 20 & $4.3 e+04$ & $1.3 e+04$ & 31 \\
\hline $\mathrm{K}^{*}$ & & $1.5 e+04$ & $9.8 e+03$ & 65 & $1.1 e+04$ & $8.7 e+03$ & 76 & & $1.9 e+04$ & $4.9 e+03$ & 26 & $1.6 e+04$ & $8.0 e+03$ & 49 \\
\hline Mg* & & $6.1 e+03$ & $4.0 e+03$ & 66 & $5.3 e+03$ & $3.6 e+03$ & 68 & & $7.0 \mathrm{e}+03$ & $2.0 e+03$ & 28 & $6.8 e+03$ & $4.1 e+03$ & 60 \\
\hline $\mathrm{Na}^{\dagger}$ & & $2.4 \mathrm{e}+03$ & $3.5 e+03$ & 140 & $2.9 e+03$ & $3.3 e+03$ & 110 & & $2.7 e+03$ & $3.7 e+03$ & 140 & $3.5 e+03$ & $3.1 e+03$ & 87 \\
\hline $\mathrm{Ca}^{*}$ & & $2.0 \mathrm{e}+03$ & $2.0 \mathrm{e}+03$ & 97 & $3.2 e+03$ & $3.2 e+03$ & 100 & & $1.4 \mathrm{e}+03$ & $1.1 e+03$ & 78 & $2.1 e+03$ & $2.5 e+03$ & 120 \\
\hline$M n^{*}$ & & $5.3 e+02$ & $4.6 e+02$ & 85 & $5.7 e+02$ & $3.9 e+02$ & 69 & & $7.0 e+02$ & $3.4 e+02$ & 49 & $7.1 e+02$ & $4.1 e+02$ & 57 \\
\hline $\mathrm{P}^{*}$ & & $8.0 e+02$ & $3.5 e+02$ & 44 & $1.3 e+03$ & $1.0 e+03$ & 79 & & $6.0 \mathrm{e}+02$ & $2.5 e+02$ & 42 & $7.3 e+02$ & $4.3 e+02$ & 60 \\
\hline $\mathrm{Ba}$ & & $3.6 e+02$ & $2.8 e+02$ & 80 & $3.6 e+02$ & $2.1 e+02$ & 57 & & $4.9 e+02$ & $1.5 e+02$ & 30 & $4.3 e+02$ & $2.1 e+02$ & 49 \\
\hline $\mathrm{S}^{*}$ & & $2.7 e+02$ & $2.6 e+02$ & 93 & $3.9 e+02$ & $3.7 e+02$ & 94 & & $1.7 e+02$ & $1.4 e+02$ & 85 & $2.5 e+02$ & $2.7 e+02$ & 110 \\
\hline $\mathrm{Rb}$ & & $1.0 e+02$ & $9.2 \mathrm{e}+01$ & 89 & $8.8 e+01$ & $6.9 e+01$ & 78 & & $1.5 e+02$ & $5.2 e+01$ & 34 & $1.3 e+02$ & $6.7 e+01$ & 51 \\
\hline $\mathrm{Zn}^{*}$ & & $7.9 e+01$ & $3.0 e+01$ & 38 & $7.6 e+01$ & $2.9 e+01$ & 38 & & $8.7 e+01$ & $2.6 e+01$ & 29 & $8.4 e+01$ & $3.5 e+01$ & 42 \\
\hline $\mathrm{Cr}$ & & $4.8 e+01$ & $3.4 \mathrm{e}+01$ & 71 & $7.1 e+01$ & $9.2 e+01$ & 130 & & $5.6 e+01$ & $3.6 e+01$ & 64 & $6.7 e+01$ & $5.2 e+01$ & 79 \\
\hline $\mathrm{La}^{\dagger}$ & & $5.0 e+01$ & $1.4 \mathrm{e}+01$ & 28 & $4.2 \mathrm{e}+01$ & $1.6 e+01$ & 38 & & $5.4 e+01$ & $9.6 e+00$ & 18 & $5.0 e+01$ & $1.8 e+01$ & 37 \\
\hline $\mathrm{Sr}$ & & $4.6 e+01$ & $2.9 e+01$ & 62 & $5.6 e+01$ & $4.4 \mathrm{e}+01$ & 79 & & $4.4 \mathrm{e}+01$ & $2.8 e+01$ & 64 & $4.9 e+01$ & $3.6 e+01$ & 72 \\
\hline $\mathrm{Ni}^{*}$ & & $3.1 e+01$ & $2.5 e+01$ & 80 & $4.4 \mathrm{e}+01$ & $5.3 e+01$ & 120 & & $3.3 e+01$ & $1.3 e+01$ & 39 & $4.1 e+01$ & $4.7 e+01$ & 110 \\
\hline $\mathrm{Cu}^{*}$ & & $3.0 e+01$ & $8.8 e+00$ & 29 & $3.7 e+01$ & $3.2 e+01$ & 86 & & $3.1 e+01$ & $1.2 e+01$ & 39 & $3.5 e+01$ & $3.0 e+01$ & 87 \\
\hline $\mathrm{Li}$ & & $2.5 e+01$ & $2.1 e+01$ & 82 & $2.5 e+01$ & $1.6 e+01$ & 63 & & $3.1 e+01$ & $1.5 e+01$ & 48 & $2.9 e+01$ & $1.3 e+01$ & 46 \\
\hline $\mathrm{Pb}$ & & $2.2 e+01$ & $8.6 e+00$ & 39 & $2.5 e+01$ & $2.0 e+01$ & 80 & & $2.8 e+01$ & $1.1 e+01$ & 40 & $3.3 e+01$ & $3.4 \mathrm{e}+01$ & 110 \\
\hline $\mathrm{Y}^{\dagger}$ & & $2.1 e+01$ & $1.2 \mathrm{e}+01$ & 57 & $2.0 e+01$ & $8.7 e+00$ & 44 & & $2.7 e+01$ & $8.0 e+00$ & 30 & $2.7 e+01$ & $1.2 \mathrm{e}+01$ & 43 \\
\hline $\mathrm{Co}^{\dagger}$ & & $1.3 e+01$ & $4.3 e+00$ & 33 & $1.3 e+01$ & $1.4 e+01$ & 110 & & $1.5 e+01$ & $5.1 e+00$ & 33 & $1.6 e+01$ & $1.2 e+01$ & 78 \\
\hline Sc & & $1.2 \mathrm{e}+01$ & $2.9 e+00$ & 23 & $1.2 \mathrm{e}+01$ & $5.8 e+00$ & 48 & & $1.4 \mathrm{e}+01$ & $2.6 e+00$ & 19 & $1.4 \mathrm{e}+01$ & $5.8 e+00$ & 41 \\
\hline Cs & & $4.2 e+00$ & $4.3 e+00$ & 100 & $6.2 e+00$ & $5.0 \mathrm{e}+00$ & 80 & & $9.3 e+00$ & $4.7 e+00$ & 50 & $8.6 e+00$ & $4.7 e+00$ & 55 \\
\hline Mo* & & $1.0 e+00$ & $5.8 e-01$ & 56 & $1.3 e+00$ & $1.3 e+00$ & 96 & & $9.4 e-01$ & $6.2 e-01$ & 67 & $1.1 e+00$ & $6.5 e-01$ & 61 \\
\hline $\mathrm{TI}$ & & $5.6 e-01$ & $2.3 e-01$ & 42 & $4.5 e-01$ & $2.4 \mathrm{e}-01$ & 53 & & $6.9 \mathrm{e}-01$ & $8.8 e-02$ & 13 & $6.1 \mathrm{e}-01$ & $2.2 \mathrm{e}-01$ & 36 \\
\hline $\mathrm{Sb}$ & & $4.2 e-01$ & $2.1 e-01$ & 51 & $4.5 e-01$ & $2.4 \mathrm{e}-01$ & 53 & & $5.3 e-01$ & $3.2 \mathrm{e}-01$ & 61 & $5.9 e-01$ & $3.6 e-01$ & 61 \\
\hline $\mathrm{Cd}$ & & $5.7 e-02$ & 2.1e-02 & 36 & $6.8 \mathrm{e}-02$ & $3.8 e-02$ & 56 & & $3.7 e-02$ & $2.9 e-02$ & 79 & $4.8 \mathrm{e}-02$ & $4.5 e-02$ & 93 \\
\hline
\end{tabular}




\subsection{Relation between Soil Geochemistry and Shoot Tip Ionome}

The relation between ionome of plants and geochemical composition of soils is mostly used to describe the element transfer. This relation is also named 'transfer-factor', 'soilplant partition coefficient', 'bio-concentration factor', 'concentration ratio', 'enrichment factor' or 'biological accumulation coefficient' (Hope 1995; Roca and Vallejo 1995; Dinelli and Lombini 1996; Rodríguez et al. 2002; Tomé et al. 2003; Chojnacka et al. 2005; Reimann et al. 2007; Goni et al. 2014). In this section the relation of plant to soil is used to I) characterize element subcompositions with similar transfer-factors and II) to identify subcompositions which log-ratios are mostly unaltered by the element transfer from the reservoir (soil) through the plant into the sink tissue.

Figure 8.3 displays the overview of simple vratios between shoot tips and corresponding subsoils. This is the most frequent type of representation for the relation between geochemical composition of soils and the ionome of plants, named 'transfer-factor', 'enrichment factor', etc. It reveals roughly the pattern of element distribution between the subsoils and the shoot tips: The macro-nutrients $\mathrm{S}, \mathrm{P}, \mathrm{K}$ and $\mathrm{Ca}$ are generally enriched in shoot tips of $C$. sinensis compared to the corresponding subsoils. Only in Assam $\mathrm{Ca}$ is not enriched in the sink tissue. The elements with small transfer-factors are all non-nutrients, micro-nutrients or main elements of the soils: Sc, Fe, REE, Li, Al, Pb, $\mathrm{Cr}$, Co, Sb, Mo, Ba, Cs, TI, Ni and $\mathrm{Cd}$. For all remaining nutrients and elements with characteristics analogous to a nutrient, $\mathrm{Rb}, \mathrm{Sr}, \mathrm{Mn}, \mathrm{Cu}, \mathrm{Mg}$ and $\mathrm{Zn}$, it depends on the regions whether the elements are enriched in the sink tissue compared to the subsoils: Especially tea grown on the nutrient-poor soils of Registro and São Miguel de Arcanjo shows greater concentrations in its shoot tips than in the soils.

The vratios of $\mathrm{Al}$ are unusual high compared to other plants, because $C$. sinensis is an Al accumulating plant (Section 2.1.3). The vratios for the beneficial REE are in contrast small. Among the non-nutrients the high vratio values of $\mathrm{Rb}$ are sticking out; they show similar values like nutrients. As described in Section 7.4, $\mathrm{Rb}$ is enriched in the shoot tips compared to the mature leaves resulting into highest concentration of a non-nutrient element in sink tissue (Figure 7.1). 


\section{Influence of Soil on the Tea lonome}

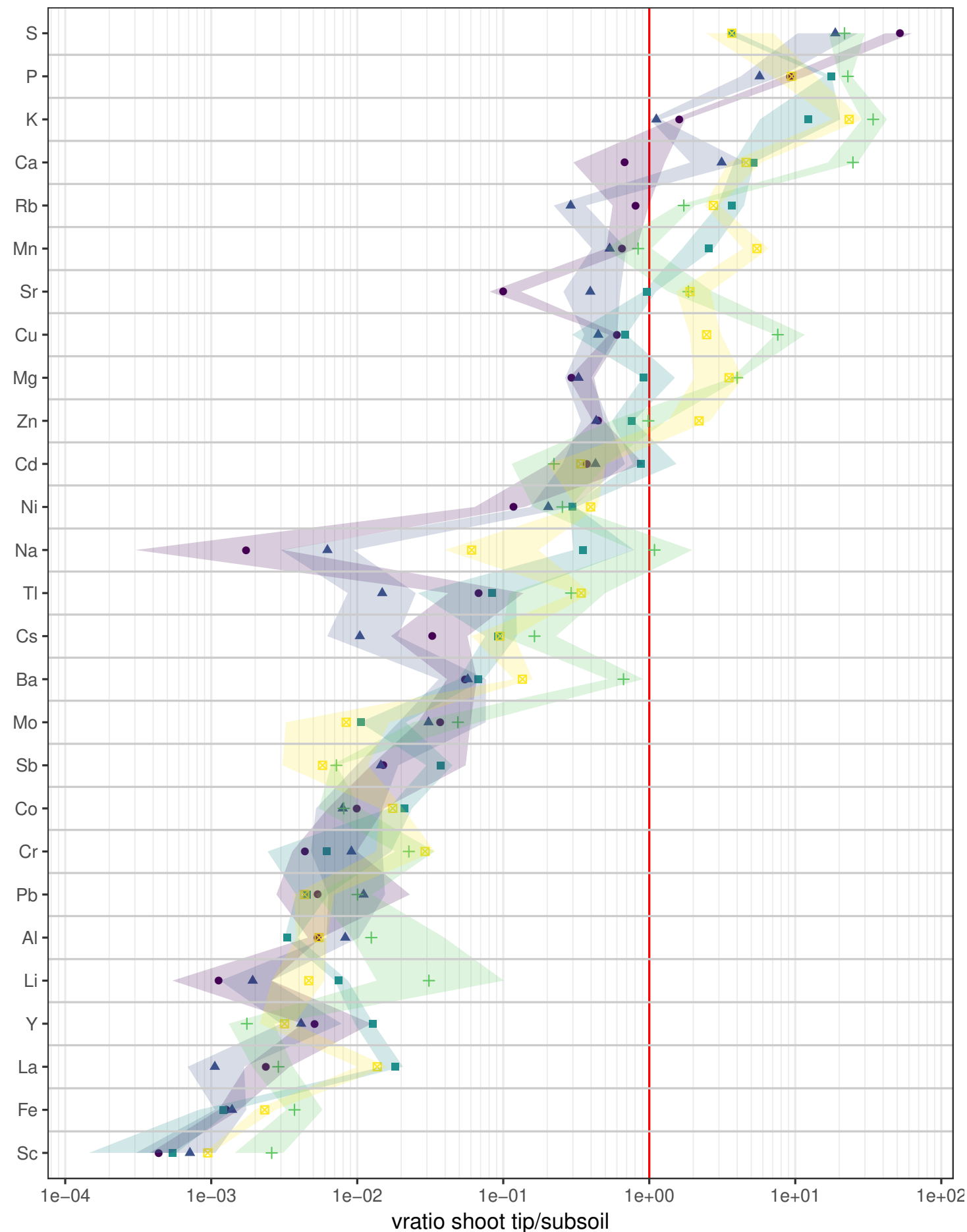

Region - Assam $\Delta$ Darjeeling $\|$ Pr Loc: Araucaria + SP Loc: Registro \SP Loc: São Miguel Arcanjo

Figure 8.3.: Simple vratios shoot tip/subsoil of corresponding pairs of samples, grouped by tea growing regions. Elements are ordered by median of vratio. Median for each sample group is marked by a colored symbol, the shaded areas mark the second and third quartile. 


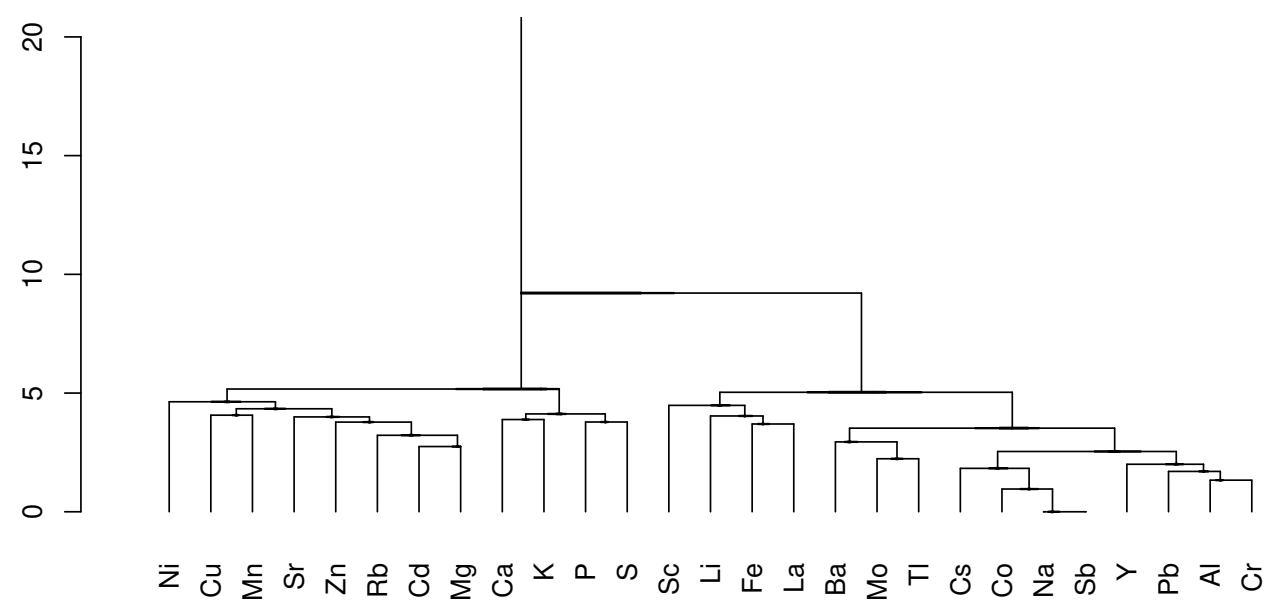

Figure 8.4.: Graph of ilr-ANOVA (CoDa dendrogram) of all shoot tip samples $(\mathrm{N}=160)$ and subsoil samples $(\mathrm{N}=96)$.

The ilr-ANOVA can be used to group the elements into subcompositions with similar transfer-factors while meeting the requirements of calculating with concentration data (Section 5.1). The graph in Figure 8.4 displays the grouping of element variables if shoot tip samples and subsoil samples from all regions in Brazil, India and Japan are included in the ilr-ANOVA. Six groups can be distinguished:

1. $\mathrm{Ca}, \mathrm{K}, \mathrm{P}$ and $\mathrm{S}$

2. $\mathrm{Cd}, \mathrm{Cu}, \mathrm{Mg}, \mathrm{Mn}, \mathrm{Ni}, \mathrm{Rb}, \mathrm{Sr}$ and $\mathrm{Zn}$

3. Ba, Mo and $\mathrm{TI}$

4. $\mathrm{Co}, \mathrm{Cs}, \mathrm{Na}$ and $\mathrm{Sb}$

5. Al, $\mathrm{Cr}, \mathrm{Pb}$ and $\mathrm{Y}$

6. Fe, La, Li and Sc

The grouping reflects roughly the order of median simple vratios in Figure 8.3 . The first group, the elements $\mathrm{Ca}, \mathrm{K}, \mathrm{P}$ and $\mathrm{S}$, form a subcomposition of elements with similar relation of concentrations between shoot tips and subsoils in respect to all other elements. The bio-availability and thereby the uptake of these elements may be influenced by soil 
management (Section 8.1.2). The second group, the subcomposition of $\mathrm{Cd}, \mathrm{Cu}, \mathrm{Mg}$, $\mathrm{Mn}, \mathrm{Ni}, \mathrm{Rb}, \mathrm{Sr}$ and $\mathrm{Zn}$, consists of elements which concentration in soils is generally less affected by soil management (Section 8.1.2). Depending on the overall situation of the soil the measures of soil management might still have an impact on the uptake of these elements. The uptake of elements of all remaining subcompositions is potentially unaffected by soil management.

The grouping of elements with regard to similarity in the relation between shoot tips and subsoils depends on the used data set. In the following two examples the total data set is split into one data set with samples only from Darjeeling, and in the second data set without the samples from Darjeeling. For both data sets an own ilr-ANOVA is calculated and displayed in CoDa dendrograms.

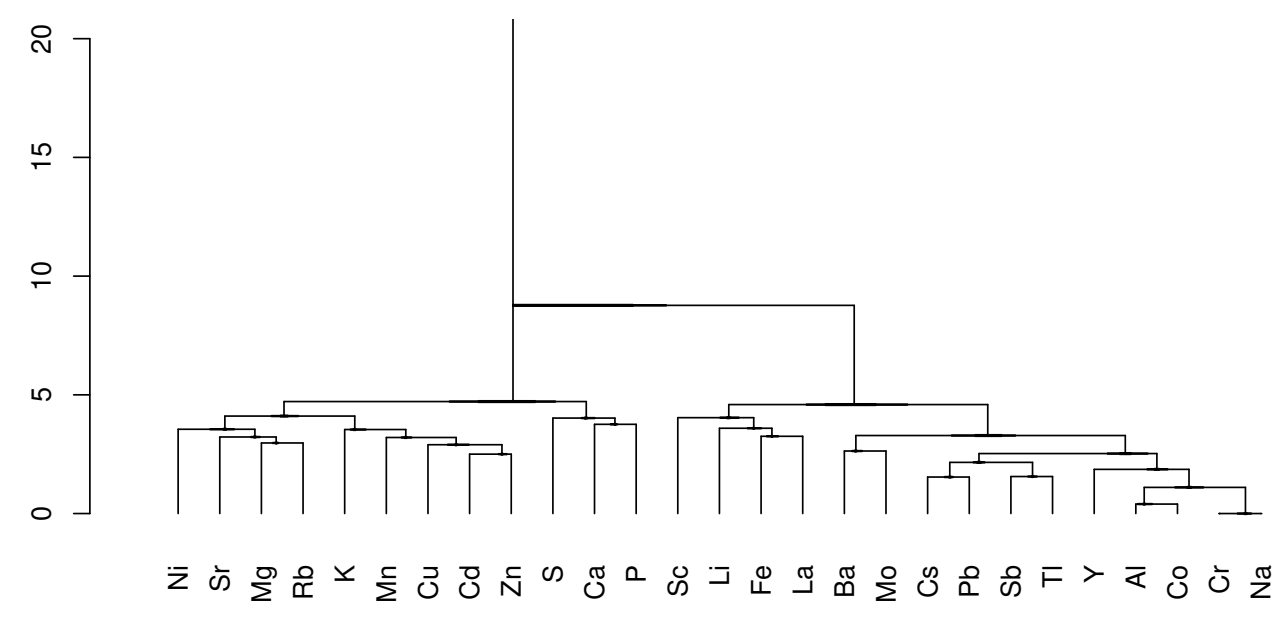

Figure 8.5.: Graph of ilr-ANOVA (CoDa dendrogram) of shoot tip samples $(\mathrm{N}=91)$ and subsoil samples $(N=71)$ of only Darjeeling.

Figure 8.5 shows the grouping of elements for comparing shoot tips and subsoils only from Darjeeling. Most subcompositions are similar to the ilr-ANOVA of the total data set, because the biggest sample group in the total data set are samples from Darjeeling. The most important difference in grouping is regrouping of the nutrient $\mathrm{K}$, which is for Darjeeling in the second group. This confirms the results from Section 8.2, that soil management in Darjeeling has only a very minor impact on $\mathrm{K}$ concentration in soils.

Figure 8.6 shows the grouping of elements for comparing shoot tips and subsoils 


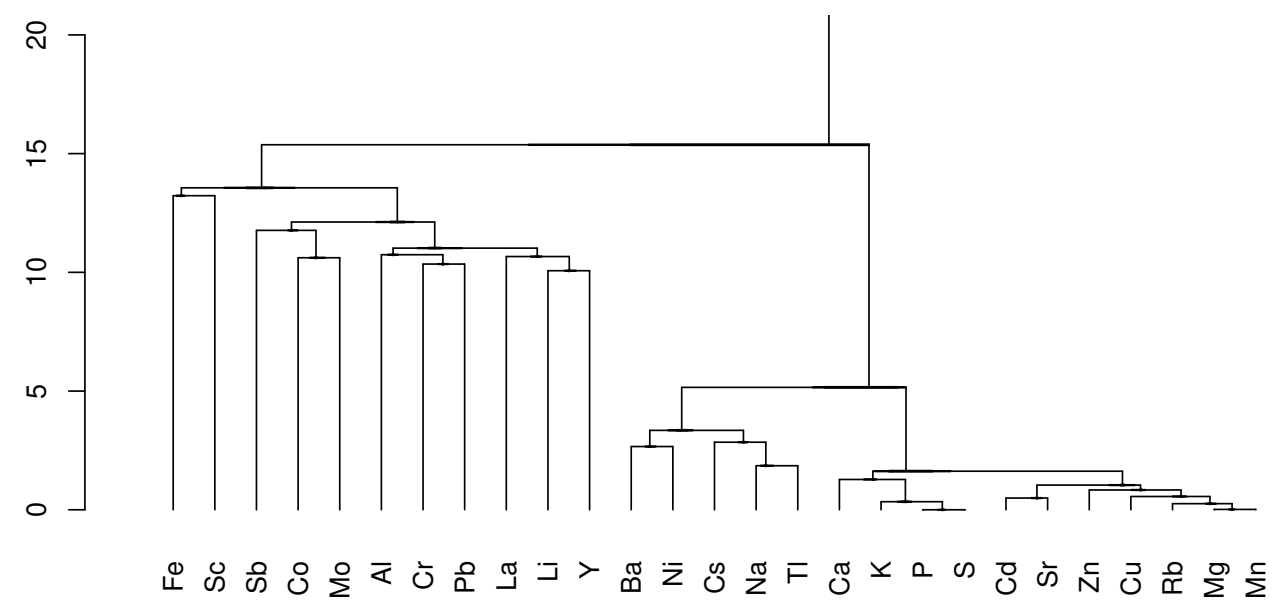

Figure 8.6.: Graph of ilr-ANOVA (CoDa dendrogram) of shoot tip samples $(\mathrm{N}=69)$ and subsoil samples $(\mathrm{N}=25)$ from all regions except Darjeeling.

without samples from Darjeeling. The first and second group are corresponding to the ilr-ANOVA of the total data set, only Ni belongs to an other group. All other element variables are differently grouped into to two groups: $\mathrm{A}$ third group of $\mathrm{Ba}, \mathrm{Cs} \mathrm{Ni}, \mathrm{Na}$ and $\mathrm{TI}$, and a fourth group of all remaining elements. This dendrogram exhibits an apparent difference between elements which are are potentially influenced in their uptake by soil management and and the agricultural 'uninteresting' elements. Especially the third and fourth subcomposition is potentially important for provenance analysis.

One preparation step for provenance analysis is to identify subcompositions which remain stable in their log-ratios during the uptake process from the element reservoir to the sink tissue. These subcompositions can be determined by comparing the distribution of pairwise log-ratios between shoot tips and corresponding soils. The results are calculated as well for topsoils as for subsoils, because for some tea growing regions geochemical composition of topsoils and subsoils have to be considered. The heatmaps of the Figures 8.7 and 8.8 display the results of the comparison between the log-ratio distributions.

Topsoils and corresponding shoot tips have following comparable log-ratio distributions:

- K, P, K S and K Ca having $\mathrm{p}$-values slightly below 0.05 


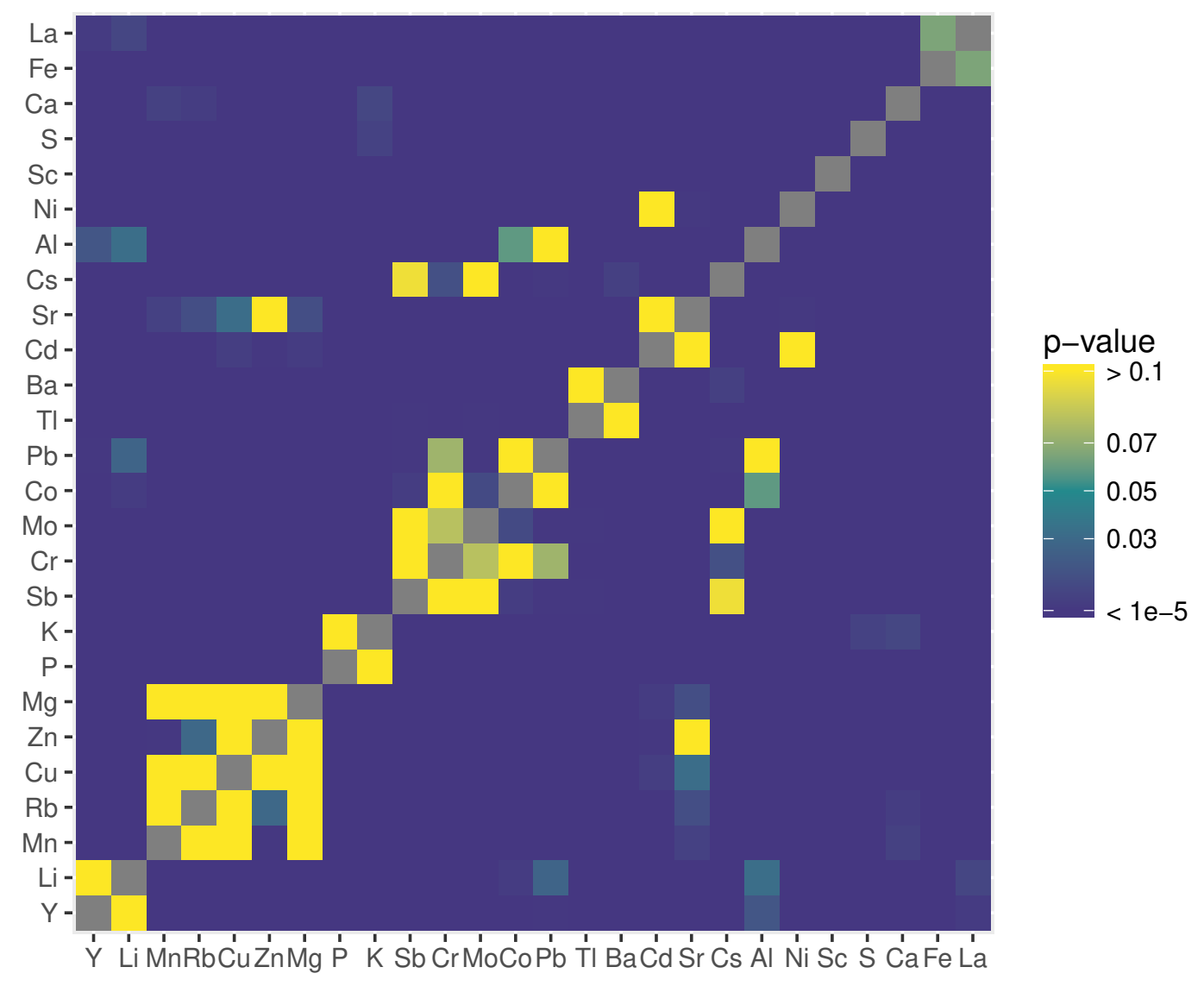

Figure 8.7.: Heatmap of p-values calculated by paired Student's $t$-test between distribution of pairwise log-ratio of shoot tip samples versus corresponding topsoils. Total number of sample pairs $N=33$, with Darjeeling $N=13$, Assam $N=6$, São Paulo $N=9$ and Paraná $N=5$. Pvalues of log-ratios of element pairs are colored blueish if they are below 0.05 (alternative hypothesis: true difference in means is not equal to 0 ) and are colored greenish to yellowish if values are above 0.05 (the means of two populations are equal).

- $\mathrm{Cu}, \mathrm{Mg}, \mathrm{Mn}, \mathrm{Rb}, \mathrm{Zn}$ with $\mathrm{Zn} \sim \mathrm{Sr}$, and without $\mathrm{Zn} \sim \mathrm{Mn}$ and $\mathrm{Zn} \sim \mathrm{Rb}$.

- $\mathrm{Cd}, \mathrm{Sr}$ and $\mathrm{Ni} \sim \mathrm{Cd}$

- Co, Cr, Mo, Pb, Sb with Cs Sb, Cs Mo, Al Pb and Al Co, but without Pb Sb, $\mathrm{Pb} \sim \mathrm{Mo}, \mathrm{Co} \sim \mathrm{Sb}$ and $\mathrm{Co} \sim \mathrm{Mo}$

- La, Fe

- Li, Y

- Ba, Tl 


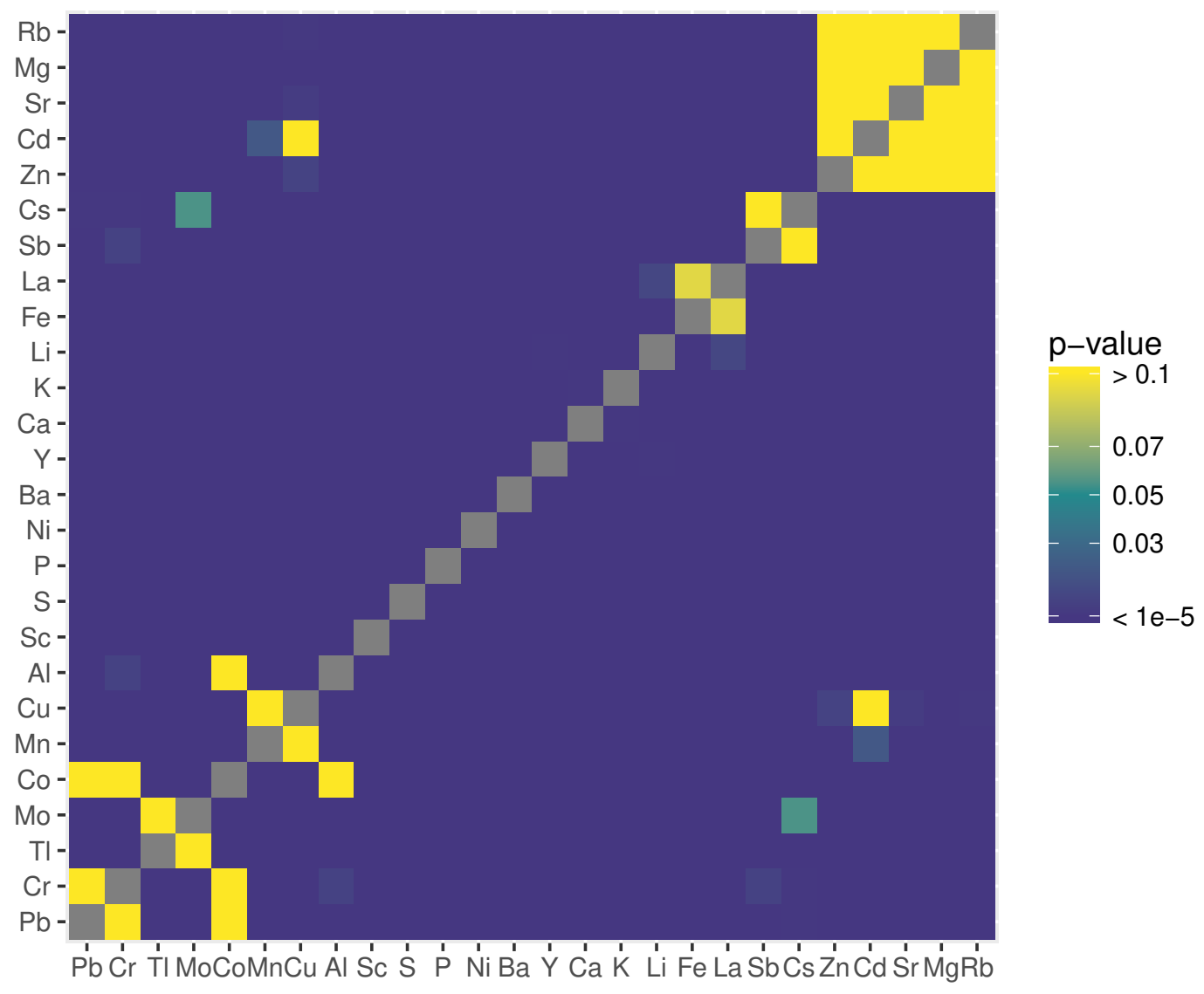

Figure 8.8.: Heatmap of $p$-values calculated by paired Student's $t$-test between distribution of pairwise log-ratio of shoot tip samples versus corresponding subsoils. Total number of sample pairs $N=81$, with Darjeeling $N=59$, Assam $N=6$, São Paulo $N=11$ and Paraná $N=5$. P-values of log-ratios of element pairs are colored blueish if they are below 0.05 (alternative hypothesis: true difference in means is not equal to 0) and are colored greenish to yellowish if values are above 0.05 (the means of two populations are equal).

Subsoils and corresponding shoot tips have following comparable log-ratio distributions:

- $\mathrm{Cd}, \mathrm{Mg}, \mathrm{Rb}, \mathrm{Sr}, \mathrm{Zn}$ with $\mathrm{Cd} \sim \mathrm{Cu}$

- $\mathrm{Mn}$ and $\mathrm{Cu}$

- Cs, Mo, Sb with Mo TI

- Co, Cr, Pb with Co Al

- Fe and La 


\section{Influence of Soil on the Tea lonome}

The subcomposition $\mathrm{Cd}, \mathrm{Cu}, \mathrm{Mg}, \mathrm{Mn}, \mathrm{Rb}, \mathrm{Sr}$ and $\mathrm{Zn}$ as similar log-ratios within the subcomposition between shoot tips and corresponding soils, as well topsoils as subsoils. Except for $\mathrm{Rb}, \mathrm{Sr}$ and $\mathrm{Cd}$ the elements are all nutrients. For several of the elements there are differences between topsoil and subsoil (Figure 8.2), especially for $\mathrm{Cu}, \mathrm{Mn}, \mathrm{Sr}$ and $\mathrm{Zn}$, leading to less comparability between subsoils and shoot tips compared to topsoils and shoot tips (Figure 8.8). Nevertheless, the element ratios within this subcomposition of shoot tips seem to generally reflect the respective element ratios in the total soil volume. Tyler (1997) describes a similar finding for $\mathrm{Rb}$ in acid soils, such as tea soils are.

The subcomposition $\mathrm{Co}, \mathrm{Cs}, \mathrm{Mo}, \mathrm{Na}$ and $\mathrm{Sb}$, combined with $\mathrm{Al}, \mathrm{Cr}$ and $\mathrm{Pb}$ is a second subcomposition where the pairwise log-ratios between shoot tips and topsoils and subsoils are comparable. Of these elements, only Mo and Co are (micro)-nutrients. Accordingly, the uptake of these elements occurs probably due to poor ion differentiation by transporter proteins rather than specific uptake. Interestingly, the log-ratios of Al Co are similar between the shoot tips and both, topsoils and subsoils. Al is by some authors described as a beneficial element for $C$. sinensis (Section 2.1.3. The data suggest, that the uptake of the beneficial elements $A l$ and Co into the sink tissue of $C$. sinensis is linked to the Al to Co ratio in the soil.

The subcomposition $\mathrm{Ca}, \mathrm{K}, \mathrm{P}$ and $\mathrm{S}$ is composed of elements of which the tea plant has high demand and which bio-availability can be strongly influenced by soil management (Section 8.1.2). The pairwise log-ratio comparison shows that the log-ratios of these elements in shoot tips are similar to the log-ratios of the corresponding topsoils, but not to the corresponding subsoils. As expected, this shows that the soil management has a major influence on the concentrations of $\mathrm{Ca}, \mathrm{K}, \mathrm{P}$ and $\mathrm{S}$ in shoot tips.

Only log-ratios with $\mathrm{Ba}$ are always different between shoot tips and corresponding soils. On the one hand, this could be an effect of varying element translocation inside the tea plant (Section 7.4.1, Figure 7.7). On the other hand, it could be an effect of differences in the $\mathrm{Ba}$ uptake into the plant, which is not yet very well understood or discussed in the bibliography, up to the author's knowledge.

The remaining subcomposition $\mathrm{Sc}, \mathrm{Li}, \mathrm{TI}, \mathrm{Fe}$ and $\mathrm{REE}$, is characterized by poor uptake of the plant. These are also all elements which can be strongly affected by APs (Chapter 6 and Figure 6.5. Although the data of the shoot tip samples had been corrected for adhering particles interpretation of log-ratios with these elements should be done with caution, e.g. through propagating the respective errors (Section 6.4). 


\subsection{Summary}

The geochemical composition of subsoils is similar between all tea soils in respect to the elements $\mathrm{Al}, \mathrm{Fe}, \mathrm{P}$ and $\mathrm{Sc}$ and less similar to the elements $\mathrm{Zn}, \mathrm{Cr}, \mathrm{Pb}$ and $\mathrm{Cd}$. The composition is different in respect to the elements $\mathrm{S}, \mathrm{Cs}, \mathrm{Ca}, \mathrm{Na}, \mathrm{Sr}, \mathrm{Rb}$, and $\mathrm{K}$. The Brazilian soils are stronger depleted in these elements than the Indian soils, except for S. The deeply weathered soils in Brazil are very poor in nutrients. The soils of Assam and Darjeeling show approximately four times greater nutrient concentrations. These differences are caused by the geochemical composition of the geological source rocks and the specific weathering and erosion patterns.

The geochemical contrast between topsoils and corresponding subsoils give a qualitative estimation about which elements are affected by soil management. In the deeply weathered soils of Brazil the concentrations of $\mathrm{P}, \mathrm{K}$ and $\mathrm{Ca}$, are strongly affected by measures of soil management, and $\mathrm{S}, \mathrm{Mg}, \mathrm{Na}, \mathrm{Mn}, \mathrm{Ba}, \mathrm{Sr}$ and $\mathrm{Cu}$ are less affected. The concentrations of the elements $P$ and $S$ are both strongly affected in less intensely weathered soils, respectively organically treated soils, e.g. the soils of Darjeeling and few locations in Assam. K, $\mathrm{Ca}$ and $\mathrm{Cu}$ are less affected in these soils. The varying distribution of heavy metals between topsoils and subsoils has to be taken in consideration for provenance analysis.

The macro-nutrients $\mathrm{S}, \mathrm{P}, \mathrm{K}$ and $\mathrm{Ca}$ are generally enriched in shoot tips of $\mathrm{C}$. sinensis compared to the corresponding subsoils. Only in Assam $\mathrm{Ca}$ is not enriched in the sink tissue. The elements with small transfer-factors are all non-nutrients, micro-nutrients or main elements of the soils: Sc, Fe, REE, Li, Al, Pb, Cr, Co, Sb, Mo, Ba, Cs, TI, Ni and Cd. For all remaining nutrients and elements with characteristics analogous to a nutrient, $\mathrm{Rb}$, $\mathrm{Sr}, \mathrm{Mn}, \mathrm{Cu}, \mathrm{Mg}$ and $\mathrm{Zn}$, it depends on the regions whether the elements are enriched in the sink tissue compared to the subsoils: Especially tea grown on the nutrient-poor soils of Registro and São Miguel de Arcanjo shows greater concentrations in its shoot tips than in the soils.

The elements can be grouped by similarity of their transfer-factors (based on ilrANOVA): One group consists of $\mathrm{Ca}, \mathrm{P}, \mathrm{S}$ and - if samples from Darjeeling are omitted also in $\mathrm{K}$. A second group consists in $\mathrm{Cd}, \mathrm{Cu}, \mathrm{Mg}, \mathrm{Mn}, \mathrm{Ni}, \mathrm{Rb}, \mathrm{Sr}$ and $\mathrm{Zn}$, and in Darjeeling also in $\mathrm{K}$. All other elements can be subsumed into one large group with several sub-groups, depending on the data set which was used to calculated the ilr-ANOVA.

There are two subcompositions which show statistically stable log-ratios during the uptake process from the element reservoir to the sink tissue: The subcomposition $\mathrm{Cd}$, 
$\mathrm{Cu}, \mathrm{Mg}, \mathrm{Mn}, \mathrm{Rb}, \mathrm{Sr}$ and $\mathrm{Zn}$ and the subcomposition $\mathrm{Co}, \mathrm{Cs}, \mathrm{Mo}, \mathrm{Na}$ and $\mathrm{Sb}$, combined with $\mathrm{Al}, \mathrm{Cr}$ and $\mathrm{Pb}$. The log-ratio distributions of the subcomposition consisting of $\mathrm{K}, \mathrm{Ca}$, $S$ and $P$ are similar only between the topsoils and the shoot tips.

In summary, the uptake of the elements $\mathrm{Ca}, \mathrm{S}, \mathrm{P}$ and in some cases $\mathrm{K}$ might be strongly influenced by the soil management and therefore, these elements are not suitable to be linked to the geological source rock. They can serve as a proxy regions with very low fertility status of the soils and/or for regions with very intensive tea cultivation. In Darjeeling uptake of $\mathrm{K}$ does not seem to be notably altered by soil management, probably because most tea gardens are submitted to organic cultivation. Log-ratios of elements of the subcomposition $\mathrm{Co}$, $\mathrm{Cs}, \mathrm{Mo}, \mathrm{Na}$ and $\mathrm{Sb}, \mathrm{Al}, \mathrm{Cr}$ and $\mathrm{Pb}$ can be correlated to the geochemical composition of the total soil volume and are under this aspect suitable for provenance analysis. The subcomposition $\mathrm{Cd}, \mathrm{Cu}, \mathrm{Mg}, \mathrm{Mn}, \mathrm{Rb}, \mathrm{Sr}$ and $\mathrm{Zn}$, consisting of nutrients and replacement elements, correlates will with the subsoil composition and is therefore suitable for provenance subcompositions which discriminate between geological source rock material. 


\section{Provenance Analysis}

In this thesis provenance analytics for tea is solely based on the ionome of the leaves. Previous publications showed that geographic discrimination of tea by element concentration is possible (Marcos et al. 1998; Fernández-Cáceres et al. 2001; Moreda-Piñeiro et al. 2001, 2003; Kelly et al. 2005; Q. Chen et al. 2009; Gonzalvez et al. 2009; Pilgrim et al. 2010). The emphasize of this chapter lies on the questions why the samples can be discriminated into geographical regions and which element subcompositions are suitable to geographically discriminate which groups of tea samples. To achieve this, the statistical provenance analysis is embedded into the framework established in the preceding chapters: The provenance is determined on basis of the 'robust' subcompositions constituted of those elements which are less affected by parameters of the tea production, such as soil management, harvesting methods, machinery or contamination by adhering particles.

The chapter will not cover a holistic model for world spanning provenance analysis of tea. As discussed in the previous chapters, the processed tea ionome is influenced by a) parameters which potentially change between seasons and b) parameter which can be treated as constants, because they are changing very slowly or change only in decades. The parameters being supposedly constants are the geological source rock, the geochemical composition of subsoils, the type of plant, seed plant, hybrid/cultivar, and the long-term climate. The parameters submitted to potentially high fluctuation are weather conditions of the respective season, soil management, like fertilizer, weeding, etc. and tea production, including shading, the plucking standard, leaf grading and used machinery for the different type of teas. The latter parameters can also significantly contribute to determining a provenance of a processed tea sample, because each tea growing region has its specific traditions and preferred tea production methods. A holistic model would include an interlacing of various statistical methods applied on the respective parameter-specific subcompositions to increase the probability of tea sample classification. For example, in the mountainous part of Darjeeling only orthodox black tea and special type of green tea is produced. In Assam nearly the whole tea produc- 


\section{Provenance Analysis}

tion is specialized in CTC black tea manufacturing, and only very minor quantities of the harvest is manufactured differently. Accordingly, the results of an analysis based on a metal subcomposition to distinguish between CTC and non-CTC production could serve as a-priori value for discriminating teas from Assam and Darjeeling. Several other methods and techniques preceding the final discrimination step had been proposed, e.g. by Moreda-Piñeiro et al. (2001) and McKenzie et al. (2010) and Gonzalvez et al. (2009, and references therein). A holistic model which includes several classification techniques in order to make the provenance analysis more robust and reliable in terms of the changing parameters of tea production is a research topic, respectively developing task, on its own.

Keeping the size of the total data set in mind, provenance analysis in this chapter is purposely based only on discrimination methods. In particular LDA has been used in this thesis. A powerful ability of this linear method is to clearly picture the influence of the variables, elements in this case, on the probability of samples to which sample group they belong to. Other, more advanced techniques, like probabilistic neural networks (McKenzie et al. 2010) or soft independent modeling of class and analogy (MoredaPiñeiro et al.2001), require larger data sets or larger samples subgroups for satisfactory results.

In the previous chapters subcompositions had been determined which are either sensitive or insensitive in respect to a tea cultivation or production parameter. It allows to rate for each element how intensely its concentration is altered by a single parameter or a combination of the parameters. Additionally, the rating is weighted based on field observations and personal communications with tea producers, traders and scientists. The combination of rating and weighting is a qualitative approach to express the robustness of each element in terms of a specific tea cultivation and production step as integer value (Table E.1): Elements with high values have a small probability to be affected by the tested parameter. For the relation tea/soil the scale is reverse; elements which show similar log-ratio distributions between shoot tip and soil have high rating-values. Thus, the sum of the values provides a qualitative criterion how suitable an element is to be used in a subcomposition which is designed to capture in the ionome the geochemical composition of the parent material of the soils. Such a subcomposition can be used to discriminate teas which had grown on contrasting soils, because the influence of tea cultivation and production parameters is minimized. The element list sorted from high suitability to low suitability is Mn, Rb, Cs, Sr, Co, Zn, Cu, Sb, La, Mg, Tl, K, Cd, Pb, Mo, $\mathrm{Cr}, \mathrm{Al}, \mathrm{Y}, \mathrm{Ni}, \mathrm{Li}, \mathrm{Fe}, \mathrm{Ca}, \mathrm{P}, \mathrm{Na}, \mathrm{S}, \mathrm{Ba}, \mathrm{Sc}$ (Table E.1). One task of a holistic model would 
be to establish a index of robustness entirely based on statistical analysis on a larger and more balanced data set. Most probably, this would also lead to a slightly different index of robustness than the qualitative approach.

In this thesis the tea provenance studies are based on subcompositions which are selected by their general suitability, respectively robustness, and less by their discriminative power in this specific data set. This contrasts with, to the author's knowledge, all published tea provenance studies, where the set of variables for the discrimination functions is selected by statistically determining the most discriminating variables of the respective data set. To show the principles of this 'robustness-approach' three case studies are presented with varying categorical variables: 1) Discrimination by countries, 2) discrimination by regions in India and 3) discrimination by geological metamorphic zones in Darjeeling. The six elements with the highest suitability/robustness rating are used as the 'standard' subcomposition: Mn, Rb, Cs, Sr, Co and Zn. This standard subcomposition will be altered by few elements to achieve a better discrimination according to knowledge about the local specifics. All samples are filtered for their approximated average leaf development stage (Section 7.4.2). The balance [Cu K | Al Ca Mn] of all samples has values between -1.2 to 0.5 . This homogenization of samples in terms of their average leaf age allows to use for example $\mathrm{Cu}, \mathrm{K}, \mathrm{Al}, \mathrm{Ca}$ and $\mathrm{Mn}$ in subcomposition for discriminating between regions. The linear discrimination is applied on ilr-transformed data.

\subsection{Countries}

The data set comprises samples of three countries: Brazil, India and Japan. Most tea production parameters vary between the countries. Additionally samples from India span the three very different tea growing region Darjeeling, Assam and Nilgiris. Applying the LDA on the pre-defined element subcompositions with the countries as categorical variable indicates which parameters do not discriminate between the countries, and which parameters could serve as proxies of origin.

Figure 9.1 displays the results of a LDA on the ilr-transformed data set of 231 samples and six elements (five ilr-variables). The three sample groups can be separated through log-linear combinations of these six elements, but the groups show an overlapping by several samples. The misclassification rate is lowest for India with only 6 of 183 samples wrongly classified as Japanese samples (Table 9.1). For Japan the misclassification rate is $37.5 \%$ with 6 samples classified to India, and for Brazil $30.8 \%$ with 12 samples 


\section{Provenance Analysis}

classified as Indian samples. Samples from Brazil and India are separated mainly along the LD1-axis and samples from Japan can be discriminated from Brazilian and Indian samples along the LD2-axis. Looking at the values of the clr-scalings (Table 9.1) the Brazilian samples differ from Indian samples by relatively higher concentrations of Cs $\mathrm{Mn}$ and $\mathrm{Zn}$ compared to $\mathrm{Rb}, \mathrm{Sr}$ and $\mathrm{Co}$. The Japanese samples differ from Brazilian and Indian samples by relatively higher concentrations of $\mathrm{Mn}, \mathrm{Zn}$ and $\mathrm{Rb}$ compared to Sr and Co.

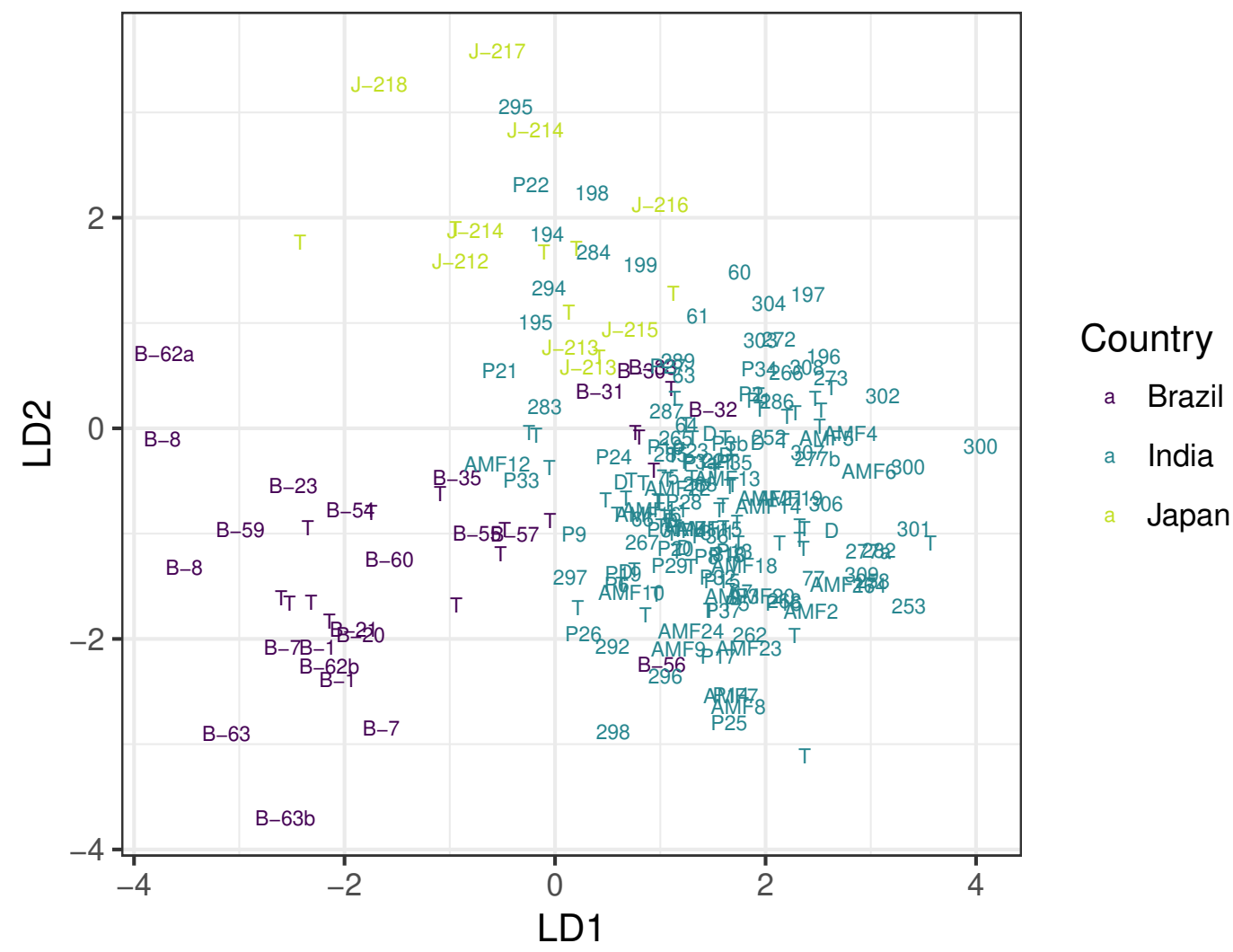

Figure 9.1.: Scatter plot of tea samples displaying the first two scores of linear discriminant functions for origin by countries. Samples are colored by country and the label denotes the sampling location of the shoot tips. Processed tea samples are labeled with 'T'. Total number of samples is $N=230$ (India $N=183$, Brazil $N=39$, Japan $N=16$ ). Clr-scalings for LD1 and LD2 and misclassifications are listed in Table 9.1

The relatively higher $\mathrm{Mn}, \mathrm{Zn}$ and $\mathrm{Rb}$ concentration in Japanese samples could be an effect of the originally igneous source rock material of the soils. The samples of Brazil plotting into the field of the Japanese samples originate from the sampling locations B-30 to B-33 of the garden Yamamotoyama do Brasil, Araucaria (Araucaria), with medium LD1 and LD2 values. These locations are situated on the hornblende-rich, tonalitic 
Table 9.1.: Clr-scalings of LDA (upper table) and misclassifications (lower table) for discrimination of tea by countries. Table corresponds to Figure 9.1. The rows of the misclassification table give the number of samples predicted into respective group.

\begin{tabular}{rrrrrrr}
\hline & Co & Cs & $\mathrm{Mn}$ & $\mathrm{Rb}$ & $\mathrm{Sr}$ & $\mathrm{Zn}$ \\
\hline LD1 & 0.51 & -1.34 & -1.17 & 1.81 & 0.45 & -0.27 \\
LD2 & -0.32 & 0.08 & 1.00 & 0.23 & -1.64 & 0.65 \\
\hline
\end{tabular}

\begin{tabular}{rrrr}
\hline & Brazil & India & Japan \\
\hline Brazil & 28 & 11 & 0 \\
India & 1 & 174 & 7 \\
Japan & 0 & 6 & 10 \\
\hline
\end{tabular}

gneisses of the Atuba-Complex, which include frequently lenses of amphibolites (Section 3.2.2. The other location of Araucaria, B-35, is situated on local, alluvial sand deposits (Figure A.10). Although this sampling location is only $600 \mathrm{~m}$ north of B-33, and all shoot tip samples from this garden have exactly the same growing conditions, this sample plots closer to all other Brazilian samples. The Indian samples 284 (UPASI, research field of South Indian Tea Association (UPASI)), 294 (Havukal) and 295 (Warwick Tea Estate (Warwick)), which also have high LD2-values, originate from the East-Nilgiris with source rock of a charnockite suite (Section 3.1.3). The charnockite formations of this area contain pyroxene granulites and doleritic dykes. Hence, one parameter constituting the LD2-function is probably mafic source rocks as parent material for the tea soils.

The selected subcomposition of $\mathrm{Mn}, \mathrm{Rb}, \mathrm{Cs}, \mathrm{Sr}$, Co and $\mathrm{Zn}$ is - from a geochemical point of view - not the most suitable selection to serve as a reliable proxy for mafic source rock for the tea plantations. A better selection includes $\mathrm{Ni}, \mathrm{Mg}$ and $\mathrm{Fe}$, which are together with $\mathrm{Co}$ and $\mathrm{Mn}$ connected to the Fe-bearing phases, e.g. altering minerals like pyroxenites and amphibolites. The biplot in Figure 9.2 is based on clr-values calculated by the subcomposition $\mathrm{Mn}, \mathrm{Rb}, \mathrm{Cs}, \mathrm{Sr}, \mathrm{Co}, \mathrm{Ni}, \mathrm{Mg}$, Fe and $\mathrm{Li}$. In this plot the labels of the elements $\mathrm{Fe}, \mathrm{Ni}$ and $\mathrm{Mn}$ are projected into one line. This indicates that the log-ratios of these three variables have a good correlation among them, i.e. that these elements are mostly controlled by the same processes. A second set of presumably co-depending elements are $\mathrm{Mn}, \mathrm{Zn}$ and $\mathrm{Mg}$. Additionally, all shoot tips with high log-ratios of $\mathrm{Mn}$, Co and $\mathrm{Ni}$ compared to other elements are grown on soils which parent material contained mafic minerals, e.g. the hornblende-biotite gneiss or charnockites (right side of Figure 


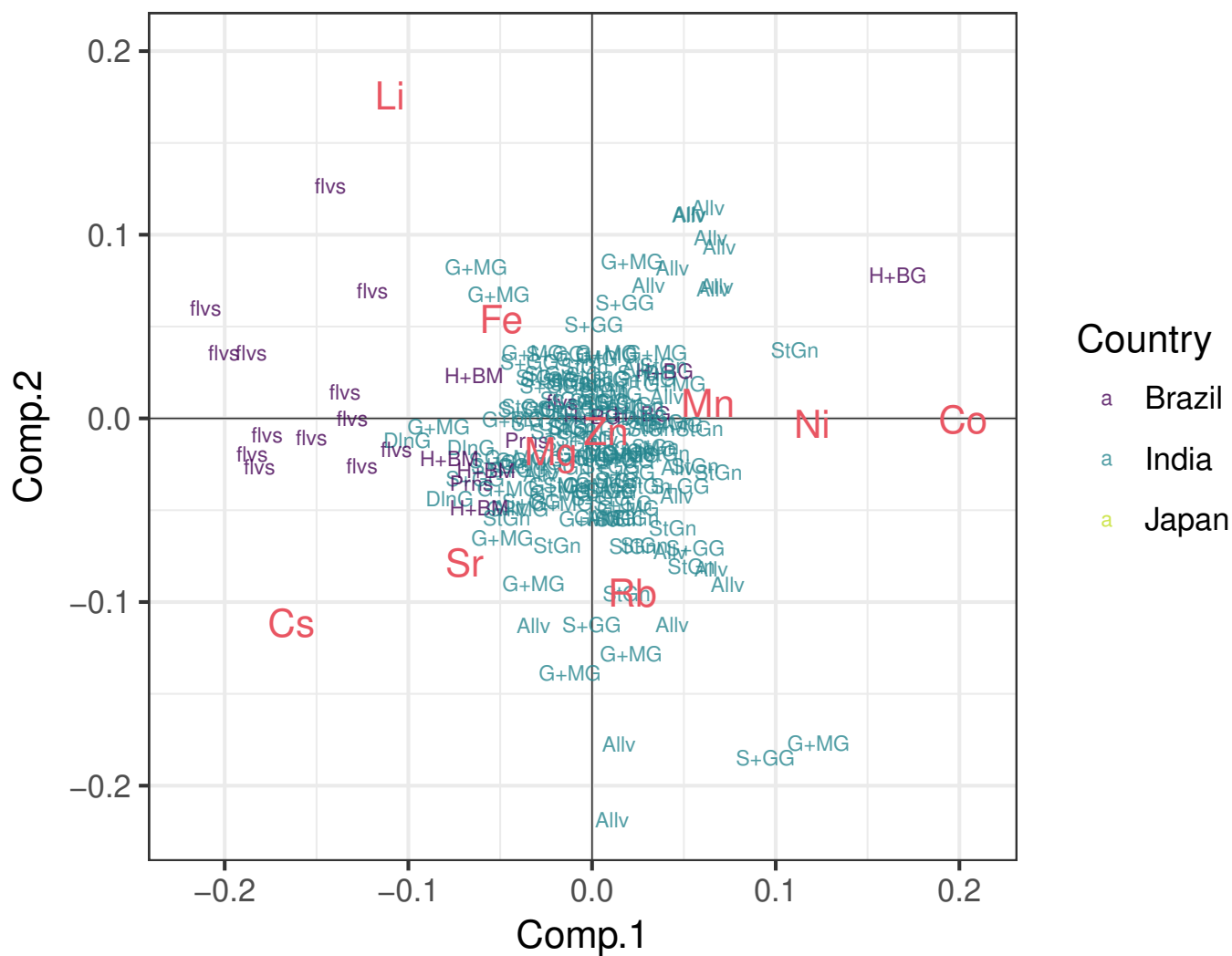

Figure 9.2.: Clr-biplot of tea ionomes of leaf samples for subcomposition $\mathrm{Mn}, \mathrm{Rb}, \mathrm{Cs}, \mathrm{Sr}, \mathrm{Co}, \mathrm{Ni}$, $\mathrm{Mg}, \mathrm{Fe}$ and $\mathrm{Li}$. Total explained variance is 0.62 . Total number of samples is $\mathrm{N}=230$ (India $\mathrm{N}=183$, Brazil $\mathrm{N}=39$, Japan $\mathrm{N}=16$ ). Labeling of samples is according to their geological source rock, or if information of those are missing, their sampling point. Abbreviations are: $\mathrm{H}+\mathrm{BG}$ hornblende+biotite gneiss, $\mathrm{G}+\mathrm{MG}$ garnet+muscovite gneiss, $\mathrm{S}+\mathrm{GG}$ staurolite+garnet gneiss, StG staurolite gneiss, $\mathrm{H}+\mathrm{BM}$ hornblende+biotite monzogranite, Chrn charnockite, Allv alluvium of Brahmaputra river, flvs fluvial sediments, Prns sediments of the Paraná Basin, T processed tea samples, others: labels of sampling points.

9.2). Shoot tips grown on fluvial sediments have the smallest log-ratios of $\mathrm{Mn}$, Co and $\mathrm{Ni}$ compared to other elements (left side of Figure 9.2). These results are a strong indicator that the $\mathrm{Mn}, \mathrm{Ni}$ and $\mathrm{Co}$ concentration in $\mathrm{C}$. sinensis are caused by altering mafic minerals. In the present data set $\mathrm{Co}$ and $\mathrm{Mn}$ may also alone serve as proxy for mafic source rocks.

The Brazilian tea samples plotting into the 'Brazilian' field are all grown on sediments as source rock. In the area of Registro (points B-7 - B-23) the outcrops are described as slightly immature, sandy to silty alluvial deposits (Section 3.2.1). In combination with the high weathering rates in the sub-tropical climate the soils are depleted in all major cations (Table 8.1). Most samples of the other Brazilian regions are grown on sedimen- 
tary source rock, except for the points B-30 - B-33 and B-55 - B-57, as described in the previous paragraph. In total 16 samples originate from sediments, 8 from gneiss and monzogranite, and 15 are processed tea samples. The LD1 function, which discriminates between Brazilian and Indian samples, is dominated by Rb versus $\mathrm{Cs}$ and $\mathrm{Mn}$ (Table 9.1). For this data set, it suggests that element uptake from sandy to silty sediments of Rb versus Cs is fundamentally different in Brazil than from soils of Japan or India. This is probably not only caused by the characteristics of the geological parent material, because the tea soils of Assam are also developed from alluvial sediments. It could be an combined effect of the geochemistry of source rock, soil types, fertilizer/manure/carbonate application, and cultivar. The latter parameter obviously has only a minor impact on the regional discrimination, because most samples of Japan are the same cultivar - C. sinensis 'yabukita' - as sampled in B-54 to B-60 in Brazil. In summary, it remains unclear whether the discrimination between Brazilian and Indian samples is purely an effect of the sedimentary source rock or rather an interaction of soil management meeting very different soil types.

The results of the LDA with the standard element subcomposition, Mn, Rb, Cs, Sr, Co and $\mathrm{Zn}$, exhibit that this subcomposition is suitable to distinguish tea samples roughly by country and by their geological source material. In some cases the fingerprint of the geological background superimposes other, regional geochemical signals caused by differing tea production systems. To enhance the discrimination between countries the element subcomposition has to be increased by elements which capture local specifics: Brazilian tea garden management includes intense fertilizer application (Section 8.1.2) and therefore $\mathrm{K}$ and $\mathrm{P}$ are included into the subcomposition. Several Japanese samples showed a significantly higher concentration of $\mathrm{Li}$ than the average ionome of tea (Figure 7.1 and 9.2 and therefore $\mathrm{Li}$ is added, too. The results of the LDA with the subcomposition of $\mathrm{Mn}, \mathrm{Rb}, \mathrm{Cs}, \mathrm{Sr}, \mathrm{Co}, \mathrm{Zn}, \mathrm{K}, \mathrm{P}$ and $\mathrm{Li}$ are displayed in Figure 9.3. In this scatter plot the samples groups are notably better discriminated than in Figure 9.1. Only eight samples of Brazil (Araucaria), two samples of India, and one sample of Japan, can not be classified into their respective group (Table 9.2). That shows that these three additional elements help to significantly improve the discrimination between the groups. Only the plants originating from mafic source rocks are still misclassified by this combination of variables and method. For a further refinement of discrimination between Brazilian and Indian samples one could either use a different, more specified set of variables, a-priori values derived from a different analysis, or a larger data set which would allow to include more variables and/or different statistical techniques. 


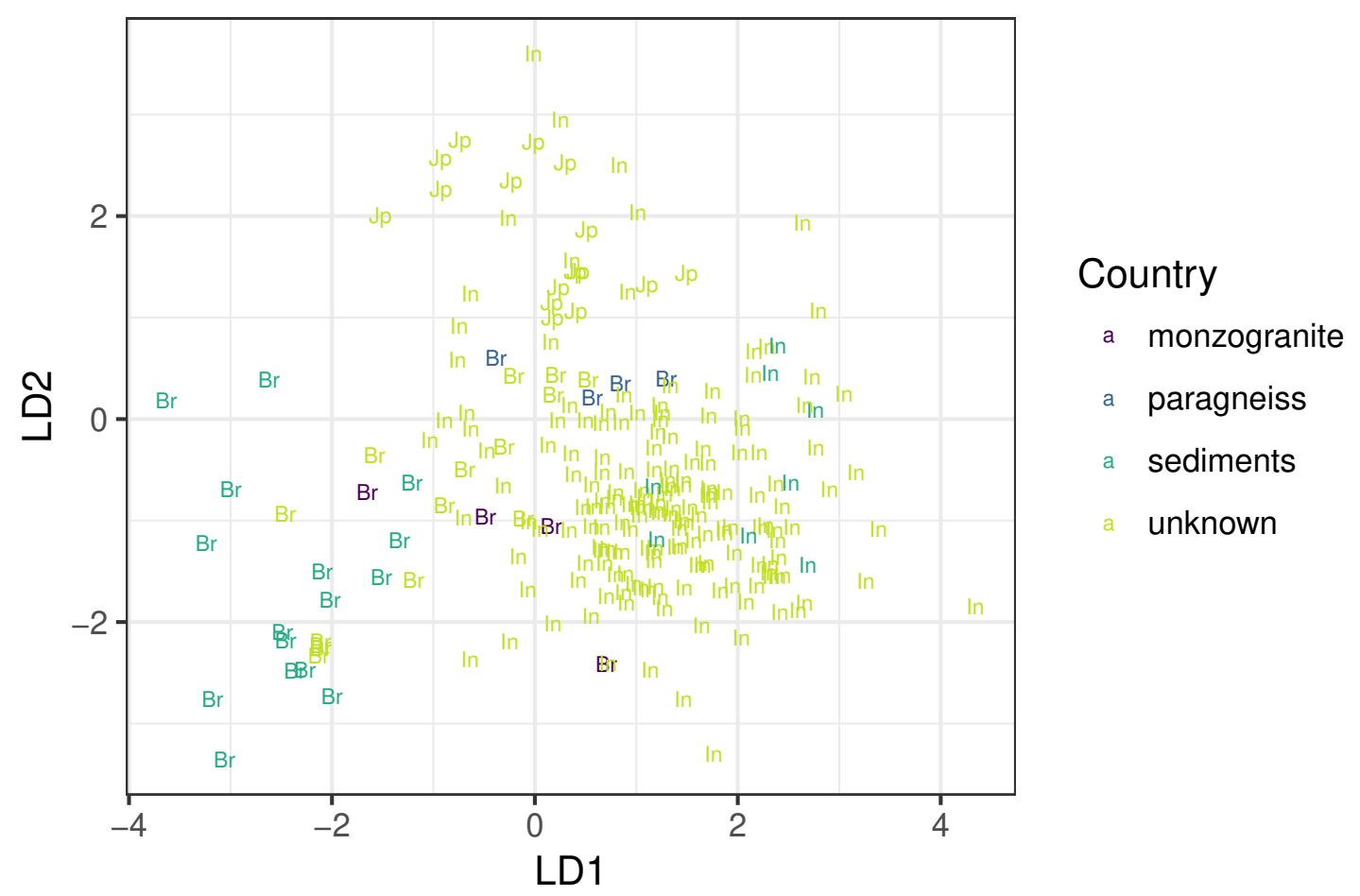

Figure 9.3.: Scatter plot of tea samples displaying the first two scores of linear discriminant functions for origin by countries, including the elements K, P and Li. Samples are colored by country and the label denotes the sampling location of the shoot tips. Processed tea samples are labeled with ' $T$ '. Total number of samples is $\mathrm{N}=231$ (India $\mathrm{N}=176$, Brazil $\mathrm{N}=39$, Japan $\mathrm{N}=16$ ). Clr-scalings for LD1 and LD2 are listed in Table 9.2

Table 9.2.: Clr-scalings of LDA (upper table) and misclassifications (lower table) for discrimination of tea by countries. The element compilation includes $\mathrm{K}, \mathrm{P}$ and $\mathrm{Li}$. Table corresponds to Figure 9.3. The rows of the misclassification table give the number of samples predicted into respective group.

\begin{tabular}{rrrrrrrrrr}
\hline & Co & $\mathrm{Cs}$ & $\mathrm{Mn}$ & $\mathrm{Rb}$ & $\mathrm{Sr}$ & $\mathrm{Zn}$ & $\mathrm{K}$ & $\mathrm{P}$ & $\mathrm{Li}$ \\
\hline LD1 & 0.44 & -0.77 & -0.61 & 1.26 & 1.09 & 1.70 & 1.61 & -4.21 & -0.50 \\
LD2 & 0.02 & -0.81 & -0.23 & 1.15 & -1.28 & -0.13 & 1.07 & -0.41 & 0.62 \\
\hline
\end{tabular}

\begin{tabular}{rrrr}
\hline & Brazil & India & Japan \\
\hline Brazil & 31 & 8 & 0 \\
India & 1 & 181 & 1 \\
Japan & 0 & 1 & 15 \\
\hline
\end{tabular}




\subsection{India}

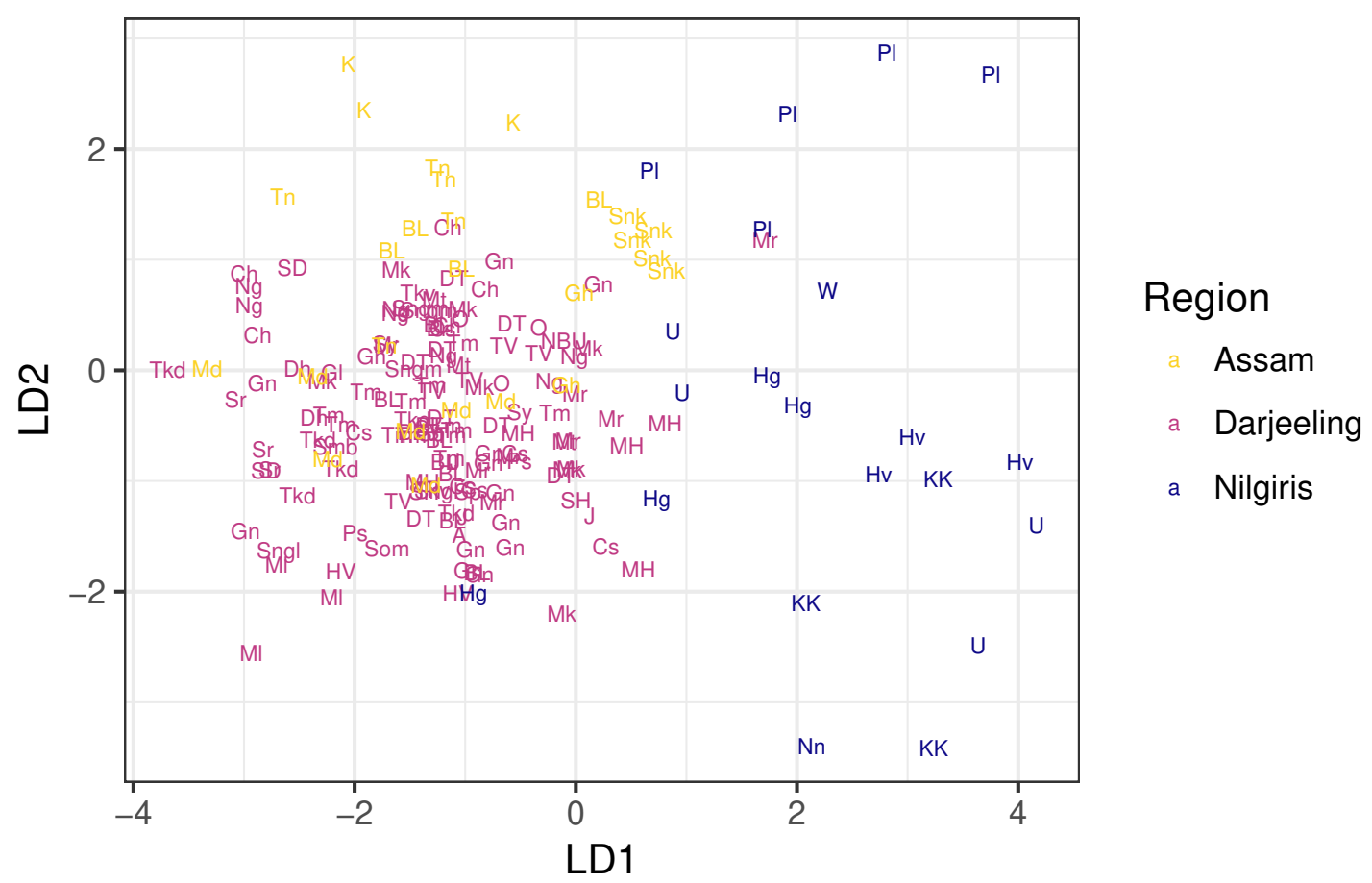

Figure 9.4.: Scatter plot of shoot tip and processed tea samples displaying the first two scores of linear discriminant functions for origin by regions of India. Samples are colored by region and the label denotes the garden of origin. Total number of samples is $N=183$ (Darjeeling $N=136$, Assam $N=26$, Nilgiris $N=21$ ). Clr-scalings for LD1 and LD2 and misclassifications are listed in Table 9.3. Abbreviations are for Nilgiris: PI Palaniappa, U UPASI, W Warwick, Hg Highfield, Hv Havukal, KK Kil Kotagiri, Nn Nonsuch.

The shoot tips and processed tea ionomes of Indian samples display in Figure 9.1 and Figure 9.3 a certain spread, but no distinct subgroups can be seen. To discriminate the regions of India the standard subcomposition $\mathrm{Mn}, \mathrm{Rb}, \mathrm{Cs}, \mathrm{Sr}$, Co and $\mathrm{Zn}$ is used, identical to the LDA in the previous section. Here, the three tea growing regions of India are taken as categorical variable for the LDA: The regions Darjeeling and Assam can not be distinguished by the standard subcomposition (Figure 9.4) and the misclassification table gives only a $35 \%$ correct classification for Assam (Table 9.3. Samples from Nilgiris display a large spread, as well along the LD1 axis as along the LD2 axis, but they can be discriminated from the other two regions with a misclassification rate of $24 \%$. 


\section{Provenance Analysis}

Table 9.3.: Clr-scalings of LDA (upper table) and misclassifications (lower table) for discrimination of tea by tea growing regions in India. Table corresponds to Figure 9.4. The rows of the misclassification table give the number of samples predicted into respective group.

\begin{tabular}{rrrrrrr}
\hline & Co & Cs & $\mathrm{Mn}$ & $\mathrm{Rb}$ & $\mathrm{Sr}$ & $\mathrm{Zn}$ \\
\hline LD1 & 0.99 & -0.22 & 0.09 & -1.35 & -0.14 & 0.62 \\
LD2 & 0.47 & -0.72 & 0.70 & 1.56 & -0.93 & -1.08 \\
\hline
\end{tabular}

\begin{tabular}{rrrr}
\hline & Assam & Darjeeling & Nilgiris \\
\hline Assam & 9 & 17 & 0 \\
Darjeeling & 0 & 135 & 1 \\
Nilgiris & 1 & 4 & 16 \\
\hline
\end{tabular}

The discrimination between tea samples from Nilgiris versus Darjeeling and Assam is controlled by the LD1 axis. The grouping is mainly achieved by log-linear combination of $\mathrm{Co}$ and $\mathrm{Zn}$ versus $\mathrm{Rb}$. The samples from Nilgiris show greater values for $\mathrm{Co}$ and $\mathrm{Zn}$ in respect to $\mathrm{Rb}$.

There are several possible reasons why log-ratios of $\mathrm{Co}$ and $\mathrm{Zn}$ versus $\mathrm{Rb}$ discriminate between Nilgiris and North India: The parameter of cultivars does obviously not induce a visible bias on discrimination by regions: The sample of Warwick consists in leaf material of a popular cultivar of Darjeeling, the 'AB2' clone; the other samples from Nilgiris, consisting in leaf material of cultivars and plants grown from seeds of $C$. sinensis var. assamica, are comparable to Assam. Nilgiris and North India differ in their climatic conditions, eg. by the monsoon pattern (Section 3.1.3). The impact of climatic parameters on geographical discrimination can not be quantified, and although it might be a notable factor for varying element uptake by $C$. sinensis, will not be discussed further on. The soils in Nilgiris are very deep with laterite subsoil and show poor nutrient status (Section 3.1.3). Therefore, most tea gardens in the Nilgiris rely on conventional soil management with regular and in cases intense usage of fertilizers (personal communication of UPASI Tea Research Foundation). Only few gardens are certified organic, e.g. Korakundah, United Nilgiri Tea Estates (Korakundah) (not sampled), or even under conversion, e.g. Highfield, or used to be organic, e.g. Palaniappa Tea Estate (Palaniappa). The samples of Darjeeling and Assam originate mainly from organic tea gardens, and soils are less intensely weathered.

In summary, the tea producing area Nilgiris exhibits a quite different setting than the northern areas, with deep, lateric soils, different monsoon pattern and contrasting source 
rock material. In Nilgiris, the soil management might have more impact on the bioavailability of elements than in e.g. in Darjeeling, where the roots of $C$. sinensis easily reach (in)to the solid rock. But Co concentration is improbable to be directly influenced by tea management, because there are no recommendation for Co-applications for tea in Nilgiris (Durairaj et al. 2015). Therefore, the relatively higher Co concentrations in the tea leaves are probably an effect of the basement rock, the charnockite suite of the Nilgiri Massif. This would point towards the same interpretation for Co concentration as in the preceding section. Hence, Nilgiris samples can most probably mainly be distinguished from other regions by elements which serve as indicators to the specific, quite unique geological setting of the area. The discrimination will gain robustness if the chosen subcomposition are combined with fertilizer indicators and indicators for the laterization of soils: The aim should be to capture the contrast between the characteristics of the soils and the soil managements.

Table 9.4.: Clr-scalings of LDA (upper table) and misclassifications (lower table) for discrimination of tea by tea growing regions in India. The element compilation includes $\mathrm{K}$ and $\mathrm{Mg}$, and excludes Mn. Table corresponds to Figure 9.5. The rows of the misclassification table give the number of samples predicted into respective group.

\begin{tabular}{rrrrrrr}
\hline & Co & Cs & Rb & Sr & Zn & K \\
\hline LD1 & 0.85 & -0.25 & -1.32 & -0.20 & 0.52 & 2.21 \\
LD2 & 0.35 & -0.40 & 0.54 & -1.47 & -2.17 & -1.12 \\
\hline & & & & & \\
\cline { 2 - 6 } & & & Assam & Darjeeling & Nilgiris \\
\cline { 2 - 6 } & Assam & 12 & 14 & 0 \\
& Darjeeling & 4 & 132 & 0 \\
& Nilgiris & 0 & 2 & 19 \\
\hline
\end{tabular}

To exemplary show how to capture the contrast between the soils by this small data set only two indicator elements are added: The element $\mathrm{K}$ is added to the standard subcomposition to capture fertilizer application and $\mathrm{Mg}$ is added to capture the contrast of laterization of soils between the regions. The element $\mathrm{Mn}$ had been excluded, because it plays no significant role for the discriminant functions (Table 9.3). The result of the LDA with the subcomposition of seven elements (six ilr-variables), $\mathrm{Ca}, \mathrm{Cs}, \mathrm{Rb}, \mathrm{Sr}, \mathrm{Zn}, \mathrm{K}$ and $\mathrm{Mg}$, is displayed in Figure 9.5. Adding the two indicator elements results into a better classification of samples from Nilgiris in comparison with samples from North India (Table 9.4, lower table) then of without them (Figure 9.4. 


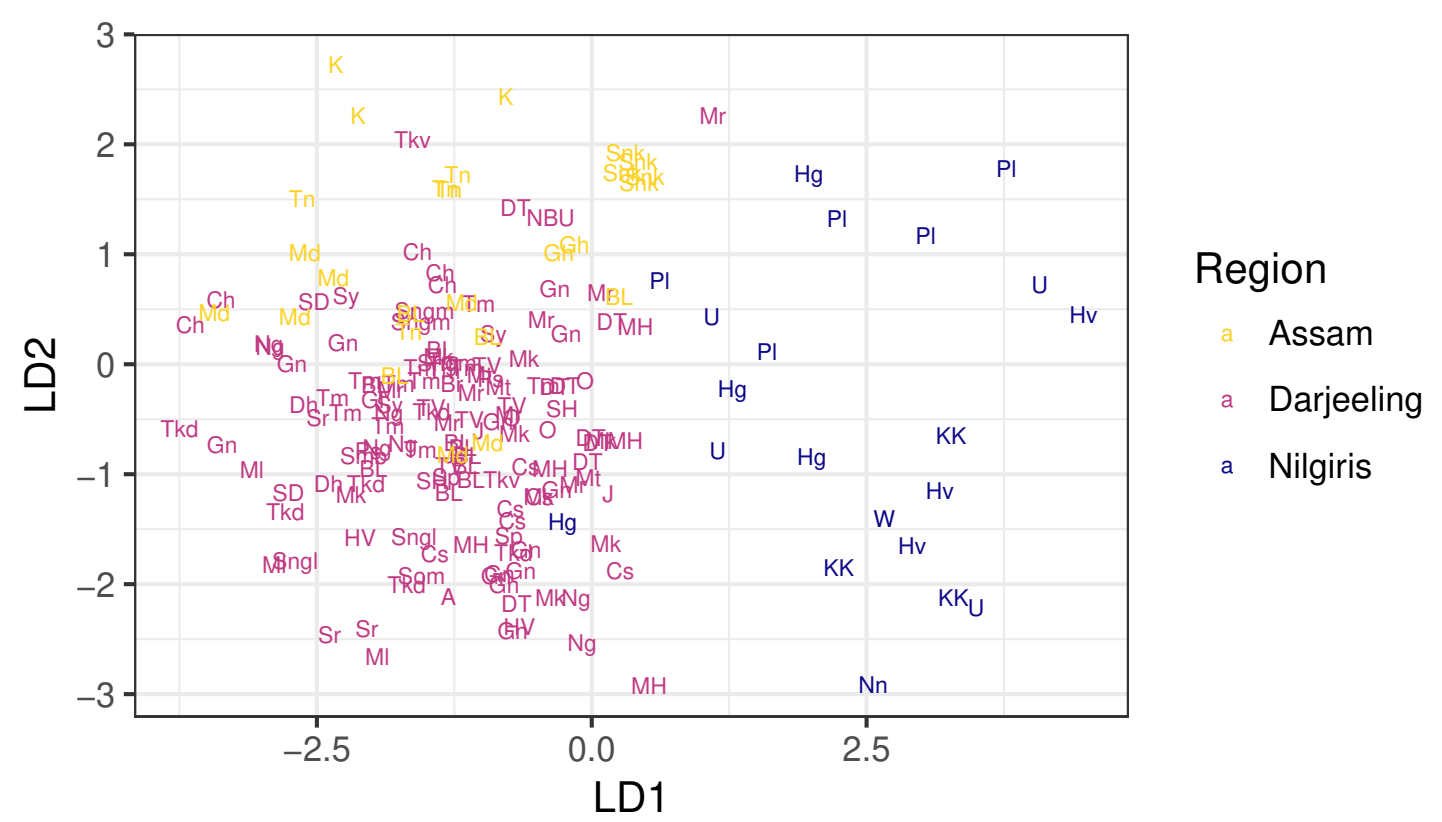

Figure 9.5.: Scatter plot of shoot tip and processed tea samples displaying the first two scores of linear discriminant functions for origin by regions of India, including the elements $\mathrm{K}$ and $\mathrm{Mg}$. Samples are colored by region and the label denotes the garden of origin. Total number of samples is $N=183$ (Darjeeling $N=136$, Assam $N=26$, Nilgiris $N=21$ ). Clr-scalings for LD1 and LD2 and misclassifications are listed in Table 9.4 Abbreviations are for Nilgiris: PI Palaniappa, U UPASI, W Warwick, Hg Highfield, Hv Havukal, KK Kil Kotagiri, Nn Nonsuch.

As before, the regions can be separated along the LD1 axis. This log-linear combination of elements is characterized roughly by $\mathrm{K}, \mathrm{Zn}$ and $\mathrm{Co}$ versus $\mathrm{Sr}, \mathrm{Rb}, \mathrm{Cs}$ and Mg. The high LD1 values of samples from Nilgiris can be interpreted as that they are relatively higher concentrated in $\mathrm{K}, \mathrm{Zn}$ and $\mathrm{Co}$ versus $\mathrm{Sr}, \mathrm{Rb}, \mathrm{Cs}$ and $\mathrm{Mg}$ than samples originating from Darjeeling or Assam. A possible source for high Co values could be the source rock material, as discussed in preceding paragraphs. High $\mathrm{K}$ concentrations are presumably caused by fertilizer application, like the recommended mixtures with muriate of potash $\mathrm{KCl}$ (Durairaj et al. 2015). A possible source of $\mathrm{Zn}$ are foliar sprays of Zinc sulphate, which is recommended to be applied four to six times every year. Both these treatments are not permitted for certified organic gardens, like most gardens in 
Darjeeling are. In contrast, the lateritic, acidic soils would be strongly depleted in soil bases, like Sr, Rb, Cs and Mg. There are no soil data of Nilgiris included in the data set, but, by field observation the soils of Paraná are similar to the soils in Nilgiris. Figure D.2 shows that the soils of Darjeeling and Assam are far less depleted in $\mathrm{Mg}, \mathrm{Sr}$, Cs and $\mathrm{Rb}$ than the soils of São Paulo and Paraná. The comparison of geochemical composition of shoot tips with corresponding subsoils in Section 8.2 shows that subcomposition Mg, $\mathrm{Rb}$ and $\mathrm{Sr}$ and the subcomposition $\mathrm{Cs}$ with $\mathrm{Sb}$ have similar log-ratios in soils and shoot tips (Figure 8.8). Resuming these findings, the low LD1-values of tea samples from Darjeeling and Assam are caused by their 'young' soils in contrast to the deeply weathered soils of Nilgiris.

The two misclassified samples of Nilgiris, point 288 in Highfield and point 301 of Palaniappa are both sampling locations where the soils are treated organically. In Highfield it was a section which was under conversion for several years, and in Palaniappa the section had been recently reactivated after several years of being overgrown. Both situations could lead to poor $\mathrm{K}$ content in the soil solution (no fertilizer) and slightly elevated bio-availability of $\mathrm{Sr}, \mathrm{Rb}, \mathrm{Cs}$ and $\mathrm{Mg}$ through increased organic content (compost, forest litter). This shows that a discrimination of tea samples originating only from organic tea gardens of Nilgiris and Darjeeling would require a subcomposition which is more specific to separate Indian tea grown on lateritic soil versus other soil types.

In summary, samples of Nilgiris can be discriminated from samples of Darjeeling and Assam due the contrasting soil geochemistry and the consequential soil management. The reasons for the spread of Nilgiris samples along the LD2 axis can not clearly be determined. There is no obvious pattern by geographical location. In Pilgrim et al. (2010) the authors have shown that samples of the Nilgiris can be separated by different tea gardens. In this publication the discrimination seems to roughly follow the geographical pattern, that samples from East and West Nilgiris plot into different groups. In contrast, in the present data set the values of the log-linear combination of LD2 points towards an influence of the conventional versus organic/integrated tea garden management: Clearly conventional gardens, like Nonsuch Estates Pvt.Limited (Nonsuch), Kil Kotagiri, Havukal, Warwick and UPASI have notably lower LD2-values than organic or gardens under conversion like Palaniappa ${ }^{1}$ and Highfield.

The discrimination of tea samples from Darjeeling and Assam requires a more spe-

\footnotetext{
${ }^{1} \mathrm{Palaniappa}$ is technically neither organic nor under conversion, but had been organic until recently, and the manager of the tea garden tries to use as less conventional products as possible (as of 2015)
} 


\section{Provenance Analysis}

cialized subcomposition, because the soil parent material and cultivation (organic/conventional) is similar for both sample groups. The data set of Darjeeling and Assam contains mostly samples from organic gardens and the soils are constituted of material derived from the Himalaya (Section 3.1.2). As described in Section 8.1.1 the geochemistry of Darjeeling and Assamese soils is very similar. The differences in the plantation setting are a) soils depth: soils in Darjeeling are partly very shallow, and roots are intruding into the solid rock, b) topography: Darjeeling is characterized by steep slopes and good drainage, while Assam is characterized by flat landscape and a drainage system is mandatory to reduce water-logging (Kabir and Das 2015), c) transport distance of source material for soils: the sediments in Assam had been transported by the river Brahmaputra, d) climate, and e) different cultivars.

To discriminate between samples from Assam and Darjeeling the subcomposition should therefore include elements which uptake is sensitive to rhizosphere - solid rock interaction, sensitive to water-logging and/or sensitive to the transport distance of source material of the soils. Elements which uptake is sensitive with respect to the climate and cultivar cannot be determined because the data set is inadequate for this purpose. As discussed in Section 7.2, an increased Al uptake could be one of the indicators for the plant's reaction to poor nutrient bio-availability, e.g. if the plant locally reduces the $\mathrm{pH}$ around the feeder root apex to mobilize nutrients from more stable phases. Regular occurring floods and seasonal changing groundwater level - like in Assam - contribute to changing Eh-pH conditions in varying soil depth. For certain elements this has a major impact on their mobility in the soils. $\mathrm{Mn}$ is one of the elements which mobility is sensitive to Eh-pH conditions in acidic soils and with very good suitability for provenance on tea. But obviously it has no discriminating power for tea samples from Assam and Darjeeling (Table 9.3). An other candidate element is $\mathrm{Tl}$, because it is general well suitable for provenance (Table E.1) and its mobility is sensitive to changing Eh-pH conditions.

It has to be noted that the mobility of $\mathrm{TI}$ in soils is still a matter of ongoing research (Grösslová et al. 2015, and references therein). Generally TI is described to have geochemically an analogous characteristics to $\mathrm{K}$. Illite clays as well as $\mathrm{Mn}$ - and Fe oxides and hydroxides are well known to have an fixating effect on TI (Kabata-Pendias 2010; Vanek et al. 2011). Also, the sorption of TI to soil organic matter (SOM) under reducing conditions is suggested to be a important parameter controlling TI bio-availability (Kabata-Pendias 2010; Grösslová et al. 2015, and references therein). Soils in Assam are described to have high content in TOC and the dominant clay mineral is illite (Section 3.1.2. The regular occurring floods and seasonal changing groundwater level contribute 
to changing Eh-pH conditions in varying soil depth.

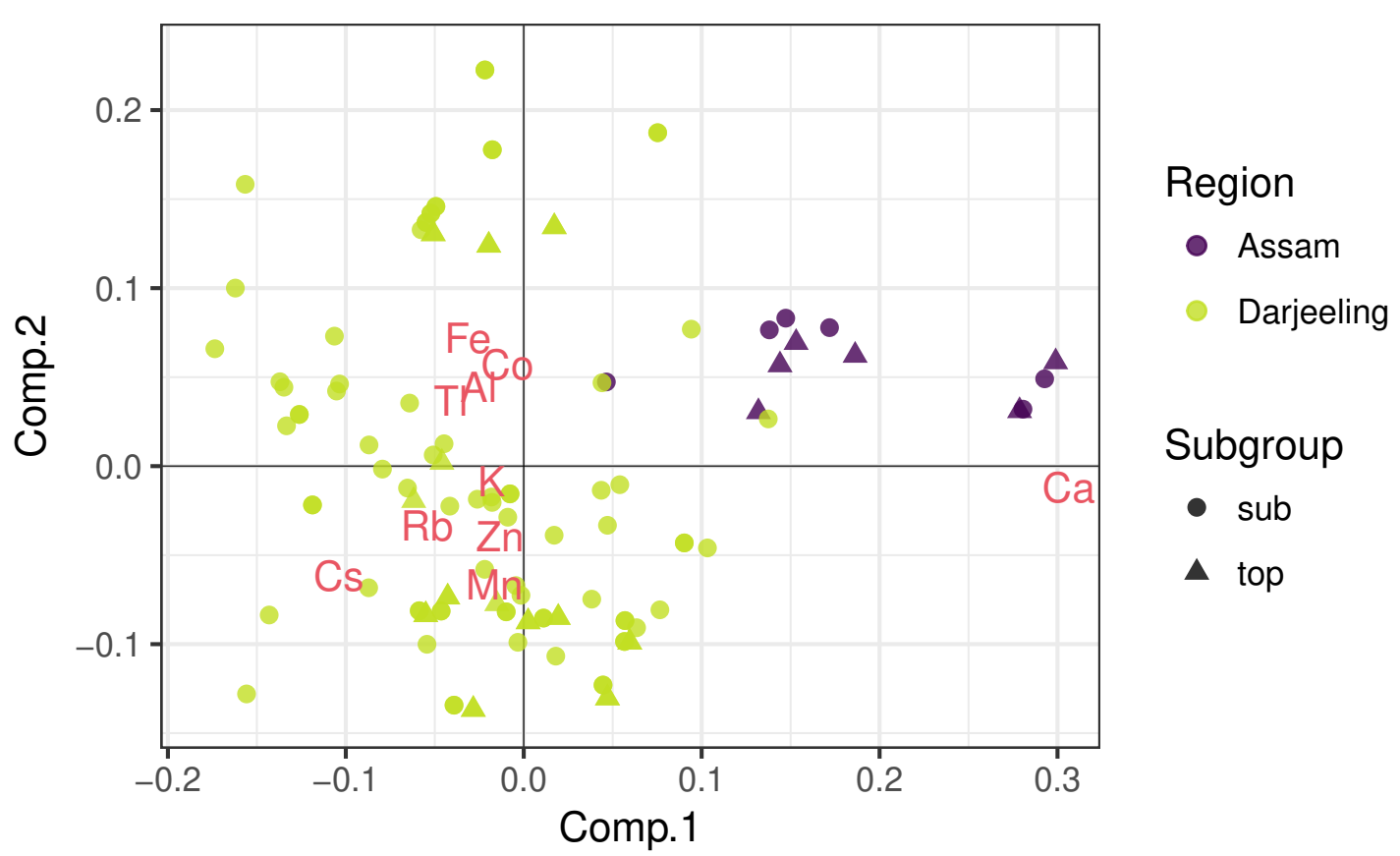

Figure 9.6.: Clr-biplot of soils from Darjeeling and Assam for the subcomposition $\mathrm{Al}, \mathrm{Ca}, \mathrm{Co}, \mathrm{Cs}$, $\mathrm{Fe}, \mathrm{K}, \mathrm{Mn}, \mathrm{Rb}, \mathrm{TI}$ and $\mathrm{Zn}$. Total explained variance is 0.77 . Total number of samples is $N=103$ (Assam $N=13$, Darjeeling $N=90$ ).

The total content of $\mathrm{TI}$ and $\mathrm{Al}$ in the soils of Assam and Darjeeling has most probably no influence on the uptake. The clr-biplot in Figure 9.6 displays that the log-ratios of $\mathrm{Al}$, Fe and $\mathrm{TI}$ have a very small spread between all soils. The main difference between the soils is the significantly higher $\mathrm{Ca}$ concentration in Assamese soils compared to Darjeeling (Figure 8.1). Supposedly, the $\mathrm{Ca}$ content increases the $\mathrm{pH}$ in the soils of Assam which would reduce the uptake of $\mathrm{Al}$ (Section 2.1.2). The role of $\mathrm{CaCO}_{3}$ for $\mathrm{TI}$ uptake is discussed diversely: On the one side $\mathrm{CaCO}_{3}$ hampers the mobility of $\mathrm{TI}$ in soils (Vanek et al. 2011), on the other it could an important source to TI (Grösslová et al. 2015). Because the source of the $\mathrm{Ca}$ in the soils of Assam is unknown, it could be either through the sediment material or the carbonate/dolomite applications, its implication for the $\mathrm{TI}$ uptake remains unclear.

Nevertheless, Figure 9.7 and Table 9.5 show that adding Al and TI to the standard sub- 


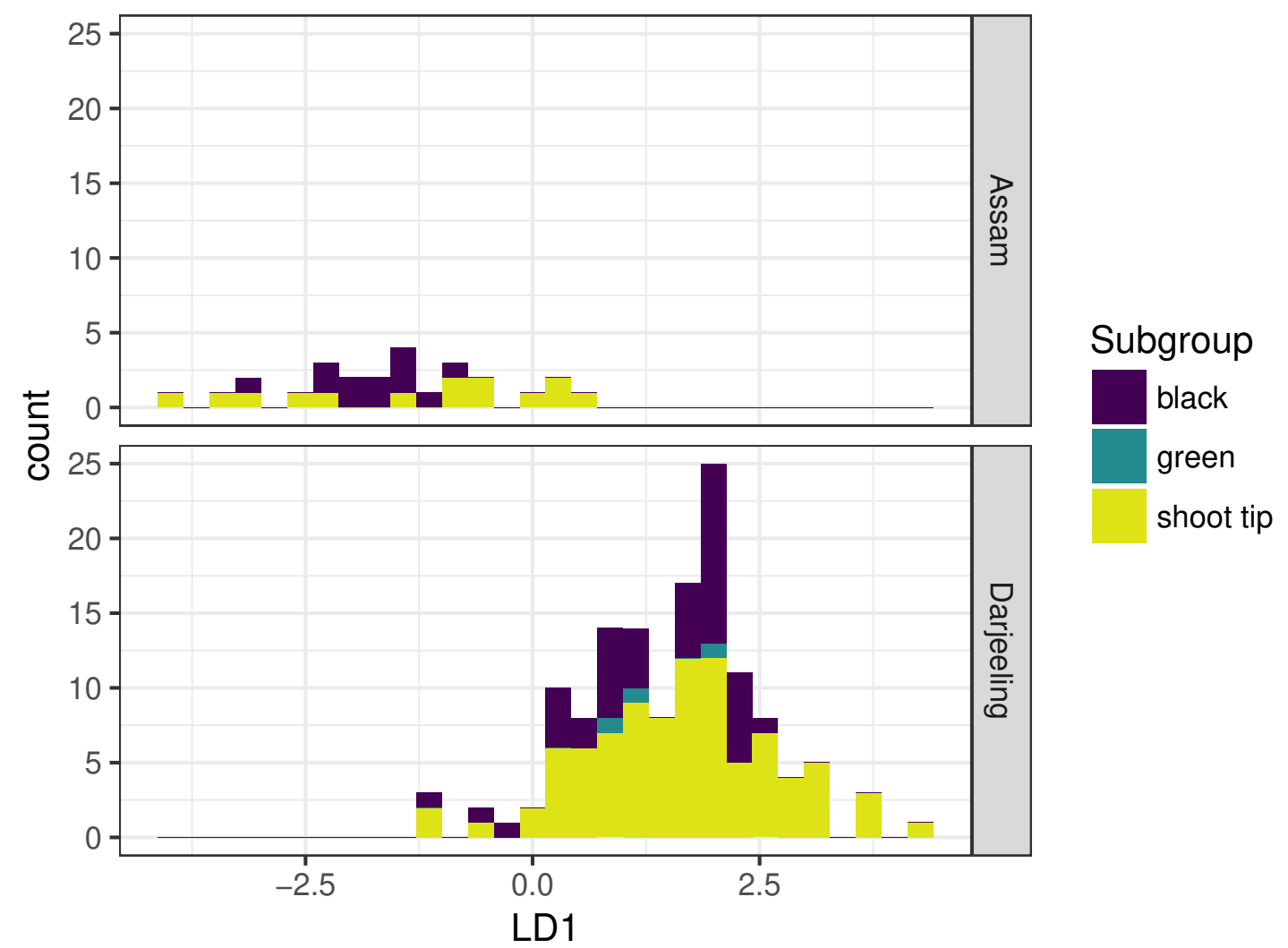

Figure 9.7.: Histogram of shoot tip and processed tea samples displaying the first score of linear discriminant function separating Darjeeling and Assam. The subcomposition includes the elements $\mathrm{Al}$ and $\mathrm{TI}$, and excludes Sr. Samples are colored by Subgroup. Total number of samples is $N=162$ (Darjeeling $N=136$, Assam $N=26$ ). Clr-scalings for LD1 and misclassifications are listed in Table 9.5

Table 9.5.: Clr-scalings of LDA (upper table) and misclassifications (lower table) for discrimination of tea from Assam and Darjeeling. The element compilation includes Al and $\mathrm{TI}$, and excludes Sr. Table corresponds to Figure 9.7). The rows of the misclassification table give the number of samples predicted into respective group.

\begin{tabular}{|c|c|c|c|c|c|c|c|}
\hline & Co & Cs & $\mathrm{Mn}$ & $\mathrm{Rb}$ & $\mathrm{Zn}$ & $\mathrm{TI}$ & $\mathrm{Al}$ \\
\hline \multirow[t]{4}{*}{ LD1 } & -0.86 & 1.06 & -0.32 & -0.94 & 1.19 & -1.29 & 1.16 \\
\hline & & & \multicolumn{2}{|c|}{ Assam } & \multicolumn{2}{|c|}{ Darjeeling } & \\
\hline & & Assa & & 22 & & 4 & \\
\hline & & arjeeli & & 5 & & & \\
\hline
\end{tabular}


composition a LDA separates the samples reasonable well according to their respective groups. Sr is excluded from the subcomposition because it is no discriminating element in this log-linear combination. The LD1 is characterized by roughly $\mathrm{Al}, \mathrm{Zn}$ and $\mathrm{Cs}$ versus $\mathrm{TI}, \mathrm{Rb}, \mathrm{Co}$ and $\mathrm{Mn}$. As expected, the positive Al values helps to discriminate Darjeeling from Assam and the negative TI values Assam from Darjeeling. The higher TI concentrations in shoot tips from Assam compared to Darjeeling (Figure 7.1) could therefore be a caused by a complex interaction of illite, SOM, water-logging and soil acidity controlling the mobility of $\mathrm{TI}$.

In summary, tea from Darjeeling can be discriminated from Assam despite very similar soil geochemistry. Elements which uptake is sensitive to $\mathrm{pH}$ and redox-conditions within a general acidic setting are good tools to capture differences of topographical situation.

\subsection{Darjeeling}

Darjeeling tea is a protected designation of origin in the European Union (Council of the European Union 2011a) and only tea grown in Darjeeling should be referred to as 'Darjeeling tea'. As demonstrated in the previous section Darjeeling tea can be discriminated from Assam tea despite very similar geological source rock material. Even more challenging is to discriminate Darjeeling from the adjacent tea producing areas in Nepal. These regions have nearly exactly the same setting of tea cultivation and production parameters including the topographical and geological setting, because the geological domains of the Himalaya orogen strike parallel to the lateral extension of the mountain range (Section 3.1.1). To figure out parameters which could possibly differentiate between East Nepal and Darjeeling the interaction of the tea plants with the their underground has to be studied.

As discussed in the preceding section, the comparably high Al concentrations in tea of Darjeeling suggests a very low $\mathrm{pH}$ in the rhizosphere. In Darjeeling, especially in tea gardens practicing an organic cultivation, the $C$. sinensis is probably forced to mobilize nutrients from the mineral fraction of the soil or even the from upper layers of the underlying rocks: The steep topography in combination with high erosion rates and locally very 'young' and shallow soils does not provide rich or stable nutrient reservoirs. Nutrient rich minerals, e.g. muscovites and plagioclase, had been described to be inhabited with bacterial and fungal communities and both are discussed to play an important role for releasing nutrients from the mineral surfaces (Gleeson et al. 2005, 2006; Koele 2012, and references therein). The coarse fraction of the soil, respectively altered upper layers 


\section{Provenance Analysis}

of the underlying rocks, is known to be one possible source of nutrients for trees, depending on the degree of weathering of the minerals (Kohler et al. 2000; Heisner et al. 2004, and references therein). Although in Darjeeling compost or other manure is regularly applied, field observations at road cuts showed that tea roots intrude up to more than two meters into solid rock. This supports the assumption that tea in Darjeeling is interacting with the weakly weathered layers of the basement rock to partly cover its nutrient and/or water supply.

Hence, in Darjeeling the ionome could be influenced by the mineral composition of the source rocks of the tea plantation areas. The geological map of Darjeeling displays that tea is mainly grown in two metamorphic zones of the MCTZ, the Grt-Mu-zone and in the St-zone, and the Daling Group (Figure 9.11). Only very few garden sections are located in the Ky-zone due to the very high elevation of this zone. Accordingly, most samples in the Darjeeling data set originate from the Grt-Mu-zone, the St-zone and the transition zone between these two zones (Table 9.6). The Daling zone crops out in the lower elevations of Darjeeling. 'Darjeeling tea' is also produced from plantations covering these lower elevations, but tea from these areas rarely achieves highest qualities. Comparing the number of samples originating of areas with the Daling Group as source rock and the areas covered by tea plantations, the Daling Group is underrepresented in this data set. For developing discrimination functions the groups with very small number of samples have to be excluded. The transition zones are also ignored for this purpose.

Table 9.6.: Number of shoot tip samples and processed tea samples by underlying source rocks in Darjeeling. 'Total N' denotes the number of all samples, including processed tea samples of gardens reaching over more than one source rock type. The second column is the number of samples which originate with high certainty from a single source rock type. Abbreviations: Grt garnet, Mu muscovite, St staurolite, Ky kyanite.

\begin{tabular}{lrr}
\hline Source rock & total N & samples with high certainty \\
\hline Alluvium & 3 & 3 \\
Daling Group & 4 & 4 \\
Grt+Mu Gneiss & 39 & 29 \\
St+Grt+Mu Gneiss & 28 & 10 \\
St Gneiss & 44 & 41 \\
Ky Gneiss & 1 & 1 \\
\hline
\end{tabular}

The geochemical composition of the soils for the standard subcomposition, including $\mathrm{K}$ and $\mathrm{Ca}$, does apparently not systematically differ between the source rock units (Figure 9.8. On the one hand this could be caused by the comparably homogeneous type 


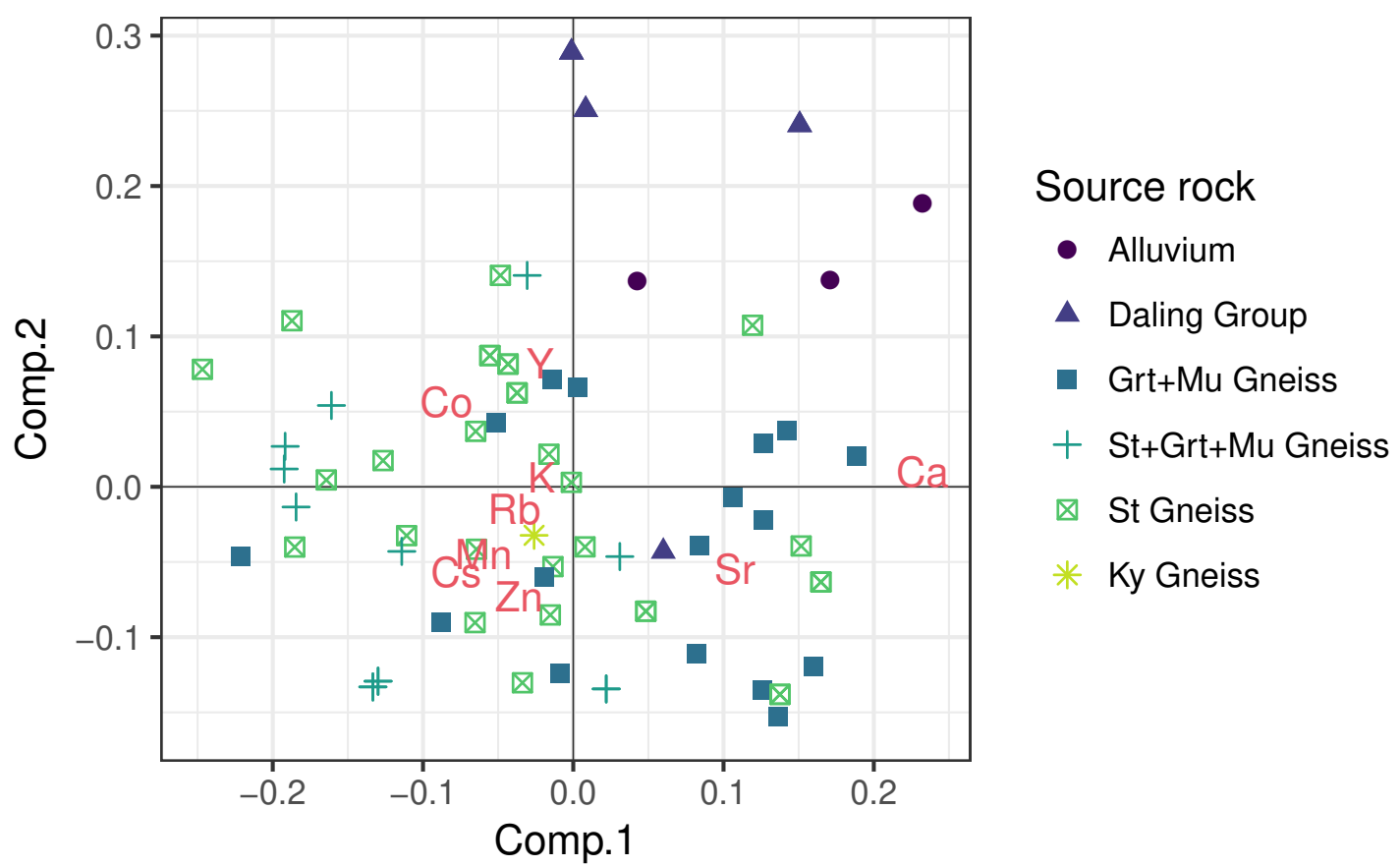

Figure 9.8.: Clr-biplot of soils from Darjeeling for the subcomposition $\mathrm{Ca}, \mathrm{Co}, \mathrm{Cs}, \mathrm{K}, \mathrm{Mn}, \mathrm{Rb}$, $\mathrm{Sr}, \mathrm{Y}$ and $\mathrm{Zn}$. Total explained variance is 0.75 . Total number of samples is $\mathrm{N}=77$. For abbreviations see caption of Table 9.6 .

of source rock: The MCTZ consists throughout whole Darjeeling of paragneisses with gradually increasing metamorphic grade from bottom to top (Section 3.1.1). On the other hand it could be a result of a homogenization of soils material through the high erosion rates and re-sedimentation of soil along the slopes.

For the LDA of tea ionome in Darjeeling the two main metamorphic zones, the Grt-Muzone and the St-zone, are used as categorical variable. Unfortunately, the samples of the two zones can not be clearly separated by the standard subcomposition. To capture the difference of mineral composition of the two zones the standard subcomposition has to be extended by main elements of the most frequent minerals. The mineral content of the Grt-Mu-zone varies strongly but the main minerals are quartz, biotite, muscovite and garnet. The mineral content of the St-zone shows also considerable variations with the main minerals being quartz, garnet, feldspar and staurolite. The feldspars are in Mottram et al. (2014a) described to be plagioclase (Section 3.1.1). Biotites and muscovites 


\section{Provenance Analysis}

belong to the mica group of the sheet silicates. The mineralogical formula for muscovite is $\mathrm{KAl}_{2}\left[\mathrm{Si}_{3} \mathrm{AlO}_{10}\right](\mathrm{OH}, \mathrm{F})_{2}$ and for biotite $\mathrm{K}\left(\mathrm{M}, \mathrm{Fe}^{2+}\right)_{3}\left[\mathrm{Si}_{3} \mathrm{AlO}_{10}\right](\mathrm{OH}, \mathrm{F})_{2}$. Hence, both micas include $\mathrm{K}$ in between their layers ('sheets') of linked $(\mathrm{SiAl}) \mathrm{O}_{4}$ tetrahedra. Plagioclase is the $\mathrm{Ca}, \mathrm{Na}$ rich member of the feldspar-group with the formula $\mathrm{Na}\left[\mathrm{AISi}_{3} \mathrm{O}_{8}\right]$ $\mathrm{Ca}\left[\mathrm{Al}_{2} \mathrm{Si}_{2} \mathrm{O}_{8}\right]$. Accordingly, $\mathrm{K}$ and $\mathrm{Ca}$ are added to the element subcomposition. In contrast to other regions most gardens in Darjeeling abstain from the use of K-containing fertilizer products and therefore the concentrations of $\mathrm{K}$ in the soils are not significantly influenced by soil management (Section 8.2 and Figure 8.2). The Ca uptake could be altered by recent application of carbonate/dolomite; only for Teesta Valley a recent application could be confirmed. Nevertheless, even for these samples no particularities in the $\mathrm{Ca}$ concentration in the ionomes could be detected.

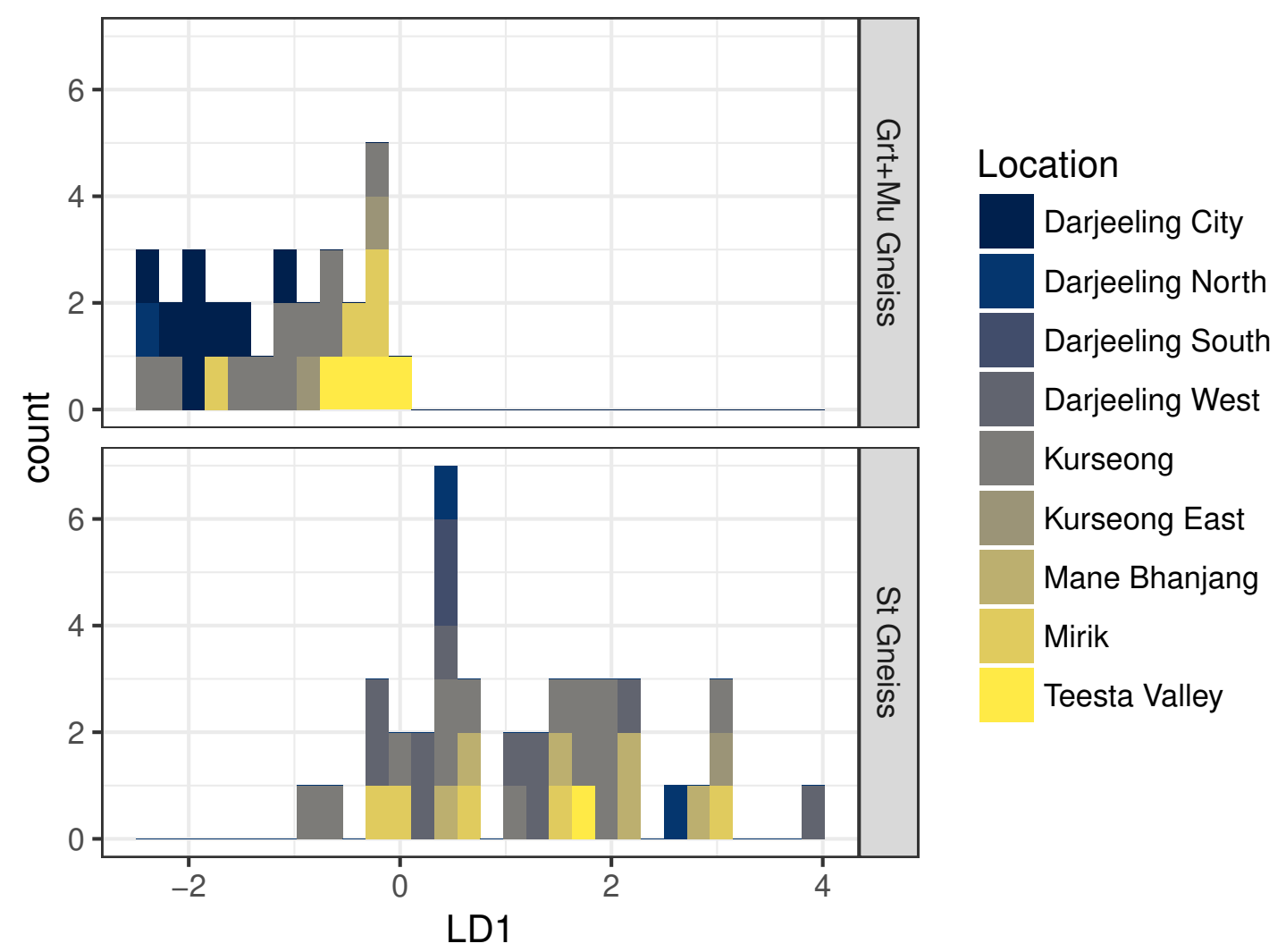

Figure 9.9.: Histogram of shoot tip and processed tea samples displaying the first score of linear discriminant function separating two metamorphic zones of Darjeeling. The element compilation includes $\mathrm{Ca}$ and $\mathrm{K}$, and excludes $\mathrm{Co}, \mathrm{Cs}$ and $\mathrm{Rb}$. Samples are colored by location in Darjeeling District. Only samples with high certainty of source rock are included (Table 9.6). Total number of samples is $N=70$ (Grt+Mu Gneiss N=29, St Gneiss N=41). For abbreviations see caption of Table 9.6. Clr-scalings for LD1 and misclassifications are listed in Table 9.7 
Table 9.7.: Clr-scalings of LDA (upper table) and misclassifications (lower table) for discrimination of tea from to metamorphic zones in Darjeeling. The element compilation includes $\mathrm{Ca}$ and $\mathrm{K}$, and excludes Co, Cs and Rb. Table corresponds to Figure E.2. The rows of the misclassification table give the number of samples predicted into respective group.

\begin{tabular}{|c|c|c|c|c|c|}
\hline & $\mathrm{Mn}$ & $\mathrm{Sr}$ & $\mathrm{Zn}$ & $\mathrm{Ca}$ & $\mathrm{K}$ \\
\hline \multirow[t]{2}{*}{ LD1 } & -2.34 & -2.69 & 0.38 & 6.26 & -1.62 \\
\hline & & Grt+l & Mu Gn & iss & St Gneiss \\
\hline \multirow{2}{*}{\multicolumn{2}{|c|}{ Grt+Mu Gneiss }} & & & 28 & 1 \\
\hline & & & & 5 & 36 \\
\hline
\end{tabular}

Figure 9.9 displays the results of a LDA with the source rocks as categorical variable and the adjusted element subcomposition including $\mathrm{Ca}$ and $\mathrm{K}$. Co, Cs and $\mathrm{Rb}$ are omitted, because they have LD1-values close to zero and apparently no discriminating power in this setting. Table 9.7 shows that with the five elements (four ilr-variables) Ca, $\mathrm{K}, \mathrm{Mn}, \mathrm{Sr}$ and $\mathrm{Zn}$ the ionome of shoot tips can be discriminated by the metamorphic grade of the parent gneisses. The discrimination can be easily enhanced if one or two trace elements are added to the subcomposition (Figure E.2, Table E.3). The coloring of the histograms indicates that the location of the sampling points has no influence on the discrimination: In both groups tea samples originate from all parts of Darjeeling District, e.g. from the southern parts like Mirik or Kurseong, or from the northern parts like Darjeeling North or Darjeeling West.

The LD1-values are characterized by $\mathrm{Ca}$ versus $\mathrm{K}, \mathrm{Mn}$ and $\mathrm{Sr}$. As expected the $\mathrm{Ca}$ weights towards the St-zone. This suggests that in the St-zone $\mathrm{Ca}$ is more easily availably for the plants than in the Grt-Mu-zone, probably due to the weathering of the plagioclase. On the other side, $\mathrm{K}$ seems to be more easily available for the plant in the Grt-Mu-zone, probably due to the high abundance of muscovites/illite/biotite. This coincides with field observations: In certain areas within the Grt-Mu-zone the soil is literally 'glittering' by micas. Interestingly, $\mathrm{Sr}$ is contrasting to $\mathrm{Ca}$ in the log-linear combination of elements for the discrimination. One possible interpretation is that in the Grt-Mu-zone the lower Ca-availability compared to the shoot tip leads to a higher $\mathrm{Sr} / \mathrm{Ca}$ ratio during uptake, because the $\mathrm{Sr}$ replaces $\mathrm{Ca}$.

Minerals of the garnet group are generally resistant to weathering processes. However, in Darjeeling the C-horizon showed weathered garnets ('red-brown dots') and in the upper layer of the soils no garnets remained. Most probably the garnets have a chemical 


\section{Provenance Analysis}

compositions between the Pyrope, $\mathrm{Mg}_{3} \mathrm{Al}_{2} \mathrm{Si}_{3} \mathrm{O}_{12}$, and Almadine, $\mathrm{Fe}_{3}{ }^{2+} \mathrm{Al}_{2} \mathrm{Si}_{3} \mathrm{O}_{12}$, end member. In the parent material of the lower elevations the garnets are not as abundant as in the zone of higher metamorphic grade. There are very few sample sets from the lowest elevations of the Grt-Mu-zone, but the data suggest that subcompositions including Mg could be sensitive to the abundance of small garnet minerals in the low- to middle grade paragneisses of Darjeeling.

In Darjeeling the metamorphic isogrades are nearly horizontally and therefore the increase in metamorphic degree correlates about with the altitude. This can be used to very roughly approximate the altitude of the tea plant's origin within the Grt-Mu-zone and the St-zone. For this purpose the subcompositions $\mathrm{Ca}, \mathrm{K}, \mathrm{Mn}, \mathrm{Sr}, \mathrm{Y}$ and $\mathrm{Zn}$ of all shoot tip data of this zone are projected by means of the LDA scalings from Figure E.2 onto a LD1 axis.

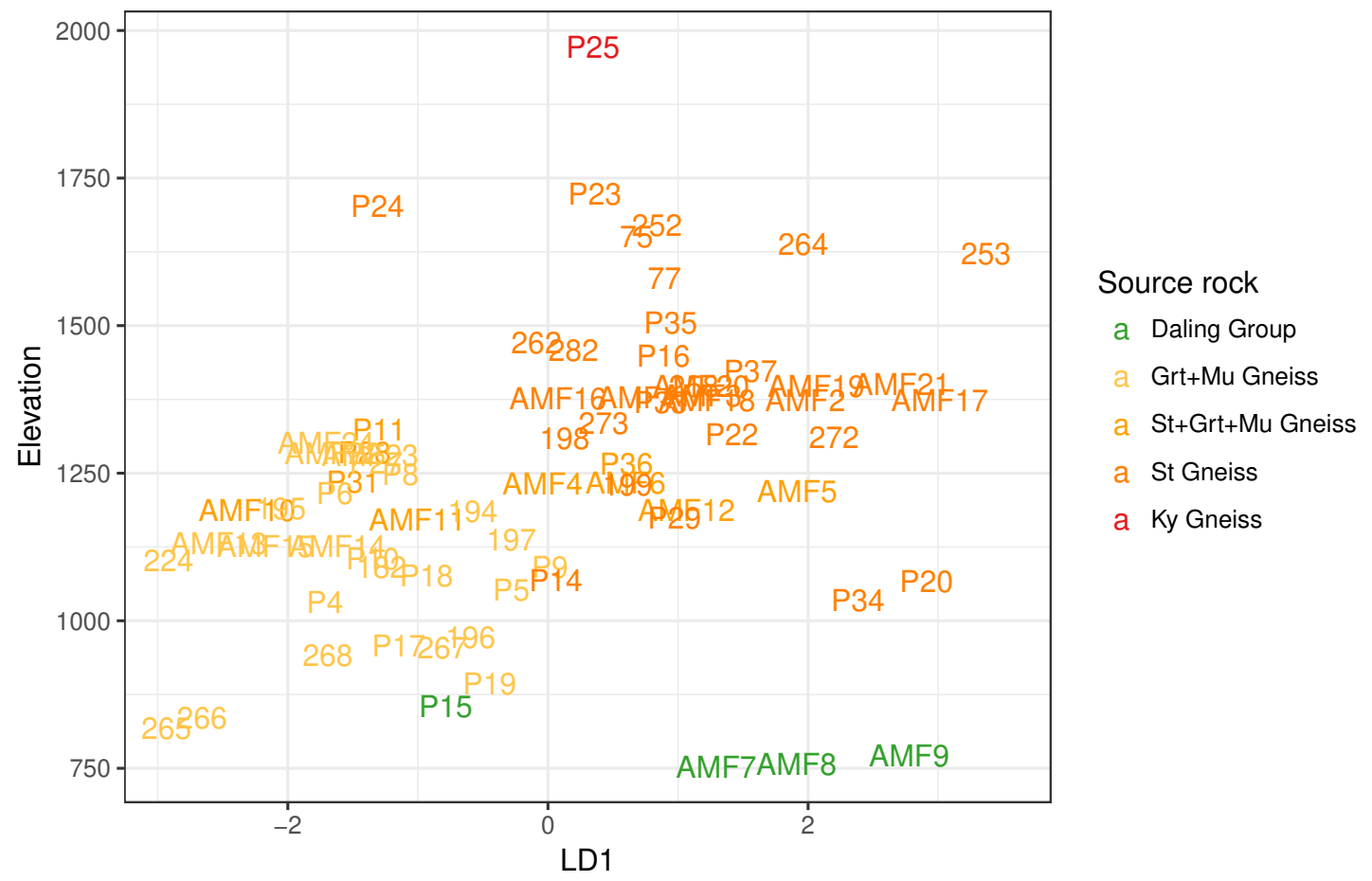

Figure 9.10.: Scatter plot for shoot tip samples of topographical elevation versus LD1 values calculated by the log-linear combination of Table 9.7. Samples are colored by geological source rock of Darjeeling District. The labels denote the sampling points. For abbreviations see caption of Table 9.6 .

In Figure 9.10 these 'LD1' values are plotted against the elevation of the sampling points. From this plot four observations can be drawn: 1) It is confirmed that within the Grt-Mu-zone and the St-zone the LD1 values very roughly increase. But estimat- 
ing the altitude of the samples by this methods will be subject to a high uncertainty. 2) But, the method is precise enough to find major tectonic offsets in this area: The three samples P14, P20 and P34 are notably 'too deep': All three of these sampling points are located on the southern slope or the northern slope of Darjeeling District. Field observations showed along the topographic 'edges' towards the southern plain or the deep valley of the Teesta River sets of steep dipping normal faults. P14 and P20 could be confirmed by field work that in these locations the source rocks are build up by units of higher grade metamorphic gneisses - presumably huge blocks which had been moved downhill through tectonic events. 3) The sample of the Ky-zone reveals medium LD1-values. The two most prominent minerals of this zone are biotite and kyanite, and accordingly the biotite could serve as a K-source for the plants, similar to the micas of the Grt-Mu-zone. Under this aspect it is logical that with increasing elevation LD1-values - which discriminate between $\mathrm{K}$ and $\mathrm{Ca}$ rich mineral phases - would again decrease. Additionally, small landslides and intense erosion is a common phenomenon in these very high and steep plantations. Depending on the situation this contributes to a massive downhill transport of fresh material of higher metamorphic degree. 4) The 'AMF' samples growing on Daling Group source rocks show 'too high' LD1 values in relation to their elevation. This data have to be very carefully interpreted because all three samples originate from the same sampling location in the Mullootar Tea Estate, Ambootia Tea Group (Mullootar). It could be a hint for poor K-availability on Daling Group source rocks: The Daling Group consists of amongst other rocks of greenish colored phyllites with a high content of chlorites. Probably one of the main minerals is the chlorite Clinochlore $\left(\left[\mathrm{Mg}, \mathrm{Fe}^{2+}\right]_{5} \mathrm{Al}\left(\mathrm{Si}_{3} \mathrm{Al}\right) \mathrm{O}_{10}(\mathrm{OH})_{8}\right)$. These minerals could be a good source for $\mathrm{Mg}$, Fe and in minor quantities Mn but not for $\mathrm{K}$. However, due to the number of samples the interpretation is highly speculative. The sampling point P15 is mapped to lie within the Daling Group, but the extrapolated contact zone between the Daling Group in the footwall and the Grt-Mu-zone in the hanging wall is not even at $50 \mathrm{~m}$ distance. Considering that this is a tectonically very active region and mapping in general is very challenging on these slopes, the plants did not necessarily grow only on Daling Group source rock material.

In summary, the log-linear combination of Table E.3 could serve in Darjeeling as a proxy for the relative mica content in geological source rock. Then again, the content in mica is related to the geological unit, LHS versus MCTZ, and the metamorphic grade of the paragneisses in the MCTZ. Because the metamorphic grade constantly increases with increasing topographical altitude this subcomposition $\mathrm{Ca}, \mathrm{K}, \mathrm{Mn}, \mathrm{Sr}, \mathrm{Y}$ and $\mathrm{Zn}$ may help to very roughly approximate the growing elevation of the tea plant. 


\section{Provenance Analysis}

Approximating the altitude will not discriminate between samples from Darjeeling and adjacent gardens in Nepal. But this case study demonstrates how sensitive the ionome of $C$. sinensis can be in terms of mineral composition of the source rock material - or soil parameters which are strongly influenced by this. If the subcompositions of these case studies are going to be extended by even more sensitive trace elements, like $\mathrm{Ba}$ or the REE, there is probably a high potential for conduction provenance analysis with a high spatial resolution. First results from a data set with samples from Nepal encourage this hypothesis. 


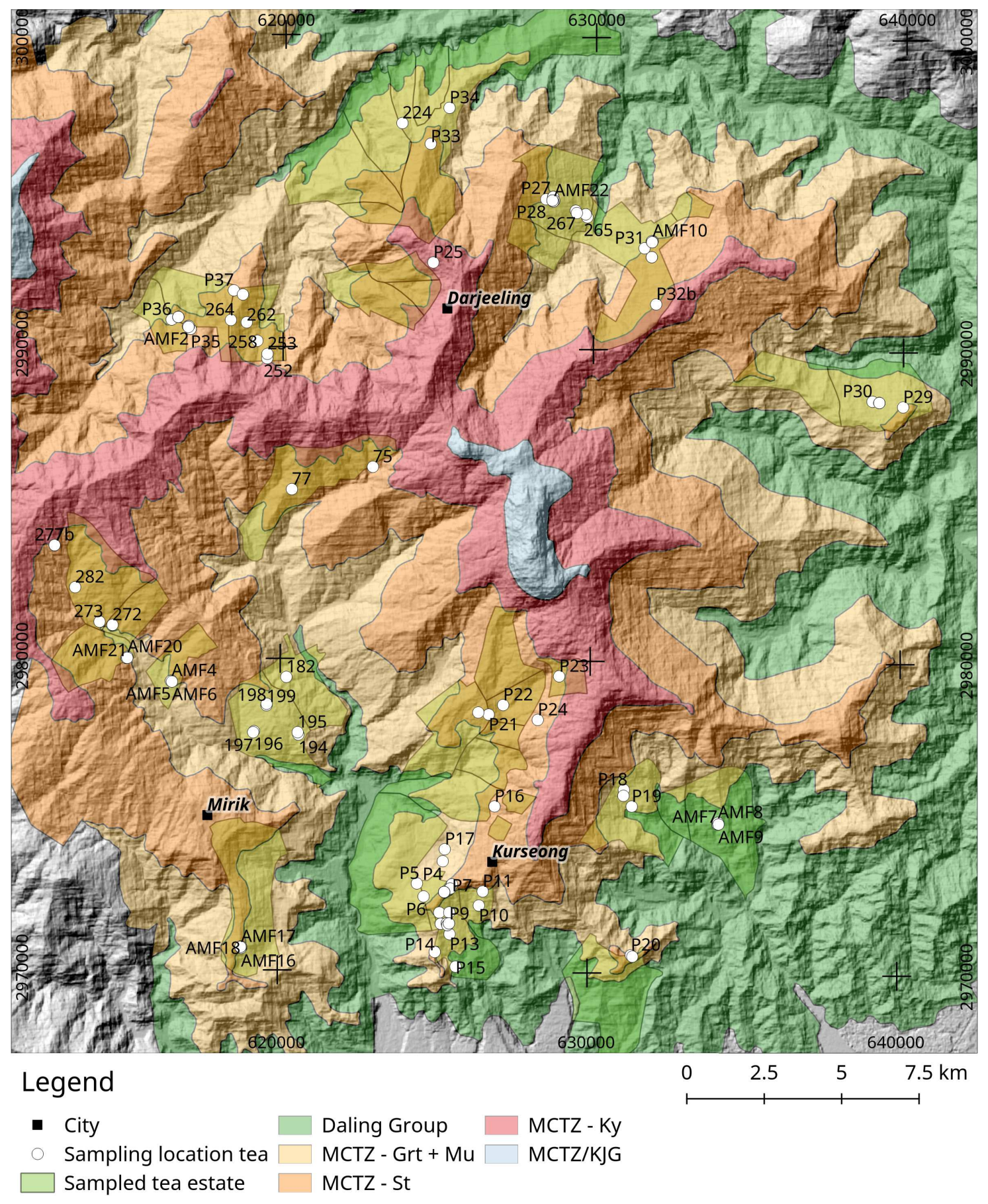

Figure 9.11.: Map of geological units in Darjeeling District with sampling locations of shoot tip samples. DEM downloaded from http://bhuvan-noeda.nrsc.gov.in, CartoDEM Ver.3, Toposheet No. G45K, projection WGS 84, UTM Zone 45R, Pseudo Mercator 


\section{Provenance Analysis}

\subsection{Summary}

The results of the chapters 6, 7 and 8 allow to estimate for each element how intensely its concentration is altered by a single parameter or a combination of parameter. Additionally, field observations and personal communications help to weight the impact of a parameter. The combination of rating elements and weighting is a qualitative approach to express the robustness of each element concentration in respect to a specific tea cultivation and production: The element list sorted from high suitability for provenance studies to low suitability is Mn, Rb, Cs, Sr, Co, Zn, Cu, Sb, La, Mg, TI, K, Cd, Pb, Mo, Cr, $\mathrm{Al}, \mathrm{Y}, \mathrm{Ni}, \mathrm{Li}, \mathrm{Fe}, \mathrm{Ca}, \mathrm{P}, \mathrm{Na}, \mathrm{S}, \mathrm{Ba}, \mathrm{Sc}$.

In this thesis the tea provenance studies are based on subcompositions which are selected by their general suitability, respectively robustness, and less by their discriminative power in this specific data set. To show the principles of this 'robustness-approach' three case studies are presented with varying categorical variables: 1) Discrimination by countries, 2) discrimination by regions in India and 3) discrimination by geological metamorphic zones in Darjeeling. The six elements with the highest suitability/robustness rating are used as the 'standard' subcomposition: $\mathrm{Mn}, \mathrm{Rb}, \mathrm{Cs}$, Sr, Co and $\mathrm{Zn}$.

The results of the LDA with the standard element subcomposition exhibit that this subcomposition is suitable to distinguish tea samples roughly by country and by their geological source material. If the standard element subcomposition is extended by elements which capture local specifics the discrimination power between countries can be increased: The results of the LDA with the subcomposition of $\mathrm{Mn}, \mathrm{Rb}, \mathrm{Cs}, \mathrm{Sr}, \mathrm{Co}, \mathrm{Zn}, \mathrm{K}$, $\mathrm{P}$ and $\mathrm{Li}$ shows that three additional elements $\mathrm{K}, \mathrm{P}$ and $\mathrm{Li}$ help significantly to improve the discrimination between the groups. Only the plants growing on soils with geological parent material containing mafic minerals are still misclassified by this combination of variables.

The samples from Darjeeling and Assam can be discriminated from samples of Nilgiris by the standard subcomposition with roughly a log-linear combination of $\mathrm{Co}$ and $\mathrm{Zn}$ versus $\mathrm{Rb}$. These results are caused by their 'young' soils in contrast to the deeply weathered soils of Nilgiris: Samples of Nilgiris can be discriminated from samples of Darjeeling and Assam due the contrasting soil geochemistry and the consequential soil management. Tea from Darjeeling can be discriminated from Assam despite very similar soil geochemistry. In both regions the soils dispaly a general acidic setting. In this case elements for which their uptake is sensitive to $\mathrm{pH}$ and redox-conditions are good tools to capture the differences of topographical situation. Accordingly, the discriminating log- 
linear combination is roughly $\mathrm{Al}, \mathrm{Zn}$ and $\mathrm{Cs}$ versus $\mathrm{TI}, \mathrm{Rb}$, Co and $\mathrm{Mn}$.

In Darjeeling the ionome is probably influenced by the mineral composition of the source rocks of the tea plantation areas. The tea is mainly grown in two metamorphic zones of the MCTZ, the Grt-Mu-zone and in the St-zone, and the Daling Group. The geochemical composition of the soils for the standard subcomposition, including $\mathrm{K}$ and $\mathrm{Ca}$, does apparently not systematically differ between the source rock units. To capture the difference of mineral composition of the two zones the standard subcomposition has to be extended by main elements of the most frequent minerals, $\mathrm{K}$ and $\mathrm{Ca}$. With the five elements (four ilr-variables) $\mathrm{Ca}, \mathrm{K}, \mathrm{Mn}, \mathrm{Sr}$ and $\mathrm{Zn}$ the ionome of shoot tips can be discriminated by the metamorphic grade of the parent gneisses. The location of the sampling points in Darjeeling has no influence on the discrimination by metamorphic zone.

The parameter of cultivars does not seem to induce a visible bias on discrimination by regions. 



\section{Conclusions and Outlook}

\subsection{Conclusions}

A world spanning provenance analysis for tea requires a statistical model which considers that tea is produced in several continents under a broad spectrum of cultivation conditions and manufacturing settings. One task of such a model is to establish methods which will help to reduce the minimal size of the training data sets required for a world wide determination of origin with a high resolution. The necessary size of the data set can be reduced if less elements for the discriminating functions are used and/or if the sample groups are more homogeneous: For example, in a data set all samples are CTC black processed teas produced of only $C$. sinensis var. assamica leaf material which was harvested manually with '2 and bud' plucking standard in shaded, organic plantations. This thesis explores the potential of using the ionome, - all the mineral nutrients and trace elements-, of shoot tips of $C$. sinensis for provenance studies and presents methods to determine suitable element subcompositions.

The ionome of tea develops through a combination of parameters of varying impact. One of the main parameters is the geochemical composition of the soils, but also important are climate, topography, soil $\mathrm{pH}$, fertilizing regimes, harvesting method, plucking standard, type of cultivar, season of harvesting, maintenance of the tea bushes, type of plantation, production machines, leaf grade of the processed tea and last but not least, blending. Some of these parameters change between seasons or years, some change within decades and some can be considered as stable. Stable parameters are the geochemical composition of soils (without the fertilizer influence), the topography, and - to some extend - the climate.

Geographical determination is most robust if based on element subcompositions of the ionome which are dominated by the stable parameters. The geographical resolution can be increased either by combining these subcompositions with elements which log-ratios are sensitive to other parameters, or by 'filtering' the data sets prior to the discriminant analysis by with respect to specific parameters. 


\section{Conclusions and Outlook}

The main result of this thesis is the determination of an element subcomposition of the ionome which is dominated by the geochemical composition of the soils and with negligible influence by other parameters. This is achieved by a combination of statistical techniques adjusted for compositional data analysis: Based on the data set used for this thesis the 'standard' subcomposition for geographical discrimination consists of $\mathrm{Mn}$, $\mathrm{Rb}, \mathrm{Cs}, \mathrm{Sr}$, Co and $\mathrm{Zn}$. To increase the discrimination power between two regions this subcomposition can be modified by adding or removing elements. For example, adding $\mathrm{TI}$ could serve as an indicator for water-logging, $\mathrm{Ni}$ and $\mathrm{Co}$ for mafic source rock material, or $\mathrm{K}$ and $\mathrm{Ca}$ as proxy for mineral composition of the underlying basement rocks.

One of the major factors affecting the elemental composition of a leaf sample is the ratio of young (sink) leaves versus mature (source) leaves. The sink/source leaf ratio in processed tea samples results from the harvesting method, plucking standard and the sorting of processed tea material after manufacturing. The average leaf age of a processed tea sample can be estimated by the value of the balanced ratio of [Cu K | Al Ca Mn]. Filtering a data set by this value helps to homogenize the data set in terms of the parameters plucking standard and sorting.

Elements which are typically influenced by fertilizer application are $\mathrm{P}$ and $\mathrm{K}$ and occasionally $\mathrm{S}$. $\mathrm{Ca}$ is potentially influenced by carbonate/dolomite applications to mitigate the $\mathrm{pH}$ of the tea soils. Although foliar applications alter other element concentrations in the leaf material as well, effects of foliar sprays containing e.g. Zn or Cu could not be shown in the present data set. In combination with other subcomposition the 'fertilizer' subcomposition can be used to indicate regions where the soil fertility status is very low and therefore intensive fertilizer application is used to meet the plant's requirements.

Processed teas can be contaminated with $\mathrm{Cr}, \mathrm{Pb}, \mathrm{Sb}, \mathrm{Sc}$ and $\mathrm{Sn}$ by machine abrasion, depending on the type of manufacturing. If the production conditions are unknown these elements should be excluded for provenance studies. In combination with other subcompositions this subcomposition could serve as a proxy for geographical region, because each tea producing region has its 'typical' type of manufacturing.

Generally, the plants' geochemical compositions can also be altered by air-borne or soil-borne adhering particles. Therefore, plant data should always be corrected for adhering particles prior to statistical analysis. Assuming that for tea the geochemical composition of the adhering particles can be approximated by the geochemical composition of the soils of the area the elements $\mathrm{Th}, \mathrm{Ta}, \mathrm{Hf}, \mathrm{Zr}, \mathrm{U}, \mathrm{Nb}$ and $\mathrm{Ti}$ should be skipped for provenance analysis. The error of their corrected values is too high for a sound usage of these values. 


\subsection{Outlook}

As mentioned in the introduction, the task of developing a holistic model for world-wide provenance studies on tea is not solved by this thesis. This thesis proposes merely statistical approaches for several of the components of such a model.

Many components of an holistic model are not discussed in this thesis. The most important one is the blending of tea. The blending can change the elemental composition of processed tea samples up to an extend that it is impossible to conduct provenance analysis. At the best, blending will give a 'blend signature' which helps to distinguish prior to any other statistical analysis - blends from tea of several regions from blends of tea originating from small, distinct areas. First data point towards such a 'blend signature', but investigating the effect of blending on the resolution of provenance studies is of uttermost importance for future projects.

In order to develop further components for a holistic model the data set has to be increased. Interesting data sets would be samples of the same region, but with contrasting cultivation techniques, sample sets of the same cultivar growing in contrasting climate conditions, samples sets where only the harvesting method differs, and generally sample sets from China, Indonesia, Sri Lanka, Kenia, Vietnam and Japan. A bigger and more diverse data set will also alter the results of this thesis.

One part of developing the holistic model will be to quantify the robustness and suitability of elements or element subcompositions in respect to certain parameters. This requires a bigger data set for the discussion of further parameters and a more complex statistical approach, e.g. through intercalating the analysis of several parameters. Several of the proposed methods have to refined, like introducing techniques which incorporate the analytical errors of each data point and meet the special requirements of compositional data.

Last but not least, future projects should include more variables. For example, the existing data set provides also lanthanide data which are not discussed in this thesis but showed encouraging results for provenance studies. The data set could also be combined with isotopic data and/or data of organic components. A better understanding of the plant/soil interaction would help to interpret the variables in terms of their impact for provenance studies. Especially interesting will be to explore the role of trace elements and elements which are in their chemical characteristics analogous to nutrients. 



\section{Bibliography}

Acharyya, Subhrangsu K. (1989). The Daling group, its nomenclature, tectonostratigraphy and structural grain: with notes on their possible equivalents. Geological Survey of India Special Publication, 22, 5-13.

Acharyya, Subhrangsu K., Subhajit Ghosh, Santanu Bose, Nibir Mandal, and Kanchan Pande (2017). Pre-Himalayan tectono-magmatic imprints in the Darjeeling-Sikkim Himalaya (DSH) constrained by $40 \mathrm{Ar} / 39$ Ar dating of muscovite. 146.

Acharyya, Subhrangsu K. and K. K. Ray (1977). "Geology of the Darjeeling-Sikkim Himalaya. Guide to Excursion No. 3". Fourth International Gondwana Symposium (Calcutta). Geological Survey of India, 1-25.

Aitchison, John (1982). The statistical analysis of compositional data. Journal of the Royal Statistical Society. Series B (Methodological), 44.2, 139-177. URL: http://www . jstor . org/ stable/2345821.

- (1986). The statistical analysis of compositional data. London, UK, UK: Chapman \& Hall, Ltd.

- (2003). "A concise guide to compositional data analysis". CDA Workshop, Girona.

Aitchison, John and Michael Greenacre (2002). Biplots for compositional data. Journal of the Royal Statistical Society, Series C (Applied Statistics), 51.4, 375-392.

Anczkiewicz, Robert, Sumit Chakraborty, Somnath Dasgupta, Dilip K. Mukhopadhyay, and Katarzyna Kołtonik (2014). Timing, duration and inversion of prograde Barrovian metamorphism constrained by high resolution Lu-Hf garnet dating: a case study from the Sikkim Himalaya, North-East India. Earth and Planetary Science Letters, 407, 70-81. DOI: 10.1016/j . epsl . 2014.09.035.

Anke, Manfred and Mathias Seifert (2004). "Titanium". Elements and Their Compounds in the Environment. Ed. by E. Merian, M. Anke, M. Innat, and M. Stoeppler. Wiley-VCH Verlag GmbH, 1125-1140. DOI: 10.1002/9783527619634.ch45.

Antal, Karoly (2017). gnumeric: Read Data from Files Readable by 'gnumeric'. URL: https : //CRAN.R-project. org/package=gnumeric

Aono, Hideya, Yoshimitsu Yanase, and Shizuo Tanaka (1981). Studies on the development and distribution of roots and its soil conservation faculty in tea plants: IV. Influences of subsoil improvement and irrigation on the development of tea roots. Japanese Journal of Crop Science, 50.3, 276-281. DOI: $10.1626 /$ jcs . 50.276.

Arkley, T. H., D. N. Munns, and C. M. Johnson (1960). Preparation of plant tissues for micronutrient analysis. removal of dust and spray contaminants. Journal of Agricultural and Food Chemistry, 8, 318-321.

Ashraf, Waqar and Atiq A. Mian (2008). Levels of selected heavy metals in black tea varieties consumed in Saudi Arabia. Bulletin of Environmental Contamination and Toxicology, 81.1, 101-104. DOI: $10.1007 / \mathrm{s} 00128-008-9402-0$

Baker, A. J. M., S. P. McGrath, C. M. D. Sidoli, and R. D. Reeves (1994). The possibility of in situ heavy metal decontamination of polluted soils using crops of metal-accumulating plants. Resour. Conserv. Recy. 11, 41-49. 


\section{Bibliography}

Banerjee, B (1992). "Botanical classification of tea". Tea. Springer, 25-51.

Bargagli, R., D. H. Brown, and L. Nelli (1995). Metal biomonitoring with mosses: Procedures for correcting for soil contamination. Environmental Pollution, 89, 169-175.

Barker, Allen V and David J Pilbeam (2015). Handbook of plant nutrition. CRC press.

Baruah, T. C., Raj Pal, S. R. Poonia, and R. S. Siyag (1983). Exchange equilibria involving aluminium versus calcium, potassium and ammonium in some acid soils of Assam. Journal of the Indian Society of Soil Science, 31.3, 387-393.

Baxter, Ivan (2015). Should we treat the ionome as a combination of individual elements, or should we be deriving novel combined traits? Journal of Experimental Botany, 66.8, 21272131. DOI: $10.1093 / \mathrm{jxb} /$ erv040

Bhagat, R. M., R. Deb Baruah, and S. Safique (2010). Climate and tea [Camellia sinensis (L.) O. Kuntze] production with special reference to north east India: A review. Journal of Environmental Research And Development, 4.

Bhattacharyya, Kathakali and Gautam Mitra (2009). A new kinematic evolutionary model for the growth of a duplex - an example from the Rangit duplex, Sikkim Himalaya, India. Gondwana Research, 16.3, 697-715. DOI: 10.1016/j.gr.2009.07.006.

- (2014). Spatial variations in deformation mechanisms along the Main Central thrust zone: implications for the evolution of the MCT in the Darjeeling - Sikkim Himalaya. Journal of Asian Earth Sciences, 96.Supplement C, 132-147. DOI: $10.1016 / j \cdot j$ seaes.2014.08.035.

Boogaart, K. Gerald van den and Raimon Tolosana-Delgado (2008). "compositions": a unified r package to analyze compositional data. Computers \& Geosciences, 34.4, 320-338. DOI: $10.1016 / \mathrm{j}$. cageo.2006.11.017

- (2013). Analyzing Compositional Data with R. Springer, Heidelberg.

Buccianti, Antonella, Glòria Mateu-Figueras, and Vera Pawlowsky-Glahn (2006). Compositional data analysis in the geosciences: from theory to practice. 264. Geological Society.

Burchfiel, Burrell C, Chen Zhiliang, Kip V Hodges, Liu Yuping, Leigh H Royden, Deng Changrong, and Xu Jiene (1992). The South Tibetan detachment system, Himalayan orogen: Extension contemporaneous with and parallel to shortening in a collisional mountain belt. Geological Society of America Special Papers, 269, 1-41.

Burg, J. P. and G. M. Chen (1984). Tectonics and structural zonation of southern Tibet, China. Nature, 311, 219-223.

Campanha, G.A. da C. and G.R. Sadowski (1999). Tectonics of the southern portion of the Ribeira Belt (Apiaí Domain). Precambrian Research, 98.1, 31-51. Dol: 10 .1016/S03019268 (99) 00027-3.

Caner, L., D. Lo Seen, Y. Gunnell, B. R. Ramesh, and G. Bourgeon (2007). Spatial heterogeneity of land cover response to climatic change in the Nilgiri highlands (Southern India) since the last glacial maximum. The Holocene, 17.2, 195-205. DoI: 10.1177/0959683607075833.

Cao, Xinde, Guiwen Zhao, Ming Yin, and Jiaxi Li (1998). Determination of ultratrace rare earth elements in tea by inductively coupled plasma mass spectrometry with microwave digestion and AG50W-x8 cation exchange chromatography. The Analyst, 123.5, 1115-1119. DOI: 10 . 1039/a708881g

Carr, Hilda Premi, E. Lombi, H. Küpper, S. P. McGrath, and Ming Hung Wong (2003). Accumulation and distribution of aluminium and other elements in tea (Camellia sinensis) leaves. Agronomie, 23.8, 705-710. Dol: 10.1051/agro:2003045.

Carr, M. K. V. (1972). The climatic requirements of the tea plant: A review. Experimental Agriculture, 8.01, 1-14. 
Carr, M. K. V. and S. Carr (1971). Water and the tea plant. Ed. by M. K. V. Carr and S. Carr. Kericho, Kenya, Tea Boards of Kenya, Uganda, and the Tanzania Tea Authority.

Carr, M. K. V., M. O. Dale, and William Stephens (1987). Yield distribution in irrigated tea (Camellia sinensis) at two sites in Eastern Africa. Experimental Agriculture, 23.01, 75-85.

Cary, E. E. (1992). Correction of elemental analysis of soil-grown wheat roots contaminated with soil. Journal of Plant Nutrition, 15.6-7, 857-869. Dol: 10.1080/01904169209364368.

Catlos, E. J., C. S. Dubey, T. Mark Harrison, and M. A. Edwards (2004). Late Miocene movement within the Himalayan Main Central Thrust shear zone, Sikkim, North-East India. Journal of Metamorphic Geology, 22.3, 207-226. DOI: 10.1111/j.1525-1314.2004.00509.x.

Censi, P., F. Cibella, E.E. Falcone, G. Cuttitta, F. Saiano, C. Inguaggiato, and V. Latteo (2017). Rare earths and trace elements contents in leaves: a new indicator of the composition of atmospheric dust. Chemosphere, 169, 342-350. DOI: 10.1016/j . chemosphere . 2016. 11.085 .

Chakraborn, SK, H Sinha, and BS Mathur (1984). Morphological and physicochemical properties of some alluvial soils of Assam. Journal of the Indian Society of Soil Science, 32.1, 128-136.

Chakraborty, Sumit, Robert Anczkiewicz, F. Gaidies, D. Rubatto, N. Sorcar, K. Faak, D.K. Mukhopadhyay, and Somnath Dasgupta (2016). A review of thermal history and timescales of tectonometamorphic processes in Sikkim Himalaya (North-East India) and implications for rates of metamorphic processes. Journal of Metamorphic Geology, 34.8, 785-803. DOI: 10 . $1111 /$ jmg. 12200 .

Chakraborty, Tapan and Parthasarathi Ghosh (2010). The geomorphology and sedimentology of the Tista megafan, Darjeeling Himalaya: Implications for megafan building processes. Geomorphology, 115.3-4, 252-266. DOI:10.1016/j.geomorph.2009.06.035

Chakravarty, D. N., G. Dev, and J. L. Sehgal (1985). Elemental distribution as an index of weathering stage for typical alluvium-derived soils of Assam. Proceedings of the Indian National Science Academy, Part A. Physical sciences, 51.5, 882-886.

Chakravarty, D. N., Jawahar L. Sehgal, and G. Dev (1979). Sand mineralogy of the alluviumderived soils of southern bank of the river Brahmaputra in Assam. Journal of the Indian Society of Soil Science, 27.4, 417-426.

Chamberlain, A. C. (1983). Fallout of lead and uptake by crops. Atmos. Environ. 17.4, 693-706. DOI: 10.1016/0004-6981(83)90416-X.

Chen, Quansheng, Jiewen Zhao, and Hao Lin (2009). Study on discrimination of roast green tea (Camellia sinensis L.) according to geographical origin by FT-NIR spectroscopy and supervised pattern recognition. Spectrochimica acta. Part A, Molecular and biomolecular spectroscopy, 72.4, 845-850. DOI: 10.1016/j . saa.2008.12.002

Chen, Yingxu, Jie Xu, Mingge Yu, Xincai Chen, and Jiyan Shi (2010). Lead contamination in different varieties of tea plant (Camellia sinensis L.) and factors affecting lead bioavailability. Journal of the Science of Food and Agriculture, 90.9, 1501-1507. DOI: 10 . 1002 / jsfa . 3974

Chen, Yingxu, Mingge Yu, Jie Xu, Xincai Chen, and Jiyan Shi (2009). Differentiation of eight tea (Camellia sinensis) cultivars in China by elemental fingerprint of their leaves. Journal of the Science of Food and Agriculture, 89.14, 2350-2355. DOI: 10.1002/ jsfa. 3716.

Chen, Yue Ming, Tsung Ming Tsao, Cheng Chung Liu, Kuo Chuan Lin, and Ming Kuang Wang (2011). Aluminium and nutrients induce changes in the profiles of phenolic substances in tea plants (Camellia sinensis). Journal of the Science of Food and Agriculture, 91.6, 1111-1117. DOI: $10.1002 /$ jsfa.4291. 


\section{Bibliography}

Cheng, Yi, Jing Wang, Jin-Bo Zhang, Christoph Müller, and Shen-Qiang Wang (2015). Mechanistic insights into the effects of $\mathrm{N}$ fertilizer application on N2O-emission pathways in acidic soil of a tea plantation. Plant and Soil, 389.1, 45-57. Dol: 10.1007/s11104-014-2343-y.

Chojnacka, K., A. Chojnacki, H. Górecka, and H. Górecki (2005). Bioavailability of heavy metals from polluted soils to plants. The Science of the total environment, 337.1-3, 175-182. DOI: 10.1016/j.scitotenv. 2004.06.009.

Cigler, Petr, Julie Olejnickova, Martin Hruby, Ladislav Csefalvay, Jiri Peterka, and Stanislav Kuzel (2010). Interactions between iron and titanium metabolism in spinach: a chlorophyll fluorescence study in hydropony. Journal of Plant Physiology, 167.18, 1592-1597.

Condie, Kent C. (2005). TTGs and adakites: are they both slab melts? Lithos, 80.1, 33-44. DOI: $10.1016 / j$.lithos.2003.11.001.

Cook, Lawrence L., Terence P. McGonigle, and Richard S. Inouye (2009). Titanium as an indicator of residual soil on arid-land plants. J. Environ. Qual. 38, 188-199.

Council of the European Union (2011a). Commission implementing Regulation (EU) no 1050/2011 of 20 october 2011 entering a name in the register of protected designations of origin and protected geographical indications (darjeeling (pgi)). Official Journal of the European Union. L, 276, 5-12. URL: https://eur-lex.europa.eu/legal-content/EN/TXT/?uri= CELEX:32011R1050

- (2011b). Directive 2011/65/EU of the European Parliament and of the Council of 8 June 2011 on the restriction of the use of certain hazardous substances in electrical and electronic equipment text with EEA relevance. Official Journal of the European Union. L, 174, 88-110. URL: https : / / eur - lex . europa . eu / legal - content / EN / TXT / ?uri = celex : $32011 \mathrm{~L} 0065$.

Dahl, David B. (2016). xtable: Export Tables to LaTeX or HTML. URL: https : / / CRAN . Rproject.org/package=xtable.

Dasgupta, Somnath, Sumit Chakraborty, and Sudipta Neogi (2009). Petrology of an inverted Barrovian sequence of metapelites in Sikkim Himalaya, India: constraints on the tectonics of inversion. American Journal of Science, 309.1, 43-84.

Dasgupta, Somnath, J. Ganguly, and S. Neogi (2004). Inverted metamorphic sequence in the Sikkim Himalayas: Crystallization history, P-T gradient and implications. Journal of Metamorphic Geology, 22.5, 395-412. DOI: 10.1111/j.1525-1314.2004.00522 .x.

Dean, John R (2014). Environmental Trace Analysis: Techniques and Applications. John Wiley \& Sons.

Deer, William Alexander, Robert Andrew Howie, and Jack Zussman (1992). An introduction to the rock-forming minerals. 2nd ed. Pearson Education Limited.

Demirel, B. and P. Scherer (2011). Trace element requirements of agricultural biogas digesters during biological conversion of renewable biomass to methane. Biomass Bioenergy, 35.3, 992-998. DOI:10.1016/j.biombioe.2010.12.022.

Dinelli, Enrico and Alessandra Lombini (1996). Metal distributions in plants growing on copper mine spoils in Northern Apennines, Italy: the evaluation of seasonal variations. Applied Geochemistry, 11.1-2, 375-385. DOI: 10.1016/0883-2927(95)00071-2.

Ding, Lin, Dalai Zhong, An Yin, Paul Kapp, and T Mark Harrison (2001). Cenozoic structural and metamorphic evolution of the eastern Himalayan syntaxis (Namche Barwa). Earth and Planetary Science Letters, 192.3, 423-438.

Dowle, Matt and Arun Srinivasan (2017). data.table: Extension of 'data.frame'. URL: https : //CRAN.R-project .org/package=data. table. 
Duce, Robert A, Gerald L Hoffman, and William H Zoller (1975). Atmospheric trace metals at remote northern and southern hemisphere sites: pollution or natural? Science, 187.4171, 5961.

Dumon, J. C. and W. H. O. Ernst (1988). Titanium in plants. Journal of Plant Physiology, 133.2, 203-209.

Durairaj, J., R. Radhakrishnan, J. B. Hudson, and N. Muraleedharan (2015). Guidelines on tea culture in south India. Ed. by The Secretary General. 9th. United Planter's Association of Southern India, Coonoor.

Eden, T. (1976). Tea. Tropical agricultural series. 3rd. Vol. 153, 31-42.

Egozcue, Juan José and Vera Pawlowsky-Glahn (2005). Groups of parts and their balances in compositional data analysis. Mathematical Geology, 37.7, 795-828. DOI:10.1007/s11004005-7381-9.

- (2011). "Basic concepts and procedures". Compositional data analysis: Theory and applications. Vol. 378. Wiley Chichester^ eUK UK, 12-27.

Egozcue, Juan José, Vera Pawlowsky-Glahn, Glòria Mateu-Figueras, and C. Barceló-Vidal (2003). Isometric logratio transformations for compositional data analysis. Mathematical Geology, 35.3, 279-300. DOI: 10.1023/A : 1023818214614.

Embrapa Solos (2013). Sistema brasileiro de classificação de solos. Centro Nacional de Pesquisa de Solos: Rio de Janeiro.

Embrapa and Emater (2006). Solos - Estado do Paraná, 1:2000 000. Ed. by Instituto de Terras, Cartografia e Geociências. Instituto de Terras, Cartografia e Geociências. URL: http : / / www . itcg · pr.gov . br/arquivos/File/Produtos_DGEO/Mapas_ITCG / PDF / Mapa_Solos.pdf (visited on 05/04/2018).

Fahrmeir, Ludwig and A. Hamerle (1984). Multivariate Statistische Verfahren. Walter de Gruyter, Berlin (D), $796 \mathrm{p}$.

Faleiros, Frederico Meira, Ginaldo Ademar da Cruz Campanha, Lucelene Martins, Silvio Roberto Farias Vlach, and Paulo M. Vasconcelos (2011). Ediacaran high-pressure collision metamorphism and tectonics of the southern Ribeira Belt (SE Brazil): evidence for terrane accretion and dispersion during Gondwana assembly. Precambrian Research, 189.3, 263-291. DOI: 10.1016/j.precamres.2011.07.013.

Fässler, E., B. H. Robinson, W. Stauffer, S. K. Gupta, A. Papritz, and R. Schulin (2010). Phytomanagement of metal-contaminated agricultural land using sunflower, maize and tobacco. Agric. Ecosyst. Environ. 136.1-2, 49-58. DOI:10.1016/j .agee.2009.11.007.

Faucon, M.-P., M. Ngoy Shutcha, and P. Meerts (2007). Revisiting copper and cobalt concentrations in supposed hyperaccumulators from SC Africa: influence of washing and metal concentrations in soil. Plant and Soil, 301.1-2, 29-36. Dol:10.1007/s11104-007-9405-3.

Fernández-Cáceres, Pedro L., María J. Martín, Fernando de Pablos, and A. Gustavo González (2001). Differentiation of tea (Camellia sinensis) varieties and their geographical origin according to their metal content. Journal of Agricultural and Food Chemistry, 49.10, 4775-4779. DOI: $10.1021 / j f 0106143$

Filzmoser, Peter and Karel Hron (2008). Correlation analysis for compositional data. Mathematical Geosciences, 41.8, 905. DOI: 10.1007/s11004-008-9196-y.

Flaten, Trond Peder (2002). Aluminium in tea-concentrations, speciation and bioavailability. Coordination Chemistry Reviews, 228.2, 385-395.

Fordham, R. (1972). Observations on the growth of roots and shoots of tea (camellia sinensis, I.) in Southern Malawi. Journal of Horticultural Science, 47.2, 221-229. DOI: 10.1080/ 00221589.1972 .11514461 


\section{Bibliography}

Froehlich, Wojciech and Leszek Starkel (1993). The effects of deforestation on slope and channel evolution in the tectonically active Darjeeling Himalaya.

Fung, Ka Fai (2003). Aluminium and fluoride concentrations of three tea varieties growing at Lantau Island, Hong Kong. Environmental Geochemistry and Health, 25.2, 219-232. DOI:10. 1023/A: 1023233226620 .

Fung, Ka Fai, Hilda Premi Carr, B. H. T. Poon, and Ming Hung Wong (2009). A comparison of aluminum levels in tea products from Hong Kong markets and in varieties of tea plants from Hong Kong and India. Chemosphere, 75.7, 955-962. DOI: $10.1016 / j$. chemosphere. 2009.01 .003

Fung, Ka Fai, Z. Q. Zhang, J.W.C Wong, and Ming Hung Wong (1999). Fluoride contents in tea and soil from tea plantations and the release of fluoride into tea liquor during infusion. Environmental Pollution, 104.2, 197-205. DOI: 10.1016/S0269-7491(98)00187-0.

Gabriel, K. Ruben (1971). The biplot graphic display of matrices with application to principal component analysis. Biometrika, 58.3, 453-467. Dol: 10.1093/biomet/58.3.453.

- (1972). Analysis of meteorological data by means of canonical decomposition and biplots. Journal of Applied Meteorology, 11.7, 1071-1077. DOI: 10 .1175/1520-0450(1972) $011<1071$ : AOMDBM>2.0.C0;2.

Gaidies, F., A. Petley-Ragan, S. Chakraborty, S. Dasgupta, and P. Jones (2015). Constraining the conditions of Barrovian metamorphism in Sikkim, India: P-T-t paths of garnet crystallization in the Lesser Himalayan Belt. Journal of Metamorphic Geology, 33.1, 23-44. DOI: 10.1111/ jmg. 12108

Gallaher, R. N., K. Gallaher, A. J. Marshall, and A. C. Marshall (2006). Mineral analysis of ten types of commercially available tea. Journal of Food Composition and Analysis, 19, S53-S57. DOI: $10.1016 / j \cdot j f c a .2006 .02 .006$.

Gansser, Augusto (1964). Geology of the Himalayas.

George, Eckhard, Horst Marschner, and Iver Jakobsen (1995). Role of arbuscular mycorrhizal fungi in uptake of phosphorus and nitrogen from soil. Critical Reviews in Biotechnology, 15.34, 257-270. DOI: $10.3109 / 07388559509147412$.

Gerzabek, M., J. Li, and K. Muck (1992). An experimental study on mass loading of soil particles on plant surface.

Ghosh, A. M. (1956). "Recent advances in the geology and structure of Eastern Himalaya". Indian Science Congress. Vol. 44, 8.

Gleeson, Deirdre B., Nicholas Clipson, Karrie Melville, Geoffrey M. Gadd, and Frank P. McDermott (2005). Characterization of fungal community structure on a weathered pegmatitic granite. Microbial Ecology, 50.3, 360. DOI: 10.1007/s00248-005-0198-8

Gleeson, Deirdre B., Nabla M. Kennedy, Nicholas Clipson, Karrie Melville, Geoffrey M. Gadd, and Frank P. McDermott (2006). Characterization of bacterial community structure on a weathered pegmatitic granite. Microbial Ecology, 51.4, 526-534. DOI: 10.1007/s00248-006-9052$\mathrm{X}$.

Goni, M. A., J. U. Ahmad, M. A. Halim, M. A. Mottalib, and D. A. Chowdhury (2014). Uptake and translocation of metals in different parts of crop plants irrigated with contaminated water from DEPZ area of Bangladesh. Bulletin of Environmental Contamination and Toxicology, 92.6, 726-732. DOI: 10.1007/s00128-014-1264-z.

Gonzalvez, A., S. Armenta, and M. de La Guardia (2009). Trace-element composition and stableisotope ratio for discrimination of foods with protected designation of origin. Trends in Analytical Chemistry, 28.11, 1295-1311. 
Gorard, Stephen (2005). Revisiting a 90-year-old debate: the advantages of the mean deviation. British Journal of Educational Studies, 53.4, 417-430. DOI: $10.1111 / \mathrm{j} .1467-8527$. 2005.00304.x.

Goscombe, Ben, David Gray, and Martin Hand (2006). Crustal architecture of the Himalayan metamorphic front in eastern Nepal. Gondwana Research, 10.3, 232-255.

Goscombe, Ben and Martin Hand (2000). Contrasting P-T paths in the Eastern Himalaya, Nepal: inverted isograds in a paired metamorphic mountain belt. Journal of Petrology, 41.12, 16731719.

Goswami, S (2005). "Inverted metamorphism in the Sikkim-Darjeeling Himalaya: structural, metamorphic and numerical studies". PhD thesis. University of Cambridge.

Gough, LP, Hansford Threlkeld Shacklette, and Arthur A Case (1979). Element concentrations toxic to plants, animals, and man. Tech. rep. US Govt. Print. Off., URL: https : / / pubs . usgs.gov/bul/1466/report.pdf.

Governement of West Bengal (2012). Darjeeling geography. URL: http : / / darjeeling . gov.in/geography.html

Greenacre, Michael J. (2010). Biplots in practice. Fundacion BBVA.

Grösslová, Zuzana, Aleš Vanek, Martin Mihaljevič, Vojtech Ettler, Maria Hojdová, Tereza Zádorová, Lenka Pavlu, Vit Penizek, Barbora Vanečková, Michael Komárek, Vladislav Chrastný, and Christopher Ash (2015). Bioaccumulation of thallium in a neutral soil as affected by solidphase association. Journal of Geochemical Exploration, 159, 208-212. DOI: $10.1016 / \mathrm{j}$. gexplo.2015.09.009.

Grujic, Djordje, Clare J. Warren, and Joseph L. Wooden (2011). Rapid synconvergent exhumation of Miocene-aged lower orogenic crust in the eastern Himalaya. Lithosphere, 3.5, 346366.

Guo, J. H., X. J. Liu, Yan Zhang, J. L. Shen, W. X. Han, W. F. Zhang, Peter Christie, K. W. T. Goulding, P. M. Vitousek, and F. S. Zhang (2010). Significant acidification in major Chinese croplands. Science, 327.5968, 1008-1010.

Gupta, Saibal, Aditi Das, Sudipta Goswami, Ananda Modak, and Suman Mondal (2010). Evidence for structural discordance in the inverted metamorphic sequence of Sikkim Himalaya: Towards resolving the Main Central Thrust controversy. Journal of the Geological Society of India, 75.1, 313-322.

Haghighi, Maryam, Salman Heidarian, and Jaime A. Teixeira da Silva (2012). The effect of titanium amendment in $\mathrm{N}$-withholding nutrient solution on physiological and photosynthesis attributes and micronutrient uptake of tomato. Biological Trace Element Research, 150.1-3, 381-390. DOI: 10.1007/s12011-012-9481-y.

Hajiboland, Roghieh, Sara Bahrami Rad, Juan Barceló, and Charlotte Poschenrieder (2013). Mechanisms of aluminum-induced growth stimulation in tea (Camellia sinensis ). Journal of Plant Nutrition and Soil Science, 176.4, 616-625. Dol: 10.1002/jpln.201200311.

Han, Wen-Yan, Yuan-Zhi Shi, Li-Feng Ma, and Jiang-Yun Ruan (2005). Arsenic, cadmium, chromium, cobalt, and copper in different types of Chinese tea. Bulletin of Environmental Contamination and Toxicology, 75.2, 272-277. DOI: 10.1007/s00128-005-0748-2.

Han, Wen-Yan, Yuan-Zhi Shi, Li-Feng Ma, Jiang-Yun Ruan, and Fang-Jie Zhao (2007). Effect of liming and seasonal variation on lead concentration of tea plant (Camellia sinensis (L.) O. Kuntze). Chemosphere, 66.1, 84-90. DoI:10.1016/j.chemosphere.2006.05.017.

Han, Wen-Yan, Fang-Jie Zhao, Yuan-Zhi Shi, Li-Feng Ma, and Jian-Yun Ruan (2006). Scale and causes of lead contamination in Chinese tea. Environmental Pollution, 139.1, 125-132. DOI: $10.1016 /$ j.envpol.2005.04.025. 


\section{Bibliography}

Harler, C. R. (1971). Tea soils. World Crops, 275.

Harris, Nigel B. W., M. Caddick, J. Kosler, S. Goswami, D. Vance, and A. G. Tindle (2004). The pressure-temperature-time path of migmatites from the Sikkim Himalaya. Journal of Metamorphic Geology, 22.3, 249-264. Dol: 10.1111/j.1525-1314.2004.00511 .x.

Harrison, R. M. and M. B. Chirgawi (1989). The assessment of air and soil as contributors of some trace metals to vegetable plants. I. Use of a filtered air growth cabinet. Science of the Total Environment, 83.1-2, 13-34. DOI: 10.1016/0048-9697(89)90003-X.

Heim, Arnold and August Gansser (1939). Central Himalaya. Hindustan Publishing; Delhi.

Heisner, Ute, Bernd Raber, and Ernst E. Hildebrand (2004). The importance of the soil skeleton for plant-available nutrients in sites of the Southern Black Forest, Germany. European Journal of Forest Research, 123.4, 249-257. DOI: 10.1007/s10342-004-0041-7.

Heiss, Mary Lou and Robert J. Heiss (2011). "The Story of Tea: A Cultural History and Drinking Guide". Random House, p. 31.

Herrador, M. Ángeles and A. Gustavo González (2001). Pattern recognition procedures for differentiation of green, black and oolong teas according to their metal content from inductively coupled plasma atomic emission spectrometry. Talanta, 53, 1249-1257. DOI:10.1016/S00399140(00)00619-6.

Hodge, Angela, Colin D. Campbell, and Alastair H. Fitter (2001). An arbuscular mycorrhizal fungus accelerates decomposition and acquires nitrogen directly from organic material. Nature, 413, 297-. DOI: $10.1038 / 35095041$.

Hoenig, M. (1995). Critical discussion of trace element analysis of plant matrices. Science of The Total Environment, 176.1, 85-91. DOI: 10 . 1016/0048-9697(95 ) 04833-2. URL: http://www.sciencedirect.com/science/article/pii/0048969795048332.

Hope, Bruce K. (1995). A review of models for estimating terrestrial ecological receptor exposure to chemical contaminants. Chemosphere, 30.12, 2267-2287. DOI: 10 . $1016 / 0045-$ 6535(95) 00100-M.

Hosseinzadegan, Sara, Winfried Nischkauer, Katharina Bica, and Andreas Limbeck (2016). Bioparticles coated with an ionic liquid for the pre-concentration of rare earth elements from microwavedigested tea samples and the subsequent quantification by ETV-ICP-OES. Analytical Methods, 8.43, 7808-7815. DOI: 10.1039/C6AY02189A.

Huang, Xin-Yuan and David E. Salt (2016). Plant ionomics: From elemental profiling to environmental adaptation. Molecular Plant, 9.6, 787-797. DoI:10.1016/j .molp.2016.05.003.

Hubert, John F (1962). A zircon-tourmaline-rutile maturity index and the interdependence of the composition of heavy mineral assemblages with the gross composition and texture of sandstones. Journal of Sedimentary Research, 32.3.

Husted, Soeren, Birgitte F. Mikkelsen, Jacob Jensen, and Niels Erik Nielsen (2004). Elemental fingerprint analysis of barley (hordeum vulgare) using inductively coupled plasma mass spectrometry, isotope-ratio mass spectrometry, and multivariate statistics. Anal. Bioanal. Chem. 378.1, 171-182. DOI: $10.1007 / \mathrm{s} 00216-003-2219-0$

Indian Tea Association (1952). Map of the tea growing districts Darjeeling and Terai.

Jabeen, Saiqa, Sahib Alam, Maria Saleem, Waqar Ahmad, Rukhsana Bibi, Farrukh S. Hamid, and Hamid U. Shah (2015). Withering timings affect the total free amino acids and mineral contents of tea leaves during black tea manufacturing. Arabian Journal of Chemistry. DOI: $10.1016 / j$.arabjc.2015.03.011.

Jaiswal, Pankaj, Cees J. van Westen, and Victor Jetten (2011). Quantitative assessment of landslide hazard along transportation lines using historical records. Landslides, 8.3, 279-291. DOI: 10.1007/s10346-011-0252-1. 
Janasi, V.A, R.J Leite, and W.R van Schmus (2001). U-pb chronostratigraphy of the granitic magmatism in the Agudos Grandes Batholith (west of São Paulo, Brazil) - implications for the evolution of the Ribeira Belt. Journal of South American Earth Sciences, 14.4, 363-376. DOI: 10.1016/S0895-9811(01)00034-7.

Jayman, T. C. Zaman and Sinnathurai Sivasubramaniam (1975). Release of bound iron and aluminium from soils by the root exudates of tea (Camellia sinensis) plants. Journal of the Science of Food and Agriculture, 26.12, 1895-1898. DOI: 10.1002/ jsfa. 2740261212.

Jin, Chong Wei, Shao Ting Du, Kai Zhang, and Xian Yong Lin (2008). Factors determining copper concentration in tea leaves produced at Yuyao County, China. Food and Chemical Toxicology, 46.6, 2054-2061. DOI: 10.1016/j.fct.2008.01.046.

Kabata-Pendias, Alina (2010). Trace elements in soils and plants. CRC press. URL: https : / / www . crcpress . com / Trace-Elements - in-Soils - and-Plants - FourthEdition/Kabata-Pendias/p/book/9781420093681.

Kabir, S. E. and A. P. Das (2015). Tea Cultivation in the plains of North East India. Regency Publications, Astral International (P) Ltd, New Delhi.

Kaiser, Brent N., Kate L. Gridley, Joanne N. Brady, Thomas Phillips, and Stephen D. Tyerman (2005). The role of molybdenum in agricultural plant production. Annals of Botany, 96.5, 745754. DOI: $10.1093 / \mathrm{aob} / \mathrm{mci} 226$

Kali, E., P. H. Leloup, N. Arnaud, G. Mahéo, Dunyi Liu, E. Boutonnet, J. Van der Woerd, Xiaohan Liu, Jing Liu-Zeng, and Haibing Li (2010). Exhumation history of the deepest central Himalayan rocks, Ama Drime range: key pressure-temperature-deformation-time constraints on orogenic models. Tectonics, 29.2, n/a-n/a. DOI:10.1029/2009TC002551

Karak, Tanmoy, Ornella Abollino, Pradip Bhattacharyya, Kishore K. Das, and Ranjit K. Paul (2011). Fractionation and speciation of arsenic in three tea gardens soil profiles and distribution of As in different parts of tea plant (Camellia sinensis L.) Chemosphere, 85.6, 948960. DOI: 10.1016/j. chemosphere.2011.06.061.

Karak, Tanmoy, Ranjit Kumar Paul, Romesh Kumar Boruah, Indira Sonar, Biswajyoti Bordoloi, Amrit Kumar Dutta, and Borsha Borkotoky (2015a). Major soil chemical properties of the major tea-growing areas in India. Pedosphere, 25.2, 316-328. DOI: 10.1016/S1002-0160(15) 60016-9.

Karak, Tanmoy, Indira Sonar, Ranjit K. Paul, Marcin Frankowski, Romesh K. Boruah, Amrit K. Dutta, and Dilip K. Das (2015b). Aluminium dynamics from soil to tea plant (Camellia sinensis L.): Is it enhanced by municipal solid waste compost application? Chemosphere, 119, 917926. DOI: 10.1016/j.chemosphere.2014.08.067.

Kellett, Dawn A., Djordje Grujic, Clare J. Warren, John M. Cottle, R. Jamieson, and T. Tenzin (2010). Metamorphic history of a syn-convergent orogen-parallel detachment: the South Tibetan detachment system, Bhutan Himalaya. Journal of Metamorphic Geology, 28.8, 785808. DOI: $10.1111 / \mathrm{j} .1525-1314.2010 .00893 . \mathrm{x}$.

Kelly, Simon, Karl Heaton, and Jurian Hoogewerff (2005). Tracing the geographical origin of food: the application of multi-element and multi-isotope analysis. Trends in Food Science \& Technology, 16, 555-567.

Koele, N. (2012). "9 The Role of the Stonesphere for the Interactions Between Mycorrhizal Fungi and Mycorrhizosphere Bacteria During Mineral Weathering". Fungal Associations. Ed. by Bertold Hock. Berlin, Heidelberg: Springer Berlin Heidelberg, 171-180. DOI: 10 . $1007 /$ 978-3-642-30826-0_9. 


\section{Bibliography}

Kohler, M., K. V. Wilpert, and E. E. Hildebrand (2000). The soil skeleton as a source for the short-term supply of "base cations" in forest soils of the Black Forest (Germany). Water, Air, and Soil Pollution, 122.1, 37-48. Dol: 10.1023/A:1005277909113.

Konishi, Shigeki, Sobun Miyamoto, and Takayuki Taki (1985). Stimulatory effects of aluminum on tea plants grown under low and high phosphorus supply. Soil Science and Plant Nutrition, 31.3, 361-368. DOI:10.1080/00380768.1985.10557443.

Kumar, R. Raj, L. Manivel, and S. Marimuthu (1998). Longevity and factors influencing photosynthesis in tea leaves. Photosynthetica, 35.1, 41-46. DOI: 10.1023/A: 1006809528852.

Kundu, Abhik, Abdul Matin, and Malay Mukul (2012). Depositional environment and provenance of Middle Siwalik sediments in Tista valley, Darjiling District, Eastern Himalaya, India. Journal of Earth System Science, 21, 73-89.

Lahiri, Siddhartha K. and Rajiv Sinha (2012). Tectonic controls on the morphodynamics of the Brahmaputra River system in the upper Assam valley, India. Geomorphology, 169-170.Supplement C, 74-85. DOI: 10.1016/j.geomorph.2012.04.012

Lal, R. K., S. Mukherjee, and D. Ackermand (1981). Deformation and Barrovian metamorphism at Takdah, Darjeeling (Eastern Himalaya). Metamorphic tectonites of the Himalaya, 231-278.

Le Fort, Patrick (1975). Himalayas: the collided range. present knowledge of the continental arc. American Journal of Science, 275.1, 44.

Leite, Renato Jordan, Larry M. Heaman, Valdecir de Assis Janasi, Lucelene Martins, and Robert A. Creaser (2007). The late- to postorogenic transition in the Neoproterozoic Agudos Grandes Granite Batholith (Apiaí Domain, SE Brazil): constraints from geology, mineralogy, and $\mathrm{U}-\mathrm{Pb}$ geochronology. Journal of South American Earth Sciences, 23.2, 193-212. DOI: 10.1016/ j.jsames.2006.09.022.

$\mathrm{Li}$, Shiyu, Haixia Li, Changliang Yang, Yandan Wang, Hui Xue, and Yanfen Niu (2016). Rates of soil acidification in tea plantations and possible causes. Agriculture, Ecosystems \& Environment, 233.Supplement C, 60-66. DOI:10.1016/j . agee.2016.08.036.

Lima, Rosiane L.S., Liv S. Severino, Ligia R. Sampaio, Valdinei Sofiatti, Jucélia A. Gomes, and Napoleão E.M. Beltrão (2011). Blends of castor meal and castor husks for optimized use as organic fertilizer. Industrial Crops and Products, 33.2, 364-368. DOI: 10.1016/j . indcrop. 2010.11 .008

Ma, Li-Feng, Yuan-Zhi Shi, and Jiang-Yun Ruan (2000). Soil pHs in the tea gardens in Jiangsu, Zhejiang, and Anhui provinces and changes of soil pH in the past decade. Chinese Journal of Soil Science, 31.5, 205-208.

MacNaeidhe, F. (1995). Procedures and precautions used in sampling techniques and analysis of trace elements in plant matrices. Science of the Total Environment, 176.1-3, 25-31.

Marcos, Ana, Andrew Fisher, Gerry Rea, and Steve J. Hill (1998). Preliminary study using trace element concentrations and a chemometrics approach to determine the geographical origin of tea. Journal of Analytical Atomic Spectrometry, 13.6, 521-525. DOI: 10.1039/a708658j.

Marimuthu, S., R. Raj Kumar, and L. Manivel (1994). Factors affecting partitioning of assimilates in tea. Journal of Nuclear Agriculture and Biology (India).

Markert, B. (1995). Sample preparation (cleaning, drying, homogenization) for trace element analysis in plant matrices. Science of the Total Environment, 176.1-3, 45-61. DOI:10.1016/ 0048-9697 (95) 04829-4.

Marschner, Horst (2002). "14 - Effect of Internal and External Factors on Root Growth and Development". Marschner's Mineral Nutrition of Higher Plants (Second Edition). Ed. by Horst Marschner. Second Edition. San Diego: Academic Press, 508-536. DOI: 10 . 1016/B9780-08-057187-4.50020-5. 
Matsuura, Hirotaka, Akiko Hokura, Fumie Katsuki, Akihide Itoh, and Hiroki Haraguchi (2001). Multielement determination and speciation of major-to-trace elements in black tea leaves by ICP-AES and ICP-MS with the aid of size exclusion chromatography. Analytical Sciences, 17.3, 391-398. DOI: 10.2116/analsci.17.391.

McKenzie, James S., José Marcos Jurado, and Fernando de Pablos (2010). Characterisation of tea leaves according to their total mineral content by means of probabilistic neural networks. Food Chemistry, 123.3, 859-864. DOI: 10.1016/j.foodchem.2010.05.007.

Melo, Mário Sérgio de (1990). "A Formação Pariquera-Açu e depósitos relacionados: sedimentação, tectônica e geomorfogênese". Portuguese. PhD thesis. São Paulo: University of São Paulo, Instituto de Geociências. DOI:10.11606/D .44.1990.tde-15092015-135545.

Mitchell, R. L. (1960). Contamination problems in soil and plant analysis. Journal of the Science of Food and Agriculture, 11, 553-560.

Mohan, A., B. F. Windley, and Michael P. Searle (1989). Geothermobarometry and development of inverted metamorphism in the Darjeeling-Sikkim region of the eastern Himalayan. Journal of Metamorphic Geology, 7.1, 95-110. DOI: 10.1111/j.1525-1314.1989.tb00577.x.

Moraes, Maria Cristina de, Sandra Garcia Gabas, Mário Sérgio de Melo, Luiz Alberto Fernandes, and Armando Márcio Coimbra (1991). Sedimentologia dos depósitos cenozóicos continentais do Baixo Vale do Rio Ribeira do Iguape, São Paulo. Revista do Instituto Geológico, 12.1-2, 39-53.

Moreda-Piñeiro, Antonio, Andrew Fisher, and Steve J. Hill (2003). The classification of tea according to region of origin using pattern recognition techniques and trace metal data. Journal of Food Composition and Analysis, 16.2, 195-211. Dol:10.1016/S0889-1575(02)001631.

Moreda-Piñeiro, Antonio, Ana Marcos, Andrew Fisher, and Steve J. Hill (2001). Evaluation of the effect of data pre-treatment procedures on classical pattern recognition and principal components analysis: A case study for the geographical classification of tea. Journal of Environmental Monitoring, 3.4, 352-360. DOI: 10.1039/b103658k.

Morita, Akio, Yousuke Fujii, and Hiromi Yokota (2001). "Effect of aluminium on exudation of organic acid anions in tea plants". Plant Nutrition. Ed. by Walter J. Horst, M. K. Schenk, A. Bürkert, N. Claassen, H. Flessa, W. B. Frommer, H. Goldbach, H.-W Olfs, V. Römheld, Burkhard Sattelmacher, U. Schmidhalter, S. Schubert, N. v. Wirén, and L. Wittenmayer. Dordrecht: Springer Netherlands, 508-509. DOI: 10.1007/0-306-47624-X_246.

Morita, Akio, Osamu Yanagisawa, Setsuko Maeda, Satoshi Takatsu, and Takashi Ikka (2011). Tea plant (Camellia sinensis L.) roots secrete oxalic acid and caffeine into medium containing aluminum. Soil Science and Plant Nutrition, 57.6, 796-802. DOI: 10 . 1080 / 00380768 . 2011.629176

Morita, Akio, Osamu Yanagisawa, Satoshi Takatsu, Setsuko Maeda, and Syuntaro Hiradate (2008). Mechanism for the detoxification of aluminum in roots of tea plant (Camellia sinensis (L.) kuntze). Phytochemistry, 69.1, 147-153. Dol:10.1016/j . phytochem.2007.06.007.

Mottram, Catherine M., Tom W. Argles, Nigel B. W. Harris, Randall R. Parrish, M. S. A. Horstwood, Clare J. Warren, and Saibal Gupta (2014a). Tectonic interleaving along the Main Central Thrust, Sikkim Himalaya. Journal of the Geological Society, 171.2, 255-268. DOI: 10.1144/ jgs2013-064.

Mottram, Catherine M., Clare J. Warren, Daniele Regis, Nick M.W. Roberts, Nigel B.W. Harris, Tom W. Argles, and Randall R. Parrish (2014b). Developing an inverted Barrovian sequence; insights from monazite petrochronology. Earth and Planetary Science Letters, 403.Supplement C, 418-431. DOI: $10.1016 /$ j.epsl.2014.07.006. 


\section{Bibliography}

Mukul, Malay (2000). The geometry and kinematics of the Main boundary Thrust and related neotectonics in the Darjiling Himalayan fold-and-thrust belt, West Bengal, India. Journal of Structural Geology, 22.9, 1261-1283. DOI:10.1016/S0191-8141(00)00032-8.

Murtagh, Fionn and Pierre Legendre (2014). Ward's hierarchical agglomerative clustering method: which algorithms implement ward's criterion? Journal of Classification, 31.3, 274-295. DOI: 10.1007/s00357-014-9161-z.

Natarajan, A., P. S. A. Reddy, J. Sehgal, and M. Velayutham (1997). Soil resources of Tamil Nadu for land-use planning. NBSS. Soils of India (46b), 88-92. URL: krishikosh .egranth . ac . in/bitstream/1/2034154/1/NBSS-4.pdf (visited on 11/12/2017).

$\varnothing$ degård, Knut E. and Walter Lund (1997). Multi-element speciation of tea infusion using cationexchange separation and size-exclusion chromatography in combination with inductively coupled plasma mass spectrometry. Journal of Analytical Atomic Spectrometry, 12.4, 403-408. DOI: $10.1039 / \mathrm{A} 606153 \mathrm{~B}$

Oliveira, J. B. d. (2014). Mapa de Solos de Estado de São Paulo. Ed. by Solo do Estado de São Paulo. Governo do Estado São Paulo, Secretaria de Agricultura e Abastecimento. URL: http://www.iac.sp.gov.br/solossp/(visited on 05/04/2018).

Oliveira, J. B. de, M. N. Rossi, and B. M. Calderano Filho (1999). Mapa pedológico do Estado de São Paulo: legenda expandida. 631.498161 M297. Empresa Brasileira de Pesquisa Agropecuaria, Campinas, SP (Brasil). Centro Nacional de Pesquisa de Solos Instituto Agronómico, Campinas, SP (Brasil).

Othieno, C. O., William Stephens, and M. K. V. Carr (1992). Yield variability at the tea research foundation of Kenya. Agricultural and Forest Meteorology, 61.3, 237-252.

Paix Mupenzi, Jean de la, Lanhai Li, Jiwen Ge, Achal Varenyam, Gabriel Habiyaremye, Nzayisenga Theoneste, and Kamanzi Emmanuel (2011). Assessment of soil degradation and chemical compositions in Rwandan tea-growing areas. Geoscience Frontiers, 2.4, 599-607. DOI: $10.1016 /$ j.gsf .2011.05.003.

Pal, D.K., P. Srivastava, S.L. Durge, and T. Bhattacharyya (2001). Role of weathering of finegrained micas in potassium management of Indian soils. Applied Clay Science, 20.1, 39-52. DOI: 10.1016/S0169-1317(01)00044-8.

Parent, Serge-Étienne, Leon Etienne Parent, Juan José Egozcue, Danilo Rozane, Amanda Hernandes, Line Lapointe, Valerie Gentile, Kristine Naess, Sebastien Marchand, Jean Lafond, Dirceu Mattos Jr, Philip Barlow, and William Natale (2013). The plant ionome revisited by the nutrient balance concept. Frontiers in Plant Science, 4, 39. DOI: $10.3389 / \mathrm{fpls}$. 2013. 00039 .

Parthasarathy, YJ and R Vaidyanathn (1974). Landforms over the Nilgiris. Journal of Geological Society of India, 15.

Pate, J. S. (1975). "Exchange of Solutes between Phloem and Xylem and Circulation in the Whole Plant". Transport in Plants I: Phloem Transport. Ed. by M. H. Zimmermann and J. A. Milburn. Berlin, Heidelberg: Springer Berlin Heidelberg, 451-473. DOI: 10 . 1007/978-3642-66161-7_19.

Pawlowsky-Glahn, Vera and Antonella Buccianti (2011). Compositional data analysis: Theory and applications. John Wiley \& Sons.

Pawlowsky-Glahn, Vera and Juan José Egozcue (2006). Compositional data and theri analysis: an introduction. Geological Society, London, Special Publications, 264, 1-10. URL: http: //sp.lyellcollection.org

Pawlowsky-Glahn, Vera, Juan José Egozcue, and Raimon Tolosana-Delgado (2015). Modeling and analysis of compositional data. John Wiley \& Sons. 
Perrotta, MM, ED Salvador, R da C LOPES, LZ D Agostino, N Peruffo, SD Gomes, LLB Sachs, VT Meira, MGM Garcia, and JV Lacerda Filho (2005). Mapa geológico do Estado de São Paulo, escala 1: 750.000. São Paulo: CPRM.

Pilgrim, Tamara S., R. John Watling, and Kliti Grice (2010). Application of trace element and stable isotope signatures to determine the provenance of tea (camellia sinensis) samples. Food Chemistry, 118.4, 921-926. DOI: 10.1016/j.foodchem .2008.08.077.

Poschenrieder, Charlotte, Benet Gunse, Isabel Corrales, and Juan Barceló (2008). A glance into aluminum toxicity and resistance in plants. Science of The Total Environment, 400.1-3, 356368. Dol: $10.1016 /$ j . scitotenv.2008.06.003

Pospiech, Solveig (2012). "Geochemical investigation on the tea plant Camellia sinensis: Implications from element soil-plant transfer factors for provenance studies - the case of Darjeeling Tea from NE-India". Diploma thesis. Georg-August-Universität Göttingen.

- (2017). ICPSubfunctions: Subfunctions for the package ICPData (in-house package).

Pospiech, Solveig and Wiebke Fahlbusch (2017). ratios: Calculating Ratios Between Two Data Sets and Correction for Adhering Particles on Plants. URL: https : //CRAN . R-project . org/package=ratios.

- (2018). ICPData: Functions to combine and work with element data from ICP-MS, ICP-OES and samplelists (in-house package).

Pospiech, Solveig, Wiebke Fahlbusch, Benedikt Sauer, Tino Pasold, and Hans Ruppert (2017). Alteration of trace element concentrations in plants by adhering particles - Methods of correction. Chemosphere, 182, 501-508. DOI: 10.1016/j.chemosphere.2017.05.038

Pospiech, Solveig, Benedikt Sauer, Wiebke Fahlbusch, Tino Pasold, Raimon Tolosana-Delgado, and Hans Ruppert (2016). "Trace Element Transfer into the Plant: What can We Learn from Element Ratios?" Goldschmidt Abstracts 2016 2532. URL: https : //goldschmidtabstracts . info/abstracts/abstractView?id=2016002450.

Pospiech, Solveig and Raimon Tolosana-Delgado (2017). "Interpretation of Soil-Plant Transfer Factors: A Log-Ratio Approach for Multi-Element Data". Goldschmidt Abstracts 20173201. URL: https : / / goldschmidtabstracts . info/abstracts / abstractView ? id= 2017005370

Powers, Peter M., Robert J. Lillie, and Robert S. Yeats (1998). Structure and shortening of the Kangra and Dehra Dun reentrants, Sub-Himalaya, India. GSA Bulletin, 110.8, 1010. DOI: 10 . 1130/0016-7606(1998) 110<1010: SASOTK>2 .3.CO;2.

QGIS Development Team (2018). QGIS Geographic Information System. Open Source Geospatial Foundation. URL: http://qgis.org.

R Core Team (2017). R: A Language and Environment for Statistical Computing. R Foundation for Statistical Computing. Vienna, Austria. URL: https://www.R-project.org/.

Raith, M, C Hengst, B Nagel, A Bhattacharya, and C Srikantappa (1988). Metamorphic conditions in the Nilgiri Granulite Terrane and the adjacent Moyar and Bhavani Shear Zones: a reevaluation.

Raith, Michael M., C. Srikantappa, Dieter Buhl, and Hermann Koehler (1999). The Nilgiri enderbites, South India: nature and age constraints on protolith formation, high-grade metamorphism and cooling history. Precambrian Research, 98.1, 129-150. DOI: 10.1016/S03019268(99) 00045-5.

Rajakumar, P., S. Sanjeevi, S. Jayaseelan, G. Isakkipandian, M. Edwin, P. Balaji, and G. Ehanthalingam (2007). Landslide susceptibility mapping in a hilly terrain using remote sensing and GIS. Journal of the Indian Society of Remote Sensing, 35.1, 31-42. DOI: 10 . 1007 / BF02991831. 


\section{Bibliography}

Rajesh, H. M. and M. Santosh (2004). Charnockitic magmatism in Southern India. Journal of Earth System Science, 113.4, 565-585. DOI:10.1007/BF02704023.

Raju, S.V. and N. Mathur (1995). Petroleum geochemistry of a part of Upper Assam Basin, India: a brief overview. Organic Geochemistry, 23.1, 55-70. DOI: $10.1016 / 0146-6380$ (94) 00104-9.

Ranganatha, V and S Natesan (1985). Potassium nutrition of tea. Potassium in Agriculture, 9811022.

Ray, Sumit Kumar (2000). Culmination zones in eastern Himalaya. Geological Survey of India Miscellaneous Publication, 55, 85-94.

Reimann, Clemens, Arnold Arnoldussen, Rognvald Boyd, Tor Erik Finne, Friedrich Koller, Øystein Nordgulen, and Peter Englmaier (2007). Element contents in leaves of four plant species (birch, mountain ash, fern and spruce) along anthropogenic and geogenic concentration gradients. Science of the Total Environment, 377.2-3, 416-433. Dol: 10.1016/j . scitotenv. 2007.02 .011

Reimann, Clemens and Patrice de Caritat (2000). Intrinsic flaws of element enrichment factors (EFs) in environmental geochemistry. Environmental Science \& Technology, 34.24, 50845091. DOI: 10.1021/es001339o.

Reimann, Clemens, Friedrich Koller, Bjørn Frengstad, Galina Kashulina, Heikki Niskavaara, and Peter Englmaier (2001). Comparison of the element composition in several plant species and their substrate from a $1500000-\mathrm{km}^{2}$ area in northern Europe. Science of the Total Environment, 278.1-3, 87-112. DOI: 10.1016/S0048-9697(00)00890-1.

Roca, M.C. and V.R. Vallejo (1995). Effect of soil potassium and calcium on caesium and strontium uptake by plant roots. Journal of Environmental Radioactivity, 28.2, 141-159. DOI: 10 . 1016/0265-931X (94)00052-X.

Rodríguez, P Blanco, F Vera Tomé, and J.C Lozano (2002). About the assumption of linearity in soil-to-plant transfer factors for uranium and thorium isotopes and 226Ra. Science of The Total Environment, 284.1-3, 167-175. DOI: 10.1016/S0048-9697(01)00877-4.

Ross, Ivan A. (2007). Medicinal plants of the world, Chemical constituents, traditional and modern medicinal uses. Vol. 3. Springer.

Ross, Jurandyr Luciano Sanches. Ribeira do Iguape Basin: morphgenesis and the environmental systems. URL: http: //www .geografia.fflch.usp.br/publicacoes/Geousp/ Geousp12/ingles/G12_i_JurandyrRoss.htm.

Roy, K. K. (1976). Some problems of tectonics and stratigraphy of the Darjeeling and Sikkim Himalayas. Geological Survey of India Miscellaneous Publication, 24, 379-394.

Ruan, Jiang-Yun, Joska Gerendas, Rolf Hardter, and Burkhard Sattelmacher (2007). Effect of nitrogen form and root-zone $\mathrm{pH}$ on growth and nitrogen uptake of tea (Camellia sinensis) plants. Annals of Botany, 99.2, 301-310. Dol: 10.1093/aob/mcl258.

Ruan, Jiang-Yun, Li-Feng Ma, and Yuan-Zhi Shi (2006). Aluminium in tea plantations: Mobility in soils and plants, and the influence of nitrogen fertilization. Environmental Geochemistry and Health, 28.6, 519-528. DOI: 10.1007/s10653-006-9047-z.

Ruan, Jiang-Yun, Li-Feng Ma, Yuan-Zhi Shi, and Wen-Yan Han (2004). The impact of pH and calcium on the uptake of fluoride by tea plants (Camellia sinensis L.) Annals of Botany, 93.1, 97-105. DOI: 10.1093/aob/mch010

Ruan, Jiang-Yun, Fusuo Zhang, and Ming Hung Wong (2000). Effect of nitrogen form and phosphorus source on the growth, nutrient uptake and rhizosphere soil property of Camellia sinensis L. Plant and Soil, 223.1, 65-73. DOI:10.1023/A: 1004882001803. 
Rudnick, R. L. and S. Gao (2003). Composition of the continental crust. Treatise on Geochemistry, 3, 1-64. DOI: 10.1016/B0-08-043751-6/03016-4.

Ruppert, Hans (1991). Natürliche Spurenmetallgehalte im Boden und ihre anthropogene überprägung. Mitteilungen der österrischen geologischen Gesellschaft, 83, 243-265.

Sahm, Hermann (1981). Biologie der Methan-Bildung. Chem. Ing. Tech. 854-863.

Salahinejad, Maryam and Fereydoon Aflaki (2010). Toxic and essential mineral elements content of black tea leaves and their tea infusions consumed in Iran. Biological Trace Element Research, 134.1, 109-117. DOI:10.1007/s12011-009-8449-z

Salt, David E. (2004). Update on plant ionomics. Plant Physiology, 136.1, 2451-2456. DOI: 10 . $1104 / \mathrm{pp} .104 .047753$

Salt, David E., Ivan Baxter, and Brett Lahner (2008). lonomics and the study of the plant ionome. Annual Review of Plant Biology, 59.1, 709-733. DoI: 10 . 1146/annurev . arplant . 59 . 032607.092942

Samac, Deborah A. and Mesfin Tesfaye (2003). Plant improvement for tolerance to aluminum in acid soils - a review. Plant Cell, Tissue and Organ Culture, 75, 189-207.

Samuel, Vinod O., M. Santosh, Shuwen Liu, Wei Wang, and K. Sajeev (2014). Neoarchean continental growth through arc magmatism in the Nilgiri Block, Southern India. Precambrian Research, 245, 146-173. DOI:10.1016/j .precamres.2014.02.002

Santos, G. H., Silvio Bhering, Itamar Bognola, R. G. Curcio, César Chagas, Waldir Carvalho, Celso Manzatto, L. D. Aglio, and S. J. de Souza (2008). Revisão e atualização do mapa de solos.

Sato, Kei, Oswaldo Siga, Allen P. Nutman, Miguel A.S. Basei, Ian McReath, and Gilberto Kaulfuss (2003). The Atuba Complex, southern South American Platform: Archean components and Paleoproterozoic to Neoproterozoic tectonothermal events. Gondwana Research, 6.2, 251263. DOI: $10.1016 / \mathrm{S} 1342-937 \mathrm{X}$ (05) 70974-6.

Sauer, Benedikt and Hans Ruppert (2013). "Bioenergy Production as an Option for Polluted Soils - A Non-phytoremediation Approach". Sustainable Bioenergy Production - An Integrated Approach. Ed. by Hans Ruppert, Martin Kappas, and Jens Ibendorf. Springer, 425-444. DOI: 10.1007/978-94-007-6642-6\{_\}14

Schachtman, Daniel P., Robert J. Reid, and S.M. Ayling (1998). Phosphorus uptake by plants: from soil to cell. Plant Physiology, 116.2, 447-453. Dol:10.1104/pp.116.2.447.

Schelling, Daniel (1992). The tectonostratigraphy and structure of the eastern Nepal Himalaya. Tectonics, 11.5, 925-943. DOI: 10.1029/92TC00213.

Schwan, W. (1980). "Shortening Structures in Eastern and North-western Himalayan Rocks". Ed. by P.S. Saklani. Today, Tomorrow's Printers, and Publishers, New Delhi.

Searle, Michael P., Richard D. Law, Laurent Godin, Kyle P. Larson, Michael J. Streule, John M Cottle, and Micah J. Jessup (2008). Defining the Himalayan main central thrust in Nepal. Journal of the Geological Society, 165.2, 523-534.

Searle, Michael P. and Adam G. Szulc (2005). Channel flow and ductile extrusion of the high Himalayan slab-the Kangchenjunga-Darjeeling profile, Sikkim Himalaya? Journal of Asian Earth Sciences, 25.1, 173-185.

Seenivasan, Subbiah, Todd Alan Anderson, and Narayanannair Muraleedharan (2016). Heavy metal content in tea soils and their distribution in different parts of tea plants, Camellia sinensis (I). O. Kuntze. Environmental Monitoring and Assessment, 188.7, 428. DOI: 10 . $1007 /$ s10661-016-5440-y. 


\section{Bibliography}

Seenivasan, Subbiah, Natarajan Manikandan, Narayanan Nair Muraleedharan, and Rajagopal Selvasundaram (2008). Heavy metal content of black teas from south India. Food Control, 19.8, 746-749. DOI:10.1016/j.foodcont.2007.07.012.

Seghal, J. (1991). West Bengal Soils, Map 1:500000. Ed. by Nagpur National Bureau of Soil Survey \& Land Use Planning. URL: https : //esdac .jrc . ec . europa . eu/content/ west-bengal-soils-sheet-3.

Selvaradjou, Senthil-Kumar, Luca Montanarella, Otto Spaargaren, and David Dent (2005). European Digital Archive on Soil Maps of the World (EuDASM)-Soil Maps of Asia (DVD-Rom version). EUR 21823 EN. Tech. rep. Office for Official Publications of the European Communities.

Seshagiri, DN, S Badrinarayanan, R Upendran, CB Lakshmikantham, and V Srinivasan (1982). The Nilgiris landslide. Miscellaneous publication, 57.

Shu, W. S., Z. Q. Zhang, C. Y. Lan, and Ming Hung Wong (2003). Fluoride and aluminium concentrations of tea plants and tea products from Sichuan Province, PR China. Chemosphere, 52.9, 1475-1482. DOI: 10.1016/S0045-6535(03) 00485-5.

Singh, Jasbir, I. P. Borah, and A. Boruah (1995). Soil characteristics under three different plant communities of North-East India. Indian Forester, 121.12, 1130-1134.

Singh, Kumar A., B. Parkash, R. Mohindra, J. V. Thomas, and A. K. Singhvi (2001). Quaternary alluvial fan sedimentation in the Dehradun valley piggyback basin, North-West Himalaya: tectonic and palaeoclimatic implications. Basin Research, 13.4, 449-471.

Singh, Shipra, Anita Pandey, and Lok Man S. Palni (2008). Screening of arbuscular mycorrhizal fungal consortia developed from the rhizospheres of natural and cultivated tea plants for growth promotion in tea [Camellia sinensis (L.) O. Kuntze]. Pedobiologia, 52.2, 119-125. DOI: $10.1016 / j$.pedobi.2008.06.001.

Singh, Sunil Kumar (2006). Spatial variability in erosion in the Brahmaputra basin: causes and impacts. Current Science, 90.9, 1272-1276.

Sinha, M. P. (2010). World Tea Production and Manufacturing. 1st. New Dehli 110020: Wishwell.

Sinha, Rajiv and Peter F. Friend (1994). River systems and their sediment flux, Indo-Gangetic plains, Northern Bihar, India. Sedimentology, 41.4, 825-845. DOI: 10.1111 / j .13653091.1994.tb01426.x.

Smith, R. I., F. J. Harvey, and M. G. R. Cannell (1990). Pattern of tea shoot growth. Experimental Agriculture, 26.2, 197-208. DOI: 10.1017/S0014479700018196

Soja, Roman and Leszek Starkel (2007). Extreme rainfalls in Eastern Himalaya and southern slope of Meghalaya Plateau and their geomorphologic impacts. Geomorphology, 84.3-4, 170180. DOI: $10.1016 / \mathrm{j}$.geomorph.2006.01.040.

Souza Lima, Elizeu de, Rafael Montanari, Lenon Henrique Lovera, Samuel Ferrari, Tatiana dos Santos da Silva, and Hernandes Andrade Queiroz (2016). Spatial correlation of productive component for peach palm crop and some physical attributes of Eutrochrept soil. Revista Brasileira de Ciências Agrárias, 11.3, 163-170. URL: http://www . redalyc .org/html/ 1190/119047705003/.

Squire, G. R. and B. A. Callander (2012). Tea plantations. Water deficits and plant growth, 6, 471-510.

Srikantappa, C., K. G. Ashamanjari, and M. Raith (1988). "Petrology and geochemistry of the high-pressure Nilgiri Granulite Terrane, Southern India". Workshop on the Deep Continental Crust of South India. Ed. by L. D. Ashwal.

Srikantappa, C, M Raith, KG Ashamanjari, and D Ackermand (1986). Pyroxenites and gabbroic rocks from the Nilgiri Granulite Terrane, Southern India. Indian Mineralogist, 27, 62-83. 
Szymczycha-Madeja, Anna, Maja Welna, and Pawel Pohl (2012). Elemental analysis of teas and their infusions by spectrometric methods. Trends in Analytical Chemistry, 35, 165-181. DOI: $10.1016 / j$.trac.2011.12.005.

Tanton, T. W. (1982). Environmental factors affecting the yield of tea (Camellia sinensis). i. effects of air temperature. Experimental Agriculture, 18.1, 47-52. DOI: 10.1017/S0014479700013417.

Tea Board India (2017). List of Small Tea Growers. URL: http: //www . teaboard . gov . in/ TEABOARDCSM/MzU $=$ (visited on 09/06/2017).

Matsumoto, Hideaki, E. Hirasawa, S. Morimura, and E. Takahashi (1976). Localization of aluminium in tea leaves. Plant Cell Physiology, 17, 627-631.

Pandey, Anita (1997). Bacillus species: The dominant bacteria of the rhizosphere of established tea bushes. Microbiological Research, 152, 359-365.

Barua, D. N. (2008). Science and Practice in Tea Culture. 2nd. Tea Research Association.

Tölgyesi, G. and B. Nagy (1990). Tájékoztaó adatok a takarmányok titántartalmáól. Magy Állatorv Lapja, 45, 226-230.

Tolosana-Delgado, Raimon and et. al. (2019).

Tolra, Roser, Katarina Vogel-Mikus, Roghieh Hajiboland, Peter Kump, Paula Pongrac, Burkhard Kaulich, Alessandra Gianoncelli, Vladimir Babin, Juan Barceló, Marjana Regvar, and Charlotte Poschenrieder (2011). Localization of aluminium in tea (Camellia sinensis) leaves using low energy X-ray fluorescence spectro-microscopy. Journal of Plant Research, 124.1, 165-172. DOI: $10.1007 / \mathrm{s} 10265-010-0344-3$.

Tomé, F Vera, M.P Blanco Rodríguez, and J.C Lozano (2003). Soil-to-plant transfer factors for natural radionuclides and stable elements in a Mediterranean area. Journal of Environmental Radioactivity, 65.2, 161-175. DOI: 10.1016/S0265-931X(02)00094-2.

Tunbridge, lan P. (1981). Sandy high-energy flood sedimentation ? Some criteria for recognition, with an example from the Devonian of SW-England. Sedimentary Geology, 28.2, 79-95. DOI: 10.1016/0037-0738(81) 90058-0.

Turgeon, Robert (1989). The sink-source transition in leaves. Annual Review of Plant Biology, $40.1,119-138$.

Tyler, Germund (1997). Influence of acidity and potassium saturation on plant uptake of indigenous soil rubidium. Environmental and Experimental Botany, 38.2, 181-186. DOI:10.1016/ S0098-8472(97) 00012-9

USDA organic (2018). URL: https: //www .usda.gov/topics/organic

Valdiya, K.S. and Jaishri Sanwal (2017). "Chapter 12 - Mysore Plateau and Nilgiri Massif". Neotectonism in the Indian Subcontinent. Ed. by K.S. Valdiya and Jaishri Sanwal. Vol. 22. Developments in Earth Surface Processes. Elsevier, 279-306. DOI: 10 . 1016/B978-0-44463971-4.00012-8.

Vanek, Aleš, Michael Komárek, Petra Vokurková, Martin Mihaljevič, Ondrej Šebek, Gabriela Panušková, Vladislav Chrastný, and Ondrej Drábek (2011). Effect of illite and birnessite on thallium retention and bioavailability in contaminated soils. Journal of Hazardous Materials, 191.1, 170-176. DOI:10.1016/j.jhazmat.2011.04.065

Venables, W. N. and B. D. Ripley (2002). Modern Applied Statistics with S. Fourth. New York: Springer. URL: http://www.stats.ox.ac.uk/pub/MASS4

Verbruggen, Nathalie, Christian Hermans, and Henk Schat (2009). Molecular mechanisms of metal hyperaccumulation in plants. The New Phytologist, 181.4, 759-776. DOI: $10.1111 / \mathrm{j}$. 1469-8137.2008.02748.x 


\section{Bibliography}

Vinichuk, M., K.J. Johanson, H. Rydin, and K. Rosén (2010). The distribution of $137 \mathrm{Cs}$, k, rb and $\mathrm{cs}$ in plants in a sphagnum-dominated peatland in eastern central sweden. Journal of Environmental Radioactivity, 101.2, 170-176. DoI:10.1016/j. jenvrad.2009.10.003

Wachira, F. N., S. Kamunya, S. Karori, R. Chalo, and T. Maritim (2013). "Chapter 1 - The Tea Plants: Botanical Aspects". Tea in Health and Disease Prevention. Ed. by Victor R. Preedy. Academic Press, 3-17. Dol: 10.1016/B978-0-12-384937-3.00001-X.

Walker, George P. L. (1972). Crystal concentration in ignimbrites. Contributions to Mineralogy and Petrology, 36.2, 135-146. DOI:10.1007/BF00371184.

Walworth, J. L. and M. E. Sumner (1987). "The Diagnosis and Recommendation Integrated System (DRIS)". Advances in Soil Science. Ed. by B. A. Stewart. New York, NY: Springer New York, 149-188.

Wan, Q., R. K. Xu, and X. H. Li (2012). Proton release by tea plant (Camellia sinensis L.) roots as affected by nutrient solution concentration and pH. Plant, Soil and Environment, 58.9, 429434.

Wang, Hui, Ren-Kou Xu, Ning Wang, and Xing-Hui Li (2010). Soil acidification of alfisols as influenced by tea cultivation in eastern China. Pedosphere, 20.6, 799-806. DOI: 10.1016/ S1002-0160(10)60070-7.

FOC (14.12.2011). Flora of China. URL: Www . eFloras . org.

Welna, Maja, Anna Szymczycha-Madeja, and Pawel Pohl (2013). A comparison of samples preparation strategies in the multi-elemental analysis of tea by spectrometric methods. Food Research International, 53.2, 922-930. DOI:10.1016/j . foodres.2013.03.030.

Wesnousky, Steven G., Senthil Kumar, R. Mohindra, and V. C. Thakur (1999). Uplift and convergence along the Himalayan Frontal Thrust of India. Tectonics, 18.6, 967-976. DOI:10.1029/ 1999TC900026.

Wiche, Oliver, Balazs Székely, Nicolai-Alexeji Kummer, Christin Moschner, and Hermann Heilmeier (2016). Effects of intercropping of oat (avena sativa I.) with white lupin (lupinus albus I.) on the mobility of target elements for phytoremediation and phytomining in soil solution. International Journal of Phytoremediation, 18.9, 900-907. DoI:10.1080/15226514.2016. 1156635.

Wickham, Hadley (2009). ggplot2: Elegant Graphics for Data Analysis. Springer-Verlag New York. URL: http: //ggplot2.org.

Wickham, Hadley, Romain Francois, Lionel Henry, and Kirill Müller (2017). dplyr: A Grammar of Data Manipulation. URL: https : //CRAN.R-project.org/package=dplyr.

Wickham, Hadley and Lionel Henry (2018). tidyr: Easily Tidy Data with 'spread()' and 'gather()' Functions. URL: https://CRAN.R-project . org/package=tidyr

Wong, Ming Hung, Z. Q. Zhang, J.W.C Wong, and C. Y. Lan (1998). Trace metal contents (Al, Cu and $\mathrm{Zn}$ ) of tea: Tea and soil from two tea plantations, and tea products from different provinces of China. Environmental Geochemistry and Health, 20, 87-94.

Xue, Dong, Huaiying Yao, and Changyong Huang (2006). Microbial biomass, N mineralization and nitrification, enzyme activities, and microbial community diversity in tea orchard soils. Plant and Soil, 288.1, 319-331. DOI:10.1007/s11104-006-9123-2.

Yamashita, Masataka, Toshio Takyu, and Tetsuji Sasa (1997). Gravitropic reaction in the growth of tea roots. Japanese Journal of Crop Science, 66.3, 472-478. DOI: $10.1626 /$ jcs . 66 . 472.

Yeats, Robert S. and Robert J. Lillie (1991). Contemporary tectonics of the Himalayan frontal fault system: Folds, blind thrusts and the 1905 Kangra earthquake. Journal of Structural Geology, 13.2, 215-225. 
Yin, An (2006). Cenozoic tectonic evolution of the Himalayan orogen as constrained by alongstrike variation of structural geometry, exhumation history, and foreland sedimentation. EarthScience Reviews, 76.1, 1-131.

Zhang, Mingkui and Liping Fang (2007). Tea plantation-induced activation of soil heavy metals. Communications in Soil Science and Plant Analysis, 38.11-12, 1467-1478. DOI: 10.1080/ 00103620701378417.

Zheng, Shao Jian, Jian Feng Ma, and Hideaki Matsumoto (1998). High aluminum resistance in buckwheat. Plant Physiology, 117, 745-751.

Zoller, William H, ES Gladney, and Robert A Duce (1974). Atmospheric concentrations and sources of trace metals at the South Pole. Science, 183.4121, 198-200. 



\section{A. Tea and Sampling Areas}

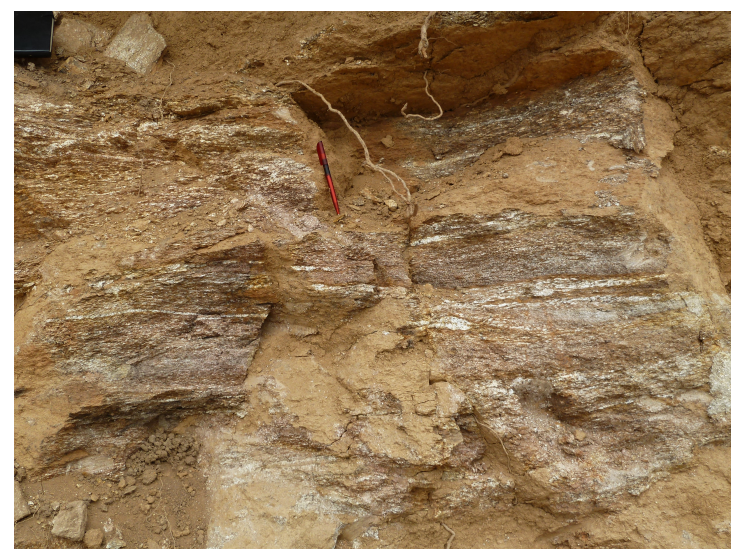

Figure A.1.: Outcrop along recently renewed street inside Nagri Farm of saprolitic schist of Main Central Thrust Zone unit, garnet-muscovite zone.

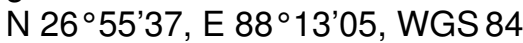

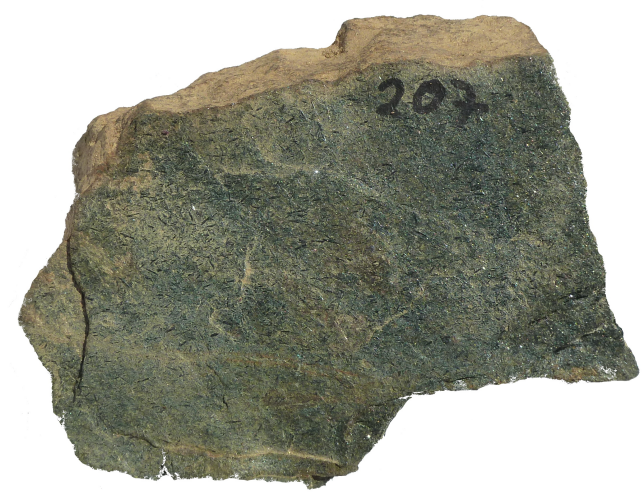

Figure A.2.: Hand specimen (length $\sim 10 \mathrm{~cm}$ ) of the Main Central Thrust Zone unit, garnetmuscovite zone, close to the footwall of the garnet-muscovite zone. Small staurolite needles are growing parallel to the foliation plane of the schist.

N $27^{\circ} 06^{\prime} 05$, E $88^{\circ} 14^{\prime} 44$, WGS 84 


\section{A. Tea and Sampling Areas}

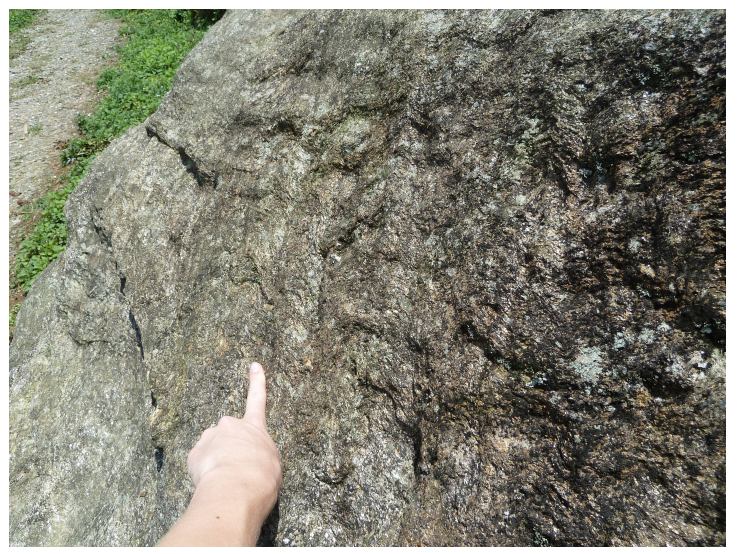

Figure A.3.: Small boulder of the Main Central Thrust Zone unit, staurolith zone, with crenulated matrix. Boulder is placed in the structurally upper level of garnet-muscovite zone.

N 2653'25, E 88¹7'91, WGS 84

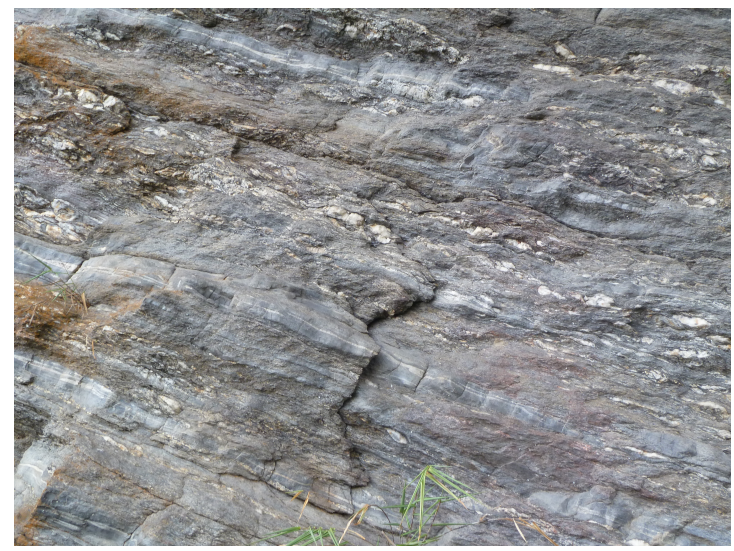

Figure A.4.: Huge boulder of the Main Central Thrust Zone unit, staurolith zone, placed in the close towards the hanging wall of the garnet-muscovite zone. See plant leaves for scale. Micaceous layers are strongly foliated while quartzo-feldspatic layers are are sheared into thin, undulating bands or boudin-like lenses.

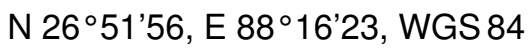

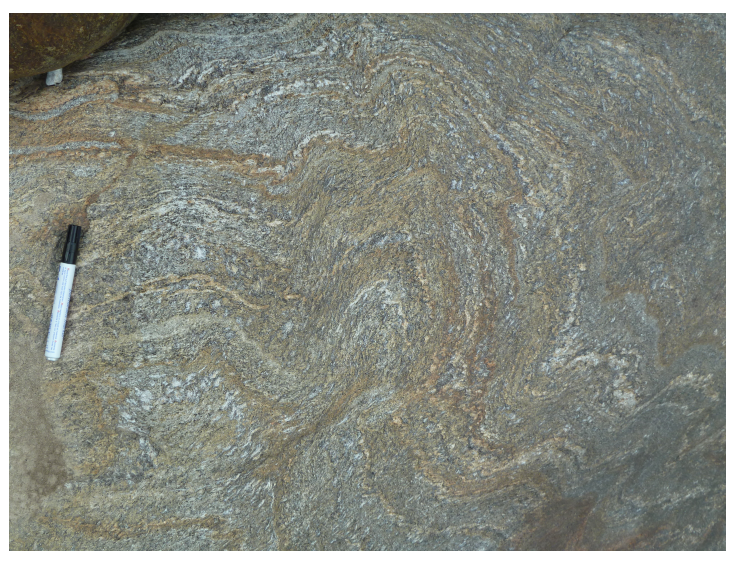

Figure A.5.: River boulder of the Main Centra Thrust Zone unit, kyanite zone. N $27^{\circ} 02^{\prime} 50$, E $88^{\circ} 10^{\prime} 25$, WGS 84

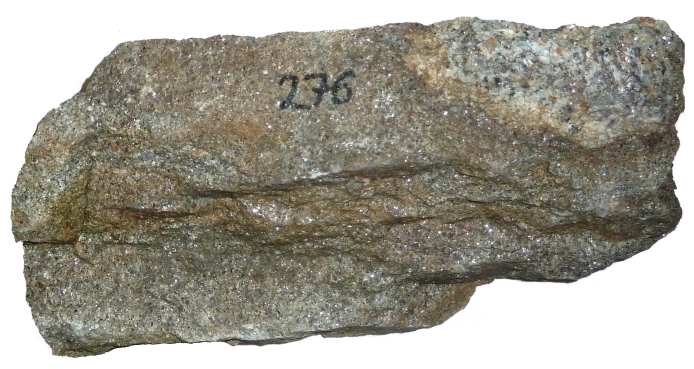

Figure A.6.: Hand specimen (length $\sim 24 \mathrm{~cm}$ ) of Main Central Thrust Zone unit, kyanite zone. In the right upper corner of the specimen is a leucosome consisting of $\mathrm{Qz} \pm \mathrm{Ky}$ $\pm \mathrm{Grt} \pm \mathrm{St}$ and accessories.

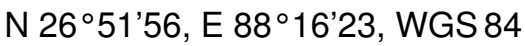


Table A.1.: List of tea estates in Darjeeling registered with the Tea Board of India to form the geographical indication 'Darjeeling' as a protected entity.

\begin{tabular}{|c|c|}
\hline Aloobari Tea Estate & Namring Tea Estate (\& Namring Upper) \\
\hline Ambiok(Hilton) Tea Estate & Nagri Farm Tea Estate (Makurjung) \\
\hline Ambootia Tea Estate & Narbada Majhua Tea Estate \\
\hline Arya Tea Estate (Sidrabong) & North Tukvar Tea Estate \\
\hline Avongrove Tea Estate & Nurbong Tea Estate \\
\hline Badamtam Tea Estate & Oaks Tea Estate \\
\hline Balasun Tea Estate & Okayti Tea Estate \\
\hline Bannockburn Tea Estate & Orange Valley Tea Estate \\
\hline Barnesbeg Tea Estate (North Tukvar) & Pandam Tea Estate \\
\hline Castleton Tea Estate & Peshok Tea Estate \\
\hline Chamong Tea Estate & Phoobsering Tea Estate \\
\hline Chongtong Tea Estate (Sirisi) & Phuguri Tea Estate \\
\hline Dhajea Tea Estate & Poobong Tea Estate \\
\hline Dilaram Tea Estate & Pussimbing Tea Estate (Minzoo) \\
\hline Dooteriah Tea Estate & Rangaroon Tea Estate \\
\hline Edenvale Tea Estate (Maharani) & Ringtong Tea Estate \\
\hline Gayabari Tea Estate (Millikthong) & Risheehat Tea Estate \\
\hline Giddhapahar Tea Estate & Rohini Tea Estate \\
\hline Gielle Tea Estate & Runglee-Rungliot Tea Estate \\
\hline Ging Tea Estate & Rungmook Tea Estate (Cedars) \\
\hline Glenburn Tea Estate & Samabeong Tea Estate \\
\hline Goomtee Tea Estate & Seepoydhoorah Tea Estate (Chamling) \\
\hline Gopaldhara Tea Estate & Seeyok Tea Estate (Spring Valley) \\
\hline Happy Valley Tea Estate & Selim Hill Tea Estate \\
\hline Jogamaya Tea Estate & Selimbong Tea Estate (Rongbong) \\
\hline Jungapana Tea Estate (Jungapana Upper) & Singbulli Tea Estate \\
\hline Kalej Valley Tea Estate & Singell Tea Estate \\
\hline Kanchaan View Tea Estate (Rungeet) & Singtam Tea Estate \\
\hline Kumai Tea Estate (Snow View) & Sivitar Tea Estate \\
\hline Lingia Tea Estate & Soom Tea Estate \\
\hline Liza Hill Tea Estate & Sourenee Tea Estate \\
\hline Longview Tea Estate (High Lands) & Springside Tea Estate \\
\hline Lopchu Tea Estate & Steinthal Tea Estate \\
\hline Mahalderam Tea Estate & Sungma Tea Estate \\
\hline Makaibari Tea Estate & Teesta Valley Tea Estate \\
\hline Margaret's Hope Tea Estate & Thurbo Tea Estate \\
\hline Marybong Tea Estate & Tindharia Tea Estate \\
\hline Mim Tea Estate & Tukvar Tea Estate (Puttabong) \\
\hline Mission Hill Tea Estate & Tukdah Tea Estate \\
\hline Mohan Majhua Tea Estate & Tumsong Tea Estate \\
\hline Moonteviot Tea Estate & Turzum Tea Estate \\
\hline Moondakotee Tea Estate & Upper Fagu Tea Estate \\
\hline Mullootar Tea Estate & Vah Tukvar Tea Estate (Shree Dwarika) \\
\hline Nagari Tea Estate & \\
\hline
\end{tabular}


Table A.2.: List of leave size classes and the respective grades of orthodox black tea

\begin{tabular}{lll}
\hline Class & Grade & Nomenclature \\
\hline \multirow{4}{*}{ Whole leaf } & FTGFOP & Fine, Tippy golden flowery orange pekoe \\
& TGFOP & Tippy golden flowery orange pekoe \\
& TGFOP 1 & Tippy golden flowery orange pekoe one \\
& GFOP & Golden flowery orange pekoe \\
& FOP & Flowery orange pekoe \\
& OP & Orange pekoe \\
\hline \multirow{4}{*}{ Brokens } & GFBOP & Golden flowery broken orange pekoe \\
& FBOP & Flowery broken orange pekoe \\
& GBOP & Golden broken orange pekoe \\
& BOP 1 & Broken orange pekoe one \\
& BPS & Broken pekoe souchang \\
\hline \multirow{4}{*}{ Fannings } & GOF & Golden orange fannings \\
& FOF & Flowery orange fannings \\
& BOPF & Broken orange pekoe fannings \\
& OF & Orange fannings \\
\hline \multirow{5}{*}{ Dust } & OPD & Orthodox pekoe dust \\
& OD & Orthodox dust \\
& OCD & Orthodox churamoni dust \\
& FD & Fine dust \\
\hline
\end{tabular}




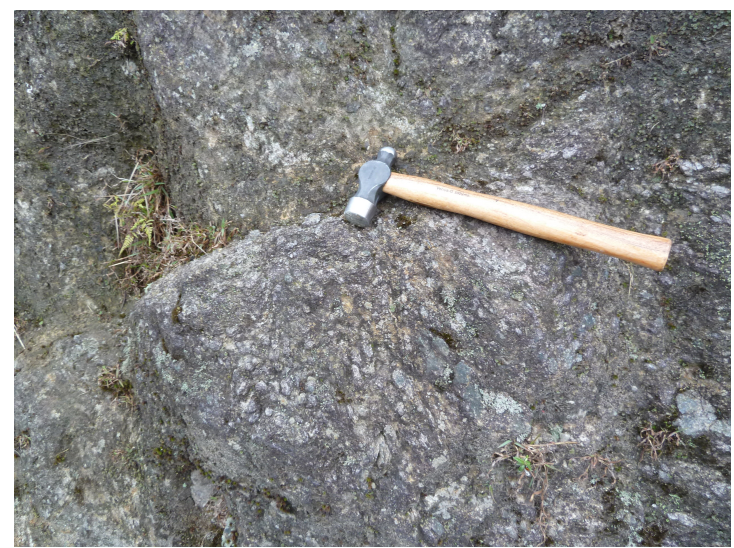

Figure A.7.: Typically weathered outcrop along a street of amphibolite grade gneisses (Kangchenjunga Gneiss (GHS) unit). The weathered aspect might trick the eye into describing the rock as "Augengneiss", but if the weathered cover is removed the outcrop looks like Figure 3.12 .

N $27^{\circ} 00^{\prime} 55$, E $88^{\circ} 15^{\prime} 08$, WGS 84

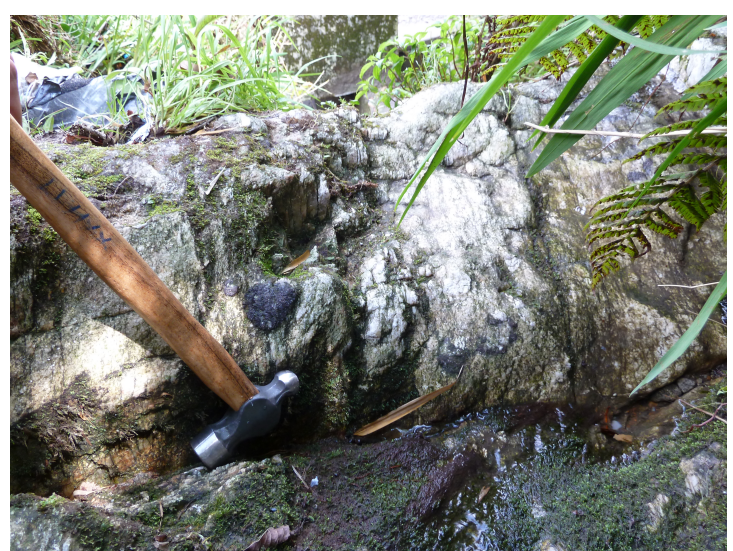

Figure A.8.: Boulder of amphibolite grade gneiss (Kangchenjunga Gneiss (GHS) unit) in a stream, $65 \mathrm{~m}$ distance to top of the ridge. Darkish mineral right above the hammer is a fist-sized garnet.

N $27^{\circ} 01^{\prime} 33$, E $88^{\circ} 15^{\prime} 45$, WGS 84

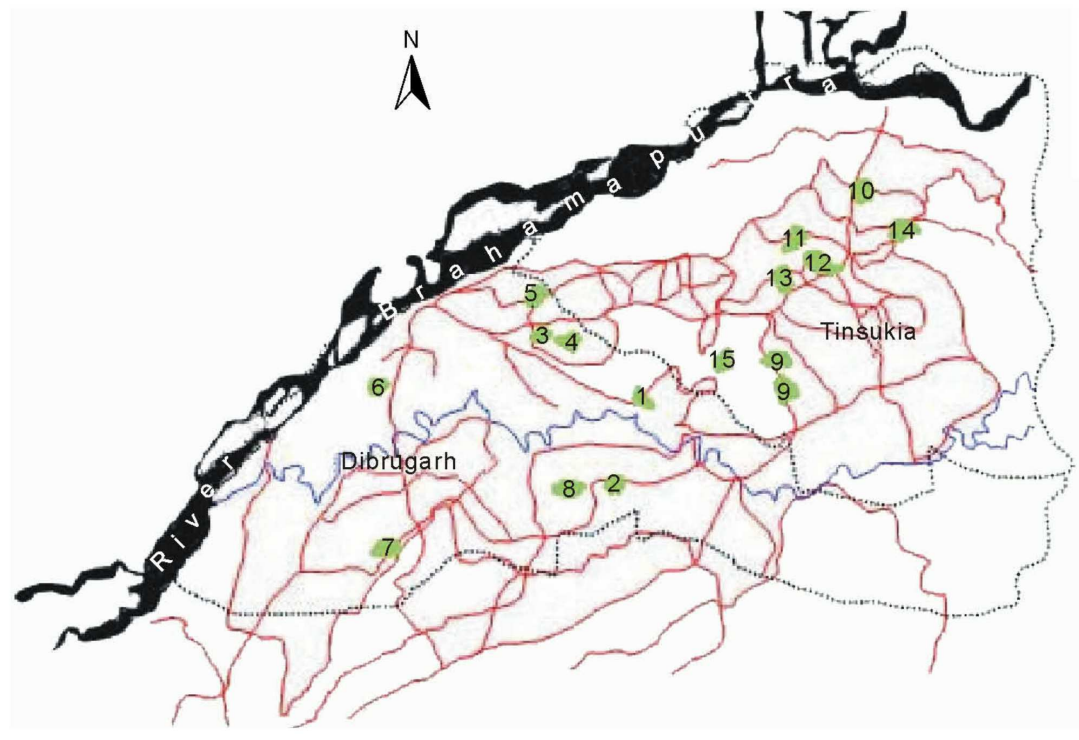

$$
\begin{aligned}
& \text { - Sampling site } \\
& \text {.....- District boundary } \\
& \text { - City or town boundary } \\
& \text { - River } \\
& \text { Tea estate } \\
& 1 \text {-Balijan } \\
& \text { 2-Desam } \\
& \text { 3-Dikom } \\
& \text { 4-Hattialli } \\
& \text { 5-Kamakhyabari } \\
& 6 \text { - Lepetkatta } \\
& \text { 7-Moran } \\
& \text { 8- Tinkong } \\
& \text { 9-Bazaloni } \\
& \text { 10-Chokhani } \\
& \text { 11-Deamoolie } \\
& \text { 12-Dhoedaam } \\
& \text { 13-Hilika } \\
& \text { 14-Koomsong } \\
& \text { 15-Mahakali }
\end{aligned}
$$

Figure A.9.: Schematic map (not in scale) of Upper Assam, India, from publication Karak et al. (2015a). The location of soil samples for the description of the tea soils in Assam are indicated by the sampling sites in this map. 


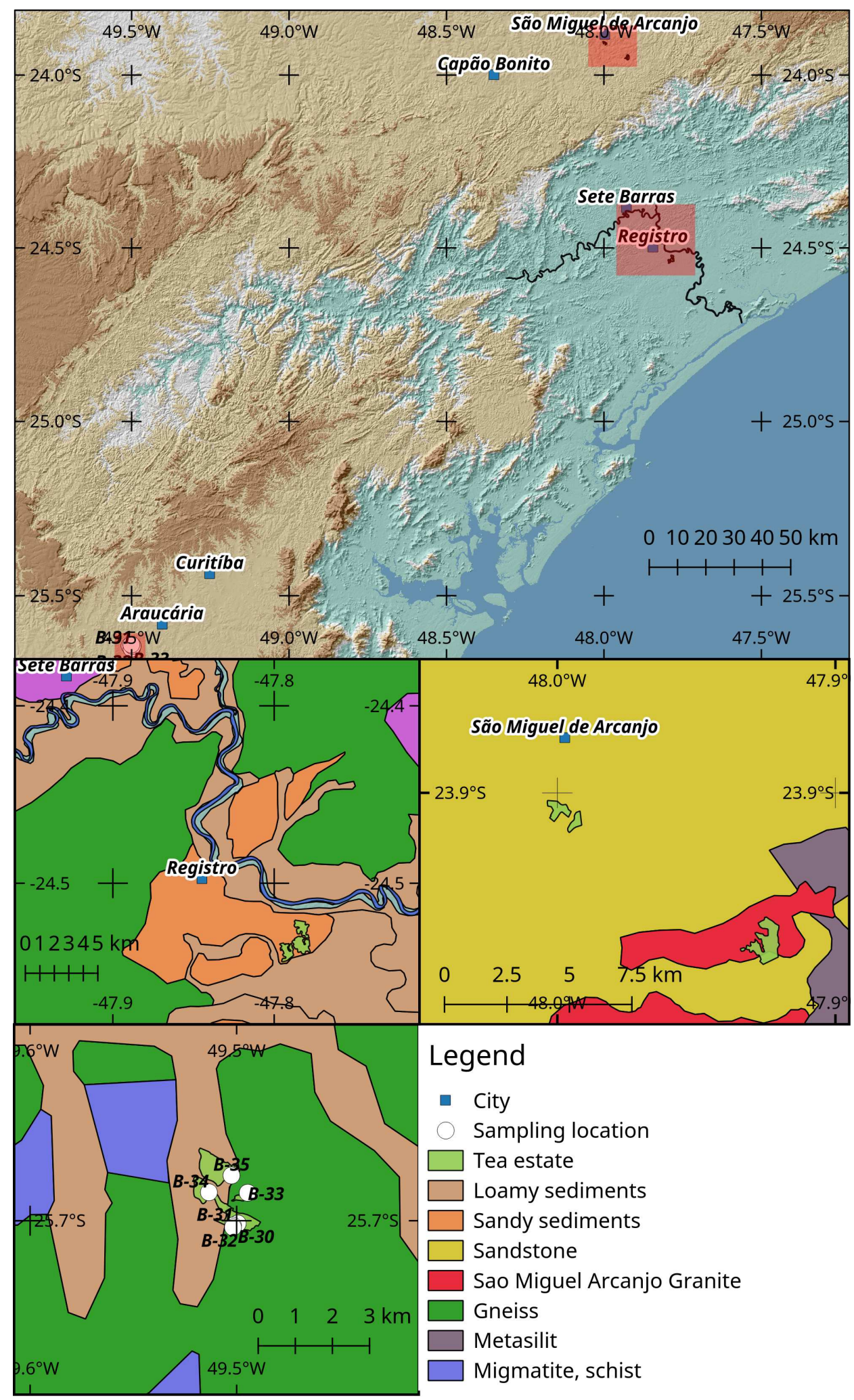

Figure A.10.: Map of tea growing regions and their geological setting in Brazil. Geological map: MM Perrotta et al. (2005). Mapa geológico do Estado de São Paulo, escala 1: 750.000. São Paulo: CPRM. Tea estates redrawn after satellite images 2017 (Google satellite). Projection WGS 84, UTM Zone 23J, Pseudo Mercator. 


\section{B. Sample Preparation and Analysis}

\section{B.1. Digestion Protocol}

\section{B.2. Protocol for Tea Brewing}

1. $100 \mathrm{~mL}$ of water, either destilled or tap water, boiled in a Perfluoroalkoxy (PFA) bottle are poured in a $100 \mathrm{~mL}$ Teflon® cup containing $2 \mathrm{~g}$ of tea.

2. The tea is brewed for $5 \mathrm{~min}$ in the cup.

3. The tea is filtered with a Teflon filter and split into two $50 \mathrm{~mL}$ units: one is used for $\mathrm{pH}$-measurements and after this the sample is dried in order to determine the amount of dissolved organic. The other $50 \mathrm{~mL}$ are stored in Polyethylen (PE) bottles and $100 \mu \mathrm{g}$ of IS is added. The left over leaf material is dried and weighed in order to determine the weight of the organic residue after cooking.

\section{B.3. Protocol for Plant Digestion}

newest version by: Benedikt Sauer, Hans Ruppert, Solveig Pospiech, Aug. 2014

1. $400 \mathrm{mg}$ to $500 \mathrm{mg}$ (best is maximum $450 \mathrm{mg}$ ) of powdered tea plant material are weighed into the Teflon $\AA$ cups of PicoTrace digestion equipment. Minimum one cup is used for an international plant standard sample like BCR-129, NJV 94-5 or GBW 10052 and minimum one cup is reserved for the blind, were no sample material is weighed in.

2. Pre-reaction: At room temperature $4 \mathrm{~mL}$ of concentrated $\mathrm{HNO}_{3}$ are added into every cup and let react for several hours (e.g. over night). Then the cups are heated up to $30^{\circ} \mathrm{C}$ within $5-10 \mathrm{~h}$ (depending on the reactivity of the powder), kept at this temperature for $12 \mathrm{~h}$, then further heated up to $50^{\circ} \mathrm{C}$ within $5-10 \mathrm{~h}$ and kept at the temperature for another $12 \mathrm{~h}$.

If the reaction slowed down after these steps $0.5 \mathrm{~mL}$ of conc. HF and $3 \mathrm{~mL}$ of conc. 
$\mathrm{HClO}_{4}$ are added. Then the cups are further heated up to $100^{\circ} \mathrm{C}$ within $2 \mathrm{~h}$ and kept at this temperature $4-5 \mathrm{~h}$ until all reactions are ceased.

3. Pressure phase I: Ontop the cups pressure plates are mounted. From the temperature of $100^{\circ} \mathrm{C}$ the cups are heated within $3 \mathrm{~h}$ up to $150^{\circ} \mathrm{C}$ and then kept at this temperature for another $15 \mathrm{~h}$.

4. Vaporization of acids: After cooling down the pressure plates are unmounted and the acids in the cups are vaporized by heating up the cups within $12 \mathrm{~h}$ to $180^{\circ} \mathrm{C}$, keeping temperature for $24 \mathrm{~h}$ and then letting cool down.

5. Pressure phase II: By now all acids should be vaporized and the residue in the cups should be dry. In every cup $0.5 \mathrm{~mL}$ of conc. $\mathrm{HNO}_{3}$ and $10 \mathrm{~mL}$ ultraclean $\mathrm{H}_{2} \mathrm{O}$ are added. For better dissolution the cups are heated up to $150^{\circ} \mathrm{C}$ within $1 \mathrm{~h}$ and kept at this temperature for $2 \mathrm{~h}$. After cooling $50 \mu \mathrm{L}$ of IS is added to every sample.

6. Solution transfer: The clear solution is transferred into a $25 \mathrm{~mL}$ narrow-necked volumetric flask, filled up to $25 \mathrm{~mL}$ with ultraclean water and then homogenised. $50 \mathrm{~mL}$ PE-bottles are pre-conditioned by a few $\mathrm{mL}$ of the digestion solution and then discarded. The rest of the solution is transferred from the volumetric flask into the sample bottles.

\section{B.4. Protocol for Soil Digestion}

newest version by: Benedikt Sauer, 2011

1. $100-120 \mathrm{mg}$ of powered soil material are weighed into the Teflon® cups of PicoTrace digestion equipment. One cup is used for the rock standard TW-45 and at least one cup is reserved for the blind solution, were no sample material is weighed in.

2. Pre-reaction: At room temperature $2 \mathrm{~mL}$ of conc. $\mathrm{HNO}_{3}$ are added into every cup and let react for several hours. Then $3 \mathrm{~mL}$ of conc. $\mathrm{HF}$ and $3 \mathrm{~mL}$ of conc. $\mathrm{HClO}_{4}$ are added.

3. Pressure phase I: Ontop the cups pressure plates are mounted and bolt down. Within $3 \mathrm{~h}$ the cups are heated up to $150^{\circ} \mathrm{C}$ and then kept at this temperature for another $5 \mathrm{~h}$. Then they are cooled down. 
4. Vaporization of acids: The pressure plates are unmounted. The acids in the cups are vaporized by heating up the cups within $6 \mathrm{~h}$ to $180^{\circ} \mathrm{C}$, keeping that temperature for $10 \mathrm{~h}$ and then they are cooled down.

5. Pressure phase II: By now all acids should be vaporized and the residues in the cups should be dry. In every cup $2 \mathrm{~mL}$ of conc. $\mathrm{HNO}_{3}, 0.6 \mathrm{~mL}$ conc. $\mathrm{HCl}$ and $10 \mathrm{~mL}$ ultraclean $\mathrm{H}_{2} \mathrm{O}$ are added. For a better dissolution the cups are heated up to $150^{\circ} \mathrm{C}$ within $1 \mathrm{~h}$ and kept at this temperature for $2 \mathrm{~h}$. After cooling down $200 \mu \mathrm{L}$ of IS are added to every sample.

6. Solution transfer: The clear and precipitation free solution are transferred into a $100 \mathrm{~mL}$ narrow-necked volumetric flask, filled up to $100 \mathrm{~mL}$ with ultraclean water and then homogenised. $100 \mathrm{~mL}$ PE-bottles are pre-conditioned by a few $\mathrm{mL}$ of the digestion solution and the discarded. The rest of the solution is transferred from the volumetric flask into the sample bottles.

\section{B.5. Calibration Solutions}

Table B.1.: Composition of Internal Standard for ICP-MS analysis (concentration given in $\mu \mathrm{g} / \mathrm{L}$ [ppb])

\begin{tabular}{cr|cc}
\hline Element & mass & Internal Standard until Aug'13 & Internal Standard after Aug'13 \\
\hline $\mathrm{Be}$ & 9 & - & 100 \\
$\mathrm{Ge}$ & 73 & 50 & - \\
$\mathrm{Rh}$ & 103 & 10 & 20 \\
$\mathrm{In}$ & 115 & 10 & 10 \\
$\mathrm{Re}$ & 185 & 20 & 10 \\
\hline
\end{tabular}


Table B.2.: Concentration of $100 \%$-calibration solution for ICP-MS analysis (concentration given in $\mathrm{mg} / \mathrm{L}[\mathrm{ppm}])$

\begin{tabular}{|c|c|}
\hline $\begin{array}{c}\text { Element } \\
(\mathrm{mg} / \mathrm{L}[\mathrm{ppm}])\end{array}$ & $\begin{array}{l}\text { calibration sol. } 100 \% \\
\left(2 \% \text { Vol. conc. } \mathrm{HNO}_{3}\right.\end{array}$ \\
\hline $\mathrm{Al}$ & 1 \\
\hline As & 0.05 \\
\hline B & 1 \\
\hline $\mathrm{Ba}$ & 0.5 \\
\hline $\mathrm{Be}$ & 0.02 \\
\hline $\mathrm{Bi}$ & 0.005 \\
\hline $\mathrm{Ca}$ & 0.5 \\
\hline $\mathrm{Cd}$ & 0.01 \\
\hline $\mathrm{Ce}$ & 0.05 \\
\hline Co & 0.05 \\
\hline $\mathrm{Cr}$ & 0.2 \\
\hline Cs & 0.01 \\
\hline $\mathrm{Cu}$ & 0.1 \\
\hline Dy & 0.004 \\
\hline $\mathrm{Er}$ & 0.004 \\
\hline Eu & 0.0025 \\
\hline $\mathrm{Fe}$ & 1 \\
\hline $\mathrm{Ga}$ & 0.2 \\
\hline Gd & 0.005 \\
\hline $\mathrm{Hf}$ & 0.02 \\
\hline Ho & 0.002 \\
\hline $\mathrm{K}$ & 1 \\
\hline $\mathrm{La}$ & 0.05 \\
\hline $\mathrm{Li}$ & 0.2 \\
\hline Lu & 0.001 \\
\hline $\mathrm{Mg}$ & 1 \\
\hline $\mathrm{Mn}$ & 0.5 \\
\hline Mo & 0.02 \\
\hline $\mathrm{Na}$ & 1 \\
\hline $\mathrm{Nb}$ & 0.1 \\
\hline $\mathrm{Nd}$ & 0.025 \\
\hline $\mathrm{Ni}$ & 0.2 \\
\hline $\mathrm{Pb}$ & 0.05 \\
\hline $\operatorname{Pr}$ & 0.01 \\
\hline $\mathrm{Rb}$ & 0.2 \\
\hline $\mathrm{Sb}$ & 0.02 \\
\hline $\mathrm{Sc}$ & 0.025 \\
\hline $\mathrm{Se}$ & 0.01 \\
\hline $\mathrm{Sm}$ & 0.005 \\
\hline Sn & 0.02 \\
\hline $\mathrm{Sr}$ & 0.5 \\
\hline $\mathrm{Ta}$ & 0.01 \\
\hline $\mathrm{Tb}$ & 0.002 \\
\hline Th & 0.025 \\
\hline $\mathrm{Ti}$ & 1 \\
\hline $\mathrm{TI}$ & 0.005 \\
\hline Tm & 0.001 \\
\hline U & 0.005 \\
\hline V & 0.2 \\
\hline W & 0.02 \\
\hline Y & 0.025 \\
\hline $\mathrm{Yb}$ & 0.005 \\
\hline $\mathrm{Zn}$ & 0.4 \\
\hline $\mathrm{Zr}$ & 0.2 \\
\hline
\end{tabular}


Table B.3.: Concentration of $100 \%$-calibration solution for soil samples and tea samples for ICPOES analysis, used until May 2015 (concentration given in $\mathrm{mg} / \mathrm{L}$ [ppm])

\begin{tabular}{|c|c|c|}
\hline $\begin{array}{c}\text { Element } \\
(\mathrm{mg} / \mathrm{L}[\mathrm{ppm}])\end{array}$ & $\begin{array}{c}\text { soil calibration sol. } 100 \% \\
\left(2 \% \text { Vol. conc. } \mathrm{HNO}_{3}\right. \\
0.5 \% \text { Vol. } 6 \mathrm{~N} \mathrm{HCl})\end{array}$ & $\begin{array}{l}\text { plant calibration sol. } 100 \% \\
\left(2 \% \text { Vol. conc. } \mathrm{HNO}_{3}\right)\end{array}$ \\
\hline $\mathrm{Al}$ & 500 & 20 \\
\hline $\mathrm{Ba}$ & 10 & 5 \\
\hline $\mathrm{Be}$ & 0.1 & 0.1 \\
\hline $\mathrm{Ca}$ & 200 & 400 \\
\hline $\mathrm{Cd}$ & 0.2 & 0.1 \\
\hline $\mathrm{Ce}$ & 1 & \\
\hline Co & 1 & 0.1 \\
\hline $\mathrm{Cr}$ & 2 & 0.1 \\
\hline $\mathrm{Cu}$ & 1 & 0.5 \\
\hline $\mathrm{Fe}$ & 400 & 10 \\
\hline $\mathrm{Ga}$ & 2 & \\
\hline $\mathrm{K}$ & 200 & 600 \\
\hline $\mathrm{La}$ & 1 & \\
\hline $\mathrm{Li}$ & 2 & \\
\hline $\mathrm{Mg}$ & 200 & 100 \\
\hline $\mathrm{Mn}$ & 10 & 6 \\
\hline Mo & 1 & 1 \\
\hline $\mathrm{Na}$ & 100 & 50 \\
\hline $\mathrm{Nb}$ & 0.5 & \\
\hline $\mathrm{Ni}$ & 2 & 1 \\
\hline $\mathrm{P}$ & 5 & 250 \\
\hline $\mathrm{Pb}$ & 2 & 1 \\
\hline$S$ & 50 & 150 \\
\hline Sc & 1 & 0.1 \\
\hline $\mathrm{Sn}$ & 0.5 & \\
\hline $\mathrm{Sr}$ & 5 & 4 \\
\hline $\mathrm{Ti}$ & 25 & 1 \\
\hline $\mathrm{V}$ & 2 & \\
\hline $\mathrm{Zn}$ & 2 & 10 \\
\hline $\mathrm{Zr}$ & 2.5 & \\
\hline
\end{tabular}


Table B.4.: Concentration of $100 \%$-calibration solution for soil samples and tea samples for ICPOES analysis, used from June 2015 onwards (concentration given in mg/L [ppm])

\begin{tabular}{|c|c|c|}
\hline $\begin{array}{c}\text { Element } \\
\text { (mg/L }[\mathrm{ppm}])\end{array}$ & $\begin{array}{c}\text { soil calibration sol. } 100 \% \\
\left(2 \% \text { Vol. conc. } \mathrm{HNO}_{3}\right. \\
0.5 \% \text { Vol. } 6 \mathrm{~N} \mathrm{HCl})\end{array}$ & $\begin{array}{l}\text { plant calibration sol. } 100 \% \\
\left(2 \% \text { Vol. conc. } \mathrm{HNO}_{3}\right)\end{array}$ \\
\hline $\mathrm{Al}$ & 200 & 20 \\
\hline $\mathrm{Ba}$ & 10 & 5 \\
\hline $\mathrm{Be}$ & 0.2 & 0.1 \\
\hline $\mathrm{Ca}$ & 100 & 400 \\
\hline $\mathrm{Cd}$ & 0.1 & 2 \\
\hline $\mathrm{Ce}$ & 1 & \\
\hline Co & 0.25 & 0.1 \\
\hline $\mathrm{Cr}$ & 0.5 & 0.1 \\
\hline $\mathrm{Cu}$ & 0.5 & 1 \\
\hline $\mathrm{Fe}$ & 100 & 50 \\
\hline $\mathrm{Ga}$ & 0.5 & \\
\hline $\mathrm{K}$ & 100 & 1000 \\
\hline La & 1 & \\
\hline $\mathrm{Li}$ & 0.5 & \\
\hline $\mathrm{Mg}$ & 50 & 100 \\
\hline $\mathrm{Mn}$ & 10 & 5 \\
\hline Mo & 0.25 & 0.25 \\
\hline $\mathrm{Na}$ & 25 & 20 \\
\hline $\mathrm{Nb}$ & 0.5 & \\
\hline $\mathrm{Ni}$ & 0.5 & 0.25 \\
\hline$P$ & 5 & 250 \\
\hline $\mathrm{Pb}$ & 0.5 & 0.25 \\
\hline$S$ & 10 & 100 \\
\hline $\mathrm{Sc}$ & 0.25 & 0.1 \\
\hline Sn & 0.25 & \\
\hline $\mathrm{Sr}$ & 2.5 & 2 \\
\hline $\mathrm{Ti}$ & 25 & 2 \\
\hline $\mathrm{V}$ & 1 & \\
\hline $\mathrm{Zn}$ & 0.5 & 2.5 \\
\hline $\mathrm{Zr}$ & 2 & \\
\hline
\end{tabular}


Table B.5.: Concentration of maximal calibration solution for soil samples and tea samples for ICP-OES analysis, used from June 2015 onwards (concentration given in $\mathrm{mg} / \mathrm{L}$ [ppm]). This calibration solution is included into the calibration curve if samples show concentrations significantly above the $100 \%$-calibration point

\begin{tabular}{ccc}
\hline $\begin{array}{c}\text { Element } \\
(\mathrm{mg} / \mathrm{L} \text { [ppm] })\end{array}$ & $\begin{array}{c}\text { soil calibration sol. Max } \\
\left(2 \% \text { Vol. conc. } \mathrm{HNO}_{3},\right. \\
0.5 \% \text { Vol. } 6 \mathrm{~N} \mathrm{HCl})\end{array}$ & $\begin{array}{c}\text { plant calibration sol. Max } \\
\left(2 \% \text { Vol. conc. } \mathrm{HNO}_{3}\right)\end{array}$ \\
\hline $\mathrm{Al}$ & 500 & 200 \\
$\mathrm{Ca}$ & 2 & 1000 \\
$\mathrm{Cr}$ & & 1 \\
$\mathrm{Cu}$ & 200 & 100 \\
$\mathrm{Fe}$ & & 400 \\
$\mathrm{Mg}$ & & 50 \\
$\mathrm{Mn}$ & & 1 \\
$\mathrm{Mo}$ & & 200 \\
$\mathrm{Na}$ & & 1 \\
$\mathrm{Ni}$ & & 1 \\
$\mathrm{~Pb}$ & 50 & 4 \\
$\mathrm{~S}$ & & 10 \\
$\mathrm{Sr}$ & 5 & 20 \\
$\mathrm{Ti}$ & & \\
$\mathrm{Zn}$ & & \\
\hline
\end{tabular}




\begin{tabular}{|c|c|c|c|c|c|c|c|c|c|c|c|}
\hline Date.digestion & Type & $\mathrm{Al}$ & $\mathrm{Ba}$ & $\mathrm{Ca}$ & $\mathrm{Cd}$ & $\mathrm{Ce}$ & Co & $\mathrm{Cr}$ & Cs & $\mathrm{Cu}$ & Dy \\
\hline 2011-07-01 & Plant & Al.396.152 & Ba.233.527 & Ca.315.887 & Cd114 & Ce140 & Co59 & Cr.267.716 & Cs133 & Cu.327.395 & Dy163 \\
\hline 2011-08-01 & Rock & Al.308.215 & Ва.233.527 & Сa.315.887 & Cd114 & Ce.413.765 & Co.228.615 & Cr.267.716 & Cs133 & Cu.327.395 & Dy163 \\
\hline 2011-08-30 & Plant & Al.396.152 & Ва.233.527 & Сa.315.887 & Cd114 & Ce140 & Co59 & Cr.267.716 & Cs133 & Cu.324.752 & Dy163 \\
\hline 2011-09-01 & Plant & Al.396.152 & Вa.233.527 & Сa.315.887 & Cd114 & Ce140 & Co59 & Cr.267.716 & Cs133 & Cu.327.395 & Dy163 \\
\hline 2014-07-24 & Plant & Al.308.215 & Ва.233.527 & Ca.315.887 & Cd114 & Ce140 & Co.230.786 & Cr.267.716 & Cs133 & Cu.327.395 & Dy 163 \\
\hline 2014-08-03 & Rock & Al.308.215 & Ва. 455.403 & Ca.315.887 & Cd114 & Ce140 & Co59 & Cr.26 & Cs133 & Cu.324.752 & Dy163 \\
\hline 2014 & Soil & 15 & $\mathrm{Ba}$ & 3 & $\mathrm{C}$ & 1 & $\mathrm{C}$ & 716 & 33 & 395 & Dy163 \\
\hline 2014-11-01 & Rock & Al.308.215 & Ва.233.527 & Ca.183.944 & Cd114 & Ce140 & Co.230.786 & Cr.2 & Cs133 & 4.754 & Dy163 \\
\hline 2014-12-01 & Plant & Al.396.152 & Ba.493.408.rad & Ca.317.933.rad & Cd.214.439 & Ce140 & Сo.230.786 & Cr.267.716 & Cs133 & Cu.327.395 & Dy163 \\
\hline $2-01$ & Rock & 215 & & & C & & 786 & 560 & Cs133 & 395 & Dy 163 \\
\hline 2015-07-09 & Plant & Al.396.152 & Вa. 455.403 & Ca.422.673.rad & Cd.214.439 & Ce140 & Co.230.786 & Cr.267.716 & Cs133 & Cu.327.395 & Dy163 \\
\hline 2016-01-27 & Rock & Al.308.215 & Ва.233.527 & Сa.422.673 & Cd114 & Ce.446.021 & Co.230.786 & Cr.206.158 & Cs133 & Cu.327.395 & Dy 163 \\
\hline 2016-05-15 & Plant & Al.396.152 & Ba137 & Сa.317.933 & Cd114 & Ce140 & Co.230.786 & Cr.267.716 & Cs133 & Cu.327.395 & Dy 163 \\
\hline 2016-08-05 & Plant & Al.396.152 & Ba135 & Сa.317.933 & Cd111 & Ce140 & Сo.230.786 & Cr052 & Cs133 & Cu.327.395 & Dy 163 \\
\hline 2016-10-05 & Plant & Al.396.152 & Ba.455.403.rad & Ca.317.933 & Cd0114 & Ce140 & Co.230.786 & Cr.205.560 & Cs133 & Cu.327.395 & Dy 163 \\
\hline 2017-03-07 & Plant & Al.396.152 & Ba.455.403 & Ca.317.933 & Cd111 & Ce140 & Co.230.786 & Cr.267.716 & Cs133 & Cu.327.395 & Dy 163 \\
\hline 2017-04-07 & Plant & Al.396.152 & Ba137 & Ca.317.933.rad & Cd0114 & Ce140 & Co.230.786 & Cr.267.716 & Cs133 & Cu.327.395 & Dy 163 \\
\hline 2017-04-14 & Plant & Al.396.152 & Ba.493.408.rad & Ca.317.933.rad & Cd111 & Ce140 & Co.230.786 & Cr.267.716 & Cs133 & Cu065 & Dy163 \\
\hline
\end{tabular}


Table B.7.: Selected element datum, continued from table B.6

\begin{tabular}{|c|c|c|c|c|c|c|c|c|c|c|c|c|}
\hline Date.digestion & $\mathrm{Er}$ & $\mathrm{Eu}$ & $\mathrm{Fe}$ & $\mathrm{Gd}$ & $\mathrm{Hf}$ & $\mathrm{Ho}$ & $\mathrm{K}$ & $\mathrm{La}$ & $\mathrm{Li}$ & $\mathrm{Lu}$ & $\mathrm{Mg}$ & $\mathrm{Mn}$ \\
\hline 2011-07-01 & Er166 & 153 & Fe.259.940 & Gd157 & $\mathrm{Hf} 177$ & 10165 & 766.491 & 139 & $\mathrm{Li} 7$ & .4175 & Mg.285.213 & Mn.257.610 \\
\hline 2011 & Er166 & Eu151 & Fe.238.863 & Gd157 & $\mathrm{Hf} 177$ & Ho165 & K.766.491 & a.408.671 & Li.670.783 & Lu175 & Mg.279.078 & 57.610 \\
\hline 2011-08-30 & Er166 & Eu153 & Fe.259.940 & Gd157 & $\mathrm{Hf} 177$ & Ho165 & K.766.491 & a139 & $\mathrm{Li} 7$ & Lu175 & Mg.285.213 & Mn.257.610 \\
\hline 201 & Er166 & Eu151 & Fe.259.940 & Gd157 & $\mathrm{Hf} 177$ & Ho165 & K.766.491 & & $\mathrm{Li} 7$ & Lu175 & Mg.285.213 & \\
\hline 201 & Er166 & Eu151 & Fe.259.940 & Gd157 & $\mathrm{Hf} 178$ & Ho165 & K.404.721 & a139 & $\mathrm{Li} 7$ & Lu175 & Mg.285.213 & 57.610 \\
\hline 2014-08-03 & Er166 & 151 & 238.204 & Gd158 & $\mathrm{Hf} 178$ & 0165 & .404 .721 & 408 & $\mathrm{Li}$ & Lu175 & Mg.279.078 & Mn 260 \\
\hline 201 & Er166 & 151 & 50 & Gd157 & $\mathrm{Ht}$ & & & & Li.67 & & & \\
\hline 2014 & Er166 & Eu151 & Fe.234.350 & Gd158 & $\mathrm{Hf} 178$ & 65 & K.7C & 9 & Li7 & 75 & 8 & 10 \\
\hline 201 & Er16e & Eu1 & & $\mathrm{Gd}$ & & & & & & & & \\
\hline 201 & & & & & & & & & $\mathrm{Li}$ & & & \\
\hline 2015- & 166 & & & Gd157 & & & $\mathrm{K}$. & & $\mathrm{Li} 7$ & & & \\
\hline 2016 & 166 & Eu151 & $\mathrm{Fe} .2$ & Gd157 & $\mathrm{Hf} 178$ & $\mathrm{Ho}$ & $\mathrm{K}$. & 3.749 & Li.67 & 75 & $\mathrm{Mc}$ & \\
\hline 2016-05-15 & Er166 & 51 & Fe.259.940 & Gd157 & $\mathrm{Hf} 178$ & Ho165 & K.766.491.rad & La139 & $\mathrm{Li} 7$ & Lu175 & Mg.285.213 & Mn.257.610 \\
\hline 2016-08-05 & Er166 & 153 & Fe.25 & 57 & $\mathrm{Hf178}$ & 165 & $d$ & 71 & Li.6 & Lu175 & 3 & $\mathrm{Mn} 055$ \\
\hline 2016-10-05 & Er166 & Eu153 & Fe.25 & Gd157 & $\mathrm{Hf} 180$ & Ho165 & $\mathrm{rad}$ & 71 & Li.6 & Lu175 & 213 & Mn.279.482 \\
\hline 2017-03-07 & Er166 & Eu153 & Fe.259.940 & Gd157 & $\mathrm{Hf} 178$ & Ho165 & K.766.491.rad & La.408.671 & Li.670.783 & Lu175 & Mg.285.213 & Mn.257.610 \\
\hline 2017-04-07 & Er166 & Eu151 & Fe.259.940 & Gd157 & $\mathrm{Hf} 180$ & Ho165 & K.766.491.rad & La.408.671 & Li.670.783 & Lu175 & Mg.285.213 & Mn.257.610 \\
\hline 2017-04-14 & Er166 & Eu151 & Fe.259.940 & Gd157 & $\mathrm{Hf} 180$ & Ho165 & K.766.491.rad & La.408.671 & Li.670.783 & Lu175 & Mg.285.213 & Mn.257.610 \\
\hline
\end{tabular}


Table B.8.: Selected element datum, continued from table B.6

\begin{tabular}{|c|c|c|c|c|c|c|c|c|c|c|c|c|}
\hline Date.digestion & Mo & $\mathrm{Na}$ & $\mathrm{Nb}$ & $\mathrm{Nd}$ & $\mathrm{Ni}$ & $P$ & $\mathrm{~Pb}$ & $\mathrm{Pr}$ & $\mathrm{Rb}$ & $S$ & $\mathrm{Sb}$ & Sc \\
\hline 2011-07-01 & Mo98 & $\mathrm{Na} 23$ & $\mathrm{Nb93}$ & Nd143 & $\mathrm{Ni60}$ & P.178.222 & $\mathrm{Pb} 208$ & Pr141 & $\mathrm{Rb} 85$ & S.181.972 & Sb121 & Sc.361.383 \\
\hline 2011-08-01 & Mo95 & Na.589.592 & Nb.309.417 & $\mathrm{Nd} 143$ & Ni.231.604 & P.214.914 & $\mathrm{Pb} 208$ & Pr141 & Rb85 & S.181.972 & Sb121 & Sc.361.383 \\
\hline 2011-08-30 & Mo98 & Na.589.592 & $\mathrm{Nb93}$ & $\mathrm{Nd} 143$ & Ni60 & P.178.222 & $\mathrm{Pb} 208$ & Pr141 & $\mathrm{Rb} 85$ & S.180.669 & Sb121 & Sc.361.383 \\
\hline 2011-09-01 & Mo98 & $\mathrm{Na} 23$ & $\mathrm{Nb93}$ & $\mathrm{Nd} 143$ & Ni.231.604 & P.178.222 & Pb208 & Pr141 & $\mathrm{Rb} 85$ & S.181.972 & Sb121 & Sc.361.383 \\
\hline 2014-07-24 & Mo98 & Na.588.995.rad & $\mathrm{Nb93}$ & Nd146 & Ni.231.604 & P.178.222 & $\mathrm{Pb} 208$ & Pr141 & $\mathrm{Rb} 85$ & S.181.972 & Sb121 & Sc.361.383 \\
\hline $2014-08-03$ & Mo98 & $\mathrm{Na}$ & $\mathrm{Nb93}$ & $\mathrm{Nd} 143$ & $N$ & P.178.222 & $\mathrm{Ph} 220$ & Pr141 & $\mathrm{Rb} 85$ & S.181.972 & 21 & 83 \\
\hline 201 & Mo98 & $\mathrm{Na}$ & $\mathrm{Nb}$ & $\mathrm{Nd}$ & Ni60 & 618 & 08 & 41 & 85 & 669 & 121 & 15 \\
\hline 2014-11-01 & Mo98 & Na.588.995.rad & $\mathrm{Nb93}$ & $\mathrm{Nd} 143$ & Ni.216.555 & P.214.914 & $\mathrm{Pb} 208$ & 41 & $\mathrm{Rb85}$ & .669 & Sb121 & \\
\hline 2014-12-01 & Mo98 & Na.588.995.rad & $\mathrm{Nb93}$ & Nd146 & Ni.231.604 & P.178.222 & $\mathrm{Pb} 208$ & Pr141 & $\mathrm{Rb} 85$ & S.181.972 & & Sc. 361.383 \\
\hline 201 & Mo98 & $\mathrm{Na}$ & 93 & & & 14 & 208 & 141 & $\mathrm{Rb} 85$ & 69 & & 372 \\
\hline 2015-07-09 & Mo98 & Na.588.995.rad & $\mathrm{Nb93}$ & $\mathrm{Nd} 146$ & Ni.231.604 & P.178.222 & Pb.220.353 & Pr141 & $\mathrm{Rb85}$ & S.181.972 & Sb121 & 1.383 \\
\hline 2016-01-27 & Mo98 & Na.588.995.rad & $\mathrm{Nb} .309 .417$ & Nd146 & Ni.221.648 & P.213.618 & Pb.220.353 & Pr141 & Rb85 & S.182.562 & Sb121 & Sc.335.372 \\
\hline 2016-05-15 & Mo98 & Na.588.995.rad & $\mathrm{Nb93}$ & $\mathrm{Nd} 146$ & Ni62 & P.178.222 & Pb208 & Pr141 & $\mathrm{Rb} 85$ & S.181.972 & Sb121 & Sc.361.383 \\
\hline 2016-08-05 & Mo98 & Na.588.995.rad & $\mathrm{Nb93}$ & $\mathrm{Nd} 146$ & $\mathrm{Ni060}$ & P.178.222 & Pb.220.353 & Pr141 & $\mathrm{Rb} 85$ & S.181.972 & Sb121 & Sc.335.372 \\
\hline 2016-10-05 & Mo98 & Na.588.995.rad & $\mathrm{Nb93}$ & $\mathrm{Nd} 146$ & Ni.231.604 & P.178.222 & $\mathrm{Pb} 206$ & Pr141 & $\mathrm{Rb} 85$ & S.181.972 & Sb121 & Sc. 361.383 \\
\hline 2017-03-07 & Mo98 & Na.588.995.rad & $\mathrm{Nb93}$ & $\mathrm{Nd} 146$ & Ni.216.555 & P.178.222 & $\mathrm{Pb} 206$ & Pr141 & $\mathrm{Rb85}$ & S.181.972 & Sb121 & Sc. 361.383 \\
\hline 2017-04-07 & Mo98 & Na.588.995.rad & $\mathrm{Nb93}$ & $\mathrm{Nd} 146$ & Ni.216.555 & P.178.222 & $\mathrm{Pb} 206$ & Pr141 & $\mathrm{Rb} 85$ & S.181.972 & Sb121 & Sc. 361.383 \\
\hline 2017-04-14 & Mo98 & Na.588.995.rad & $\mathrm{Nb93}$ & $\mathrm{Nd} 144$ & $\mathrm{Ni060}$ & P.178.222 & Pb.220.353 & Pr141 & $\mathrm{Rb} 85$ & S.181.972 & Sb121 & Sc.361.383 \\
\hline
\end{tabular}


Table B.9.: Selected element datum, continued from table B.6

\begin{tabular}{|c|c|c|c|c|c|c|c|c|c|c|c|c|c|}
\hline Date.digestion & $\mathrm{Sm}$ & $\mathrm{Sr}$ & $\mathrm{Ta}$ & $\mathrm{Tb}$ & Th & $\mathrm{Ti}$ & $\mathrm{Tl}$ & $\mathrm{Tm}$ & $U$ & $\mathrm{Y}$ & $\mathrm{Yb}$ & $\mathrm{Zn}$ & $\mathrm{Zr}$ \\
\hline 2011-07-01 & Sm147 & Sr.407.771 & Ta181 & Tb159 & Th232 & Ti.336.122 & Tl205 & Tm169 & U238 & Y89 & Yb173 & Zn.213.857 & Zr90 \\
\hline 2011-08-01 & Sm147 & Sr.421.552 & Ta181 & Tb159 & Th232 & Ti.334.903 & Tl205 & Tm169 & U238 & Y89 & Yb173 & Zn.213.857 & Zr.343.823 \\
\hline 2011-08-30 & Sm147 & Sr.407.771 & Ta181 & Tb159 & Th232 & Ti.336.122 & Tl205 & Tm169 & U238 & Y89 & Yb173 & Zn.213.857 & Zr90 \\
\hline 2011-09-01 & Sm147 & Sr.407.771 & Ta181 & Tb159 & Th232 & Ti.336.122 & Tl205 & Tm169 & U238 & Y89 & Yb173 & Zn.213.857 & Zr90 \\
\hline 2014-07-24 & Sm152 & Sr.232.235 & Ta181 & Tb159 & Th232 & Ti.336.122 & Tl205 & Tm169 & U238 & Y89 & Yb172 & Zn.213.857 & Zr90 \\
\hline 2014-08-03 & Sm147 & Sr.421.552 & Ta181 & Tb159 & Th232 & Ti.336.122 & Tl205 & Tm169 & U238 & Y89 & Yb172 & Zn.206.200 & Zr.34 \\
\hline 2014 & Sm152 & Sr.460.733 & $\mathrm{T}$ & $\mathrm{Tb}$ & Th232 & Ti49 & Tl205 & $\mathrm{Tm}$ & U238 & Y89 & 74 & 258 & Zr.3 \\
\hline 2014-11-01 & Sm147 & Sr.460.733 & Ta181 & Tb159 & Th232 & Ti.334.941 & TI205 & Tm169 & U238 & Y89 & 74 & 857 & Zr.343.823 \\
\hline 2014-12-01 & Sm147 & Sr88 & Ta181 & Tb159 & Th232 & Ti.336.122 & Tl205 & Tm169 & U238 & Y89 & Yb172 & Zn.213.857 & Zr90 \\
\hline 201 & 147 & $\mathrm{Sr}$ & Ta181 & Tb159 & Th232 & Ti.36 & & Tm169 & U238 & Y89 & Yb174 & 857 & Zr.34 \\
\hline 201 & Sm147 & Sr.421.552.rad & Ta181 & Tb159 & Th232 & Ti.336.122 & Tl205 & Tm169 & U238 & Y89 & Yb172 & 357 & Zr90 \\
\hline 2016-01-27 & Sm147 & Sr.460.733 & Ta181 & Tb159 & Th232 & Ti.336.122 & Tl205 & Tm169 & U238 & Y89 & Yb174 & 857 & Zr.343.823 \\
\hline 2016-05-15 & Sm147 & Sr.421.552.rad & Ta181 & Tb159 & Th232 & Ti.336.122 & Tl205 & Tm169 & U238 & Y89 & Yb172 & Zn.213.857 & Zr90 \\
\hline 2016 & 47 & $\mathrm{~S}$ & $\mathrm{~T}$ & 159 & Th & Ti.336.122 & Tl205 & 9 & 8 & Y89 & 72 & Zn.213.857 & Zr90 \\
\hline 2016-10-05 & Sm147 & Sr.421.552.rad & Ta181 & Tb159 & Th232 & Ti.336.122 & Tl205 & Tm169 & U238 & Y89 & Yb172 & Zn.213.857 & Zr9o \\
\hline 2017-03-07 & Sm152 & Sr.421.552.rad & Ta181 & Tb159 & Th232 & Ti.336.122 & Tl205 & Tm169 & U238 & Y89 & Yb172 & Zn.213.857 & Zr90 \\
\hline 2017-04-07 & Sm147 & Sr88 & Ta181 & Tb159 & Th232 & Ti.336.122 & Tl205 & Tm169 & U238 & Y89 & Yb172 & Zn.213.857 & Zr90 \\
\hline 2017-04-14 & Sm147 & Sr88 & Ta181 & Tb159 & Th232 & Ti.336.122 & Tl205 & Tm169 & U238 & Y89 & Yb172 & Zn.213.857 & Zr90 \\
\hline
\end{tabular}


B. Sample Preparation and Analysis

B.6. Reference Samples 
Table B.10.: Certified values of the international and in-house reference materials

\begin{tabular}{|c|c|c|c|c|c|}
\hline & $\begin{array}{l}\text { BCR- } 129 \\
\text { hay powder }\end{array}$ & $\begin{array}{c}\text { GBW } 10052 \\
\text { Tea }\end{array}$ & $\begin{array}{c}\text { NCS DC } 73349 \\
\text { bush twigs and } \\
\text { leaves }\end{array}$ & $\begin{array}{l}\text { NJV 94-5 } \\
\text { wood fuel }\end{array}$ & $\begin{array}{c}\text { Clayey Shale } 59 \\
\text { Wissenbacher } \\
\text { Tonschiefer }\end{array}$ \\
\hline $\mathrm{Al}$ & 112 & - & 2000 & 260 & 81500 \\
\hline As & - & 0.27 & 1.25 & 0.80 & 8.5 \\
\hline $\mathrm{Ba}$ & - & 41 & 18 & - & 380 \\
\hline B & - & 14.1 & 38.0 & - & - \\
\hline $\mathrm{Be}$ & - & 0.025 & 0.051 & - & 3 \\
\hline $\mathrm{Bi}$ & - & 0.040 & 0.023 & - & 0.27 \\
\hline $\mathrm{Ca}$ & 6400 & 12100 & 16800 & 3500 & 25400 \\
\hline $\mathrm{Cd}$ & - & 0.076 & 0.380 & 0.270 & 0.23 \\
\hline $\mathrm{Ce}$ & - & 0.81 & 2.20 & - & 81 \\
\hline Co & 0.12 & 0.30 & 0.41 & - & 18 \\
\hline $\mathrm{Cr}$ & 2.50 & 0.92 & 2.60 & 0.80 & 105 \\
\hline Cs & - & 0.58 & 0.27 & - & 7.8 \\
\hline $\mathrm{Cu}$ & 10.0 & 24.0 & 6.6 & 2.2 & 35 \\
\hline Dy & - & 0.065 & 0.130 & - & 5.5 \\
\hline $\mathrm{Er}$ & - & 0.037 & - & - & 3.1 \\
\hline $\mathrm{Eu}$ & - & 0.022 & 0.039 & - & 1.2 \\
\hline $\mathrm{Fe}$ & 114 & 322 & 1070 & 70 & 45000 \\
\hline $\mathrm{Ga}$ & - & - & - & - & 21 \\
\hline $\mathrm{Gd}$ & - & 0.076 & 0.190 & - & 5.9 \\
\hline $\mathrm{Ge}$ & - & 0.015 & - & - & - \\
\hline $\mathrm{Hf}$ & - & - & 0.15 & - & 4.7 \\
\hline Ho & - & 0.013 & 0.033 & - & 1.1 \\
\hline $\mathrm{K}$ & 33800 & 15500 & 9200 & 900 & 30300 \\
\hline $\mathrm{La}$ & - & 0.54 & 1.25 & - & 40 \\
\hline $\mathrm{Li}$ & - & 0.52 & 2.60 & - & 48 \\
\hline $\mathrm{Lu}$ & - & 0.0062 & 0.0110 & - & 0.44 \\
\hline $\mathrm{Mg}$ & 1450 & 2200 & 4800 & 300 & 23500 \\
\hline $\mathrm{Mn}$ & 72 & 1170 & 61 & 210 & 550 \\
\hline Mo & 1.00 & 0.11 & 0.28 & - & 0.48 \\
\hline $\mathrm{Na}$ & 3490 & 100 & 19600 & 40 & 6600 \\
\hline $\mathrm{Nb}$ & - & 0.05 & - & - & 16 \\
\hline $\mathrm{Nd}$ & - & 0.35 & 1.00 & - & 38 \\
\hline $\mathrm{Ni}$ & - & 5.4 & 1.7 & - & 72 \\
\hline$P$ & 2360 & 2800 & 1000 & 210 & 393 \\
\hline $\mathrm{Pb}$ & - & 1.60 & 47.00 & 0.68 & 38 \\
\hline $\operatorname{Pr}$ & - & 0.093 & 0.240 & - & 9.3 \\
\hline $\mathrm{Rb}$ & 50.0 & 89.0 & 4.5 & - & 155 \\
\hline S & 3160 & 4200 & 7300 & 180 & 4000 \\
\hline $\mathrm{Sb}$ & - & 0.052 & 0.095 & - & 1 \\
\hline Sc & 0.017 & 0.070 & 0.320 & - & 14 \\
\hline $\mathrm{Se}$ & 0.025 & 0.100 & 0.120 & - & 0.7 \\
\hline $\mathrm{Sm}$ & - & 0.066 & 0.190 & - & 6.9 \\
\hline Sn & - & 0.17 & 0.27 & - & 3.3 \\
\hline $\mathrm{Sr}$ & 22.1 & 36.0 & 246.0 & - & 90 \\
\hline $\mathrm{Ta}$ & - & - & - & - & 2 \\
\hline $\mathrm{Tb}$ & - & 0.0114 & 0.0250 & - & 0.85 \\
\hline Th & - & 0.079 & 0.360 & - & 13 \\
\hline $\mathrm{Ti}$ & 3.8 & 21.0 & 95.0 & 2.4 & 4300 \\
\hline TI & - & 0.057 & - & - & 0.77 \\
\hline $\mathrm{Tm}$ & - & 0.0059 & - & - & 0.5 \\
\hline$U$ & - & 0.047 & 0.120 & - & 3.3 \\
\hline V & - & 0.6 & 2.4 & - & 105 \\
\hline W & - & - & 0.06 & - & 2 \\
\hline$Y$ & - & 0.52 & 0.68 & - & 31 \\
\hline $\mathrm{Yb}$ & - & 0.038 & 0.063 & - & 2.9 \\
\hline $\mathrm{Zn}$ & 32.1 & 35.0 & 55.0 & 38.0 & 115 \\
\hline $\mathrm{Zr}$ & - & - & - & - & 180 \\
\hline
\end{tabular}


Table B.11.: Results (in ppm) of references samples of the selected element analysis of ICP-MS and ICP-OES. Please note: This table includes all measured elements, including the elements of poor analytical quality.

\begin{tabular}{|c|c|c|c|c|c|c|c|c|c|c|c|c|}
\hline Type & Species & ProjNr & Date.digestion & $\mathrm{Al}$ & As & $B$ & $\mathrm{Ba}$ & $\mathrm{Bi}$ & $\mathrm{Ca}$ & $\mathrm{Cd}$ & $\mathrm{Ce}$ & Co \\
\hline Plant & NJV 94-5 (wood fuel) & SOL-njv1_mt1 & $2011-07-01$ & 219 & 0.505 & $<$ LOD & 28.5 & 0.00463 & 3060 & 0.238 & 0.0585 & 0.0619 \\
\hline Plant & NJV 94-5 (wood fuel) & SOL-njv2_mt1 & $2011-07-01$ & 252 & 0.714 & $<$ LOD & 28.8 & 0.00469 & 3070 & 0.223 & 0.0529 & 0.0629 \\
\hline Plant & BCR- 129 (hay powder) & SOL-bcr_tee 1 & $2011-08-30$ & 118 & 0.705 & 284 & 11.4 & 0.00156 & 6310 & 0.0955 & 0.191 & 0.0699 \\
\hline Plant & NJV 94-5 (wood fuel) & SOL-njv_tee1 & $2011-08-30$ & 256 & 0.807 & 904 & 31.2 & 0.00601 & 3270 & 0.241 & 0.0498 & 0.0644 \\
\hline Plant & BCR- 129 (hay powder) & SOL-bcr_bl1 & 2011-09-01 & 128 & 0.0618 & 434 & 11.4 & 0.000726 & 6200 & 0.0983 & 0.142 & 0.0797 \\
\hline Plant & NJV 94-5 (wood fuel) & SOL-njv1_bl1 & 2011-09-01 & 236 & 1.24 & $<$ LOD & 28.8 & 0.00567 & 3070 & 0.192 & 0.0392 & 0.0591 \\
\hline Plant & NJV 94-5 (wood fuel) & SOL-njv2_bl1 & $2011-09-01$ & 237 & 0.742 & 116 & 30.4 & 0.00538 & 3210 & 0.206 & 0.0415 & 0.0631 \\
\hline Plant & GBW 10052 (Tea) & SOL-gbw1_mt2 & $2012-01-10$ & 1500 & 0.238 & 103 & 39 & 0.0308 & 5880 & 0.0757 & 0.811 & 0.28 \\
\hline Plant & GBW 10052 (Tea) & SOL-gbw3_mt2 & $2012-01-10$ & 1570 & 0.256 & 76.7 & 37.6 & 0.00339 & 6030 & 0.0729 & 0.781 & 0.272 \\
\hline Plant & NJV 94-5 (wood fuel) & SOL-njv_mt2 & $2012-01-10$ & 241 & 0.862 & 30.2 & 30.1 & 0.00531 & 1530 & 0.238 & 0.0408 & 0.0682 \\
\hline Plant & GBW 10052 (Tea) & SOL-gbw_tee2 & $2014-07-24$ & 1660 & 0.288 & 16.8 & 38.8 & 0.0386 & 11200 & 0.0769 & 0.95 & 0.267 \\
\hline Plant & GBW 10052 (Tea) & SOL-gbw_tee3 & $2014-12-01$ & 1780 & 0.308 & 15.2 & 41.8 & 0.0342 & 12600 & 0.06 & 0.895 & 0.262 \\
\hline Plant & GBW 10052 (Tea) & SOL-gbw1_tee4 & 2015-07-09 & 1760 & 0.59 & 16 & 38.9 & 0.0376 & 11300 & 0.0624 & 0.962 & 0.251 \\
\hline Plant & GBW 10052 (Tea) & SOL-gbw2_tee4 & 2015-07-09 & 1740 & 0.564 & 15.4 & 39.5 & 0.0359 & 11400 & 0.0659 & 0.871 & 0.281 \\
\hline Plant & GBW 10052 (Tea) & SOL-gbw_mt3 & $2016-05-15$ & 1730 & 0.27 & 2.48 & 40.4 & 0.0314 & 12100 & 0.0757 & 0.839 & 0.279 \\
\hline Plant & GBW 10052 (Tea) & SOL-gbw_mt4 & $2016-08-05$ & 1770 & 0.274 & 4.62 & 40.2 & 0.0268 & 11800 & 0.0722 & 0.805 & 0.279 \\
\hline Plant & GBW 10052 (Tea) & SOL-gbw1_tee5 & $2016-10-05$ & 1720 & 0.31 & 7.37 & 39.7 & 0.0389 & 11600 & 0.101 & 1.3 & 0.245 \\
\hline Plant & GBW 10052 (Tea) & SOL-gbw2_tee5 & 2016-10-05 & 1710 & 0.311 & 8.02 & 40.8 & 0.0302 & 11700 & 0.0779 & 0.88 & 0.273 \\
\hline Plant & NCS DC 73349 (bush leaves) & SOL-ncs_tee5 & 2016-10-05 & 1990 & 1.18 & 8.67 & 16.9 & 0.0127 & 15800 & 0.718 & 2.31 & 0.367 \\
\hline Plant & GBW 10052 (Tea) & SOL-gbw_tee6 & 2017-03-07 & 1720 & 0.281 & 4.71 & 39 & 0.0243 & 11700 & 0.0608 & 0.774 & 0.234 \\
\hline Plant & NCS DC 73349 (bush leaves) & SOL-ncs_tee6 & 2017-03-07 & 2110 & 1.17 & 6.93 & 16.7 & 0.0136 & 16800 & 0.713 & 2.51 & 0.367 \\
\hline Plant & GBW 10052 (Tea) & SOL-gbw_tee7 & 2017-04-07 & 1740 & 0.312 & 8.57 & 38.4 & 0.0305 & 11600 & 0.0778 & 0.844 & 0.228 \\
\hline Plant & NCS DC 73349 (bush leaves) & SOL-ncs_tee7 & 2017-04-07 & 2150 & 1.38 & 6.5 & 17.5 & 0.0125 & 17600 & 0.677 & 2.02 & 0.387 \\
\hline Plant & GBW 10052 (Tea) & SOL-gbw_mt5 & 2017-04-14 & 1760 & 0.319 & 6.51 & 41 & 0.0288 & 11900 & 0.0791 & 0.811 & 0.233 \\
\hline Rock & Clayey Shale 59 & SOL-tw_bd1 & 2011-08-01 & 78900 & 6.6 & $<$ LOD & 375 & 0.351 & 25400 & 0.199 & 78.2 & 22.5 \\
\hline Rock & Clayey Shale 45 & SOL-tw_bd2 & 2014-08-03 & 81900 & 7.05 & $<\mathrm{LOD}$ & 378 & 0.283 & 26100 & 0.204 & 93 & 17.4 \\
\hline Rock & Clayey Shale 45 & SOL-tw_bd3 & 2014-11-01 & 82000 & 7.69 & $<$ LOD & 371 & 0.25 & 26500 & 0.157 & 82.7 & 18.1 \\
\hline Rock & Clayey Shale 45 & SOL-tw_bd4 & $2015-02-01$ & 82200 & 7.71 & $<$ LOD & 371 & 0.244 & 26500 & 0.165 & 84.4 & 18.4 \\
\hline Rock & Clayey Shale 45 & SOL-tw_bd5 & 2016-01-27 & 81200 & 10.6 & $<$ LOD & 360 & 0.25 & 26500 & 0.178 & 84.8 & 17 \\
\hline
\end{tabular}


Table B.12.: Reference samples, continued from table B.11

\begin{tabular}{|c|c|c|c|c|c|c|c|c|c|c|c|c|c|c|c|}
\hline ProjNr & $\mathrm{Cr}$ & Cs & $\mathrm{Cu}$ & Dy & $\mathrm{Er}$ & $\mathrm{Eu}$ & $\mathrm{Fe}$ & $\mathrm{Ga}$ & $\mathrm{Gd}$ & $\mathrm{Hf}$ & $\mathrm{Ho}$ & $\mathrm{K}$ & $\mathrm{La}$ & $\mathrm{Li}$ & $\mathrm{Lu}$ \\
\hline SOL-njv1_mt1 & 0.419 & 0.0605 & 1.64 & 0.00313 & 0.00161 & $<$ LOD & 49.2 & 3.11 & 0.00467 & 0.00423 & 0.00063 & 943 & 0.051 & 0.0224 & 0.000257 \\
\hline SOL-njv2_mt1 & 0.554 & 0.0412 & 1.96 & 0.00263 & 0.00138 & $<$ LOD & 50.3 & 2.67 & 0.00385 & 0.00385 & 0.000503 & 975 & 0.0415 & 0.0248 & 0.000201 \\
\hline SOL-bcr_tee1 & 2.31 & 0.0802 & 9.39 & 0.00854 & 0.00328 & 0.002 & 113 & 5.57 & 0.0125 & 0.00685 & 0.00151 & 33500 & 0.122 & 0.0606 & 0.000515 \\
\hline SOL-njv_tee1 & 0.512 & 0.0527 & 1.89 & 0.00309 & 0.00186 & $<\mathrm{LOD}$ & 57.1 & 13.7 & 0.00397 & 0.00265 & 0.000691 & 1020 & 0.0403 & 0.0445 & 0.000293 \\
\hline SOL-bcr_bl1 & 2.51 & 0.0737 & 9.23 & 0.0063 & 0.00292 & 0.00177 & 113 & 0.95 & 0.00825 & 0.00614 & 0.00107 & 32500 & 0.0888 & 0.0366 & 0.000437 \\
\hline SOL-njv1_bl1 & 0.624 & 0.0383 & 2.23 & 0.00265 & 0.00133 & $<\mathrm{LOD}$ & 49.9 & 2.63 & 0.00352 & 0.00435 & 0.000465 & 965 & 0.0339 & 0.0193 & 0.000167 \\
\hline SOL-njv2_bl1 & 0.487 & 0.0397 & 1.79 & 0.00228 & 0.00122 & $<\mathrm{LOD}$ & 59.5 & 2.54 & 0.00319 & 0.00162 & 0.00046 & 1000 & 0.035 & 0.0246 & 0.00021 \\
\hline SOL-gbw1_mt2 & 0.551 & 0.554 & 21.8 & 0.0561 & 0.0332 & 0.0105 & 162 & 4.56 & 0.06 & 0.0981 & 0.011 & 7250 & 0.588 & 0.521 & 0.00474 \\
\hline SOL-gbw3_mt2 & 0.339 & 0.527 & 21.9 & 0.0595 & 0.036 & 0.0131 & 176 & 4.09 & 0.0636 & 0.0469 & 0.0117 & 7330 & 0.57 & 0.53 & 0.00534 \\
\hline SOL-njv_mt2 & 0.228 & 0.0435 & 1.94 & 0.00231 & 0.00111 & $<\mathrm{LOD}$ & 22.7 & 3.22 & 0.00268 & 0.00197 & 0.000448 & 271 & 0.035 & 0.0302 & 0.000149 \\
\hline SOL-gbw_tee2 & 1.52 & 0.632 & 23.9 & 0.0688 & 0.0394 & 0.0156 & 318 & 1.18 & 0.0761 & 0.196 & 0.0143 & 14400 & 0.603 & 0.655 & 0.00619 \\
\hline SOL-gbw_tee3 & 1.32 & 0.581 & 25.7 & 0.0642 & 0.037 & 0.0192 & 339 & 1.37 & 0.0699 & 0.243 & 0.0132 & 15400 & 0.646 & 0.607 & 0.00579 \\
\hline SOL-gbw1_tee4 & 2.32 & 0.61 & 25.4 & 0.0715 & 0.0384 & 0.0225 & 331 & 0.944 & 0.0755 & 0.168 & 0.0142 & 15100 & 0.687 & 0.635 & 0.00619 \\
\hline SOL-gbw2_tee4 & 1.42 & 0.589 & 25.6 & 0.0642 & 0.0356 & 0.0203 & 332 & 1.1 & 0.0666 & 0.149 & 0.0129 & 15400 & 0.636 & 0.637 & 0.00557 \\
\hline SOL-gbw_mt3 & 0.932 & 0.556 & 25.3 & 0.0626 & 0.0383 & 0.0147 & 320 & 0.825 & 0.07 & 0.233 & 0.0136 & 15600 & 0.514 & 0.579 & 0.00574 \\
\hline SOL-gbw_mt4 & 1.19 & 0.542 & 25.9 & 0.0569 & 0.0347 & 0.0105 & 324 & 0.19 & 0.0621 & 0.25 & 0.0117 & 16500 & 0.519 & 0.541 & 0.00559 \\
\hline SOL-gbw1_tee5 & 0.9 & 0.817 & 25.4 & 0.0857 & 0.0496 & 0.0162 & 324 & 0.946 & 0.0948 & 0.151 & 0.0172 & 15000 & 0.543 & 0.529 & 0.00774 \\
\hline SOL-gbw2_tee5 & 0.832 & 0.598 & 25.2 & 0.0608 & 0.0361 & 0.0104 & 312 & 0.665 & 0.0668 & 0.0841 & 0.0123 & 15900 & 0.516 & 0.524 & 0.00565 \\
\hline SOL-ncs_tee5 & 2.1 & 0.265 & 5.79 & 0.153 & 0.0818 & 0.0345 & 991 & 1.6 & 0.175 & 0.044 & 0.0293 & 9790 & 1.07 & 2.55 & 0.011 \\
\hline SOL-gbw_tee6 & 0.778 & 0.464 & 25 & 0.0507 & 0.0298 & 0.00827 & 301 & 0.195 & 0.0545 & 0.196 & 0.0103 & 16100 & 0.587 & 0.54 & 0.00468 \\
\hline SOL-ncs_tee6 & 2.11 & 0.287 & 6.07 & 0.16 & 0.0857 & 0.0371 & 1030 & 0.457 & 0.186 & 0.115 & 0.0309 & 10200 & 1.13 & 2.68 & 0.0118 \\
\hline SOL-gbw_tee7 & 1.07 & 0.516 & 25.2 & 0.0592 & 0.0358 & 0.0186 & 319 & 0.64 & 0.0655 & 0.207 & 0.0128 & 15600 & 0.515 & 0.556 & 0.00516 \\
\hline SOL-ncs_tee7 & 1.99 & 0.251 & 6.16 & 0.137 & 0.0746 & 0.0375 & 1180 & 1.52 & 0.155 & 0.0929 & 0.0282 & 10300 & 1.01 & 2.71 & 0.00952 \\
\hline SOL-gbw_mt5 & 0.811 & 0.496 & 23.1 & 0.0577 & 0.0348 & 0.0186 & 320 & 0.631 & 0.0622 & 0.229 & 0.0124 & 15800 & 0.514 & 0.569 & 0.00496 \\
\hline SOL-tw_bd1 & 48.9 & 7.6 & 41.4 & 5.25 & 2.64 & 1.11 & 45000 & 30.1 & 5.82 & 6.45 & 1.05 & 30500 & 49.4 & 40.2 & 0.417 \\
\hline SOL-tw_bd2 & 51.2 & 7.99 & 38.5 & 6.07 & 3.25 & 1.28 & 47800 & 26.7 & 6.55 & 4.76 & 1.22 & 26800 & 49.8 & 59.7 & 0.488 \\
\hline SOL-tw_bd3 & 107 & 7.59 & 34.8 & 5.3 & 2.92 & 1.15 & 45400 & 23.6 & 5.96 & 4.04 & 1.05 & 30700 & 45.2 & 49.8 & 0.443 \\
\hline SOL-tw_bd4 & 40.9 & 7.6 & 36.5 & 5.56 & 3.07 & 1.18 & 45300 & 98.6 & 6.18 & 4.35 & 1.1 & 30800 & 46 & 50.2 & 0.46 \\
\hline SOL-tw_bd5 & 107 & 7 & 35.2 & 5.13 & 2.69 & 1.14 & 43900 & 22.6 & 6.12 & 3.91 & 1.01 & 29000 & 42.9 & 47.9 & 0.435 \\
\hline
\end{tabular}


Table B.13.: Reference samples, continued from table B.11

\begin{tabular}{|c|c|c|c|c|c|c|c|c|c|c|c|c|c|c|c|c|}
\hline ProjNr & $\mathrm{Mg}$ & $\mathrm{Mn}$ & Mo & $\mathrm{Na}$ & $\mathrm{Nb}$ & $\mathrm{Nd}$ & $\mathrm{Ni}$ & $P$ & $\mathrm{~Pb}$ & $\operatorname{Pr}$ & $\mathrm{Rb}$ & $S$ & $\mathrm{Sb}$ & Sc & $\mathrm{Se}$ & $\mathrm{Sm}$ \\
\hline SOL-njv1_mt1 & 268 & 171 & 0.0132 & 26.5 & 0.00682 & 0.0231 & 0.317 & 197 & 0.435 & 0.00659 & 4.8 & 145 & 0.0178 & 0.00425 & $<\mathrm{LOD}$ & 0.00424 \\
\hline SOL-njv2_mt1 & 272 & 173 & 0.0124 & 28.8 & 0.00543 & 0.0211 & 0.297 & 201 & 0.476 & 0.00557 & 5.14 & 147 & 0.0159 & 0.00471 & $<\mathrm{LOD}$ & 0.00376 \\
\hline SOL-bcr_tee1 & 1480 & 81.4 & 0.866 & 3570 & 0.0307 & 0.087 & 4.54 & 2310 & 1.39 & 0.0223 & 34.8 & 2960 & 0.141 & 0.0179 & $<\mathrm{LOD}$ & 0.0148 \\
\hline SOL-njv_tee1 & 290 & 183 & 0.0174 & 40.1 & 0.0093 & 0.0199 & 0.405 & 214 & 0.601 & 0.00554 & 5.86 & 180 & 0.016 & 0.014 & $<\mathrm{LOD}$ & 0.0039 \\
\hline SOL-bcr_bl1 & 1450 & 80.5 & 1.05 & 2470 & 0.0247 & 0.0654 & 4.28 & 2270 & 1.27 & 0.0164 & 39.7 & 2860 & 0.0744 & 0.0113 & $<\mathrm{LOD}$ & 0.0125 \\
\hline SOL-njv1_bl1 & 274 & 172 & 0.0161 & 38.1 & 0.00508 & 0.0163 & 0.12 & 201 & 0.548 & 0.00452 & 4.64 & 145 & 0.0163 & LOD & 14.8 & 0.00309 \\
\hline SOL-njv2_bl1 & 287 & 181 & 0.0119 & 31.4 & 0.00702 & 0.0173 & 0.13 & 214 & 0.514 & 0.0046 & 4.85 & 154 & 0.013 & $<\mathrm{LOD}$ & 4.78 & 0.00307 \\
\hline SOL-gbw1_mt2 & 1450 & 953 & 0.0714 & 99.4 & 0.0694 & 0.328 & 5.26 & 1330 & 1.41 & 0.0853 & 79.5 & 2070 & 0.0598 & 0.0355 & 0.0898 & 0.0625 \\
\hline SOL-gbw3_mt2 & 1440 & 342 & 0.0118 & 92.7 & 0.0163 & 0.331 & 4.91 & 1380 & 1.23 & 0.0873 & 85.2 & 2160 & 0.0378 & 0.0319 & 0.0374 & 0.0629 \\
\hline SOL-njv_mt2 & 215 & 138 & 0.0146 & 41.1 & 0.00732 & 0.0171 & 0.336 & 84.5 & 0.524 & 0.00472 & 5.28 & 74.7 & 0.0166 & $<\mathrm{LOD}$ & $<\mathrm{LOD}$ & 0.00301 \\
\hline SOL-gbw_tee2 & 2000 & 1200 & 0.0886 & $<\mathrm{LOD}$ & 0.102 & 0.372 & 5.11 & 2540 & 1.66 & 0.104 & 96.8 & 4070 & 0.0661 & 0.0773 & 0.462 & 0.071 \\
\hline SOL-gbw_tee3 & 2050 & 1200 & 0.0913 & 104 & 0.105 & 0.367 & 5.72 & 2570 & 1.53 & 0.101 & 89.1 & 4150 & $<$ LOD & 0.0818 & 0.49 & 0.0707 \\
\hline SOL-gbw1_tee4 & 2040 & 1210 & 0.107 & 107 & 0.0765 & 0.399 & 6.06 & 2540 & 1.72 & 0.108 & 89.5 & 4120 & 0.0478 & 0.0823 & 0.339 & 0.0 \\
\hline SOL-gbw2_tee4 & 2050 & 1220 & 0.0855 & 102 & 0.0893 & 0.361 & 5.59 & 2550 & 1.65 & 0.0986 & 88.5 & 4120 & 0.0584 & 0.0797 & 0.416 & 0.0702 \\
\hline SOL-gbw_mt3 & 2110 & 1280 & 0.0842 & 119 & 0.11 & 0.345 & 5.51 & 2740 & 1.61 & 0.095 & 118 & 4220 & 0.0652 & 0.0865 & 0.293 & 0.0701 \\
\hline SOL-gbw_mt4 & 2110 & 1520 & 0.0875 & 108 & 0.111 & 0.321 & 5.58 & 2750 & 1.52 & 0.0897 & 100 & 4500 & 0.0479 & 0.0812 & 0.0553 & 0.064 \\
\hline SOL-gbw1_tee5 & 2040 & 1060 & 0.132 & 110 & 0.12 & 0.514 & 6.18 & 2690 & 1.86 & 0.144 & 140 & 4430 & 0.0742 & 0.0814 & 0.0783 & 0.102 \\
\hline SOL-gbw2_tee5 & 2070 & 1050 & 0.0040 & 109 & 0 & 0. & 6.02 & 2720 & 1.38 & & 105 & 4450 & 0.0579 & 0.0822 & 581 & 069 \\
\hline SOL-ncs_tee5 & 4430 & 56.3 & 0.4 & 17900 & 0.409 & 0.985 & 1.4 & 897 & 42.5 & 0.269 & 4.73 & 7150 & 0.103 & 0.283 & 0.087 & 0.195 \\
\hline SOL-gbw_tee6 & 2040 & 1210 & 0.0786 & 110 & 0.094 & 0.296 & 6.53 & 2650 & 1.09 & 0.0844 & 80.7 & 4290 & 0.0483 & 0.0807 & 0.041 & 0.0647 \\
\hline SOL-ncs_tee 6 & 4590 & 58.9 & 0.413 & 18500 & 0.454 & 1.0 & 1.37 & 927 & 44.8 & 0.2 & 5.1 & 7440 & 0.111 & 0.3 & 869 & 0.213 \\
\hline SOL-gbw_tee7 & 2030 & 1250 & 0.103 & 108 & 0.116 & 0.3 & 6.19 & 2670 & 1.44 & & 95.4 & 4390 & 0.0533 & 86 & 0.0553 & 0.0728 \\
\hline SOL-ncs_tee7 & 4840 & 60.8 & 0.407 & 18500 & 0.483 & 0.925 & 1.5 & 922 & 38.3 & 0.238 & 4.57 & 7760 & 0.101 & 0.293 & 0.0883 & 0.176 \\
\hline SOL-gbw_mt5 & 2080 & 1280 & 0.104 & 108 & 0.131 & 0.345 & 5.78 & 2710 & 1.55 & 0.095 & 95.2 & 4500 & 0.0537 & 0.0829 & 0.0552 & 0.065 \\
\hline SOL-tw_bd1 & 22900 & 523 & 0.382 & 6360 & 19.2 & 35.5 & 70.9 & 383 & 44.1 & 9.59 & 178 & 4250 & 1.35 & 14.5 & $<\mathrm{LOD}$ & 6.45 \\
\hline SOL-tw_bd2 & 23200 & 555 & 0.384 & 6170 & 17.8 & 40.4 & 72.3 & 384 & 36.9 & 11.1 & 165 & 4340 & 1.11 & 14.8 & $<\mathrm{LOD}$ & 7.7 \\
\hline SOL-tw_bd3 & 22600 & 538 & 0.392 & 6630 & 17.3 & 36 & 71.7 & 398 & 34.1 & 10 & 152 & 4330 & 1.08 & 16.9 & 2.42 & 6.48 \\
\hline SOL-tw_bd4 & 22700 & 532 & 0.339 & 6740 & 16.7 & 36.7 & 72.6 & 396 & 36.6 & 10.2 & 154 & 4320 & 1.13 & 15.3 & 2.9 & 6.67 \\
\hline SOL-tw_bd5 & 23000 & 536 & 0.444 & 6640 & 17.4 & 36.7 & 67.6 & 366 & 37.8 & 9.85 & 142 & 4690 & 0.923 & 14 & 2.21 & 6.81 \\
\hline
\end{tabular}


Table B.14.: Reference samples, continued from table B.11

\begin{tabular}{|c|c|c|c|c|c|c|c|c|c|c|c|c|c|c|c|}
\hline ProjNr & Sn & $\mathrm{Sr}$ & $\mathrm{Ta}$ & $\mathrm{Tb}$ & Th & $\mathrm{Ti}$ & $\mathrm{TI}$ & $\mathrm{Tm}$ & $U$ & $\mathrm{~V}$ & W & $\mathrm{Y}$ & $\mathrm{Yb}$ & $\mathrm{Zn}$ & $\mathrm{Zr}$ \\
\hline SOL-njv1_mt1 & 0.0174 & 10.3 & 0.000356 & 0.00048 & 0.00592 & 2.24 & 0.134 & 0.00025 & 0.00354 & 0.164 & 0.127 & 0.0113 & 0.00407 & 34.9 & 0.164 \\
\hline SOL-njv2_mt1 & 0.011 & 10.5 & 0.000304 & 0.000421 & 0.00825 & 1.53 & 0.138 & 0.000211 & 0.00407 & 0.135 & 0.151 & 0.0148 & 0.00316 & 34.9 & 0.186 \\
\hline SOL-bcr_tee1 & 0.0547 & 21.5 & 0.00634 & 0.00159 & 0.0435 & 6.1 & 0.00609 & 0.000574 & 0.0179 & $<\mathrm{LOD}$ & 0.0583 & 0.0431 & 0.0079 & 28.6 & 0.258 \\
\hline SOL-njv_tee1 & 0.0276 & 11.3 & 0.00173 & 0.000512 & 0.00698 & 2.63 & 0.168 & 0.000239 & 0.00467 & $<\mathrm{LOD}$ & 0.355 & 0.0196 & 0.00515 & 38 & 0.112 \\
\hline SOL-bcr_bl1 & 0.0591 & 21.3 & 0.00475 & 0.00111 & 0.0305 & 5.31 & 0.00538 & 0.000442 & 0.0111 & 1.07 & 0.042 & 0.0365 & 0.00683 & 27.5 & 0.283 \\
\hline SOL-njv1_bl1 & 0.00921 & 10.6 & 0.000874 & 0.000382 & 0.00725 & 1.73 & 0.152 & 0.000199 & 0.00465 & 0.37 & 0.372 & 0.0159 & 0.00341 & 34.8 & 0.161 \\
\hline SOL-njv2_bl1 & 0.013 & 10.9 & 0.00107 & 0.000397 & 0.00683 & 2.35 & 0.154 & 0.000186 & 0.00438 & 0.35 & 0.0285 & 0.015 & 0.00421 & 36.8 & 0.0587 \\
\hline SOL-gbw1_mt2 & 0.19 & 31.3 & 0.00414 & 0.00923 & 0.149 & 28 & 0.0458 & 0.00502 & 0.105 & 0.746 & 0.0208 & 0.614 & 0.068 & 31.1 & 4.37 \\
\hline SOL-gbw3_mt2 & 0.0254 & 31.6 & 0.000447 & 0.0101 & 0.111 & 33.8 & 0.0423 & 0.00537 & 0.0899 & 0.73 & $<\mathrm{LOD}$ & 0.582 & 0.0738 & 34.7 & 2.13 \\
\hline SOL-njv_mt2 & 0.00713 & 10.9 & 0.000194 & 0.000342 & 0.00652 & 2.33 & 0.161 & 0.000179 & 0.00398 & $<\mathrm{LOD}$ & 0.147 & 0.0171 & 0.00261 & 36.1 & 0.0822 \\
\hline SOL-gbw_tee2 & 0.248 & 30.4 & 0.00794 & 0.0112 & 0.0906 & 28 & 0.0531 & 0.00606 & 0.0591 & 4.55 & 0.0832 & 0.684 & 0.0405 & 31.9 & 9.68 \\
\hline SOL-gbw_tee3 & 0.267 & 35.2 & 0.00802 & 0.011 & 0.113 & 29.4 & 0.0497 & 0.00569 & 0.0593 & 3.38 & 0.0427 & 0.647 & 0.0398 & 32.2 & 10.3 \\
\hline SOL-gbw1_tee4 & 0.14 & 33.8 & 0.00653 & 0.0125 & 0.0868 & 27.5 & 0.0512 & 0.00661 & 0.0574 & 3.03 & 0.0491 & 0.722 & 0.0408 & 32.9 & 7.28 \\
\hline SOL-gbw2_tee4 & 0.133 & 34.4 & 0.00561 & 0.0113 & 0.0781 & 26.6 & 0.0506 & 0.00572 & 0.0537 & 3.53 & 0.0299 & 0.681 & 0.038 & 32.8 & 6.96 \\
\hline SOL-gbw_mt3 & 0.237 & 31.4 & 0.00851 & 0.0106 & 0.0833 & 32.1 & 0.046 & 0.0061 & 0.0526 & 3.19 & 0.0493 & 0.642 & 0.0406 & 35.4 & 9.93 \\
\hline SOL-gbw_mt4 & 0.208 & 33.7 & 0.00777 & 0.00912 & 0.0743 & 32.1 & 0.0435 & 0.00536 & 0.0497 & 0.599 & 0.0422 & 0.643 & 0.037 & 34.6 & 11.6 \\
\hline SOL-gbw1_tee5 & 0.251 & 33.2 & 0.00443 & 0.0138 & 0.115 & 31 & 0.0602 & 0.00754 & 0.0691 & 0.649 & 0.12 & 0.971 & 0.0526 & 33.7 & 8.65 \\
\hline SOL-gbw2_tee5 & 0.198 & 31.9 & 0.00366 & 0.00979 & 0.0765 & 28.9 & 0.0459 & 0.0056 & 0.051 & 0.644 & 0.0462 & 0.689 & 0.0384 & 34.4 & 5.21 \\
\hline SOL-ncs_tee5 & 0.372 & 245 & 0.00998 & 0.0251 & 0.366 & 114 & 0.0202 & 0.0114 & 0.132 & 2.26 & 0.0657 & 0.989 & 0.0758 & 51.4 & 2.25 \\
\hline SOL-gbw_tee6 & 0.255 & 33.8 & 0.00717 & 0.00804 & 0.0725 & 29.6 & 0.0362 & 0.00455 & 0.041 & 0.65 & 0.041 & 0.54 & 0.0313 & 33.5 & 9.31 \\
\hline SOL-ncs_tee6 & 0.259 & 269 & 0.0285 & 0.0266 & 0.4 & 120 & 0.0214 & 0.012 & 0.136 & 2.4 & 0.0768 & 0.983 & 0.0805 & 53 & 4.75 \\
\hline SOL-gbw_tee7 & 0.393 & 36.2 & 0.00786 & 0.0107 & 0.0948 & 26.3 & 0.0468 & 0.00517 & 0.056 & 0.641 & 0.0974 & 0.626 & 0.0359 & 33.5 & 11.4 \\
\hline SOL-ncs_tee7 & 0.51 & 267 & 0.0318 & 0.0255 & 0.429 & 125 & 0.0212 & 0.00992 & 0.133 & 2.48 & 0.15 & 0.801 & 0.067 & 54 & 3.84 \\
\hline SOL-gbw_mt5 & 0.247 & 35.2 & 0.00907 & 0.0104 & 0.0922 & 34.7 & 0.0466 & 0.00503 & 0.0556 & 0.664 & 0.057 & 0.615 & 0.0347 & 34.6 & 11.4 \\
\hline SOL-tw_bd1 & 4.42 & 85.5 & 2.89 & 0.812 & 33.2 & 4190 & 0.84 & 0.422 & 8.1 & 109 & 277 & 37.7 & 6.15 & 113 & 153 \\
\hline SOL-tw_bd2 & 3.97 & 89.3 & 1.17 & 0.977 & 14.8 & 4280 & 0.839 & 0.494 & 3.63 & 111 & 1.71 & 32.4 & 3.44 & 126 & 169 \\
\hline SOL-tw_bd3 & 3.69 & 92.8 & 1.13 & 0.878 & 14 & 4230 & 0.794 & 0.429 & 3.5 & 108 & 1.45 & 29.3 & 3.06 & 117 & 134 \\
\hline SOL-tw_bd4 & 3.71 & 94.2 & 1.01 & 0.913 & 14.2 & 4260 & $<\mathrm{LOD}$ & 0.463 & 3.62 & 109 & 1.47 & 30.7 & 3.11 & 116 & 146 \\
\hline SOL-tw_bd5 & 3.69 & 89.8 & 1.07 & 0.842 & 12.7 & 4220 & 0.71 & 0.396 & 3.31 & 106 & 1.25 & 27.5 & 2.98 & 118 & 129 \\
\hline
\end{tabular}


Table B.15.: Accuracy (in \%) of references samples of the selected element analysis of ICP-MS and ICP-OES. Please note: This table contains all measured elements, including the elements of poor analytical quality.

\begin{tabular}{|c|c|c|c|c|c|c|c|c|c|c|c|c|}
\hline Type & Species & ProjNr & Date.digestion & Al & As & B & $\mathrm{Ba}$ & $\mathrm{Bi}$ & $\mathrm{Ca}$ & $\mathrm{Cd}$ & $\mathrm{Ce}$ & Co \\
\hline Plant & NJV 94-5 (wood fuel) & SOL-njv1_mt1 & 2011-07-01 & -15.8 & -36.8 & & & & -12.5 & -11.8 & & \\
\hline Plant & NJV 94-5 (wood fuel) & SOL-njv2_mt1 & 2011-07-01 & -3.0 & -10.7 & & & & -12.4 & -17.6 & & \\
\hline Plant & BCR- 129 (hay powder) & SOL-bcr_tee 1 & 2011-08-30 & 5.2 & & & & & -1.4 & & & -41.8 \\
\hline Plant & NJV 94-5 (wood fuel) & SOL-njv_tee1 & 2011-08-30 & -1.5 & 0.9 & & & & -6.5 & -10.8 & & \\
\hline Plant & GBW 10052 (Tea) & SOL-gbw1_mt2 & $2012-01-10$ & & -11.7 & 630.5 & -5.0 & -23.0 & -51.4 & -0.4 & 0.1 & -6.7 \\
\hline Plant & GBW 10052 (Tea) & SOL-gbw3_mt2 & $2012-01-10$ & & -5.2 & 444.1 & -8.2 & -91.5 & -50.2 & -4.1 & -3.6 & -9.2 \\
\hline Plant & NJV 94-5 (wood fuel) & SOL-njv_mt2 & 2012-01-10 & -7.2 & 7.8 & & & & -56.4 & -11.7 & & \\
\hline Plant & GBW 10052 (Tea) & SOL-gbw_tee2 & 2014-07-24 & & 6.5 & 19.2 & -5.5 & -3.6 & -7.5 & 1.1 & 17.3 & -11.1 \\
\hline Plant & GBW 10052 (Tea) & SOL-gbw_tee3 & 2014-12-01 & & 14.2 & 8.2 & 1.9 & -14.4 & 4.2 & -21.0 & 10.5 & -12.6 \\
\hline Plant & GBW 10052 (Tea) & SOL-gbw1_tee4 & 2015-07-09 & & 118.4 & 13.2 & -5.1 & -6.0 & -6.9 & -17.8 & 18.7 & -16.4 \\
\hline Plant & GBW 10052 (Tea) & SOL-gbw2_tee4 & 2015-07-09 & & 108.8 & 9.2 & -3.6 & -10.1 & -5.5 & -13.3 & 7.5 & -6.3 \\
\hline Plant & GBW 10052 (Tea) & SOL & 201 & & -0.2 & -82.4 & -1.5 & -21.5 & -0.0 & -0.4 & 3.5 & -7.0 \\
\hline Plant & GBW 10052 (Tea) & SOL-gbw_mt4 & 2016-08-05 & & 1.4 & -67.2 & -2.0 & -33.1 & -2.6 & -5.0 & -0.6 & -6.9 \\
\hline Plant & GBW 10052 (Tea) & SOL-gbw1_tee5 & 2016-10-05 & & 14.6 & -47.8 & -3.1 & -2.6 & -4.2 & 33.4 & 61.0 & -18.2 \\
\hline Plant & GBW 10052 (Tea) & SOL-gbw2_tee5 & 2016-10-05 & & 15.1 & -43.1 & -0.5 & -24.5 & -3.5 & 2.5 & 8.7 & -9.0 \\
\hline Plant & NCS DC 73349 (bush leaves) & SOL-ncs_tee5 & 2016-10-05 & -0.6 & -5.2 & -77.2 & -6.4 & -44.6 & -6.1 & 89.0 & 5.0 & -10.4 \\
\hline Plant & GBW 10052 (Tea) & SOL-gbw_tee6 & 2017-03-07 & & 4.1 & -66.6 & -4.9 & -39.3 & -3.1 & -20.0 & -4.4 & -22.1 \\
\hline Plant & NCS DC 73349 (bu & & & 5.5 & -6.4 & & -7.2 & & -0.2 & & 13.9 & -10.5 \\
\hline Plant & GBW 10052 (Tea) & SOL-gbw_tee7 & 2017-04-07 & & 15.6 & -39.2 & -6.3 & -23.7 & -4.1 & 2.3 & 4.2 & -24.1 \\
\hline Plant & NCS DC 73349 (bush leaves) & SOL-ncs_tee7 & 2017-04-07 & 7.3 & 10.0 & -82.9 & -2.7 & -45.5 & 4.6 & 78.2 & -8.0 & -5.7 \\
\hline Plant & GBW 10052 (Tea) & SOL-gbw_mt5 & 2017-04-14 & & 18.2 & -53.8 & 0.1 & -28.0 & -2.0 & 4.1 & 0.2 & -22.4 \\
\hline Rock & Clayey Shale 59 & SOL-tw_bd1 & 2011-08-01 & -3.2 & -22.3 & & -1.4 & 29.9 & -0.0 & -13.6 & -3.4 & 25.0 \\
\hline Rock & Clayey Shale 45 & SOL-tw_bd2 & 2014-08-03 & 0.5 & -17.0 & & -0.6 & 4.8 & 2.6 & -11.5 & 14.8 & -3.1 \\
\hline Rock & Clayey Shale 45 & SOL-tw_bd3 & 2014-11-01 & 0.6 & -9.5 & & -2.4 & -7.4 & 4.3 & -31.8 & 2.1 & 0.5 \\
\hline Rock & Clayey Shale 45 & SOL-tw_bd4 & 2015-02-01 & 0.9 & -9.3 & & -2.4 & -9.6 & 4.3 & -28.3 & 4.2 & 2.2 \\
\hline Rock & Clayey Shale 45 & AMA-tw_ama1 & $2016-01-27$ & 0.1 & -0.9 & & -5.9 & -33.5 & 3.1 & -42.0 & 8.5 & -5.4 \\
\hline Rock & Clayey Shale 45 & SOL-tw_bd5 & $2016-01-27$ & -0.4 & 24.9 & & -5.1 & -7.3 & 4.4 & -22.6 & 4.7 & -5.3 \\
\hline Rock & JLk- 1 (lake sediment) & AMA-jlk1_ama1 & $2016-01-27$ & 1.8 & 9.6 & -190.7 & -0.6 & -62.2 & 3.3 & -35.0 & 10.8 & 0.7 \\
\hline
\end{tabular}


Table B.16.: Accuracy, continued from table B.15

\begin{tabular}{|c|c|c|c|c|c|c|c|c|c|c|c|c|c|c|c|c|}
\hline ProjNr & $\mathrm{Cr}$ & Cs & $\mathrm{Cu}$ & Dy & $\mathrm{Er}$ & $\mathrm{Eu}$ & $\mathrm{Fe}$ & $\mathrm{Ga}$ & $\mathrm{Gd}$ & $\mathrm{Hf}$ & $\mathrm{Ho}$ & $\mathrm{K}$ & $\mathrm{La}$ & $\mathrm{Li}$ & $\mathrm{Lu}$ & $\mathrm{Mg}$ \\
\hline SOL-njv1_mt1 & -47.7 & & -25.5 & & & & -29.7 & & & & & 4.7 & & & & -10.7 \\
\hline SOL-njv2_mt1 & -30.8 & & -10.9 & & & & -28.2 & & & & & 8.3 & & & & -9.3 \\
\hline SOL-bcr_tee1 & -7.8 & & -6.1 & & & & -0.9 & & & & & -0.9 & & & & 1.8 \\
\hline SOL-njv_tee1 & -36.0 & & -14.1 & & & & -18.5 & & & & & 12.9 & & & & -3.4 \\
\hline SOL-gbw1_mt2 & -40.1 & -4.5 & -9.2 & -13.7 & -10.3 & -52.3 & -49.6 & & -21.0 & & -15.4 & -53.2 & 9.0 & 0.1 & -23.6 & -34.2 \\
\hline SOL-gbw3_mt2 & -63.2 & -9.1 & -8.8 & -8.5 & -2.6 & -40.3 & -45.4 & & -16.4 & & -9.7 & -52.7 & 5.6 & 1.9 & -13.9 & -34.4 \\
\hline SOL-njv_mt2 & -71.4 & & -12.0 & & & & -67.5 & & & & & -69.9 & & & & -28.2 \\
\hline SOL-gbw_tee2 & 65.0 & 9.0 & -0.3 & 5.8 & 6.6 & -29.0 & -1.2 & & 0.1 & & 10.1 & -6.8 & 11.6 & 26.0 & -0.2 & -9.3 \\
\hline SOL-gbw_tee3 & 43.0 & 0.2 & 7.3 & -1.2 & 0.1 & -12.9 & 5.3 & & -8.0 & & 1.6 & -0.9 & 19.6 & 16.8 & -6.6 & -6.7 \\
\hline SOL-gbw1_tee4 & 151.7 & 5.2 & 5.8 & 10.0 & 3.8 & 2.2 & 2.9 & & -0.6 & & 9.2 & -2.6 & 27.1 & 22.0 & -0.2 & -7.2 \\
\hline SOL-gbw2_tee4 & 54.3 & 1.6 & 6.7 & -1.3 & -3.9 & -7.7 & 3.2 & & -12.4 & & -0.7 & -0.8 & 17.8 & 22.5 & -10.2 & -6.7 \\
\hline SOL-gbw_mt3 & 1.3 & -4.1 & 5.5 & -3.7 & 3.6 & -33.0 & -0.6 & & -7.9 & & 4.9 & 0.5 & -4.8 & 11.3 & -7.4 & -4.2 \\
\hline SOL-gbw_mt4 & 29.4 & -6.6 & 7.7 & -12.5 & -6.3 & -52.5 & 0.6 & & -18.3 & & -10.2 & 6.2 & -3.9 & 4.0 & -9.9 & -4.0 \\
\hline SOL-gbw1_tee5 & -2.2 & 40.8 & 6.0 & 31.8 & 34.2 & -26.4 & 0.6 & & 24.7 & & 32.0 & -3.1 & 0.5 & 1.7 & 24.8 & -7.5 \\
\hline SOL-gbw2_tee5 & -9.5 & 3.1 & 5.2 & -6.4 & -2.3 & -52.6 & -3.0 & & -12.1 & & -5.1 & 2.7 & -4.4 & 0.7 & -8.9 & -6.0 \\
\hline SOL-ncs_tee5 & -19.1 & -1.9 & -12.3 & 18.0 & & -11.4 & -7.4 & & -8.0 & -70.7 & -11.3 & 6.4 & -14.8 & -1.8 & -0.1 & -7.8 \\
\hline SOL-gbw_tee 6 & -15.4 & -20.0 & 4.0 & -22.0 & -19.4 & -62.4 & -6.4 & & -28.3 & & -20.7 & 3.9 & 8.7 & 3.9 & -24.6 & -7.3 \\
\hline SOL-ncs_tee6 & -18.7 & 6.1 & -8.1 & 23.3 & & -4.8 & -3.5 & & -1.9 & -23.1 & -6.3 & 10.7 & -9.7 & 3.1 & 7.2 & -4.3 \\
\hline SOL-gbw_tee7 & 16.2 & -11.1 & 4.9 & -8.9 & -3.2 & -15.4 & -1.0 & & -13.8 & & -1.8 & 0.8 & -4.7 & 6.8 & -16.9 & -7.6 \\
\hline SOL-ncs_tee7 & -23.4 & -7.2 & -6.6 & 5.5 & & -3.9 & 10.3 & & -18.5 & -38.1 & -14.6 & 12.3 & -19.5 & 4.3 & -13.5 & 0.8 \\
\hline SOL-gbw_mt5 & -11.8 & -14.5 & -3.8 & -11.2 & -5.8 & -15.3 & -0.5 & & -18.2 & & -4.6 & 3.2 & -4.7 & 9.3 & -20.0 & -5.3 \\
\hline SOL-tw_bd1 & -53.4 & -2.6 & 18.2 & -4.5 & -14.7 & -7.9 & 0.1 & 43.2 & -1.4 & 37.2 & -4.6 & 0.6 & 23.5 & -16.2 & -5.1 & -2.7 \\
\hline SOL-tw_bd2 & -51.2 & 2.5 & 10.1 & 10.3 & 4.8 & 6.5 & 6.3 & 27.3 & 11.1 & 1.3 & 10.6 & -11.5 & 24.5 & 24.4 & 10.8 & -1.2 \\
\hline SOL-tw_bd3 & 2.3 & -2.7 & -0.6 & -3.7 & -5.7 & -4.1 & 1.0 & 12.5 & 1.0 & -14.0 & -4.3 & 1.5 & 13.0 & 3.7 & 0.7 & -3.9 \\
\hline SOL-tw_bd4 & -61.0 & -2.5 & 4.2 & 1.0 & -1.0 & -1.3 & 0.6 & 369.4 & 4.8 & -7.5 & 0.1 & 1.6 & 15.1 & 4.6 & 4.5 & -3.4 \\
\hline AMA-tw_ama1 & -51.9 & -24.9 & 4.4 & -20.7 & -23.6 & -21.6 & -0.8 & 11.9 & -18.6 & -27.7 & -19.9 & -2.8 & 14.1 & 2.1 & -14.5 & -7.0 \\
\hline SOL-tw_bd5 & 1.7 & -10.3 & 0.6 & -6.7 & -13.3 & -5.1 & -2.5 & 7.7 & 3.8 & -16.7 & -8.2 & -4.3 & 7.2 & -0.2 & -1.2 & -2.0 \\
\hline AMA-jlk1_ama1 & -25.8 & -8.8 & 6.0 & -10.9 & -7.8 & -15.7 & -1.4 & 16.3 & 2.3 & -27.9 & 12.5 & -2.5 & 6.5 & 0.1 & -14.3 & 1.3 \\
\hline
\end{tabular}




\begin{tabular}{|c|c|c|c|c|c|c|c|c|c|c|c|c|c|c|c|c|}
\hline ProjNr & $\mathrm{Mn}$ & Mo & $\mathrm{Na}$ & $\mathrm{Nb}$ & $\mathrm{Nd}$ & $\mathrm{Ni}$ & $P$ & $\mathrm{~Pb}$ & $\operatorname{Pr}$ & $\mathrm{Rb}$ & $S$ & $\mathrm{Sb}$ & Sc & $\mathrm{Se}$ & $\mathrm{Sm}$ & Sn \\
\hline SOL-njv1_mt1 & -18.6 & & -33.7 & & & & -6.3 & -36.1 & & & -19.7 & & & & & \\
\hline SOL-njv2_mt1 & -17.5 & & -27.9 & & & & -4.3 & -30.0 & & & -18.4 & & & & & \\
\hline SOL-bcr_tee1 & 13.0 & -13.4 & 2.3 & & & & -2.0 & & & -30.3 & -6.3 & & 5.0 & -100.0 & & \\
\hline SOL-njv_tee1 & -12.7 & & 0.3 & & & & 1.7 & -11.7 & & & -0.1 & & & & & \\
\hline SOL-gbw1_mt2 & -18.6 & -35.1 & -0.6 & 38.9 & -6.2 & -2.6 & -52.6 & -12.2 & -8.3 & -10.7 & -50.7 & 15.0 & -49.2 & -10.2 & -5.3 & 12.0 \\
\hline SOL-gbw3_mt2 & -70.8 & -89.2 & -7.3 & -67.4 & -5.3 & -9.1 & -50.7 & -23.0 & -6.1 & -4.3 & -48.6 & -27.3 & -54.5 & -62.6 & -4.6 & -85.1 \\
\hline SOL-njv_mt2 & -34.3 & & 2.9 & & & & -59.8 & -23.0 & & & -58.5 & & & & & \\
\hline SOL-gbw_tee2 & 2.6 & -19.5 & & 103.0 & 6.3 & -5.4 & -9.2 & 3.5 & 11.5 & 8.8 & -3.1 & 27.1 & 10.4 & 362.4 & 7.6 & 46.0 \\
\hline SOL-gbw_tee3 & 2.9 & -17.0 & 3.6 & 109.5 & 4.7 & 6.0 & -8.2 & -4.5 & 9.1 & 0.1 & -1.3 & & 16.9 & 390.0 & 7.1 & 57.2 \\
\hline SOL-gbw1_tee4 & 3.3 & -2.7 & 6.6 & 53.1 & 14.1 & 12.3 & -9.2 & 7.6 & 16.2 & 0.5 & -1.9 & -8.1 & 17.6 & 238.5 & 20.4 & -17.7 \\
\hline SOL-gbw2_tee4 & 4.1 & -22.3 & 1.6 & 78.6 & 3.0 & 3.5 & -8.9 & 2.8 & 6.1 & -0.6 & -1.8 & 12.3 & 13.9 & 316.1 & 6.4 & -21.9 \\
\hline SOL-gbw_mt3 & 9.3 & -23.4 & 18.7 & 120.1 & -1.5 & 2.1 & -2.2 & 0.4 & 2.2 & 32 & 0.4 & 25.4 & & 192 & 6.2 & 39.6 \\
\hline SOL-gbw_mt4 & 29.6 & -20.5 & 8.1 & 122.9 & -8.4 & 3.3 & -1.8 & -5.1 & -3.6 & 12.7 & 7.2 & -7.8 & 16.0 & -44.7 & -3.0 & 22.5 \\
\hline SOL-gbw1_tee5 & -9.3 & 20.5 & 9.7 & 139.4 & 46.9 & 14.5 & -4.0 & 16.3 & 54.9 & 57.8 & 5.5 & 42.7 & 16.3 & -21.7 & 54.2 & 47.4 \\
\hline SOL-gbw2_tee5 & -10.0 & -13.8 & 8.9 & 58.6 & 0.0 & 11.4 & -3.0 & -14.0 & 5.9 & 17.9 & 5.8 & 11.3 & 17 & -41 & 4.5 & 16.6 \\
\hline SOL-r & -7.7 & 42.8 & -8.7 & & -1.5 & -17.9 & -10.3 & -9.6 & 12.2 & & -2.0 & 8.8 & -11 & -27 & 2.9 & 37.8 \\
\hline SOL-gbw_tee6 & 3.1 & -28.6 & 10.0 & 87.9 & -15.4 & 210 & -5.4 & -32.2 & 02 & 00 & 2.2 & 71 & 15.2 & -59.0 & 00 & 49.8 \\
\hline SOL- & -3.4 & & -5.7 & & & -19.4 & -7.3 & -4.7 & 21.0 & & 2.2 & & & & 12.2 & -4.0 \\
\hline w_tee7 & 6.8 & -6.0 & 7.7 & 131.7 & 0.6 & 14.7 & -4.7 & -9.8 & 7.0 & 7.2 & 4.6 & 2.5 & 12.3 & -44.7 & 10.4 & 131.1 \\
\hline SOL-ncs_tee7 & -0.4 & 45.3 & -5.5 & & -7.5 & -11.6 & -7.8 & -18.5 & -1.0 & & 6.3 & 6.7 & -8.5 & -26.4 & -7.4 & 88.8 \\
\hline SOL-gbw_mt5 & 9.1 & -5.1 & 7.7 & 162.6 & -1.5 & 7.0 & -3.2 & -3.2 & 2.2 & 7.0 & 7.1 & 3.3 & 18.4 & -44.8 & -1.4 & 45.0 \\
\hline SOL-tw_bd1 & -4.9 & -20.3 & -3.7 & 19.8 & -6.7 & -1.6 & -2.5 & 16.0 & 3.2 & 14.7 & 6.3 & 35.2 & 3.2 & -171.0 & -6.5 & 34.0 \\
\hline SOL-tw_bd2 & 0.9 & -20.0 & -6.6 & 11.4 & 6.4 & 0.4 & -2.3 & -2.8 & 19.8 & 6.4 & 8.4 & 11.4 & 5.5 & -134.9 & 11.6 & 20.3 \\
\hline SOL-tw_bd3 & -2.2 & -18.4 & 0.5 & 8.1 & -5.2 & -0.4 & 1.2 & -10.1 & 7.7 & -1.6 & 8.1 & 7.6 & 20.9 & 246.1 & -6.1 & 11.9 \\
\hline SOL-tw_bd4 & -3.3 & -29.4 & 2.1 & 4.2 & -3.4 & 0.8 & 0.6 & -3.6 & 9.7 & -0.9 & 8.1 & 13.3 & 9.1 & 313.7 & -3.3 & 12.4 \\
\hline AMA-tw_ama1 & -3.5 & -20.4 & -0.2 & 15.3 & -20.9 & -4.9 & -8.1 & -0.8 & -13.3 & -24.6 & 15.1 & -19.3 & 0.3 & 288.7 & -19.3 & -9.0 \\
\hline SOL-tw_bd5 & -2.5 & -7.4 & 0.7 & 8.7 & -3.5 & -6.2 & -6.8 & -0.6 & 5.9 & -8.4 & 17.4 & -7.7 & -0.3 & 215.3 & -1.2 & 11.9 \\
\hline AMA-jlk1_ama1 & -2.3 & -33.8 & 5.1 & 9.6 & -14.9 & 7.2 & 2.5 & 5.9 & -6.2 & -25.7 & 14.2 & -11.7 & -4.7 & 123.5 & -19.8 & -16.8 \\
\hline
\end{tabular}


Table B.18.: Accuracy, continued from table B.15

\begin{tabular}{|c|c|c|c|c|c|c|c|c|c|c|c|c|c|c|}
\hline ProjNr & $\mathrm{Sr}$ & $\mathrm{Ta}$ & $\mathrm{Tb}$ & Th & $\mathrm{Ti}$ & $\mathrm{TI}$ & $\mathrm{Tm}$ & $U$ & V & W & $\mathrm{Y}$ & $\mathrm{Yb}$ & $\mathrm{Zn}$ & $\mathrm{Zr}$ \\
\hline SOL-njv1_mt1 & & & & & -6.6 & & & & & & & & -8.1 & \\
\hline SOL-njv2_mt1 & & & & & -36.2 & & & & & & & & -8.1 & \\
\hline SOL-bcr_tee1 & -2.5 & & & & 60.6 & & & & & & & & -10.9 & \\
\hline SOL-njv_tee1 & & & & & 9.6 & & & & & & & & 0.1 & \\
\hline SOL-gbw1_mt2 & -12.9 & & -19.0 & 88.8 & 33.4 & -19.6 & -14.9 & 124.3 & 24.4 & & 18.2 & 79.1 & -11.1 & \\
\hline $\begin{array}{l}\text { SOL-gbw3_mt2 } \\
\text { SOL-njv_mt2 }\end{array}$ & -12.3 & & -11.6 & 40.6 & $\begin{array}{l}61.1 \\
-3.0\end{array}$ & -25.7 & -8.9 & 91.3 & 21.7 & & 12.0 & 94.2 & $\begin{array}{l}-0.9 \\
-5.1\end{array}$ & \\
\hline SOL-gbw_tee2 & -15.6 & & -1.9 & 14.7 & 33.3 & -6.9 & 2.8 & 25.7 & 658.7 & & 31.6 & 6.5 & -8.8 & \\
\hline SOL-gbw_tee3 & -2.2 & & -3.2 & 43.7 & 40.0 & -12.9 & -3.5 & 26.3 & 463.3 & & 24.4 & 4.7 & -8.0 & \\
\hline SOL-gbw1_tee4 & -6.2 & & 9.7 & 9.9 & 31.1 & -10.1 & 12.1 & 22.2 & 404.3 & & 38.8 & 7.3 & -6.1 & \\
\hline SOL-gbw2_tee4 & -4.4 & & -1.2 & -1.1 & 26.9 & -11.2 & -3.1 & 14.2 & 487.7 & & 31.0 & 0.0 & -6.3 & \\
\hline SOL-gbw_mt3 & -12.8 & & -7.0 & 5.4 & 52.6 & -19.3 & 3.5 & 11.9 & 432.2 & & 23.5 & 6.7 & 1.1 & \\
\hline SOL-gbw_mt4 & -6.3 & & -20.0 & -6.0 & 52.9 & -23.6 & -9.2 & 5.8 & -0.1 & & 23.6 & -2.6 & -1.1 & \\
\hline SOL-gbw1_tee5 & -7.9 & & 21.4 & 46.1 & 47.7 & 5.6 & 27.8 & 47.1 & 8.1 & & 86.7 & 38.3 & -3.8 & \\
\hline SOL-gbw2_tee5 & -11.4 & & -14.1 & -3.2 & 37.5 & -19.5 & -5.0 & 8.4 & 7.4 & & 32.4 & 1.1 & -1.6 & \\
\hline SOL-ncs_tee5 & -0.3 & & 0.3 & 1.6 & 20.3 & & & 9.7 & -5.7 & 9.4 & 45.4 & 20.3 & -6.5 & \\
\hline SOL-gbw_tee6 & -6.2 & & -29.5 & -8.2 & 40.9 & -36.4 & -23.0 & -12.8 & 8.3 & & 3.8 & -17.7 & -4.3 & \\
\hline SOL-ncs_tee6 & 9.2 & & 6.3 & 11.1 & 26.6 & & & 13.7 & -0.0 & 28.1 & 44.6 & 27.8 & -3.6 & \\
\hline SOL-gbw_tee7 & 0.6 & & -5.8 & 20.0 & 25.4 & -18.0 & -12.4 & 19.1 & 6.9 & & 20.4 & -5.6 & -4.3 & \\
\hline SOL-ncs_tee7 & 8.6 & & 3.2 & 19.0 & 31.6 & & & 11.2 & 3.4 & 149.9 & 17.9 & 6.3 & -1.8 & \\
\hline SOL-gbw_mt5 & -2.1 & & -8.6 & 16.7 & 65.1 & -18.3 & -14.7 & 18.4 & 10.7 & & 18.3 & -8.8 & -1.2 & \\
\hline SOL-tw_bd1 & -5.0 & 44.4 & -4.5 & 155.7 & -2.5 & 9.1 & -15.6 & 145.5 & 3.5 & 13763.8 & 21.5 & 111.9 & -1.6 & -14.7 \\
\hline SOL-tw_bd2 & -0.8 & -41.3 & 15.0 & 13.8 & -0.4 & 8.9 & -1.1 & 10.0 & 5.6 & -14.7 & 4.4 & 18.6 & 9.6 & -6.1 \\
\hline SOL-tw_bd3 & 3.1 & -43.5 & 3.3 & 7.7 & -1.6 & 3.1 & -14.3 & 6.1 & 3.2 & -27.4 & -5.4 & 5.4 & 2.2 & -25.3 \\
\hline SOL-tw_bd4 & 4.7 & -49.7 & 7.4 & 9.3 & -0.8 & & -7.5 & 9.6 & 3.3 & -26.4 & -1.0 & 7.1 & 1.2 & -19.0 \\
\hline AMA-tw_ama1 & -0.8 & -55.8 & -13.2 & -18.5 & -2.0 & -25.5 & -25.3 & -17.9 & 0.2 & -26.4 & -22.1 & -12.0 & -0.9 & -27.1 \\
\hline SOL-tw_bd5 & -0.3 & -46.4 & -0.9 & -2.0 & -1.9 & -7.8 & -20.8 & 0.2 & 0.7 & -37.5 & -11.4 & 2.6 & 2.8 & -28.4 \\
\hline AMA-jlk1_ama1 & -4.3 & -35.6 & -22.4 & -19.0 & 1.3 & -41.5 & -8.7 & -17.4 & -0.7 & 392.8 & -15.5 & -16.6 & -2.9 & -25.2 \\
\hline
\end{tabular}


Table B.19.: Precision (in \%) of reference samples of the selected element analysis of ICP-MS and ICP-OES. Please note: This table contains all measured elements, including the elements of poor analytical quality.

\begin{tabular}{|c|c|c|c|c|c|c|c|c|c|c|c|c|}
\hline Type & Species & ProjNr & Date.digestion & $\mathrm{Al}$ & As & $B$ & $\mathrm{Ba}$ & $\mathrm{Bi}$ & $\mathrm{Ca}$ & $\mathrm{Cd}$ & $\mathrm{Ce}$ & Co \\
\hline Plant & NJV 94-5 (wood fuel) & SOL-njv1_mt1 & 2011-07-01 & 7.4 & 32.6 & 105.2 & 1.2 & 13.5 & 0.2 & 3.4 & 28.2 & 1.9 \\
\hline Plant & NJV 94-5 (wood fuel) & SOL-njv2_mt1 & 2011-07-01 & 6.6 & 4.7 & 105.3 & 0.2 & 12.3 & 0.0 & 3.4 & 15.9 & 0.2 \\
\hline Plant & BCR- 129 (hay powder) & SOL-bcr_tee 1 & $2011-08-30$ & 8.4 & 72.0 & 95.7 & 0.2 & 31.8 & 0.9 & 1.0 & 43.3 & 23.5 \\
\hline Plant & NJV 94-5 (wood fuel) & SOL-njv_tee1 & $2011-08-30$ & 8.2 & 7.8 & 6056.3 & 8.3 & 12.4 & 6.7 & 4.5 & 9.1 & 2.2 \\
\hline Plant & GBW 10052 (Tea) & SOL-gbw1_mt2 & $2012-01-10$ & 5.5 & 10.4 & 1238.6 & 4.6 & 0.5 & 1.3 & 2.2 & 4.0 & 8.9 \\
\hline Plant & GBW 10052 (Tea) & SOL-gbw3_mt2 & $2012-01-10$ & 1.1 & 3.8 & 897.0 & 7.9 & 89.1 & 1.3 & 3.7 & 7.5 & 11.4 \\
\hline Plant & NJV 94-5 (wood fuel) & SOL-njv_mt2 & $2012-01-10$ & 3.5 & 7.5 & 105.2 & 2.3 & 0.7 & 3.2 & 8.3 & 10.6 & 8.3 \\
\hline Plant & GBW 10052 (Tea) & SOL-gbw_tee2 & $2014-07-24$ & 1.7 & 8.2 & 118.5 & 4.2 & 24.5 & 2.2 & 1.7 & 12.6 & 3.2 \\
\hline Plant & GBW 10052 (Tea) & SOL-gbw_tee3 & $2014-12-01$ & 4.5 & 16.0 & 98.2 & 1.9 & 10.5 & 6.8 & 15.7 & 6.0 & 1.4 \\
\hline Plant & GBW 10052 (Tea) & SOL-gbw1_tee4 & 2015-07-09 & 3.3 & 48.6 & 107.4 & 0.4 & 21.4 & 0.2 & 12.3 & 13.9 & 3.0 \\
\hline Plant & GBW 10052 (Tea) & SOL-gbw2_tee4 & 2015-07-09 & 1.8 & 42.1 & 100.0 & 2.2 & 16.1 & 1.7 & 7.4 & 3.1 & 8.6 \\
\hline Plant & GBI & t3 & 20 & 1.1 & 1.4 & 67.8 & 3.2 & 1.4 & 4.7 & 0.2 & 0.7 & 7.9 \\
\hline Plant & GBW 10052 (Tea) & SOL-gbw_mt4 & $2016-08-05$ & 3.4 & 9.0 & 39.9 & 1.7 & 13.6 & 2.1 & 4.6 & 4.6 & 8.0 \\
\hline Plant & GBW 10052 (Tea) & SOL-gbw1_tee5 & & 1.0 & 15.0 & 4.3 & 0.0 & 25.7 & 0.4 & 30.3 & 54.5 & 5.2 \\
\hline Plant & GBW 10052 (Tea) & SOL-gbw2_tee5 & 2016-10-05 & 0.3 & 15.4 & 4.3 & 2.6 & 2.5 & 1.1 & 0.1 & 4.3 & 5.5 \\
\hline Plant & NCS DC 73349 (bush leaves) & SOL-ncs_tee5 & $2016-10-05$ & 0.7 & 4.2 & 1153.8 & 0.3 & 6.0 & 2.4 & 3.7 & 2.7 & 4.8 \\
\hline Plant & GBW 10052 (Tea) & SOL & 2017-03-07 & 0.5 & 4.3 & 38.8 & 0.6 & 21.7 & 1.5 & 19.7 & 8.3 & 9.6 \\
\hline & & & & & & & 3.7 & 0.7 & 3.7 & 5.8 & 11.4 & 4.9 \\
\hline Plant & GBW 10052 (Tea) & SOL-gbw_tee7 & 2017-04-07 & 2.1 & 3.8 & 11.4 & 4.9 & 1.5 & 1.6 & 2.2 & 3.2 & 11.9 \\
\hline Plant & NCS DC 73349 (bush leaves) & SOL-ncs_tee7 & 2017-04-07 & 7.1 & 9.2 & 839.8 & 1.2 & 7.5 & 5.8 & 2.3 & 10.0 & 0.2 \\
\hline Plant & GBW 10052 (Tea) & SOL-gbw_mt5 & 2017-04-14 & 3.3 & 6.1 & 15.4 & 0.1 & 7.0 & 0.6 & 4.5 & 3.9 & 10.0 \\
\hline Rock & Clayey Shale 59 & SOL-tw_bd1 & 2011-08-01 & 4.2 & 81.0 & 164.2 & 3.2 & 88.3 & 3.2 & 88.0 & 4.2 & 4.2 \\
\hline Rock & Clayey Shale 45 & SOL-tw_bd2 & 2014-08-03 & 0.0 & 8.4 & 33.1 & 4.1 & 16.6 & 0.0 & 14.4 & 12.4 & 4.2 \\
\hline Rock & Clay & & $-11-01$ & 0.0 & 0.1 & 97.6 & 2.9 & 3.0 & 0.3 & 11.8 & 4.2 & 3.6 \\
\hline Rock & Clayey Shale 45 & SOL-tw_bd4 & $2015-02-01$ & 0.3 & 0.1 & 97.5 & 2.9 & 0.5 & 0.3 & 7.3 & 2.1 & 5.0 \\
\hline Rock & Clayey Shale 45 & AMA-tw_ama1 & $2016-01-27$ & 0.4 & 9.4 & 3.2 & 0.8 & 26.0 & 3.2 & 25.1 & 7.0 & 2.6 \\
\hline Rock & Clayey Shale 45 & SOL-tw_bd5 & $2016-01-27$ & 0.9 & 37.9 & 65.1 & 0.0 & 3.2 & 1.3 & 3.2 & 3.2 & 2.5 \\
\hline Rock & JLk- 1 (lake sediment) & AMA-jlk1_ama1 & $2016-01-27$ & 0.9 & 1.9 & 6.9 & 3.2 & 21.2 & 1.9 & 24.6 & 9.4 & 3.2 \\
\hline
\end{tabular}


Table B.20.: Precision, continued from table B.19

\begin{tabular}{|c|c|c|c|c|c|c|c|c|c|c|c|c|c|c|c|c|}
\hline ProjNr & $\mathrm{Cr}$ & Cs & $\mathrm{Cu}$ & Dy & $\mathrm{Er}$ & $\mathrm{Eu}$ & $\mathrm{Fe}$ & $\mathrm{Ga}$ & $\mathrm{Gd}$ & $\mathrm{Hf}$ & Ho & $\mathrm{K}$ & $\mathrm{La}$ & $\mathrm{Li}$ & Lu & $\mathrm{Mg}$ \\
\hline SOL-njv1_mt1 & 16.2 & 42.9 & 14.5 & 18.7 & 18.4 & 29.2 & 1.8 & 7.5 & 26.8 & 30.4 & 30.2 & 2.8 & 35.5 & 9.3 & 25.2 & 2.3 \\
\hline SOL-njv2_mt1 & 10.9 & 2.7 & 2.3 & 0.4 & 1.9 & 28.2 & 0.4 & 7.5 & 4.4 & 18.4 & 3.9 & 0.5 & 10.1 & 0.4 & 2.1 & 0.8 \\
\hline SOL-bcr_tee1 & 9.1 & 3.2 & 1.0 & 17.8 & 1.8 & 49.6 & 3.5 & 97.6 & 38.0 & 5.5 & 14.4 & 7.6 & 52.2 & 11.2 & 8.8 & 4.0 \\
\hline SOL-njv_tee1 & 2.5 & 24.5 & 4.7 & 17.2 & 37.1 & 100.0 & 13.9 & 106.5 & 7.7 & 18.4 & 42.9 & 4.8 & 6.9 & 79.7 & 42.8 & 5.7 \\
\hline SOL-gbw1_mt2 & 37.4 & 1.8 & 16.5 & 8.0 & 8.1 & 26.0 & 49.0 & 5.5 & 10.1 & 35.3 & 14.7 & 54.4 & 9.4 & 10.3 & 15.3 & 0.2 \\
\hline SOL-gbw3_mt2 & 61.5 & 6.6 & 16.2 & 2.5 & 0.3 & 7.5 & 44.8 & 5.5 & 4.8 & 35.3 & 9.1 & 53.9 & 6.1 & 8.7 & 4.5 & 0.2 \\
\hline SOL-njv_mt2 & 54.3 & 2.7 & 2.9 & 12.3 & 18.4 & 28.2 & 54.6 & 11.4 & 27.2 & 39.4 & 7.5 & 72.0 & 7.0 & 22.2 & 27.5 & 6.9 \\
\hline SOL-gbw_tee2 & 72.5 & 12.0 & 4.2 & 12.7 & 9.1 & 7.5 & 0.1 & 13.4 & 14.0 & 2.7 & 10.9 & 3.2 & 12.1 & 12.9 & 10.7 & 2.1 \\
\hline SOL-gbw_tee3 & 49.5 & 3.0 & 5.4 & 5.3 & 2.5 & 31.8 & 6.5 & 30.8 & 4.7 & 27.4 & 2.3 & 0.5 & 20.1 & 4.7 & 3.6 & 0.6 \\
\hline SOL-gbw1_tee4 & 163.1 & 8.1 & 4.0 & 17.2 & 6.3 & 54.6 & 4.0 & 9.6 & 13.1 & 12.1 & 10.0 & 2.3 & 27.7 & 9.3 & 10.7 & 0.1 \\
\hline SOL-gbw2_tee4 & 61.3 & 4.4 & 4.8 & 5.2 & 1.6 & 39.6 & 4.4 & 5.7 & 0.3 & 21.8 & 4.2 & 0.4 & 18.3 & 9.8 & 0.3 & 0.6 \\
\hline SOL-gbw_mt3 & 5.9 & 1.4 & 3.7 & 2.6 & 6.1 & 1.4 & 0.5 & 21.0 & 4.8 & 22.2 & 5.6 & 0.9 & 4.4 & 0.3 & 2.7 & 3.3 \\
\hline SOL-gbw_mt4 & 6.8 & 4.0 & 5.8 & 6.8 & 4.1 & 26.3 & 1.8 & 43.8 & 7.0 & 30.8 & 9.5 & 6.6 & 0.5 & 0.0 & 4.2 & 3.6 \\
\hline SOL-gbw1_tee5 & 8.1 & 44.7 & 4.1 & 40.4 & 37.4 & 14.1 & 1.7 & 47.8 & 41.9 & 24.6 & 33.0 & 2.8 & 5.2 & 2.2 & 38.5 & 0.2 \\
\hline SOL-gbw2_tee5 & 2.2 & 5.9 & 3.3 & 0.3 & 2.2 & 26.5 & 1.9 & 3.8 & 3.2 & 58.2 & 4.4 & 3.1 & 2.2 & 3.1 & 1.1 & 1.4 \\
\hline SOL-ncs_tee5 & 2.2 & 4.3 & 1.2 & 1.9 & 2.8 & 3.2 & 0.8 & 5.3 & 1.7 & 58.4 & 0.3 & 1.7 & 0.2 & 1.3 & 3.1 & 0.7 \\
\hline SOL-gbw_tee6 & 11.6 & 17.8 & 2.2 & 16.9 & 17.5 & 41.8 & 5.4 & 47.7 & 18.4 & 2.3 & 20.1 & 4.3 & 13.7 & 3.2 & 16.3 & 3.2 \\
\hline SOL- & 7.4 & 12.9 & 3.6 & 6.5 & 7.7 & 4.1 & 0.4 & 7.4 & 8.4 & 22.9 & 5.3 & 5.8 & 5.8 & 3.7 & 10.6 & 3.1 \\
\hline SOL-gbw_tee7 & 21.4 & 8.7 & 3.1 & 3.0 & 0.8 & 28.0 & 0.2 & 4.2 & 1.9 & 3.0 & 1.1 & 1.2 & 0.3 & 2.8 & 7.7 & 0.3 \\
\hline SOL-ncs_tee7 & 1.2 & 1.3 & 5.2 & 8.9 & 6.3 & 3.8 & 18.1 & 3.2 & 9.9 & 12.1 & 4.0 & 7.4 & 5.7 & 4.9 & 10.7 & 8.6 \\
\hline SOL-gbw_mt5 & 7.8 & 12.2 & 1.9 & 5.4 & 3.6 & 28.2 & 0.6 & 1.4 & 6.9 & 13.7 & 3.9 & 2.4 & 0.3 & 5.2 & 11.2 & 2.1 \\
\hline SOL-tw_bd1 & 3.2 & 86.4 & 3.2 & 92.5 & 91.6 & 92.7 & 2.2 & 4.2 & 92.7 & 90.3 & 92.8 & 2.2 & 4.2 & 3.2 & 72.5 & 3.2 \\
\hline SOL-tw_bd2 & 34.3 & 7.0 & 3.2 & 12.5 & 8.5 & 11.0 & 3.1 & 12.6 & 16.5 & 12.4 & 13.7 & 1.8 & 17.6 & 27.4 & 9.3 & 2.7 \\
\hline SOL-tw_bd3 & 37.2 & 1.6 & 0.8 & 1.8 & 2.4 & 0.0 & 0.9 & 0.5 & 5.8 & 4.6 & 1.5 & 0.7 & 8.7 & 6.2 & 0.7 & 0.0 \\
\hline SOL-tw_bd4 & 47.7 & 1.8 & 0.3 & 3.0 & 2.5 & 4.6 & 1.3 & 3.7 & 9.8 & 2.6 & 3.0 & 0.9 & 10.7 & 7.1 & 3.1 & 2.2 \\
\hline AMA-tw_ama1 & 35.8 & 21.6 & 0.6 & 19.2 & 20.8 & 18.3 & 2.6 & 1.0 & 17.9 & 19.9 & 17.6 & 3.6 & 9.7 & 3.7 & 15.6 & 1.2 \\
\hline SOL-tw_bd5 & 35.8 & 6.4 & 3.1 & 4.9 & 10.3 & 1.1 & 4.3 & 4.7 & 4.6 & 7.6 & 5.6 & 5.1 & 3.1 & 5.9 & 2.5 & 4.1 \\
\hline AMA-jlk1_ama1 & 4.2 & 20.2 & 2.6 & 18.0 & 16.8 & 16.0 & 1.8 & 3.5 & 15.7 & 23.1 & 17.2 & 2.5 & 8.1 & 4.0 & 14.1 & 0.2 \\
\hline
\end{tabular}




\begin{tabular}{|c|c|c|c|c|c|c|c|c|c|c|c|c|c|c|c|c|c|}
\hline ProjNr & $\mathrm{Mn}$ & Mo & $\mathrm{Na}$ & $\mathrm{Nb}$ & $\mathrm{Nd}$ & $\mathrm{Ni}$ & $\mathrm{P}$ & $\mathrm{Pb}$ & $\mathrm{Pr}$ & $\mathrm{Rb}$ & $S$ & $\mathrm{Sb}$ & $\mathrm{Sc}$ & $\mathrm{Se}$ & Sm & Sn & $\mathrm{Sr}$ \\
\hline SOL-njv1_mt1 & 1.1 & 5.0 & 23.7 & 1.4 & 24.6 & 3.3 & 4.2 & 16.3 & 28.4 & 3.9 & 0.8 & 10.2 & 262.6 & 650.8 & 23.8 & 45.1 & 2.2 \\
\hline SOL-njv2_mt1 & 0.2 & 10.9 & 17.1 & 21.6 & 13.4 & 3.3 & 0.0 & 8.3 & 8.6 & 2.9 & 0.7 & 1.7 & 301.5 & 661.5 & 9.8 & 8.2 & 0.3 \\
\hline SOL-bcr_tee1 & 7.6 & 6.8 & 22.5 & 3.7 & 14.1 & 2.2 & 2.4 & 6.6 & 34.8 & 14.7 & 2.3 & 72.0 & 3.7 & 100.0 & 31.0 & 9.9 & 2.3 \\
\hline SOL-njv_tee1 & 6.0 & 25.3 & 14.7 & 34.4 & 7.0 & 32.0 & 6.3 & 15.7 & 8.0 & 17.3 & 6.8 & 1.0 & 1090.2 & 100.0 & 13.9 & 130.3 & 7.0 \\
\hline SOL-gbw1_mt2 & 13.8 & 14.7 & 7.2 & 26.1 & 6.0 & 8.6 & 49.9 & 1.4 & 12.7 & 12.1 & 33.1 & 3.3 & 56.5 & 68.4 & 10.8 & 6.5 & 10.3 \\
\hline SOL-gbw3_mt2 & 69.1 & 85.9 & 2.2 & 82.7 & 5.1 & 14.7 & 47.9 & 13.6 & 10.6 & 5.8 & 30.3 & 34.7 & 60.9 & 86.8 & 10.2 & 87.6 & 9.7 \\
\hline SOL-njv_mt2 & 2.2 & 5.0 & 18.3 & 5.8 & 7.9 & 31.1 & 57.9 & 0.9 & 8.0 & 5.8 & 55.7 & 2.8 & 268.6 & 100.0 & 12.2 & 40.6 & 0.5 \\
\hline SOL-gbw_tee2 & 0.8 & 5.8 & & 8.0 & 5.7 & 15.1 & 4.0 & 16.2 & 6.1 & 7.0 & 1.9 & 14.2 & 5.3 & 62.9 & 0.2 & 21.9 & 3.2 \\
\hline SOL-gbw_tee3 & 0.5 & 9.0 & 3.8 & 11.5 & 4.1 & 4.9 & 3.0 & 7.2 & 3.9 & 1.4 & 0.2 & & 0.2 & 72.6 & 0.9 & 28.3 & 0.8 \\
\hline SOL-gbw1_tee4 & 0.1 & 27.8 & 1.0 & 18.5 & 13.5 & 0.7 & 4.0 & 12.3 & 10.6 & 1.1 & 0.7 & 17.5 & 0.8 & 19.2 & 13.4 & 31.3 & 5.1 \\
\hline SOL-gbw2_tee4 & 0.7 & 2.1 & 5.6 & 5.0 & 2.4 & 7.1 & 3.7 & 7.3 & 0.9 & 2.2 & 0.7 & 0.9 & 2.3 & 46.5 & 0.3 & 34.8 & 7.1 \\
\hline SOL-gbw_mt3 & 5.7 & 0.6 & 10.2 & 17.2 & 2.1 & 4.2 & 3.3 & 12.7 & 2.7 & 30.5 & 1.6 & 12.6 & 6.0 & 3.2 & 2.2 & 16.5 & 2.3 \\
\hline SOL-gbw_mt4 & 3.6 & 4.5 & 0.4 & 18.6 & 8.9 & 3.7 & 3.8 & 1.0 & 8.2 & 10.9 & 8.4 & 17.2 & 0.7 & 4.2 & 8.6 & 3.2 & 5.0 \\
\hline SOL-gbw1_tee5 & 14.4 & 58.3 & 1.9 & 27.4 & 46.1 & 2.7 & 1.5 & 33.9 & 47.5 & 55.2 & 6.7 & 28.2 & 0.2 & 41.6 & 45.2 & 15.3 & 3.2 \\
\hline SOL-gbw2_tee5 & 14.9 & 13.3 & 1.1 & 15.6 & 0.6 & 0.0 & 2.6 & 1.0 & 0.8 & 16.1 & 7.1 & 3.2 & 0.7 & 5.1 & 1.5 & 8.8 & 0.7 \\
\hline SOL-ncs_tee5 & 11.0 & 35.9 & 7.3 & 4.3 & 0.3 & 5.0 & 2.6 & 1.3 & 0.6 & 16.9 & 0.6 & 2.4 & 2.6 & 3.9 & 2.9 & 15.8 & 2.9 \\
\hline SOL-gbw_tee6 & 0.3 & 6.1 & 2.1 & 3.2 & 15.9 & 6.3 & 4.2 & 21.9 & 13.6 & 10.8 & 3.4 & 16.5 & 1.2 & 25.8 & 9.1 & 116.8 & 5.1 \\
\hline SOL-ncs_tee6 & 2.2 & 40.4 & 4.3 & 15.8 & 7.0 & 3.5 & 0.7 & 4.0 & 8.5 & 26.1 & 4.6 & 10.0 & 3.7 & 3.8 & 11.9 & 15.9 & 6.3 \\
\hline SOL-gbw_tee7 & 3.3 & 23.5 & 0.0 & 23.3 & 2.2 & 0.7 & 0.8 & 3.8 & 1.8 & 5.5 & 5.7 & 7.9 & 3.7 & 0.0 & 4.0 & 92.9 & 3.6 \\
\hline SOL-ncs_tee7 & 3.1 & 38.3 & 4.1 & 23.1 & 6.4 & 5.9 & 0.1 & 11.0 & 11.2 & 12.9 & 9.1 & 0.4 & 0.8 & 5.4 & 7.4 & 2.1 & 9.2 \\
\hline SOL-gbw_mt5 & 5.5 & 24.7 & 0.0 & 39.7 & 2.4 & 0.2 & 2.3 & 1.0 & 2.8 & 5.3 & 8.4 & 7.2 & 1.6 & 0.2 & 7.1 & 18.4 & 0.8 \\
\hline SOL-tw_bd1 & 3.2 & 96.0 & 4.2 & 2.2 & 92.6 & 2.2 & 4.2 & 88.1 & 93.0 & 87.9 & 4.2 & 89.0 & 3.2 & 23.2 & 92.8 & 88.9 & 3.2 \\
\hline SOL-tw_bd2 & 4.0 & 9.5 & 0.0 & 3.1 & 14.1 & 3.2 & 1.9 & 3.4 & 10.4 & 9.1 & 5.0 & 4.9 & 4.5 & 109.1 & 13.4 & 7.4 & 3.1 \\
\hline SOL-tw_bd3 & 0.8 & 11.7 & 1.1 & 0.1 & 1.7 & 0.8 & 0.3 & 1.3 & 0.7 & 0.8 & 3.2 & 1.2 & 2.7 & 9.9 & 4.6 & 0.1 & 3.4 \\
\hline SOL-tw_bd4 & 0.4 & 3.4 & 2.8 & 3.5 & 3.7 & 0.4 & 0.3 & 8.7 & 1.2 & 1.6 & 0.0 & 6.6 & 8.7 & 7.7 & 1.8 & 0.4 & 5.0 \\
\hline AMA-tw_ama1 & 0.5 & 8.9 & 0.4 & 1.6 & 18.1 & 1.6 & 0.9 & 5.5 & 20.1 & 22.7 & 0.0 & 24.0 & 2.2 & 1.2 & 18.0 & 18.5 & 0.6 \\
\hline SOL-tw_bd5 & 0.4 & 26.7 & 1.3 & 4.1 & 4.2 & 0.3 & 0.4 & 5.7 & 2.4 & 6.1 & 1.9 & 13.1 & 0.6 & 17.9 & 0.3 & 0.2 & 2.2 \\
\hline AMA-jlk1_ama1 & 2.2 & 16.9 & 4.2 & 2.4 & 16.0 & 3.2 & 0.7 & 1.0 & 19.6 & 22.7 & 5.2 & 21.3 & 4.2 & Inf & 14.7 & 17.1 & 2.2 \\
\hline
\end{tabular}


Table B.22.: Precision, continued from table B.19

\begin{tabular}{|c|c|c|c|c|c|c|c|c|c|c|c|c|c|}
\hline ProjNr & $\mathrm{Ta}$ & $\mathrm{Tb}$ & Th & $\mathrm{Ti}$ & $\mathrm{TI}$ & $\mathrm{Tm}$ & $U$ & V & W & Y & $\mathrm{Yb}$ & $\mathrm{Zn}$ & $\mathrm{Zr}$ \\
\hline SOL-njv1_mt1 & 42.1 & 17.4 & 14.2 & 12.9 & 12.7 & 22.3 & 16.3 & 9.8 & 14.2 & 26.8 & 8.8 & 0.0 & 20.0 \\
\hline SOL-njv2_mt1 & 50.6 & 2.9 & 19.4 & 22.9 & 9.6 & 2.8 & 3.6 & 9.8 & 1.3 & 4.0 & 15.6 & 0.0 & 36.0 \\
\hline SOL-bcr_tee1 & 87.7 & 18.8 & 102.1 & 0.7 & 13.4 & 3.8 & 117.6 & 100.0 & 38.6 & 5.2 & 7.3 & 6.3 & 4.7 \\
\hline SOL-njv_tee1 & 181.1 & 25.1 & 1.0 & 32.6 & 9.9 & 16.6 & 10.5 & 100.0 & 138.9 & 26.6 & 37.6 & 8.9 & 18.0 \\
\hline SOL-gbw1_mt2 & 38.6 & 11.4 & 76.4 & 18.7 & 1.7 & 11.8 & 91.0 & 76.3 & 51.8 & 4.3 & 4.1 & 7.8 & 47.3 \\
\hline SOL-gbw3_mt2 & 93.4 & 3.3 & 31.4 & 1.9 & 9.1 & 5.6 & 62.9 & 76.8 & 122.3 & 9.3 & 4.1 & 2.7 & 74.3 \\
\hline SOL-njv_mt2 & 68.4 & 16.3 & 5.6 & 4.9 & 5.4 & 12.6 & 5.8 & 162.8 & 1.3 & 10.7 & 30.3 & 5.4 & 39.7 \\
\hline SOL-gbw_tee2 & 17.8 & 7.3 & 7.2 & 0.5 & 13.9 & 6.5 & 7.0 & 44.6 & 79.9 & 6.5 & 1.7 & 4.7 & 16.9 \\
\hline SOL-gbw_tee3 & 18.9 & 6.0 & 34.2 & 4.6 & 6.6 & 2.2 & 7.5 & 7.3 & 7.7 & 0.7 & 3.2 & 3.9 & 24.9 \\
\hline SOL-gbw1_tee4 & 3.1 & 20.1 & 2.7 & 2.1 & 10.0 & 16.1 & 4.1 & 3.9 & 6.1 & 12.4 & 2.4 & 1.9 & 12.0 \\
\hline SOL-gbw2_tee4 & 16.7 & 8.1 & 7.6 & 5.3 & 8.6 & 0.4 & 2.7 & 12.0 & 35.3 & 6.1 & 4.5 & 2.1 & 15.9 \\
\hline SOL-gbw_mt3 & 26.2 & 1.8 & 1.5 & 14.0 & 1.2 & 7.2 & 4.7 & 1.4 & 6.7 & 2.2 & 1.9 & 5.6 & 19.9 \\
\hline SOL & 15.3 & 12.5 & 12.2 & 14.1 & 6.6 & 5.9 & 9.9 & 4.2 & 8.8 & 0.1 & 7.0 & 3.4 & 40.7 \\
\hline SOL-gbw1_tee5 & 34.3 & 32.9 & 36.5 & 10.3 & 29.3 & 32.4 & 25.3 & 3.2 & 160.6 & 51.2 & 32.1 & 0.5 & 4.5 \\
\hline SOL-gbw2_tee5 & 45.7 & 6.0 & 9.5 & 2.7 & 1.4 & 1.6 & 7.6 & 0.7 & 2.2 & 7.2 & 3.5 & 2.8 & 37.1 \\
\hline SOL-ncs_tee5 & 62.5 & 3.4 & 5.4 & 8.1 & 1.5 & 3.2 & 5.8 & 3.9 & 13.0 & 17.4 & 1.8 & 2.7 & 35.5 \\
\hline SOL-gbw_tee6 & 6.3 & 22.8 & 14.2 & 5.2 & 22.2 & 20.2 & 25.7 & 0.2 & 11.4 & 16.0 & 21.4 & 4.2 & 12.5 \\
\hline SOL-ncs_tee6 & 7.0 & 2.4 & 3.5 & 13.7 & 4.5 & 5.2 & 2.3 & 1.9 & 1.8 & 16.7 & 8.1 & 0.3 & 36.0 \\
\hline SOL-gbw_tee7 & 16.6 & 3.1 & 12.1 & 6.3 & 0.4 & 9.2 & 1.4 & 1.1 & 110.8 & 2.5 & 9.8 & 0.0 & 37.6 \\
\hline SOL-ncs_tee7 & 19.4 & 1.7 & 10.8 & 18.2 & 3.5 & 12.9 & 4.5 & 5.4 & 98.6 & 4.9 & 10.1 & 2.2 & 9.9 \\
\hline SOL-gbw_mt5 & 34.6 & 4.2 & 9.0 & 23.3 & 4.2 & 11.6 & 0.8 & 2.4 & 23.4 & 4.2 & 12.9 & 3.2 & 37.2 \\
\hline SOL-tw_bd1 & 93.8 & 92.2 & 88.9 & 2.2 & 86.5 & 92.5 & 2.2 & 3.2 & 89.7 & 79.3 & 92.7 & 4.2 & 4.2 \\
\hline SOL-tw_bd2 & 4.0 & 11.6 & 12.7 & 1.6 & 13.7 & 8.8 & 8.9 & 2.2 & 14.1 & 5.5 & 12.2 & 1.7 & 22.9 \\
\hline SOL-tw_bd3 & 4.2 & 0.3 & 6.7 & 0.9 & 7.6 & 5.6 & 5.0 & 1.9 & 2.8 & 4.4 & 3.2 & 2.6 & 2.2 \\
\hline SOL-tw_bd4 & 11.0 & 4.2 & 8.2 & 1.1 & & 1.9 & 8.5 & 4.2 & 1.4 & 4.2 & 1.7 & 3.5 & 6.0 \\
\hline AMA-tw_ama1 & 21.8 & 15.8 & 19.3 & 0.1 & 22.3 & 17.7 & 18.8 & 1.1 & 1.5 & 21.3 & 16.5 & 5.5 & 4.6 \\
\hline SOL-tw_bd5 & 5.2 & 3.8 & 2.9 & 0.1 & 3.7 & 12.9 & 0.9 & 0.6 & 16.3 & 10.4 & 2.6 & 3.2 & 6.3 \\
\hline AMA-jlk1_ama1 & 23.1 & 15.8 & 19.0 & 3.2 & 21.7 & 19.3 & 20.6 & 2.2 & 3.2 & 21.7 & 14.7 & 1.9 & 10.4 \\
\hline
\end{tabular}





\section{Mathematical Tools}

Simple log-ratio:

$$
\ln \left(\frac{x_{i}}{x_{j}}\right)=\ln \left(x_{i}\right)-\ln \left(x_{j}\right)
$$

additive log-ratio (Aitchison 1986):

$$
\operatorname{alr}(\mathbf{x}):=\left[\ln \left(\frac{x_{1}}{x_{D}}\right), \ln \left(\frac{x_{2}}{x_{D}}\right), \cdots, \ln \left(\frac{x_{D-1}}{x_{D}}\right)\right]
$$

centered log-ratio (Aitchison 1986):

$$
\operatorname{clr}(\mathbf{x}):=\left[\ln \left(\frac{x_{1}}{g_{m}(\mathbf{x})}\right), \cdots, \ln \left(\frac{x_{D}}{g_{m}(\mathbf{x})}\right)\right] \quad, \quad g_{m}(\mathbf{x}):=\sqrt[D]{x_{1} \cdot x_{2} \cdots x_{D}}
$$

Balanced log-ratio (Egozcue and Pawlowsky-Glahn 2005):

$$
\left[r_{1}, \ldots, r_{N} \mid s_{1}, \ldots, s_{M}\right]:=\sqrt{\frac{N \cdot M}{N+M}} \cdot \ln \left(\frac{\sqrt[N]{r_{1} \cdot r_{2} \cdots r_{N}}}{\sqrt[M]{s_{1} \cdot s_{2} \cdot s_{M}}}\right)
$$



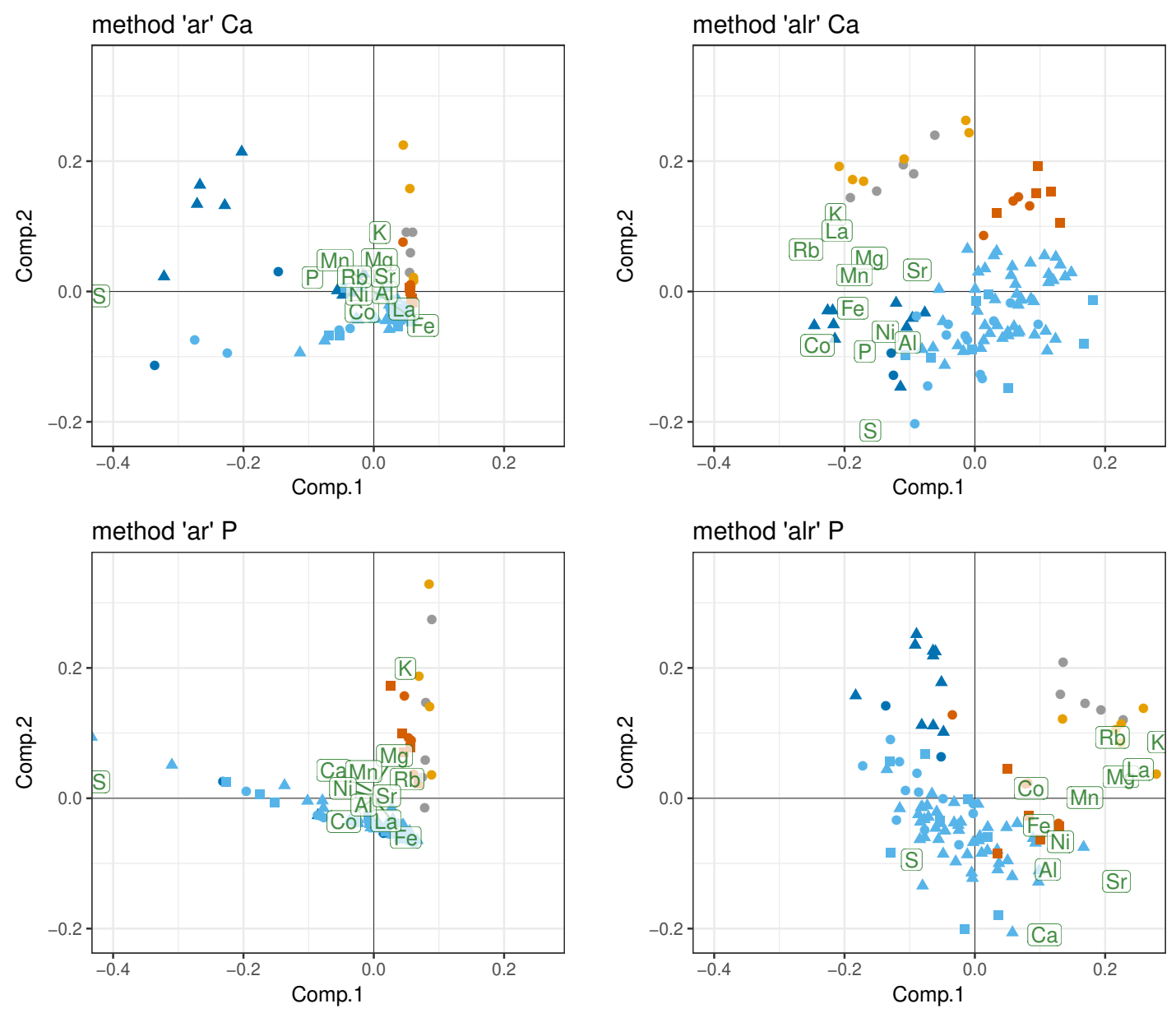

Region

Cultivation

- Darjeeling • Assam - Curitíba • Arcanjo, SP • Registro, SP

- conventional $\boldsymbol{\Delta}$ organic

under conversion

Figure C.1.: Biplots of the PCA of vratios between shoot tips of $C$. sinensis and corresponding soil analysis. Labels of elements do not overlap for visibility. 


\section{Geochemical Characterization of Tea and Soil}
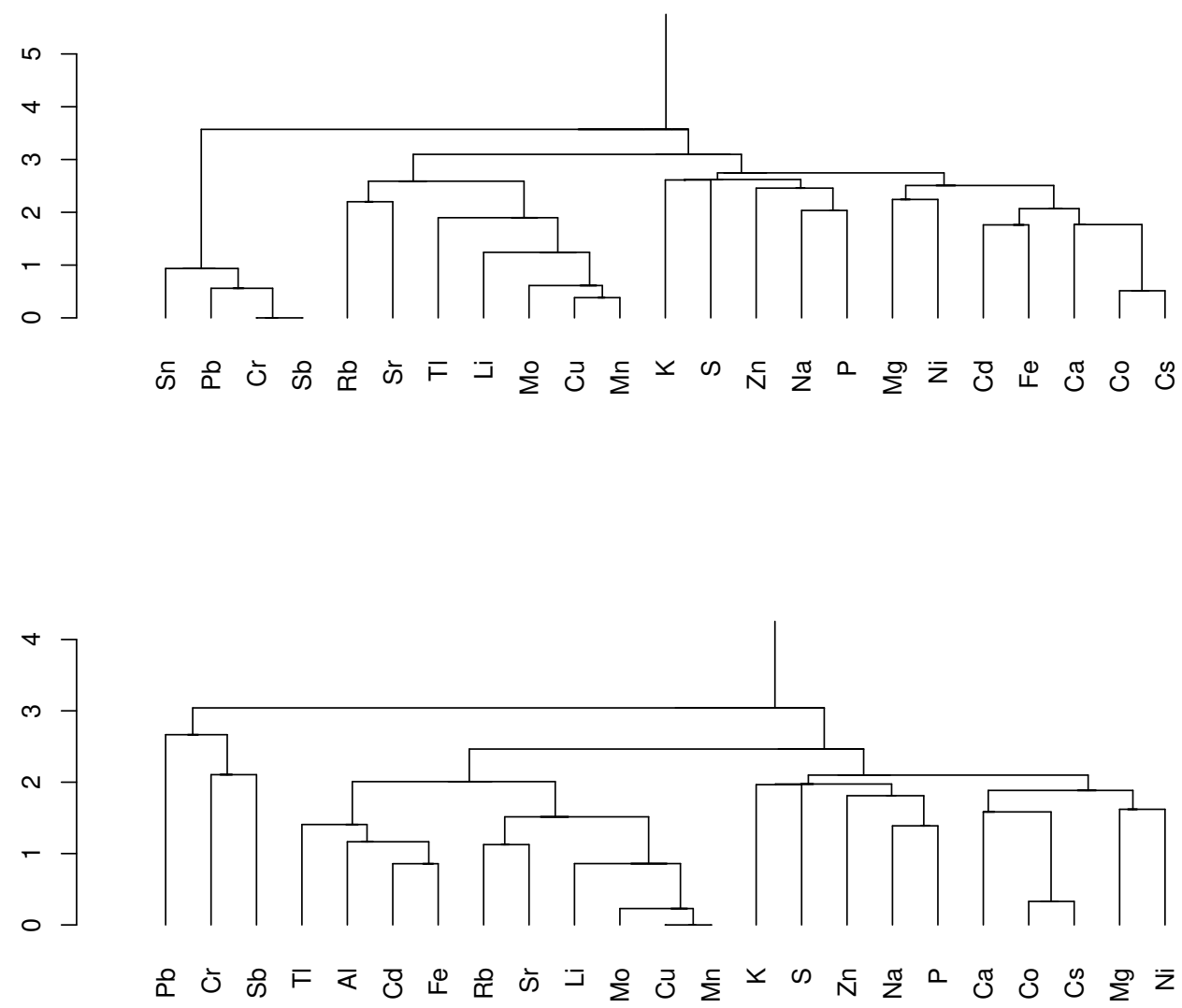

Figure D.1.: Graph of ilr-ANOVA (CoDa dendrogram) for shoot tip samples and black processed teas. For calculating the ilr-ANOVA an element subcomposition was used which excludes elements with high probability to be altered by correction for AP (Section 6.4) and by leaf development stage (Section 7.4.1), but including $\mathrm{Sn}$ in the upper graph, and including $\mathrm{Al}$ in the lower graph. 
Table D.1.: List of elements by parameter which affect log-ratios of element concentrations.

\begin{tabular}{lll}
\hline Parameter & Affected elements & Unaffected element sub-composition \\
\hline $\begin{array}{l}\text { Correction for adhering } \\
\text { particles }\end{array}$ & $\mathrm{Th}, \mathrm{Ta}, \mathrm{Hf}, \mathrm{Zr}, \mathrm{U}, \mathrm{Nb}, \mathrm{Ti}$ & $\mathrm{Al}, \mathrm{Ba}, \mathrm{Ca}, \mathrm{Cd}, \mathrm{Ce}, \mathrm{Co}, \mathrm{Cr}, \mathrm{Cs}, \mathrm{Cu}, \mathrm{Dy}, \mathrm{Er}$, \\
& & $\mathrm{Eu}, \mathrm{Fe}, \mathrm{Gd}, \mathrm{Ho}, \mathrm{K}, \mathrm{La}, \mathrm{Li}, \mathrm{Lu}, \mathrm{Mg}, \mathrm{Mn}, \mathrm{Mo}$, \\
& & $\mathrm{Na}, \mathrm{Nd}, \mathrm{Ni}, \mathrm{P}, \mathrm{Pb}, \mathrm{Pr}, \mathrm{Rb}, \mathrm{S}, \mathrm{Sb}, \mathrm{Sc}, \mathrm{Sm}$, \\
Leaf & $\mathrm{Sr}, \mathrm{Tb}, \mathrm{Tl}, \mathrm{Tm}, \mathrm{Y}, \mathrm{Yb}, \mathrm{Zn}$ \\
stage & $\mathrm{Al}, \mathrm{Sc}, \mathrm{Ba}, \mathrm{Y}, \mathrm{La}, \mathrm{Ce}, \mathrm{Pr}, \mathrm{Nd}, \mathrm{Sm}, \mathrm{Eu}, \mathrm{Gd}$, & $\mathrm{Ca}, \mathrm{Cd}, \mathrm{Co}, \mathrm{Cr}, \mathrm{Cs}, \mathrm{Cu}, \mathrm{Fe}, \mathrm{K}, \mathrm{Li}, \mathrm{Mg}, \mathrm{Mn}$, \\
Tea manufacturing & $\mathrm{Tb}, \mathrm{Dy}, \mathrm{Ho}, \mathrm{Er}, \mathrm{Tm}, \mathrm{Yb}, \mathrm{Lu}$ & $\mathrm{Mo}, \mathrm{Na}, \mathrm{Ni}, \mathrm{P}, \mathrm{Pb}, \mathrm{Rb}, \mathrm{S}, \mathrm{Sb}, \mathrm{Sr}, \mathrm{Tl}, \mathrm{Zn}$ \\
& $\mathrm{Sb}, \mathrm{Pb}, \mathrm{Cr}, \mathrm{Sc}$ & $\mathrm{Ca}, \mathrm{Cd}, \mathrm{Co}, \mathrm{Cs}, \mathrm{Cu}, \mathrm{Fe}, \mathrm{K}, \mathrm{Li}, \mathrm{Mg}, \mathrm{Mn}, \mathrm{Mo}$, \\
Soil management & $\mathrm{S}, \mathrm{P}, \mathrm{Ca}, \mathrm{K})$ & $\mathrm{Na}, \mathrm{Ni}, \mathrm{P}, \mathrm{Rb}, \mathrm{S}, \mathrm{Sr}, \mathrm{TI}, \mathrm{Zn}$ \\
& & $\mathrm{Cd}, \mathrm{Co}, \mathrm{Cs}, \mathrm{Cu}, \mathrm{Fe}, \mathrm{K}), \mathrm{Li}, \mathrm{Mg}, \mathrm{Mn}, \mathrm{Mo}$, \\
Intra-location variability & $\mathrm{Al}, \mathrm{Ba}, \mathrm{Cd}, \mathrm{Cr}, \mathrm{Cu}, \mathrm{Fe}, \mathrm{Li}, \mathrm{Mg}, \mathrm{Mo}, \mathrm{P}, \mathrm{S}, \mathrm{Sc}, \mathrm{Ca}, \mathrm{Co}, \mathrm{Cs}, \mathrm{K}, \mathrm{La}, \mathrm{Mn}, \mathrm{Na}, \mathrm{Ni}, \mathrm{Pb}, \mathrm{Sb}, \mathrm{Tl}$, \\
& $\mathrm{Sr}, \mathrm{Zn}$ & $\mathrm{Y}$ \\
\hline
\end{tabular}


Table D.2.: List of elements by nutrient status for $C$. sinensis.

\begin{tabular}{ll}
\hline & elements \\
\hline macro-nutrients & $\mathrm{K}, \mathrm{Ca}, \mathrm{P}, \mathrm{Mg}, \mathrm{S}$ \\
micro-nutrients & $\mathrm{Cu}, \mathrm{Fe}, \mathrm{Mn}, \mathrm{Mo}, \mathrm{Ni}, \mathrm{Zn}$ \\
beneficial elements & $\mathrm{Al}, \mathrm{Co}, \mathrm{La}, \mathrm{Y}, \mathrm{Na}, \mathrm{Ce}, \mathrm{Pr}, \mathrm{Nd}, \mathrm{Sm}, \mathrm{Eu}, \mathrm{Gd}, \mathrm{Tb}, \mathrm{Dy}, \mathrm{Ho}, \mathrm{Er}$, \\
& $\mathrm{Tm}, \mathrm{Yb}, \mathrm{Lu}$ \\
non-nutrients & $\mathrm{Ba}, \mathrm{Cd}, \mathrm{Cr}, \mathrm{Cs}, \mathrm{Li}, \mathrm{Pb}, \mathrm{Rb}, \mathrm{Sb}, \mathrm{Sc}, \mathrm{Sr}, \mathrm{Tl}$ \\
\hline
\end{tabular}


D. Geochemical Characterization of Tea and Soil

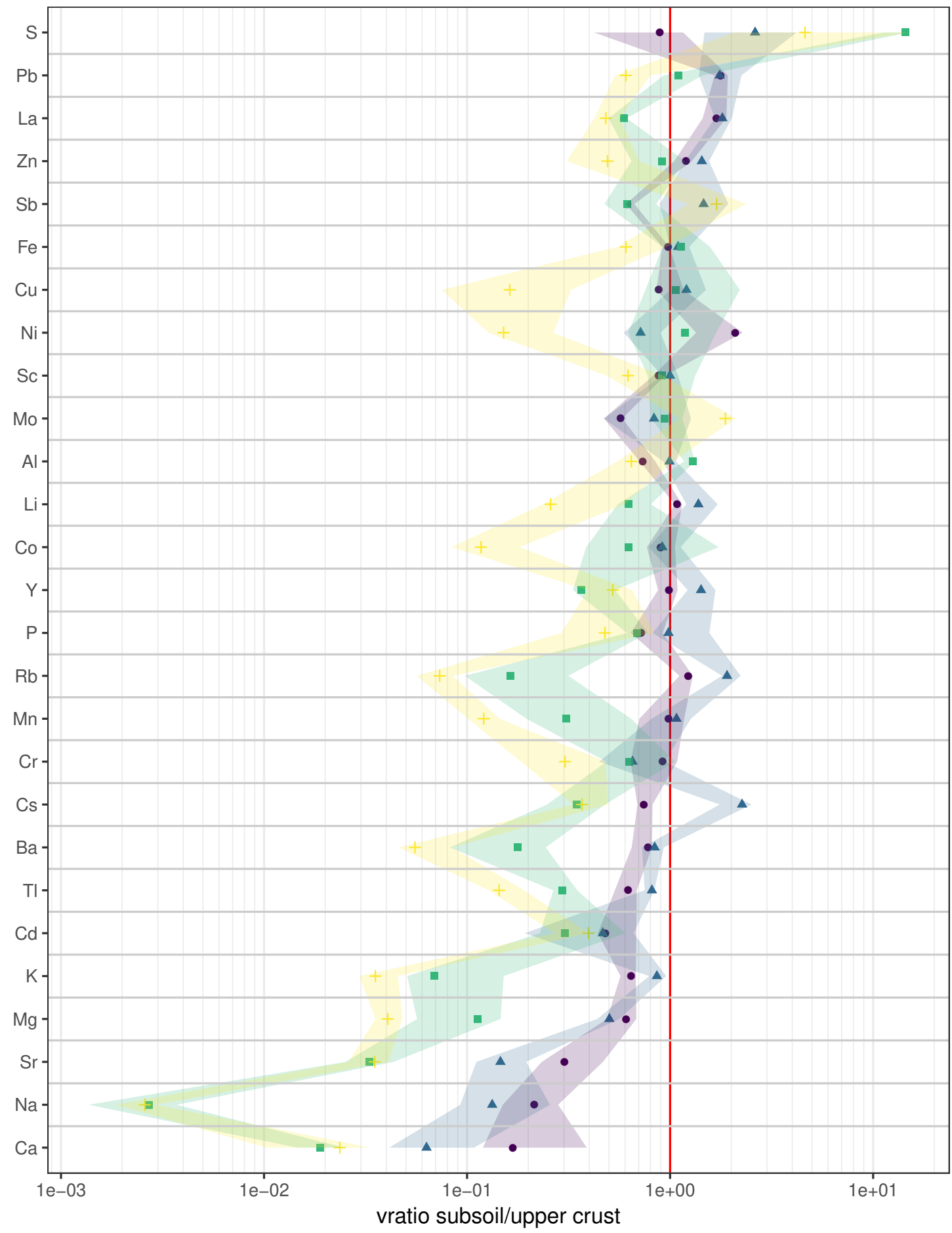

Region • Assam \ Darjeeling | Paraná + São Paulo

Figure D.2.: Vratios of subsoil/upper crust, grouped by tea growing regions. Elements are ordered by median of vratio. 


\section{E. Provenance Analysis}


Table E.1.: List of elements for suitability for provenance analysis: Each element has for each influencing paramter a weight. If the parameter influences the element strongly the weight is set to zero. If the parameter has no influence on the element concentration the weight is high For each the parameter the weight are multiplite with a factor, depending on the the impact these factors are 1,2 , or 3 . The sum of all weights per element is an indicator how robust the respective element is pro provenance analysis, the higher the value, the better.

\begin{tabular}{|c|c|c|c|c|c|c|c|c|c|}
\hline & $\begin{array}{l}\text { intra-garden } \\
\text { variability }\end{array}$ & $\begin{array}{l}\text { sink/source } \\
\text { pairwise } \\
\text { log-ratio }\end{array}$ & $\begin{array}{l}\text { sink/source } \\
\text { pairwise } \\
\text { log-ratio sum } \\
\text { p-value }\end{array}$ & $\begin{array}{l}\text { sink/source } \\
\text { distribution }\end{array}$ & manufacturing & $\begin{array}{c}\text { soil } \\
\text { managment }\end{array}$ & $\begin{array}{c}\text { sink/subsoil } \\
\text { pairwise } \\
\text { log-ratio }\end{array}$ & $\begin{array}{l}\text { sink/topsoil } \\
\text { pairwise } \\
\text { log-ratio }\end{array}$ & sum \\
\hline $\mathrm{Al}$ & 0 & 0 & 0 & 4 & 6 & 6 & 3 & 1 & 20 \\
\hline $\mathrm{Ba}$ & 1 & 0 & 0 & 0 & 6 & 6 & 0 & 1 & 14 \\
\hline $\mathrm{Ca}$ & 2 & 4 & 1 & 4 & 6 & 0 & 0 & 1 & 18 \\
\hline $\mathrm{Cd}$ & 0 & 2 & 0 & 0 & 6 & 6 & 6 & 2 & 22 \\
\hline Co & 2 & 4 & 1 & 0 & 6 & 6 & 6 & 2 & 27 \\
\hline $\mathrm{Cr}$ & 1 & 4 & 1 & 0 & 0 & 6 & 6 & 2 & 20 \\
\hline Cs & 2 & 4 & 1 & 4 & 6 & 6 & 6 & 1 & 30 \\
\hline $\mathrm{Cu}$ & 0 & 4 & 1 & 4 & 3 & 6 & 6 & 2 & 26 \\
\hline $\mathrm{Fe}$ & 0 & 0 & 0 & 0 & 6 & 6 & 6 & 1 & 19 \\
\hline K & 2 & 4 & 1 & 4 & 6 & 3 & 0 & 2 & 22 \\
\hline La & 2 & 4 & 0 & 0 & 6 & 6 & 6 & 1 & 25 \\
\hline $\mathrm{Li}$ & 1 & 4 & 1 & 0 & 6 & 6 & 0 & 1 & 19 \\
\hline $\mathrm{Mg}$ & 0 & 4 & 0 & 0 & 6 & 6 & 6 & 2 & 24 \\
\hline $\mathrm{Mn}$ & 2 & 4 & 1 & 4 & 6 & 6 & 6 & 2 & 31 \\
\hline Mo & 1 & 2 & 1 & 0 & 6 & 6 & 3 & 2 & 21 \\
\hline $\mathrm{Na}$ & 2 & 2 & 0 & 0 & 6 & 6 & 0 & 0 & 16 \\
\hline $\mathrm{Ni}$ & 2 & 4 & 1 & 0 & 6 & 6 & 0 & 0 & 19 \\
\hline$P$ & 0 & 4 & 1 & 4 & 6 & 0 & 0 & 2 & 17 \\
\hline $\mathrm{Pb}$ & 2 & 4 & 1 & 0 & 0 & 6 & 6 & 2 & 21 \\
\hline $\mathrm{Rb}$ & 1 & 4 & 1 & 4 & 6 & 6 & 6 & 2 & 30 \\
\hline S & 0 & 2 & 0 & 4 & 6 & 0 & 0 & 2 & 14 \\
\hline $\mathrm{Sb}$ & 2 & 4 & 1 & 4 & 0 & 6 & 6 & 2 & 25 \\
\hline Sc & 0 & 0 & 0 & 0 & 6 & 6 & 0 & 0 & 12 \\
\hline $\mathrm{Sr}$ & 0 & 4 & 1 & 4 & 6 & 6 & 6 & 2 & 29 \\
\hline TI & 2 & 4 & 1 & 0 & 6 & 6 & 3 & 1 & 23 \\
\hline Y & 2 & 4 & 0 & 0 & 6 & 6 & 0 & 1 & 19 \\
\hline $\mathrm{Zn}$ & 0 & 4 & 1 & 0 & 6 & 6 & 6 & 2 & 25 \\
\hline
\end{tabular}




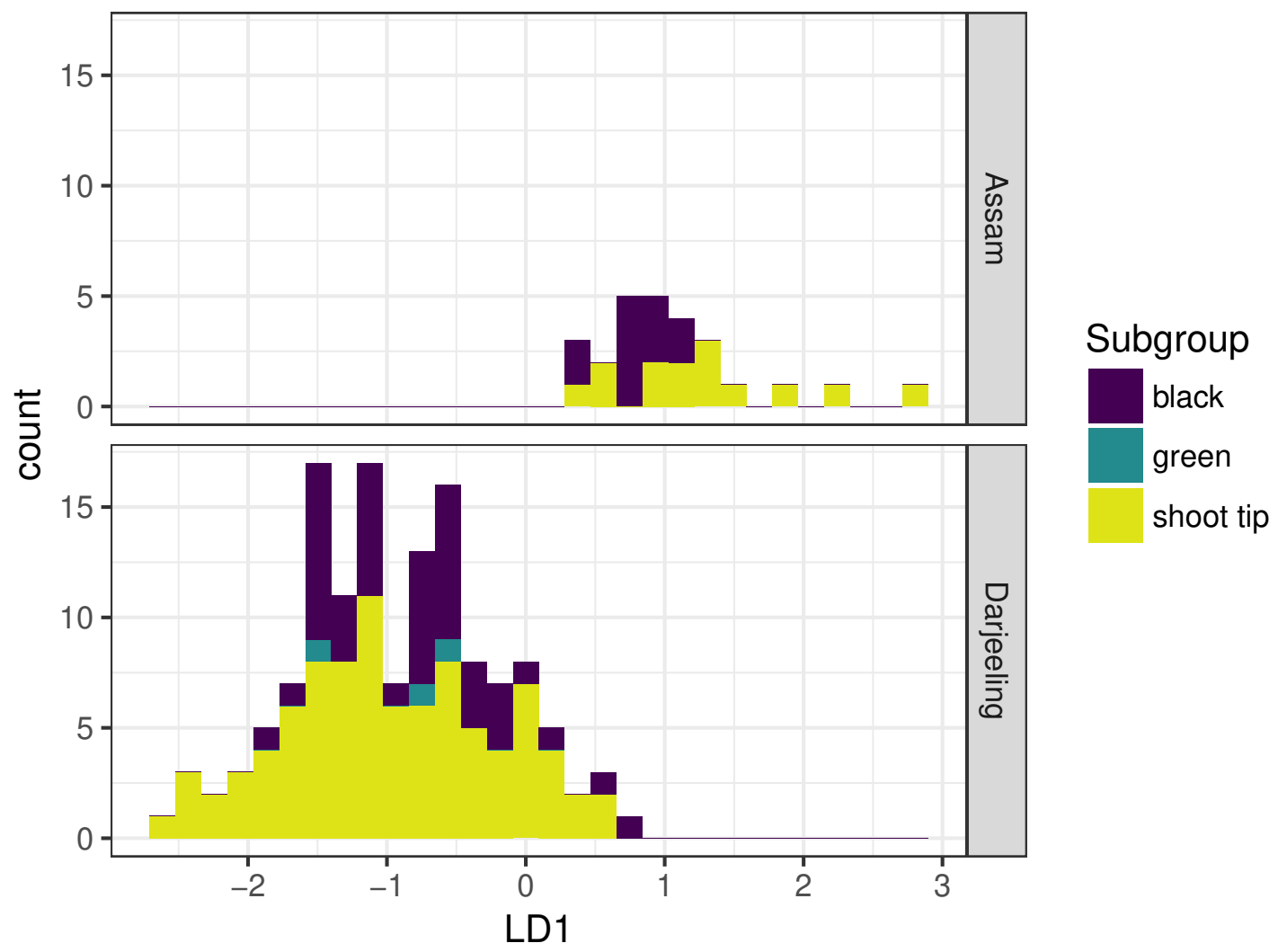

Figure E.1.: Histogram of the first score of weighted linear discriminant function for shoot tip and processed tea samples from Darjeeling and Assam. The subcomposition includes the elements Al, Mg and $\mathrm{Tl}$, and excludes Rb. Samples are colored by Subgroup. Total number of samples is $N=162$ (Darjeeling $N=136$, Assam $N=26$ ). Clr-scalings for LD1 and misclassifications are listed in Table E.2.

Table E.2.: Clr-scalings of weighted LDA (upper table) and misclassifications (lower table) for discrimination of tea from Assam and Darjeeling. The element compilation includes $\mathrm{Al}, \mathrm{Tl}$ and $\mathrm{Mg}$, and excludes Rb. Table corresponds to Figure E.1. The rows of the misclassification table give the number of samples predicted into respective group.

\begin{tabular}{|c|c|c|c|c|c|c|c|c|}
\hline & Co & Cs & $\mathrm{Mn}$ & $\mathrm{Sr}$ & $\mathrm{Zn}$ & $\mathrm{Mg}$ & $\mathrm{Al}$ & $\mathrm{TI}$ \\
\hline \multirow[t]{4}{*}{1} & -0.26 & 0.02 & -0.10 & 0.84 & 0.86 & -1.78 & 1.07 & -0.65 \\
\hline & & & & Assar & $\overline{D z}$ & jeeling & & \\
\hline & & & Assam & 1 & 4 & 12 & & \\
\hline & & & eeling & & 0 & 136 & & \\
\hline
\end{tabular}




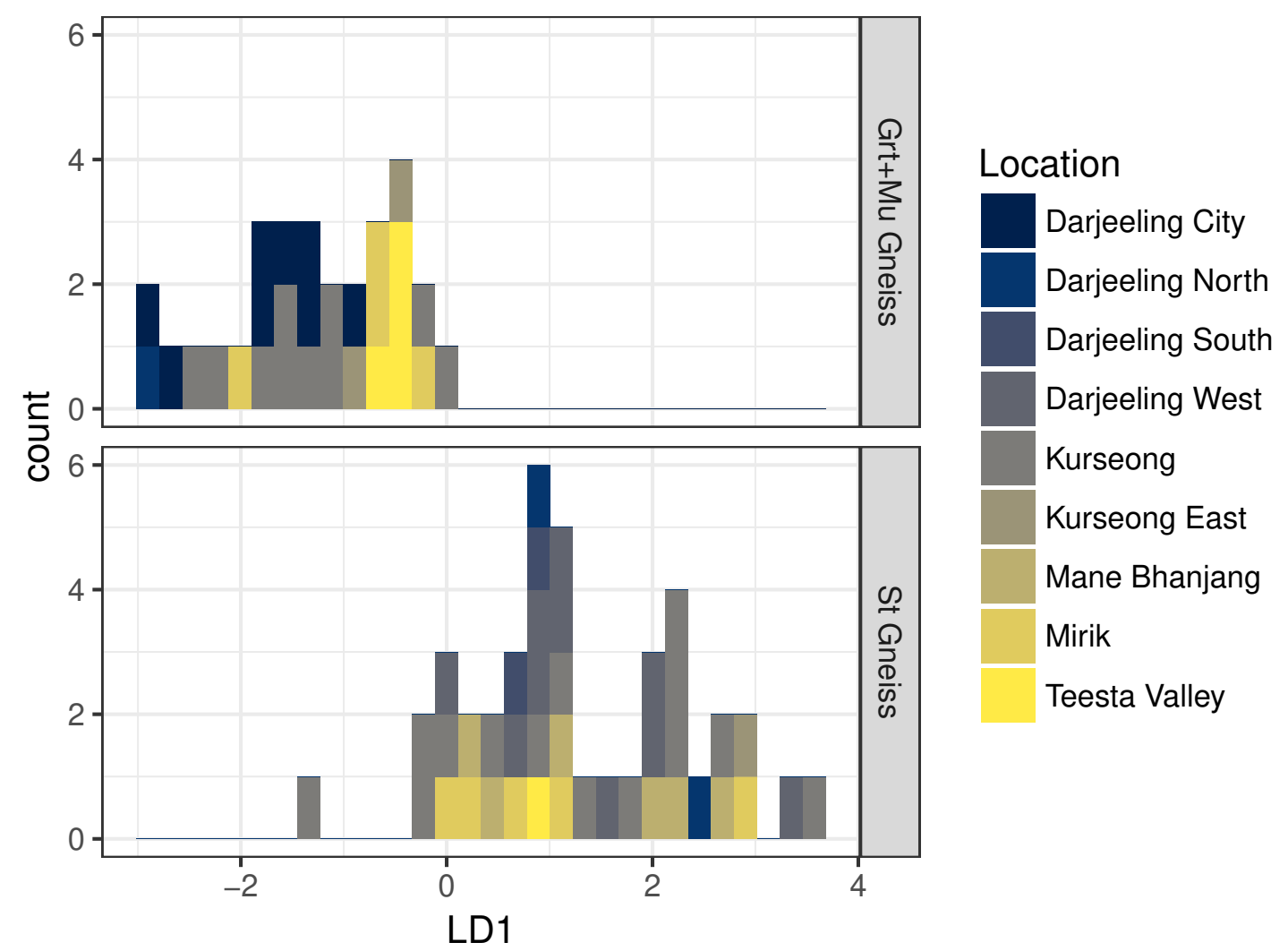

Figure E.2.: Histogram of shoot tip and processed tea samples displaying the first score of linear discriminant function separating two metamorphic zones of Darjeeling. The element compilation includes $\mathrm{Ca}, \mathrm{K}$ and $\mathrm{Y}$, and excludes $\mathrm{Co}$, $\mathrm{Cs}$ and $\mathrm{Rb}$. Samples are colored by location in Darjeeling District. Only samples with high certainty of source rock are included (Table 9.6). Total number of samples is $N=71$ (Grt+Mu Gneiss N=29, St Gneiss N=41). For abbreviations see caption of Table 9.6. Clr-scalings for LD1 and misclassifications are listed in Table E.3.

Table E.3.: Clr-scalings of LDA (upper table) and misclassifications (lower table) for discrimination of tea from to metamorphic zones in Darjeeling. The element compilation includes $\mathrm{Ca}$, $\mathrm{K}$ and $\mathrm{Y}$, and excludes $\mathrm{Co}$ and $\mathrm{Cs}$. Table corresponds to Figure E.2). The rows of the misclassification table give the number of samples predicted into respective group.

\begin{tabular}{|c|c|c|c|c|c|c|}
\hline & $\mathrm{Mn}$ & $\mathrm{Sr}$ & $\mathrm{Zn}$ & $\mathrm{Ca}$ & $\mathrm{K}$ & $\bar{Y}$ \\
\hline LD1 & -2.45 & -2.89 & 0.85 & 5.82 & -2.08 & 0.75 \\
\hline & & \multicolumn{3}{|c|}{ Grt+Mu Gneiss } & \multicolumn{2}{|c|}{ St Gneiss } \\
\hline \multicolumn{3}{|c|}{ Grt+Mu Gneiss } & & 28 & & 1 \\
\hline & St Gn & & & 3 & & 38 \\
\hline
\end{tabular}




\section{Curriculum Vitae}

Name Solveig Ingrid Pospiech

Birth 8. January 1986 Heidelberg, Germany

\section{School}

$1996-2004 \quad$ St.Raphael-Gymnasium, Heidelberg, Germany

$2000-2004 \quad$ Participant of Heidelberger Life-Science Lab

Summer 2003 Participation in 1st International Summer Science Camp of XLab, Göttingen, Germany

Summer $2004 \quad$ Participation in 36th ISSI (Dr. Bessie F. Lawrence International Summer Science Institute) of the Weizmann Institute, Israel

July $2004 \quad$ Participation in the 55. Nobel Laureate Meeting in Lindau

18. Nobel Laureate Meeting of Physics 


\section{Study - Diploma}

10/2004-09/2007 Study of Geosciences with specialization in Geophysics at Friedrich-Schiller-University Jena, Germany

10/2007-07/2008 Study at Universidad Complutense Madrid (UCM), Spain, Sección Departamental de Astronomía y Geodesia

08/2008-09/2008 Internship at UAF/AVO (University of Alaska Fairbanks/ Alaska Volcanic Observatory), Supervisor: Dr. Jessica Larsen

10/2008 - 05/2012 Study of Geosciences with specialization in Geochemistry at Georg-August-University Göttingen, Germany

08/2009-10/2010 Geological Mapping Project in SE-Sweden: Geologische Kartierung metamorpher und granitoider Gesteine der Transskandinavischen Magmatischen Zone, NE' Vätervik (SE-Schweden), Grade 2,0

02/2011-04/2012 Diploma Thesis Geochemical investigation on the tea plant Camellia sinensis: Implications from element soil-plant transfer factors for provenance studies - the case of Darjeeling Tea from NE-India, Grade 1,0

\section{Study - PhD}

12/2012 - 07/2018 PhD Study at Georg-August-University Göttingen, Germany 12/2012 - 03/2016 PhD - Scholarship of the Stiftung der Deutschen Wirtschaft 